Universidade de São Paulo

Instituto de Astronomia, Geofísica e Ciências Atmosféricas

Departamento de Geofísica

Nelson Ricardo Coelho Flores Zuniga

NONHYPERBOLIC MULTIPARAMETRIC TRAVEL-TIME APPROXIMATION FOR CONVERTED-WAVE AND OBN DATA

São Paulo

2021 

Nelson Ricardo Coelho Flores Zuniga

\title{
NONHYPERBOLIC MULTIPARAMETRIC TRAVEL-TIME APPROXIMATION FOR CONVERTED-WAVE AND OBN DATA
}

\author{
Tese apresentada ao Instituto de \\ Astronomia, Geofísica e Ciências \\ Atmosféricas da Universidade de São Paulo \\ como parte dos requisitos para obtenção do \\ título de Doutor em Ciências na área de \\ Geofísica.
}

Área de Concentração: Geofísica

Orientador: Prof. Dr. Viacheslav Ivanovich Priimenko

Co-orientador: Prof. Dr. Fernando Brenha

Ribeiro

"Versão Corrigida. O original encontra-se disponível na Unidade."

São Paulo 



\section{ACKNOWLEDGEMENTS}

At first, I would like to thank my advisor, Prof. Dr. Viacheslav Ivanovich Priimenko, my co-advisor, Prof. Dr. Fernando Brenha Ribeiro, and my supervisor during the period at the Delft University of Technology, Prof. Dr. Deyan Draganov.

I would like to thank all the members of the teaching staff of my $\mathrm{PhD}$, my Master's and my Bachelor's.

I would like to thank the teachers who were part of my high school and my basic education.

I would like to thank the Geophysics department of the Instituto de Astronomia Geofísica e Ciências Atmosféricas of the Universidade de São Paulo, and also thank the Faculty of Civil Engineering and Geosciences of the Delft University of Technology for the opportunity of obtaining this degree.

This work was kindly supported by CAPES and CNPq.

I would like to thank my colleagues for being together in this journey.

I would like to thank my friends, my parents and my sisters for always supporting me, encouraging me and believing I could become even better.

I would like to thank my friend Filipe Lima Marinho for the thorough grammar revision. 
"If I can see much farther, it's because I am standing on the shoulders of giants"

-Isaac Newton 


\section{ABSTRACT}

\section{ZUNIGA, N. R. C. F. Nonhyperbolic multiparametric travel-time approximation}

for converted-wave and OBN data. 2021. 277 p. Tese (Doutorado em Ciências) — Instituto de Astronomia, Geofísica e Ciências Atmosféricas, Universidade de São Paulo, São Paulo, 2021 .

To obtain a more accurate stratigraphic model, it is important to perform a reliable velocity analysis by fitting the calculated travel-time curve to the recorded one. The main challenge to perform this step in the offshore seismic survey is the fact there are many factors which present characteristics that generate nonhyperbolicity in travel-time events. For this reason, it is necessary to use an approximation which is able to control the effects of the nonhyperbolicity so that performing an efficient velocity analysis is possible.

The main proposition of this thesis was to develop a generalization of a nonhyperbolic multiparametric travel-time approximation, which can control the nonhyperbolicity associated to heterogeneity of layered media and long offsets, wave conversion, and difference of datum between source and receiver. This work proposes a numerical study which aims to analyse the complexity of the objective function, the quality and efficiency of the travel-time curve fitting with different approximations, using different optimization algorithms and using the L1- and L2-norm. Three of the five models studied in this thesis were elaborated from well logs data of the Santos Basin and showed different characteristics making the proposed analysis more complex. The other two models were adapted to test some limits of the nonhyperbolic approximation (including the proposed one). Another important proposition was the development of an automated picking technique to obtain the reflection events related to the interface between the bottom of the salt and the top of the reservoir (target reflection). That technique was also part of a set of tests to obtain information of phase variation.

It was possible to determine how complex each approximation is with the set of information obtained by the residual function maps analysis. The quality of the fitting and the efficiency of each approximation were also analysed. The optimization algorithm which showed the best results was determined. The difference between the use of L1-norm and L2- 
norm was also studied and it was determined which one is better to work for each kind of inverse problem. With all these analyses, it was possible to identify which approximation along with which optimization algorithm present the best results for each reflection event of each model after the inversion. The automated picking technique proved to be able to obtain the target reflection seismic events and information of phase variation.

Keywords: nonhyperbolic, multicomponent, converted wave, OBN, phase shift. 


\title{
RESUMO
}

\author{
ZUNIGA, N. R. C. F. Aproximação não-hiperbólica multiparamétrica de tempos \\ de trânsito para onda convertida e dado OBN. 2021. 277 p. Tese (Doutorado em Ciências) \\ — Instituto de Astronomia, Geofísica e Ciências Atmosféricas, Universidade de São Paulo, \\ São Paulo, 2021.
}

Para obter um modelo estratigráfico mais preciso, é importante realizar uma confiável análise de velocidades, ajustando a curva de tempos de trânsito calculada com a curva observada. O principal desafio para realizar esta etapa em um levantamento sísmico marítimo é o fato de haver diversos fatores que apresentam características que geram nãohiperbolicidade nos eventos de tempos de trânsito. Por esta razão, é necessário usar uma aproximação que seja capaz de controlar os efeitos da não-hiperbolicidade para que seja possível realizar uma eficiente análise de velocidades.

A principal proposta dessa tese foi desenvolver uma generalização de uma aproximação não-hiperbólica multiparamétrica de tempos de trânsito, que possa controlar a não-hiperbolicidade associada à heterogeneidade em meios estratificados e longos afastamentos, conversão de onda e diferença de profundidade entre fonte e receptor. Um estudo numérico foi proposto visando analisar a complexidade das funções objetivo, a qualidade e eficiência do ajuste das curvas de tempos de trânsito com diferentes aproximações, utilizando diferentes algoritmos de otimização e utilizando normas L1 e L2. Três dos cinco modelos estudados nesta tese foram elaborados de perfis de poços da Bacia de Santos e mostraram diferentes características, fazendo a análise proposta mais complexa. Os outros dois modelos foram adaptados para testar alguns limites das aproximações nãohiperbólicas (incluindo a aqui proposta). Outra proposta importante foi o desenvolvimento de uma técnica de seleção automática para obter as reflexões relacionadas às interfaces entre a base do sal e o topo do reservatório (reflexão alvo). Esta técnica foi também parte de um conjunto de testes para obter informações de variação de fase.

Foi possível determinar o quão complexa cada aproximação é com o conjunto de informações obtido pela análise de mapas residuais de função objetivo. A qualidade de ajuste 
e a eficiência de cada aproximação também foram analisadas. Foram determinados os algoritmos de otimização que mostraram os melhores resultados. Foi estudada a diferença entre as normas L1 e L2, e foi determinada a melhor para se trabalhar com cada tipo de problema inverso. Com todas essas análises, foi possível identificar qual aproximação junto de qual algoritmo de otimização apresenta os melhores resultados para cada evento de reflexão de cada modelo depois da inversão. A técnica de seleção automática provou ser apta para obter os eventos sísmicos de reflexão almejados e informações de variação de fase.

Palavras chave: não-hiperbólico, multicomponente, onda convertida, OBN, mudança de fase. 


\section{LIST OF FIGURES}

Figure 1: Elastic deformation and particle motion associated with the passage of P-waves. (Kearey, Brooks and Hill, 2002).

Figure 2: Elastic deformation and particle motion associated with the passage of S-waves. (Kearey, Brooks and Hill, 2002).

Figure 3: Representation of the recorded components in a Cartesian coordinate system (XYZ). The red star represents the sources and the blue triangle represents the receivers. (Zuniga, 2017)

Figure 4: (a) Wave reflection at the common mid-point (CMP); (b) PSv wave reflection at the common conversion point (CCP). (Zuniga, 2017).

Figure 5: PP and PS wave reflection events with the use of OBN technology. (Zuniga, 2017).

Figure 6: Schematization demonstrating how the RMS velocity differs from the set of interval velocities as the offset is increased. Solid lines represent the interval velocities and the dotted lines represent the RMS velocity. (Zuniga, 2017).

Figure 7: Velocity profiles of $\mathrm{P}$ wave, $\mathrm{S}$ wave, and $\mathrm{Vp} / \mathrm{Vs}$ ratio related to the depths of the Model 1 85

Figure 8: Ray tracing schematization of the PP reflection event from Model 1. 86

Figure 9: Ray tracing schematization of the PS reflection event from Model 1. 86

Figure 10: Travel-time curve of the PP reflection event from Model 1. 87

Figure 11: Travel-time curve of the PS reflection event from Model 1. 87

Figure 12: Velocity profiles of $\mathrm{P}$ wave, $\mathrm{S}$ wave, and $\mathrm{Vp} / \mathrm{Vs}$ ratio related to the depths of the Model 2 88

Figure 13: Ray tracing schematization of the PP reflection event from Model 2. 89

Figure 14: Ray tracing schematization of the PS reflection event from Model 2. 89

Figure 15: Travel-time curve of the PP reflection event from Model 2. 90

Figure 16: Travel-time curve of the PS reflection event from Model 2. 90 
Figure 17: Velocity profiles of $\mathrm{P}$ wave, $\mathrm{S}$ wave, and $\mathrm{Vp} / \mathrm{Vs}$ ratio related to the depths of the Model 3

Figure 18: Ray tracing schematization of the PP reflection event from Model 3. 92

Figure 19: Ray tracing schematization of the PS reflection event from Model 3. 92

Figure 20: Travel-time curve of the PP reflection event from Model 3. 93

Figure 21: Travel-time curve of the PS reflection event from Model 3. 93

Figure 22: Velocity profiles of $\mathrm{P}$ wave, $\mathrm{S}$ wave, and $\mathrm{Vp} / \mathrm{Vs}$ ratio related to the depths of the Model 4 94

Figure 23: Ray tracing schematization of the PP reflection event from Model 4. 95

Figure 24: Ray tracing schematization of the PS reflection event from Model 4. 95

Figure 25: Travel-time curve of the PP reflection event from Model 4 96

Figure 26: Travel-time curve of the PS reflection event from Model 4. 96

Figure 27: Velocity profiles of $\mathrm{P}$ wave, $\mathrm{S}$ wave, and $\mathrm{Vp} / \mathrm{Vs}$ ratio related to the depths of the Model 5 97

Figure 28: Ray tracing schematization of the PP reflection event from Model 5. 98

Figure 29: Ray tracing schematization of the PS reflection event from Model 5. 98

Figure 30: Travel-time curve of the PP reflection event from Model 5. 99

Figure 31: Travel-time curve of the PS reflection event from Model 5 99

Figure 32: Two-dimensional example showing the sparseness of a solution when using the L1-norm and the L2-norm. 105

Figure 33: The residual function map which demonstrates the complexity of the Malovichko (1978) approximation of the PP reflection event with L2-norm. Red dispersions represent the global minimum region, and the blue dispersions, the local minimum regions.

Figure 34: The residual function map which demonstrates the complexity of the Malovichko (1978) approximation of the PS reflection event with L2-norm. Red dispersions represent the global minimum region, and the blue dispersions, the local minimum regions. 109 
Figure 35: The residual function map which demonstrates the complexity of the Malovichko (1978) approximation of the PP reflection event with L1-norm. Red dispersions represent the global minimum region, and the blue dispersions, the local minimum regions. 110

Figure 36: The residual function map which demonstrates the complexity of the Malovichko (1978) approximation of the PS reflection event with L1-norm. Red dispersions represent the global minimum region, and the blue dispersions, the local minimum regions. 110

Figure 37: The residual function map which demonstrates the complexity of the Alkhalifah and Tsvankin (1995) approximation of the PP reflection event with L2-norm. Red dispersions represent the global minimum region, and the blue dispersions, the local minimum regions.

Figure 38: The residual function map which demonstrates the complexity of the Alkhalifah and Tsvankin (1995) approximation of the PS reflection event with L2-norm. Red dispersions represent the global minimum region, and the blue dispersions, the local minimum regions.

Figure 39: The residual function map which demonstrates the complexity of the Alkhalifah and Tsvankin (1995) approximation of the PP reflection event with L1-norm. Red dispersions represent the global minimum region, and the blue dispersions, the local minimum regions.

Figure 40: The residual function map which demonstrates the complexity of the Alkhalifah and Tsvankin (1995) approximation of the PS reflection event with L1-norm. Red dispersions represent the global minimum region, and the blue dispersions, the local minimum regions.

Figure 41: The residual function map which demonstrates the complexity of the Ursin and Stovas (2006) approximation of the PP reflection event with L2-norm. Red dispersions represent the global minimum region, and the blue dispersions, the local minimum regions.

Figure 42: The residual function map which demonstrates the complexity of the Ursin and Stovas (2006) approximation of the PS reflection event with L2-norm. Red dispersions represent the global minimum region, and the blue dispersions, the local minimum regions. 
Figure 43: The residual function map which demonstrates the complexity of the Ursin and Stovas (2006) approximation of the PP reflection event with L1-norm. Red dispersions represent the global minimum region, and the blue dispersions, the local minimum regions.

Figure 44: The residual function map which demonstrates the complexity of the Ursin and Stovas (2006) approximation of the PS reflection event with L1-norm. Red dispersions represent the global minimum region, and the blue dispersions, the local minimum regions.

Figure 45: The residual function map which demonstrates the complexity of the Blias (2009) approximation of the PP reflection event with L2-norm. Red dispersions represent the global minimum region, and the blue dispersions, the local minimum regions

Figure 46: The residual function map which demonstrates the complexity of the Blias (2009) approximation of the PS reflection event with L2-norm. Red dispersions represent the global minimum region, and the blue dispersions, the local minimum regions 115

Figure 47: The residual function map which demonstrates the complexity of the Blias (2009) approximation of the PP reflection event with L1-norm. Red dispersions represent the global minimum region, and the blue dispersions, the local minimum regions

Figure 48: The residual function map which demonstrates the complexity of the Blias (2009) approximation of the PS reflection event with L1-norm. Red dispersions represent the global minimum region, and the blue dispersions, the local minimum regions

Figure 49: The residual function map which demonstrates the complexity of the Muir and Dellinger (1985) approximation of the PP reflection event with L2-norm. Red dispersions represent the global minimum region, and the blue dispersions, the local minimum regions.

Figure 50: The residual function map which demonstrates the complexity of the Muir and Dellinger (1985) approximation of the PS reflection event with L2-norm. Red dispersions represent the global minimum region, and the blue dispersions, the local minimum regions.

Figure 51: The residual function map which demonstrates the complexity of the Muir and Dellinger (1985) approximation of the PP reflection event with L1-norm. Red dispersions 
represent the global minimum region, and the blue dispersions, the local minimum regions.

Figure 52: The residual function map which demonstrates the complexity of the Muir and Dellinger (1985) approximation of the PS reflection event with L1-norm. Red dispersions represent the global minimum region, and the blue dispersions, the local minimum regions.

Figure 53: The residual function map which demonstrates the complexity of the Li and Yuan (2001) approximation of the PP reflection event with L2-norm. Red dispersions represent the global minimum region, and the blue dispersions, the local minimum regions

Figure 54: The residual function map which demonstrates the complexity of the Li and Yuan (2001) approximation of the PS reflection event with L2-norm. Red dispersions represent the global minimum region, and the blue dispersions, the local minimum regions

Figure 55: The residual function map which demonstrates the complexity of the Li and Yuan (2001) approximation of the PP reflection event with L1-norm. Red dispersions represent the global minimum region, and the blue dispersions, the local minimum regions

Figure 56: The residual function map which demonstrates the complexity of the Li and Yuan (2001) approximation of the PS reflection event with L1-norm. Red dispersions represent the global minimum region, and the blue dispersions, the local minimum regions

Figure 57: The residual function map which demonstrates the complexity of the approximation proposed in this work of the PP reflection event with L2-norm. Red dispersions represent the global minimum region, and the blue dispersions, the local minimum regions. 121

Figure 58: The residual function map which demonstrates the complexity of the approximation proposed in this work of the PS reflection event with L2-norm. Red dispersions represent the global minimum region, and the blue dispersions, the local minimum regions.

Figure 59: The residual function map which demonstrates the complexity of the approximation proposed in this work of the PP reflection event with L1-norm. Red dispersions represent the global minimum region, and the blue dispersions, the local minimum regions. 122 
Figure 60: The residual function map which demonstrates the complexity of the approximation proposed in this work of the PS reflection event with L1-norm. Red dispersions represent the global minimum region, and the blue dispersions, the local minimum regions.

Figure 61: The residual function map which demonstrates the complexity of the Malovichko (1978) approximation of the PP reflection event with L2-norm. Red dispersions represent the global minimum region, and the blue dispersions, the local minimum regions.

Figure 62: The residual function map which demonstrates the complexity of the Malovichko (1978) approximation of the PS reflection event with L2-norm. Red dispersions represent the global minimum region, and the blue dispersions, the local minimum regions

Figure 63: The residual function map which demonstrates the complexity of the Malovichko (1978) approximation of the PP reflection event with L1-norm. Red dispersions represent the global minimum region, and the blue dispersions, the local minimum regions

Figure 64: The residual function map which demonstrates the complexity of the Malovichko (1978) approximation of the PS reflection event with L1-norm. Red dispersions represent the global minimum region, and the blue dispersions, the local minimum regions. 125

Figure 65: The residual function map which demonstrates the complexity of the Alkhalifah and Tsvankin (1995) approximation of the PP reflection event with L2-norm. Red dispersions represent the global minimum region, and the blue dispersions, the local minimum regions.

Figure 66: The residual function map which demonstrates the complexity of the Alkhalifah and Tsvankin (1995) approximation of the PS reflection event with L2-norm. Red dispersions represent the global minimum region, and the blue dispersions, the local minimum regions.

Figure 67: The residual function map which demonstrates the complexity of the Alkhalifah and Tsvankin (1995) approximation of the PP reflection event with L1-norm. Red dispersions represent the global minimum region, and the blue dispersions, the local minimum regions.

Figure 68: The residual function map which demonstrates the complexity of the Alkhalifah and Tsvankin (1995) approximation of the PS reflection event with L1-norm. Red dispersions 
represent the global minimum region, and the blue dispersions, the local minimum regions.

Figure 69: The residual function map which demonstrates the complexity of the Ursin and Stovas (2006) approximation of the PP reflection event with L2-norm. Red dispersions represent the global minimum region, and the blue dispersions, the local minimum regions.

Figure 70: The residual function map which demonstrates the complexity of the Ursin and Stovas (2006) approximation of the PS reflection event with L2-norm. Red dispersions represent the global minimum region, and the blue dispersions, the local minimum regions.

Figure 71: The residual function map which demonstrates the complexity of the Ursin and Stovas (2006) approximation of the PP reflection event with L1-norm. Red dispersions represent the global minimum region, and the blue dispersions, the local minimum regions.

Figure 72: The residual function map which demonstrates the complexity of the Ursin and Stovas (2006) approximation of the PS reflection event with L1-norm. Red dispersions represent the global minimum region, and the blue dispersions, the local minimum regions.

Figure 73: The residual function map which demonstrates the complexity of the Blias (2009) approximation of the PP reflection event with L2-norm. Red dispersions represent the global minimum region, and the blue dispersions, the local minimum regions

Figure 74: The residual function map which demonstrates the complexity of the Blias (2009) approximation of the PS reflection event with L2-norm. Red dispersions represent the global minimum region, and the blue dispersions, the local minimum regions

Figure 75: The residual function map which demonstrates the complexity of the Blias (2009) approximation of the PP reflection event with L1-norm. Red dispersions represent the global minimum region, and the blue dispersions, the local minimum regions

Figure 76: The residual function map which demonstrates the complexity of the Blias (2009) approximation of the PS reflection event with L1-norm. Red dispersions represent the global minimum region, and the blue dispersions, the local minimum regions 131 
Figure 77: The residual function map which demonstrates the complexity of the Muir and Dellinger (1985) approximation of the PP reflection event with L2-norm. Red dispersions represent the global minimum region, and the blue dispersions, the local minimum regions.

Figure 78: The residual function map which demonstrates the complexity of the Muir and Dellinger (1985) approximation of the PS reflection event with L2-norm. Red dispersions represent the global minimum region, and the blue dispersions, the local minimum regions.

Figure 79: The residual function map which demonstrates the complexity of the Muir and Dellinger (1985) approximation of the PP reflection event with L1-norm. Red dispersions represent the global minimum region, and the blue dispersions, the local minimum regions.

Figure 80: The residual function map which demonstrates the complexity of the Muir and Dellinger (1985) approximation of the PS reflection event with L1-norm. Red dispersions represent the global minimum region, and the blue dispersions, the local minimum regions.

Figure 81: The residual function map which demonstrates the complexity of the Li and Yuan (2001) approximation of the PP reflection event with L2-norm. Red dispersions represent the global minimum region, and the blue dispersions, the local minimum regions.

Figure 82: The residual function map which demonstrates the complexity of the Li and Yuan (2001) approximation of the PS reflection event with L2-norm. Red dispersions represent the global minimum region, and the blue dispersions, the local minimum regions. 134

Figure 83: The residual function map which demonstrates the complexity of the Li and Yuan (2001) approximation of the PP reflection event with L1-norm. Red dispersions represent the global minimum region, and the blue dispersions, the local minimum regions. 135

Figure 84: The residual function map which demonstrates the complexity of the Li and Yuan (2001) approximation of the PS reflection event with L1-norm. Red dispersions represent the global minimum region, and the blue dispersions, the local minimum regions. 135

Figure 85: The residual function map which demonstrates the complexity of the approximation proposed in this work of the PP reflection event with L2-norm. Red 
dispersions represent the global minimum region, and the blue dispersions, the local minimum regions.

Figure 86: The residual function map which demonstrates the complexity of the approximation proposed in this work of the PS reflection event with L2-norm. Red dispersions represent the global minimum region, and the blue dispersions, the local minimum regions. 136

Figure 87: The residual function map which demonstrates the complexity of the approximation proposed in this work of the PP reflection event with L1-norm. Red dispersions represent the global minimum region, and the blue dispersions, the local minimum regions.

Figure 88: The residual function map which demonstrates the complexity of the approximation proposed in this work of the PS reflection event with L1-norm. Red dispersions represent the global minimum region, and the blue dispersions, the local minimum regions. 137

Figure 89: The residual function map which demonstrates the complexity of the Malovichko (1978) approximation of the PP reflection event with L2-norm. Red dispersions represent the global minimum region, and the blue dispersions, the local minimum regions.

Figure 90: The residual function map which demonstrates the complexity of the Malovichko (1978) approximation of the PS reflection event with L2-norm. Red dispersions represent the global minimum region, and the blue dispersions, the local minimum regions

Figure 91: The residual function map which demonstrates the complexity of the Malovichko (1978) approximation of the PP reflection event with L1-norm. Red dispersions represent the global minimum region, and the blue dispersions, the local minimum regions.

Figure 92: The residual function map which demonstrates the complexity of the Malovichko (1978) approximation of the PS reflection event with L1-norm. Red dispersions represent the global minimum region, and the blue dispersions, the local minimum regions.

Figure 93: The residual function map which demonstrates the complexity of the Alkhalifah and Tsvankin (1995) approximation of the PP reflection event with L2-norm. Red dispersions represent the global minimum region, and the blue dispersions, the local minimum regions. 
Figure 94: The residual function map which demonstrates the complexity of the Alkhalifah and Tsvankin (1995) approximation of the PS reflection event with L2-norm. Red dispersions represent the global minimum region, and the blue dispersions, the local minimum regions.

Figure 95: The residual function map which demonstrates the complexity of the Alkhalifah and Tsvankin (1995) approximation of the PP reflection event with L1-norm. Red dispersions represent the global minimum region, and the blue dispersions, the local minimum regions.

Figure 96: The residual function map which demonstrates the complexity of the Alkhalifah and Tsvankin (1995) approximation of the PS reflection event with L1-norm. Red dispersions represent the global minimum region, and the blue dispersions, the local minimum regions.

Figure 97: The residual function map which demonstrates the complexity of the Ursin and Stovas (2006) approximation of the PP reflection event with L2-norm. Red dispersions represent the global minimum region, and the blue dispersions, the local minimum regions.

Figure 98: The residual function map which demonstrates the complexity of the Ursin and Stovas (2006) approximation of the PS reflection event with L2-norm. Red dispersions represent the global minimum region, and the blue dispersions, the local minimum regions.

Figure 99: The residual function map which demonstrates the complexity of the Ursin and Stovas (2006) approximation of the PP reflection event with L1-norm. Red dispersions represent the global minimum region, and the blue dispersions, the local minimum regions.

Figure 100: The residual function map which demonstrates the complexity of the Ursin and Stovas (2006) approximation of the PS reflection event with L1-norm. Red dispersions represent the global minimum region, and the blue dispersions, the local minimum regions.

Figure 101: The residual function map which demonstrates the complexity of the Blias (2009) approximation of the PP reflection event with L2-norm. Red dispersions represent the global minimum region, and the blue dispersions, the local minimum regions. 
Figure 102: The residual function map which demonstrates the complexity of the Blias (2009) approximation of the PS reflection event with L2-norm. Red dispersions represent the global minimum region, and the blue dispersions, the local minimum regions

Figure 103: The residual function map which demonstrates the complexity of the Blias (2009) approximation of the PP reflection event with L1-norm. Red dispersions represent the global minimum region, and the blue dispersions, the local minimum regions 146

Figure 104: The residual function map which demonstrates the complexity of the Blias (2009) approximation of the PS reflection event with L1-norm. Red dispersions represent the global minimum region, and the blue dispersions, the local minimum regions

Figure 105: The residual function map which demonstrates the complexity of the Muir and Dellinger (1985) approximation of the PP reflection event with L2-norm. Red dispersions represent the global minimum region, and the blue dispersions, the local minimum regions.

Figure 106: The residual function map which demonstrates the complexity of the Muir and Dellinger (1985) approximation of the PS reflection event with L2-norm. Red dispersions represent the global minimum region, and the blue dispersions, the local minimum regions.

Figure 107: The residual function map which demonstrates the complexity of the Muir and Dellinger (1985) approximation of the PP reflection event with L1-norm. Red dispersions represent the global minimum region, and the blue dispersions, the local minimum regions.

Figure 108: The residual function map which demonstrates the complexity of the Muir and Dellinger (1985) approximation of the PS reflection event with L1-norm. Red dispersions represent the global minimum region, and the blue dispersions, the local minimum regions.

Figure 109: The residual function map which demonstrates the complexity of the Li and Yuan (2001) approximation of the PP reflection event with L2-norm. Red dispersions represent the global minimum region, and the blue dispersions, the local minimum regions

Figure 110: The residual function map which demonstrates the complexity of the Li and Yuan (2001) approximation of the PS reflection event with L2-norm. Red dispersions represent the global minimum region, and the blue dispersions, the local minimum regions 
Figure 111: The residual function map which demonstrates the complexity of the Li and Yuan (2001) approximation of the PP reflection event with L1-norm. Red dispersions represent the global minimum region, and the blue dispersions, the local minimum regions.

Figure 112: The residual function map which demonstrates the complexity of the Li and Yuan (2001) approximation of the PS reflection event with L1-norm. Red dispersions represent the global minimum region, and the blue dispersions, the local minimum regions. 150

Figure 113: The residual function map which demonstrates the complexity of the approximation proposed in this work of the PP reflection event with L2-norm. Red dispersions represent the global minimum region, and the blue dispersions, the local minimum regions. 151

Figure 114: The residual function map which demonstrates the complexity of the approximation proposed in this work of the PS reflection event with L2-norm. Red dispersions represent the global minimum region, and the blue dispersions, the local minimum regions. 151

Figure 115: The residual function map which demonstrates the complexity of the approximation proposed in this work of the PP reflection event with L1-norm. Red dispersions represent the global minimum region, and the blue dispersions, the local minimum regions. .152

Figure 116: The residual function map which demonstrates the complexity of the approximation proposed in this work of the PS reflection event with L1-norm. Red dispersions represent the global minimum region, and the blue dispersions, the local minimum regions. 152

Figure 117: The residual function map which demonstrates the complexity of the Malovichko (1978) approximation of the PP reflection event with L2-norm. Red dispersions represent the global minimum region, and the blue dispersions, the local minimum regions. 155

Figure 118: The residual function map which demonstrates the complexity of the Malovichko (1978) approximation of the PS reflection event with L2-norm. Red dispersions represent the global minimum region, and the blue dispersions, the local minimum regions. 155

Figure 119: The residual function map which demonstrates the complexity of the Malovichko (1978) approximation of the PP reflection event with L1-norm. Red dispersions represent the global minimum region, and the blue dispersions, the local minimum regions. 156 
Figure 120: The residual function map which demonstrates the complexity of the Malovichko (1978) approximation of the PS reflection event with L1-norm. Red dispersions represent the global minimum region, and the blue dispersions, the local minimum regions. 156

Figure 121: The residual function map which demonstrates the complexity of the Alkhalifah and Tsvankin (1995) approximation of the PP reflection event with L2-norm. Red dispersions represent the global minimum region, and the blue dispersions, the local minimum regions.

Figure 122: The residual function map which demonstrates the complexity of the Alkhalifah and Tsvankin (1995) approximation of the PS reflection event with L2-norm. Red dispersions represent the global minimum region, and the blue dispersions, the local minimum regions.

Figure 123: The residual function map which demonstrates the complexity of the Alkhalifah and Tsvankin (1995) approximation of the PP reflection event with L1-norm. Red dispersions represent the global minimum region, and the blue dispersions, the local minimum regions.

Figure 124: The residual function map which demonstrates the complexity of the Alkhalifah and Tsvankin (1995) approximation of the PS reflection event with L1-norm. Red dispersions represent the global minimum region, and the blue dispersions, the local minimum regions.

Figure 125: The residual function map which demonstrates the complexity of the Ursin and Stovas (2006) approximation of the PP reflection event with L2-norm. Red dispersions represent the global minimum region, and the blue dispersions, the local minimum regions.

Figure 126: The residual function map which demonstrates the complexity of the Ursin and Stovas (2006) approximation of the PS reflection event with L2-norm. Red dispersions represent the global minimum region, and the blue dispersions, the local minimum regions.

Figure 127: The residual function map which demonstrates the complexity of the Ursin and Stovas (2006) approximation of the PP reflection event with L1-norm. Red dispersions represent the global minimum region, and the blue dispersions, the local minimum regions. 
Figure 128: The residual function map which demonstrates the complexity of the Ursin and Stovas (2006) approximation of the PS reflection event with L1-norm. Red dispersions represent the global minimum region, and the blue dispersions, the local minimum regions.

Figure 129: The residual function map which demonstrates the complexity of the Blias (2009) approximation of the PP reflection event with L2-norm. Red dispersions represent the global minimum region, and the blue dispersions, the local minimum regions

Figure 130: The residual function map which demonstrates the complexity of the Blias (2009) approximation of the PS reflection event with L2-norm. Red dispersions represent the global minimum region, and the blue dispersions, the local minimum regions

Figure 131: The residual function map which demonstrates the complexity of the Blias (2009) approximation of the PP reflection event with L1-norm. Red dispersions represent the global minimum region, and the blue dispersions, the local minimum regions.

Figure 132: The residual function map which demonstrates the complexity of the Blias (2009) approximation of the PS reflection event with L1-norm. Red dispersions represent the global minimum region, and the blue dispersions, the local minimum regions.

Figure 133: The residual function map which demonstrates the complexity of the Muir and Dellinger (1985) approximation of the PP reflection event with L2-norm. Red dispersions represent the global minimum region, and the blue dispersions, the local minimum regions.

Figure 134: The residual function map which demonstrates the complexity of the Muir and Dellinger (1985) approximation of the PS reflection event with L2-norm. Red dispersions represent the global minimum region, and the blue dispersions, the local minimum regions.

Figure 135: The residual function map which demonstrates the complexity of the Muir and Dellinger (1985) approximation of the PP reflection event with L1-norm. Red dispersions represent the global minimum region, and the blue dispersions, the local minimum regions.

Figure 136: The residual function map which demonstrates the complexity of the Muir and Dellinger (1985) approximation of the PS reflection event with L1-norm. Red dispersions 
represent the global minimum region, and the blue dispersions, the local minimum regions.

Figure 137: The residual function map which demonstrates the complexity of the Li and Yuan (2001) approximation of the PP reflection event with L2-norm. Red dispersions represent the global minimum region, and the blue dispersions, the local minimum regions

Figure 138: The residual function map which demonstrates the complexity of the Li and Yuan (2001) approximation of the PS reflection event with L2-norm. Red dispersions represent the global minimum region, and the blue dispersions, the local minimum regions

Figure 139: The residual function map which demonstrates the complexity of the Li and Yuan (2001) approximation of the PP reflection event with L1-norm. Red dispersions represent the global minimum region, and the blue dispersions, the local minimum regions

Figure 140: The residual function map which demonstrates the complexity of the Li and Yuan (2001) approximation of the PS reflection event with L1-norm. Red dispersions represent the global minimum region, and the blue dispersions, the local minimum regions 166

Figure 141: The residual function map which demonstrates the complexity of the approximation proposed in this work of the PP reflection event with L2-norm. Red dispersions represent the global minimum region, and the blue dispersions, the local minimum regions.

Figure 142: The residual function map which demonstrates the complexity of the approximation proposed in this work of the PS reflection event with L2-norm. Red dispersions represent the global minimum region, and the blue dispersions, the local minimum regions.

Figure 143: The residual function map which demonstrates the complexity of the approximation proposed in this work of the PP reflection event with L1-norm. Red dispersions represent the global minimum region, and the blue dispersions, the local minimum regions.

Figure 144: The residual function map which demonstrates the complexity of the approximation proposed in this work of the PS reflection event with L1-norm. Red dispersions represent the global minimum region, and the blue dispersions, the local minimum regions. 168 
Figure 145: The residual function map which demonstrates the complexity of the Malovichko (1978) approximation of the PP reflection event with L2-norm. Red dispersions represent the global minimum region, and the blue dispersions, the local minimum regions.

Figure 146: The residual function map which demonstrates the complexity of the Malovichko (1978) approximation of the PS reflection event with L2-norm. Red dispersions represent the global minimum region, and the blue dispersions, the local minimum regions. 170

Figure 147: The residual function map which demonstrates the complexity of the Malovichko (1978) approximation of the PP reflection event with L1-norm. Red dispersions represent the global minimum region, and the blue dispersions, the local minimum regions.

Figure 148: The residual function map which demonstrates the complexity of the Malovichko (1978) approximation of the PS reflection event with L1-norm. Red dispersions represent the global minimum region, and the blue dispersions, the local minimum regions.

Figure 149: The residual function map which demonstrates the complexity of the Alkhalifah and Tsvankin (1995) approximation of the PP reflection event with L2-norm. Red dispersions represent the global minimum region, and the blue dispersions, the local minimum regions.

Figure 150: The residual function map which demonstrates the complexity of the Alkhalifah and Tsvankin (1995) approximation of the PS reflection event with L2-norm. Red dispersions represent the global minimum region, and the blue dispersions, the local minimum regions.

Figure 151: The residual function map which demonstrates the complexity of the Alkhalifah and Tsvankin (1995) approximation of the PP reflection event with L1-norm. Red dispersions represent the global minimum region, and the blue dispersions, the local minimum regions.

Figure 152: The residual function map which demonstrates the complexity of the Alkhalifah and Tsvankin (1995) approximation of the PS reflection event with L1-norm. Red dispersions represent the global minimum region, and the blue dispersions, the local minimum regions.

Figure 153: The residual function map which demonstrates the complexity of the Ursin and Stovas (2006) approximation of the PP reflection event with L2-norm. Red dispersions 
represent the global minimum region, and the blue dispersions, the local minimum regions.

Figure 154: The residual function map which demonstrates the complexity of the Ursin and Stovas (2006) approximation of the PS reflection event with L2-norm. Red dispersions represent the global minimum region, and the blue dispersions, the local minimum regions.

Figure 155: The residual function map which demonstrates the complexity of the Ursin and Stovas (2006) approximation of the PP reflection event with L1-norm. Red dispersions represent the global minimum region, and the blue dispersions, the local minimum regions.

Figure 156: The residual function map which demonstrates the complexity of the Ursin and Stovas (2006) approximation of the PS reflection event with L1-norm. Red dispersions represent the global minimum region, and the blue dispersions, the local minimum regions.

Figure 157: The residual function map which demonstrates the complexity of the Blias (2009) approximation of the PP reflection event with L2-norm. Red dispersions represent the global minimum region, and the blue dispersions, the local minimum regions

Figure 158: The residual function map which demonstrates the complexity of the Blias (2009) approximation of the PS reflection event with L2-norm. Red dispersions represent the global minimum region, and the blue dispersions, the local minimum regions

Figure 159: The residual function map which demonstrates the complexity of the Blias (2009) approximation of the PP reflection event with L1-norm. Red dispersions represent the global minimum region, and the blue dispersions, the local minimum regions

Figure 160: The residual function map which demonstrates the complexity of the Blias (2009) approximation of the PS reflection event with L1-norm. Red dispersions represent the global minimum region, and the blue dispersions, the local minimum regions

Figure 161: The residual function map which demonstrates the complexity of the Muir and Dellinger (1985) approximation of the PP reflection event with L2-norm. Red dispersions represent the global minimum region, and the blue dispersions, the local minimum regions. 
Figure 162: The residual function map which demonstrates the complexity of the Muir and Dellinger (1985) approximation of the PS reflection event with L2-norm. Red dispersions represent the global minimum region, and the blue dispersions, the local minimum regions.

Figure 163: The residual function map which demonstrates the complexity of the Muir and Dellinger (1985) approximation of the PP reflection event with L1-norm. Red dispersions represent the global minimum region, and the blue dispersions, the local minimum regions.

Figure 164: The residual function map which demonstrates the complexity of the Muir and Dellinger (1985) approximation of the PS reflection event with L1-norm. Red dispersions represent the global minimum region, and the blue dispersions, the local minimum regions.

Figure 165: The residual function map which demonstrates the complexity of the Li and Yuan (2001) approximation of the PP reflection event with L2-norm. Red dispersions represent the global minimum region, and the blue dispersions, the local minimum regions.

Figure 166: The residual function map which demonstrates the complexity of the Li and Yuan (2001) approximation of the PS reflection event with L2-norm. Red dispersions represent the global minimum region, and the blue dispersions, the local minimum regions.

Figure 167: The residual function map which demonstrates the complexity of the Li and Yuan (2001) approximation of the PP reflection event with L1-norm. Red dispersions represent the global minimum region, and the blue dispersions, the local minimum regions.

Figure 168: The residual function map which demonstrates the complexity of the Li and Yuan (2001) approximation of the PS reflection event with L1-norm. Red dispersions represent the global minimum region, and the blue dispersions, the local minimum regions.

Figure 169: The residual function map which demonstrates the complexity of the approximation proposed in this work of the PP reflection event with L2-norm. Red dispersions represent the global minimum region, and the blue dispersions, the local minimum regions.

Figure 170: The residual function map which demonstrates the complexity of the approximation proposed in this work of the PS reflection event with L2-norm. Red 
dispersions represent the global minimum region, and the blue dispersions, the local minimum regions.

Figure 171: The residual function map which demonstrates the complexity of the approximation proposed in this work of the PP reflection event with L1-norm. Red dispersions represent the global minimum region, and the blue dispersions, the local minimum regions. 183

Figure 172: The residual function map which demonstrates the complexity of the approximation proposed in this work of the PS reflection event with L1-norm. Red dispersions represent the global minimum region, and the blue dispersions, the local minimum regions.

Figure 173: Relative error in travel-time between the observed curve and the calculated curve with each approximation of the PP reflection event with L2-norm and IMFIL optimization algorithm

Figure 174: Relative error in travel-time between the observed curve and the calculated curve with each approximation of the PS reflection event with L2-norm and IMFIL optimization algorithm

Figure 175: Relative error in travel-time between the observed curve and the calculated curve with each approximation of the PP reflection event with L1-norm and IMFIL optimization algorithm

Figure 176: Relative error in travel-time between the observed curve and the calculated curve with each approximation of the PS reflection event with L1-norm and IMFIL optimization algorithm. 188

Figure 177: Relative error in travel-time between the observed curve and the calculated curve with each approximation of the PP reflection event with L2-norm and FMINSEARCH optimization algorithm

Figure 178: Relative error in travel-time between the observed curve and the calculated curve with each approximation of the PS reflection event with L2-norm and FMINSEARCH optimization algorithm.

Figure 179: Relative error in travel-time between the observed curve and the calculated curve with each approximation of the PP reflection event with L1-norm and FMINSEARCH optimization algorithm. 
Figure 180: Relative error in travel-time between the observed curve and the calculated curve with each approximation of the PS reflection event with L1-norm and FMINSEARCH optimization algorithm.

Figure 181: Relative error in travel-time between the observed curve and the calculated curve with each approximation of the PP reflection event with L2-norm and SID-PSM optimization algorithm. 191

Figure 182: Relative error in travel-time between the observed curve and the calculated curve with each approximation of the PS reflection event with L2-norm and SID-PSM optimization algorithm.

Figure 183: Relative error in travel-time between the observed curve and the calculated curve with each approximation of the PP reflection event with L1-norm and SID-PSM optimization algorithm.

Figure 184: Relative error in travel-time between the observed curve and the calculated curve with each approximation of the PS reflection event with L1-norm and SID-PSM optimization algorithm. 192

Figure 185: Relative error in travel-time between the observed curve and the calculated curve with each approximation of the PP reflection event with L2-norm and MCS optimization algorithm.

Figure 186: Relative error in travel-time between the observed curve and the calculated curve with each approximation of the PS reflection event with L2-norm and MCS optimization algorithm.

Figure 187: Relative error in travel-time between the observed curve and the calculated curve with each approximation of the PP reflection event with L1-norm and MCS optimization algorithm.

Figure 188: Relative error in travel-time between the observed curve and the calculated curve with each approximation of the PS reflection event with L1-norm and MCS optimization algorithm.

Figure 189: Relative error in travel-time between the observed curve and the calculated curve with each approximation of the PP reflection event with L2-norm and TOMLAB/LGO optimization algorithm. 
Figure 190: Relative error in travel-time between the observed curve and the calculated curve with each approximation of the PS reflection event with L2-norm and TOMLAB/LGO optimization algorithm.

Figure 191: Relative error in travel-time between the observed curve and the calculated curve with each approximation of the PP reflection event with L1-norm and TOMLAB/LGO optimization algorithm. 196

Figure 192: Relative error in travel-time between the observed curve and the calculated curve with each approximation of the PS reflection event with L1-norm and TOMLAB/LGO optimization algorithm.

Figure 193: Relative error in travel-time between the observed curve and the calculated curve with each approximation of the PP reflection event with L2-norm and IMFIL optimization algorithm.

Figure 194: Relative error in travel-time between the observed curve and the calculated curve with each approximation of the PS reflection event with L2-norm and IMFIL optimization algorithm. 198

Figure 195: Relative error in travel-time between the observed curve and the calculated curve with each approximation of the PP reflection event with L1-norm and IMFIL optimization algorithm.

Figure 196: Relative error in travel-time between the observed curve and the calculated curve with each approximation of the PS reflection event with L1-norm and IMFIL optimization algorithm.

Figure 197: Relative error in travel-time between the observed curve and the calculated curve with each approximation of the PP reflection event with L2-norm and FMINSEARCH optimization algorithm.

Figure 198: Relative error in travel-time between the observed curve and the calculated curve with each approximation of the PS reflection event with L2-norm and FMINSEARCH optimization algorithm.

Figure 199: Relative error in travel-time between the observed curve and the calculated curve with each approximation of the PP reflection event with L1-norm and FMINSEARCH optimization algorithm. 
Figure 200: Relative error in travel-time between the observed curve and the calculated curve with each approximation of the PS reflection event with L1-norm and FMINSEARCH optimization algorithm.

Figure 201: Relative error in travel-time between the observed curve and the calculated curve with each approximation of the PP reflection event with L2-norm and SID-PSM optimization algorithm. 202

Figure 202: Relative error in travel-time between the observed curve and the calculated curve with each approximation of the PS reflection event with L2-norm and SID-PSM optimization algorithm. 202

Figure 203: Relative error in travel-time between the observed curve and the calculated curve with each approximation of the PP reflection event with L1-norm and SID-PSM optimization algorithm.

Figure 204: Relative error in travel-time between the observed curve and the calculated curve with each approximation of the PS reflection event with L1-norm and SID-PSM optimization algorithm. 203

Figure 205: Relative error in travel-time between the observed curve and the calculated curve with each approximation of the PP reflection event with L2-norm and MCS optimization algorithm. 204

Figure 206: Relative error in travel-time between the observed curve and the calculated curve with each approximation of the PS reflection event with L2-norm and MCS optimization algorithm. 204

Figure 207: Relative error in travel-time between the observed curve and the calculated curve with each approximation of the PP reflection event with L1-norm and MCS optimization algorithm. 205

Figure 208: Relative error in travel-time between the observed curve and the calculated curve with each approximation of the PS reflection event with L1-norm and MCS optimization algorithm. 205

Figure 209: Relative error in travel-time between the observed curve and the calculated curve with each approximation of the PP reflection event with L2-norm and TOMLAB/LGO optimization algorithm. 
Figure 210: Relative error in travel-time between the observed curve and the calculated curve with each approximation of the PS reflection event with L2-norm and TOMLAB/LGO optimization algorithm.

Figure 211: Relative error in travel-time between the observed curve and the calculated curve with each approximation of the PP reflection event with L1-norm and TOMLAB/LGO optimization algorithm.

Figure 212: Relative error in travel-time between the observed curve and the calculated curve with each approximation of the PS reflection event with L1-norm and TOMLAB/LGO optimization algorithm.

Figure 213: Relative error in travel-time between the observed curve and the calculated curve with each approximation of the PP reflection event with L2-norm and IMFIL optimization algorithm.

Figure 214: Relative error in travel-time between the observed curve and the calculated curve with each approximation of the PS reflection event with L2-norm and IMFIL optimization algorithm. 209

Figure 215: Relative error in travel-time between the observed curve and the calculated curve with each approximation of the PP reflection event with L1-norm and IMFIL optimization algorithm

Figure 216: Relative error in travel-time between the observed curve and the calculated curve with each approximation of the PS reflection event with L1-norm and IMFIL optimization algorithm.

Figure 217: Relative error in travel-time between the observed curve and the calculated curve with each approximation of the PP reflection event with L2-norm and FMINSEARCH optimization algorithm.

Figure 218: Relative error in travel-time between the observed curve and the calculated curve with each approximation of the PS reflection event with L2-norm and FMINSEARCH optimization algorithm.

Figure 219: Relative error in travel-time between the observed curve and the calculated curve with each approximation of the PP reflection event with L1-norm and FMINSEARCH optimization algorithm. 
Figure 220: Relative error in travel-time between the observed curve and the calculated curve with each approximation of the PS reflection event with L1-norm and FMINSEARCH optimization algorithm.

Figure 221: Relative error in travel-time between the observed curve and the calculated curve with each approximation of the PP reflection event with L2-norm and SID-PSM optimization algorithm. 213

Figure 222: Relative error in travel-time between the observed curve and the calculated curve with each approximation of the PS reflection event with L2-norm and SID-PSM optimization algorithm

Figure 223: Relative error in travel-time between the observed curve and the calculated curve with each approximation of the PP reflection event with L1-norm and SID-PSM optimization algorithm.

Figure 224: Relative error in travel-time between the observed curve and the calculated curve with each approximation of the PS reflection event with L1-norm and SID-PSM optimization algorithm. 214

Figure 225: Relative error in travel-time between the observed curve and the calculated curve with each approximation of the PP reflection event with L2-norm and MCS optimization algorithm.

Figure 226: Relative error in travel-time between the observed curve and the calculated curve with each approximation of the PS reflection event with L2-norm and MCS optimization algorithm.

Figure 227: Relative error in travel-time between the observed curve and the calculated curve with each approximation of the PP reflection event with L1-norm and MCS optimization algorithm. 216

Figure 228: Relative error in travel-time between the observed curve and the calculated curve with each approximation of the PS reflection event with L1-norm and MCS optimization algorithm.

Figure 229: Relative error in travel-time between the observed curve and the calculated curve with each approximation of the PP reflection event with L2-norm and TOMLAB/LGO optimization algorithm. 
Figure 230: Relative error in travel-time between the observed curve and the calculated curve with each approximation of the PS reflection event with L2-norm and TOMLAB/LGO optimization algorithm.

Figure 231: Relative error in travel-time between the observed curve and the calculated curve with each approximation of the PP reflection event with L1-norm and TOMLAB/LGO optimization algorithm. 218

Figure 232: Relative error in travel-time between the observed curve and the calculated curve with each approximation of the PS reflection event with L1-norm and TOMLAB/LGO optimization algorithm.

Figure 233: Relative error in travel-time between the observed curve and the calculated curve with each approximation of the PP reflection event with L2-norm and IMFIL optimization algorithm

Figure 234: Relative error in travel-time between the observed curve and the calculated curve with each approximation of the PS reflection event with L2-norm and IMFIL optimization algorithm 220

Figure 235: Relative error in travel-time between the observed curve and the calculated curve with each approximation of the PP reflection event with L1-norm and IMFIL optimization algorithm

Figure 236: Relative error in travel-time between the observed curve and the calculated curve with each approximation of the PS reflection event with L1-norm and IMFIL optimization algorithm.

Figure 237: Relative error in travel-time between the observed curve and the calculated curve with each approximation of the PP reflection event with L2-norm and FMINSEARCH optimization algorithm. 222

Figure 238: Relative error in travel-time between the observed curve and the calculated curve with each approximation of the PS reflection event with L2-norm and FMINSEARCH optimization algorithm.

Figure 239: Relative error in travel-time between the observed curve and the calculated curve with each approximation of the PP reflection event with L1-norm and FMINSEARCH optimization algorithm. 
Figure 240: Relative error in travel-time between the observed curve and the calculated curve with each approximation of the PS reflection event with L1-norm and FMINSEARCH optimization algorithm.

Figure 241: Relative error in travel-time between the observed curve and the calculated curve with each approximation of the PP reflection event with L2-norm and SID-PSM optimization algorithm. 224

Figure 242: Relative error in travel-time between the observed curve and the calculated curve with each approximation of the PS reflection event with L2-norm and SID-PSM optimization algorithm

Figure 243: Relative error in travel-time between the observed curve and the calculated curve with each approximation of the PP reflection event with L1-norm and SID-PSM optimization algorithm.

Figure 244: Relative error in travel-time between the observed curve and the calculated curve with each approximation of the PS reflection event with L1-norm and SID-PSM optimization algorithm. 225

Figure 245: Relative error in travel-time between the observed curve and the calculated curve with each approximation of the PP reflection event with L2-norm and MCS optimization algorithm. 226

Figure 246: Relative error in travel-time between the observed curve and the calculated curve with each approximation of the PS reflection event with L2-norm and MCS optimization algorithm.

Figure 247: Relative error in travel-time between the observed curve and the calculated curve with each approximation of the PP reflection event with L1-norm and MCS optimization algorithm.

Figure 248: Relative error in travel-time between the observed curve and the calculated curve with each approximation of the PS reflection event with L1-norm and MCS optimization algorithm

Figure 249: Relative error in travel-time between the observed curve and the calculated curve with each approximation of the PP reflection event with L2-norm and TOMLAB/LGO optimization algorithm. 
Figure 250: Relative error in travel-time between the observed curve and the calculated curve with each approximation of the PS reflection event with L2-norm and TOMLAB/LGO optimization algorithm.

Figure 251: Relative error in travel-time between the observed curve and the calculated curve with each approximation of the PP reflection event with L1-norm and TOMLAB/LGO optimization algorithm.

Figure 252: Relative error in travel-time between the observed curve and the calculated curve with each approximation of the PS reflection event with L1-norm and TOMLAB/LGO optimization algorithm.

Figure 253: Relative error in travel-time between the observed curve and the calculated curve with each approximation of the PP reflection event with L2-norm and IMFIL optimization algorithm.

Figure 254: Relative error in travel-time between the observed curve and the calculated curve with each approximation of the PS reflection event with L2-norm and IMFIL optimization algorithm 231

Figure 255: Relative error in travel-time between the observed curve and the calculated curve with each approximation of the PP reflection event with L1-norm and IMFIL optimization algorithm

Figure 256: Relative error in travel-time between the observed curve and the calculated curve with each approximation of the PS reflection event with L1-norm and IMFIL optimization algorithm.

Figure 257: Relative error in travel-time between the observed curve and the calculated curve with each approximation of the PP reflection event with L2-norm and FMINSEARCH optimization algorithm.

Figure 258: Relative error in travel-time between the observed curve and the calculated curve with each approximation of the PS reflection event with L2-norm and FMINSEARCH optimization algorithm.

Figure 259: Relative error in travel-time between the observed curve and the calculated curve with each approximation of the PP reflection event with L1-norm and FMINSEARCH optimization algorithm. 
Figure 260: Relative error in travel-time between the observed curve and the calculated curve with each approximation of the PS reflection event with L1-norm and FMINSEARCH optimization algorithm.

Figure 261: Relative error in travel-time between the observed curve and the calculated curve with each approximation of the PP reflection event with L2-norm and SID-PSM optimization algorithm. 235

Figure 262: Relative error in travel-time between the observed curve and the calculated curve with each approximation of the PS reflection event with L2-norm and SID-PSM optimization algorithm.

Figure 263: Relative error in travel-time between the observed curve and the calculated curve with each approximation of the PP reflection event with L1-norm and SID-PSM optimization algorithm.

Figure 264: Relative error in travel-time between the observed curve and the calculated curve with each approximation of the PS reflection event with L1-norm and SID-PSM optimization algorithm. 236

Figure 265: Relative error in travel-time between the observed curve and the calculated curve with each approximation of the PP reflection event with L2-norm and MCS optimization algorithm.

Figure 266: Relative error in travel-time between the observed curve and the calculated curve with each approximation of the PS reflection event with L2-norm and MCS optimization algorithm.

Figure 267: Relative error in travel-time between the observed curve and the calculated curve with each approximation of the PP reflection event with L1-norm and MCS optimization algorithm. 238

Figure 268: Relative error in travel-time between the observed curve and the calculated curve with each approximation of the PS reflection event with L1-norm and MCS optimization algorithm 238

Figure 269: Relative error in travel-time between the observed curve and the calculated curve with each approximation of the PP reflection event with L2-norm and TOMLAB/LGO optimization algorithm. 
Figure 270: Relative error in travel-time between the observed curve and the calculated curve with each approximation of the PS reflection event with L2-norm and TOMLAB/LGO optimization algorithm.

Figure 271: Relative error in travel-time between the observed curve and the calculated curve with each approximation of the PP reflection event with L1-norm and TOMLAB/LGO optimization algorithm. 240

Figure 272: Relative error in travel-time between the observed curve and the calculated curve with each approximation of the PS reflection event with L1-norm and TOMLAB/LGO optimization algorithm.

Figure 273: Seismogram of the Model 1 with no noise. The red lines represent the automated picks for the PP and PSv events.

Figure 274: Seismogram of the Model 1 with signal-noise ratio of $90 \%$. The red lines represent the automated picks for the PP and PSv events.

Figure 275: Seismogram of the Model 2 with no noise. The red lines represent the automated picks for the PP and PSv events. 258

Figure 276: Seismogram of the Model 2 with signal-noise ratio of 90\%. The red lines represent the automated picks for the PP and PSv events.

Figure 277: Seismogram of the Model 3 with no noise. The red lines represent the automated picks for the PP and PSv events.

Figure 278: Seismogram of the Model 3 with signal-noise ratio of 90\%. The red lines represent the automated picks for the PP and PSv events.

Figure 279: Seismogram of the Model 4 with no noise. The red lines represent the automated picks for the PP and PSv events....

Figure 280: Seismogram of the Model 4 with no noise with signal-noise ratio of $90 \%$. The red lines represent the automated picks for the PP and PSv events. 263

Figure 281: Seismogram of the Model 5 with no noise. The red lines represent the automated picks for the PP and PSv events.

Figure 282: Seismogram of the Model 5 with signal-noise ratio of 90\%. The red lines represent the automated picks for the PP and PSv events. 
Figure 283: Seismograms of the four models used to perform the tests of PVO analysis. (A) Critical angle of around 37 degrees, (B) same critical angle, but with semi-Gaussian wavelet, (C) critical angle of around 30 degrees and (D) critical angle of around 44 degrees 266

Figure 284: The variation for each trace/angle shown versus the global minimum, amplitude and peak frequency (A) of the first and (B) of the second models from the PVO analysis...267 Figure 285: The variation for each trace/angle shown versus the global minimum, amplitude and peak frequency (A) of the third and (B) of the fourth models from the PVO analysis. ..268 Figure 286: The analytically calculated instantaneous phase versus the incident angle of (A) the first and second models, (B) third model and (C) the fourth model 269

Figure 287: The reflection coefficient and the transmission coefficient versus the incidence angle. The red dashed line represents the critical angle of the first and second models. 270 Figure 288: The reflection coefficient and the transmission coefficient versus the incidence angle. The red dashed line represents the critical angle of the third model.

Figure 289: The reflection coefficient and the transmission coefficient versus the incidence angle. The red dashed line represents the critical angle of the fourth model. 272 


\section{LIST OF TABLES}

Table 1: Processing time (in seconds) of each inversion routine of the Model 1.

Table 2: Processing time (in seconds) of each inversion routine of the Model 2. 274

Table 3: Processing time (in seconds) of each inversion routine of the Model 3. 275

Table 4: Processing time (in seconds) of each inversion routine of the Model 4. 276

Table 5: Processing time (in seconds) of each inversion routine of the Model 5. 277 


\section{CONTENTS}

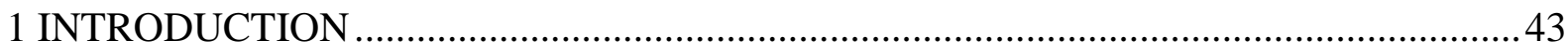

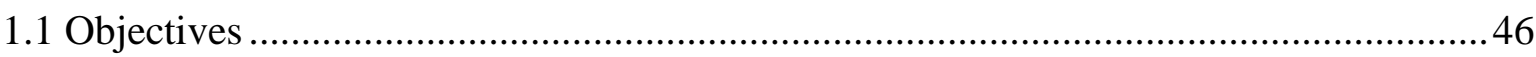

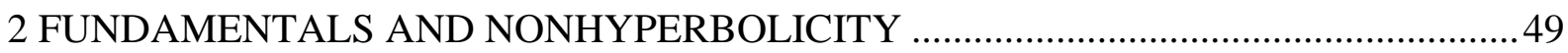

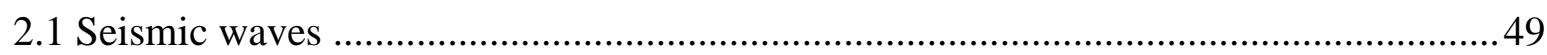

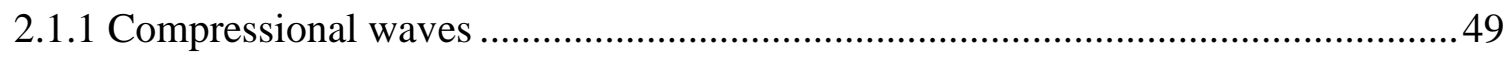

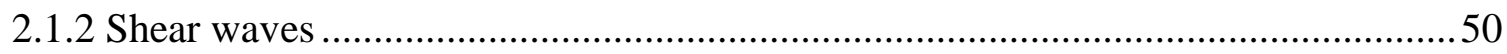

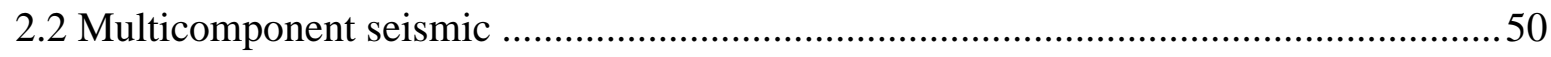

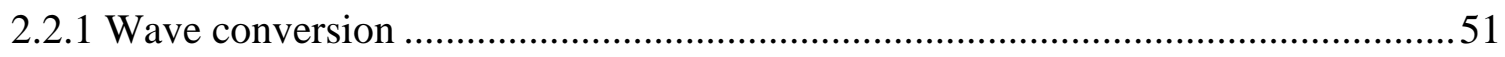

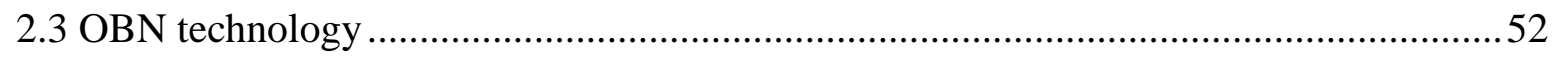

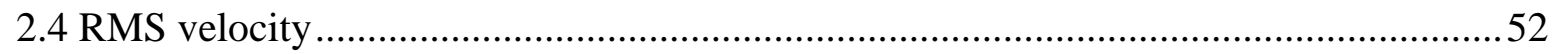

3 NONHYPERBOLIC EXPRESSIONS TO PERFORM VELOCITY ANALYSIS ..............55

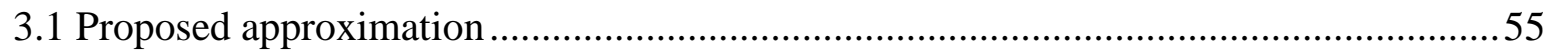

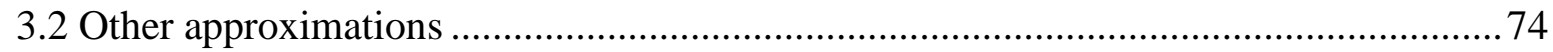

4 MODELLING COMPLEX STRUCTURES AND GEOMETRIES ….............................. 79

4.1 Automated picking using spectral recomposition ............................................. 80

4.1.1 PVO analysis to find critical reflection using spectral recomposition .................... 82

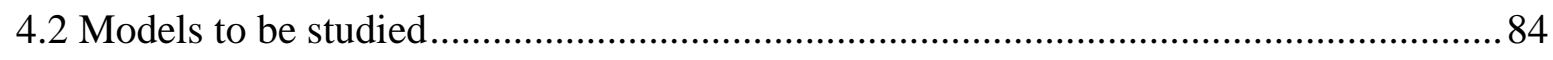

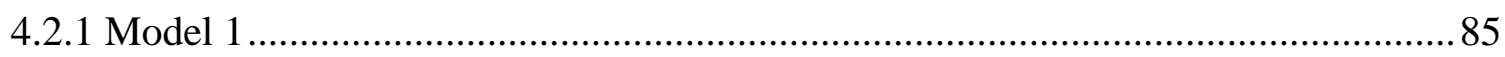

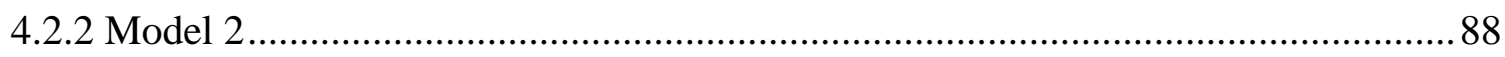

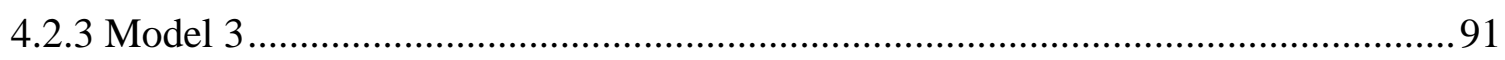

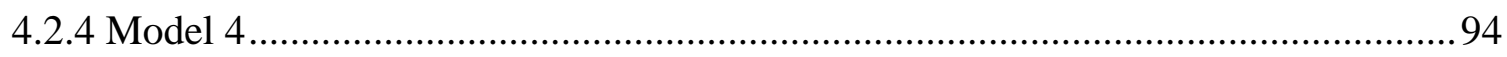

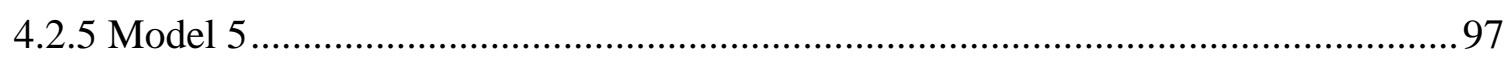

5 COMPLEXITY ANALYSIS OF THE OBJECIVE FUNCTION .................................. 101

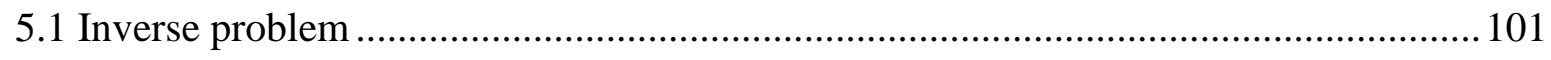




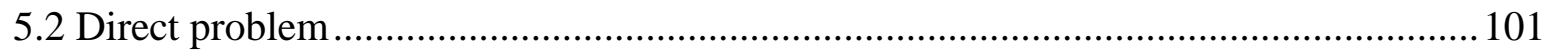

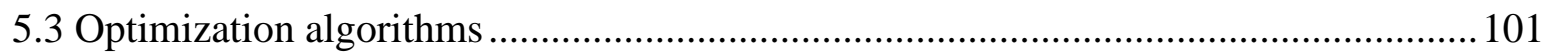

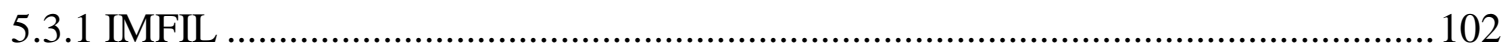

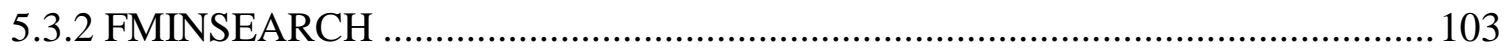

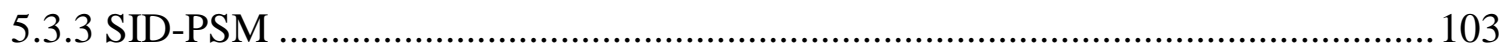

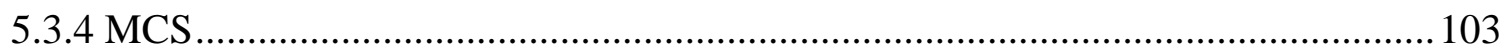

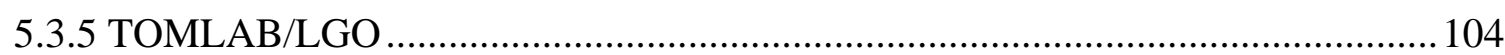

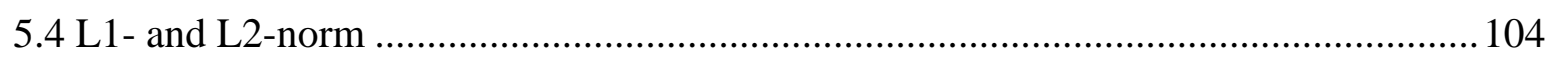

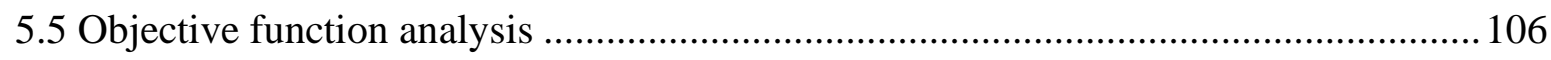

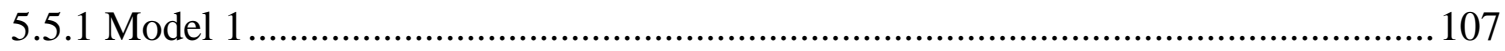

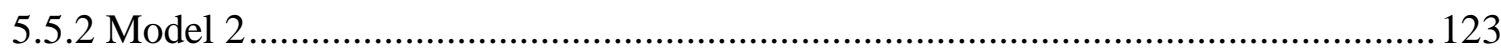

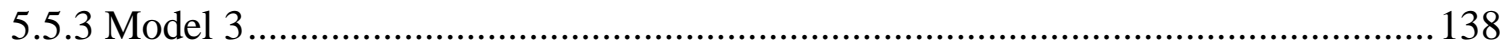

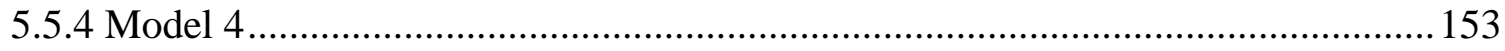

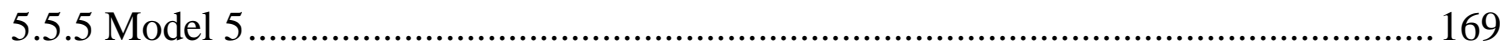

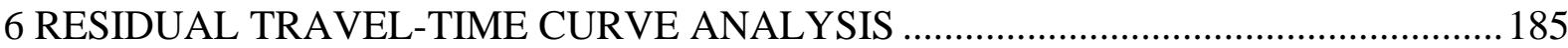

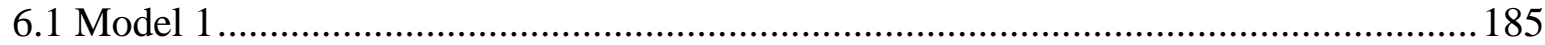

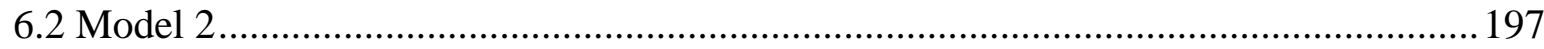

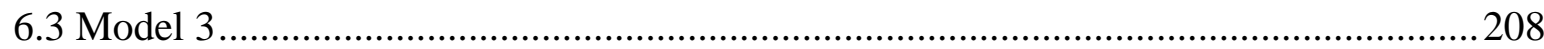

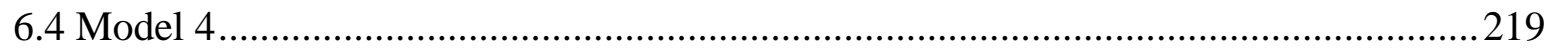

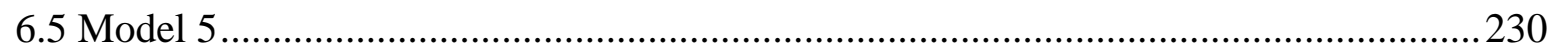

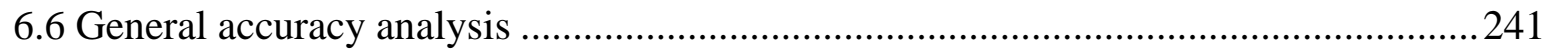

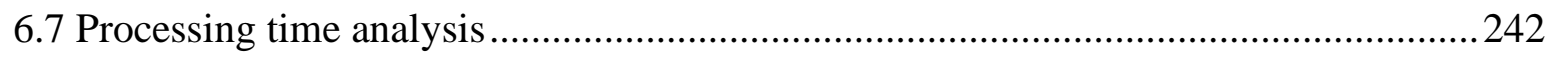

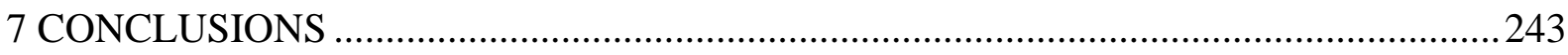

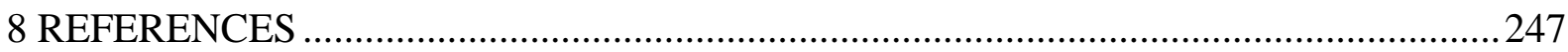

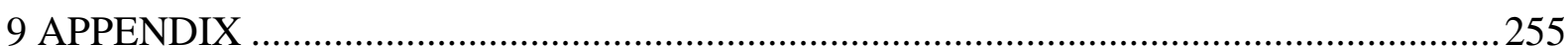

9.1 Appendix A - Seismogram of the pre salt models ..............................................255 
9.2 Appendix B - PVO analysis of SvSv reflection events..........................................266

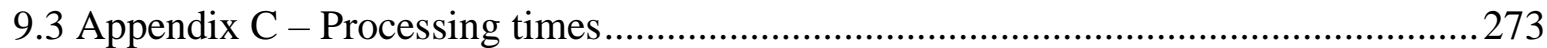




\section{INTRODUCTION}

The seismic method aims at characterizing the geology of a region, frequently with the objective of exploring hydrocarbon reservoirs. For this purpose, the method analyzes recordings of elastic waves produced by artificial sources. The seismic waves that return to the surface travel through the subsurface until they reach an interface, where they reflect and refract due to the differences between their physical properties of the overlying and underlying layers. Then, it is possible to extract information of the waves received, which allows to construct an image of the subsurface.

To record the seismic waves, many receivers are placed on the surface at different offsets from the source. There are two kinds of receivers: geophones, which are the receivers able to detect the velocity of the ground movement; and hydrophones, which detect the pressure variation in aquatic media. Different acquisition geometries are proposed to group the receivers into arrays, which depend on the objectives of the seismic acquisition (Sheriff and Geldart, 1995).

The seismic method is broadly used for the hydrocarbon exploration (Kearey, Brooks and Hill, 2002). The reason is that the seismic method is capable of mapping interfaces and other structures in the seismic sections, which allows constructing a stratigraphic and geological model (Kearey, Brooks and Hill, 2002).

Though the seismic method was originally conceived for hydrocarbon exploration, it is also used for mineral exploration, hydrogeology and environmental science. Even nearsurface seismic, in spite of many similarities, presents some different characteristics due to the depth scale of hydrocarbon exploration. In particular, near-surface seismic involves low multiplicity of the CMP (Common Mid-Point) survey, strong interferences of ground roll for short offsets and changes in the waveform due to the supercritical reflections.

The compressional P-waves, recorded conventionally in seismic surveys, are obtained with vertical geophones, unlike the multicomponent seismic, which is able to determine the polarization of shear S-waves by the three component geophones, one vertical and two orthogonally arrayed (Bokhonok, 2010; Zuniga, 2017). 
Multi-component seismic data have shown themselves extremely important for improving the subsurface characterization, because they contain more complete information about the elastic wave field than single-component data. The use of multicomponent seismic provides many enhancements — such as the determination of $\mathrm{Vp} / \mathrm{Vs}$ ratio to mapping the lithological variation (Pickett, 1963, Stewart et al., 2002, Agudelo et al., 2013), the possibility of obtaining the seismic sections even with a weak P reflectivity (Pickett, 1963, Stewart et al., 2002, Agudelo et al., 2013), the possibility of a better resolution of the seismic image (Stewart et al., 2003; Meier and Lee, 2009, Ursenbach et al., 2013), a better imaging of high angle faults (Purnell, 1992, Stewart et al., 2003, Wang et al., 2014), a better imaging through gas cloud (Granli el al. 1995), a better fluid discrimination (Qian et al., 2007), and monitoring the variations of the 4D seismic (Spitz et al., 2000).

Multicomponent seismic has been proven to be able to overcome some of the limitations of the conventional seismic (Kendall, 2006; Davis, 2001), and for this reason, it was expected that multicomponent seismic would become the leading technique for both onshore and offshore surveys (Davis, 2001; Hardage et al., 2011).

The steps to perform seismic processing have their complexity increased significantly when the converted wave is also analysed (Bokhonok, 2010). This happens because the incident wave presents a different velocity than the reflected wave, which results in an asymmetric ray tracing. The asymmetric ray tracing, generated by the wave conversion, produces a nonhyperbolic travel-time event. Moreover, Zuniga (2017) discussed about a number of possible causes of the nonhyperbolicity in a seismic event.

Vertical heterogeneity, combined with long offsets between source and receiver, is another factor able to generate a nonhyperbolic event, once there is a difference between the interval velocity and the RMS (Root Mean Square) velocity, and this discrepancy increases with larger offsets (Malovichko, 1978; Zuniga, 2017).

The use of OBN (Ocean Bottom Nodes) technology, even providing the PS event information, produces a different kind of ray tracing asymmetry than the one produced by the wave conversion (Zuniga, 2015 and 2017). In this case, the asymmetric seismic reflection event is generated by the difference between the datum of the source (on the ocean surface) and the receivers (on the seabed), which presents a difference between the distance traveled by the incident and the reflected rays (Zuniga, 2017). However, using the OBN technology 
enables the acquisition of converted waves, which was impossible to obtain with the conventional technology (streamer) on offshore surveys, once the S-waves cannot travel through Newtonian fluids, as water.

The different causes of the nonhyperbolicity in a general form have been studied in recent decades in different works (Malovichko, 1978; Blias, 1983 and 2009; GonzalezSerrano and Claerbout, 1984; Muir and Dellinger, 1985; Castle, 1988 and 1994; Nowroozi, 1989; Slotboom, 1990; Tsvankin and Thomsen, 1994; Alkhalifah and Tsvankin, 1995; Sain and Kaila, 1996; Li and Yuan, 1999, 2001 and 2002; Cheret et al., 2000; Causse et al., 2000; Tsvankin and Grechka, 2000a,b; Fomel and Grechka, 2000 and 2001; Tsvankin, 2001; Leiderman et al., 2003; Silva et al., 2003; Ursin and Stovas, 2006; Abedi and Riahi, 2016). Most of these works aimed at developing travel-time approximations capable of describing the nonhyperbolicity generated by one or more causes, so as to perform the velocity analysis of a nonhyperbolic event.

Morover, there were some works intended to compare the different approximations for different cases during the velocity analysis (Aleixo and Schleicher, 2010; Golikov and Stovas, 2012; Zuniga et al., 2015 and 2016; Zuniga, 2017).

Through the analysis of the developed approximations, with tests in real and theoretical models, it is possible to conclude that there is no approximation capable of fitting travel-times equally well for all models. However, some approximations presented better results than the others, and also demonstrated to be much more general than the others (Zuniga et al., 2015 and 2016; Zuniga, 2017).

Despite presenting good results, even the approximations which showed the best results in previous works have difficulties to describe some of some nonhyperbolic effects. For this reason, it is necessary to develop a more general approximation, capable of describing the effects that the other approximations have problems to deal with.

Starting with the analysis of Malovichko (1978), it is possible to understand how reaching an approximation can control the vertical heterogeneity. Blias' (2009) approximation is a strong complement to the vertical heterogeneity study. The studies from Tsvankin and Thomsen (1994), Alkhalifah and Tsvankin (1995) and Thomsen (1999) help with the effects of anisotropy. The Li and Yuan (2001) equation is a more general approximation and, for this reason, it is an important manner to combine multiple parameters in order to describe different 
nonhyperbolic effects. The difference of datum between source and receiver can be considered in the approximation proposed by Wang and Pham (2001).

A more general approximation must consider the effects of vertical heterogeneity for large offsets, anisotropy, wave conversion and the difference of datum between source and receiver.

After the development of an approximation capable of considering these causes of nonhyperbolicity, it must be compared to the other approximations to observe in which aspects the proposed one provides better results than the others.

The analysis must be performed by a comparison of curve fitting between the observed and the calculated travel-time curves from the reflection events. Considering the fact that the approximations have more than two parameters, it must be treated as an inverse problem according to an optimization criterion. The comparison of optimization algorithms must also be performed to determine the one which can minimize the processing time with a good accuracy.

Not only the residual travel-time must be compared, but also the complexity of the approximations, to understand the capacity of being unimodal or multimodal, which affects the processing time. Another important analysis is the comparison between L1-norm and L2norm to find out if there is some decrease of the processing time or difficulty of reaching the global minimum.

With all these analyses, it is possible to find if the proposed approximation can describe the nonhyperbolic effects and present better results than the other approximations.

\subsection{Objectives}

To develop an approximation which considers the nonhyperbolicity associated with vertical heterogeneity, anisotropy, wave conversion and difference of datum between source and receiver.

To study the complexity of the objective function to understand its topology, then find if it is unimodal or multimodal. This information is important to select which kind of 
optimization algorithm must be used and if the data processing can take much processing time.

To perform the velocity analysis comparing the proposed approximation with the others to find if it can fit the calculated travel-time curve with the observed curve with a lower residual travel-time.

To make this possible, an automated pick to obtain the travel-time curve of the target interface is also proposed by using the spectral recomposition to find the specific wavelet of each trace from the target event.

As a complementary study, the proposition of investigating the use of the spectral recomposition also provides important information concerning the wave phase, which allows for a deeper understanding of the critical and post critical reflection. 


\section{FUNDAMENTALS AND NONHYPERBOLICITY}

The nonhyperbolicity is a behaviour caused by wave behaviour and characteristics of the medium. These factors act generating interferences in the conventional hyperbolic behaviour of a travel-time curve. As the conventional seismic processing considers the events hyperbolic, techniques which can overcome this difficulty must be used, once the travel-time curves are not hyperbolic in many real situations.

In this chapter, some general fundamentals are presented aiming to understand the different causes for nonhyperbolicity as it was shown by Zuniga (2017).

\subsection{Seismic waves}

The seismic waves are energy which propagates through the Earth and are originated by earthquakes or artificial sources. The body waves travel through material media and can be reflected or refracted in interfaces between layers or geological structures, depending on the difference of the physical properties (Kearey, Brooks and Hill, 2002).

\subsubsection{Compressional waves}

The compressional waves, also called P-waves, travel in a longitudinal way, the same direction of the particle motion and wave propagation. It travels by compressing and distending the medium by which the wave propagates (Figure 1). Then, the P-wave travels faster than the other kinds of body waves, and can travel through any kind of material medium, whether solid, liquid or gas.

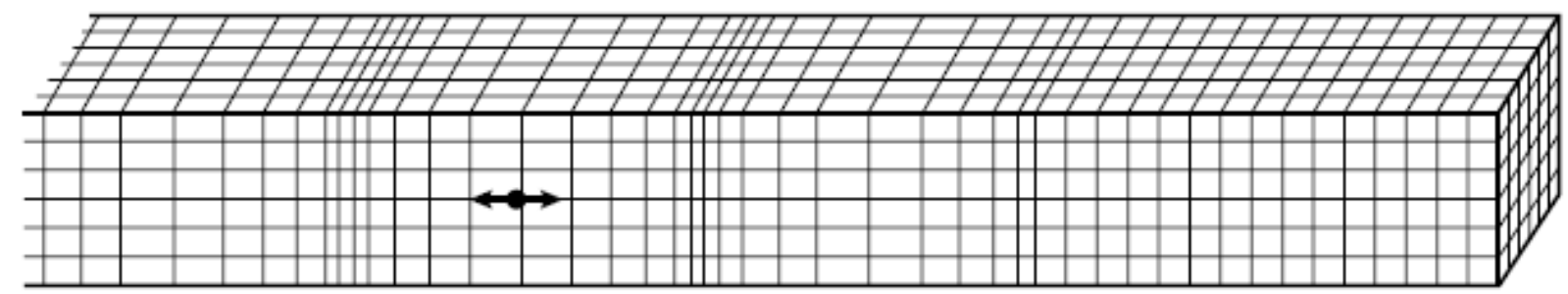

Figure 1: Elastic deformation and particle motion associated with the passage of P-waves. (Kearey, Brooks and Hill, 2002). 


\subsubsection{Shear waves}

The shear waves (S-waves) travel in a transversal form, with the direction of the particle motion perpendicular to the wave propagation axis, shearing the medium by which they travel (Figure 2). Then, the S-wave travels through the medium with an inferior velocity than the P-wave, and it is not capable of traveling through liquid and gas.

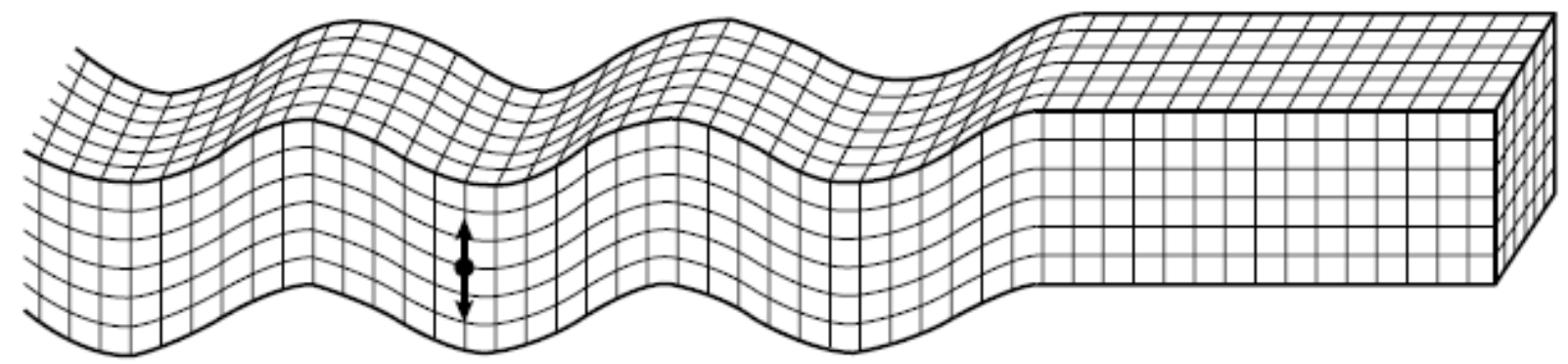

Figure 2: Elastic deformation and particle motion associated with the passage of S-waves. (Kearey, Brooks and Hill, 2002)

\subsection{Multicomponent seismic}

Multicomponent seismic differs from the conventional seismic by the use of not only P-wave record, but also Sv-waves (vertically polarized S-waves), and Sh-waves (horizontally polarized S-waves), which can be separated from P-wave movement. Therefore, converted waves events as PSv and SvP can be recorded.

The seismic processing for the multicomponent seismic data is much more complicated, because five processing sequences are necessary (Figure 3), one for each kind of reflection event (PP, SvSv, ShSh, PSv and SvP), once each one represents different frequency and velocity contents. Besides, each kind of reflection event presents different magnitudes of random and coherent noises. 


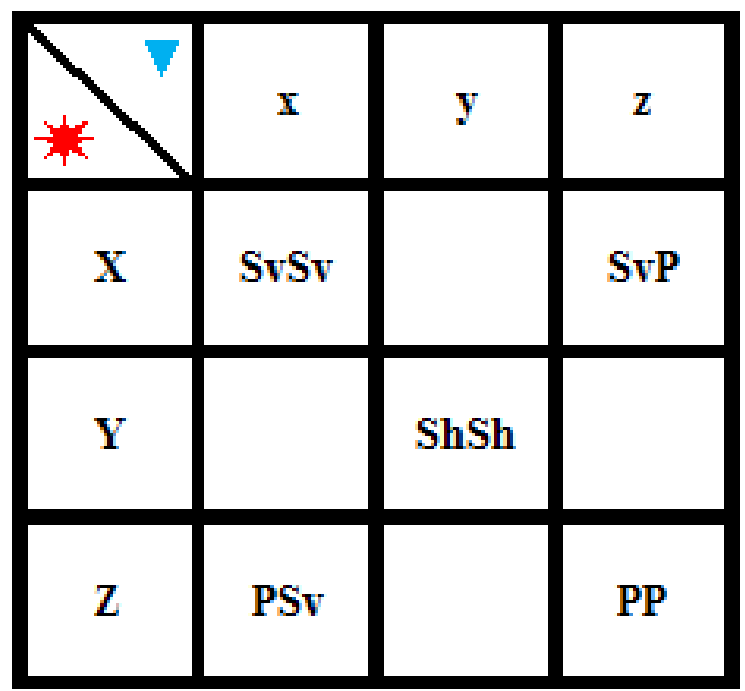

Figure 3: Representation of the recorded components in a Cartesian coordinate system (XYZ). The red star represents the sources and the blue triangle represents the receivers. (Zuniga, 2017).

\subsubsection{Wave conversion}

When a P-wave reaches an interface between two layers, it can reflect or refract as an S-wave (and vice versa), and then generate a converted wave. Hence, in a case of converted wave event, the incident wave ray has a velocity and an angle different than the emergent wave ray (Figure 4). This kind of situation results in a nonhyperbolic event due to the ray traces asymmetry between the incident ray and the emergent ray.

(a)

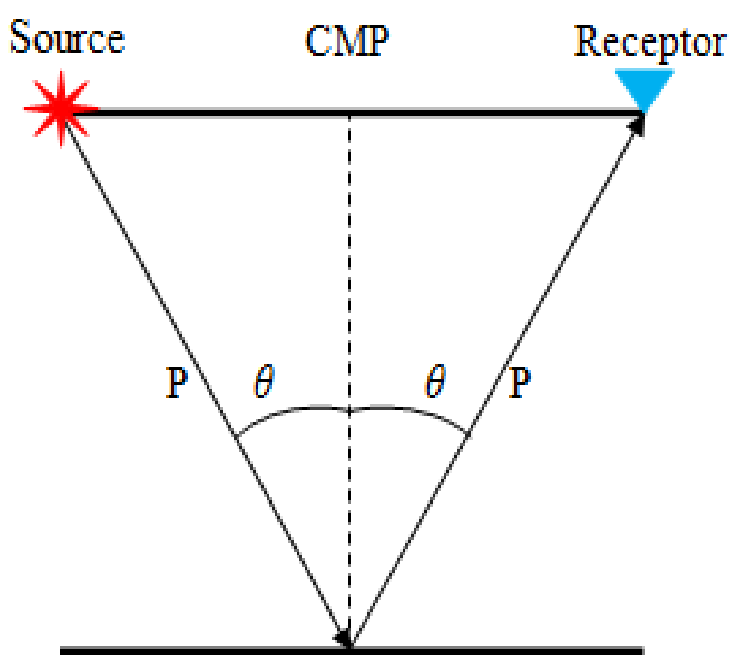

(b)

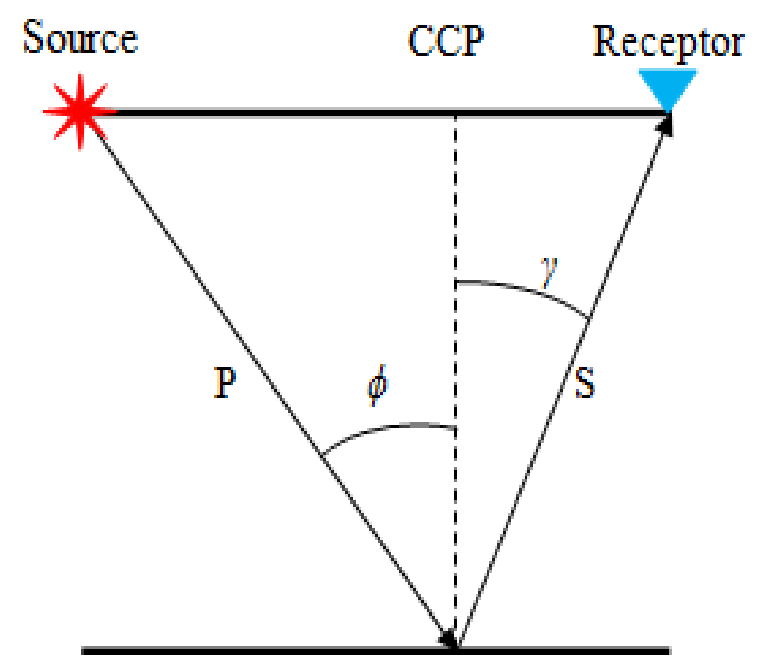

Figure 4: (a) Wave reflection at the common mid-point (CMP); (b) PSv wave reflection at the common conversion point (CCP). (Zuniga, 2017). 


\subsection{OBN technology}

The OBN (Ocean Bottom Nodes) technology uses the source on the surface and the receivers on the bottom of the ocean (Figure 5). The conventional offshore acquisition technology (Streamer) is different due to the fact it uses the source and the receiver on the surface of the ocean, which makes acquiring multicomponent seismic data impossible, once the S-waves cannot travel through the water. However, the using of OBN technology generates a ray trace asymmetry concerning the difference of datum between source and receiver resulting in a nonhyperbolic event.

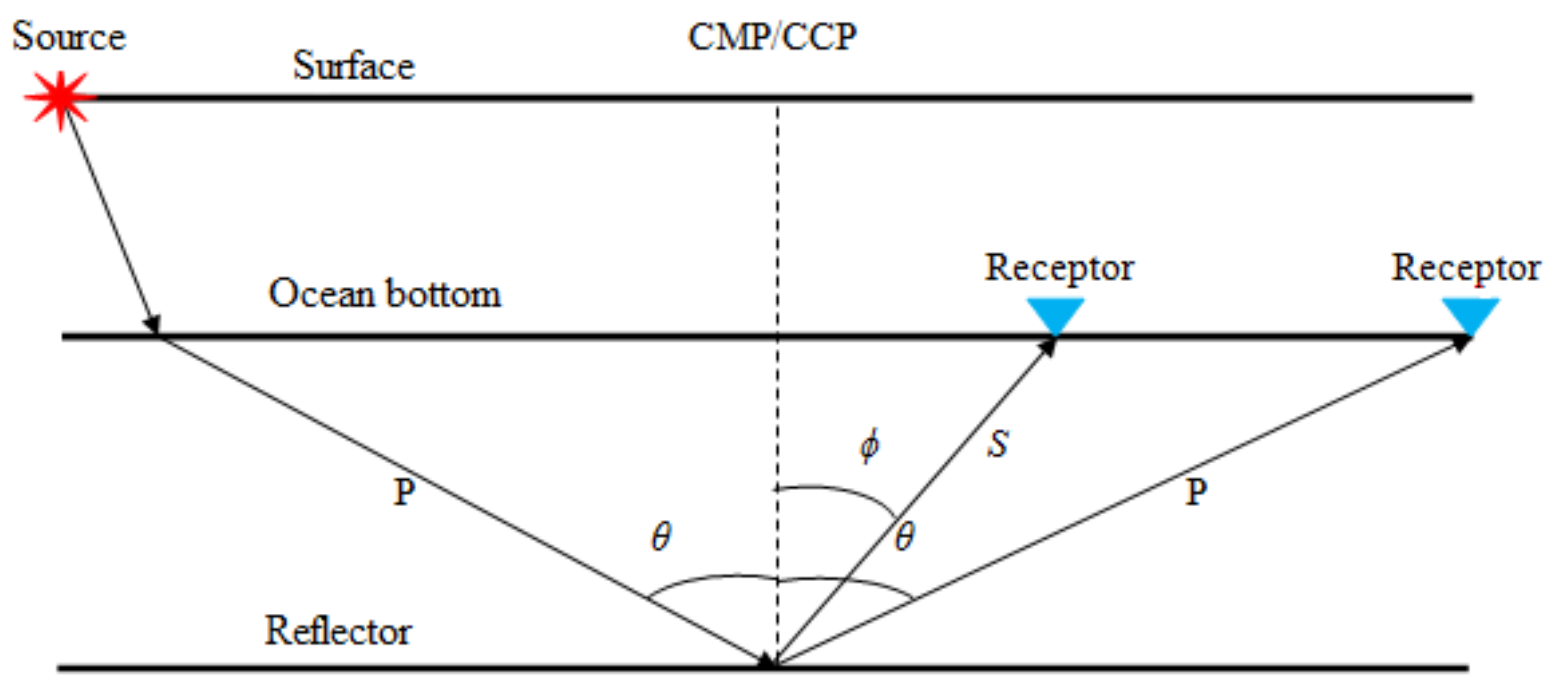

Figure 5: PP and PS wave reflection events with the use of OBN technology. (Zuniga, 2017).

\subsection{RMS velocity}

The RMS (Root Mean Square) velocity is the quadratic mean of the interval velocities. As, during the seismic processing, the RMS velocity is used, it is natural to have an error associated to the difference between the RMS velocity and the set of interval velocities. This error increases while the offset is increased (Figure 6), and then for offsets larger than the reflector depth, the hyperbolic approach is not valid anymore. 


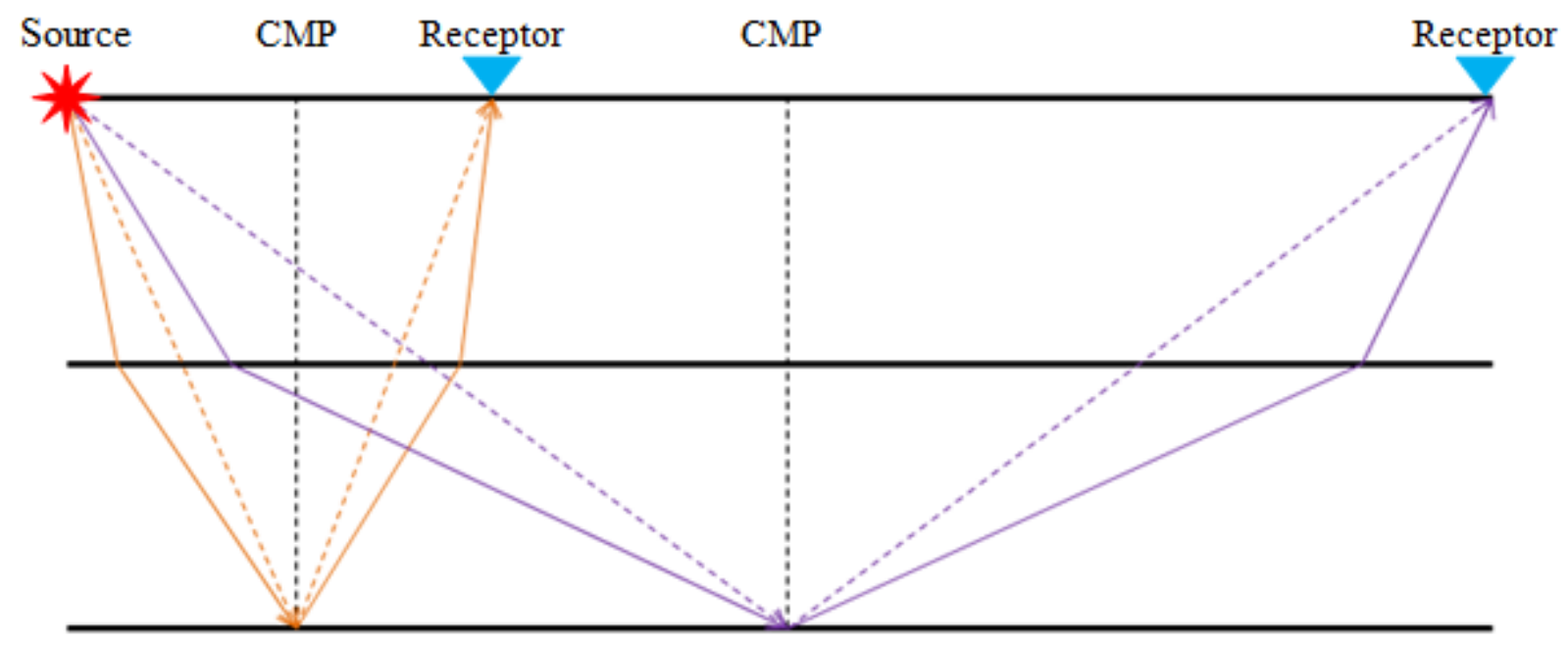

Figure 6: Schematization demonstrating how the RMS velocity differs from the set of interval velocities as the offset is increased. Solid lines represent the interval velocities and the dotted lines represent the RMS velocity. (Zuniga,2017). 


\section{NONHYPERBOLIC EXPRESSIONS TO PERFORM VELOCITY} ANALYSIS

Nonhyperbolic travel-time events cannot be described by the conventional hyperbola expression, and, for this reason, it is necessary to use approximations which are able to describe the nonhyperbolicity. Most of them use one more parameter to describe the nonhyperbolicity related to their specific origin.

In this work, a generalization of a nonhyperbolic multiparametric travel-time approximation for converted-wave is proposed to consider the also the nonhyperbolic effect generated by the difference of datum between source and receivers.

\subsection{Proposed approximation}

The main complication to process multicomponent seismic data concerns the ray tracing asymmetry associated to the converted wave. However, we can describe this relation between the incident P-wave ray and the reflected S-wave ray, with Snell's law,

$$
\frac{\sin \theta_{P}}{V_{P}}=\frac{\sin \theta_{S}}{V_{S}}=p
$$

where $V_{P}$ is the $\mathrm{P}$-wave velocity, $V_{S}$ is the $\mathrm{S}$-wave velocity, $\theta_{P}$ is the angle between the $\mathrm{P}$-wave incident ray and the normal component, $\theta_{S}$ is the angle between the S-wave reflected emergent ray and the normal component, and $p$ is the ray parameter which is constant along the ray for isotropic media. The ray parameter for this situation is described by

$$
p=\frac{d t_{C}}{d x}
$$

where $t_{C}$ is the arrival time of the converted wave at an offset $x$.

The sum of the one-way oblique travel-time through the layer for the P-wave $\left(t_{P}\right)$ and the one-way oblique travel-time through the layer for the $S$-wave $\left(t_{S}\right)$ results in the converted wave arrival time, written as 


$$
t_{C}=t_{P}+t_{S}
$$

The time which an incident ray of a P-wave takes to reach the reflector is described by

$$
t_{P}=\frac{z}{V_{P} \cos \theta_{P}}
$$

where $z$ is the reflector depth considering the source and the receiver at the same datum.

There is also the time that the reflected ray of an S-wave takes to reach the receiver after the reflection, described as

$$
t_{S}=\frac{z}{V_{S} \cos \theta_{S}}
$$

The $t_{C}$ is therefore described as the sum of the Equations 4 and 5, and can be written as

$$
t_{C}=\frac{z}{V_{P} \cos \theta_{P}}+\frac{z}{V_{S} \cos \theta_{S}}
$$

Similar to Equation 6, the offset $x$ is given by

$$
x=V_{P} t_{P} \sin \theta_{P}+V_{S} t_{S} \sin \theta_{S}=p V_{P}^{2} t_{P}+p V_{S}^{2} t_{S} .
$$

For more complex cases, the approximations derived from the Taylor series must be used (Tsvankin and Thomsen, 1994; Alkhalifah and Tsvankin, 1995; Thomsen, 1999). The expansion of the expression in $t^{2}$ versus $x^{2}$ results in

$$
t_{C}^{2}=t_{C 0}^{2}+\frac{x^{2}}{V_{C 2}^{2}}+A_{4} x^{4}+\cdots
$$

where $A_{4}$ is a non-independent term from the expansion. The $A_{4}$ is going to be later explained in this chapter. $V_{C 2}$ denotes the RMS stacking velocity of the converted wave.

To consider the converted wave travel-time for a zero offset $\left(t_{C 0}\right)$, the relation must be initially written as

$$
t_{C 0}=t_{P 0}+t_{S 0}
$$


where $t_{P 0}$ and $t_{S 0}$ are, for a zero offset, the one-way travel-time of a P-wave and an S-wave, respectively.

The Equation 9 can be expressed and rewritten as

$$
t_{C 0}=t_{P 0}+\frac{t_{P 0} t_{S 0}}{t_{P 0}}=t_{P 0}\left(1+\frac{t_{S 0}}{t_{P 0}}\right)
$$

Thomsen (1999) introduced a parameter as the ratio between $t_{P 0}$ and $t_{S 0}$, written as

$$
\gamma_{0}=\frac{V_{P}}{V_{S}}=\frac{\frac{Z}{t_{P 0}}}{\frac{Z}{t_{S 0}}}=\frac{t_{S 0}}{t_{P 0}} .
$$

Substituting Equation 11 into Equation 10 gives

$$
t_{C 0}=t_{P 0}\left(1+\gamma_{0}\right)
$$

For anisotropic cases, the short-spread moveout velocities are affected by the anisotropy. Tsvankin and Thomsen (1994) proposed that this influence could be characterized by the relation among the vertical $\mathrm{P}$-wave and $\mathrm{S}$-wave velocities and the independent anisotropic parameters $\delta$ and $\sigma$. These relations are given by

$$
V_{P 2}^{2}=V_{P 0}^{2}(1+2 \delta)
$$

and

$$
V_{S 2}^{2}=V_{S 0}^{2}(1+\sigma)
$$

Here, $V_{P 2}$ and $V_{S 2}$ are $\mathrm{P}$-wave and $\mathrm{S}$-wave RMS stacking velocities, respectively.

Tsvankin and Thomsen (1994) also determined that if there are many anisotropic layers, the squared vertical velocities should be considered as the RMS vertical velocities.

The ratio between $V_{P 2}$ and $V_{S 2}$ was introduced by Thomsen (1999) as

$$
\gamma_{2}=\frac{V_{P 2}}{V_{S 2}}
$$

Thomsen (1999) introduced the ratio between the squared $\gamma_{2}$ from the Equation 15 and $\gamma_{0}$ from Equation 11 as 


$$
\gamma_{e f f}=\frac{\gamma_{2}^{2}}{\gamma_{0}}
$$

Replacing Equation 15 into Equation 16 gives

$$
\gamma_{e f f}=\frac{\gamma_{2}^{2}}{\gamma_{0}}=\frac{\frac{V_{P 2}^{2}}{V_{S 2}^{2}}}{\frac{t_{S 0}}{t_{P 0}}}=\frac{V_{P 2}^{2}}{V_{S 2}^{2}} \frac{1}{\gamma_{0}} .
$$

For a multilayered media, Li and Yuan (2001 and 2003) described the one-way traveltime of a P-wave and an S-wave for a zero offset as

$$
t_{P 0}=\sum_{i=1}^{n} \Delta t_{P 0 i}
$$

and

$$
t_{S 0}=\sum_{i=1}^{n} \Delta t_{S 0 i}
$$

Where $\Delta t_{P 0 i}$ and $\Delta t_{S 0 i}$ are the travel-times of each layer for, respectively, P-waves and Swaves.

Introducing $V_{P 2}^{2}$ and $V_{S 2}^{2}$, respectively, into Equations 18 and 19 and reorganizing them gives

$$
V_{P 2}^{2}=\frac{1}{t_{P 0}} \sum_{i=1}^{n} V_{P 2}^{2} \Delta t_{P 0 i}
$$

and

$$
V_{S 2}^{2}=\frac{1}{t_{S 0}} \sum_{i=1}^{n} V_{S 2}^{2} \Delta t_{S 0 i}
$$

Then, these relations with Equation 9 give

$$
t_{C 0} V_{C 2}^{2}=t_{P 0} V_{P 2}^{2}+t_{S 0} V_{S 2}^{2}
$$


Replacing Equation 12 into Equation 22 gives

$$
t_{P 0}\left(1+\gamma_{0}\right) V_{C 2}^{2}=t_{P 0} V_{P 2}^{2}+t_{S 0} V_{S 2}^{2}
$$

Rearranging Equation 23 gives

$$
V_{C 2}^{2}=\frac{t_{P 0} V_{P 2}^{2}+t_{P 0} \gamma_{0} V_{S 2}^{2}}{t_{P 0}\left(1+\gamma_{0}\right)},
$$

and therefore it results in

$$
V_{C 2}^{2}=\frac{V_{P 2}^{2}+\gamma_{0} V_{S 2}^{2}}{\left(1+\gamma_{0}\right)}
$$

Rearranging Equation 25 into

$$
V_{S 2}^{2}=\frac{\left(1+\gamma_{0}\right) V_{C 2}^{2}-V_{P 2}^{2}}{\gamma_{0}}
$$

and replacing it into Equation 17 gives

$$
\gamma_{e f f}=\frac{V_{P 2}^{2}}{\frac{\left(1+\gamma_{0}\right) V_{C 2}^{2}-V_{P 2}^{2}}{\gamma_{0}} \gamma_{0}},
$$

and simplifying it results in

$$
\gamma_{e f f}=\frac{V_{P 2}^{2}}{\left(1+\gamma_{0}\right) V_{C 2}^{2}-V_{P 2}^{2}}
$$

The rearrangement of Equation 28 to evidence $V_{P 2}^{2}$ is given by

$$
V_{P 2}^{2}=\gamma_{e f f}\left[\left(1+\gamma_{0}\right) V_{C 2}^{2}-V_{P 2}^{2}\right]
$$

and distributing $\gamma_{e f f}$ gives

$$
V_{P 2}^{2}=\gamma_{e f f}\left(1+\gamma_{0}\right) V_{C 2}^{2}-\gamma_{e f f} V_{P 2}^{2}
$$

and hence results in

$$
V_{P 2}^{2}+\gamma_{e f f} V_{P 2}^{2}=\gamma_{e f f}\left(1+\gamma_{0}\right) V_{C 2}^{2}
$$


and evidencing $V_{P 2}^{2}$ gives

$$
V_{P 2}^{2}\left(1+\gamma_{e f f}\right)=\gamma_{e f f}\left(1+\gamma_{0}\right) V_{C 2}^{2}
$$

which finally leads to

$$
V_{P 2}^{2}=\frac{V_{C 2}^{2} \gamma_{e f f}\left(1+\gamma_{0}\right)}{\left(1+\gamma_{e f f}\right)}
$$

Rearranging Equation 17 into

$$
V_{S 2}^{2}=\frac{V_{P 2}^{2}}{\gamma_{0} \gamma_{e f f}},
$$

and replacing Equation 33 into Equation 34 gives

$$
V_{S 2}^{2}=\frac{V_{C 2}^{2} \gamma_{e f f}\left(1+\gamma_{0}\right)}{\left(1+\gamma_{e f f}\right) \gamma_{0} \gamma_{e f f}},
$$

and simplifying it results in

$$
V_{S 2}^{2}=\frac{V_{C 2}^{2}\left(1+\gamma_{0}\right)}{\left(1+\gamma_{e f f}\right) \gamma_{0}}
$$

Introducing the anisotropic parameters $\eta$ and $\zeta$, proposed by Tsvankin and Thomsen (1994) and used by Alkhalifah and Tsvankin (1995), which respectively control the anisotropic behaviour in long-spread P-wave reaction moveout, and controls the anisotropic behaviour in intermediate-spread S-wave moveout, there are

$$
\eta_{i}=\frac{\epsilon_{i}-\delta_{i}}{\left(1+2 \delta_{i}\right)^{2}}\left(1+\frac{2 \delta_{i}}{1-\frac{V_{S 0 i}^{2}}{V_{P 0 i}^{2}}}\right)
$$

and

$$
\zeta_{i}=\frac{\sigma_{i}}{\left(1+2 \sigma_{i}\right)^{2}}\left(1+\frac{2 \delta_{i}}{1-\frac{V_{S 0 i}^{2}}{V_{P 0 i}^{2}}}\right)
$$


where $\sigma$ is described by

$$
\sigma_{i}=\gamma_{0}^{2}\left(\epsilon_{i}-\delta_{i}\right)
$$

and where $\epsilon$ quantifies the difference between the wave velocities along the symmetry axis and perpendicular to the symmetry axis, and $\delta$ describes the $\mathrm{P}$-wave propagation for angles close to the symmetry axis.

Alkhalifah and Tsvankin (1995) found that the effect of the term $V_{S 0 i}^{2} / V_{P 0 i}^{2}$ is ignorable for long-spread P-wave propagation, which reduces the Equation 37 to

$$
\eta_{i}=\frac{\epsilon_{i}-\delta_{i}}{\left(1+2 \delta_{i}\right)^{2}}\left(1+2 \delta_{i}\right)=\frac{\epsilon_{i}-\delta_{i}}{1+2 \delta_{i}}
$$

Yuan (2002) observed that, for intermediate-spread S-wave propagation, the effect of the term $V_{S 0 i}^{2} / V_{P 0 i}^{2}$ can also be ignored for Equation 38, and after rearranging, it gives

$$
\zeta_{i}=\gamma_{e f f}^{2} \eta_{i}
$$

To generate the generalized equation, the conversion-point offset $\left(x_{c}\right)$ for a converted wave ray which was converted at the bottom of the $n$-th layer (the layer above the reflection) and was reflected emerging at offset $x$ must be considered. It can be described by

$$
x_{c}=x\left(c_{0}+\frac{c_{2} x^{2}}{1+c_{3} x^{2}}\right) \text {, }
$$

where Thomsen (1999) described that $c_{3}=c_{2} /\left(1-c_{0}\right)$.

The coefficients $c_{0}$ and $c_{2}$ must be derived, and it starts with the consideration proposed by Li and Yuan (2003) that $x_{P}=x_{C}$. Then there is the relation of the total offset ray path described as

$$
x=x_{P}+x_{S}
$$

where 


$$
x_{P}=\sum_{i=1}^{n} \Delta x_{P i}=p \sum_{i=1}^{n} \mathrm{U}_{P i} \Delta t_{P i}
$$

and

$$
x_{S}=\sum_{i=1}^{n} \Delta x_{S i}=p \sum_{i=1}^{n} \mathrm{U}_{S i} \Delta t_{S i}
$$

The $U_{i}$ can be described as

$$
U_{i}=\frac{w_{1 i}}{p}
$$

where $w_{1 i}$ is the horizontal component of the group velocity vector ( $\mathrm{Li}$ and Yuan, 2001 and 2003).

As $x_{P}$ is an odd function of $x$, the Taylor expansion of the conversion point offset $x_{P}$ at the total offset $x$ can be described by

$$
x_{P}=\lim _{x \rightarrow 0} \sum_{k=0}^{\infty} \mathrm{c}_{2 k} x^{2 k+1}=\lim _{p \rightarrow 0} \sum_{k=0}^{\infty} \mathrm{c}_{2 k} x^{2 k+1},
$$

where

$$
c_{k 2}=\frac{1}{(2 k+1) !} \frac{d^{2 k+1} x_{p}}{d x^{2 k+1}}
$$

For $k=0, \mathrm{c}_{0}$ can be described by

$$
\mathrm{c}_{0}=\lim _{p \rightarrow 0} \frac{d x_{p}}{d x}=\lim _{p \rightarrow 0} \frac{d x_{p}}{d p} \frac{d p}{d x} .
$$

From Equations 43, 44, 45 and 46,

$$
\frac{d x_{p}}{d p} \frac{d p}{d x}=\frac{b_{1}}{b_{2}}
$$

where 


$$
b_{1}=\sum_{i=1}^{n} U_{P i} \Delta t_{P i}+p \frac{d}{d p}\left(\sum_{i=1}^{n} U_{P i} \Delta t_{P i}\right)
$$

and

$$
b_{2}=\sum_{i=1}^{n} U_{P i} \Delta t_{P i}+p \frac{d}{d p}\left(\sum_{i=1}^{n} U_{P i} \Delta t_{P i}\right)+\sum_{i=1}^{n} U_{S i} \Delta t_{S i}+p \frac{d}{d p}\left(\sum_{i=1}^{n} U_{S i} \Delta t_{S i}\right),
$$

and where the differential terms are given by

$$
p \frac{d}{d p}\left(\sum_{i=1}^{n} U_{P i} \Delta t_{P i}\right)=p \sum_{i=1}^{n} \frac{d U_{P i}}{d p} \Delta t_{P i}+p^{2} \sum_{i=1}^{n} U_{P i}^{2} \Delta t_{P i}
$$

and

$$
p \frac{d}{d p}\left(\sum_{i=1}^{n} U_{S i} \Delta t_{S i}\right)=p \sum_{i=1}^{n} \frac{d U_{S i}}{d p} \Delta t_{S i}+p^{2} \sum_{i=1}^{n} U_{S i} \Delta t_{i}
$$

Tsvankin and Thomsen (1994) adapted for VTI media and found

$$
\lim _{p \rightarrow 0} U_{P i}=v_{P 2 i}^{2}=v_{P 0 i}^{2}\left(1+2 \delta_{i}\right)
$$

and

$$
\lim _{p \rightarrow 0} U_{S i}=v_{S 2 i}^{2}=v_{S 0 i}^{2}\left(1+2 \sigma_{i}\right) .
$$

and they also found

$$
\lim _{p \rightarrow 0} \frac{1}{p} \frac{d U_{P i}}{d p}=\lim _{p \rightarrow 0} \frac{d^{2} U_{P i}}{d p^{2}}=8 v_{P 0 i}^{4}\left(\epsilon_{i}-\delta_{i}\right)\left(1+\frac{2 \delta_{i}}{1-\frac{v_{S 0 i}^{2}}{v_{P 0 i}^{2}}}\right)
$$

and 


$$
\lim _{p \rightarrow 0} \frac{1}{p} \frac{d U_{S i}}{d p}=\lim _{p \rightarrow 0} \frac{d^{2} U_{S i}}{d p^{2}}=-8 v_{S 0 i}^{4} \sigma_{i}\left(1+\frac{2 \delta_{i}}{1-\frac{v_{S 0 i}^{2}}{v_{P 0 i}^{2}}}\right)
$$

For $k=1, c_{2}$ can be described by

$$
c_{2}=\frac{1}{6} \lim _{p \rightarrow 0} \frac{d^{3} x_{P}}{d x^{3}}
$$

where

$$
\frac{d^{3} x_{P}}{d x^{3}}=\frac{d}{d p}\left(\frac{d}{d p}\left(\frac{d x_{P}}{d p} \frac{d p}{d x}\right) \frac{d p}{d x}\right) \frac{d p}{d x}
$$

which is the same as

$$
\frac{d^{3} x_{P}}{d x^{3}}=\frac{B_{1}\left[3 B_{2}+p \frac{d}{d p} B_{2}+\frac{d}{d p}\left(p^{2} B_{3}\right)\right]-B_{4}\left(2 B_{5}+p B_{2}+p^{2} B_{3}\right)}{B_{1}^{5}}
$$

where the coefficients $B_{1}, B_{2}, B_{3}, B_{4}$ and $B_{5}$ are

$$
\begin{gathered}
B_{1}=e_{P}+p f_{P}+e_{S}+p f_{S}, \\
B_{2}=e_{S} g_{P}-e_{P} g_{S}, \\
B_{3}=f_{S} g_{P}-f_{P} g_{S}, \\
B_{4}=f_{P}+p g_{P}+2 f_{S}+p g_{S}
\end{gathered}
$$

and

$$
B_{5}=e_{S} f_{P}-e_{P} g f_{S},
$$

and where the coefficients $e, f$ and $g$ are described as

$$
\begin{gathered}
e=\sum_{i=0}^{n} U_{i} \Delta t_{i}, \\
f=\frac{d e}{d p}=\frac{d}{d p} \sum_{i=0}^{n} U_{i} \Delta t_{i}=\sum_{i=0}^{n} \frac{d U_{i}}{d p} \Delta t_{i}+p \sum_{i=0}^{n} U_{i}^{2} \Delta t_{i},
\end{gathered}
$$


and

$$
g=\frac{d f}{d p}=\sum_{i=0}^{n} U_{i}^{2} \Delta t_{i}=\sum_{i=0}^{n} \frac{d^{2} U_{i}}{d p^{2}} \Delta t_{i}+3 p \sum_{i=0}^{n} U_{i}^{2} \frac{d U_{i}}{d p} \Delta t_{i}+p^{2} \sum_{i=0}^{n} U_{i}^{3} \Delta t_{i}
$$

From Equations 55 and 56 and for the limit of $p \rightarrow 0$, it is possible to find that

$$
\begin{aligned}
& \lim _{p \rightarrow 0} e_{P}=\sum_{i=0}^{n} v_{P 2 i}^{2} \Delta t_{P 0 i}=V_{P 2}^{2} t_{P 0} \\
& \lim _{p \rightarrow 0} e_{S}=\sum_{i=0}^{n} v_{S 2 i}^{2} \Delta t_{S 0 i}=V_{S 2}^{2} t_{S 0}
\end{aligned}
$$

and

$$
\lim _{p \rightarrow 0} f_{P}=\lim _{p \rightarrow 0} f_{S}=0 .
$$

Also, that

$$
\lim _{p \rightarrow 0} g_{P}=\lim _{p \rightarrow 0} \sum_{i=0}^{n} U_{P i}^{2} \Delta t_{P i}+\lim _{p \rightarrow 0} \sum_{i=0}^{n} \frac{d^{2} U_{P i}}{d p^{2}} \Delta t_{P i}
$$

and

$$
\lim _{p \rightarrow 0} g_{s}=\lim _{p \rightarrow 0} \sum_{i=0}^{n} U_{S i}^{2} \Delta t_{S i}+\lim _{p \rightarrow 0} \sum_{i=0}^{n} \frac{d^{2} U_{S i}}{d p^{2}} \Delta t_{S i}
$$

and that they can also be respectively described as

$$
\lim _{p \rightarrow 0} g_{P}=\sum_{i=0}^{n} v_{P 2 i}^{4} \Delta t_{P 0 i}+\sum_{i=0}^{n} 8 v_{P 0 i}^{4}\left(\epsilon_{i}-\delta_{i}\right)\left(1+\frac{2 \delta_{i}}{1-\frac{v_{S 0 i}^{2}}{v_{P 0 i}^{2}}}\right) \Delta t_{P i}
$$

and 


$$
\lim _{p \rightarrow 0} g_{S}=\sum_{i=0}^{n} v_{S 2 i}^{4} \Delta t_{S 0 i}+\sum_{i=0}^{n}-8 v_{S 0 i}^{4} \sigma_{i}\left(1+\frac{2 \delta_{i}}{1-\frac{v_{S 0 i}^{2}}{v_{P 0 i}^{2}}}\right) \Delta t_{P i}
$$

Replacing Equations 70 and 71 into Equation 76, the $c_{2}$ becomes

$$
c_{2}=\frac{1}{6} \lim _{p \rightarrow 0} \frac{d^{3} x_{P}}{d x^{3}}=\lim _{p \rightarrow 0} \frac{e_{S} g_{P}-e_{P} g_{S}}{2\left(e_{P}+e_{S}\right)^{4}},
$$

and $c_{2}$ can also be written as

$$
c_{2}=\frac{1}{2}\left(\frac{Q_{1}}{Q_{3}}-\frac{Q_{2}}{Q_{4}}\right)
$$

where

$$
\begin{gathered}
Q_{1}=V_{S 2}^{2} t_{S 0} \sum_{i=0}^{n}\left\{\Delta t_{P 0 i} v_{P 0 i}^{4}\left[\left(1+2 \delta_{i}\right)^{2}+8\left(\epsilon_{i}-\delta_{i}\right)\left(1+\frac{2 \delta_{i}}{1+\frac{v_{S 0 i}^{2}}{v_{P 0 i}^{2}}}\right)\right]\right\}, \\
Q_{2}=-V_{P 2}^{2} t_{P 0} \sum_{i=0}^{n}\left\{\Delta t_{S 0 i} v_{S 0 i}^{4}\left[\left(1+2 \sigma_{i}\right)^{2}+8 \sigma_{i}\left(1+\frac{2 \delta_{i}}{1+\frac{v_{S 0 i}^{2}}{v_{P 0 i}^{2}}}\right)\right]\right\}, \\
Q_{3}=V_{P n 2}^{2} t_{P 0}+V_{S n 2}^{2} t_{S 0},
\end{gathered}
$$

and

$$
Q_{4}=V_{P 2}^{2} t_{P 0}+V_{S 2}^{2} t_{S 0}
$$

Replacing the Equations from 36 to 39 into the extended Equation 49 gives

$$
c_{0}=\sum_{i=1}^{n} V_{P 2 i}^{2} \Delta t_{P 0 i} /\left(\sum_{i=1}^{n} V_{P 2 i}^{2} \Delta t_{P 0 i}+\sum_{i=1}^{n} V_{S 2 i}^{2} \Delta t_{P 0 i}\right) .
$$


Replacing Equations 37 and 38 into the extended Equation 78 gives

$$
\begin{gathered}
c_{2}=\left\{\left(\sum_{i=1}^{n} V_{S 2 i}^{2} \Delta t_{S 0 i}\right)\left[\sum_{i=1}^{n} V_{P 2 i}^{2} \Delta t_{P 0 i}\left(1+8 \eta_{i}\right)\right]\right. \\
\left.-\left(\sum_{i=1}^{n} V_{P 2 i}^{2} \Delta t_{P 0 i}\right)\left[\sum_{i=1}^{n} V_{S 2 i}^{2} \Delta t_{S 0 i}\left(1+8 \zeta_{i}\right)\right]\right\} \\
\times \frac{1}{2}\left(\sum_{i=1}^{n} V_{P 2 i}^{2} \Delta t_{P 0 i}+\sum_{i=1}^{n} V_{S 2 i}^{2} \Delta t_{S 0 i}\right)^{-4} .
\end{gathered}
$$

Replacing Equations 9, 11 and from 15 to 22 into Equations 83 and 84, and introducing the parameters

$$
\eta_{e f f}=\frac{1}{8 t_{P 0} V_{P 2}^{4}}\left[\sum_{i=1}^{n} V_{P 2 i}^{4}\left(1+8 \eta_{i}\right) \Delta t_{P 0 i}-t_{P 0} V_{P 2}^{4}\right]
$$

and

$$
\zeta_{\text {eff }}=\frac{1}{8 t_{S 0} V_{S 2}^{4}}\left[t_{S 0} V_{S 2}^{4}-\sum_{i=1}^{n} V_{S 2 i}^{4}\left(1-8 \zeta_{i}\right) \Delta t_{S 0 i}\right]
$$

results in

$$
c_{0}=\frac{\gamma_{e f f}}{1+\gamma_{e f f}}
$$

and

$$
c_{2}=\frac{\gamma_{e f f}\left(1+\gamma_{0}\right)}{2 t_{C 0}^{2} V_{C 2}^{2} \gamma_{0}\left(1+\gamma_{e f f}\right)^{3}}\left[\gamma_{0} \gamma_{e f f}-1+8\left(\eta_{e f f} \gamma_{0} \gamma_{e f f}+\zeta_{e f f}\right)\right]
$$

Li and Yuan (2003) verified that $\eta_{e f f}$, which is the effective anisotropic parameter for a P-wave in a multilayered media, is the same introduced by Alkhalifah and Tsvankin (1995). They also verified that $\zeta_{\text {eff }}$ is the corresponding parameter for vertical $\mathrm{S}$-wave in a layered VTI media. Hence, $\eta_{\text {eff }}$ can be reduced to $\eta$ and $\zeta_{\text {eff }}$ can be reduced to $\zeta$, for a single-layered VTI medium. Then, replacing Equations 40 and 41 into Equation 88, the latter is reduced to 


$$
c_{2}=\frac{\gamma_{e f f}\left(1+\gamma_{0}\right)}{2 t_{C 0}^{2} V_{C 2}^{2} \gamma_{0}\left(1+\gamma_{e f f}\right)^{3}}\left[\gamma_{0} \gamma_{e f f}-1+8 \gamma_{e f f} \eta\left(\gamma_{0}+\gamma_{e f f}\right)\right]
$$

In a case for a layered isotropic medium, Li and Yuan (2003) considered

$$
\eta_{e f f}=\frac{1}{8 t_{P 0} V_{P 2}^{4}}\left[\sum_{i=1}^{n} V_{P 2 i}^{4} \Delta t_{P 0 i}-t_{P 0} V_{P 2}^{4}\right]
$$

and

$$
\zeta_{e f f}=\frac{1}{8 t_{S 0} V_{S 2}^{4}}\left[t_{S 0} V_{S 2}^{4}-\sum_{i=1}^{n} V_{S 2 i}^{4} \Delta t_{S 0 i}\right]
$$

Li and Yuan (2003) found that for this case, $\eta_{\text {eff }}$ and $\zeta_{\text {eff }}$ control respectively the residual effects on $\mathrm{P}$-waves and $\mathrm{S}$-waves caused by layering. Therefore, to disregard these effects, the Equation 89 can be reduced to

$$
c_{2}=\frac{\gamma_{e f f}\left(1+\gamma_{0}\right)\left(\gamma_{0} \gamma_{e f f}-1\right)}{2 t_{C 0}^{2} V_{C 2}^{2} \gamma_{0}\left(1+\gamma_{e f f}\right)^{3}} .
$$

Working with the generalized equation proposed by Tsvankin and Thomsen (1994) for $t_{C}$, the converted wave moveout which suffered the conversion at the bottom of the $n$-th layer and emerged at the offset $x$, it is found that

$$
t_{C}^{2}=t_{C 0}^{2}+\frac{x^{2}}{V_{C 2}^{2}}+\frac{A_{4} x^{4}}{1+A_{5} x^{2}} .
$$

To find the non-independent parameter $A_{4}$, the development proposed by Tsvankin and Thomsen (1994) and Li and Yuan (2003) must be followed. Initially considering

$$
A_{4}=t_{C 0} V_{C 2}^{4}-\left[\sum_{i=1}^{n}\left(H_{P i}+v_{P 2 i}^{4}\right) \Delta t_{P 0 i}+\sum_{i=1}^{n}\left(H_{S i}+v_{S 2 i}^{4}\right) \Delta t_{S 0 i}\right] / 4 t_{C 0}^{3} V_{C 2}^{8},
$$

where 


$$
H_{P i}=8 v_{P 2 i}^{4}\left(\epsilon_{i}-\delta_{i}\right)\left(1+\frac{2 \delta_{i}}{1-\frac{1}{\gamma_{0 i}^{2}}}\right)
$$

and

$$
H_{S i}=-8 v_{S 2 i}^{4} \sigma_{i}\left(1+\frac{2 \delta_{i}}{1-\frac{1}{\gamma_{0 i}^{2}}}\right)
$$

Replacing Equations from 37 to 39 into Equations 95 and 96, it is possible to obtain

$$
H_{P i}=8 v_{P 2 i}^{4} \eta_{i}
$$

and

$$
H_{S i}=-8 v_{S 2 i}^{4} \zeta_{i}
$$

Introducing the definitions of Equation 85 and 86 respectively into Equations 97 and 98 results in

$$
\sum_{i=1}^{n}\left(H_{P i}+v_{P 2 i}^{4}\right) \Delta t_{P 0 i}=\left(1-8 \eta_{e f f}\right) t_{P 0} V_{P 2}^{4}
$$

and

$$
\sum_{i=1}^{n}\left(H_{S i}+v_{S 2 i}^{4}\right) \Delta t_{S 0 i}=\left(1-8 \zeta_{e f f}\right) t_{S 0} V_{S 2}^{4}
$$

With the replacement of the Equations 99 and 100 into Equation 94, it is possible to obtain

$$
A_{4}=\frac{t_{C 0} V_{C 2}^{4}-\left[\left(1-8 \eta_{e f f}\right) t_{P 0} V_{P 2}^{4}+\left(1-8 \zeta_{e f f}\right) t_{S 0} V_{S 2}^{4}\right]}{4 t_{C 0}^{3} V_{C 2}^{8}}
$$


Replacing and rearranging the parameters $V_{C 2}, V_{P 2}, V_{S 2}, t_{C 0}, t_{P 0}$ and $t_{S 0}$ into Equation 101 give

$$
A_{4}=\frac{\left(\gamma_{0} \gamma_{e f f}-1\right)^{2}+8\left(1+\gamma_{0}\right)\left(\eta_{e f f} \gamma_{0} \gamma_{e f f}^{2}-\zeta_{e f f}\right)}{4 t_{C 0}^{2} V_{C 2}^{4} \gamma_{0}\left(1+\gamma_{e f f}\right)^{2}}
$$

The non-independent coefficient $A_{5}$ is given by

$$
A_{5}=\frac{A_{4}}{\frac{1}{V_{P h}^{2}}-\frac{1}{V_{C 2}^{2}}}
$$

where $V_{P h}$ is the horizontal P-wave velocity.

Combining the parameters $\eta_{e f f}, \gamma_{e f f}, \gamma_{0}$ and $\zeta_{e f f}$ gives

$$
\chi_{e f f}=\eta_{e f f} \gamma_{0} \gamma_{e f f}^{2}-\zeta_{e f f}
$$

The Equation 104, for a single VTI layer, can be reduced to

$$
\chi=\eta \gamma_{e f f}^{2}\left(\gamma_{0}-1\right)
$$

Replacing Equation 105 into Equation 102 results in

$$
A_{4}=\frac{\left(\gamma_{0} \gamma_{e f f}-1\right)^{2}+8 \chi_{e f f}\left(1+\gamma_{0}\right)}{4 t_{C 0}^{2} V_{C 2}^{4} \gamma_{0}\left(1+\gamma_{e f f}\right)^{2}}
$$

Li and Yuan (2003) suggested an empirical relation between $\zeta_{\text {eff }}$ and $\chi_{\text {eff }}$, given by

$$
V_{P h}=V_{P 2} \sqrt{1+2 \eta}
$$

and where it can be approximated to

$$
V_{P h}=V_{P 2} \sqrt{1+\frac{2 \chi_{e f f}}{\gamma_{e f f}^{2}\left(\gamma_{0}-1\right)}}
$$

The Equation 108 is a good approximation for layered media, even being considered only for single VTI media. Thus, replacing Equations 106 and 108 into Equations 33 and 103 results in 


$$
A_{5}=\frac{A_{4} V_{C 2}^{2} \gamma_{e f f}\left(1+\gamma_{0}\right)\left[\left(\gamma_{0}-1\right) \gamma_{e f f}^{2}+2 \chi_{e f f}\right]}{\gamma_{e f f}^{2}\left(\gamma_{0}-1\right)\left(1-\gamma_{0} \gamma_{e f f}\right)-2 \gamma_{e f f} \chi_{e f f}\left(1+\gamma_{0}\right)} .
$$

For the case of a single VTI layer, it is possible to obtain an $A_{4}$, described as

$$
A_{4}=\frac{-1}{t_{C 0}^{2} V_{C 2}^{2}\left(1+\gamma_{e f f}\right)^{2}}\left[\frac{2 \eta \gamma_{e f f}^{2}\left(\gamma_{0}^{2}-1\right)}{\gamma_{0}}+\frac{\left(\gamma_{2}^{2}-1\right)^{2}}{4 \gamma_{0}}\right] \text {. }
$$

However, for isotropic media, $\gamma_{e f f}=\gamma_{0}$ and $\chi_{e f f}=0$. Thus, the coefficients $A_{4}$ and $A_{5}$ can be reduced to

$$
A_{4}=\frac{-\left(\gamma_{0}-1\right)^{2}}{4 t_{C 0}^{2} V_{C 2}^{4} \gamma_{0}}
$$

and

$$
A_{5}=\frac{A_{4} V_{C 2}^{2} \gamma_{0}}{1-\gamma_{0}}
$$

As the approximations for isotropic cases showed good results in previous works, even a simplified version is a reliable approximation to be considered with three parameters. However, it does not consider a difference of datum between source and receiver, which leads to the use of the relation proposed by Wang and Pham (2001). Initially, they consider that the total travel-time is given by

$$
t=t_{W D}+t_{L S},
$$

where $t_{W D}$ is the one-way travel-time of the P-wave ray along the water depth, and the $t_{L S}$ is the travel-time of the P-wave from the bottom of the ocean to the reflection point and then reflected as $\mathrm{S}$-wave to the receptor. The reflection point as the bottom of the $n$-th layer is considered, once Wang and Pham (2001) do not consider stratified media as their initial premises.

Considering the same relations applied to the distances and using the Pythagoras theorem, it is possible to find

$$
x_{W D}^{2}=\left(t_{W D} V_{W D}\right)^{2}=\left(x-x_{L S}\right)^{2}+z_{W D}^{2},
$$


where $x_{W D}$ is the distance that the $\mathrm{P}$-wave ray traveled until reaching the bottom of the ocean, $V_{W D}$ is the velocity of the P-wave in the water, $x_{L S}$ is offset between the point at which the Pwave reached the bottom of the ocean and the receiver which received the S-wave, and $z_{W D}$ is the water depth.

Rearranging the Equation 114 gives

$$
t_{W D}=\frac{\sqrt{\left(x-x_{L S}\right)^{2}+z_{W D}^{2}}}{V_{W D}} .
$$

The conventional static shift approach assumes $x_{L S}$ equal to $x$. However, considering a straight ray path from the source until reaching the reflection point can be written as

$$
x_{L S}=\frac{x}{1+\frac{Z_{W D}}{t_{C 0} V_{C 2}}} \text {. }
$$

For a stratified medium and with the consideration of a different ray inclination in the water, the relation becomes

$$
x_{L S}=\frac{x}{1+\frac{z_{W D} V_{W D}}{t_{C 0} V_{C 2}^{2}}}
$$

Distinctly from what Wang and Pham (2001) proposed, it can be considered for ultradeep reservoirs as

$$
x=x_{L S}\left(1+\frac{z_{W D} V_{W D}}{t_{C 0} V_{C 2}^{2}}\right) .
$$

Replacing the Equation 118 into Equation 93 gives

$$
t_{C}^{2}=t_{C 0}^{2}+\frac{x^{2}}{V_{C 2}^{2}}\left(1+\frac{z_{W D} V_{W D}}{t_{C 0} V_{C 2}^{2}}\right)^{2}+\frac{A_{4} x^{4}}{1+A_{5} x^{2}\left(1+\frac{z_{W D} V_{W D}}{t_{C 0} V_{C 2}^{2}}\right)^{2}}\left(1+\frac{z_{W D} V_{W D}}{t_{C 0} V_{C 2}^{2}}\right)^{4}
$$

As the depth increases, the hyperbolic term suffers less influence by the shifting, which allows to disregard it for the term $x^{2} / V_{C 2}^{2}$, and reduce the Equation 119 to 


$$
t_{C}^{2}=t_{C 0}^{2}+\frac{x^{2}}{V_{C 2}^{2}}+\frac{A_{4} x^{4}}{1+A_{5} x^{2}\left(1+\frac{z_{W D} V_{W D}}{t_{C 0} V_{C 2}^{2}}\right)^{2}}\left(1+\frac{z_{W D} V_{W D}}{t_{C 0} V_{C 2}^{2}}\right)^{4}
$$

Replacing Equations 111 and 112 into Equation 120 results in

$$
t_{C}^{2}=t_{C 0}^{2}+\frac{x^{2}}{V_{C 2}^{2}}+\frac{-\left(\gamma_{0}-1\right)^{2} x^{4}\left(1+\frac{z_{W D} V_{W D}}{t_{C 0} V_{C 2}^{2}}\right)^{4}}{4 t_{C 0}^{2} V_{C 2}^{4} \gamma_{0}\left[1-\frac{\gamma_{0} V_{C 2}^{2}\left(\gamma_{0}-1\right)^{2} x^{2}}{4 t_{C 0}^{2} V_{C 2}^{4} \gamma_{0}\left(1-\gamma_{0}\right)}\left(1+\frac{z_{W D} V_{W D}}{t_{C 0} V_{C 2}^{2}}\right)^{2}\right]},
$$

and after that, distributing the terms gives

$$
\begin{gathered}
t_{C}^{2}=t_{C 0}^{2}+\frac{x^{2}}{V_{C 2}^{2}} \\
++\frac{-\left(\gamma_{0}-1\right)^{2} x^{4}\left(1+\frac{z_{W D} V_{W D}}{t_{C 0} V_{C 2}^{2}}\right)^{4}}{\left[4 t_{C 0}^{2} V_{C 2}^{4} \gamma_{0}-\frac{\left(4 t_{C 0}^{2} V_{C 2}^{4} \gamma_{0}\right) \gamma_{0} V_{C 2}^{2}\left(\gamma_{0}-1\right)^{2} x^{2}}{4 t_{C 0}^{2} V_{C 2}^{4} \gamma_{0}\left(1-\gamma_{0}\right)}\left(1+\frac{z_{W D} V_{W D}}{t_{C 0} V_{C 2}^{2}}\right)^{2}\right]}
\end{gathered}
$$

and then it can be rewritten as

$$
t_{C}^{2}=t_{C 0}^{2}+\frac{x^{2}}{V_{C 2}^{2}}+\frac{-\left(\gamma_{0}-1\right)^{2} x^{4}\left(1+\frac{z_{W D} V_{W D}}{t_{C 0} V_{C 2}^{2}}\right)^{4}}{\left[4 t_{C 0}^{2} V_{C 2}^{4} \gamma_{0}-\frac{\gamma_{0} V_{C 2}^{2}\left(\gamma_{0}-1\right)\left(\gamma_{0}-1\right) x^{2}}{\left(1-\gamma_{0}\right)}\left(1+\frac{z_{W D} V_{W D}}{t_{C 0} V_{C 2}^{2}}\right)^{2}\right]},
$$

and then as

$$
t_{C}^{2}=t_{C 0}^{2}+\frac{x^{2}}{V_{C 2}^{2}}+\frac{-\left(\gamma_{0}-1\right)^{2} x^{4}\left(1+\frac{z_{W D} V_{W D}}{t_{C 0} V_{C 2}^{2}}\right)^{4}}{\left[4 t_{C 0}^{2} V_{C 2}^{4} \gamma_{0}+\frac{\gamma_{0} V_{C 2}^{2}\left(1-\gamma_{0}\right)\left(\gamma_{0}-1\right) x^{2}}{\left(1-\gamma_{0}\right)}\left(1+\frac{z_{W D} V_{W D}}{t_{C 0} V_{C 2}^{2}}\right)^{2}\right]}
$$

Simplifying Equation 124 gives

$$
t_{C}^{2}=t_{C 0}^{2}+\frac{x^{2}}{V_{C 2}^{2}}+\frac{-\left(\gamma_{0}-1\right)^{2} x^{4}\left(1+\frac{z_{W D} V_{W D}}{t_{C 0} V_{C 2}^{2}}\right)^{4}}{\left[4 t_{C 0}^{2} V_{C 2}^{4} \gamma_{0}+\gamma_{0} V_{C 2}^{2}\left(1-\gamma_{0}\right) x^{2}\left(1+\frac{z_{W D} V_{W D}}{t_{C 0} V_{C 2}^{2}}\right)^{2}\right]},
$$


and rearranging it gives

$$
t=\sqrt{t_{C 0}^{2}+\frac{x^{2}}{V_{C 2}^{2}}+\frac{-\left(\gamma_{0}-1\right)^{2} x^{4}\left(1+\frac{z_{W D} V_{W D}}{t_{C 0} V_{C 2}^{2}}\right)^{4}}{\gamma_{0} V_{C 2}^{2}\left[4 t_{C 0}^{2} V_{C 2}^{2}+\left(1-\gamma_{0}\right) x^{2}\left(1+\frac{z_{W D} V_{W D}}{t_{C 0} V_{C 2}^{2}}\right)^{2}\right]}} .
$$

Replacing and introducing the generic parameters result in

$$
t=\sqrt{t_{0}^{2}+\frac{x^{2}}{V^{2}}+\frac{-(\gamma-1)^{2} x^{4}\left(1+\frac{z_{W D} V_{W D}}{t_{0} V^{2}}\right)^{4}}{\gamma V^{2}\left[4 t_{0}^{2} V^{2}+(1-\gamma) x^{2}\left(1+\frac{z_{W D} V_{W D}}{t_{0} V^{2}}\right)^{2}\right]}},
$$

an approximation with five parameters, three parameters to be recovered and two a priori parameters $Z_{W D}$ and $V_{W D}$.

\subsection{Other approximations}

Each nonhyperbolic approximation used in this thesis has idiosyncratic characteristics, and an additional parameter which aims to perform a better fit considering different premises. These approximations were already previously tested for different models and problems by Zuniga (2015, 2017 and 2018) and Zuniga et al. (2015, 2016, 2017, 2018, 2019a, 2019b and 2019c).

Eight approximations were chosen for this study. The first one is the hyperbola equation, proposed by Dix (1955).

$$
t=\sqrt{t_{0}^{2}+\frac{x^{2}}{v^{2}}}
$$

where $x$ is the vector of offsets between source and receiver, $t_{0}$ is the zero-offset travel-time and $v$ is the RMS (Root Mean Square) velocity.

However, this approximation is treated in this thesis only for a comparison effect, due to the fact it is insufficient for the strong nonhyperbolic conditions studied, once it considers only hyperbolic events. 
The shifted hyperbola (Malovichko, 1978) has the proposition of controlling the effect of large offsets in inhomogeneous media using the $S$ parameter. This equation was also studied and derived by Castle (1988 and 1994) and de Bazelaire (1988), and more recently tested for VTI media (Zuniga et al., 2015), for strong heterogeneity (Zuniga, 2015), and for offshore OBN data (Zuniga, 2017; Zuniga et al., 2015, 2016, 2017, 2018 and 2019a). The shifted hyperbola can be described as

$$
t=t_{0}^{2}\left(1-\frac{1}{S}\right)+\frac{1}{S} \sqrt{t_{0}^{2}+\frac{S x^{2}}{v^{2}}}
$$

where $S$ is the heterogeneity parameter which depends on $\mu_{4}$ and $\mu_{2}$ by the relation

$$
S=\frac{\mu_{4}}{\mu_{2}^{2}} \text {. }
$$

Here, $\mu_{j}(j=2,4)$ is the $j$-th velocity momentum described by

$$
\mu_{j=} \sum_{k=1}^{n} t_{k} v_{k}^{j} / \sum_{k=1}^{n} t_{k}
$$

where $v_{k}$ is the interval velocity of the $k$-th layer and $t_{k}$ is the travel-time of the $k$-th layer.

The next approximation was proposed by Slotboom (1990) to perform the velocity analysis for a nonhyperbolic behaviour from conversion of a $\mathrm{P}$-wave to a vertical S-wave. Once it has no additional parameter to control the nonhyperbolicity in a better way, it brought a less accurate set of results in previous works (Zuniga, 2015; Zuniga et al., 2019), even though it shows a low processing time (Zuniga, 2017).

$$
t=\frac{t_{0}}{2}+\sqrt{\frac{t_{0}^{2}}{2}+\frac{x^{2}}{2 v^{2}}}
$$

However, this approximation is presented just to explain the evolution of the development concerning the nonhyperbolic approximations, once it was tested in previews works and has never shown significant results for complex models (Zuniga et al., 2015 and 2016; Zuniga, 2017).

The following approximation is a very popular equation, broadly used in the petroleum industry. It was proposed by Alkhalifah and Tsvankin (1995) to deal with 
nonhyperbolicity originated in VTI (Vertical Transversal Isotropy) media by anisotropy. This approximation was strongly tested by Zuniga (2015 and 2017).

$$
t=\sqrt{t_{0}^{2}+\frac{x^{2}}{v^{2}}-\frac{2 \eta x^{4}}{v^{2}\left[t_{0}^{2} v^{2}+(1+2 \eta) x^{2}\right]}}
$$

where $\eta$ parameter quantifies the nonhyperbolicity of the event. This parameter represents a determined anisotropy and it is function of the Thomsen anisotropic parameters (Thomsen, 1986) described as

$$
\eta=\frac{\varepsilon-\delta}{1+2 \delta}
$$

where $\varepsilon$ quantifies the difference between the wave velocities along the symmetry axis and perpendicular to the symmetry axis, and $\delta$ represents the propagation of $\mathrm{P}$-wave for angles close to the symmetry axis.

Similar to the shifted hyperbola, the next equation proposes to use the heterogeneity parameter, but in this case, it aims to use the fractional condition of the approximation to obtain a more accurate result of the velocity, once it is more related to the $S$ parameter. However, for this approximation, the authors (Ursin and Stovas, 2006) proposed that the $S$ parameter is expressed in a quasi-acoustic case as a function of the Thomsen anisotropic parameters (Thomsen, 1986). For this reason, it showed a better result concerning the velocity recovery, even when, on the other hand, the topology of the function is sensitive to the model variation (Zuniga et al., 2018, 2019b and 2019c).

$$
t=\sqrt{t_{0}^{2}+\frac{x^{2}}{v^{2}}-\frac{(S-1) x^{4}}{4 v^{4}\left(t_{0}^{2}+\frac{(S-1)}{2} \frac{x^{2}}{v^{2}}\right)}}
$$

The same problem was observed for the approximation proposed by Blias (2009), which also uses the $S$ parameter of heterogeneity, in a similar way to the equations proposed by Malovichko (1978) and by Ursin and Stovas (2006). To develop this approximation, the author performed several numerical tests related to the walkway vertical seismic profile (VSP). In this case, the approximation was very sensitive to the additional parameter and 
velocity (Zuniga et al., 2018, 2019b and 2019c), however, it had a very complex topology and was strongly dependent on the model.

$$
t=\frac{1}{2} \sqrt{t_{0}^{2}+\frac{1-\sqrt{S-1}}{v^{2}} x^{2}}+\frac{1}{2} \sqrt{t_{0}^{2}+\frac{1+\sqrt{S-1}}{v^{2}} x^{2}}
$$

Different from the other approximations, the next one, proposed by Muir and Dellinger (1985), considers the anellipticity parameter $(f)$ to deal with the nonhyperbolicity generated by the variation of the wavefront when it varies from a spherical shape and tends to the elliptical shape (Fomel and Grechka (2000). The problem observed before for this equation is that the global and local minimum regions are too similar, which makes the search for the global minimum even harder (Zuniga, 2017).

$$
t=\sqrt{t_{0}^{2}+\frac{x^{2}}{v^{2}}-\frac{f_{\circ}\left(1-f_{\circ}\right) x^{4}}{v^{2}\left(v^{2} t_{0}^{2}+f_{0} x^{2}\right)}}
$$

Different from all previous approximations in this work, the one proposed by $\mathrm{Li}$ and Yuan (2001), aims to consider the CP (Conversion Point) to control the effects of a nonhyperbolicity associated to the wave conversion. For this, the $\gamma$ parameter is used as the nonhyperbolic parameter.

$$
t=\sqrt{t_{0}^{2}+\frac{x^{2}}{v^{2}}-\frac{(\gamma-1)}{\gamma v^{2}} \frac{(\gamma-1) x^{4}}{4 t_{0}^{2} v^{2}+(\gamma-1) x^{2}}}
$$

where $\gamma$ is the ratio between the squared P-wave stacking velocity $v_{P 2}$ and the squared converted wave stacking velocity $v_{C 2}$, written as

$$
\gamma=\frac{v_{P 2}^{2}}{v_{C 2}^{2}}=\frac{\gamma_{e f f}\left(1+\gamma_{0}\right)}{\left(1+\gamma_{e f f}\right)}
$$

Here, $\gamma_{e f f}$ is the relation between $\gamma_{2}$ and $\gamma_{0}$, given by

$$
\gamma_{e f f}=\frac{\gamma_{2}^{2}}{\gamma_{0}}
$$


where $\gamma_{2}$ is the ratio between the stacking P-wave and stacking S-wave, and $\gamma_{0}$ is the ratio between P-wave velocity and S-wave velocity, which travel along the normal component.

The parameter from Li and Yuan (2001) approximation was tested by Yuan (2002) for a maximum offset-depth ratio of $1.5(x / z=1.5)$ and it brought results that stimulated several studies for the different applications of this approximation (Zuniga, 2015, 2017 and 2018; Zuniga et al., 2017, 2019a and 2019b). Yuan (2002) found, for this equation, the proper value of $x / z$ is 2.7 in a heterogeneous isotropic media, however, it was found that, for OBN condition, the equation works very well for higher values of $x / z$ (Zuniga, 2017; Zuniga et al., 2019a). 


\section{MODELLING COMPLEX STRUCTURES AND GEOMETRIES}

There are several different geological scenarios intended to model the offshore structures, and for this reason it is necessary to understand these different conditions. This work proposes to test five different models; three of them are ultra-deep carbonate reservoirs sealed by salt (pre salt), which were partially tested by Zuniga (2017), while the two new tested models are shallower reservoirs composed by sandstone and sealed by shale (post salt). The analysed data are both PP and PS reflection events from the interface between the salt base and the top of the reservoir. The three carbonate reservoirs were modelled using well log data from the pre salt from the Santos Basin, which provided the modelling performed by using stratigraphic and lithological information. For the two sandstone reservoirs, the modelling was performed to create fictional reservoirs inspired by post salt reservoirs from the Campos Basin. These two fictional models were generated aiming to test the nonhyperbolic multiparametric approximations in conditions which make them work on their thresholds, once this condition provides a RMS velocity much different than the real velocity, which was tested by Zuniga et al. (2018, 2019b and 2019c).

It is essential to use the P-wave velocity (Vp), S-wave velocity (Vs) and the ratio between the P-wave velocity and $\mathrm{S}$-wave velocity $(\mathrm{Vp} / \mathrm{Vs})$ as the main elastic parameters to perform the tests proposed in this work, since it is necessary to recover the value of velocity by identifying the travel-time of the waves. For the two shallower models, the values of these parameters were based on the references which provide a set of physical parameters for different lithology obtained empirically for different conditions of depth and pressure (e.g. Han, 1986; Lucet, 1989; Geertsma, 1961; Jizba, 1991; Strandenes, 1991; Blangy, 1992; Cadoret, 1993; Yale and Jameison, 1994; Mavko et al., 2009).

Zuniga (2017) generated the complete velocity profile as a function of depth using the MATLAB Toolbox for ray tracing modelling proposed by Margrave (2000 and 2003). It was performed by using the ray tracing method aiming to define how each reflection event travels through the layers. However, once it is necessary to model structures with geometry of acquisition where there is a difference of datum between source and receiver, it is also necessary to use a more complex tool, as the software of $2 \mathrm{D}$ wave field modelling by finite differences proposed by Thorbecke and Draganov (2012). 


\subsection{Automated picking using spectral recomposition}

To perform the velocity analysis using the proposed method, it is necessary to extract the travel-time curve from the target reflection event (the PP and PS reflection events from the interface between the bottom of the salt and the top of the reservoir). For this reason, a technique to perform an automated pick of the travel-times from the seismogram trace by trace, even in the presence of significant random noise, is proposed.

As the pre salt from the Santos Basin presents a very thick salt layer above the reservoir, there is a significant difference concerning the depth - and therefore concerning the arrival time - between the strongest reflections related to the interfaces above the reservoir and the one related to the target interface, the interface between bottom of the salt and the top of the reservoir. For this reason, it is easier to perform the travel-time pick for the target interface when it has less interference from other reflections of other interfaces.

So, a technique capable of performing the inversion of the frequency spectrum for each trace aiming to recover the information of amplitude and frequency using the spectral recomposition approach (Tomasso et al., 2010; Cai et al., 2013) is proposed. It was performed before in different situations aiming only to recover this kind of information to obtain a better geological characterization (Tomasso et al., 2010; Cai et al., 2013). However, until now, it has not being applied to compare the wavelets and specifically to find a peak frequency of a random noise or of a signal.

The seismic spectrum can be described as a sum of different Ricker components (Tomasso et al., 2010), described as

$$
d(f) \approx \sum_{i=1}^{n} a_{i} \psi_{i}\left(m_{i}, f\right)
$$

The $d(f)$ is the spectrum of a seismic trace, $f$ is the frequency, and $a_{1}$ and $m_{1}$ are, respectively, the amplitude and the peak frequency of the $i$-th Ricker spectrum component, given by

$$
R(f)=a \psi(m, f)=a \frac{f^{2}}{m^{2}} \exp \left(-\frac{f^{2}}{m^{2}}\right)
$$


With this description of the wavelet, it is possible to perform an inversion fitting the calculated frequency spectrum to the observed one of all wavelets found in a trace. Certainly, the frequency spectrum is found after a Fourier transform of the wavelets is performed in the analysed trace. The optimization algorithms used are going to be better described in subsequent chapters.

So, for the first trace, the curve fitting of the calculated spectrum to all spectra of the frequencies found this way is performed; and then, with each pair of information of frequency and amplitude, they can be compared to each other and to the frequency used by the seismic source. With an automated comparison of the values of peak frequency and amplitude, and cross correlation among each wavelet, it is possible to find which of those spectra are related to the random noise or to the signal of the reflection. Once there is only one target signal related to the travel-time of the reflection on this trace and several related to the random noise, the spectrum of the signal must be different from all the other random noise spectra. That is the main reason to consider this technique to obtain only reflections well separated in time; otherwise, reflections too close to each other may be ill-picked.

After finding the wavelet of the signal for the first trace, the technique does the same for the next trace; however, it considers some constraints to perform a more accurate picking. The first of the constraints is to consider the arrival time of a trace always higher than the previous one, once the next arrival must take more time to be received than the previous one. The second constraint is to compare the difference between the arrival time of the analysed trace and the arrival time of the previous trace. It is necessary to restrict the time of the target wavelet between the two arrival times around it, once it cannot be lower than the previous arrival time and higher than the next one. This difference limit can be set according to different kinds of models due to the fact some models can present a lithology which can generate a more horizontalized reflection event and therefore a narrower time window between the previous and the next trace. The third constraint is to compare the peak frequency and the amplitude and cross correlate the spectrum of the signal of the trace analysed to the previous trace. This constraint aims to compare the spectrum of the signal of the event in a trace to the previous one, once they must be very similar to each other and different from the random noise spectrum. All difference limits can be set according to the different characteristics of the medium, seismic source or kind of propagation (PP, ShSh, SvSv, SP or PS), once each of these characteristics can present different sets of arrival times and wavelets, 
and therefore it is possible to restrict a specific area of the seismogram aiming to find the target information more quickly.

The seismogram may also be muted on the parts in which the reflection event is not expected to appear. It is important to help the algorithm to find less wavelet to fit, decreasing the possibility of picking a wrong spectrum and increasing the processing time. However, it should be applied only in regions of the seismogram which certainly does not affect the target reflections; otherwise it could mute important information of the target reflection.

After performing the technique for each trace and obtaining each frequency spectrum associated to the target reflection signal, it is possible, after performing the inverse Fourier transform for each trace, to find the arrival time of each wavelet of the reflection signal. With each arrival time of the reflection event, there is the travel-time curve of the same reflection event. That procedure was performed for each PP and PS reflection event of the interface between the bottom of the sealing layer and the top of the reservoir for each model, and the two reflection events (PP and PS) of each of the five models could be found (9.1 Appendix A). Then, it is possible to perform the velocity analysis by fitting the calculated travel-time curve to the observed one (extracted with this technique).

\subsubsection{PVO analysis to find critical reflection using spectral recomposition}

It is possible to observe that, in cases where there is a critical reflection, the phase begins to significantly vary after the critical reflection. For this reason, it is proposed to apply the same technique used to perform the automated picking, but this time to extract the events with phase shifting. Once the travel-time curve is extracted, the same idea can be applied in a different way. So, another technique is proposed in this thesis, and this technique is based on performing the inversion of the spectrum of a trace using the Ricker spectrum as the calculated curve, which provides the information of the amplitude and peak frequency. However, in a condition where there is a phase shift, the Ricker wavelet is gradually distorted into its Hilbert transform, which is very similar to a semi-Gaussian wavelet, due to the fact of both being odd functions. Once the phase is getting shifted, the observed wavelet is becoming more disparate from the Ricker wavelet and becoming closer to a semi-Gaussian wavelet, which results in a higher global minimum value. It happens because the error of the calculated curve represented by the mathematical model of the Ricker spectrum starts to be fitted to a 
wavelet closer to a semi-Gaussian wavelet. It happens because the Ricker wavelet spectrum is being used to calculate the spectrum of the wavelets in the traces. For this reason, it was expected that the trace with the highest global minimum value was related to the critical angle. This is very interesting for the PVO (Phase Variation with Offset) analysis, once it is possible to find the critical angle (CA) data driven and then find some elastic properties like Poison ratio when it is made for PP and SS reflection events of a layer. It can also be useful to calculate the velocity ratios for each studied layer and then, after finding the velocity of the first one, calculate the velocity of each underlying layer. In this work, it was tested for $\mathrm{SvSv}$ reflection events with different critical angles, aiming to find the critical angles of these events data driven. Four models were proposed to be tested: the first model with 37 degrees of critical angle; the second model is the same as the first one except that a semi-Gaussian wavelet was used rather than a Ricker wavelet; the third model presents 30 degrees of critical angle; and the fourth model with 44 degrees of critical angle.

In this study, it was possible to observe that the value of the global minimum error (the error between the calculated spectrum and the observed frequency spectrum) along each trace varies as it gets closer to the critical angle. The global minimum value increases abruptly as it reaches the $\mathrm{CA}$ and then abruptly decreases for the angles higher than the $\mathrm{CA}$. It happens for the three models with different CA (37, 30 and 44 degrees). However, for the second model tested, the CA is related to the first lowest minimum value. It happens due to the Ricker wavelet used to calculate the spectrum being very similar to the first wavelets of the models 1 , 3 and 4, and when the wavelets start to become different from the Ricker spectrum they stop to fit as well. For the model 2, the opposite happens, once the first wavelets are semiGaussian and are being calculated with a Ricker wavelet spectrum, so the error starts to decrease when the wavelets start to become more similar to the calculated Ricker wavelets. So, for any value of CA (with a Ricker wavelet), it may be explained by the fact that the phase variation gets more disparate from the Ricker wavelet shape as it gets closer to the critical point, and therefore, the error increases as the Ricker wavelet is considered for calculating the spectrum. So, in the CA, the worst fitting is observed. Therefore, the peak of maximum error is associated to the critical angle, and for higher and lower angles, it starts to decrease and show a better fitting.

The same is observed to the peak frequency value and the amplitude value $(9.2$ Appendix B - Figure 284 and 285). But this time, for all the four models. The amplitude and 
peak frequency have their highest values observed in the critical angle. However, for the amplitude, it is observed that, after the critical angle, its values decrease gradually, while for the peak frequency, the values decrease abruptly and then increase again, getting slightly more stable. For the frequency, the increase of the value can be possibly explained by interference from the refracted event which starts to get separated from the reflected one. For the Ricker wavelet, it was also tested for higher and lower critical angles. It showed the same behaviour of the global minimum, amplitude and frequency variations with the incident angle. When the same study was performed for a semi-Gaussian wavelet (Gaussian's first derivative or Ricker's integral), the value of the first minimum peak right before the maximum peak showed to be related to the critical angle rather than the maximum peak. However, the amplitude and the frequency showed the same behaviour shown by the Ricker wavelet with the same critical angle.

Additionally, it is important to prove and show how the reflection and the phase behave around the CA. For each trace (consequently each angle), the theoretical instant phase shows the angle when the phase changes for each model (9.2 Appendix B - Figure 286). Another way to show this is to theoretically calculate the reflection (RC) and the transmission (TC) coefficients using the Zoeppritz equations, once the RC reaches its maximum and becomes stable immediately after the critical angle, and the TC reaches its maximum value exactly in the CA (9.2 Appendix B - Figure 287, 288 and 289).

\subsection{Models to be studied}

The first three models are conventionally found in the pre salt environment, a very thick salt sealing layer above a carbonate reservoir layer. However, the other two models tested are sandstone reservoirs sealed by shale, so a broader range of results can be found. For all the following models, the depth of the source is 5 metres, the number of receivers is 100 , and the minimum offset between source and receiver, in the same way as the offset between receivers, is 150 metres. The five models used in this work present only vertical velocity variation, and not varying horizontally. 


\subsubsection{Model 1}

For the first model, it is possible to observe a very conventional structure from the pre salt from Santos Basin, with more than 2000 metres of water depth and around 2000 metres of salt sealing the reservoir. This salt structure is composed by one anhydrite $\left(\mathrm{CaSO}_{4}\right)$ layer between two halite $(\mathrm{NaCl})$ layers. The anhydrite layer has higher density and higher P-wave and S-wave propagation than the other two salt layers (Figure 7). The receivers are coupled at 2157 metres of depth on the bottom of the ocean and the salt layer begins at a depth of 2761 metres and ends at the depth of 5172 metres, where the top of the carbonate reservoir is. The ray tracings of this model with the described geometry of acquisition are presented by the Figures 8 and 9, while the travel-time curves are represented by the Figures 10 and 11 .
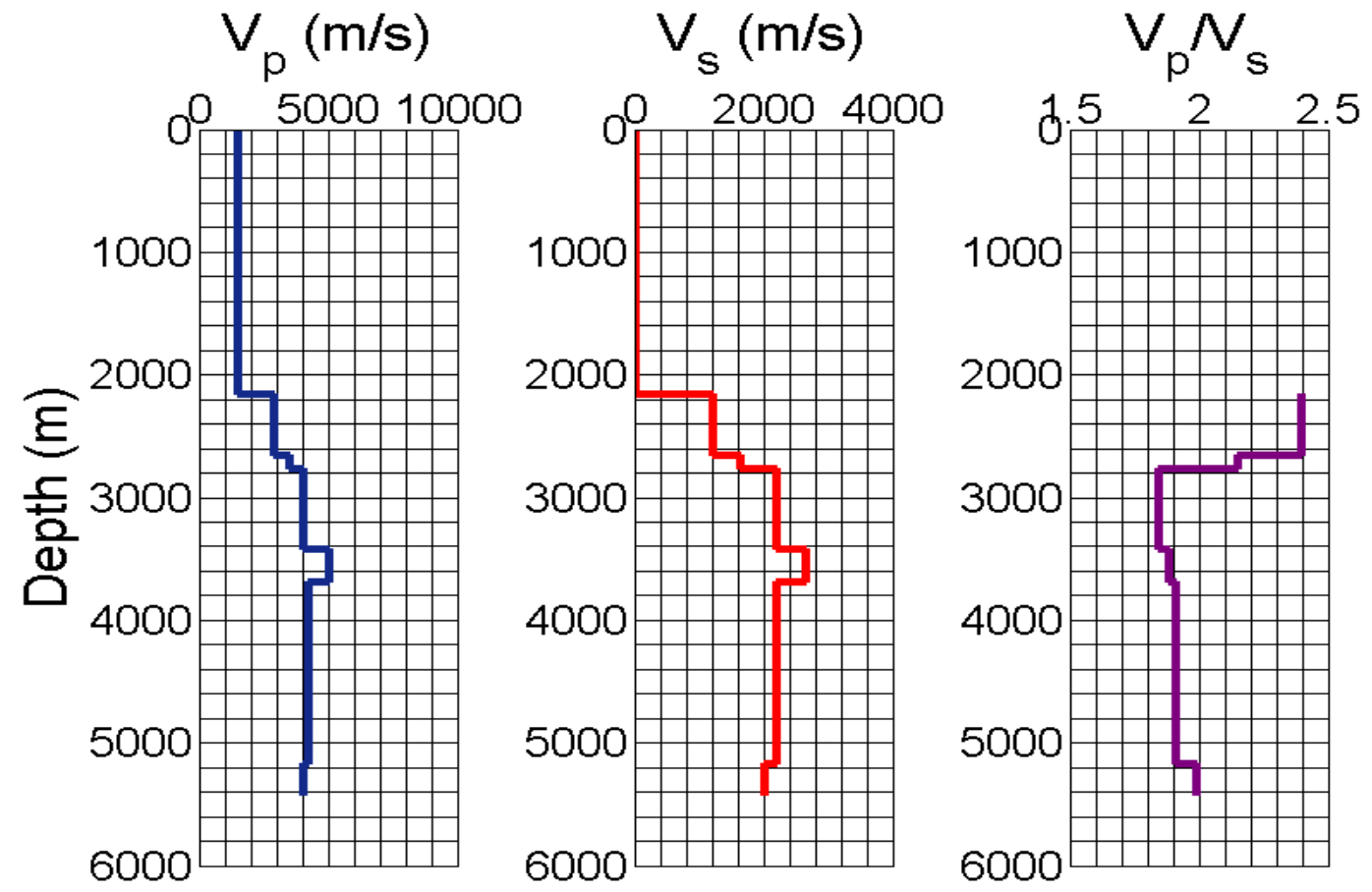

Figure 7: Velocity profiles of $\mathrm{P}$ wave, $\mathrm{S}$ wave, and $\mathrm{Vp} / \mathrm{Vs}$ ratio related to the depths of the Model 1. 


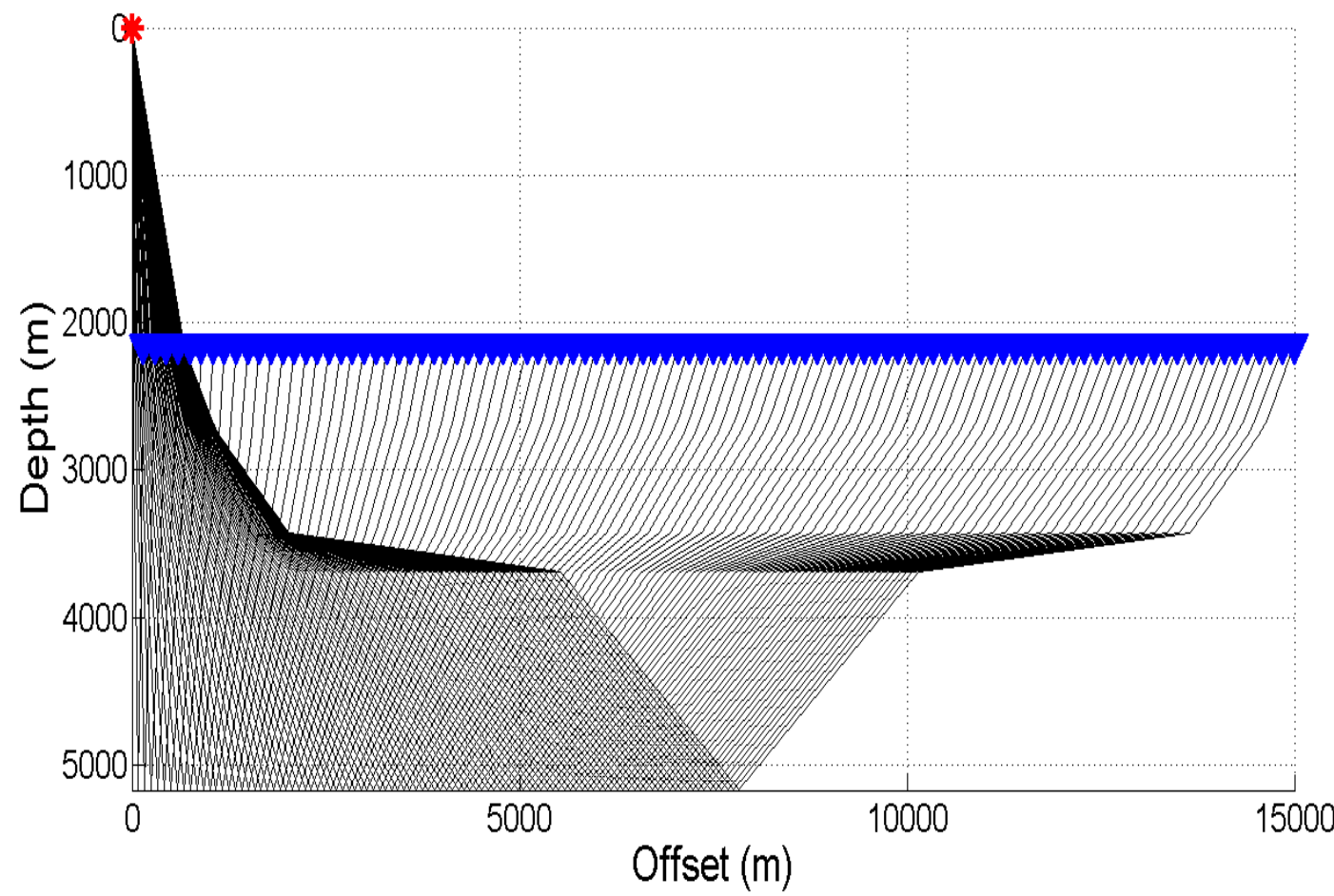

Figure 8: Ray tracing schematization of the PP reflection event from Model 1.

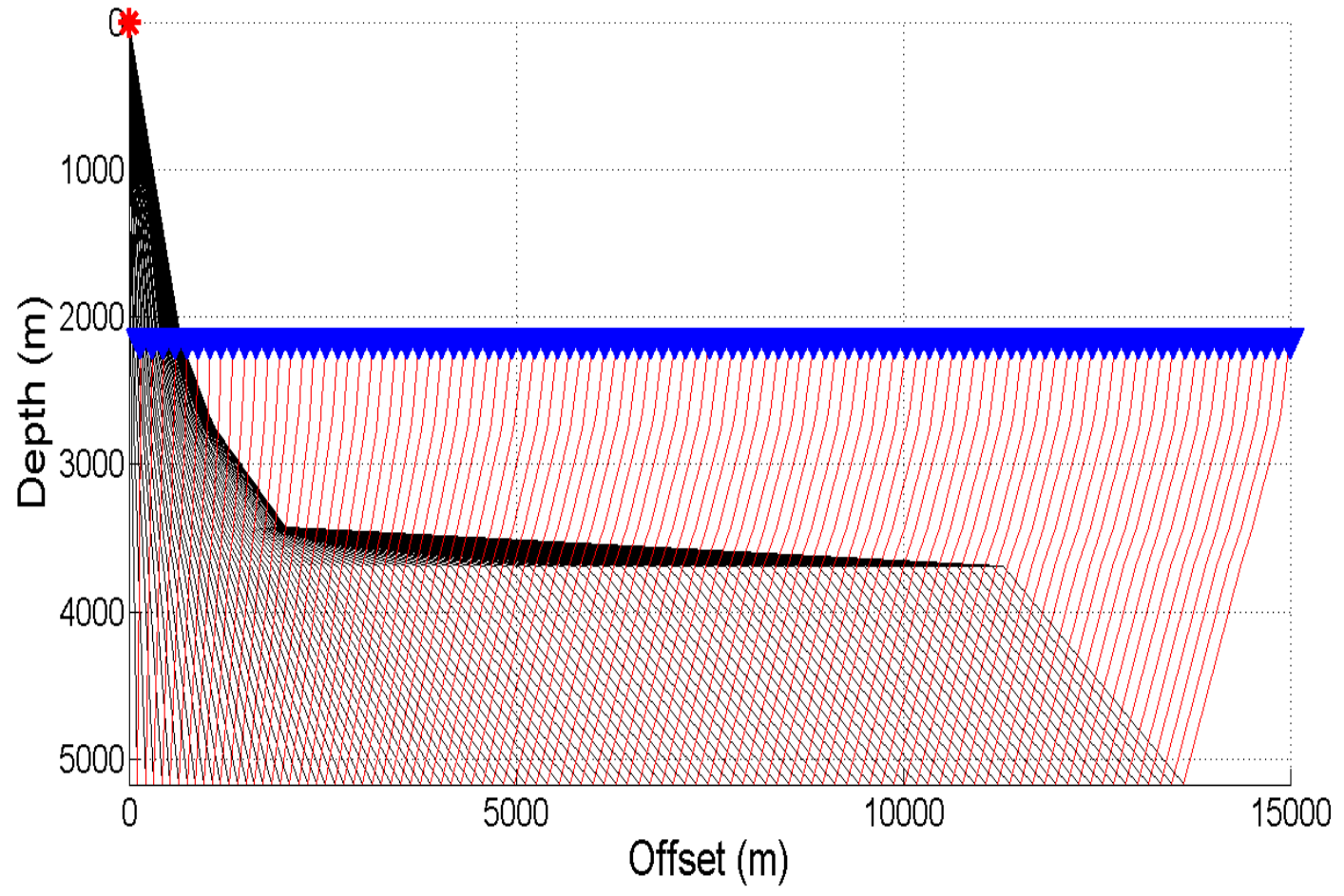

Figure 9: Ray tracing schematization of the PS reflection event from Model 1. 


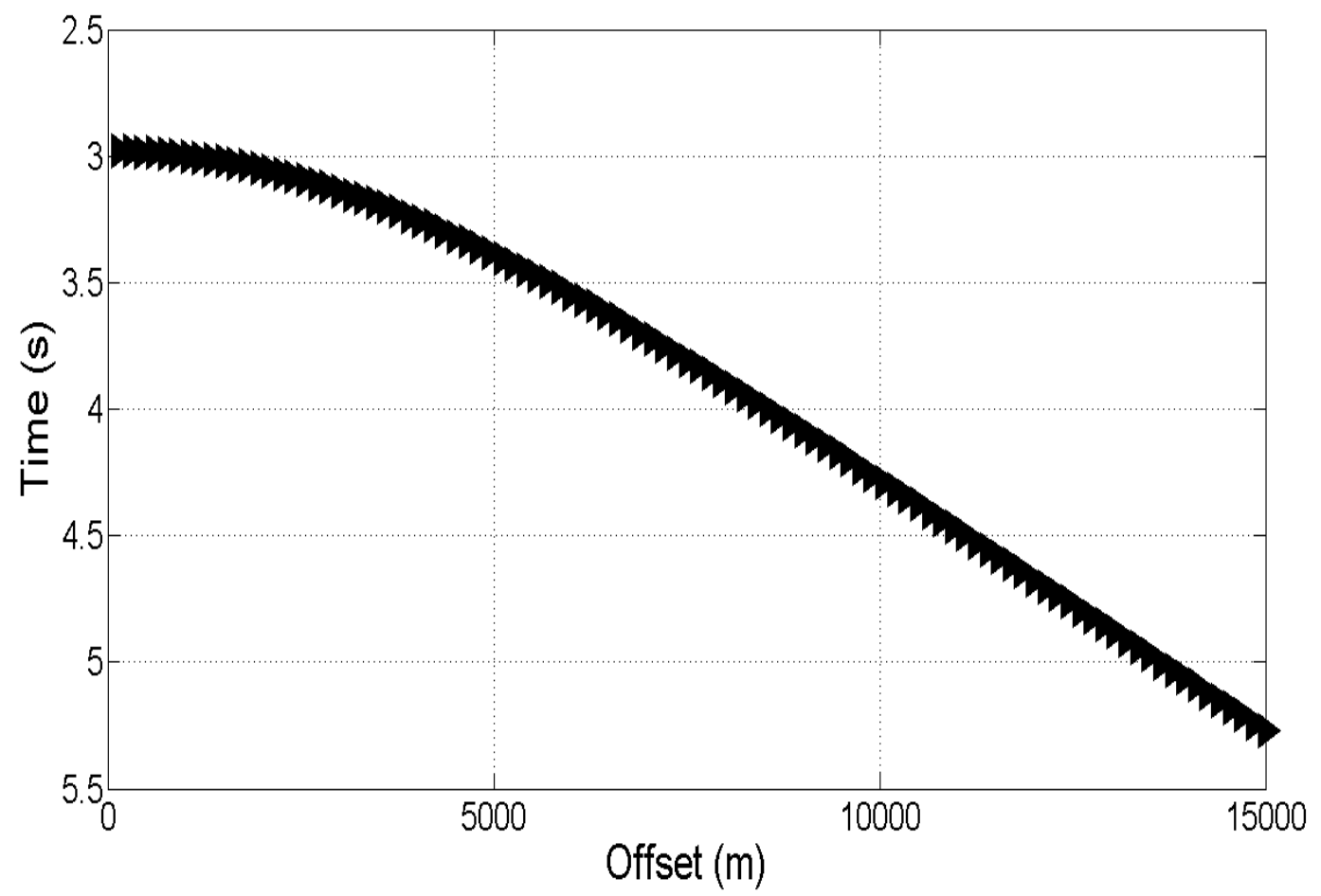

Figure 10: Travel-time curve of the PP reflection event from Model 1.

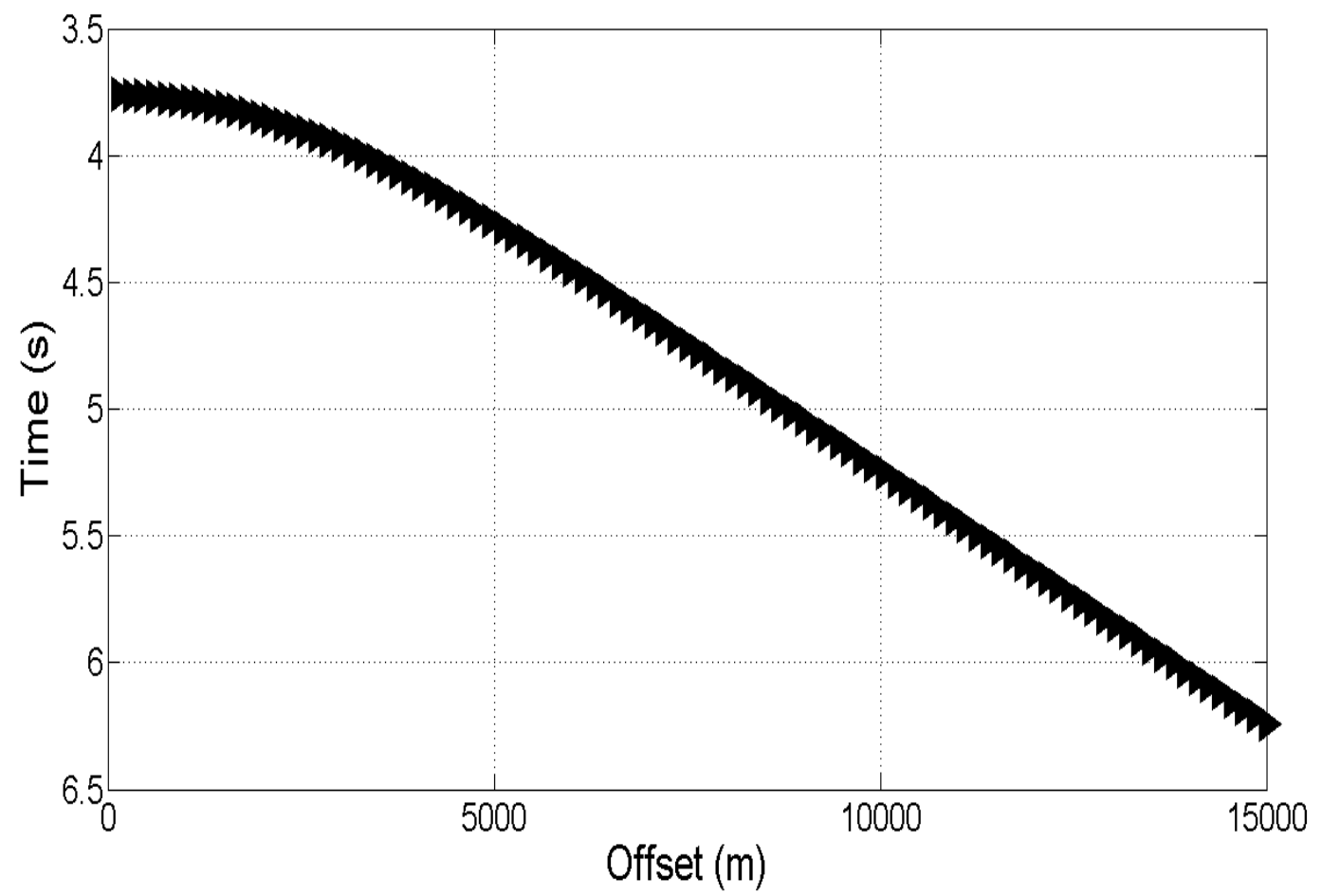

Figure 11: Travel-time curve of the PS reflection event from Model 1. 


\subsubsection{Model 2}

The second model is also from pre salt from Santos Basin, and, as the first model, it presents a salt structure thicker than 2000 metres; however, in this case, the salt layer starts to appear at a depth of 3139 metres and ends at a depth of 5005 metres, where the top of the carbonate reservoir is. The main difference of this model in comparison to the previous one is that, in this case, in the salt structure, despite being composed by two halite layers and an anhydrite layer in between them, the anhydrite layer is much thicker (more than 1000 metres), while the halite layers are thinner (Figure 12). The receivers are coupled at a depth of 2101 metres on the water bottom as it can be seen in the Figures 13 and 14. The reflection events of PP and PS waves are shown in Figures 15 and 16.
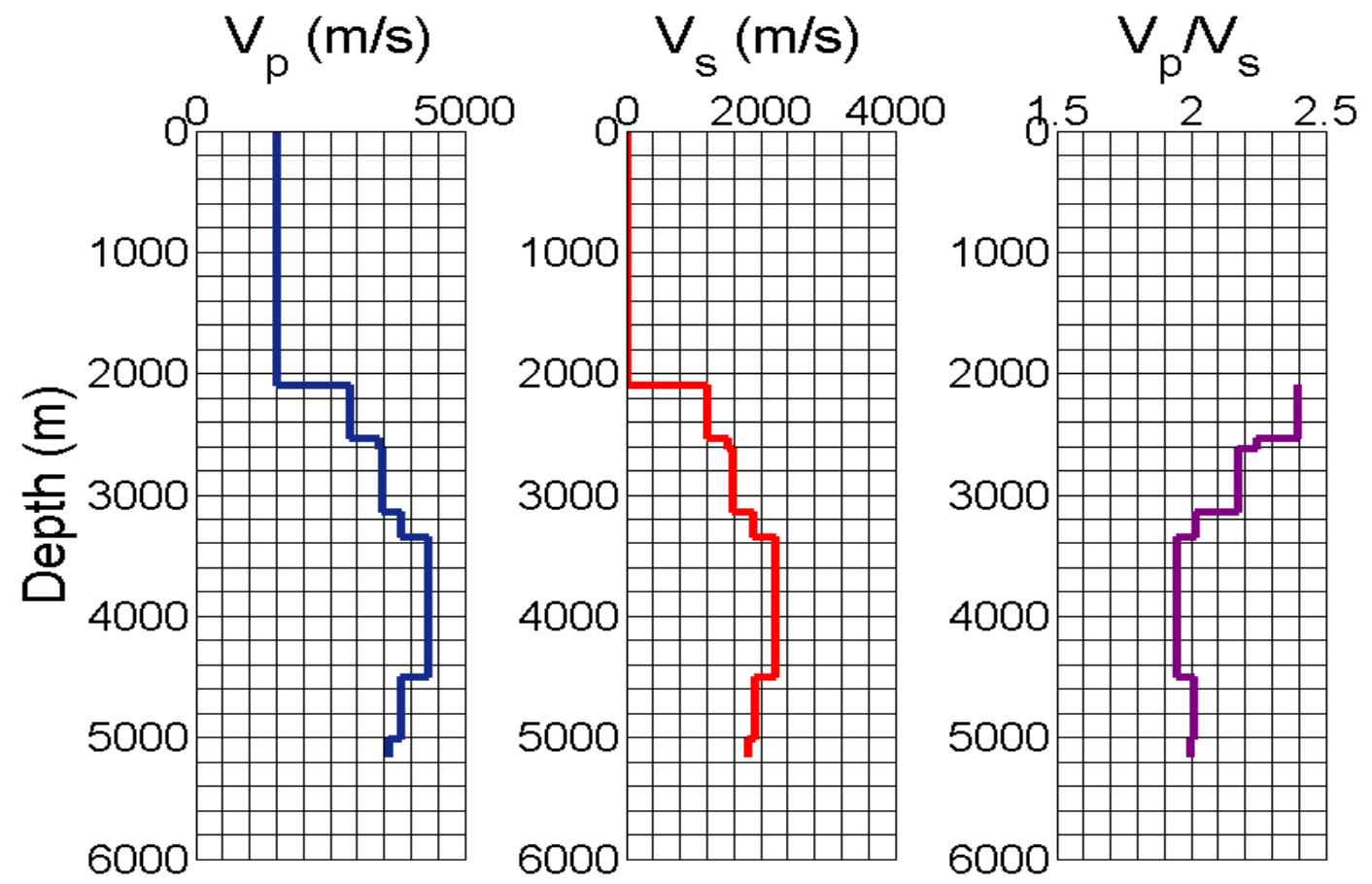

Figure 12: Velocity profiles of $\mathrm{P}$ wave, $\mathrm{S}$ wave, and $\mathrm{Vp} / \mathrm{Vs}$ ratio related to the depths of the Model 2. 


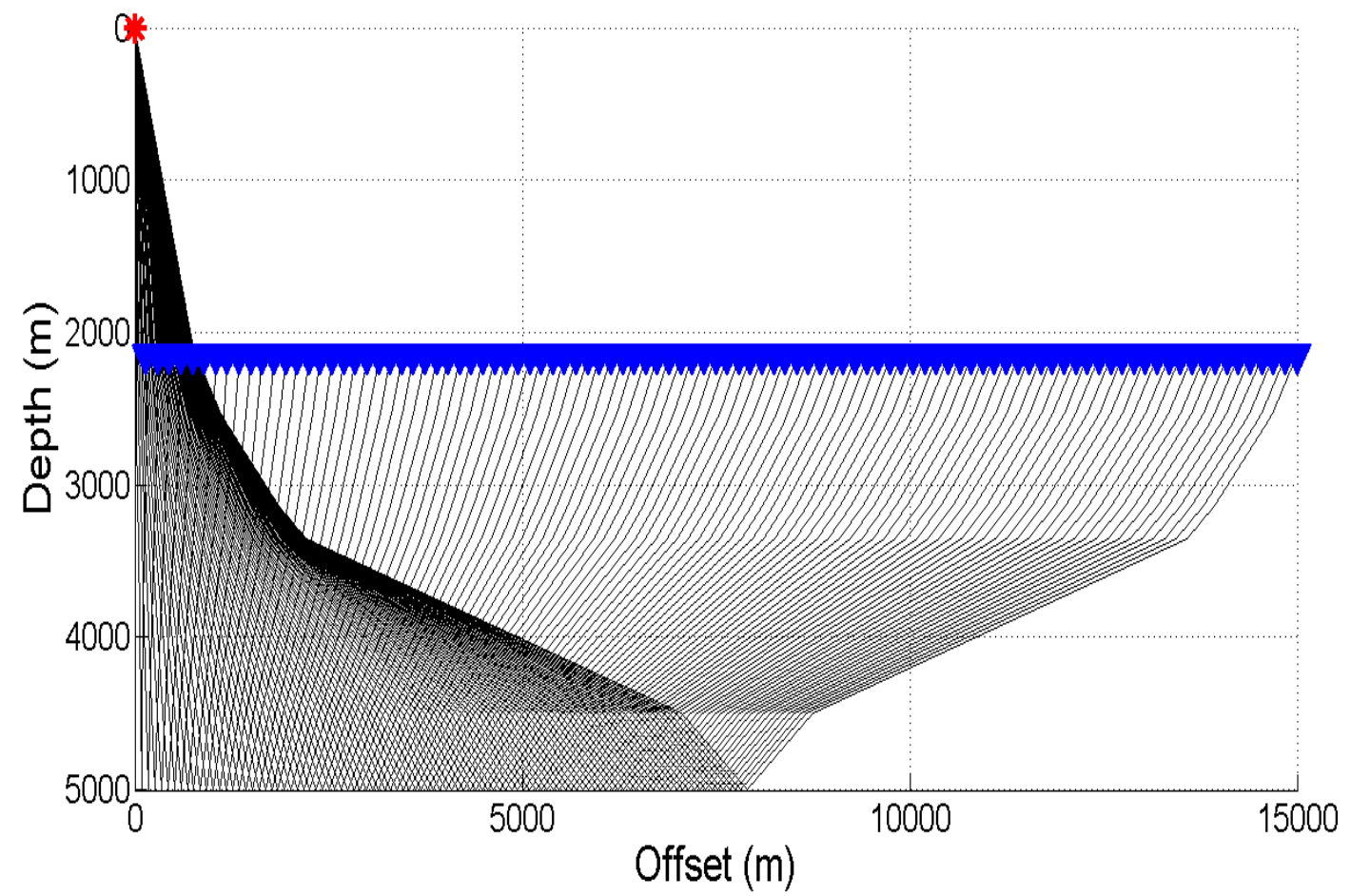

Figure 13: Ray tracing schematization of the PP reflection event from Model 2.

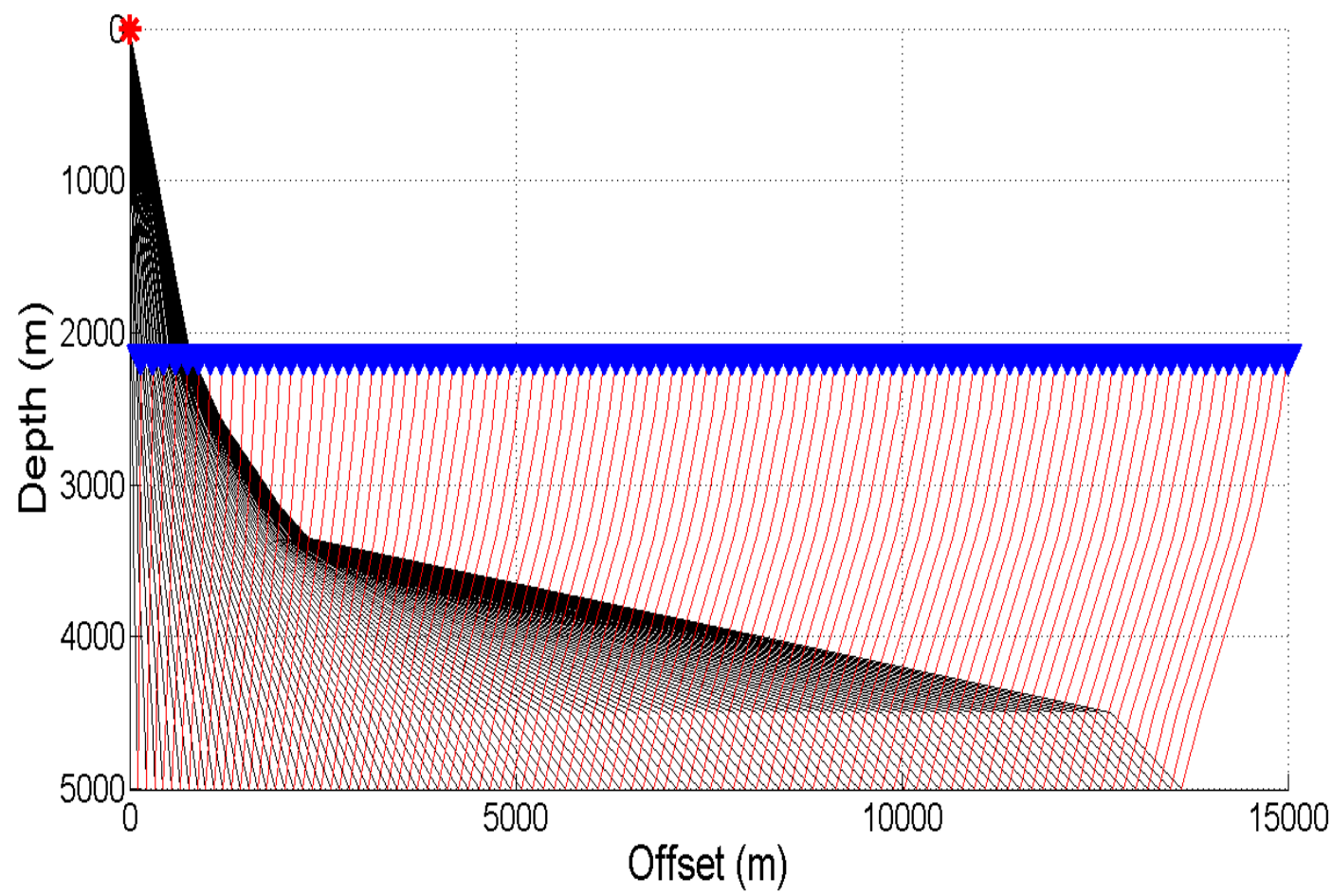

Figure 14: Ray tracing schematization of the PS reflection event from Model 2. 


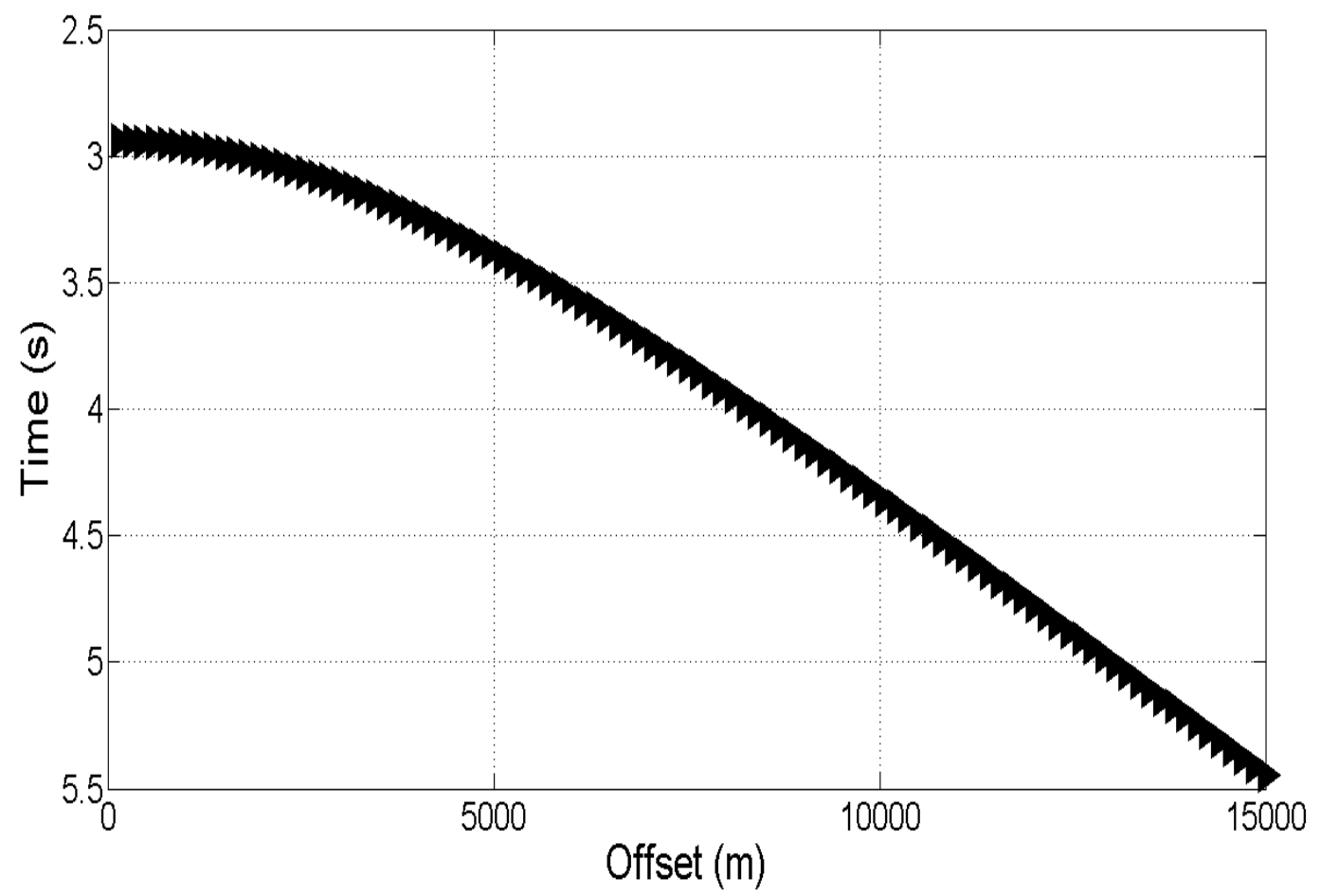

Figure 15: Travel-time curve of the PP reflection event from Model 2.

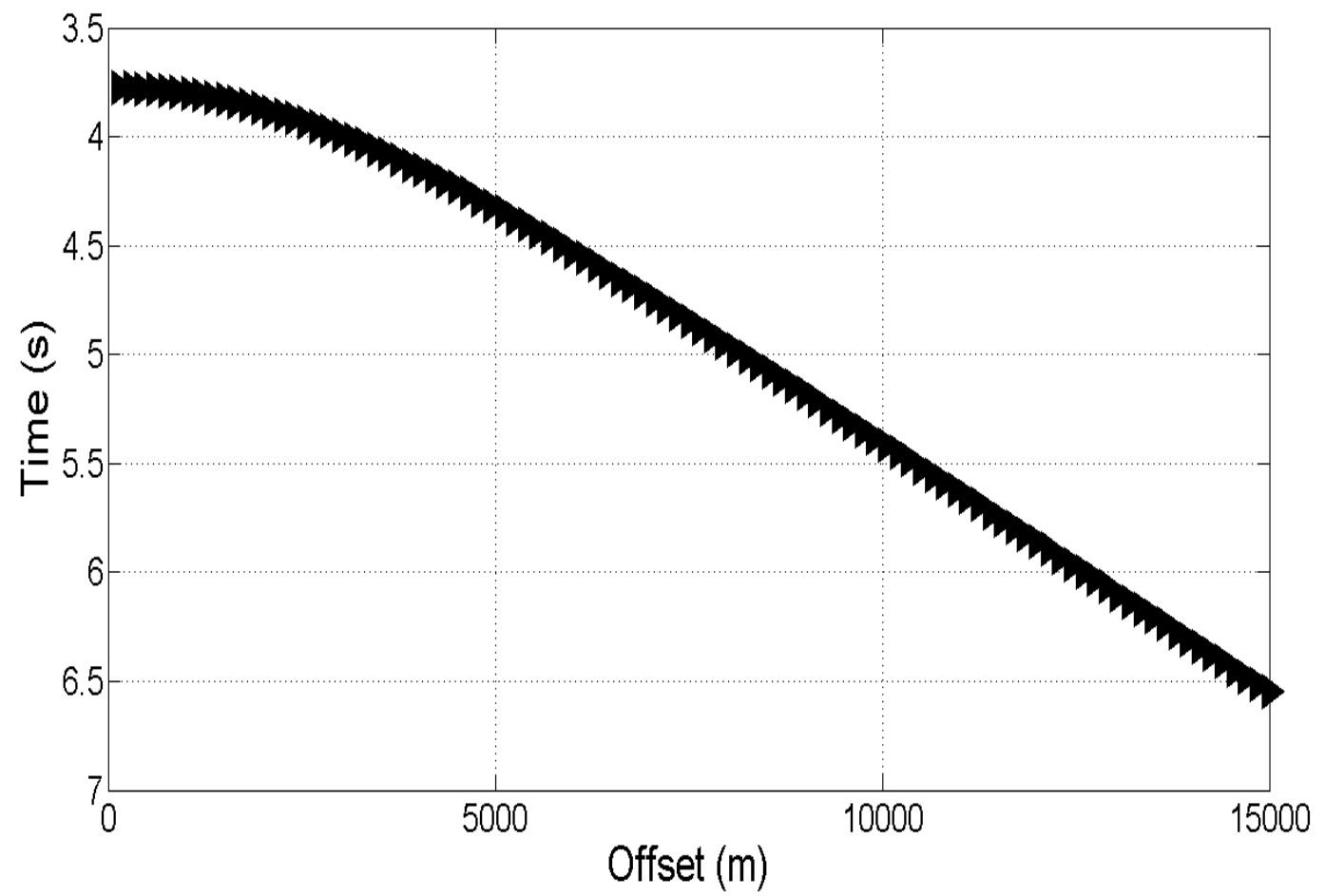

Figure 16: Travel-time curve of the PS reflection event from Model 2. 


\subsubsection{Model 3}

Differently from the models shown previously, the third model presents a very peculiar characteristic concerning the salt structure: The halite layer is in between two anhydrite layers. The salt structure also presents a thinner total thickness, something around 1500 metres, starting at 3590 metres of depth and the bottom of the salt reaching the top of the carbonate reservoir at 4949 metres of depth (Figure 17). This kind of velocity variation, with a velocity abnormally lower than it should be, found between two very dense salt structures, can cause a strong distortion on the ray tracing (Figures 18 and 19) and therefore generates a stronger nonhyperbolicity on the reflection events (Figures 20 and 21). The receivers are coupled at the ocean bottom, where they are at a depth of 2159 metres.

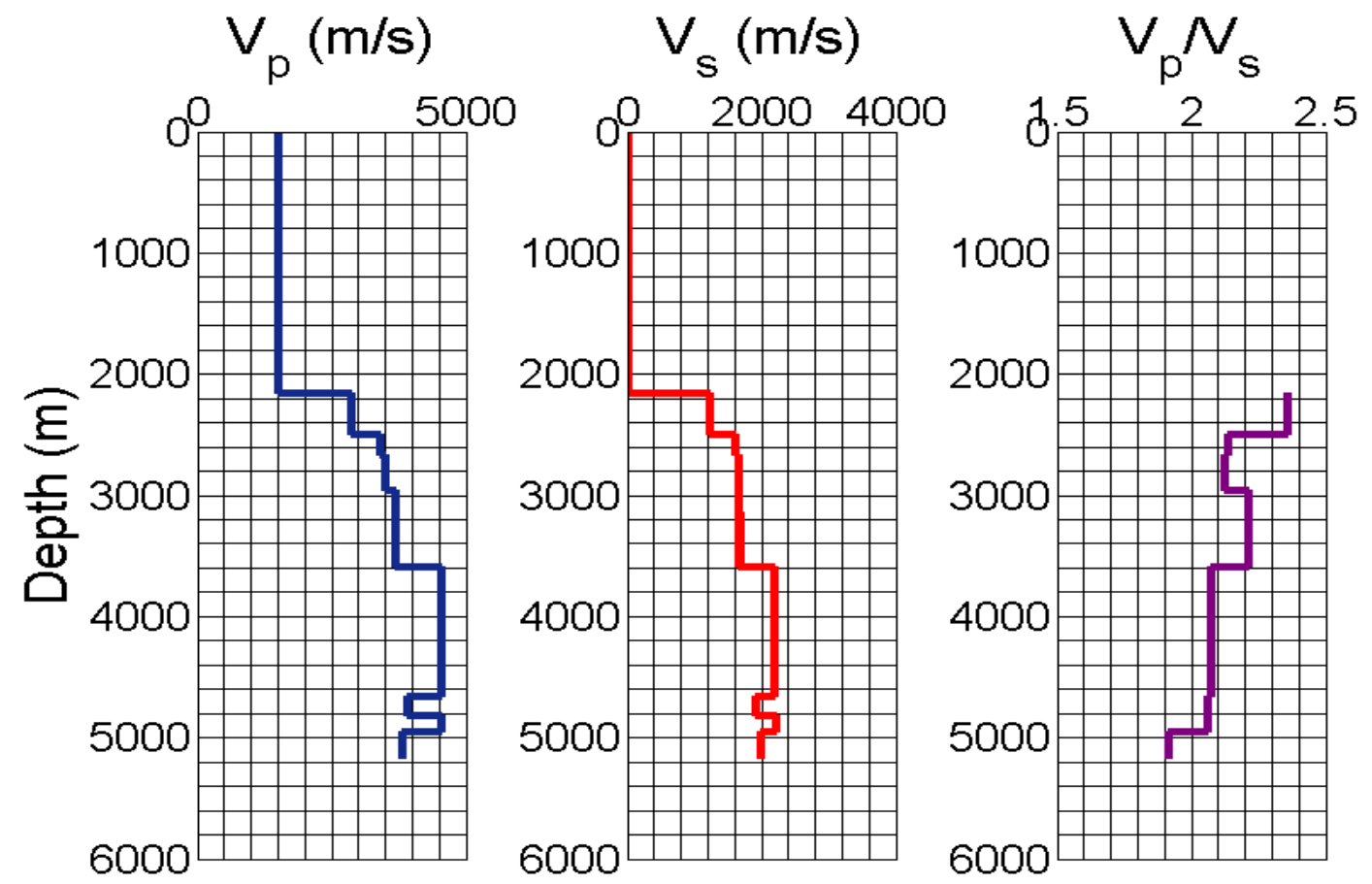

Figure 17: Velocity profiles of $\mathrm{P}$ wave, $\mathrm{S}$ wave, and $\mathrm{Vp} / \mathrm{Vs}$ ratio related to the depths of the Model 3. 


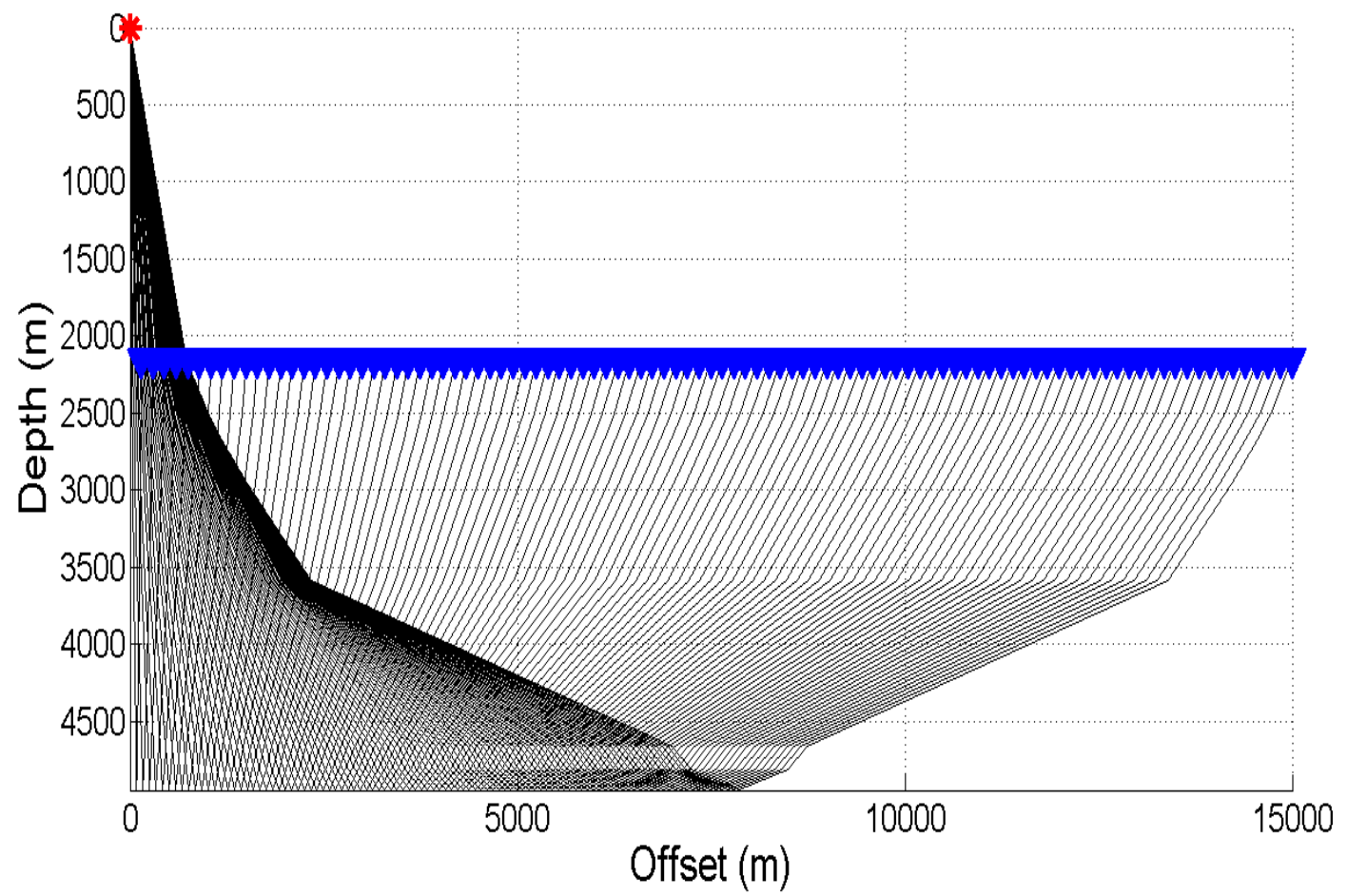

Figure 18: Ray tracing schematization of the PP reflection event from Model 3.

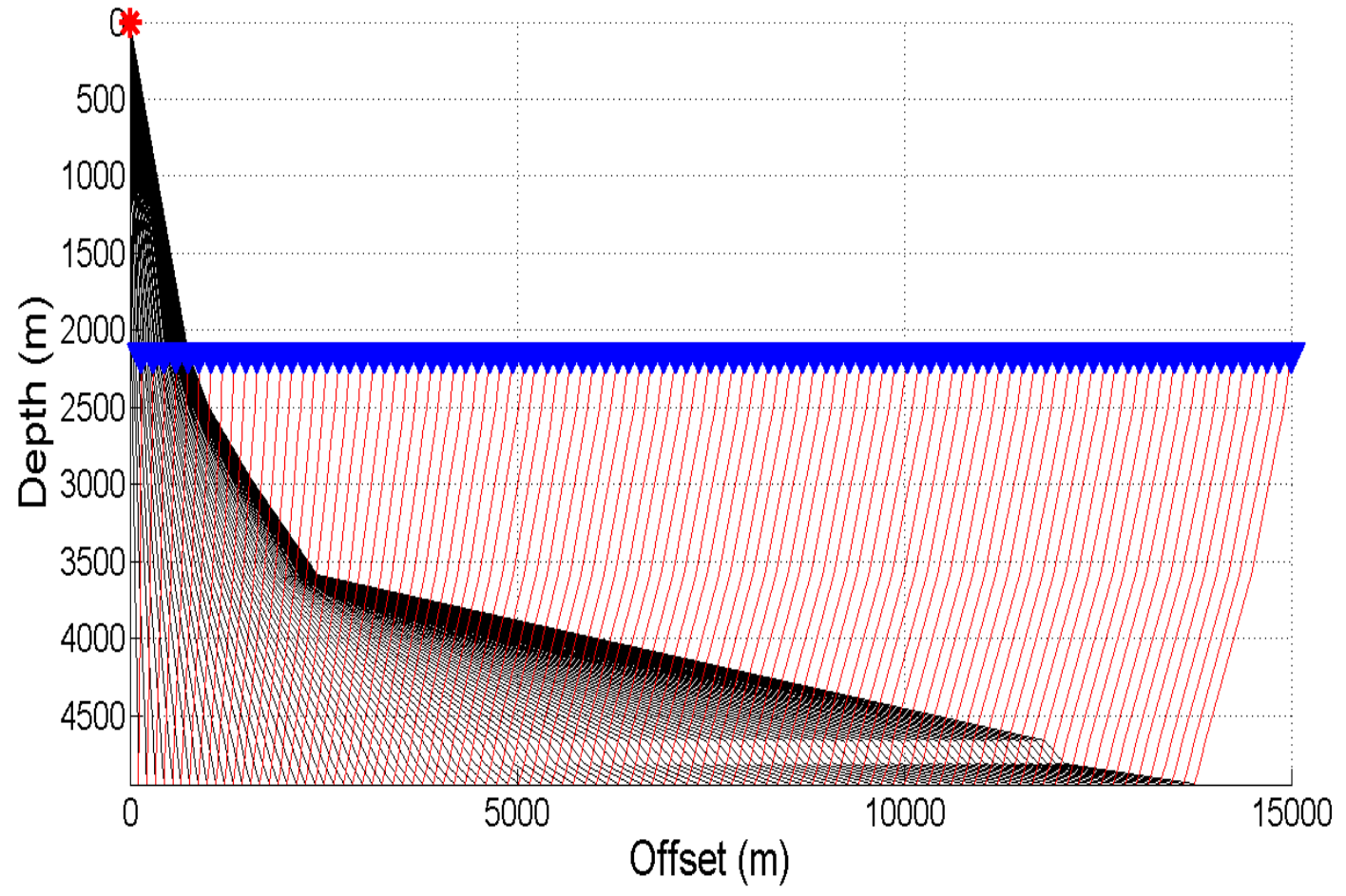

Figure 19: Ray tracing schematization of the PS reflection event from Model 3. 


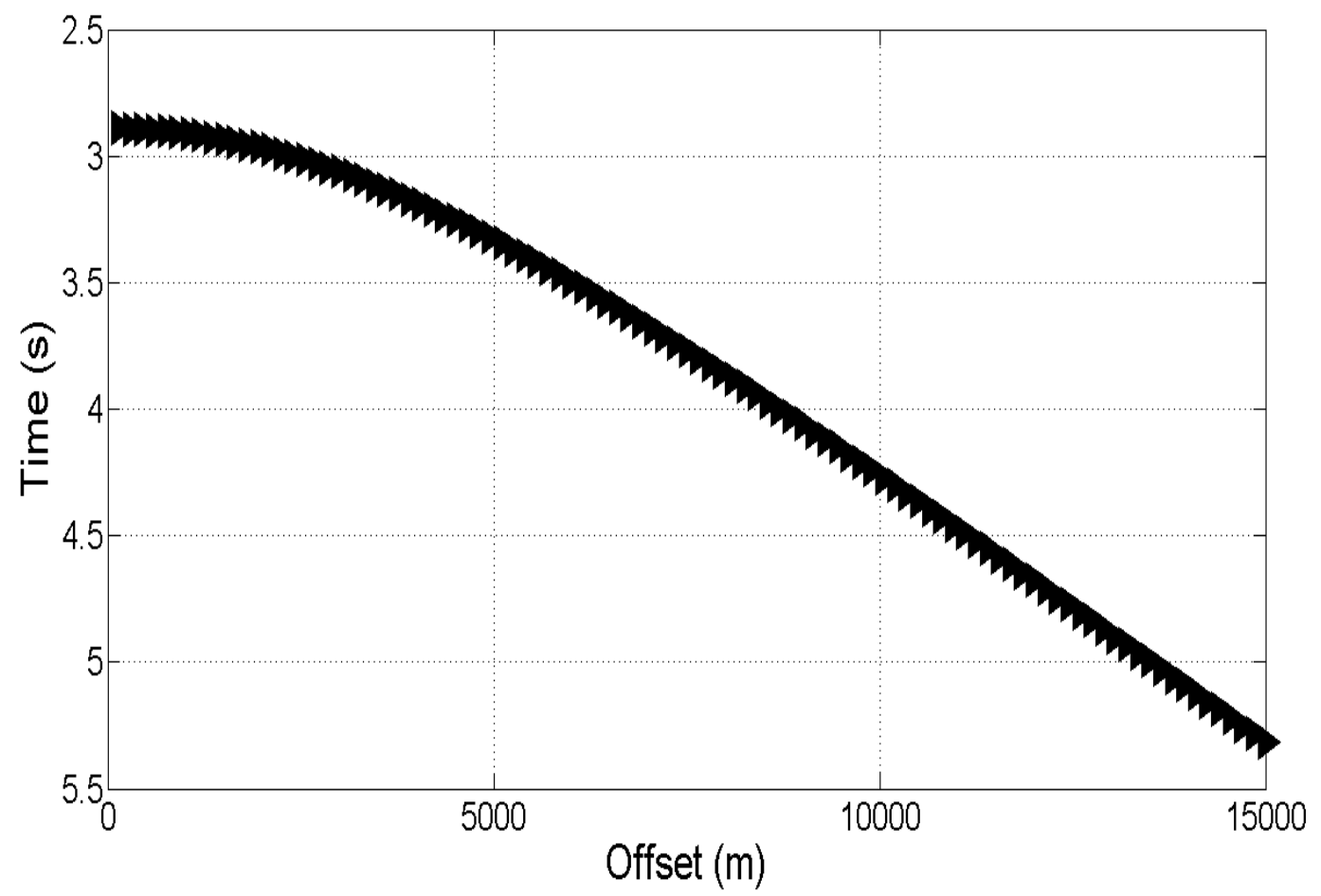

Figure 20: Travel-time curve of the PP reflection event from Model 3.

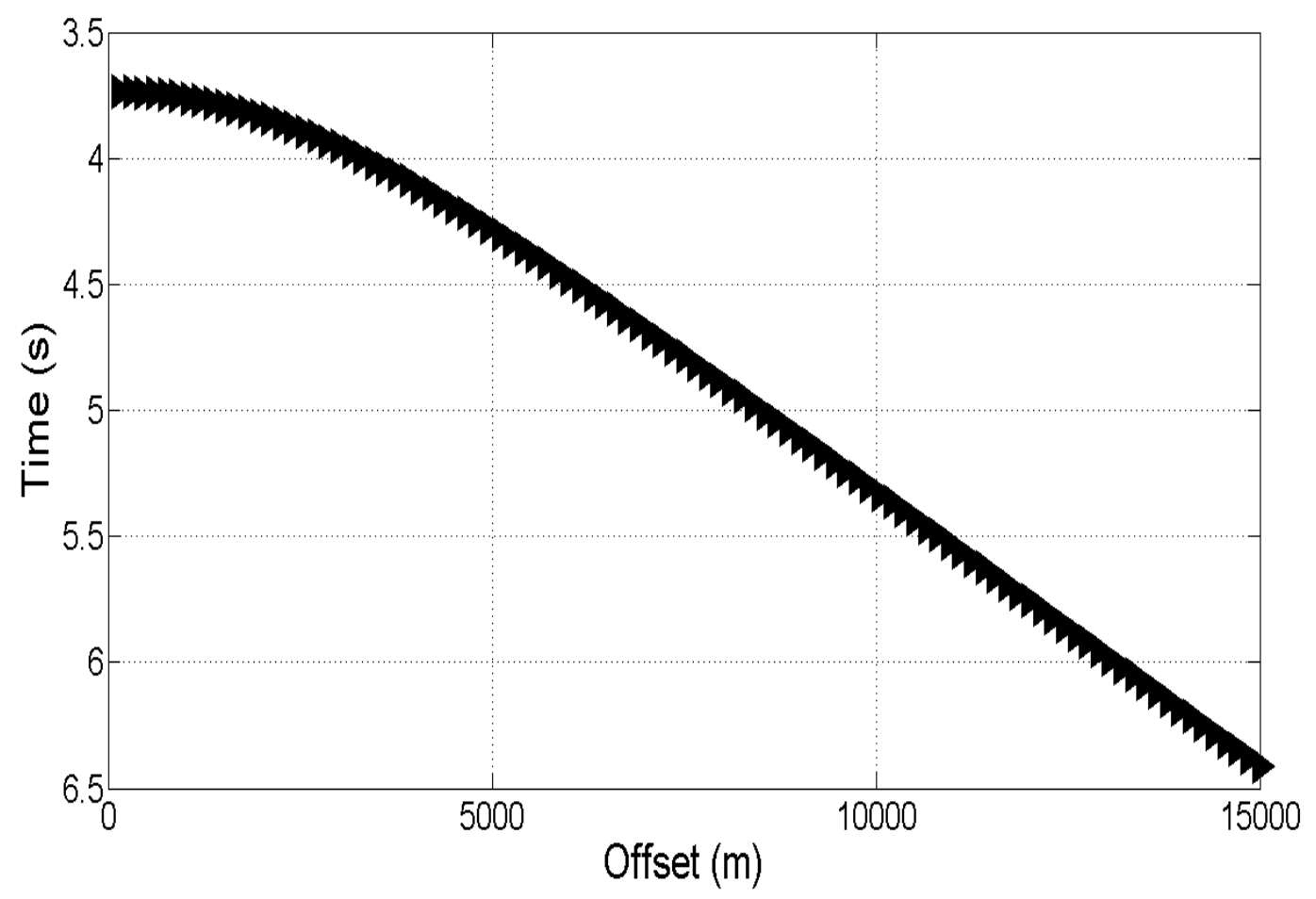

Figure 21: Travel-time curve of the PS reflection event from Model 3. 


\subsubsection{Model 4}

The Model 4 is a conventional sandstone reservoir sealed by shale. This structure has a water depth of 2050 metres and a shallower reservoir in comparison to the other models. The depth of the top of the sealing layer is 3220 metres and the top of the reservoir is at a depth of 3524 metres (Figure 22). For this case, the same application of OBN technology is considered, so the receivers are coupled at the ocean bottom, as it is shown by Figures 23 and 24. Despite not being a complex stratigraphy or lithology, this model represents a challenge concerning the RMS velocity, once it has a very high offset-depth relation, which can also increase the nonhyperbolicity of the reflection events (Figures 25 and 26).

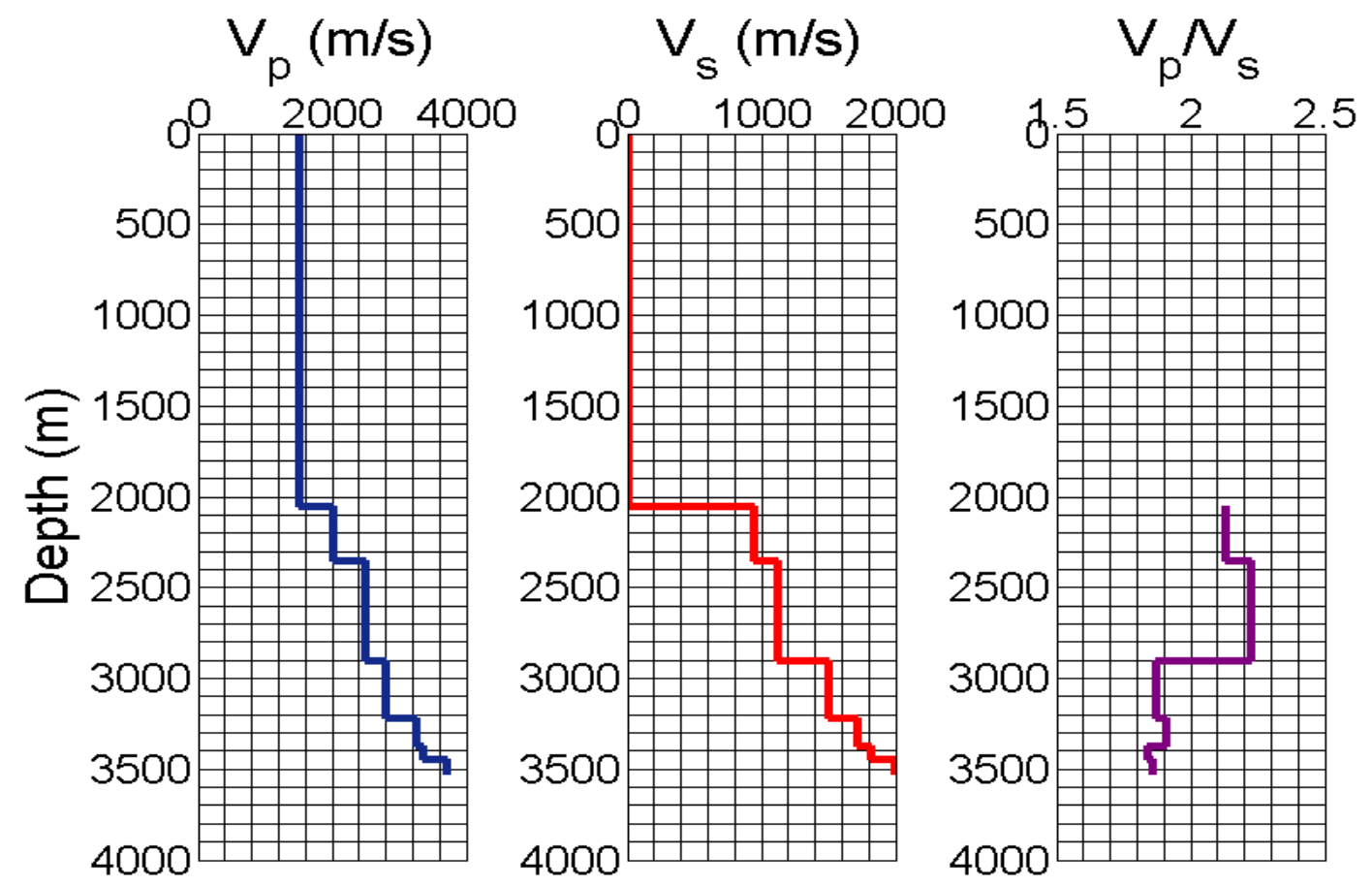

Figure 22: Velocity profiles of $\mathrm{P}$ wave, $\mathrm{S}$ wave, and $\mathrm{Vp} / \mathrm{Vs}$ ratio related to the depths of the Model 4. 


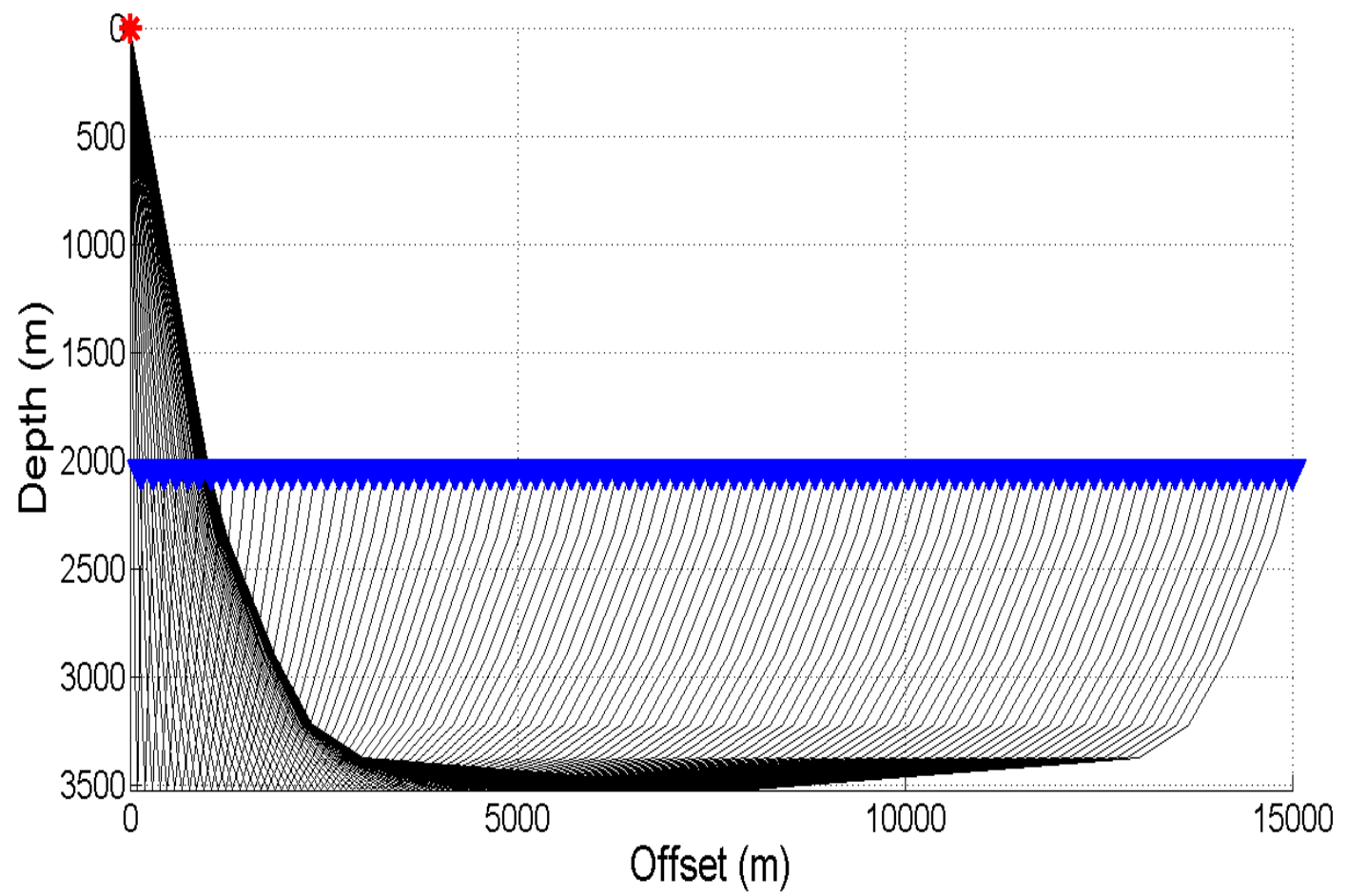

Figure 23: Ray tracing schematization of the PP reflection event from Model 4.

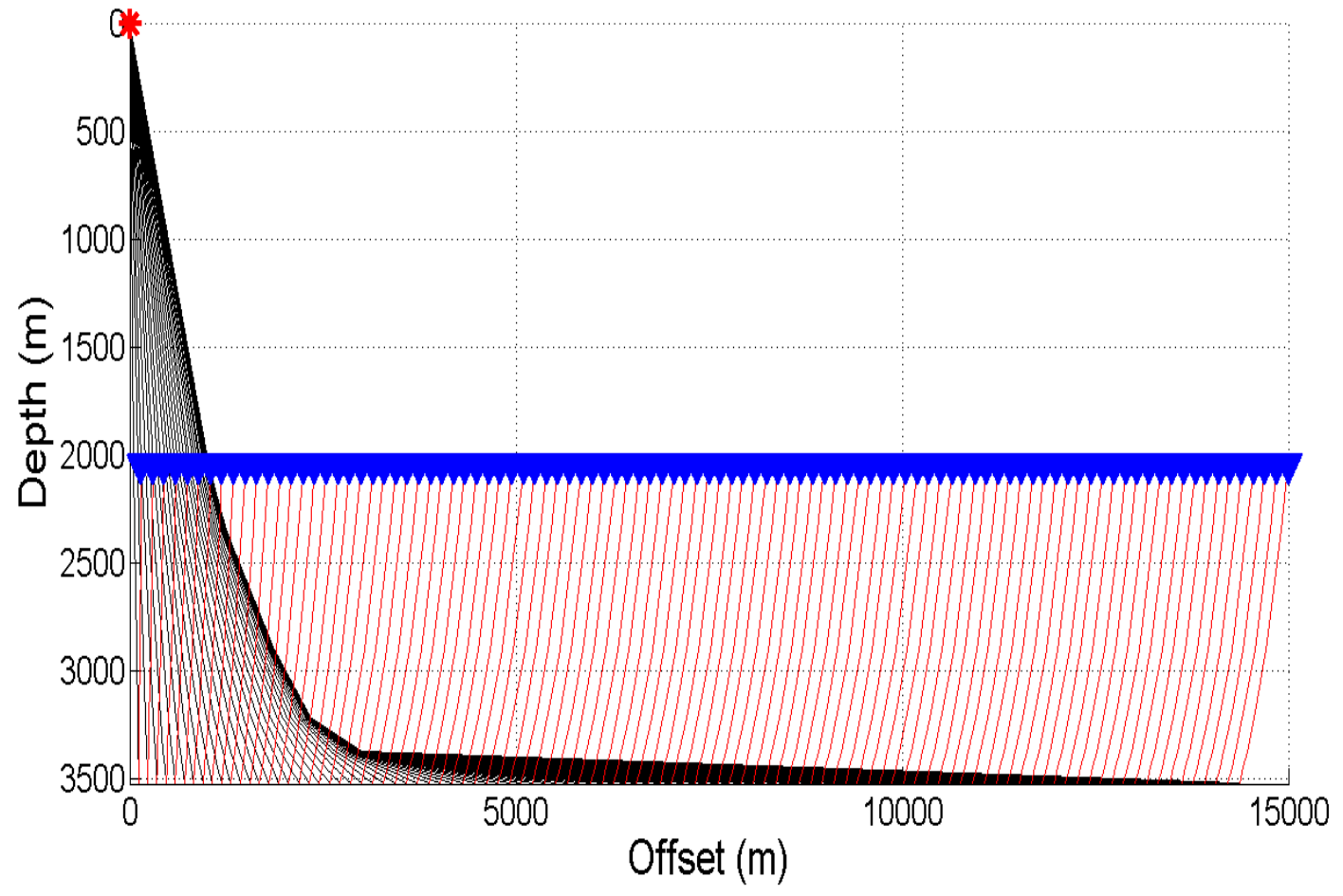

Figure 24: Ray tracing schematization of the PS reflection event from Model 4. 


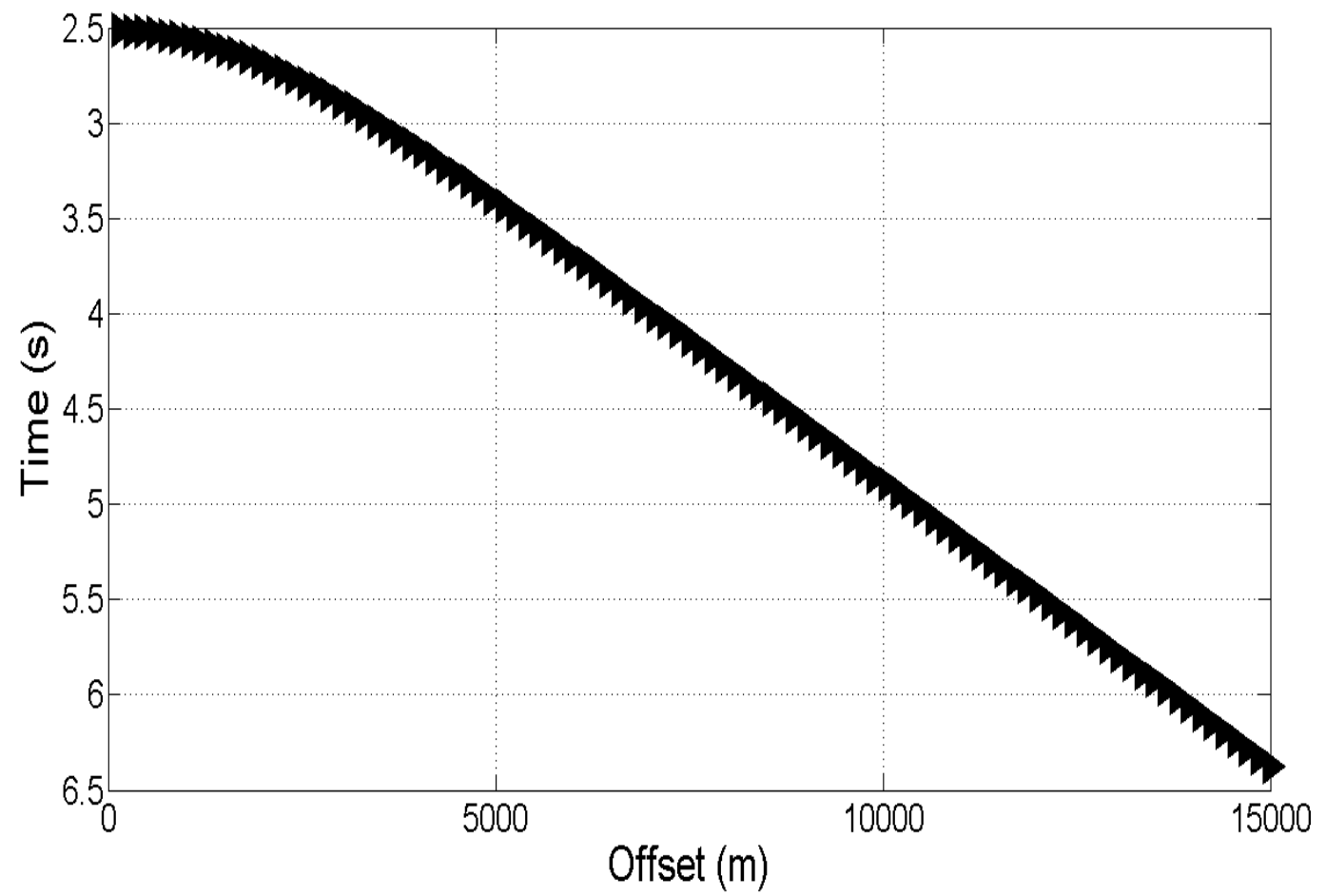

Figure 25: Travel-time curve of the PP reflection event from Model 4.

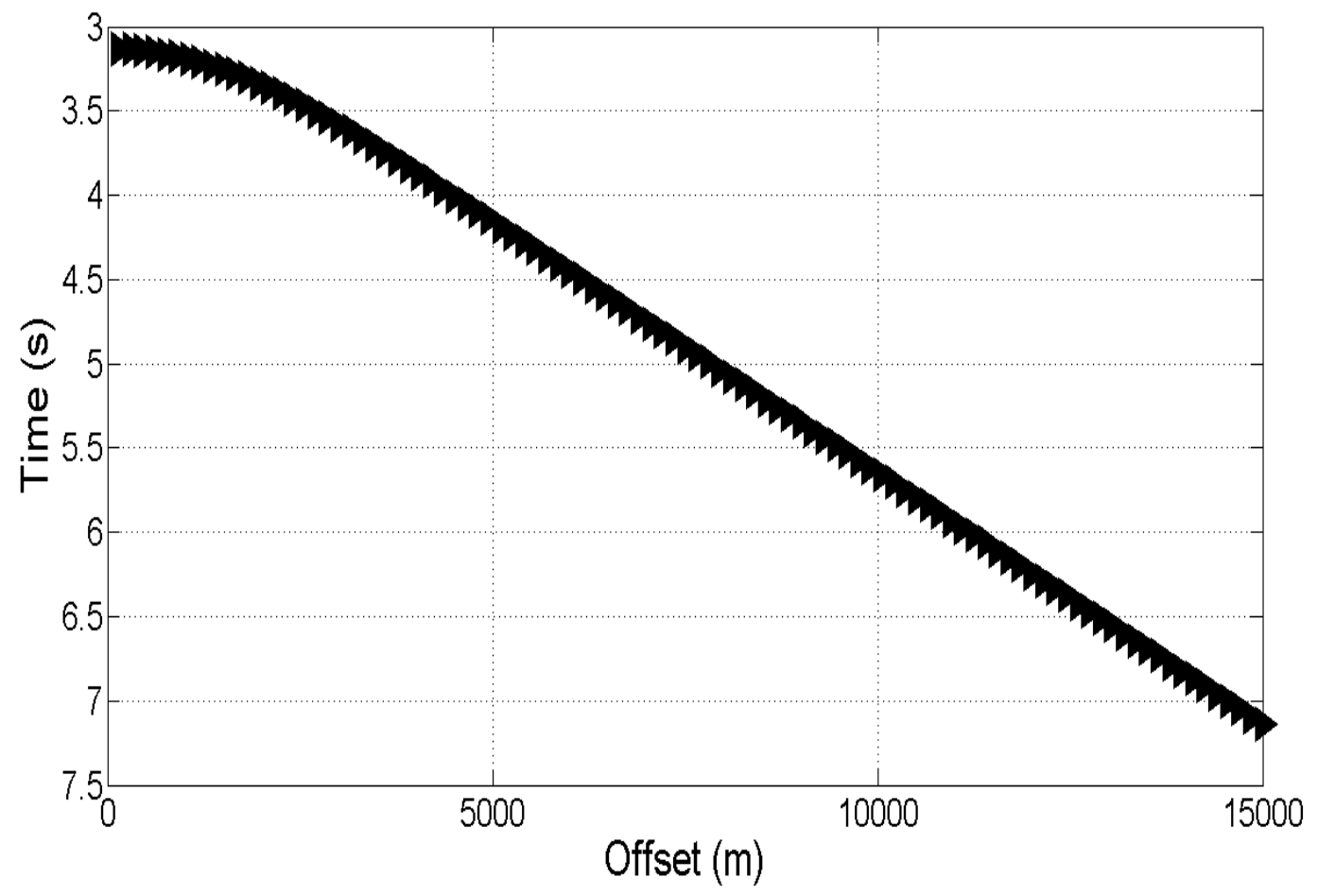

Figure 26: Travel-time curve of the PS reflection event from Model 4. 


\subsubsection{Model 5}

Concerning the sealing structure, the Model 5 is very similar to the Model 4. However, the fifth model has a shallower reservoir than the others, with a water depth of 1052 metres, the sealing shale layer starting at a depth of 2357 metres and the total depth of the reservoir is 2594 metres (Figure 27). Similarly to the Model 5, it is possible to observe the not so frequent variation of the inclination concerning the ray tracing (Figures 28 and 29). However, the fact that the target layer is so shallow for this geometry of acquisition, the RMS velocity can distort the reflection events even more (Figures 30 and 31).

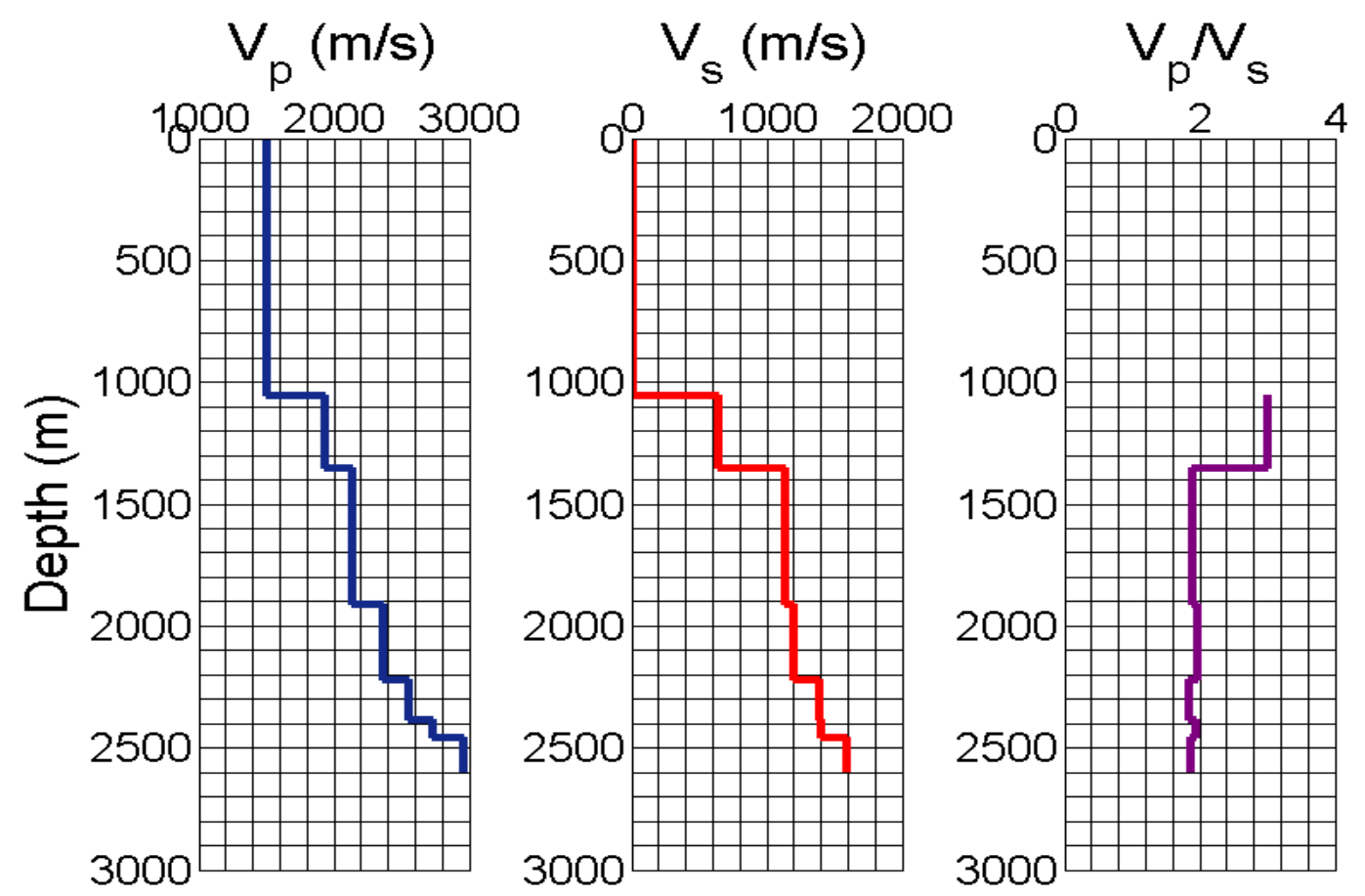

Figure 27: Velocity profiles of $\mathrm{P}$ wave, $\mathrm{S}$ wave, and $\mathrm{Vp} / \mathrm{Vs}$ ratio related to the depths of the Model 5. 


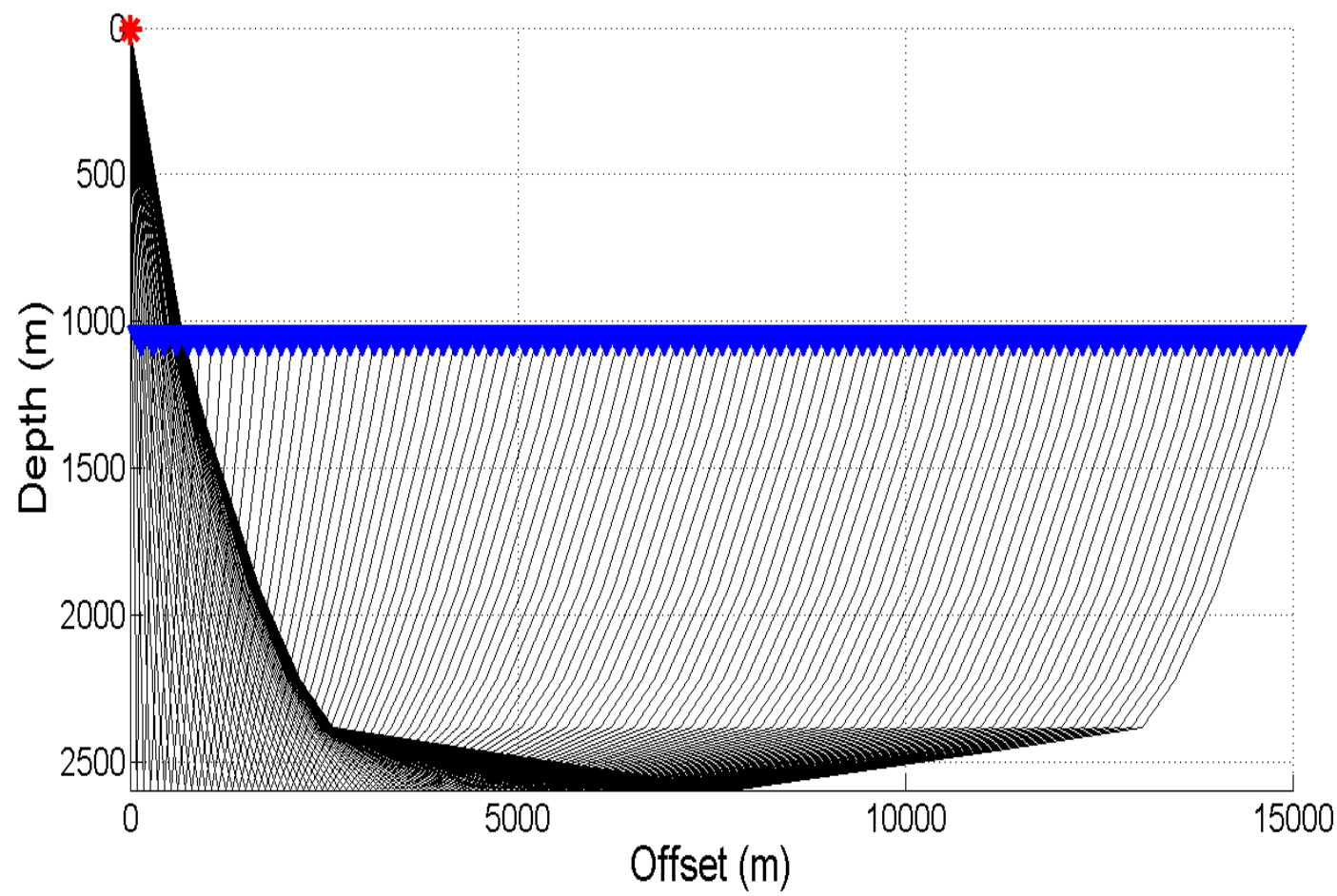

Figure 28: Ray tracing schematization of the PP reflection event from Model 5.

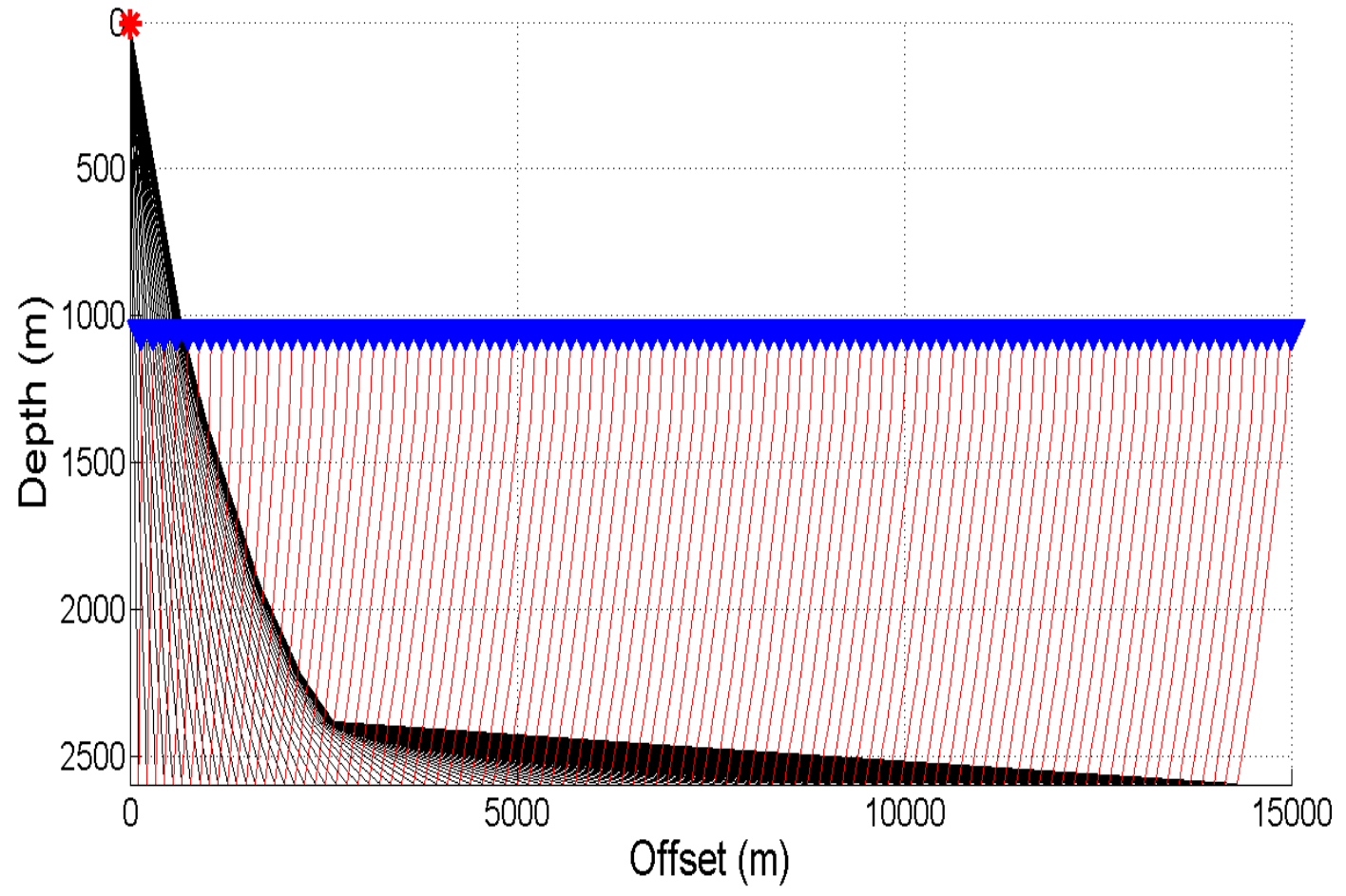

Figure 29: Ray tracing schematization of the PS reflection event from Model 5. 


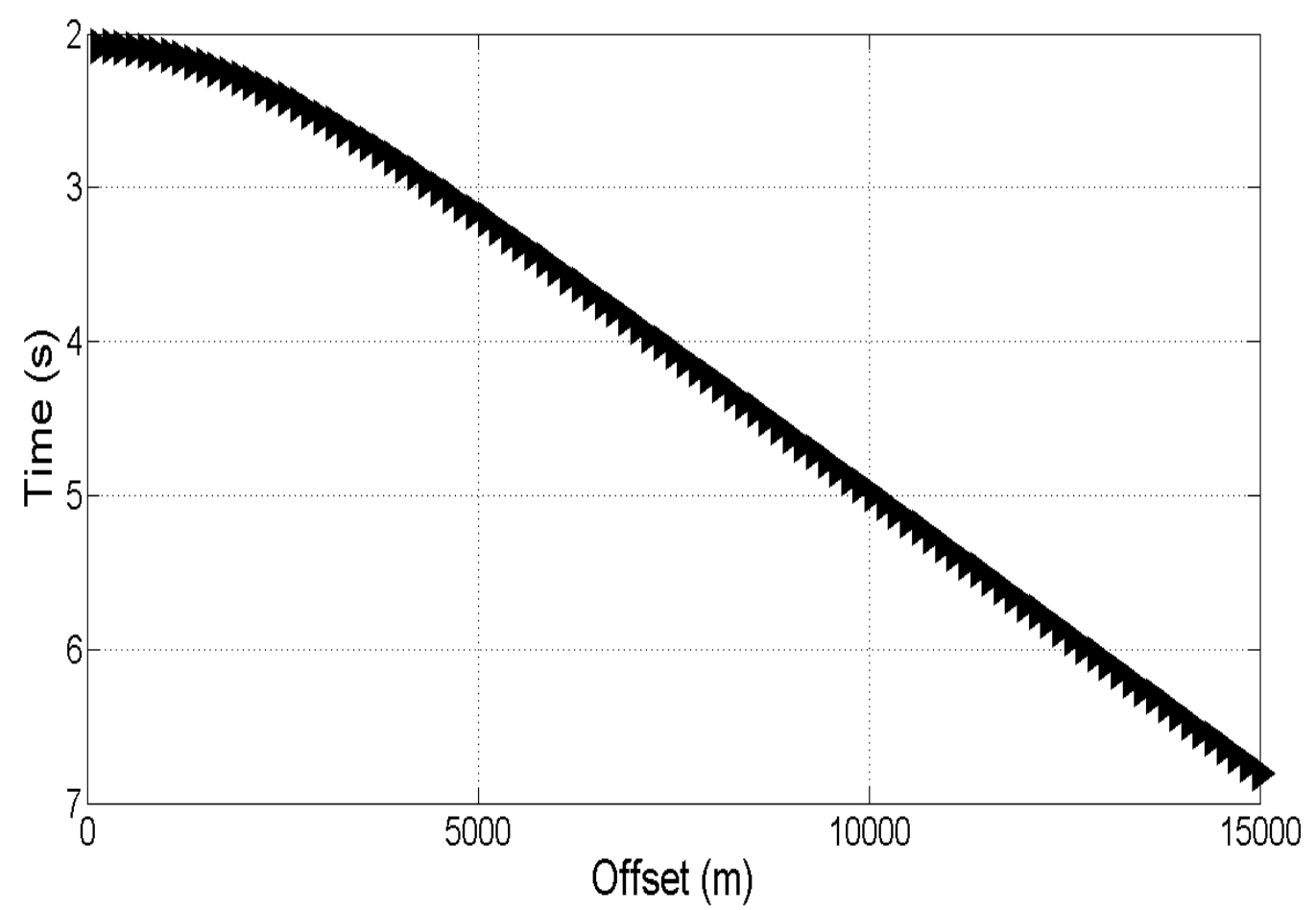

Figure 30: Travel-time curve of the PP reflection event from Model 5.

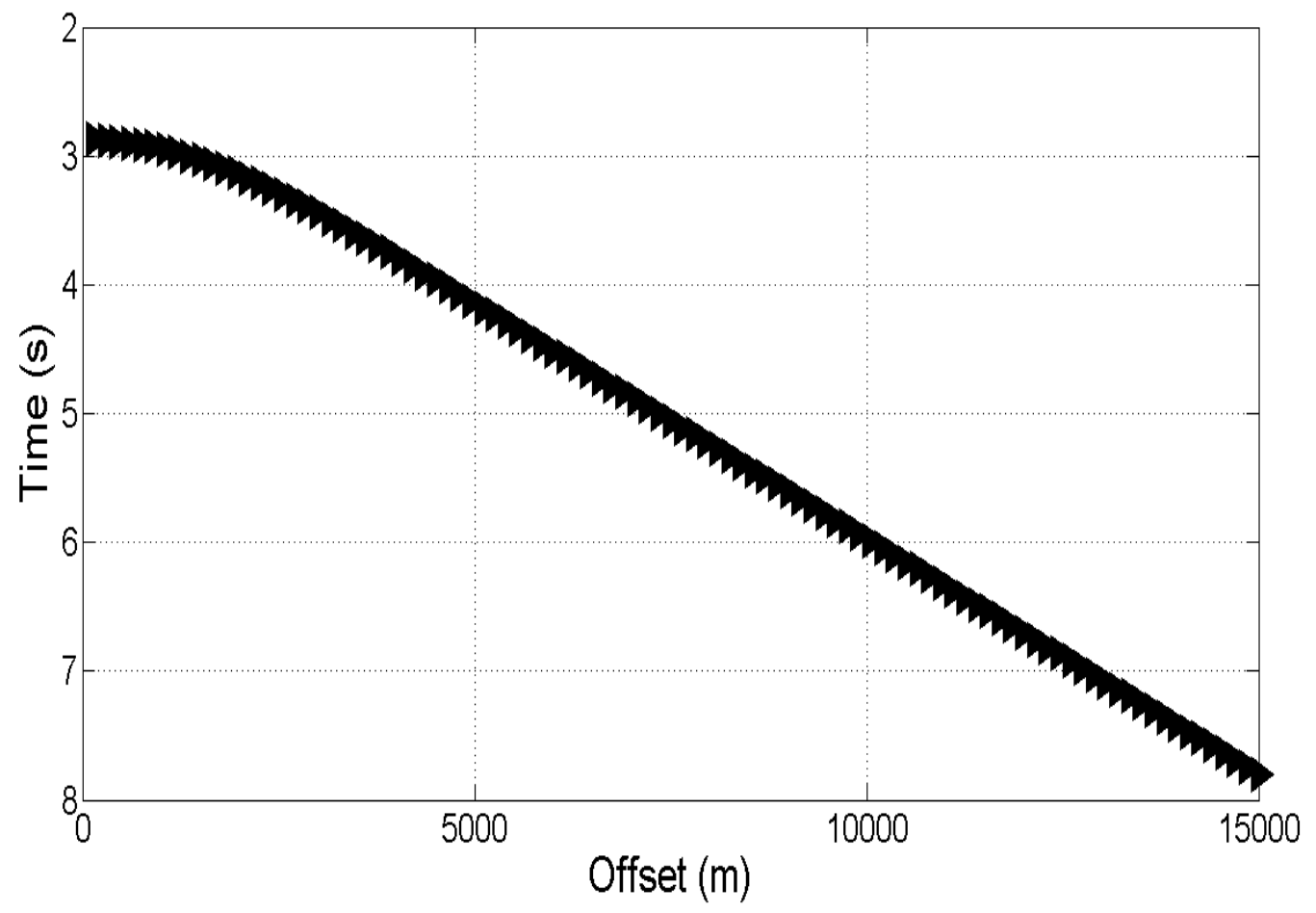

Figure 31: Travel-time curve of the PS reflection event from Model 5 


\section{COMPLEXITY ANALYSIS OF THE OBJECIVE FUNCTION}

\subsection{Inverse problem}

The mathematical inverse problem consists in obtaining, or calculating, the unknown cause of a known effect (Duarte, 2010; Aster, Borches and Thurber, 2012). It is necessary to know the precedence of the data to apply the inversion problem in an effective way, once the quality of the model analysis is dependent on the a priori information (Tarantola, 2005).

In this work, the proposed inversion is based on the curve fit between the calculated curve with each approximation and the observed travel-time curve in the seismic record. The travel-times are the effect and, from them, it is possible to determine the cause - in other words, it is possible to reach the information necessary to characterize the model.

\subsection{Direct problem}

The direct problem consists in mathematically calculating the effect of a known cause. It is related to the inverse problems, since it allows calculating an ensemble of effects generated by different models intended to represent a phenomenon, or perturbations in a single model (Duarte, 2010; Aster, Borches and Thurber, 2012).

It is proposed that the direct problem starts from the obtainment of the travel-times from a reflection PP or PS event making use of the ray tracing methods. The modelling was performed for multi-layered media, with large offsets between source and receivers, in offshore and ultra-depth water (depths larger than 1500 metres), and using the OBN technology to obtain converted waves.

\subsection{Optimization algorithms}

The main objective of the optimization is to reach the best option, in a set of options of input values to be used in the procedure, to find an extremum of a specified function (Horst, Pardalos and Thoai, 2000). In this work, it is aimed to reach the minimum point. 
The processes of optimization usually generate an improvement in each interaction until reaching the convergence in the minimum point of the function. However, several models can present more than one solution, due to its nonlinearity. This is common when an inversion problem is being solved, a situation in which the focus is to obtain the best possible approximation of the parameters to be estimated.

In this work, five known optimization algorithms from the literature were selected. Three of them (IMFL, FMINSEARCH and SID-PSM) are local search optimization algorithms, which were used with the multi-start procedure aiming to run the optimization algorithm several times, once they could not efficiently find a global minimum region among more than one minimum region, and, therefore, not providing a reliable solution. With the multi-start procedure, the optimization restarts its interactions from different random initial points. When the problem is unimodal, it can be easily applied without the multi-start procedure. For the multimodal problems, an alternative for the multi-start procedure is to use global search optimization algorithms, as MCS and TOMLAB/LGO.

The optimization algorithm, along with the norm, is the responsible to find the minimum region by minimizing the error between the calculated and the observed data. So, the calculation of the data with the approximation used for the initial parameters related to a random starting point on the topography of the function is performed, and then the difference between the calculated curve and the observed curve using one of the two norms is compared. If the minimum value found in the interaction is higher than the value defined initially as the minimum acceptable value, the optimization goes on for a next interaction until it reaches a minimum value lower than the value set as a minimum acceptable value. Therefore, the last interaction finishes and the last comparison between the observed and calculated data is performed. So, it provides the lowest error for a specific approximation with a specific algorithm minimized with a specific norm, as presented by Zuniga (2019a and 2019b).

\subsubsection{IMFIL}

IMFIL (Implicit Filtering) is an implementation, in MATLAB, of the implicit filtering algorithm, a local search algorithm with a deterministic sampling method for boundconstrained optimization. This kind of algorithm is useful to minimize functions which are 
non-smooth, noisy, random or discontinuous (Winslow et al., 1991; Gilmore and Kelley 1995; Kelley, 2011).

\subsubsection{FMINSEARCH}

FMINSEARCH (Find Minimum Search) is a MATLAB implementation of the Nelder-Mead algorithm (Nelder and Mead, 1965), which is based on the simplex method (Spendley et al., 1962; Lagarias et al., 1998). This local search algorithm is focused in the use of a simplex, a polytope of $n+1$ vertices in $n$ dimensions with edges of the same size, and it is useful for unconstrained optimization problems (Coxeter, 1948; Lewis, Torczon and Trosset, 2000).

\subsubsection{SID-PSM}

SID-PSM (Simplex Derivative - Pattern Search Method) is a local search algorithm implemented in MATLAB, based on a pattern search method with the pool step guided by simplex derivatives (Custódio and Vicente, 2007 and 2008). This kind of algorithm was created to solve unconstrained and constrained problems, and each search step is based on the optimization of quadratic surrogate models (Custódio et al., 2010).

As the optimization methods shown previously are local search algorithms, it is necessary to use the multi-start procedure which is based on performing several inversions starting from different sets of initial points (Kan and Timmer, 1978a and 1978b; Telraky and Sotirov, 2010 and 2013).

\subsubsection{MCS}

MCS (Multilevel Coordinate Search) is a MATLAB implementation of the algorithm for global optimization of bound-constrained problems (Neumaier et al., 2005). This algorithm is based on performing the partition of the search space into boxes with an evaluated base point. The global local search is balanced by a multilevel approach, according to which box is assigned a level s that is an increasing function of the number of times that the box was processed (Huyer and Neumaier, 1999). 


\subsubsection{TOMLAB/LGO}

The TOMLAB/LGO is a TOMLAB solver implemented in MATLAB. This kind of solver provides access to several derivative-free optimization solvers (Jones, 2001; Holmström et al., 2007a and 2007b; Holmström et al., 2008). The LGO (Local and Global Optimization) solver used is a combination of global and local nonlinear solvers that implements a combination of Lipschitzian-based branch-and-bound with deterministic and stochastic local search (Pintér, 1995; Pintér et al., 2006).

Other optimization algorithms were considered; however, for the similarities in the operations and in the results concerning the selected methods, some algorithms, such as SNOBFIT (Huyer and Neumaier, 2008), PSWARM (Vaz and Vicente, 2007), CMA (Hansen, 2006; Hansen, 2016), among others (Rios and Sahinidis, 2013), were not used in this work.

\subsection{L1- and L2-norm}

A norm is, concerning the mathematics, the total length or the total size of, respectively, a set of vectors in a vector space or a set of matrices in a matrix space. A norm is essentially a function, for example, which assigns a strictly positive length or size to each vector in a vector space; however, it is not valid to the zero vector, due to the fact that, in this case, the length is assigned as zero. So, the higher the norm is, the bigger the value in the vector or in the matrix is (Treves, 1967). The norm is also known as Euclidian distance, among other names.

When the L2-norm is used, also known as least squares, it is based on the minimization of the sum of the square of the differences between the observed value and the estimated value. On the other hand, the L1-norm is known as least absolute deviation or least absolute errors. For this case, the minimization is performed minimizing the sum of the absolute differences between the observed value and the estimated value (Khaleelulla, 1982). The difference between the L1- and L2-norm is represented in Figure 32. 
L1

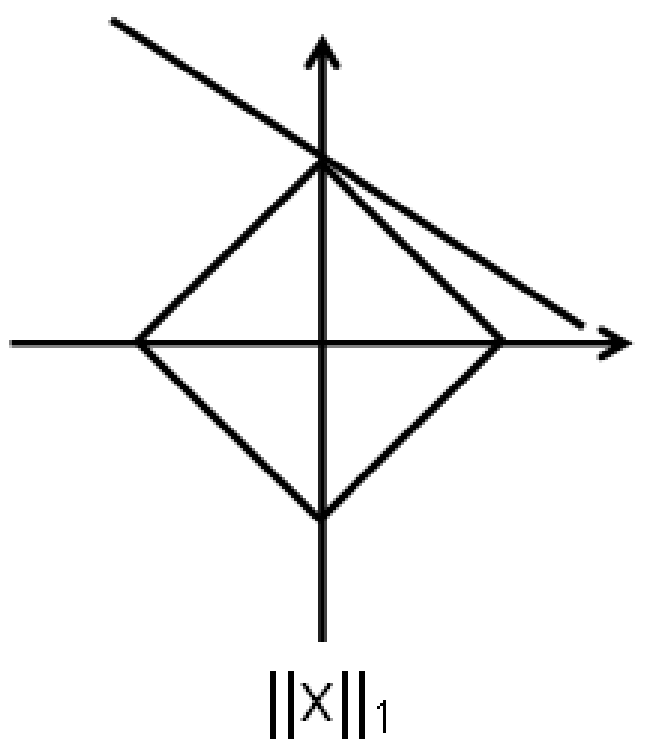

L2

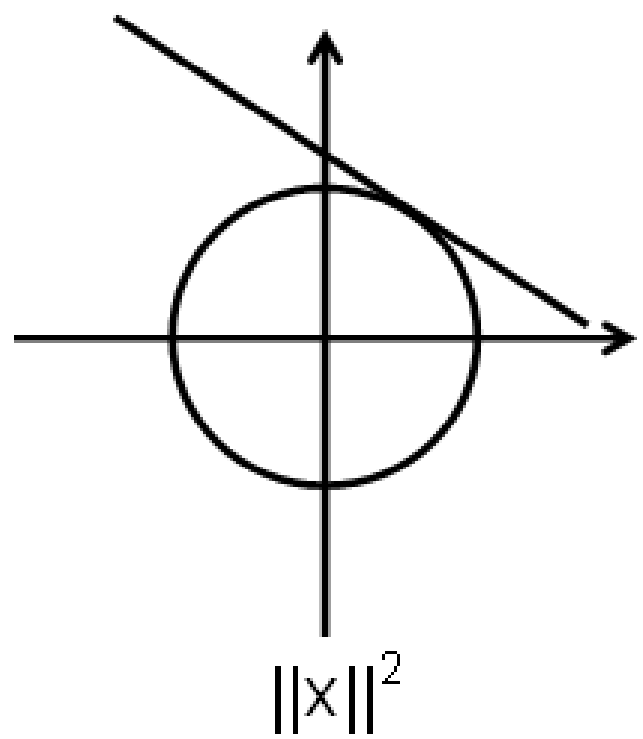

Figure 32: Two-dimensional example showing the sparseness of a solution when using the L1-norm and the L2norm.

The L2-norm is the most popular one, and it is used in many fields of physics and engineering. Even though the solution of least squares method is easy to compute, it may be not the best solution, due to its smooth nature. For this reason, in many cases, it is hard to find a solution which is single and, at the same time, the best one for the problem. However, the L1-norm, in some cases, can provide a better set of results due to its not-smooth nature, once the unicity is stronger in this kind of problem. Even though the L1-norm minimization has a similar form to the L2-norm, it is much harder to solve. Due to the absence of a smooth behaviour in this kind of function, the search for the best solution must be performed directly by seeking the means of the computed solutions (Bourbaki, 1987).

Even though it is difficult to find a solution with the L1-norm, the increase of the computational processing power in the last decades allowed overcoming these limitations. Then, it is possible to use it as direct search, and also to divide the space in parts of L1-norm and other parts of L2-norm.

In this case of comparing the optimization algorithms, it was necessary to select derivative free algorithms, once algorithms which use derivatives would not work for L1- 
norm. For this reason, it was necessary to select optimization algorithms which are able to be used for both norms, as it was presented by Zuniga et al. (2019b and 2019c).

\subsection{Objective function analysis}

The objective function is the function which defines the quality of a solution in relation to its variables.

The method used to make the estimation of the parameters of the travel-time approximations possible is based on the implementation of an algorithm aiming to optimize the fit for the reflection PP and PS events.

The least squares method is the standard approach to approximate solutions. This method consists in reaching the best fit for a set of data aiming to minimize the sums of the squares of the differences between the estimated values and the observed values (Stigler, 1981). In this work, the least squares method was used to perform the inversion to measure how close the estimated travel-times and the observed travel-times are. With the minimum value of the function, there is the optimum value of the function, once the sum of the squares of the differences between the observed and estimated travel-times is the least possible.

With this, it is necessary to select the search algorithm; however, it is essential to analyse how complex the problem is. This analysis can be performed by observing the residual function maps (Larsen, 1999, Kurt, 2007). It is appropriated to use this kind of analysis for problems with two variables. However, in the cases with three or more variables, it is not possible to have reliable information concerning the complexity of the function. It is associated to the fact that the construction of the map has two parameters as variables (velocity and the nonhyperbolicity parameter), and the third parameter (the time for zero offset) is fixed in its value associated to the minimum value, the closest value from the correct one. To overcome these limitations, a method which consists in analysing the dispersions of the results obtained from several inversions was adopted. The results obtained are plotted in a hyperplane, where the vertical and horizontal axes are the related parameters, and the third dimension is the representation of the minimum values of the objective function (Bokhonok, 2010; Zuniga, 2015). 


\subsubsection{Model 1}

It was possible to observe that the Malovichko (1978) approximation showed a homogeneous topological structure concerning the objective function. Also, it showed to be a unimodal approximation in studied cases (Figures 33 and 34). Generically, all approximations showed a PS converted wave event with the structure displaced along the values of the axes, and this displacement is associated to the difference between the parameters of the conventional event and the converted event. Even with the L1-norm, the variation in the structure was subtle. However, the difference between the maximum region and the global minimum region became more abrupt and with a narrower global minimum region (Figures 35 and 36).

The approximation proposed by Alkhalifah and Tsvankin (1995) showed the same kind of variation between the PP and PS events, and for the L2- and L1-norm (Figures 37, 38, 39 and 40).

Ursin and Stovas (2006) approximation for this model showed to have a unimodal statistical distribution, in the same way as previous works with similar models. This approximation showed the same variations between the Figures 41 and 42, and Figures 43 and 44.

The Figures 45 and 46 showed the same variation between a PP and a PS reflection event, which can also be seen concerning the variations between the L2- and L1-norm (Figures 47 and 48). Further, this approximation (Blias, 2009), which presented the same characteristics of the Ursin and Stovas (2006) one, also showed to be unimodal for this model.

The approximation developed by Muir and Dellinger (1985) presented a multimodal behaviour. However, the variation between the PP and PS reflection events was different than the others (Figures 49 and 50). Its variation was not only concerning the displacement of the objective function structure, but there was also a variation of the structural position between the global and local minimum region. This factor is due to the local and global minimum values being so close to each other. The variation concerning the L2- and L1-norm (Figures 51 and 52) presented the same kind of variation as the other approximations.

The approximation proposed by $\mathrm{Li}$ and Yuan (2001) showed a multimodal behaviour and only a structural variation between the PP and PS events (Figures 53 and 54), and the same variation between L2- and L1-norm as the other approximations (Figures 55 and 56). 
The approximation proposed in this work showed the same characteristics and variations as the approximation proposed by $\mathrm{Li}$ and Yuan (2001), due to the fact that the development of this approximation shares the same origin conserving some characteristics and behaviours (Figures 57, 58, 59 and 60). The main difference is concerning the topological structure of the objective function which presented an additional parameter as a less sensible variable than what is shown by Li and Yuan (2001) approximation. 


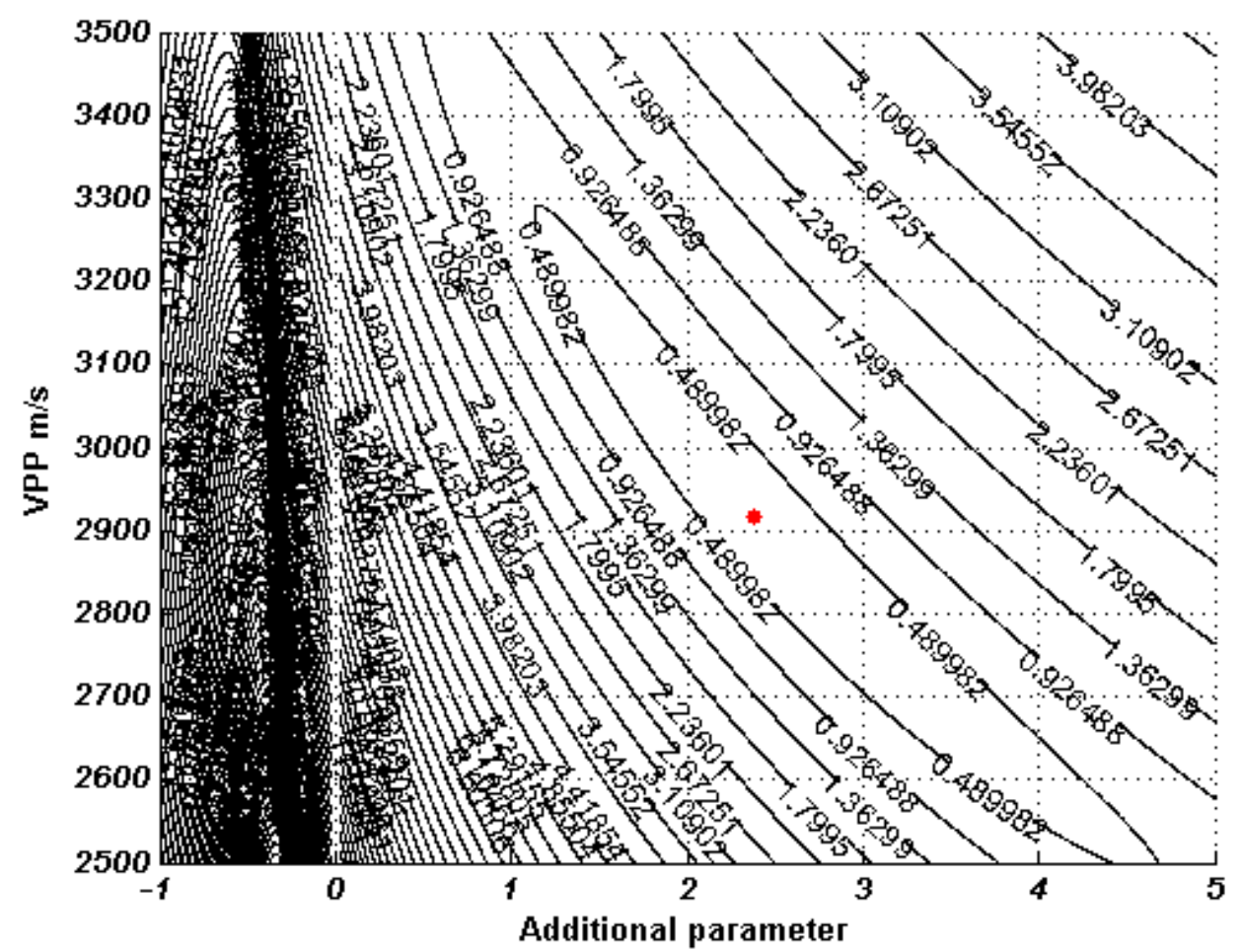

Figure 33: The residual function map which demonstrates the complexity of the Malovichko (1978) approximation of the PP reflection event with L2-norm. Red dispersions represent the global minimum region, and the blue dispersions, the local minimum regions.

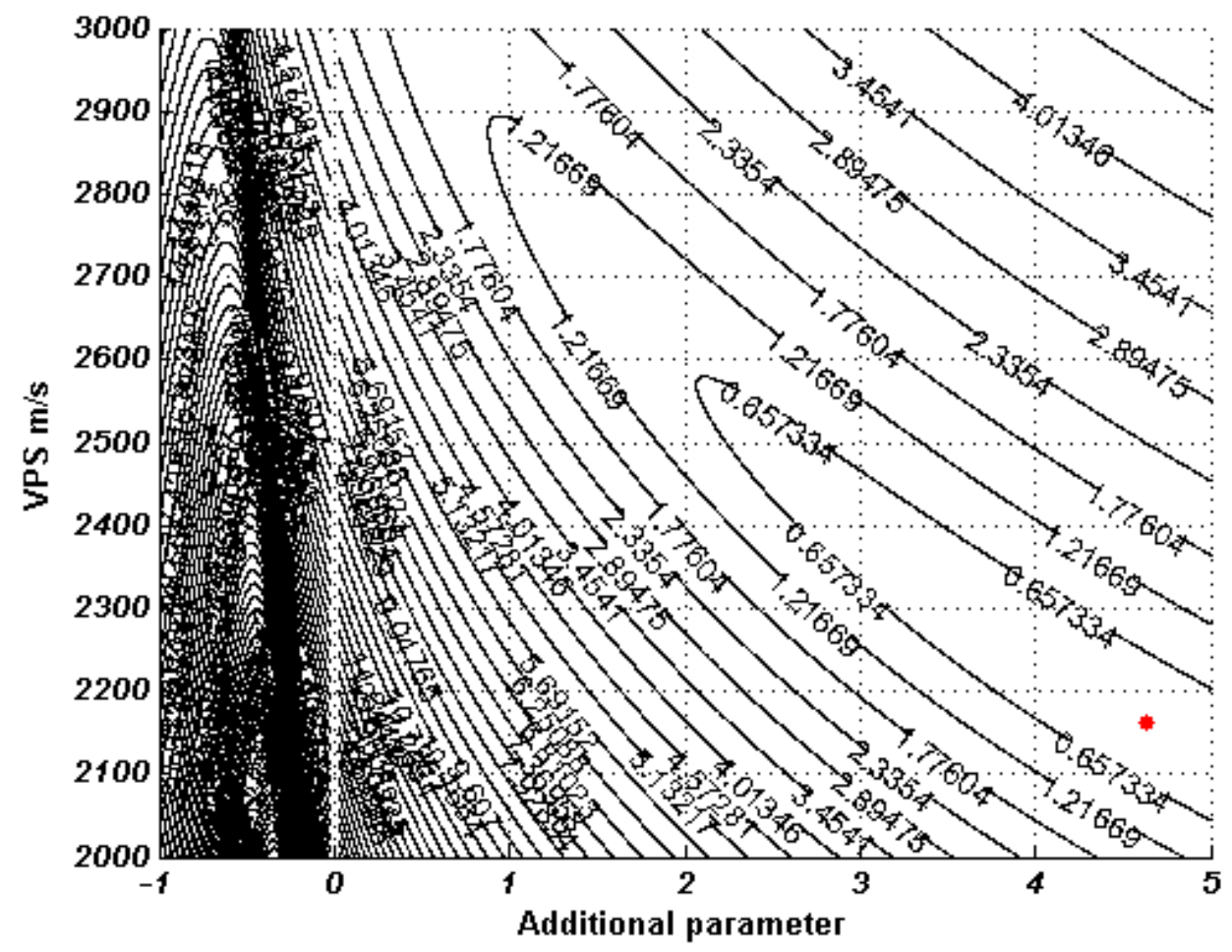

Figure 34: The residual function map which demonstrates the complexity of the Malovichko (1978) approximation of the PS reflection event with L2-norm. Red dispersions represent the global minimum region, and the blue dispersions, the local minimum regions. 


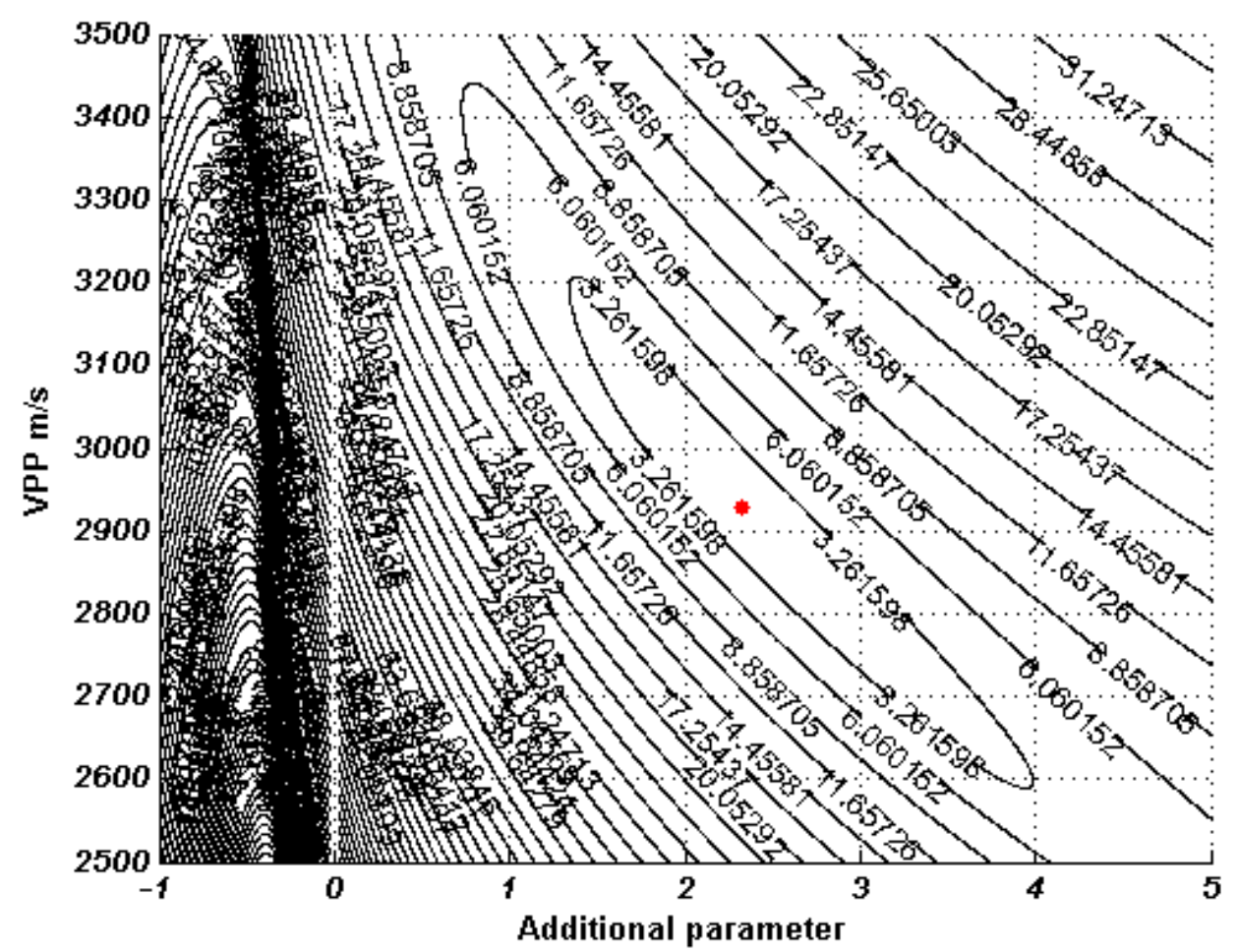

Figure 35: The residual function map which demonstrates the complexity of the Malovichko (1978) approximation of the PP reflection event with L1-norm. Red dispersions represent the global minimum region, and the blue dispersions, the local minimum regions.

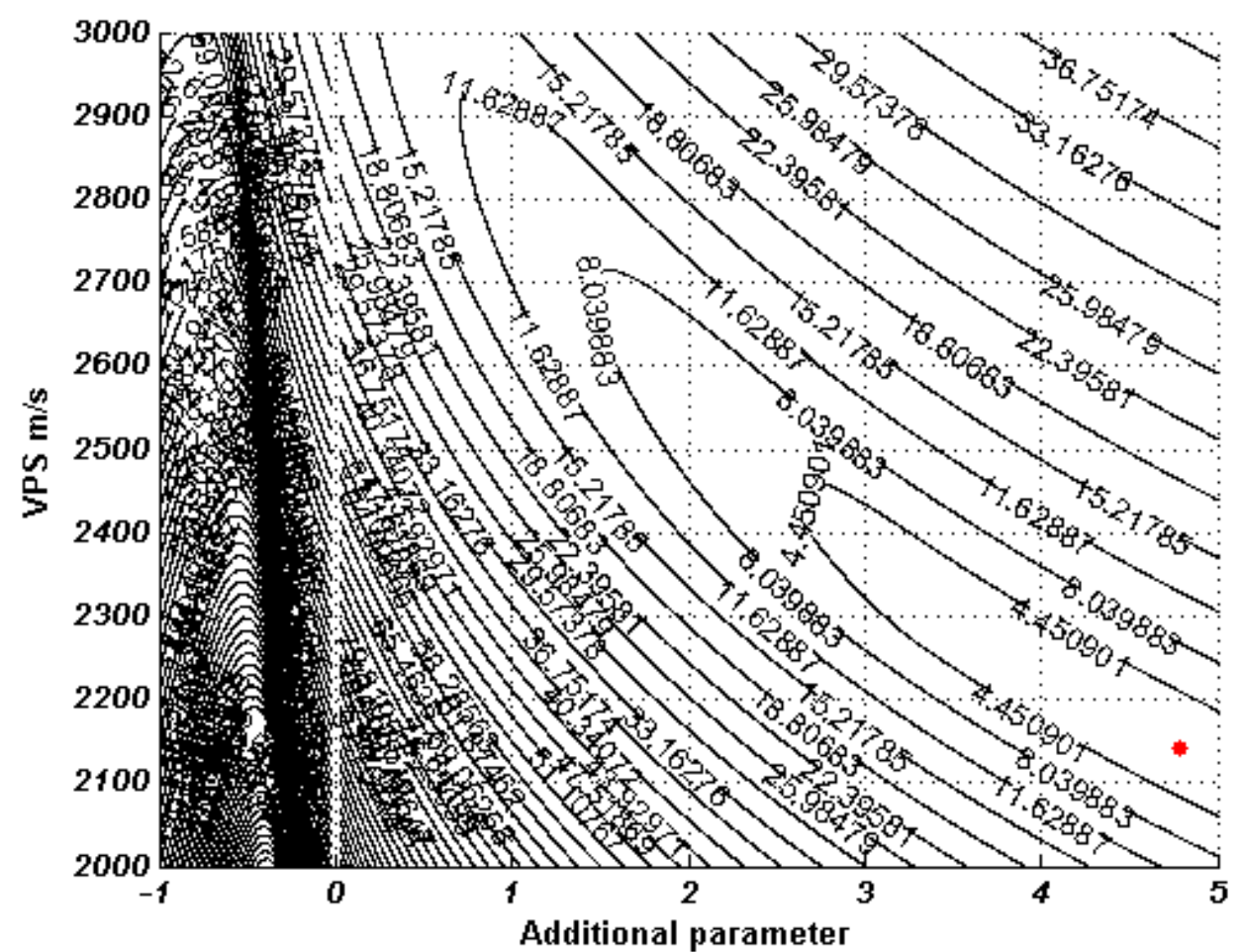

Figure 36: The residual function map which demonstrates the complexity of the Malovichko (1978) approximation of the PS reflection event with L1-norm. Red dispersions represent the global minimum region, and the blue dispersions, the local minimum regions. 


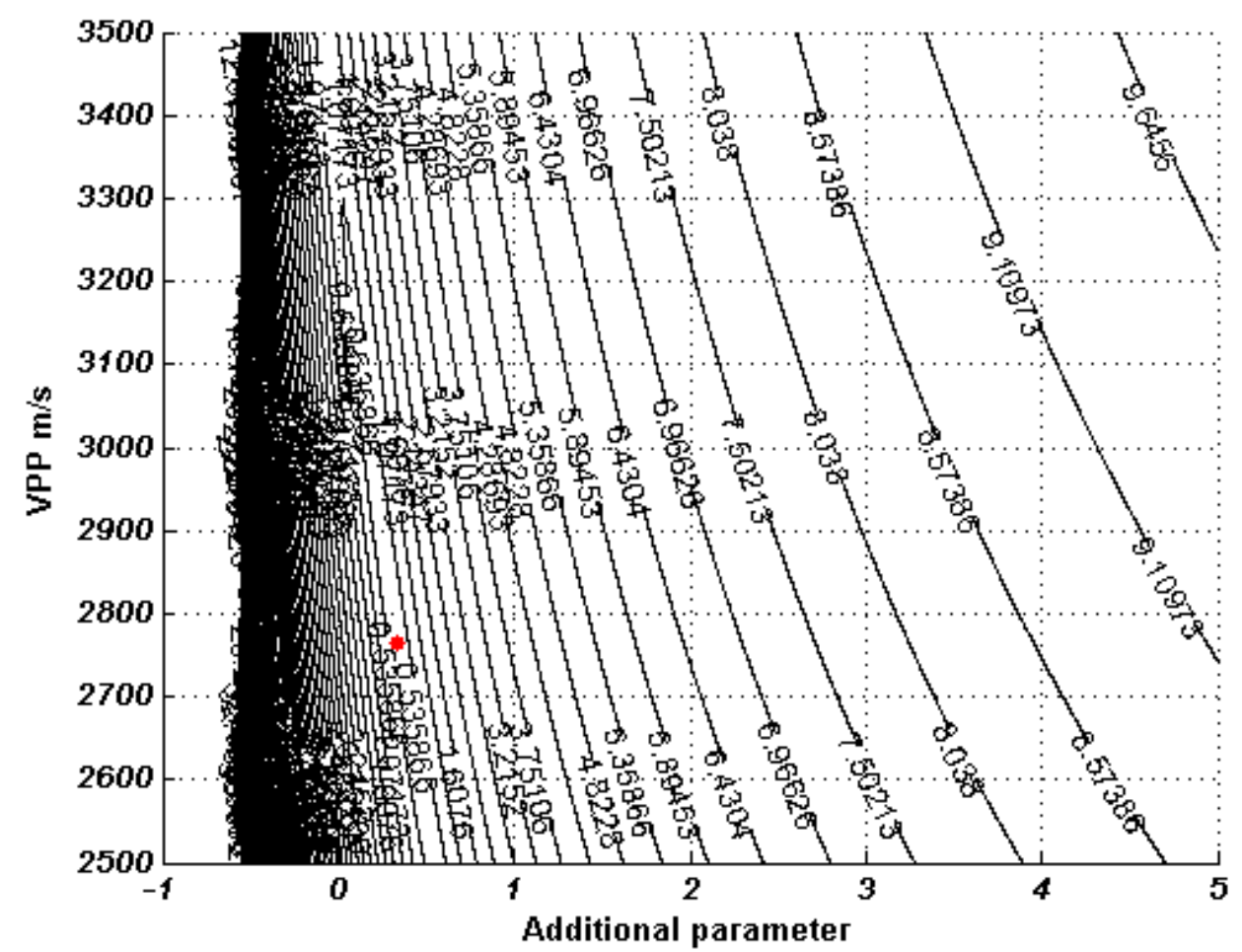

Figure 37: The residual function map which demonstrates the complexity of the Alkhalifah and Tsvankin (1995) approximation of the PP reflection event with L2-norm. Red dispersions represent the global minimum region, and the blue dispersions, the local minimum regions.

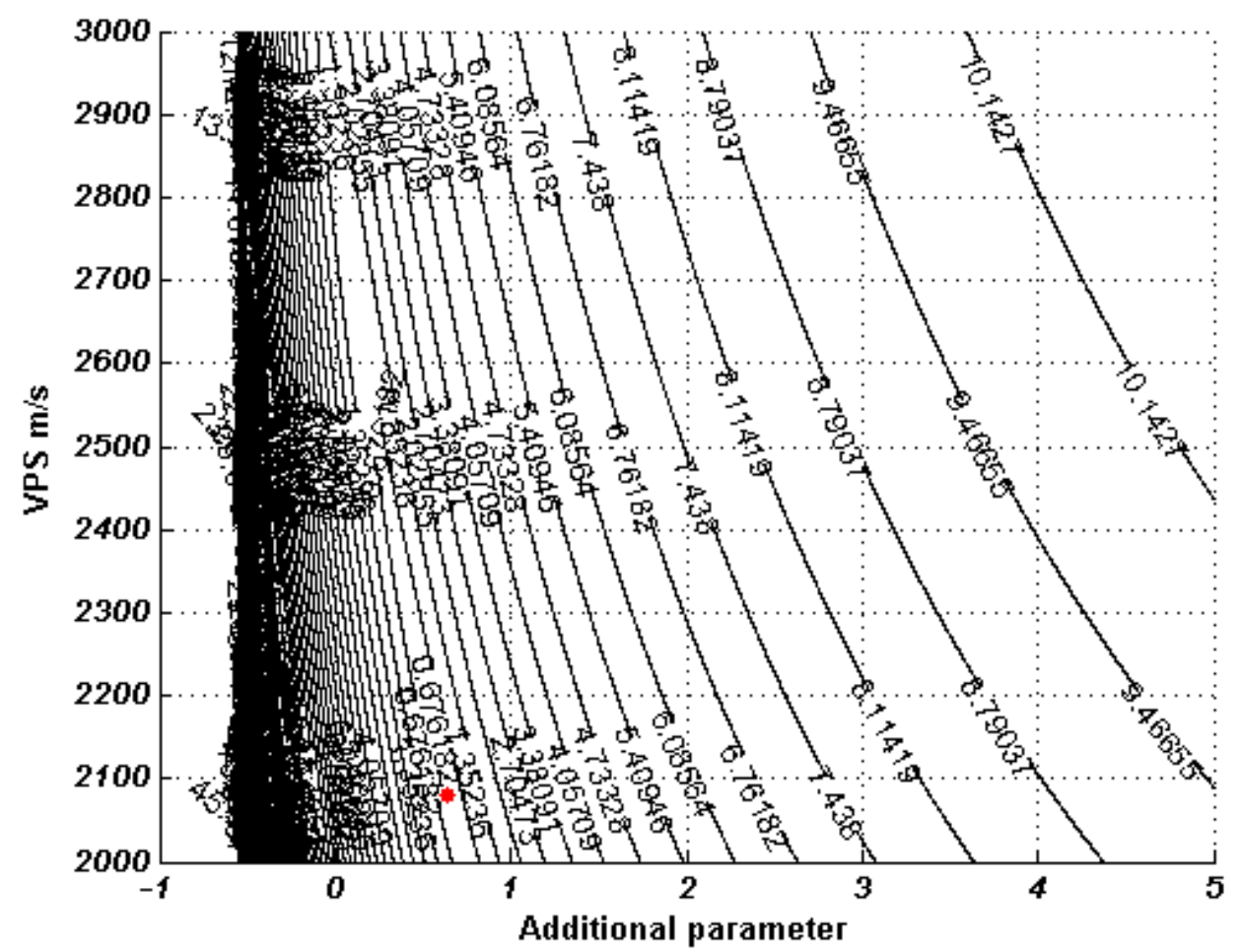

Figure 38: The residual function map which demonstrates the complexity of the Alkhalifah and Tsvankin (1995) approximation of the PS reflection event with L2-norm. Red dispersions represent the global minimum region, and the blue dispersions, the local minimum regions. 


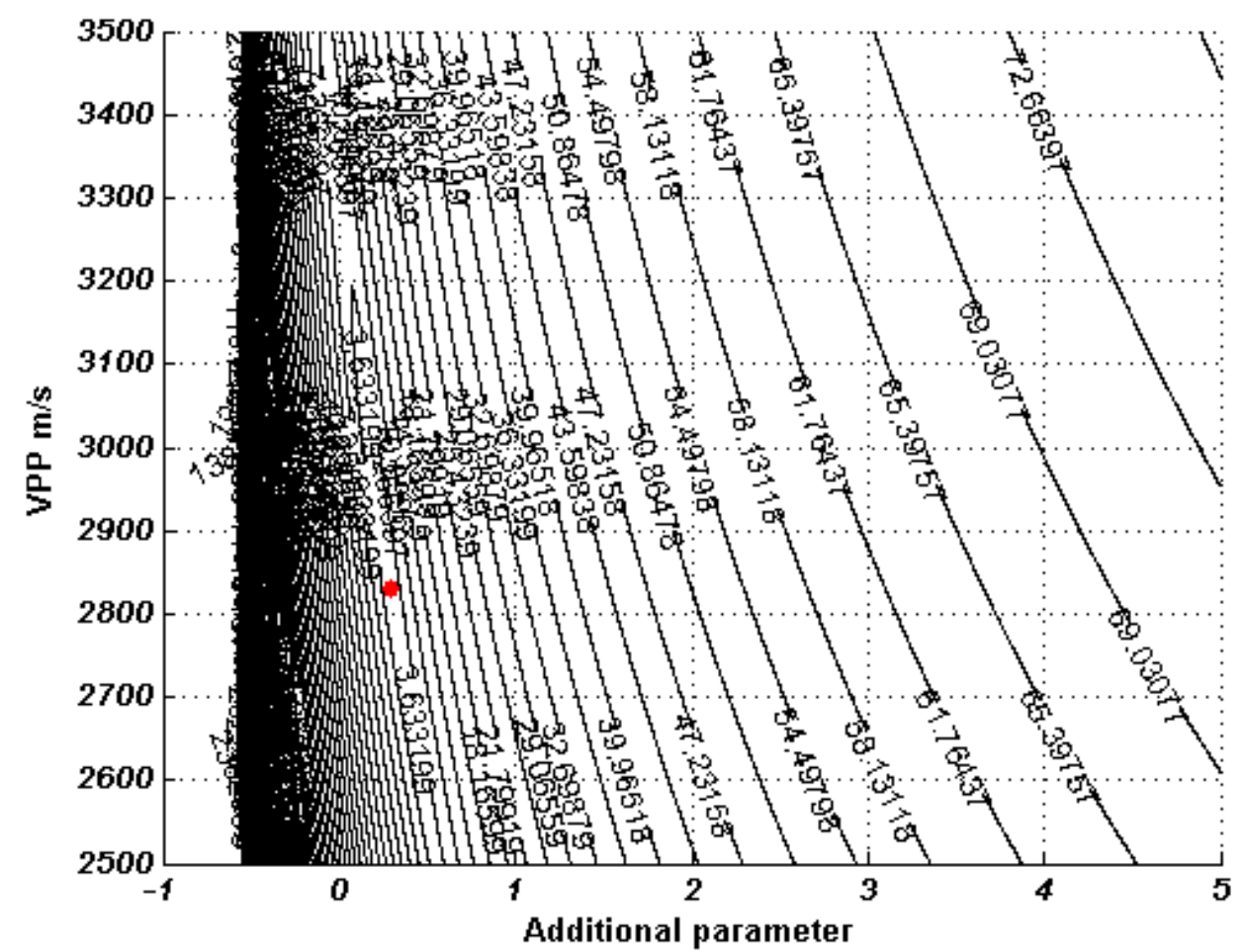

Figure 39: The residual function map which demonstrates the complexity of the Alkhalifah and Tsvankin (1995) approximation of the PP reflection event with L1-norm. Red dispersions represent the global minimum region, and the blue dispersions, the local minimum regions.

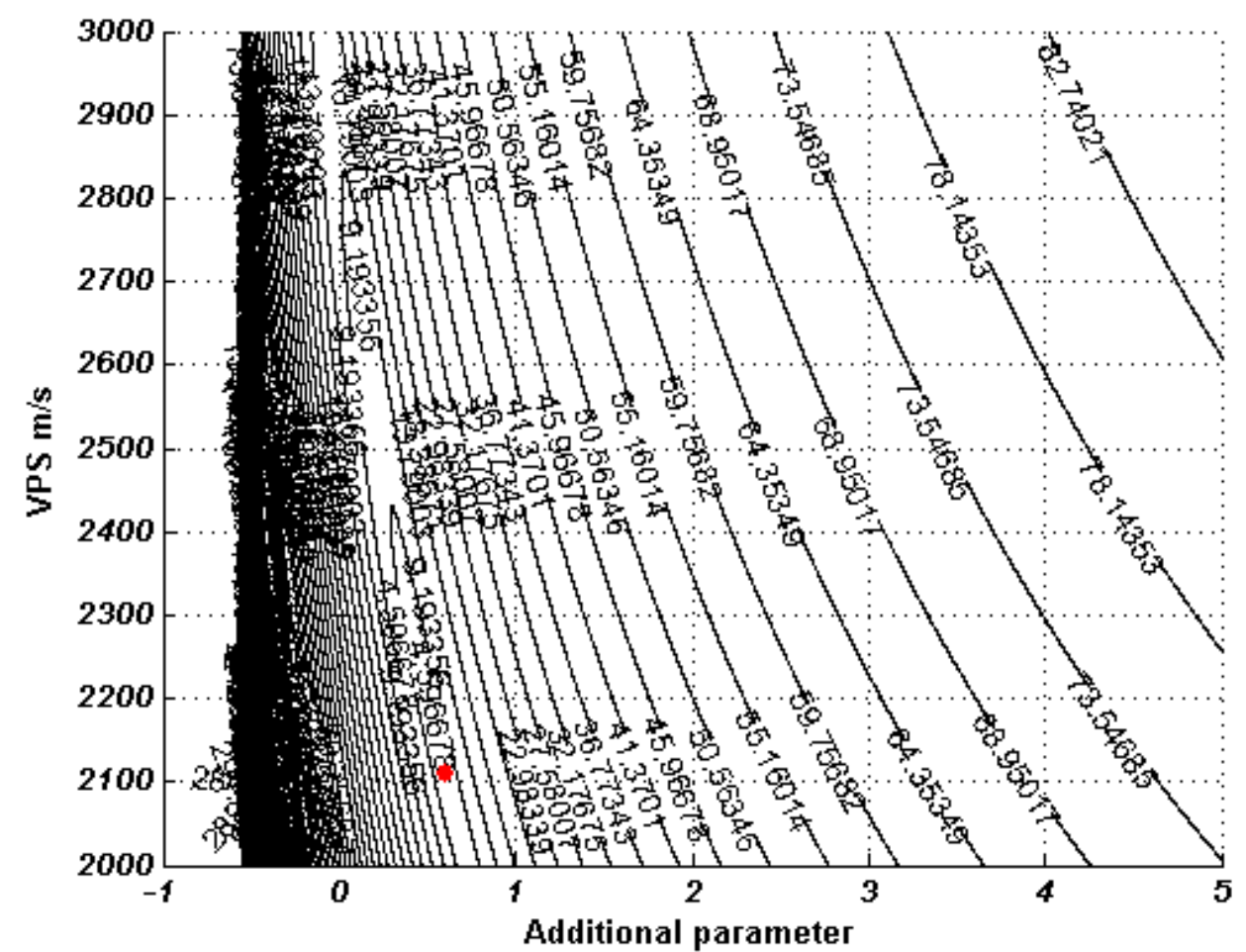

Figure 40: The residual function map which demonstrates the complexity of the Alkhalifah and Tsvankin (1995) approximation of the PS reflection event with L1-norm. Red dispersions represent the global minimum region, and the blue dispersions, the local minimum regions. 


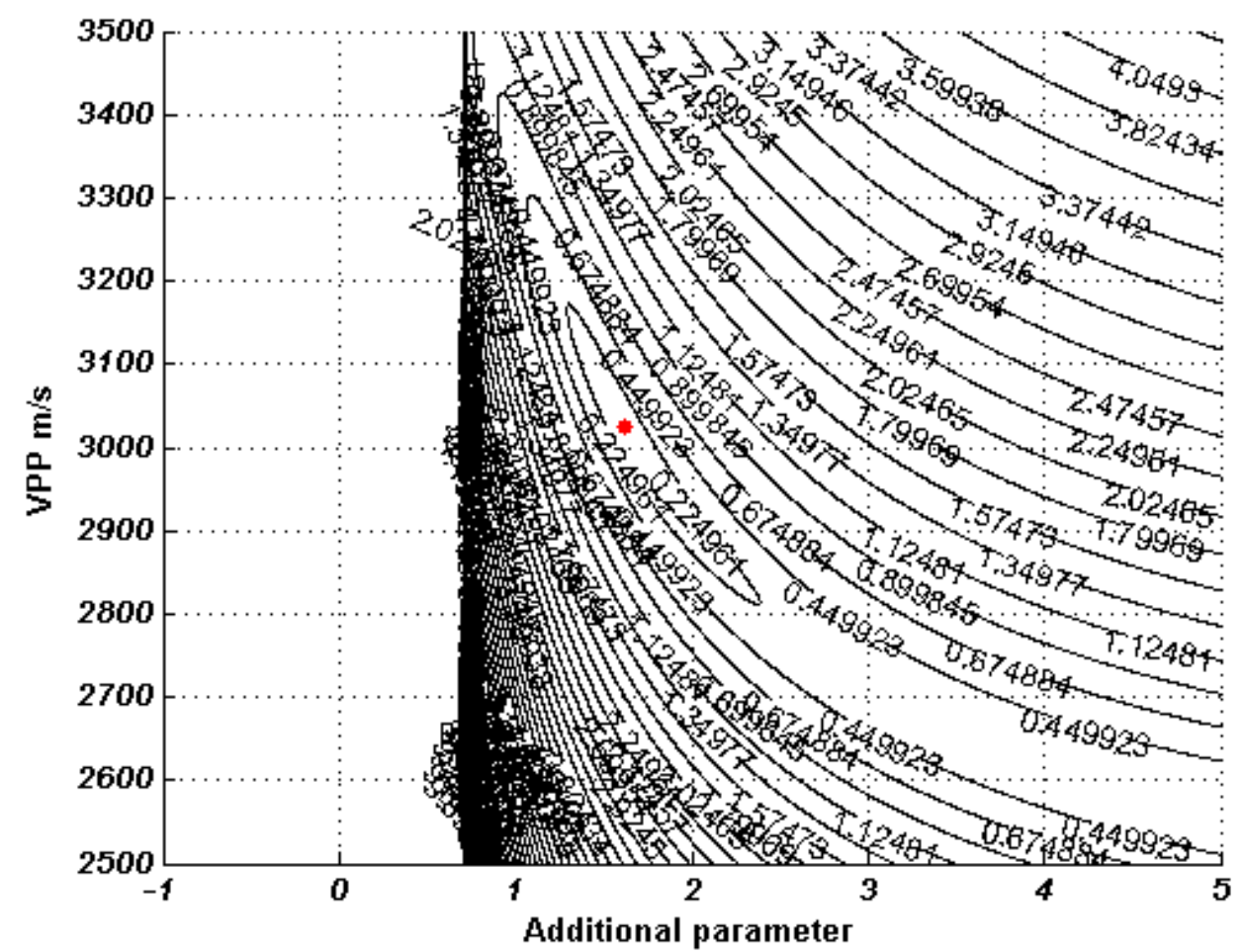

Figure 41: The residual function map which demonstrates the complexity of the Ursin and Stovas (2006) approximation of the PP reflection event with L2-norm. Red dispersions represent the global minimum region, and the blue dispersions, the local minimum regions.

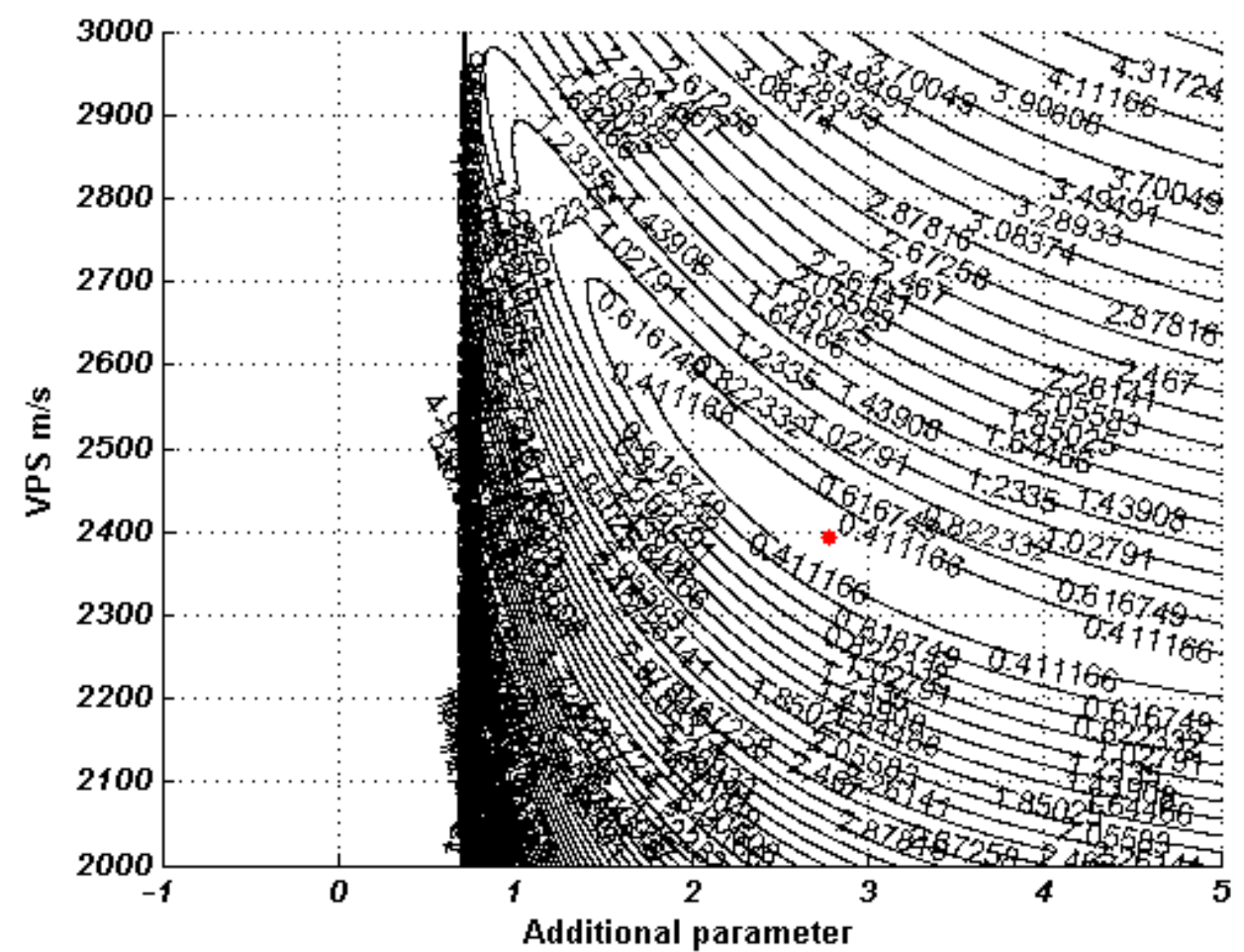

Figure 42: The residual function map which demonstrates the complexity of the Ursin and Stovas (2006) approximation of the PS reflection event with L2-norm. Red dispersions represent the global minimum region, and the blue dispersions, the local minimum regions. 


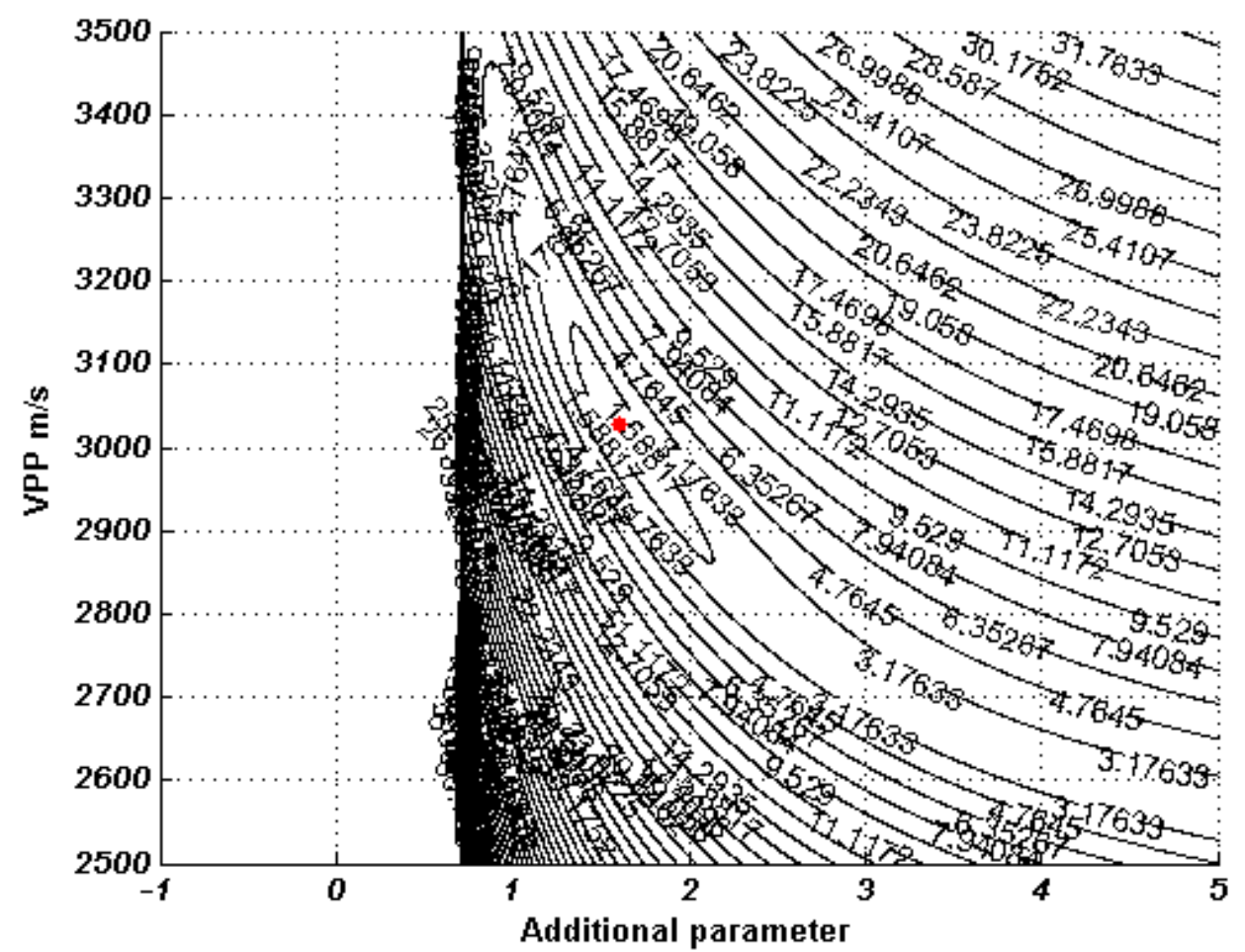

Figure 43: The residual function map which demonstrates the complexity of the Ursin and Stovas (2006) approximation of the PP reflection event with L1-norm. Red dispersions represent the global minimum region, and the blue dispersions, the local minimum regions.

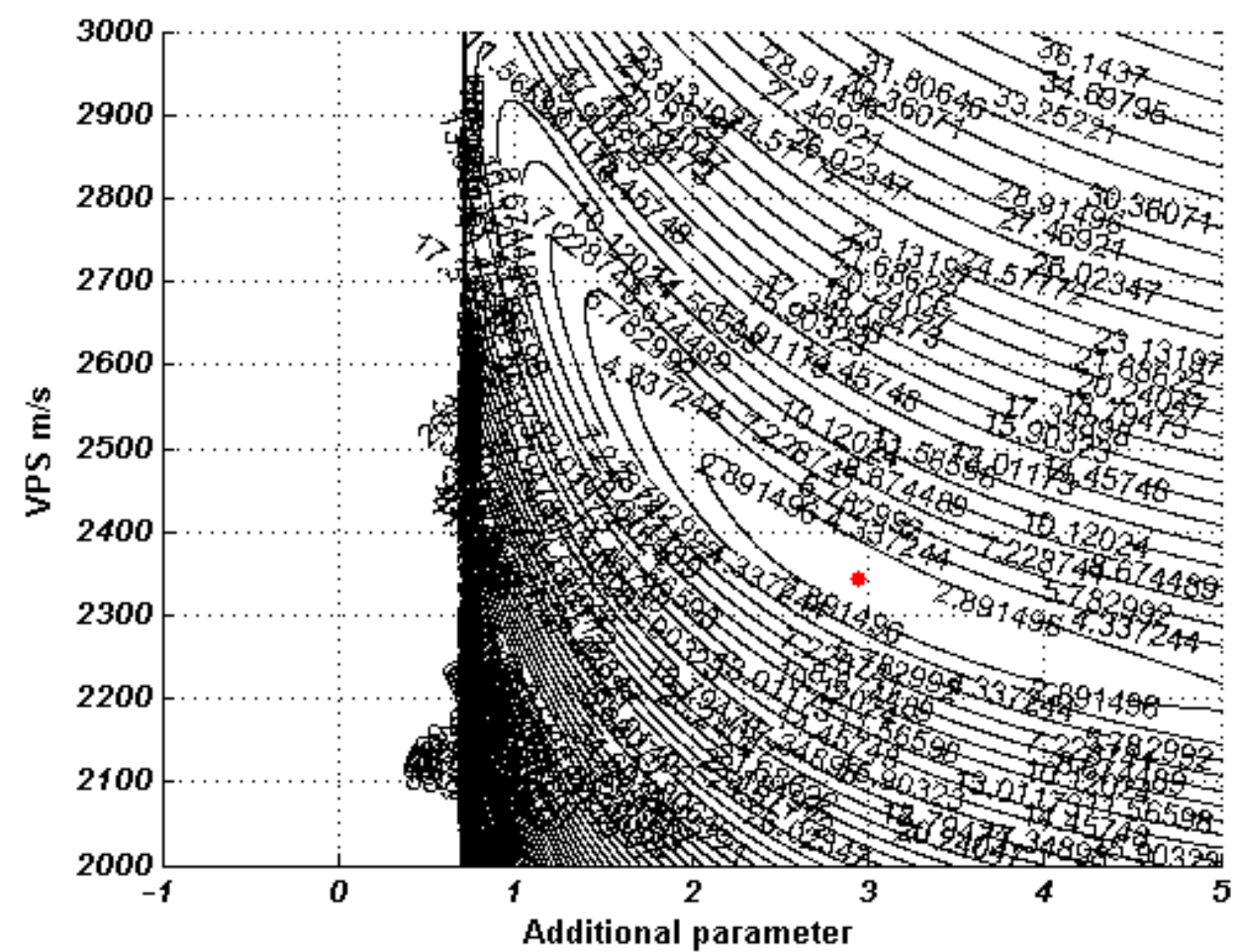

Figure 44: The residual function map which demonstrates the complexity of the Ursin and Stovas (2006) approximation of the PS reflection event with L1-norm. Red dispersions represent the global minimum region, and the blue dispersions, the local minimum regions. 


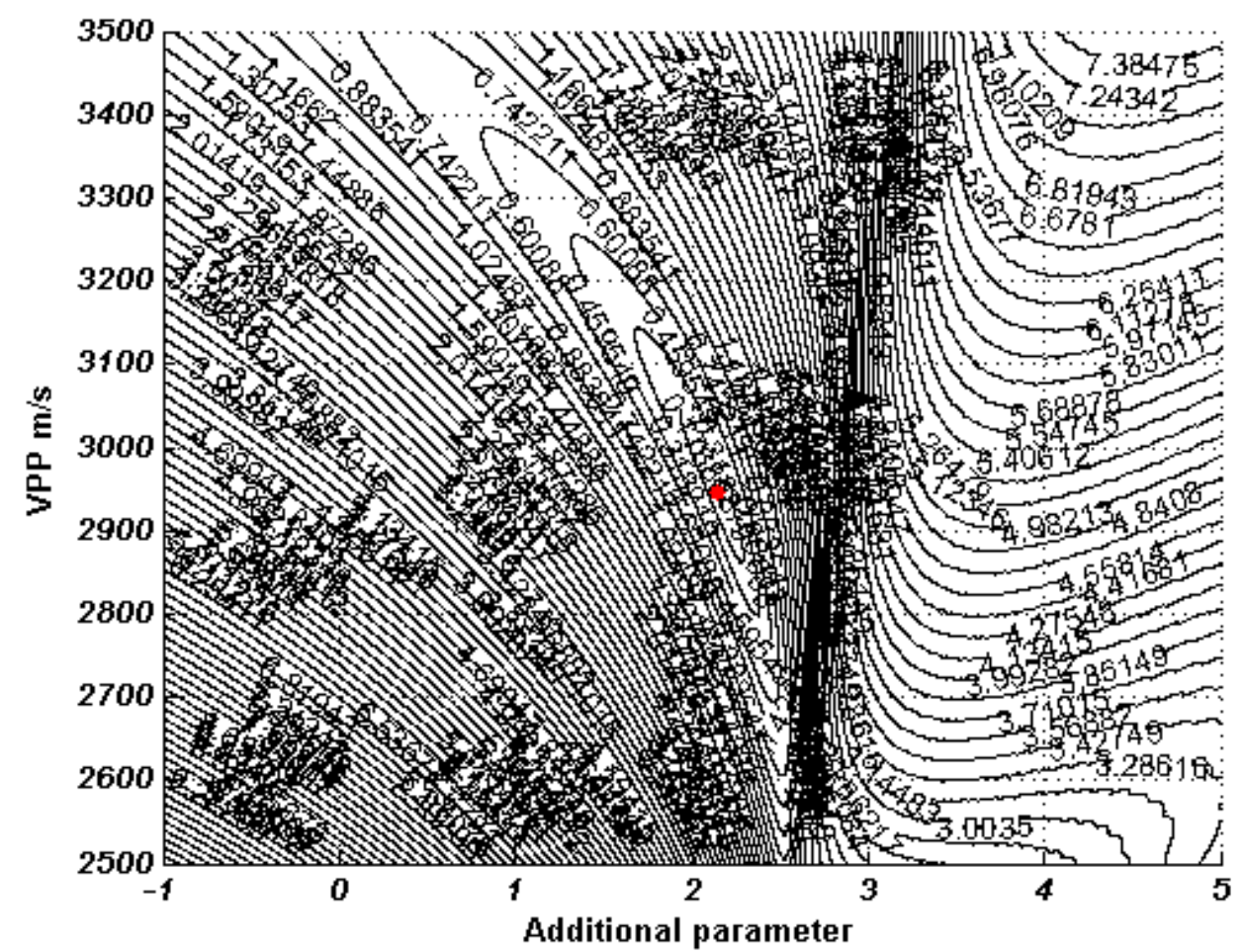

Figure 45: The residual function map which demonstrates the complexity of the Blias (2009) approximation of the PP reflection event with L2-norm. Red dispersions represent the global minimum region, and the blue dispersions, the local minimum regions.

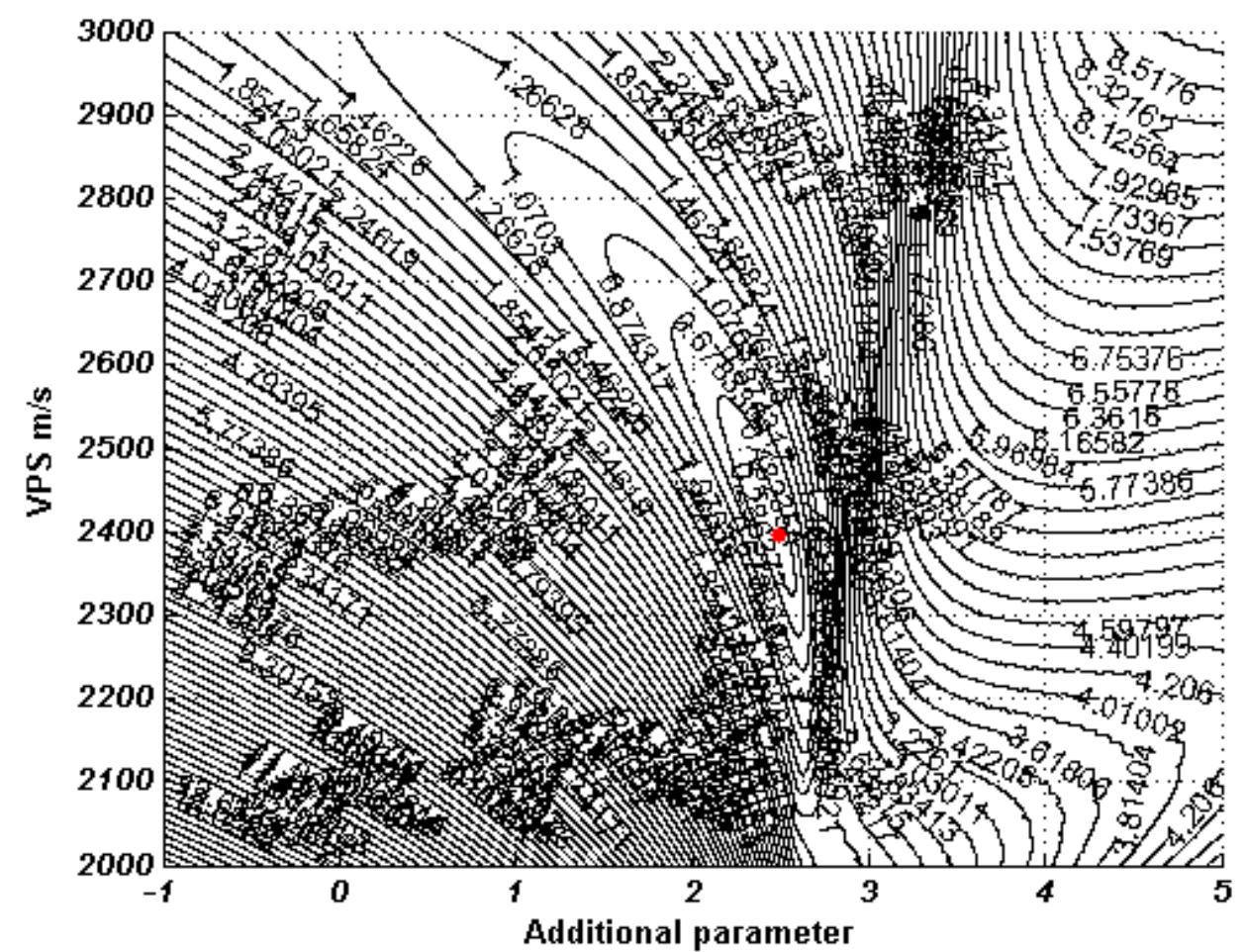

Figure 46: The residual function map which demonstrates the complexity of the Blias (2009) approximation of the PS reflection event with L2-norm. Red dispersions represent the global minimum region, and the blue dispersions, the local minimum regions. 


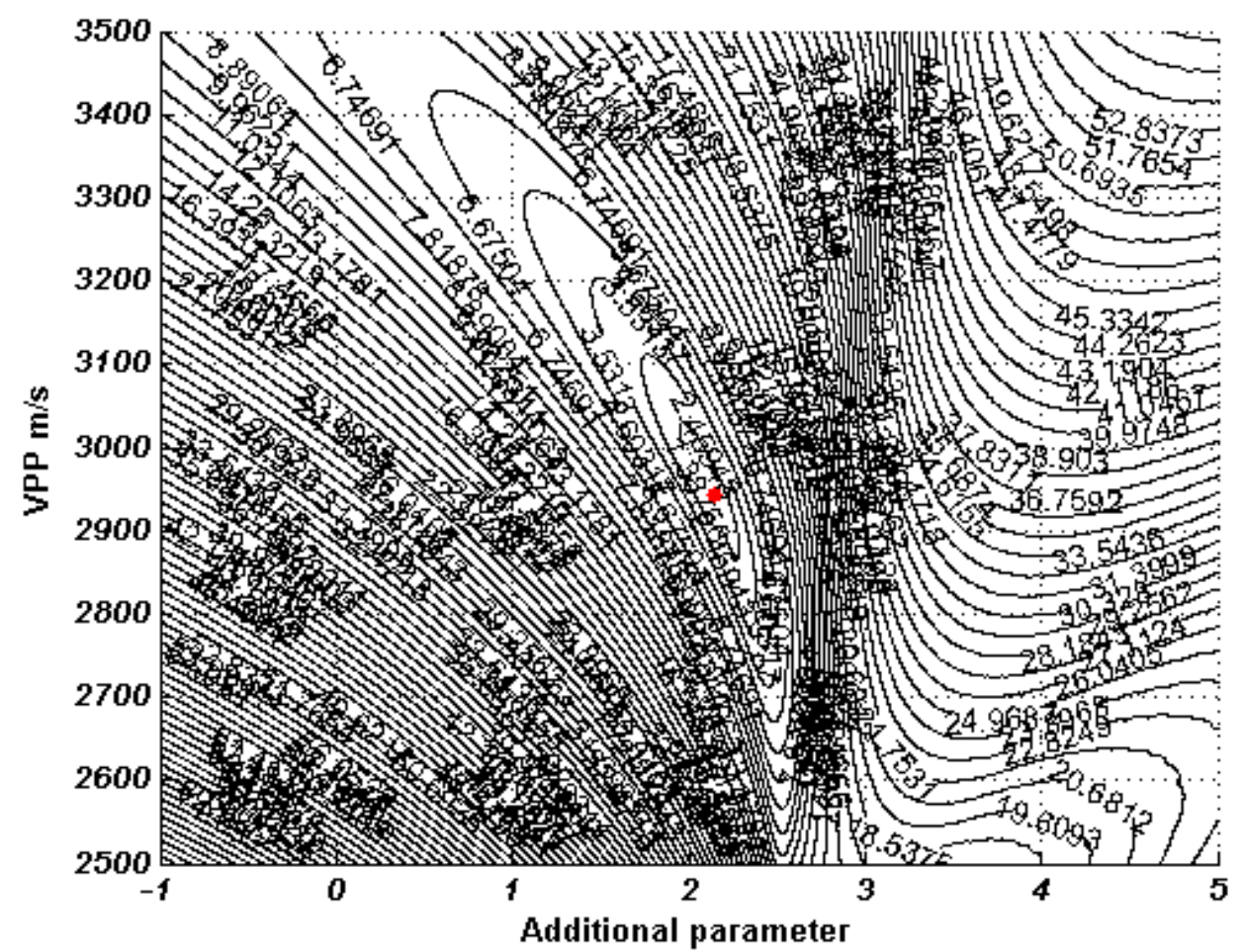

Figure 47: The residual function map which demonstrates the complexity of the Blias (2009) approximation of the PP reflection event with L1-norm. Red dispersions represent the global minimum region, and the blue dispersions, the local minimum regions.

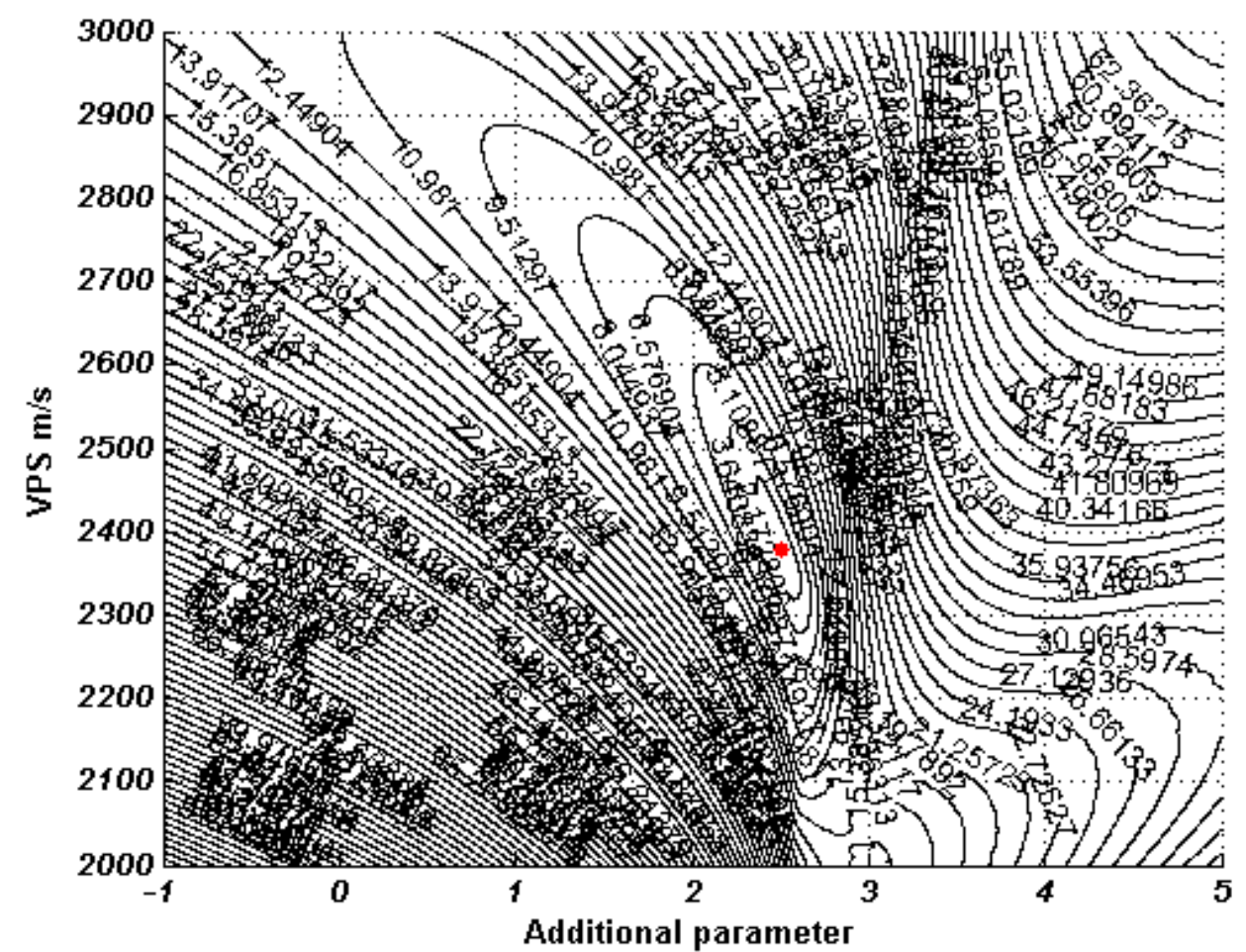

Figure 48: The residual function map which demonstrates the complexity of the Blias (2009) approximation of the PS reflection event with L1-norm. Red dispersions represent the global minimum region, and the blue dispersions, the local minimum regions. 


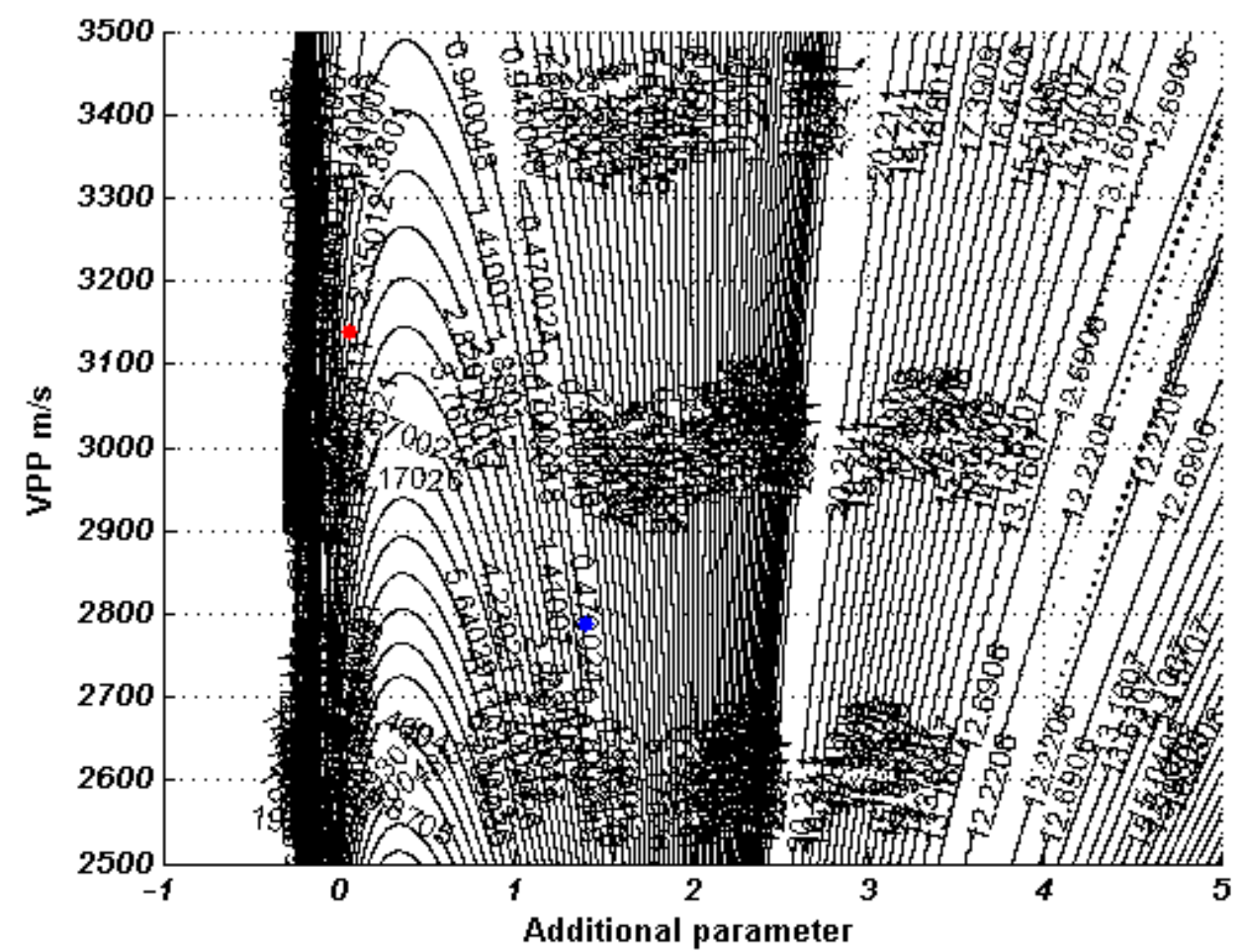

Figure 49: The residual function map which demonstrates the complexity of the Muir and Dellinger (1985) approximation of the PP reflection event with L2-norm. Red dispersions represent the global minimum region, and the blue dispersions, the local minimum regions.

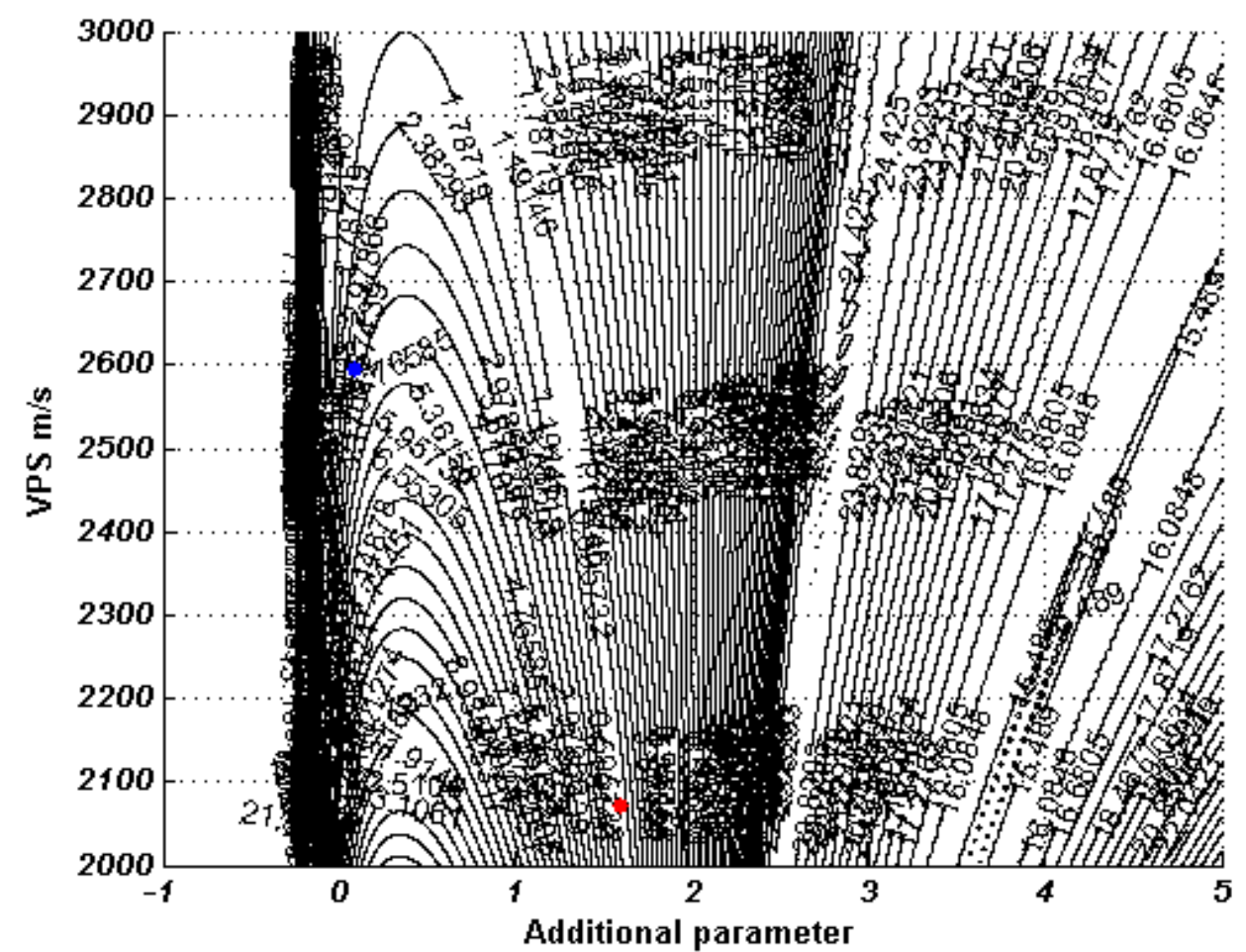

Figure 50: The residual function map which demonstrates the complexity of the Muir and Dellinger (1985) approximation of the PS reflection event with L2-norm. Red dispersions represent the global minimum region, and the blue dispersions, the local minimum regions. 


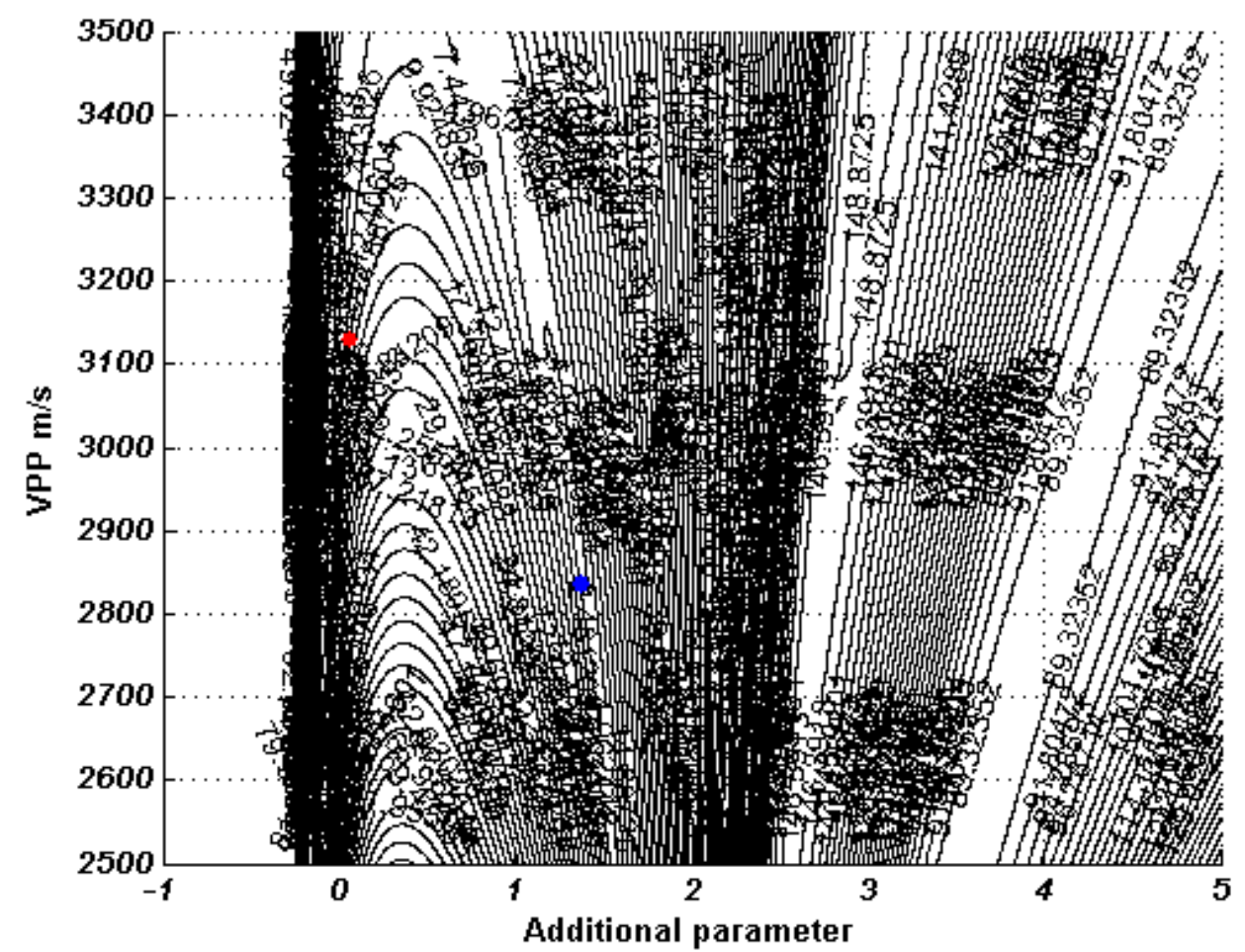

Figure 51: The residual function map which demonstrates the complexity of the Muir and Dellinger (1985) approximation of the PP reflection event with L1-norm. Red dispersions represent the global minimum region, and the blue dispersions, the local minimum regions.

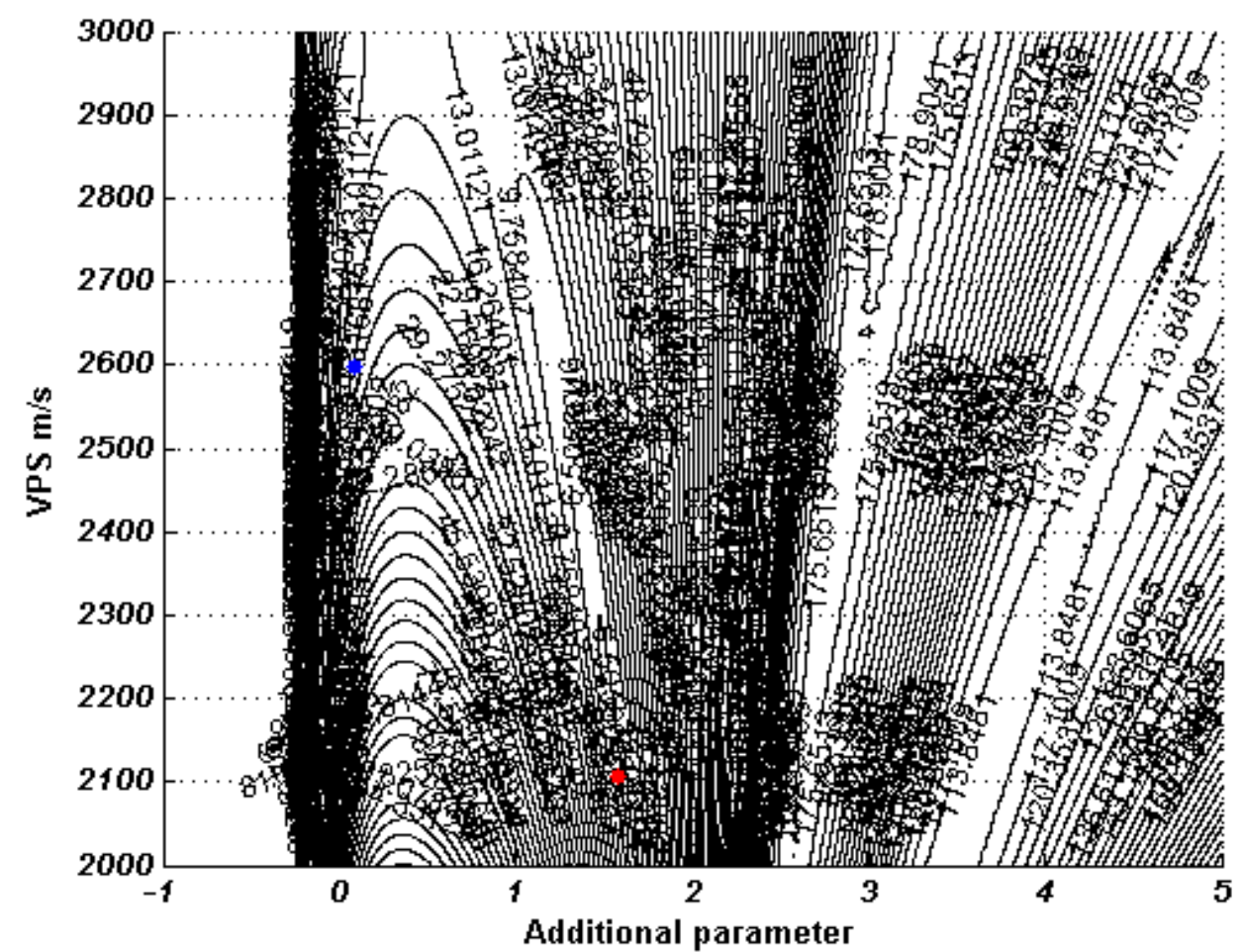

Figure 52: The residual function map which demonstrates the complexity of the Muir and Dellinger (1985) approximation of the PS reflection event with L1-norm. Red dispersions represent the global minimum region, and the blue dispersions, the local minimum regions. 


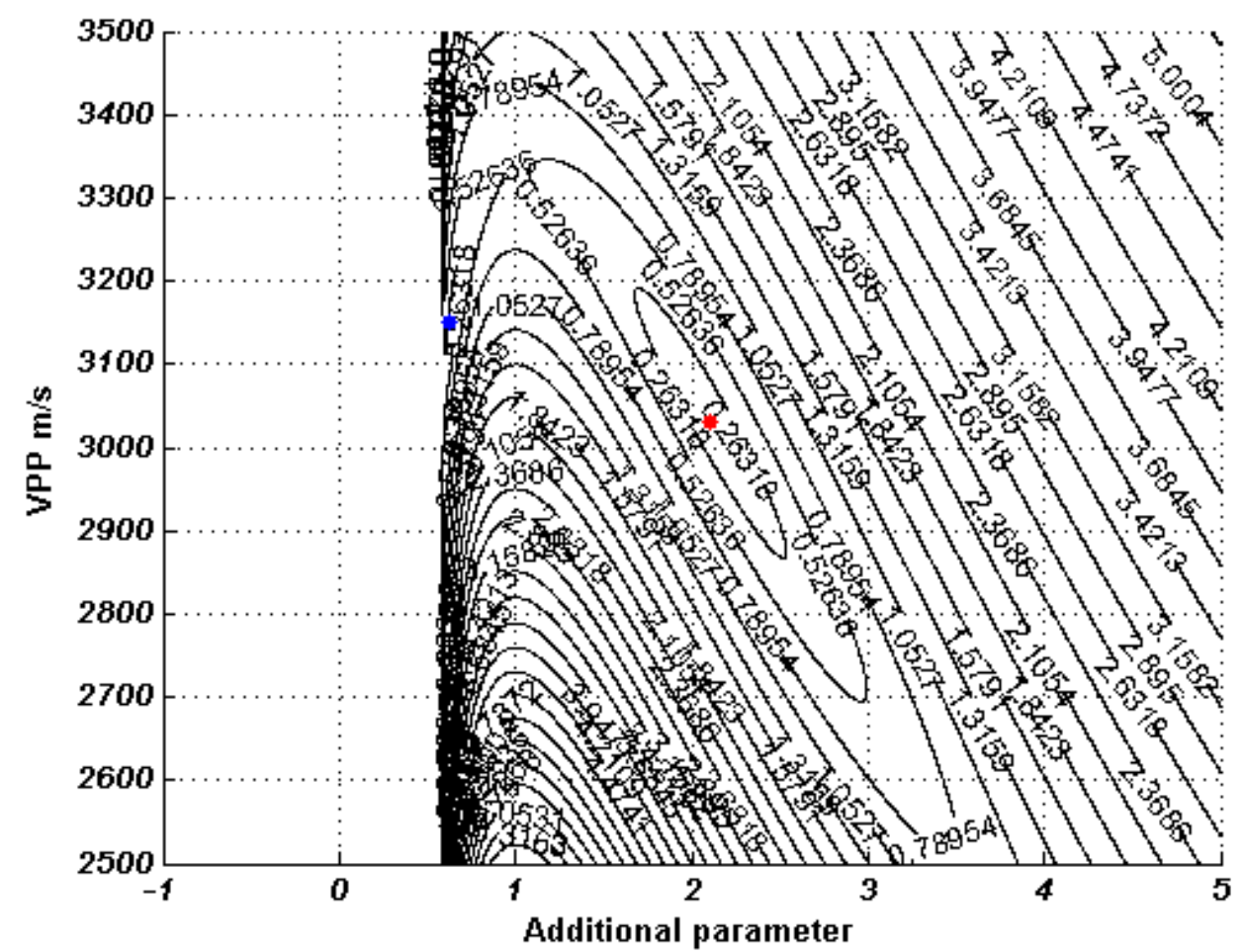

Figure 53: The residual function map which demonstrates the complexity of the Li and Yuan (2001) approximation of the PP reflection event with L2-norm. Red dispersions represent the global minimum region, and the blue dispersions, the local minimum regions.

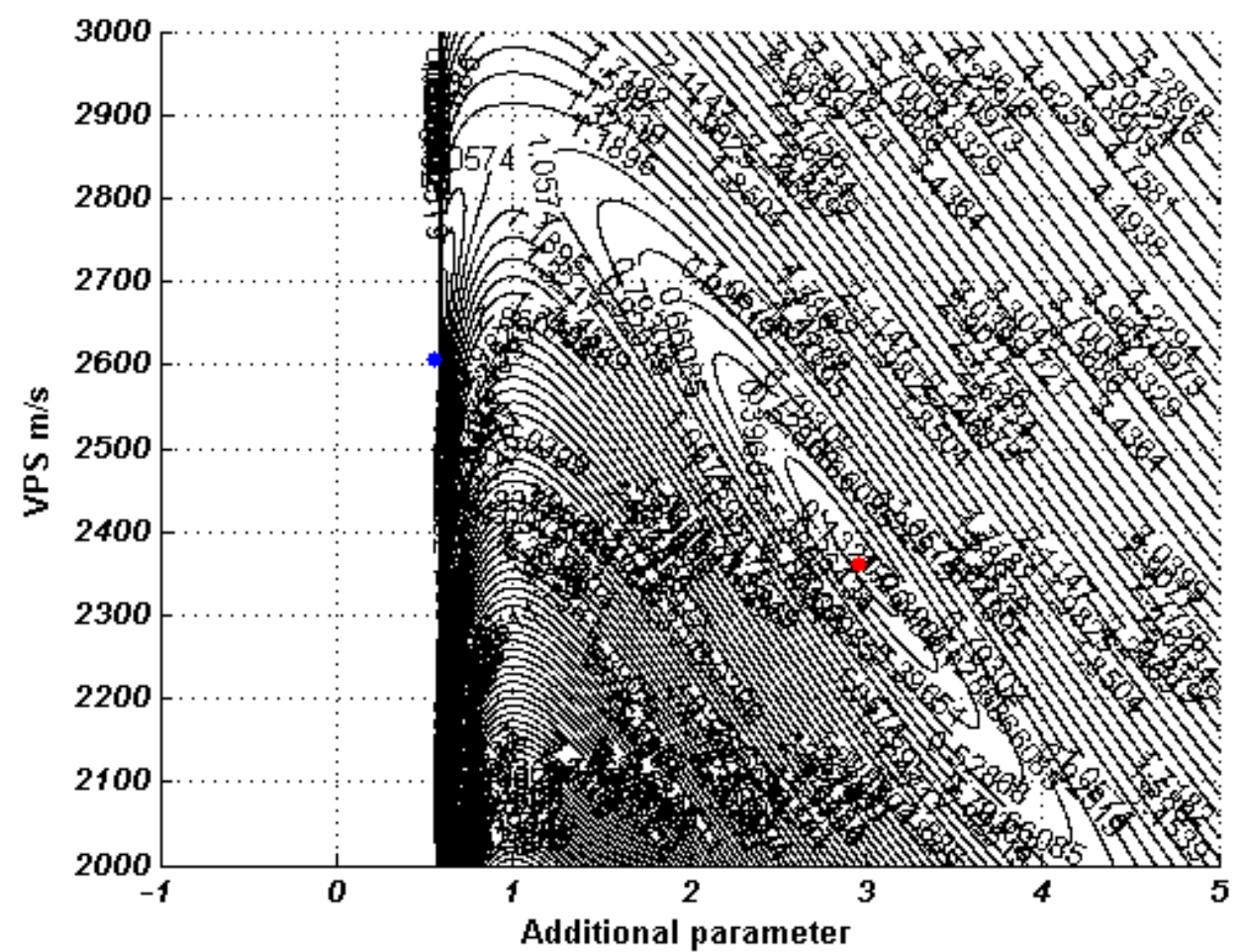

Figure 54: The residual function map which demonstrates the complexity of the Li and Yuan (2001) approximation of the PS reflection event with L2-norm. Red dispersions represent the global minimum region, and the blue dispersions, the local minimum regions. 


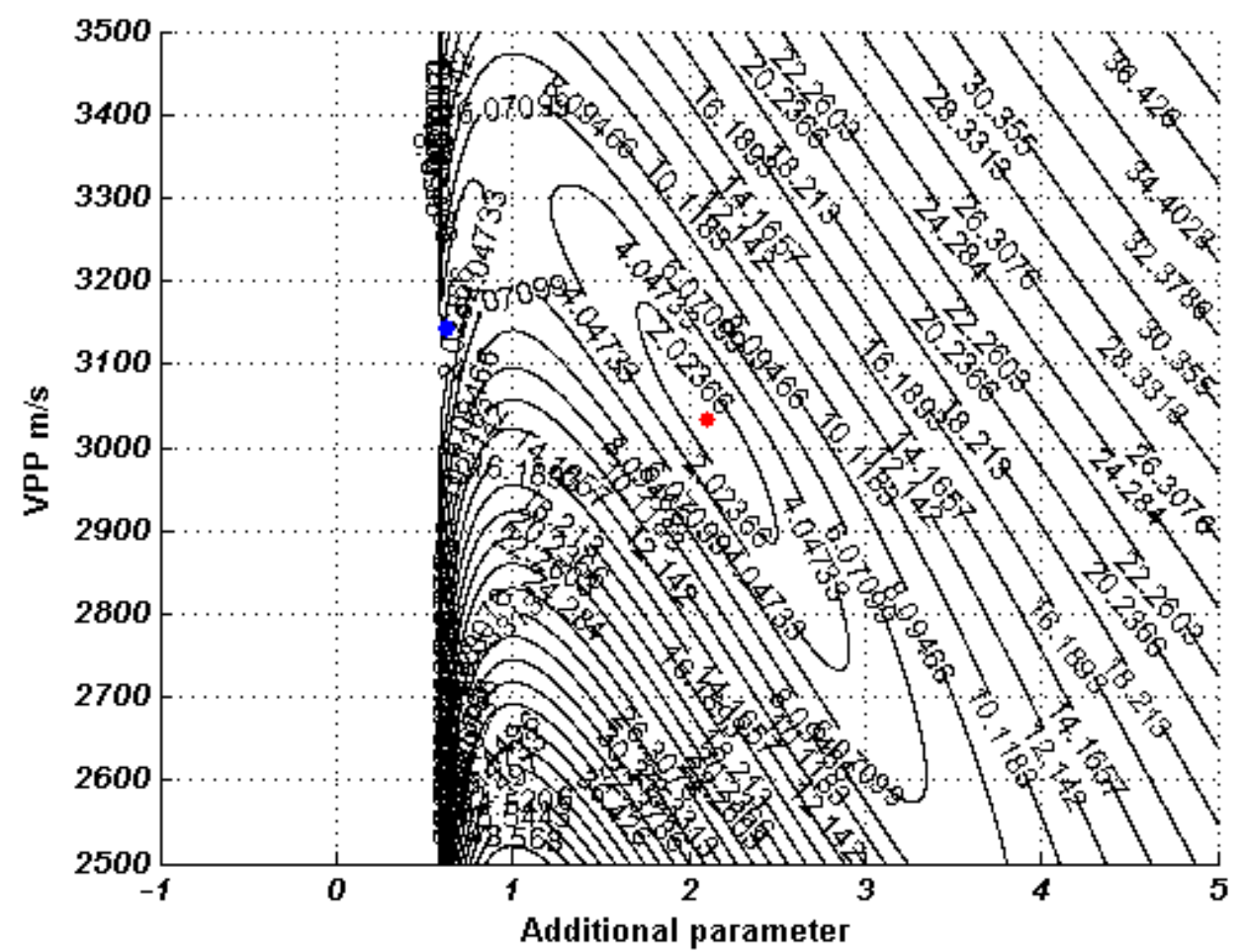

Figure 55: The residual function map which demonstrates the complexity of the Li and Yuan (2001) approximation of the PP reflection event with L1-norm. Red dispersions represent the global minimum region, and the blue dispersions, the local minimum regions.

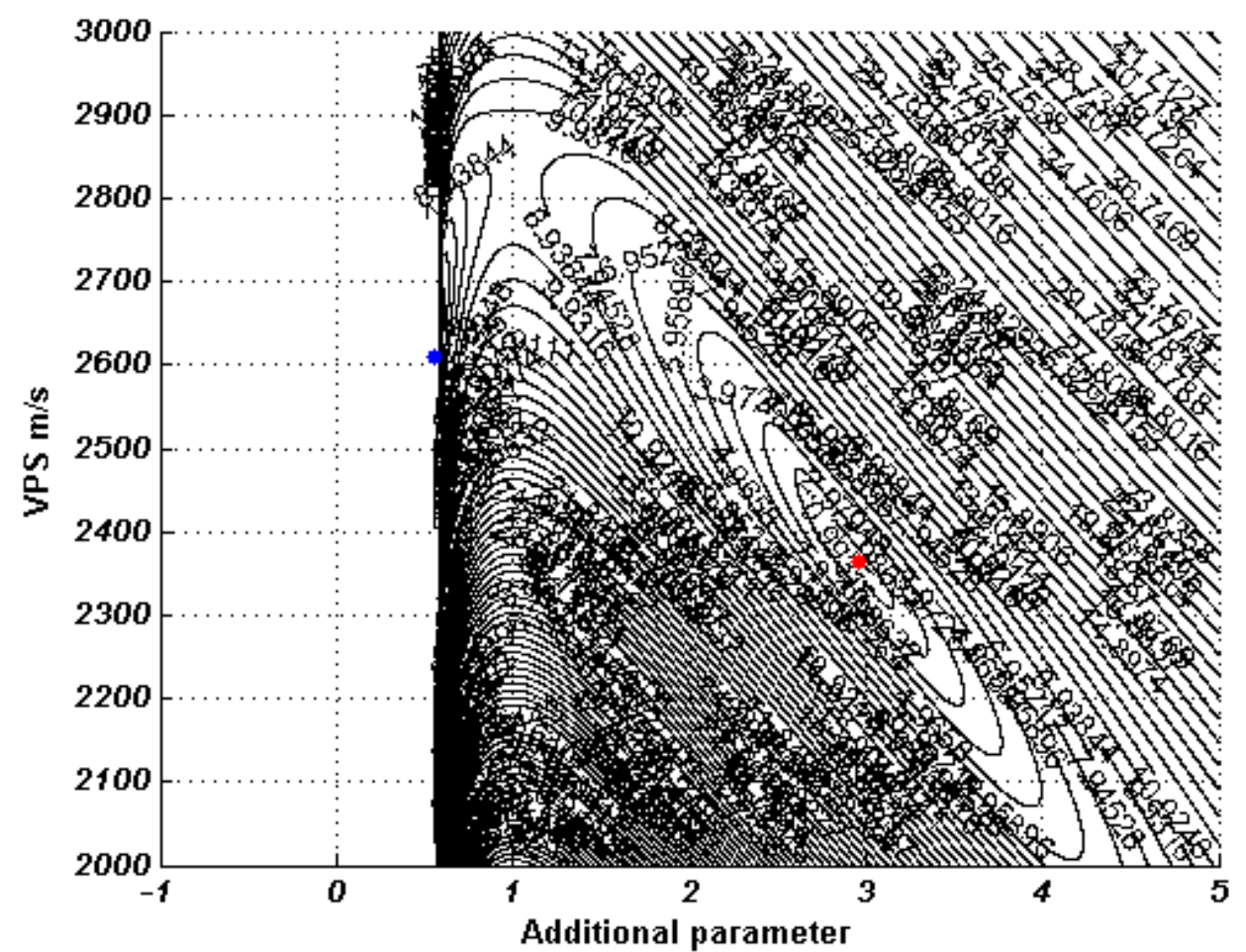

Figure 56: The residual function map which demonstrates the complexity of the Li and Yuan (2001) approximation of the PS reflection event with L1-norm. Red dispersions represent the global minimum region, and the blue dispersions, the local minimum regions. 


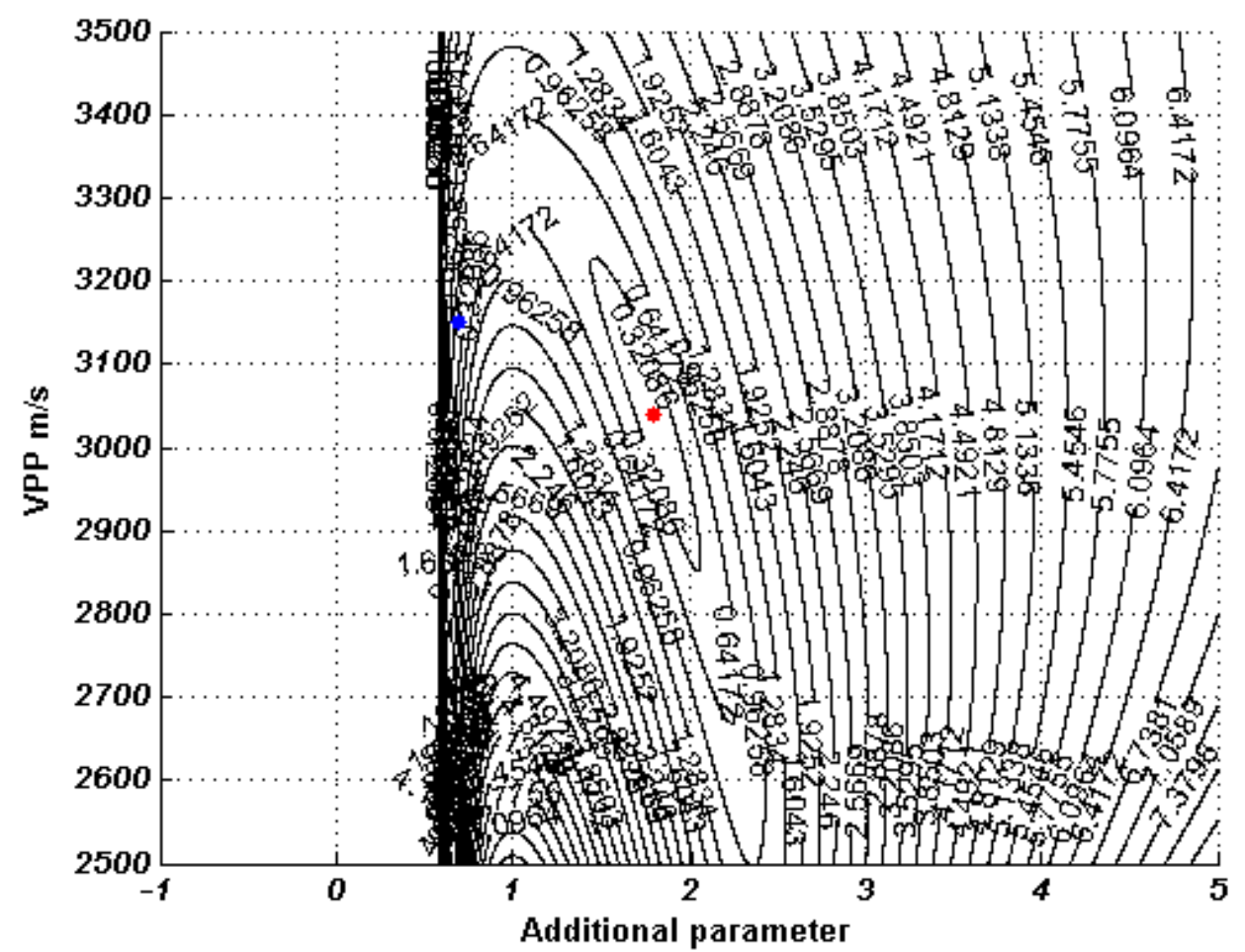

Figure 57: The residual function map which demonstrates the complexity of the approximation proposed in this work of the PP reflection event with L2-norm. Red dispersions represent the global minimum region, and the blue dispersions, the local minimum regions.

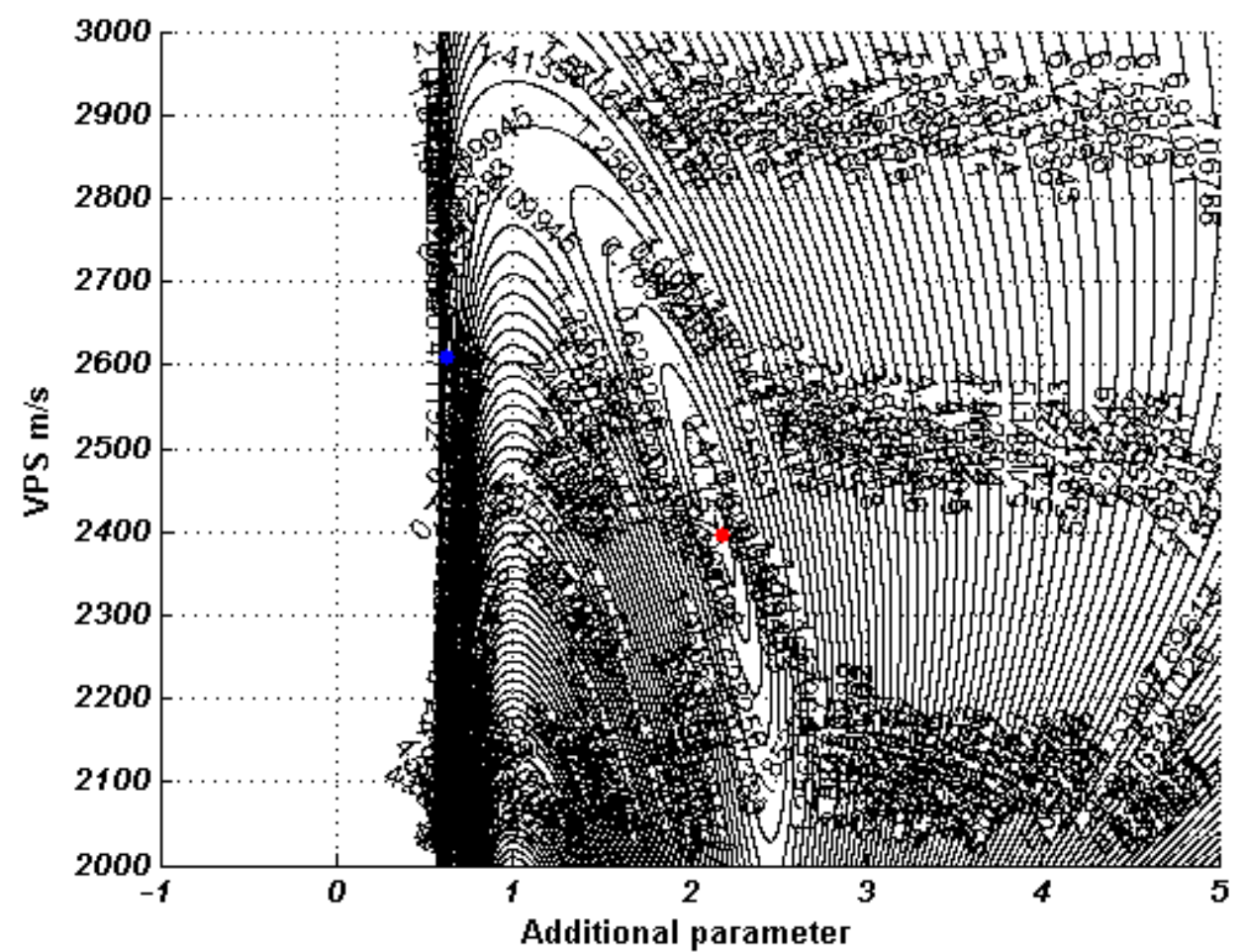

Figure 58: The residual function map which demonstrates the complexity of the approximation proposed in this work of the PS reflection event with L2-norm. Red dispersions represent the global minimum region, and the blue dispersions, the local minimum regions. 


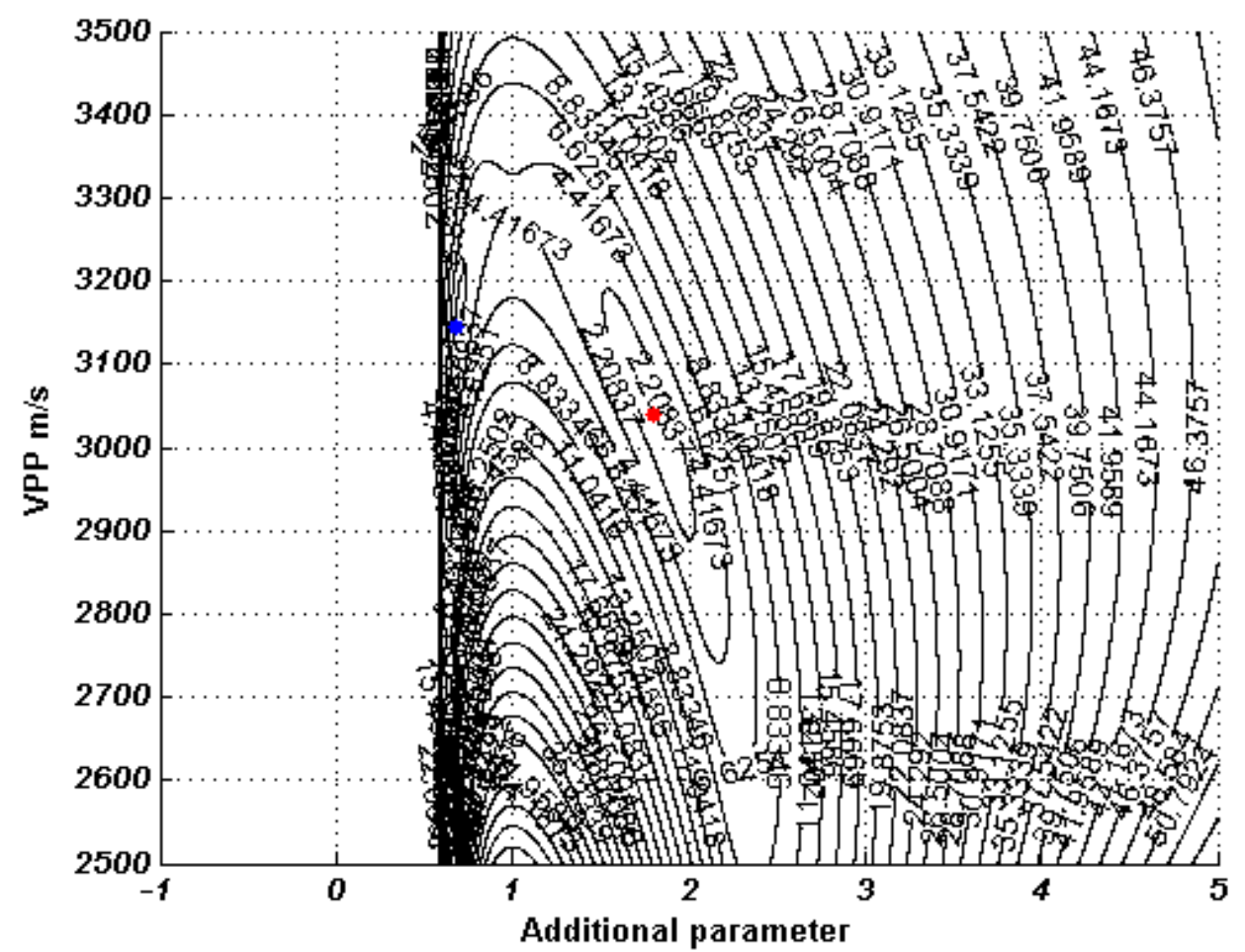

Figure 59: The residual function map which demonstrates the complexity of the approximation proposed in this work of the PP reflection event with L1-norm. Red dispersions represent the global minimum region, and the blue dispersions, the local minimum regions.

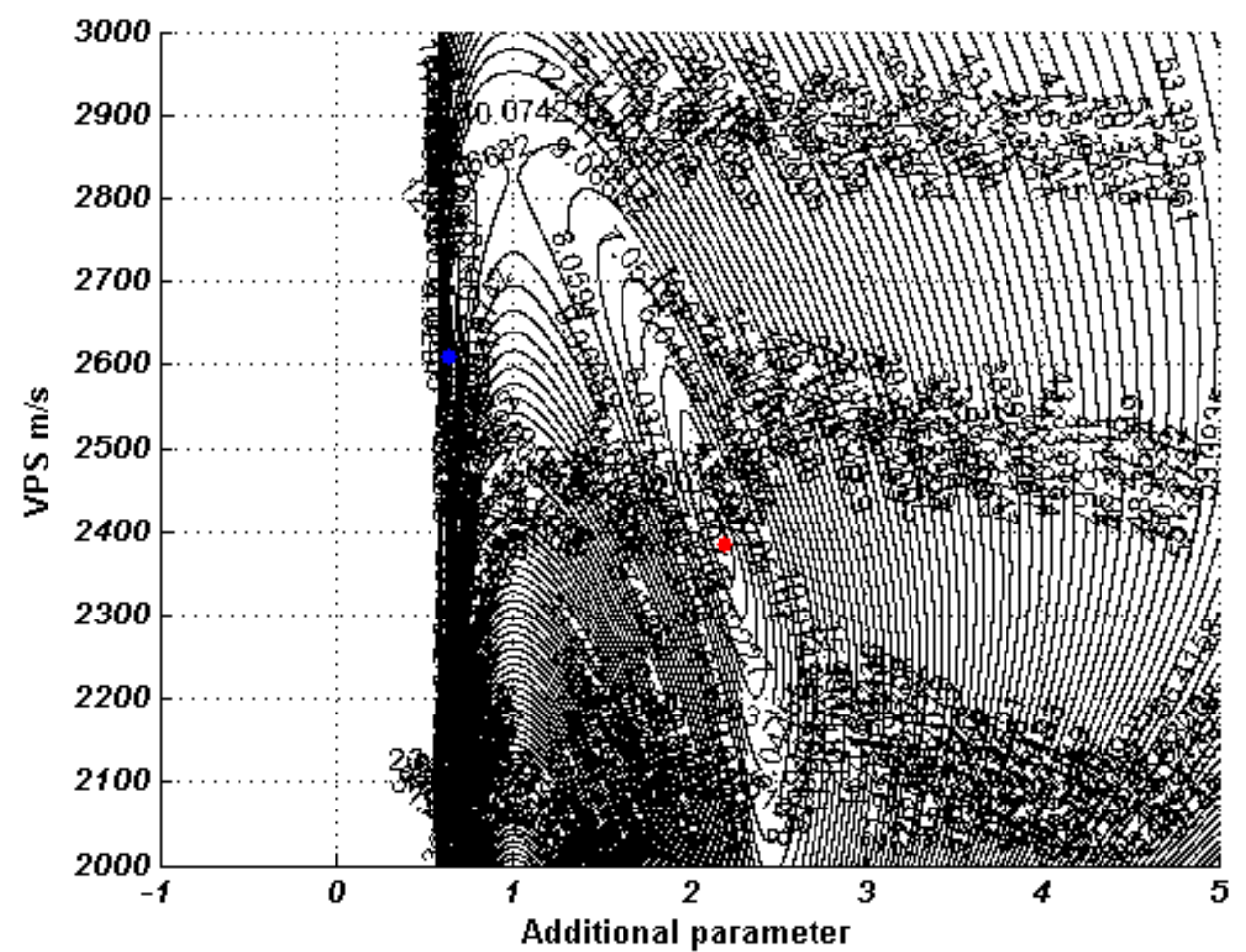

Figure 60: The residual function map which demonstrates the complexity of the approximation proposed in this work of the PS reflection event with L1-norm. Red dispersions represent the global minimum region, and the blue dispersions, the local minimum regions. 


\subsubsection{Model 2}

With a model which is slightly more complex, subtle differences in the topological structure could be observed. However, the same variations could be seen for the Malovichko (1978) approximation concerning the distribution of the contour lines of the structure between the PP and PS events (Figures 61 and 62). And in the Figures 63 and 64, it is possible to observe, again, the gradient of the curves becoming stronger between the L2- and L1-norm. Furthermore, the approximation showed to be, once again, a unimodal approximation.

The Alkhalifah and Tsvankin (1995) approximation showed to be unimodal and presented the same characteristics as before (Figures 65, 66, 67 and 68), except for the subtle differences in the topological structure.

The approximation proposed by Ursin and Stovas (2006) again showed the same characteristics and variations as the previous model (Figures 69, 70, 71 and 72).

For the approximation proposed by Blias (2009), the same kind of variations as the previous approximation could be observed (Figures 73, 74, 75 and 76).

The Muir and Dellinger (1985) approximation showed the same curious variation it presented previously concerning the PP and PS event (Figures 77 and 78). It also presented the same multimodal behaviour and variation between the norms (Figures 79 and 80).

The approximation presented by $\mathrm{Li}$ and Yuan (2001) showed all the same characteristics and variations it showed in Model 1 (Figures 81, 82, 83 and 84).

The proposed approximation showed the same complex characteristics and behaviours concerning the topological structure of the objective function, and also presented similar variations as before (Figures $85,86,87$ and 88 ). 


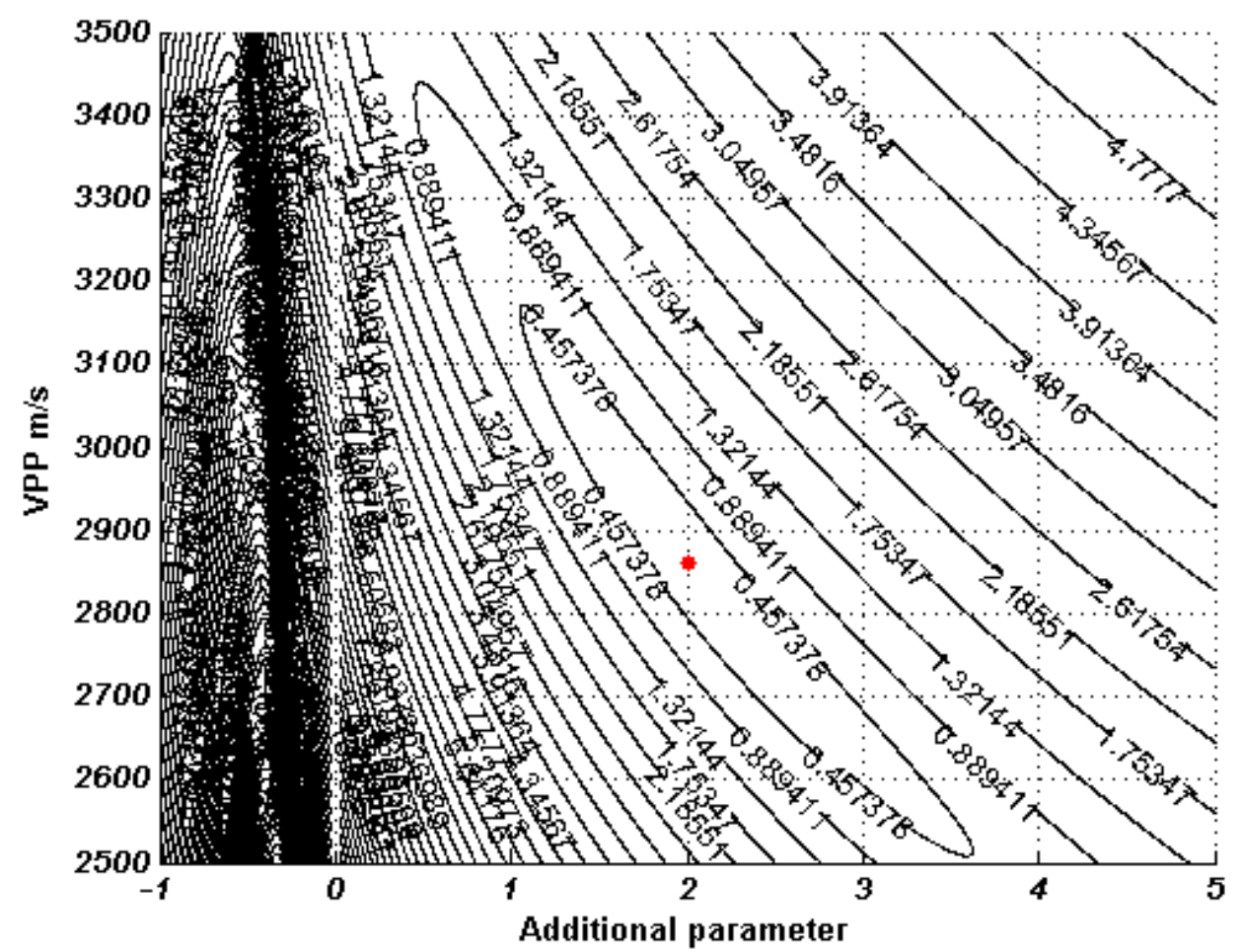

Figure 61: The residual function map which demonstrates the complexity of the Malovichko (1978) approximation of the PP reflection event with L2-norm. Red dispersions represent the global minimum region, and the blue dispersions, the local minimum regions.

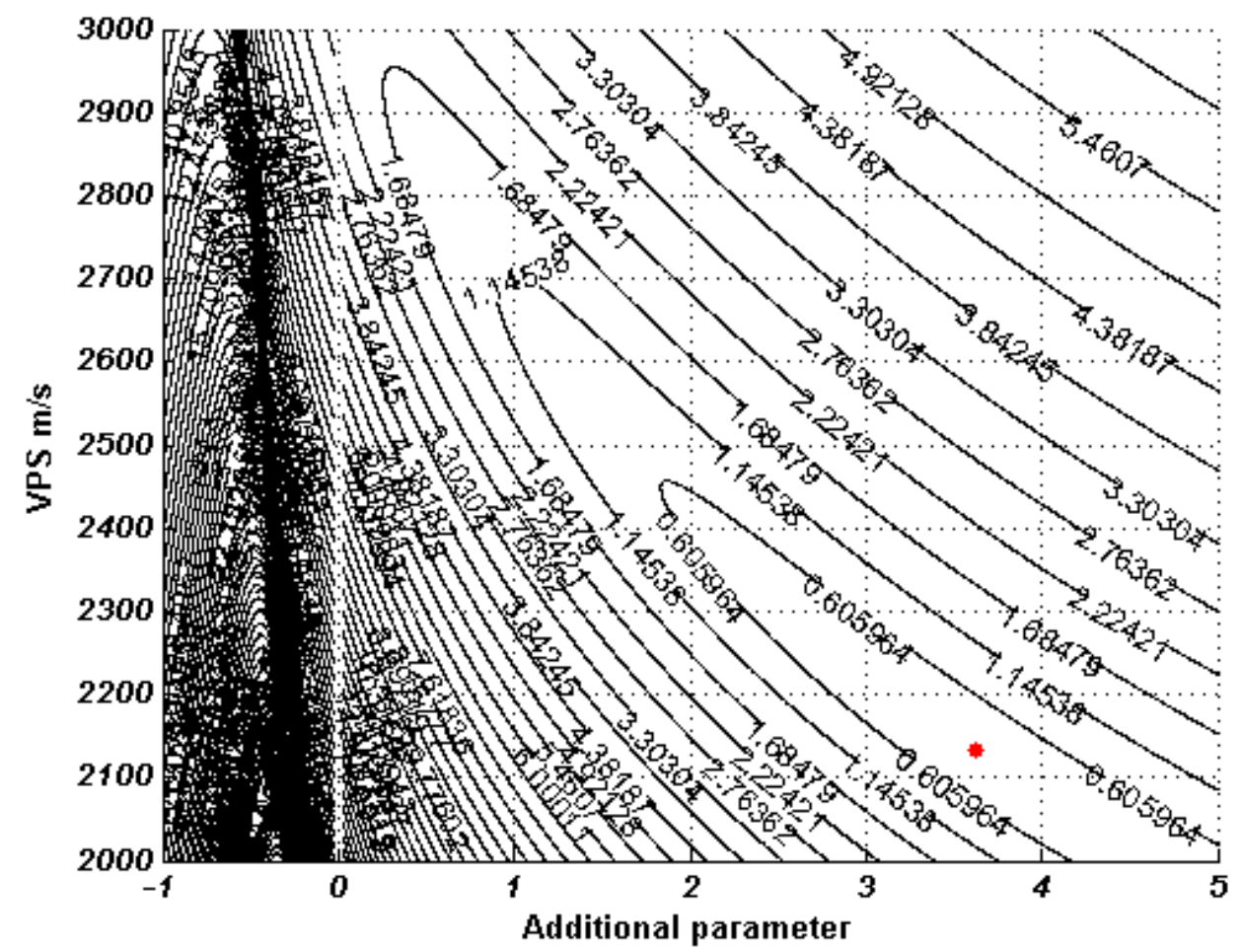

Figure 62: The residual function map which demonstrates the complexity of the Malovichko (1978) approximation of the PS reflection event with L2-norm. Red dispersions represent the global minimum region, and the blue dispersions, the local minimum regions. 


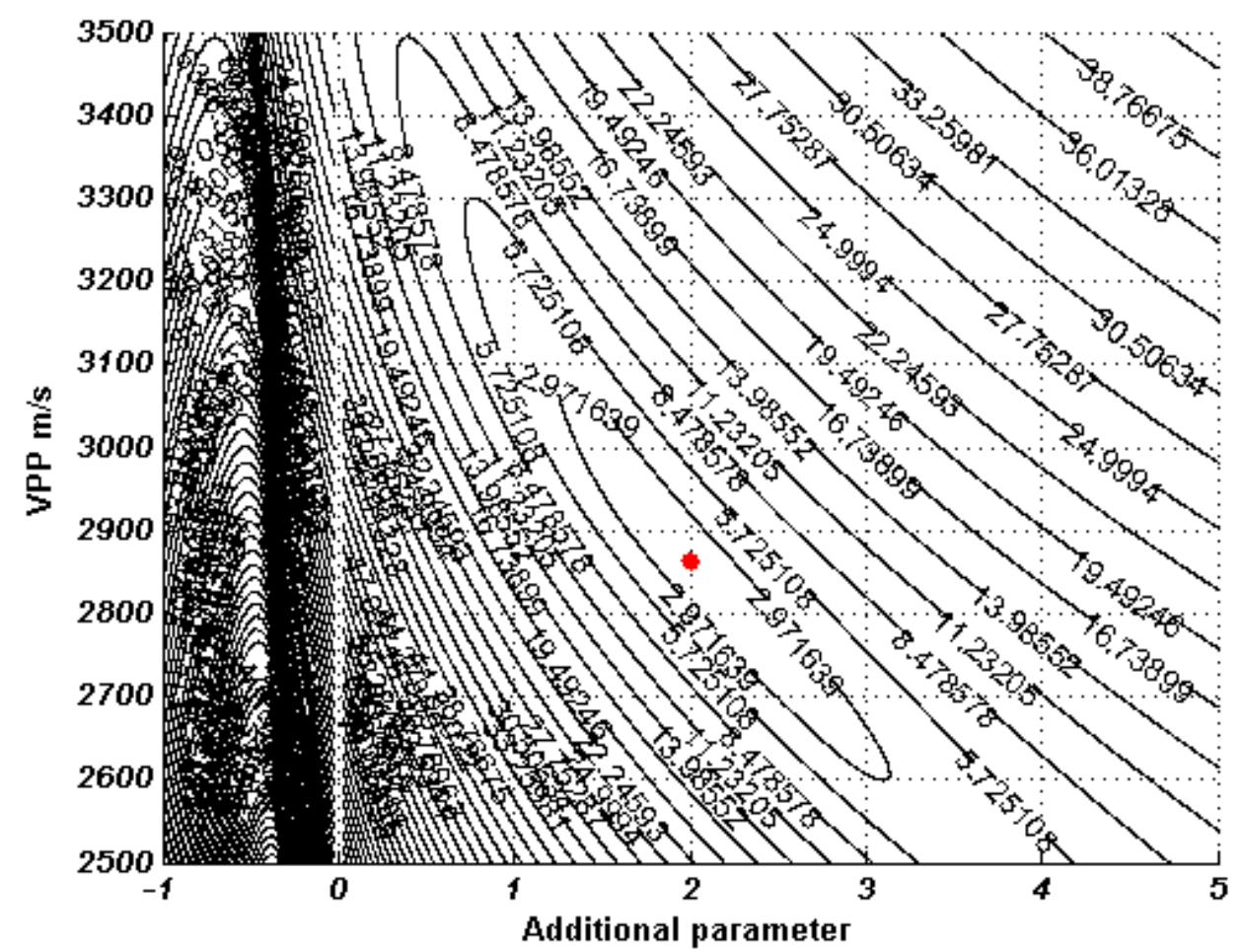

Figure 63: The residual function map which demonstrates the complexity of the Malovichko (1978) approximation of the PP reflection event with L1-norm. Red dispersions represent the global minimum region, and the blue dispersions, the local minimum regions.

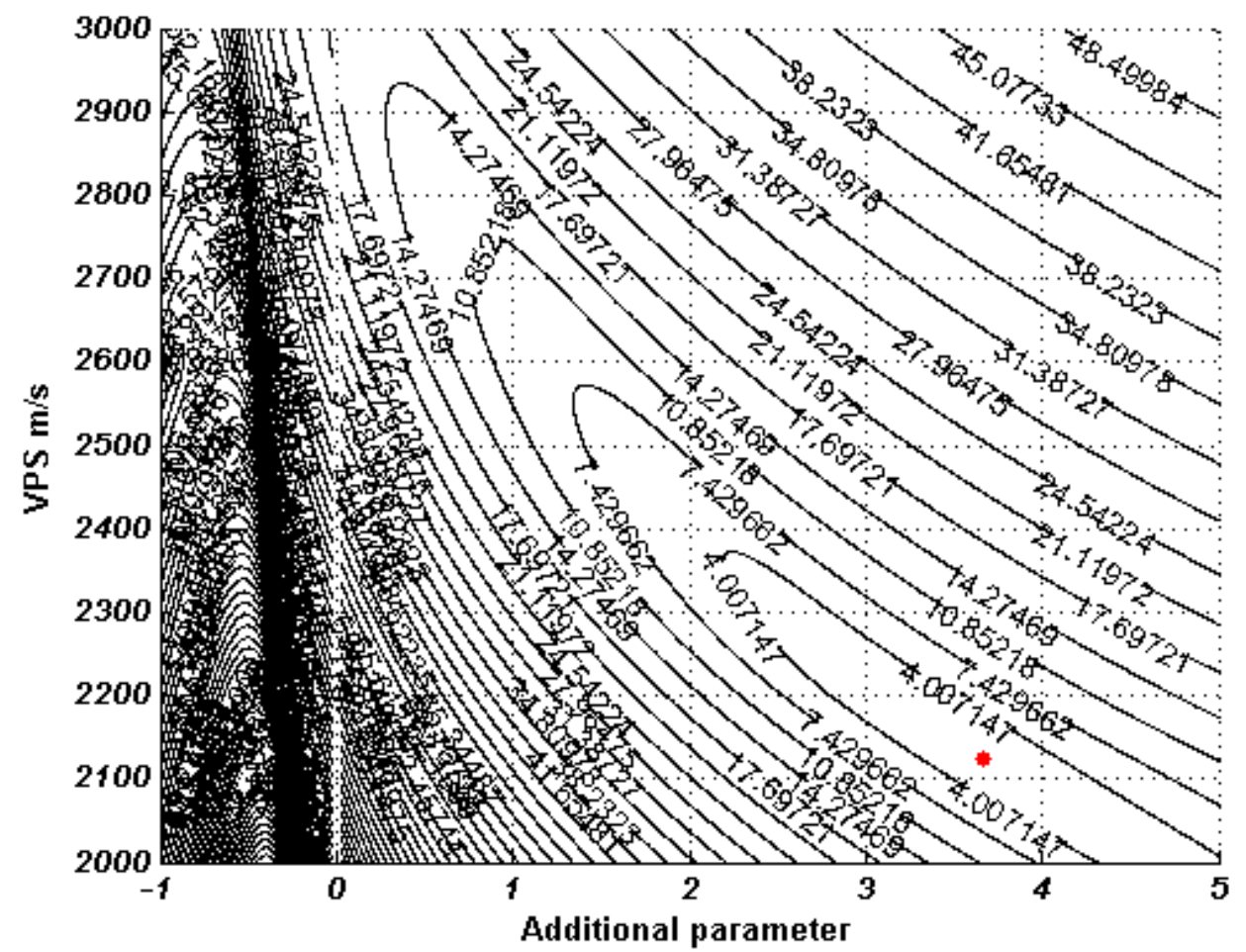

Figure 64: The residual function map which demonstrates the complexity of the Malovichko (1978) approximation of the PS reflection event with L1-norm. Red dispersions represent the global minimum region, and the blue dispersions, the local minimum regions. 


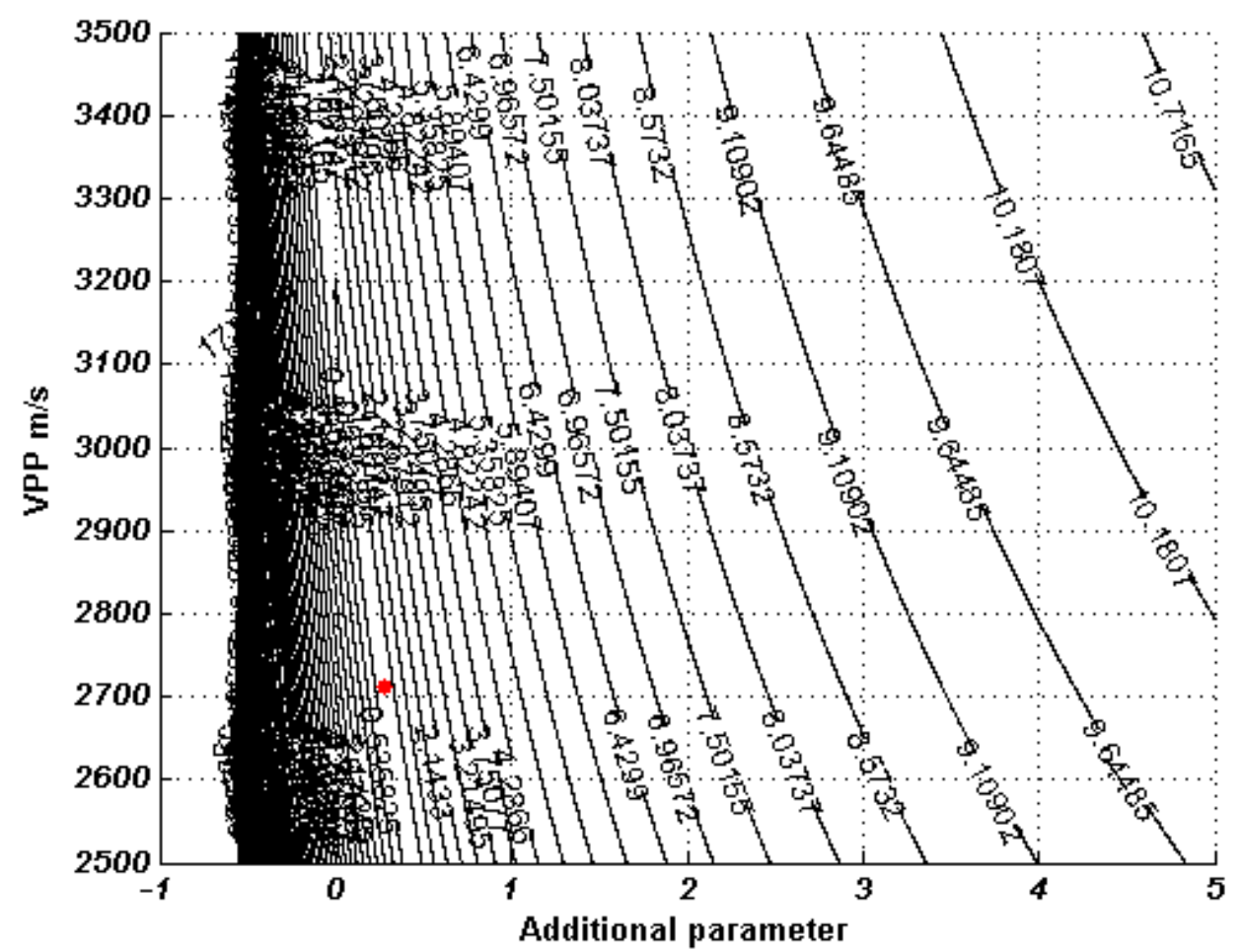

Figure 65: The residual function map which demonstrates the complexity of the Alkhalifah and Tsvankin (1995) approximation of the PP reflection event with L2-norm. Red dispersions represent the global minimum region, and the blue dispersions, the local minimum regions.

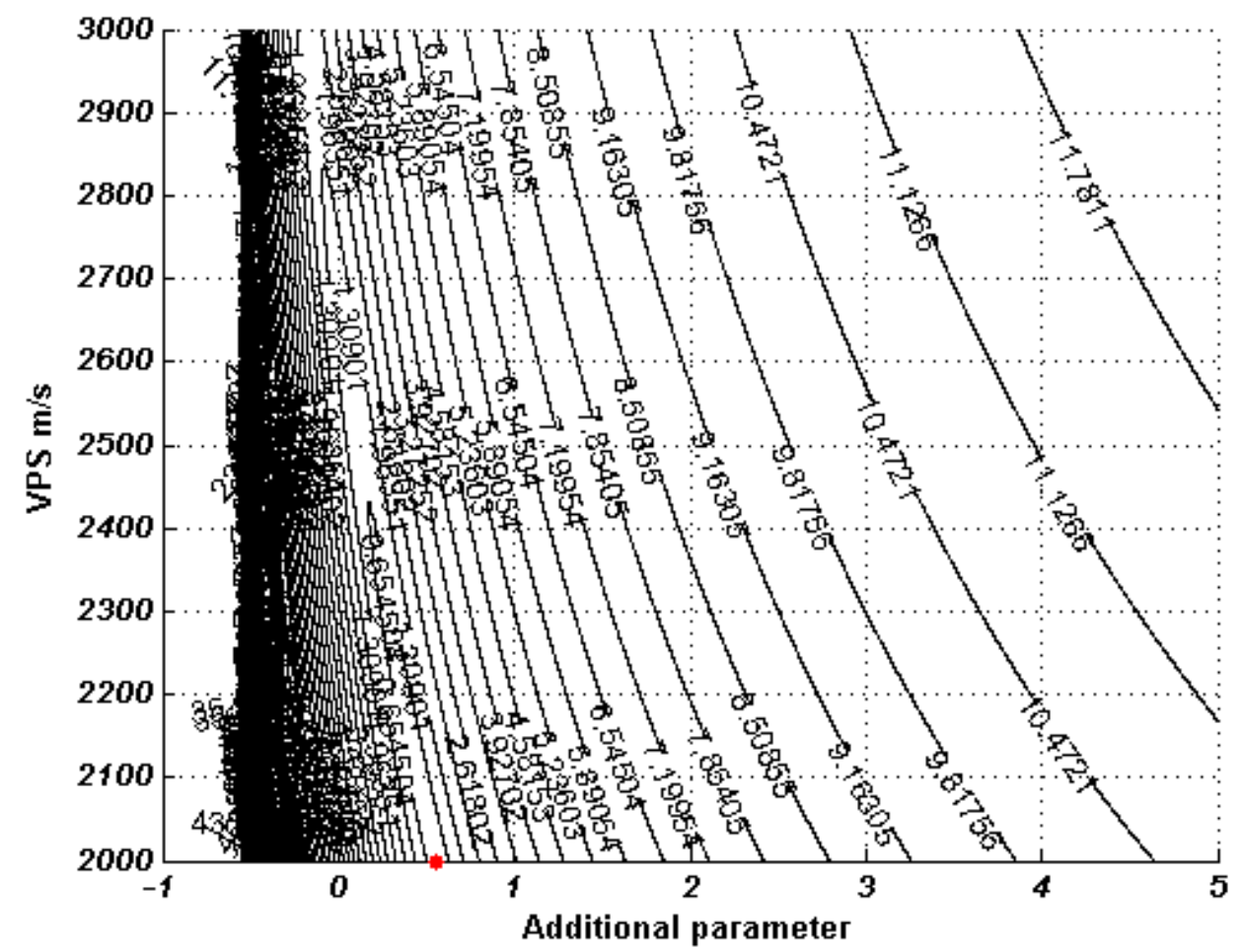

Figure 66: The residual function map which demonstrates the complexity of the Alkhalifah and Tsvankin (1995) approximation of the PS reflection event with L2-norm. Red dispersions represent the global minimum region, and the blue dispersions, the local minimum regions. 


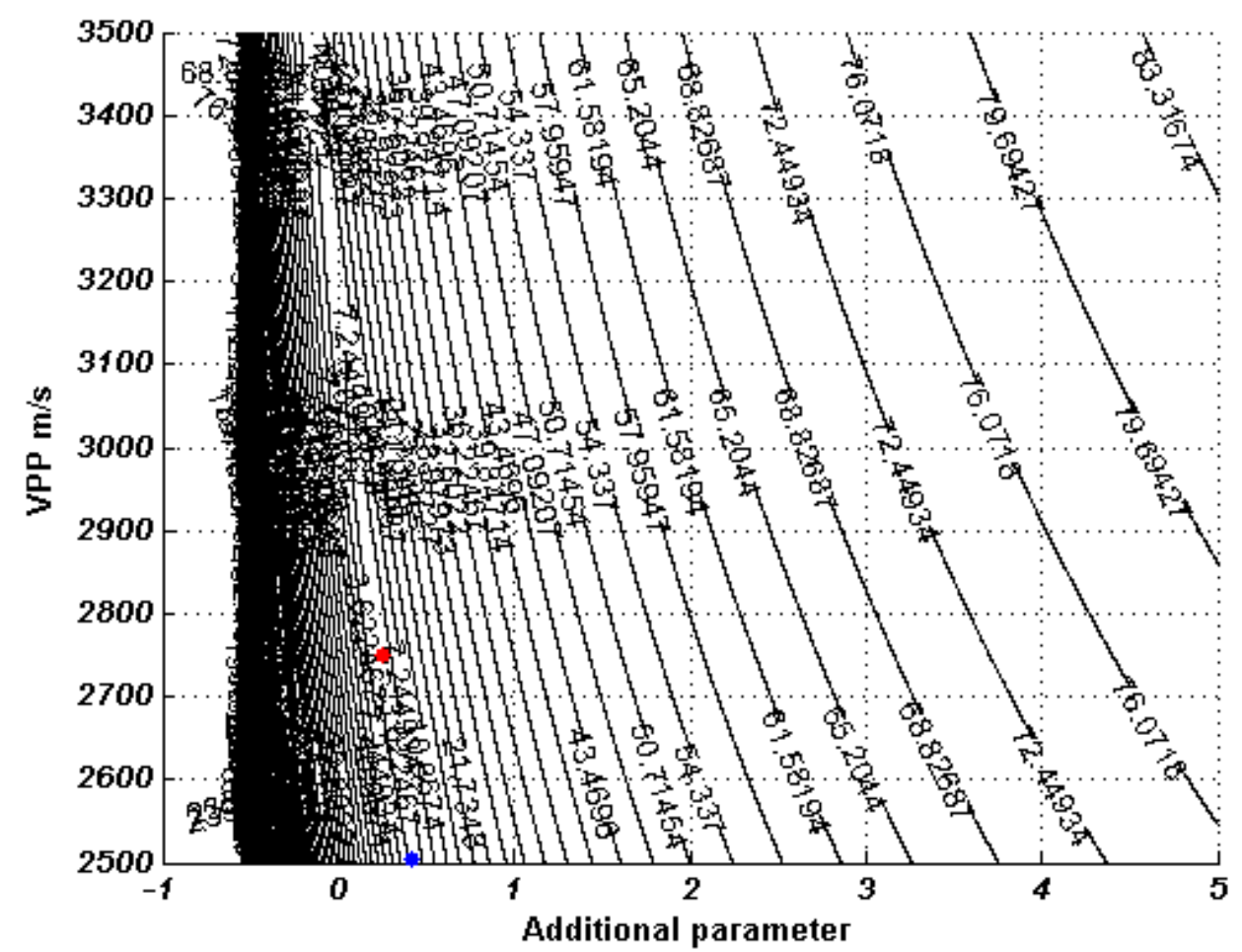

Figure 67: The residual function map which demonstrates the complexity of the Alkhalifah and Tsvankin (1995) approximation of the PP reflection event with L1-norm. Red dispersions represent the global minimum region, and the blue dispersions, the local minimum regions.

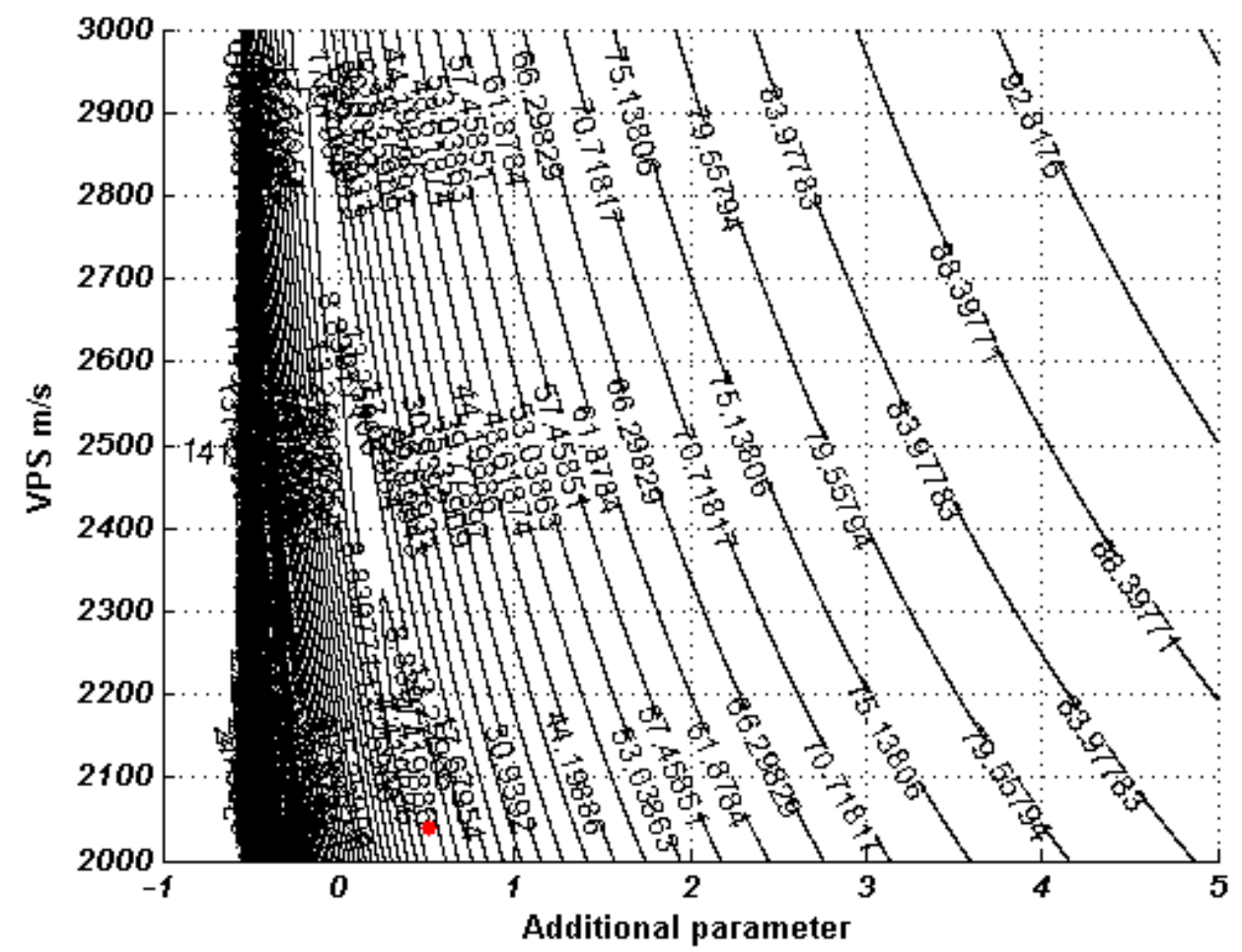

Figure 68: The residual function map which demonstrates the complexity of the Alkhalifah and Tsvankin (1995) approximation of the PS reflection event with L1-norm. Red dispersions represent the global minimum region, and the blue dispersions, the local minimum regions. 


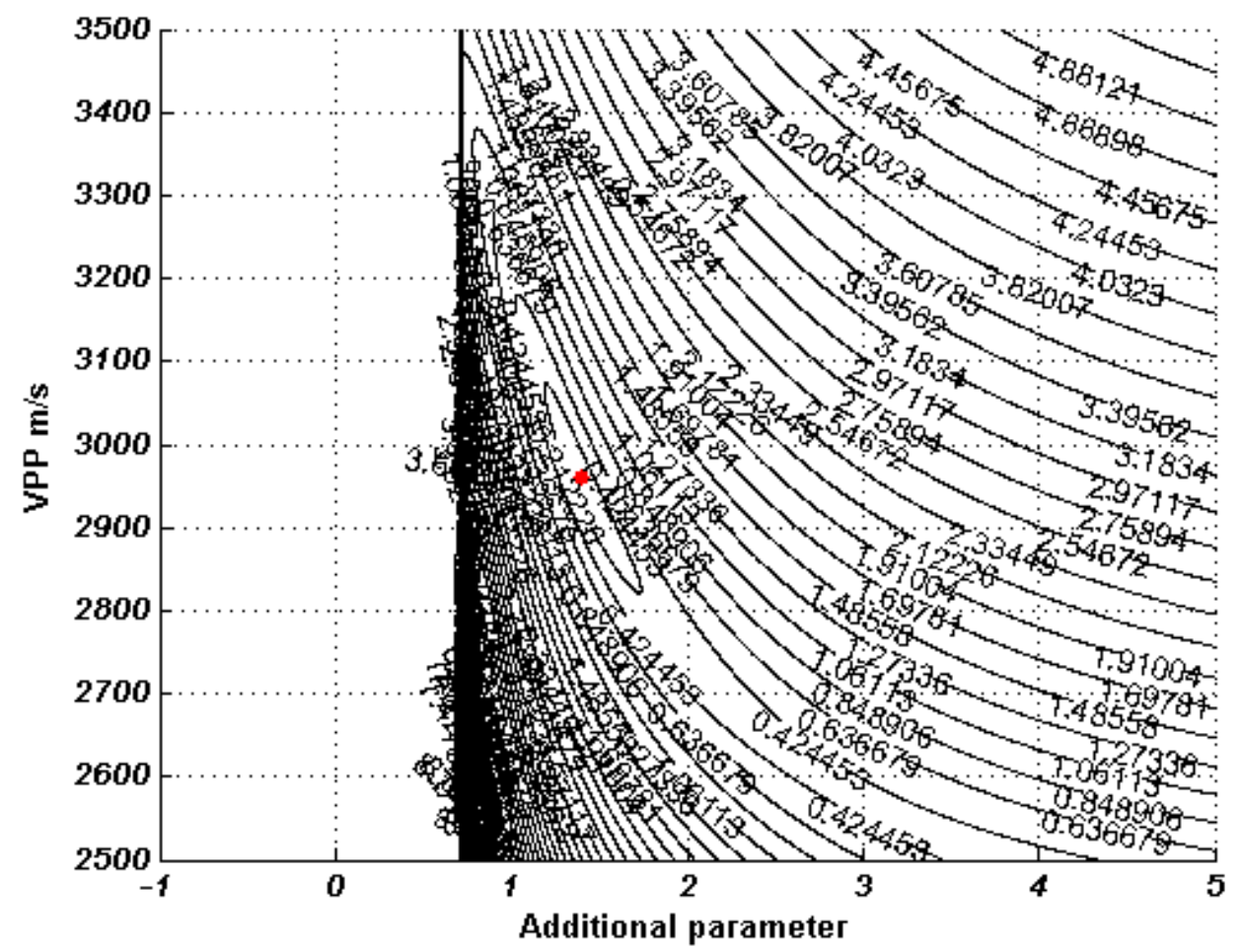

Figure 69: The residual function map which demonstrates the complexity of the Ursin and Stovas (2006) approximation of the PP reflection event with L2-norm. Red dispersions represent the global minimum region, and the blue dispersions, the local minimum regions.

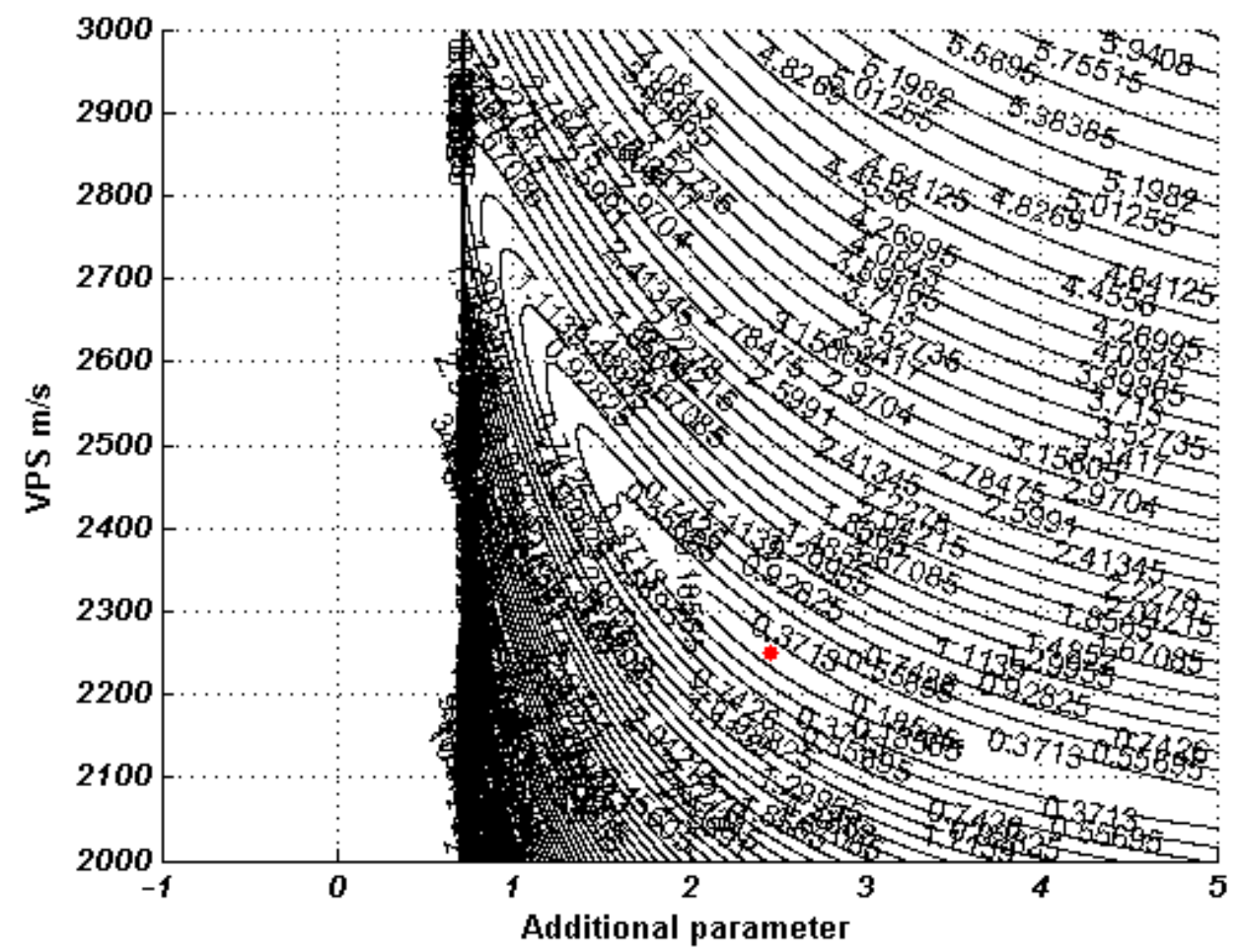

Figure 70: The residual function map which demonstrates the complexity of the Ursin and Stovas (2006) approximation of the PS reflection event with L2-norm. Red dispersions represent the global minimum region, and the blue dispersions, the local minimum regions. 


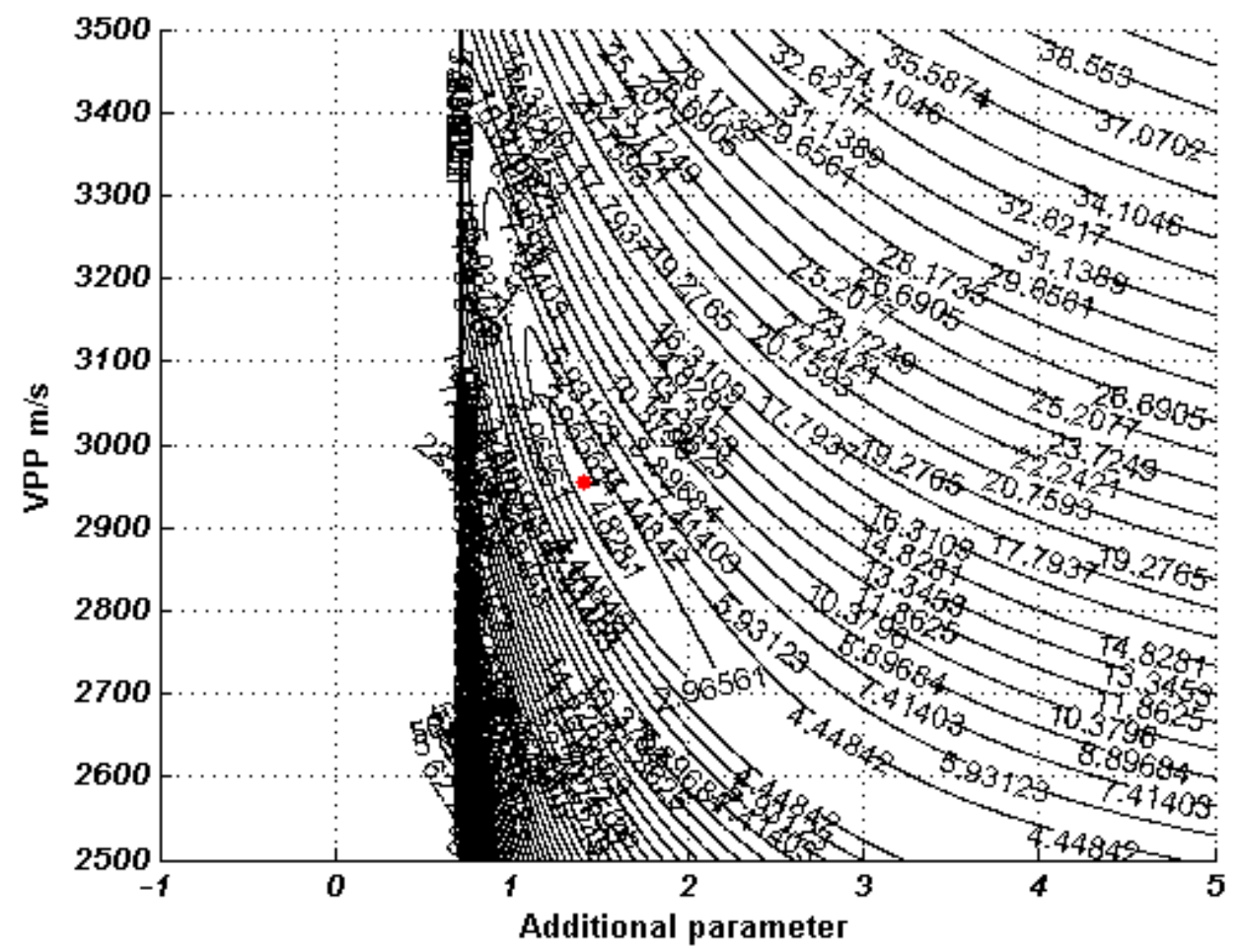

Figure 71: The residual function map which demonstrates the complexity of the Ursin and Stovas (2006) approximation of the PP reflection event with L1-norm. Red dispersions represent the global minimum region, and the blue dispersions, the local minimum regions.

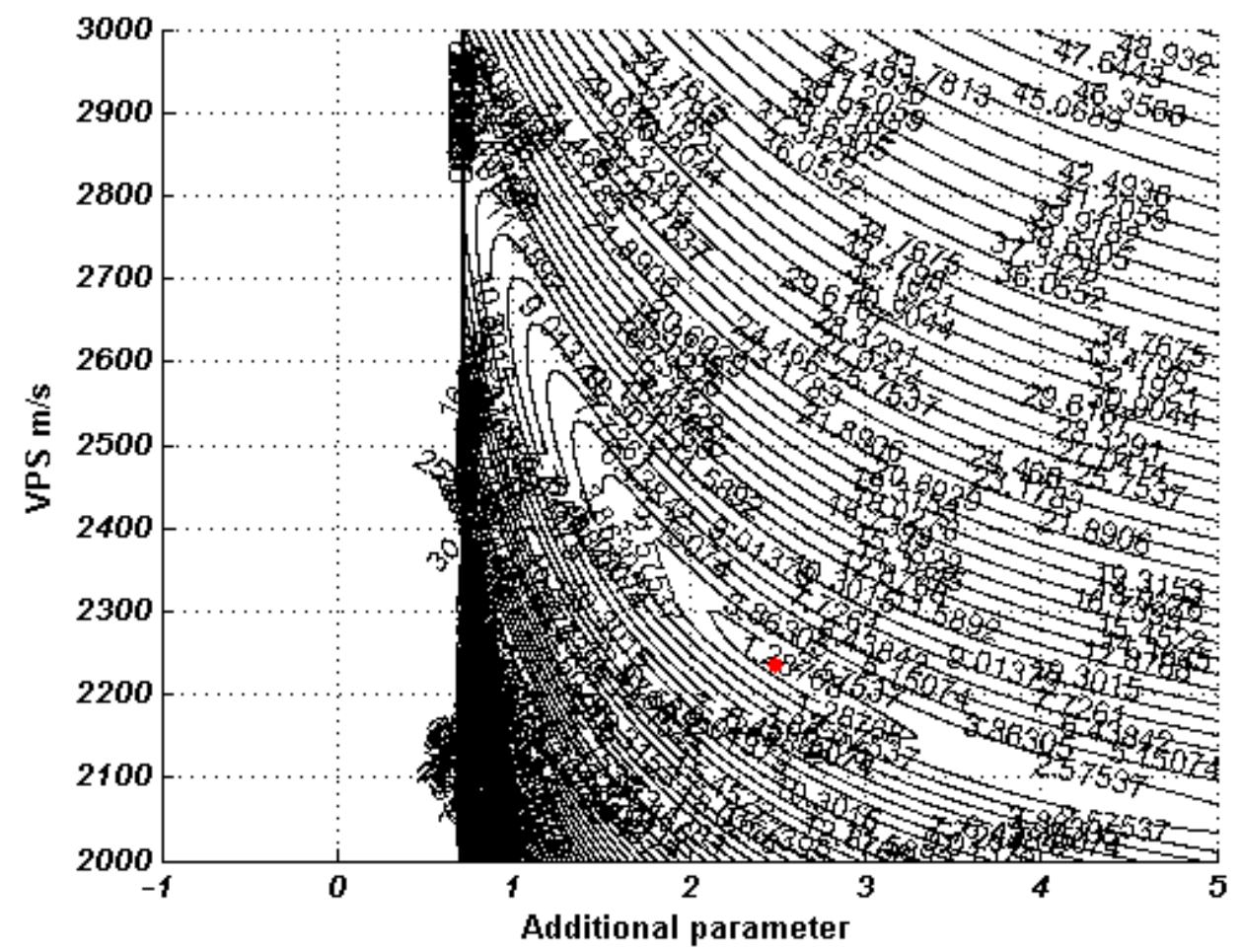

Figure 72: The residual function map which demonstrates the complexity of the Ursin and Stovas (2006) approximation of the PS reflection event with L1-norm. Red dispersions represent the global minimum region, and the blue dispersions, the local minimum regions. 


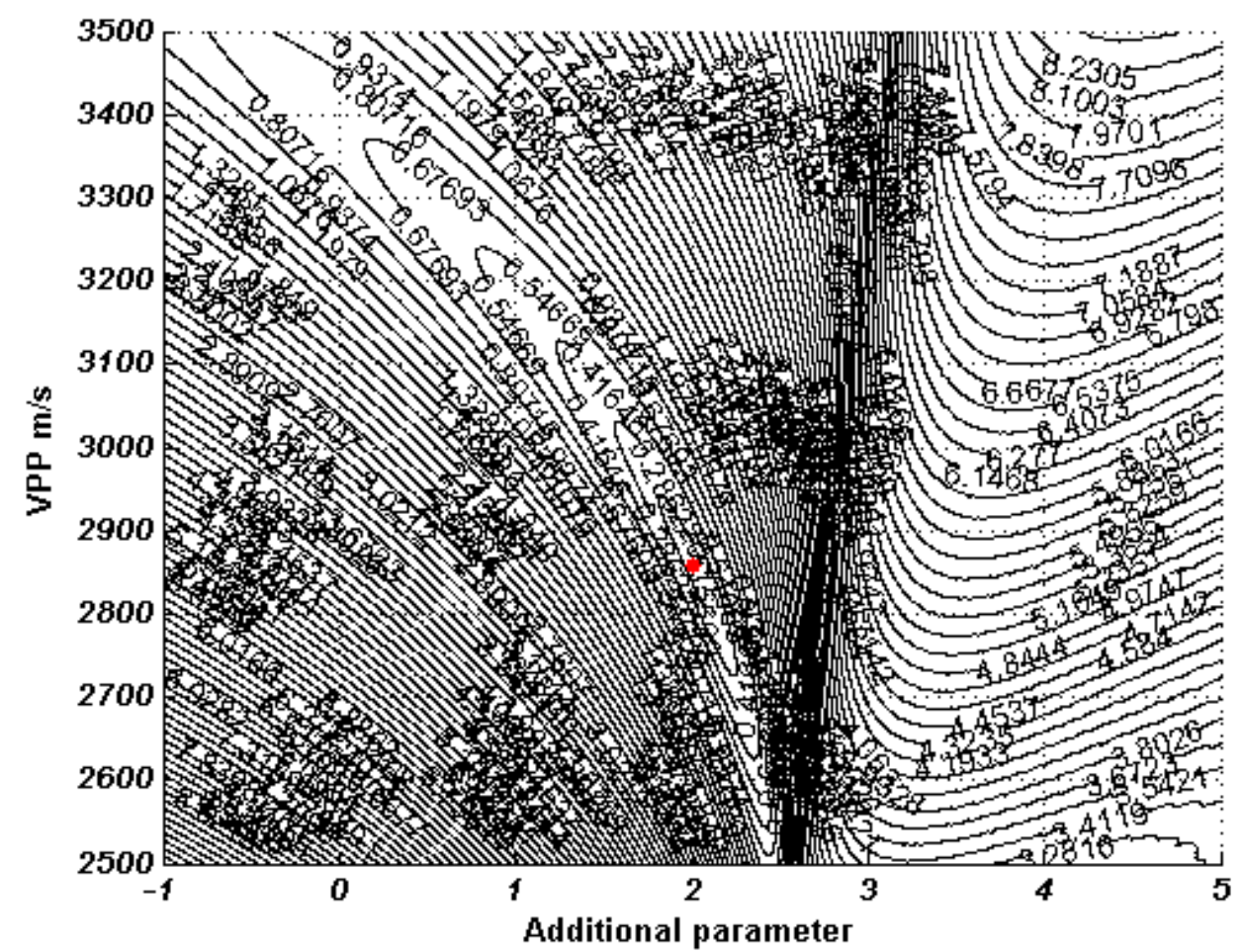

Figure 73: The residual function map which demonstrates the complexity of the Blias (2009) approximation of the PP reflection event with L2-norm. Red dispersions represent the global minimum region, and the blue dispersions, the local minimum regions.

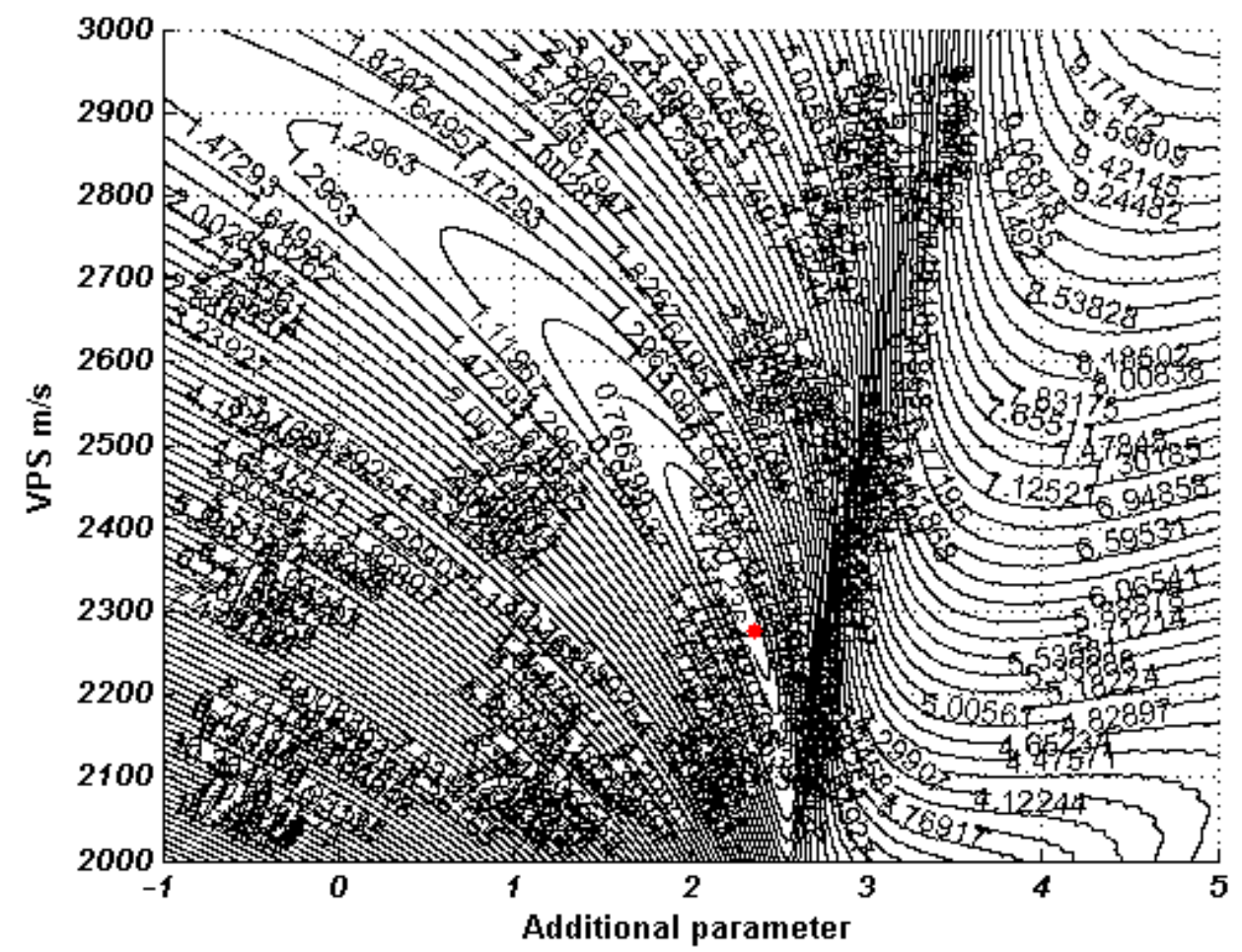

Figure 74: The residual function map which demonstrates the complexity of the Blias (2009) approximation of the PS reflection event with L2-norm. Red dispersions represent the global minimum region, and the blue dispersions, the local minimum regions. 


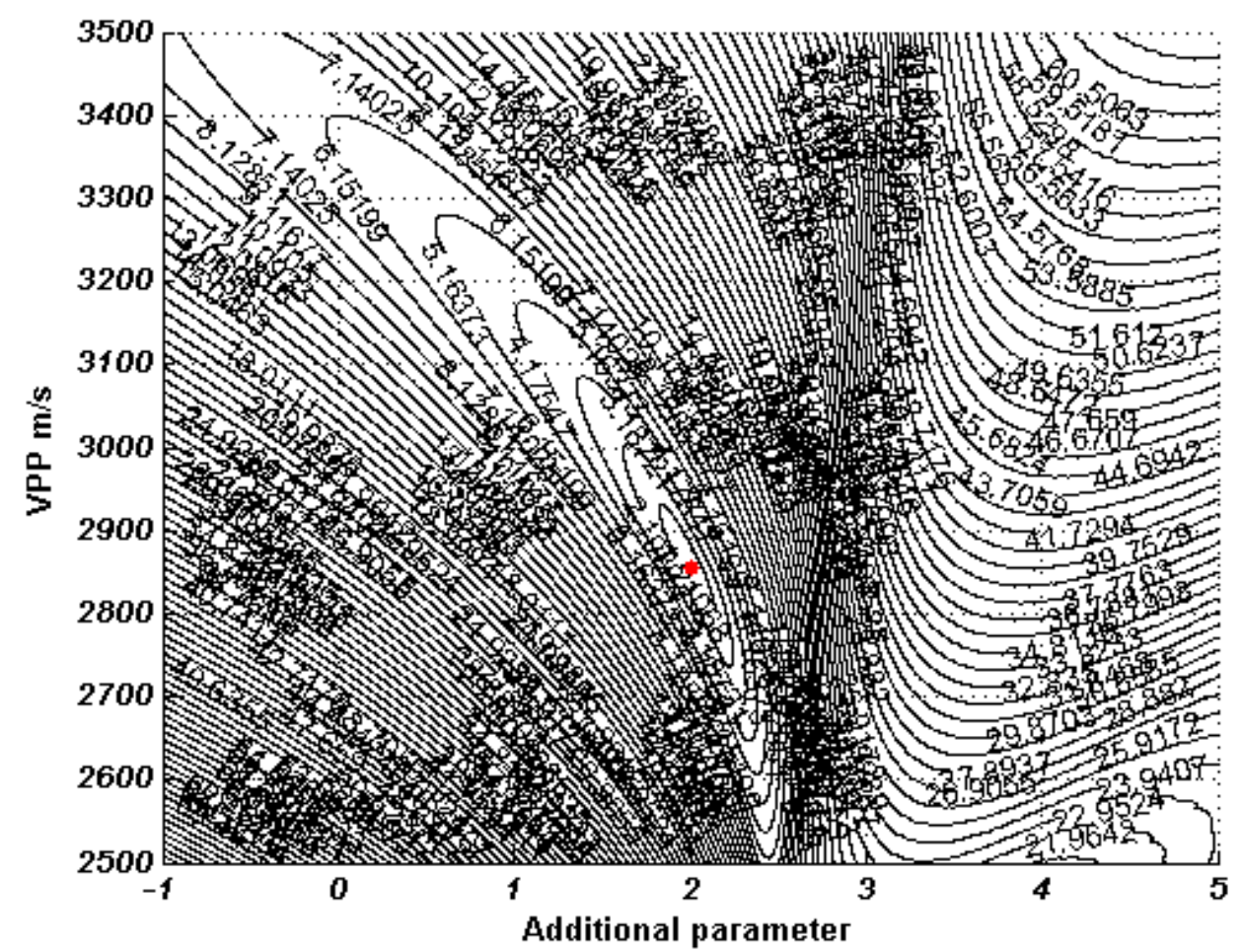

Figure 75: The residual function map which demonstrates the complexity of the Blias (2009) approximation of the PP reflection event with L1-norm. Red dispersions represent the global minimum region, and the blue dispersions, the local minimum regions.

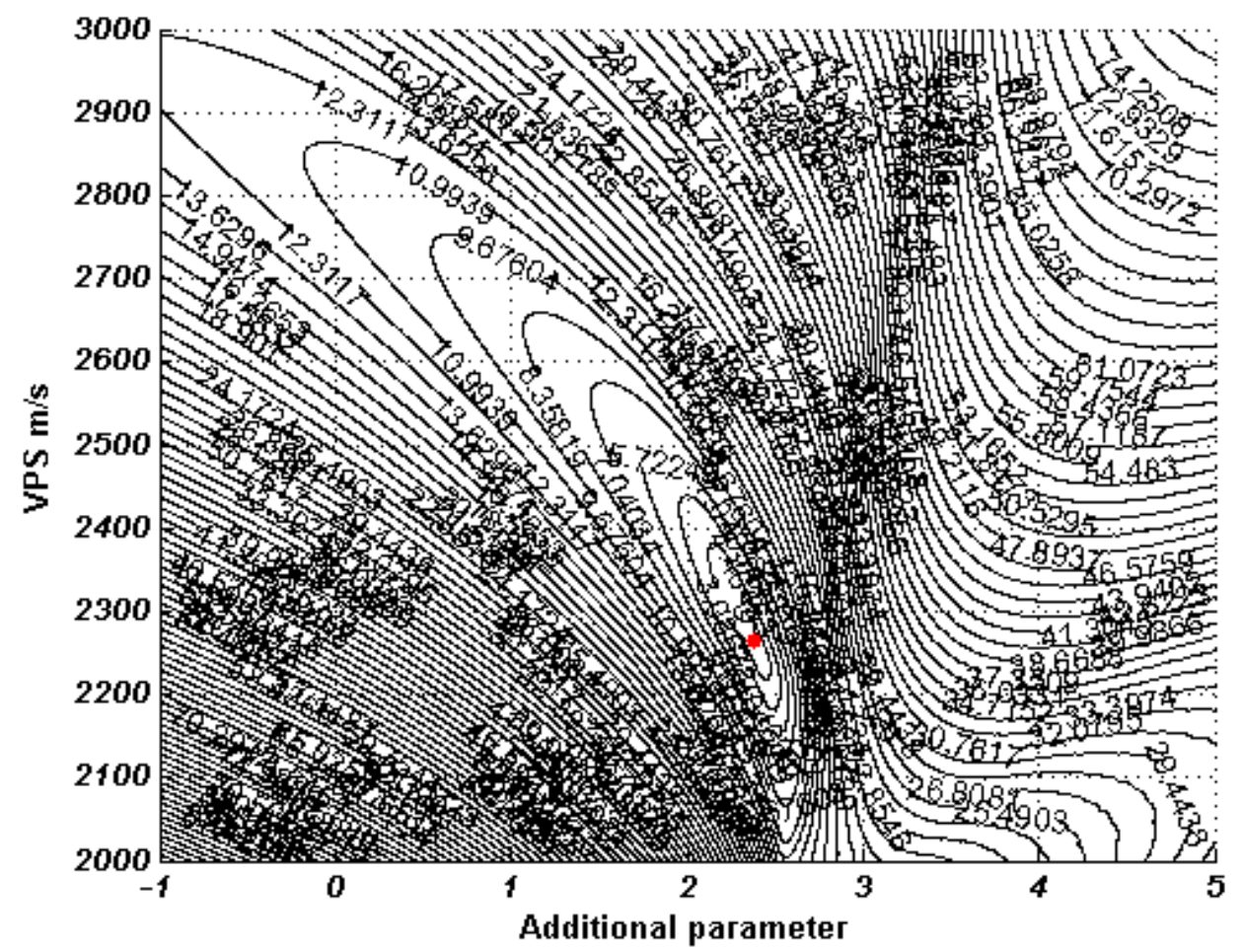

Figure 76: The residual function map which demonstrates the complexity of the Blias (2009) approximation of the PS reflection event with L1-norm. Red dispersions represent the global minimum region, and the blue dispersions, the local minimum regions. 


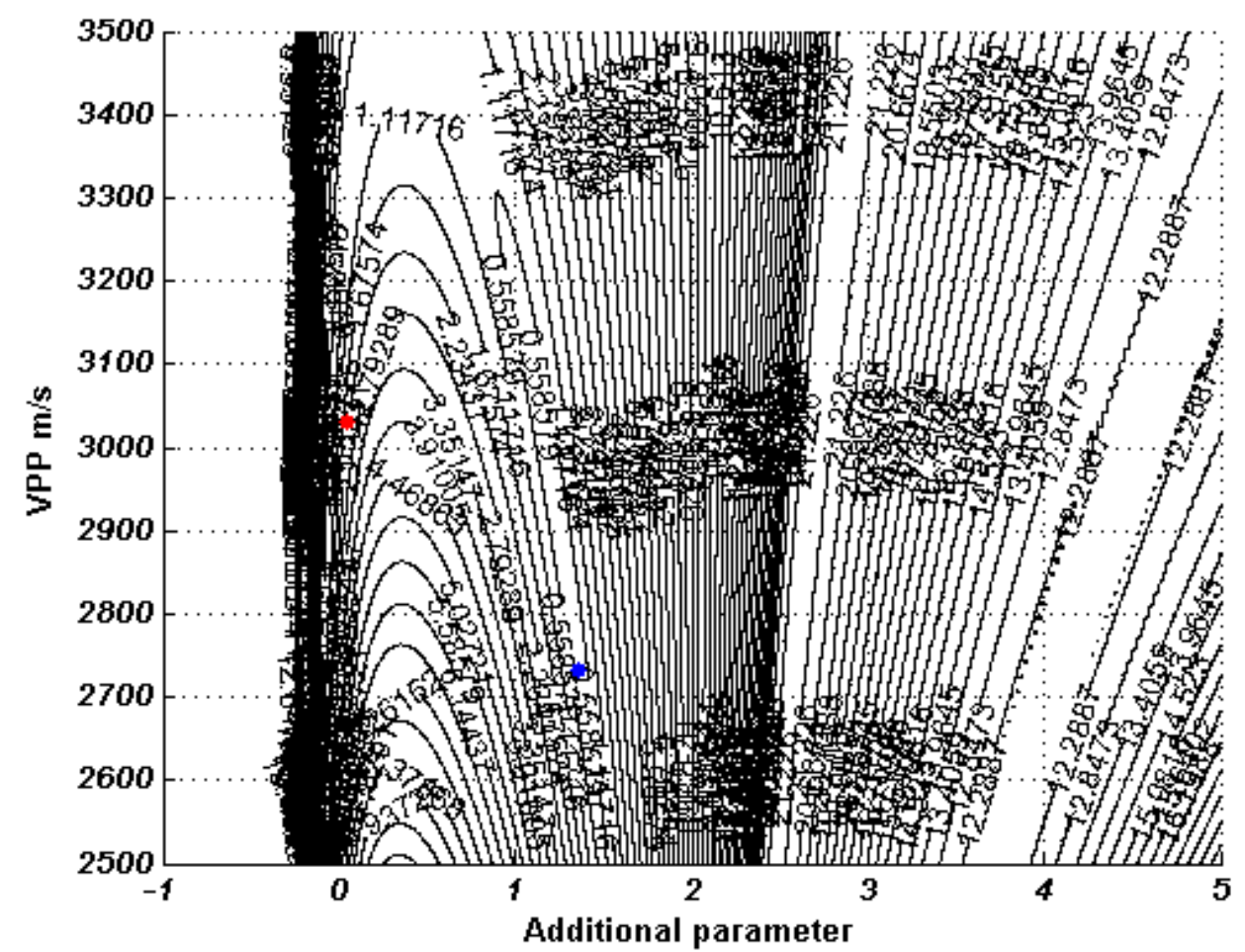

Figure 77: The residual function map which demonstrates the complexity of the Muir and Dellinger (1985) approximation of the PP reflection event with L2-norm. Red dispersions represent the global minimum region, and the blue dispersions, the local minimum regions.

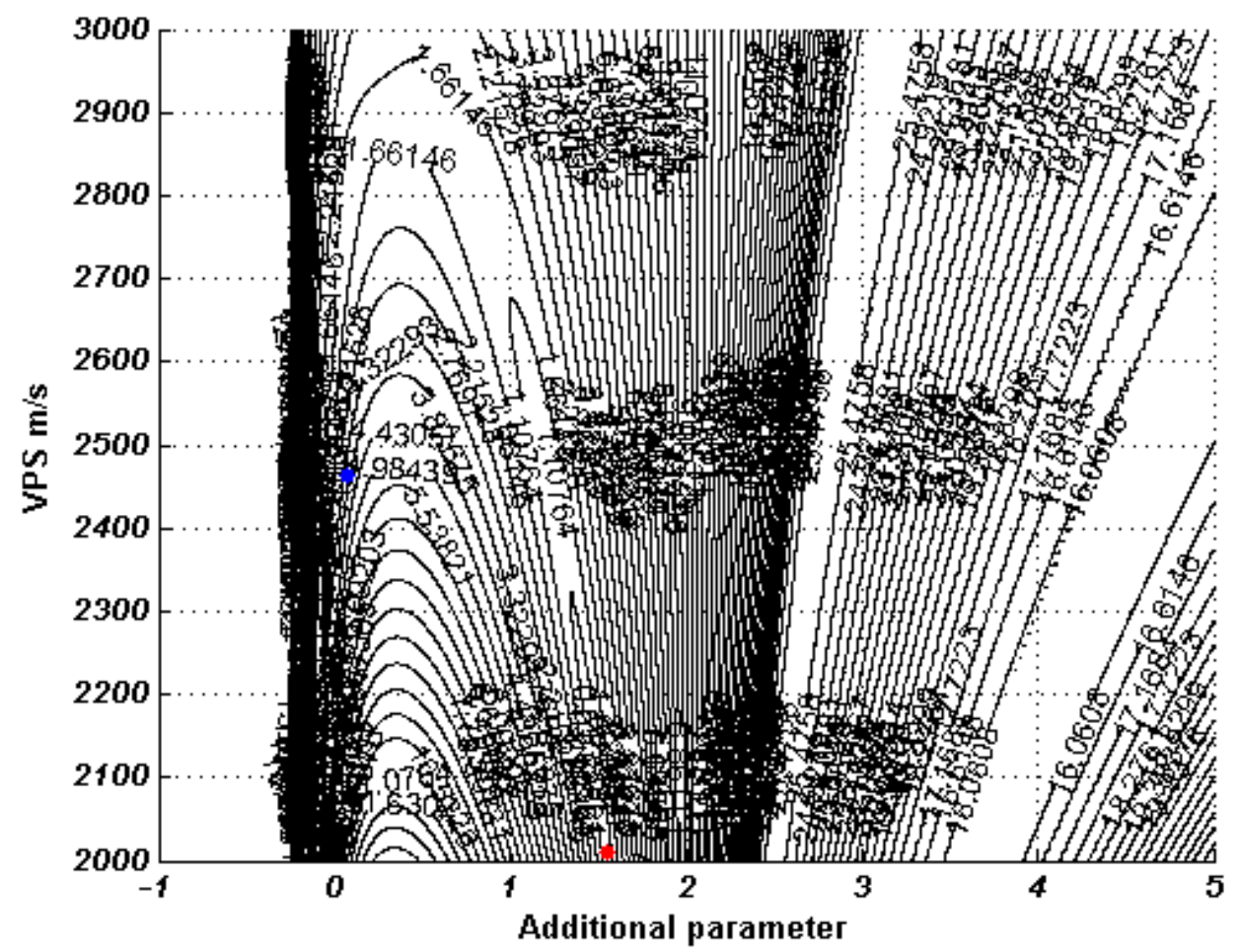

Figure 78: The residual function map which demonstrates the complexity of the Muir and Dellinger (1985) approximation of the PS reflection event with L2-norm. Red dispersions represent the global minimum region, and the blue dispersions, the local minimum regions. 


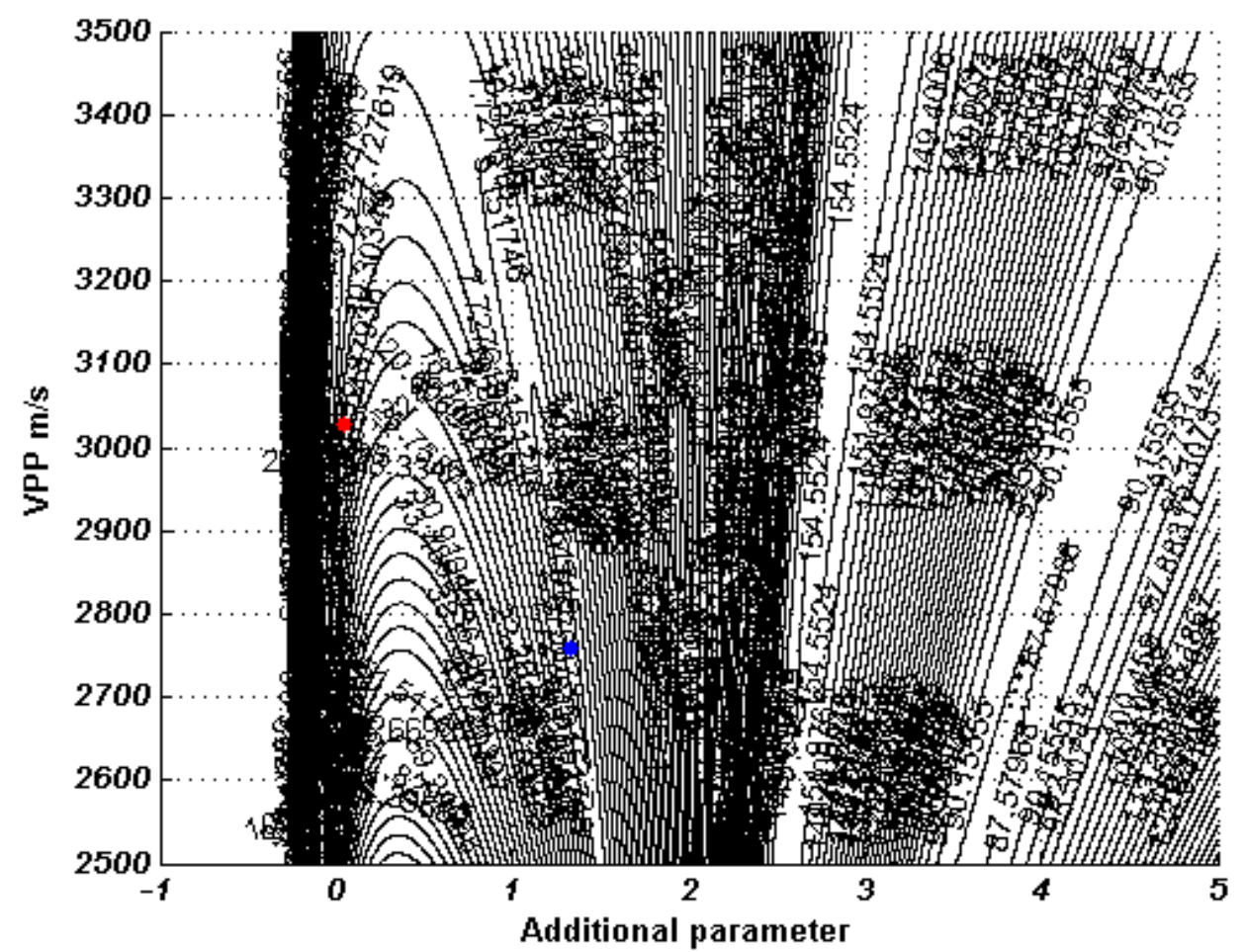

Figure 79: The residual function map which demonstrates the complexity of the Muir and Dellinger (1985) approximation of the PP reflection event with L1-norm. Red dispersions represent the global minimum region, and the blue dispersions, the local minimum regions.

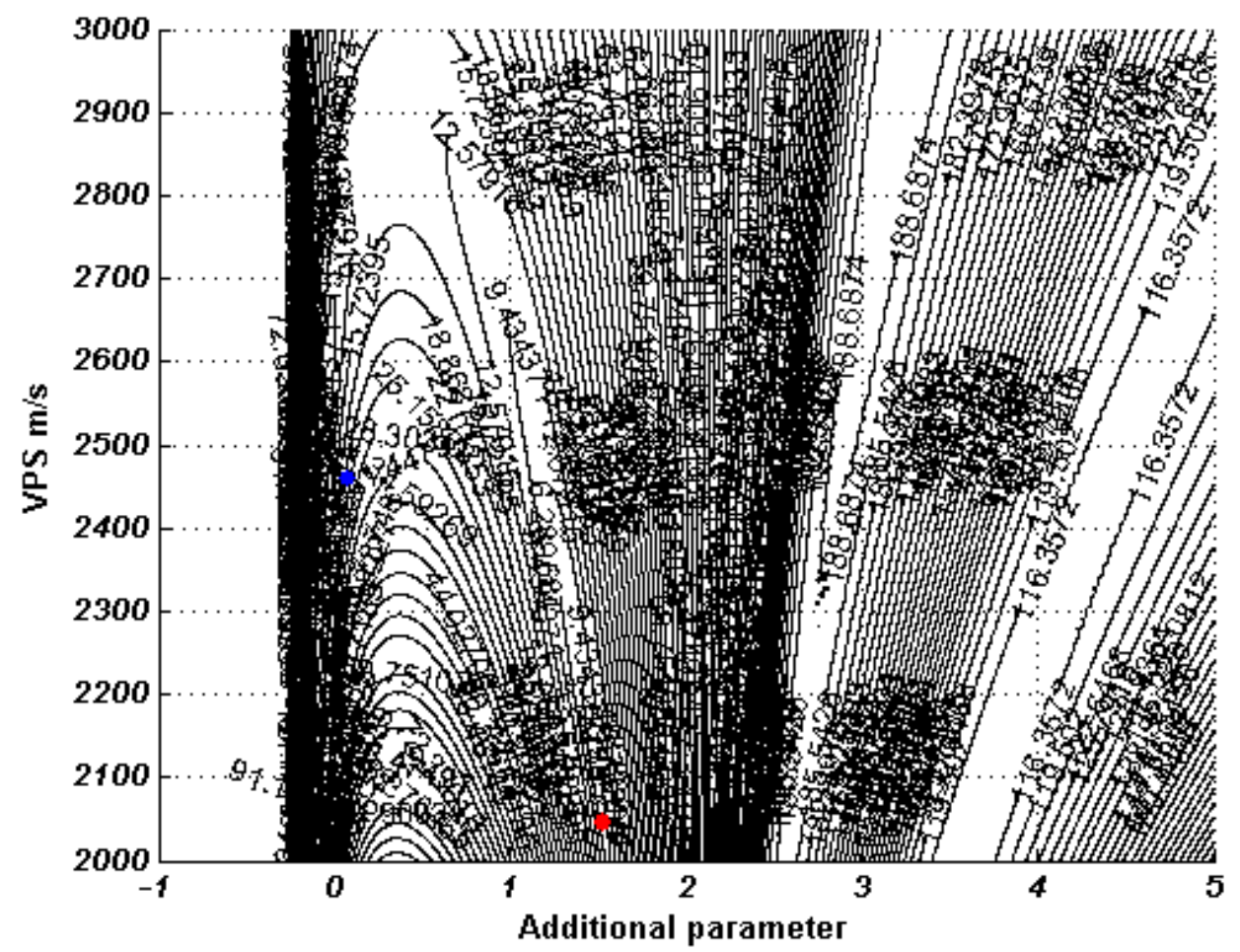

Figure 80: The residual function map which demonstrates the complexity of the Muir and Dellinger (1985) approximation of the PS reflection event with L1-norm. Red dispersions represent the global minimum region, and the blue dispersions, the local minimum regions. 


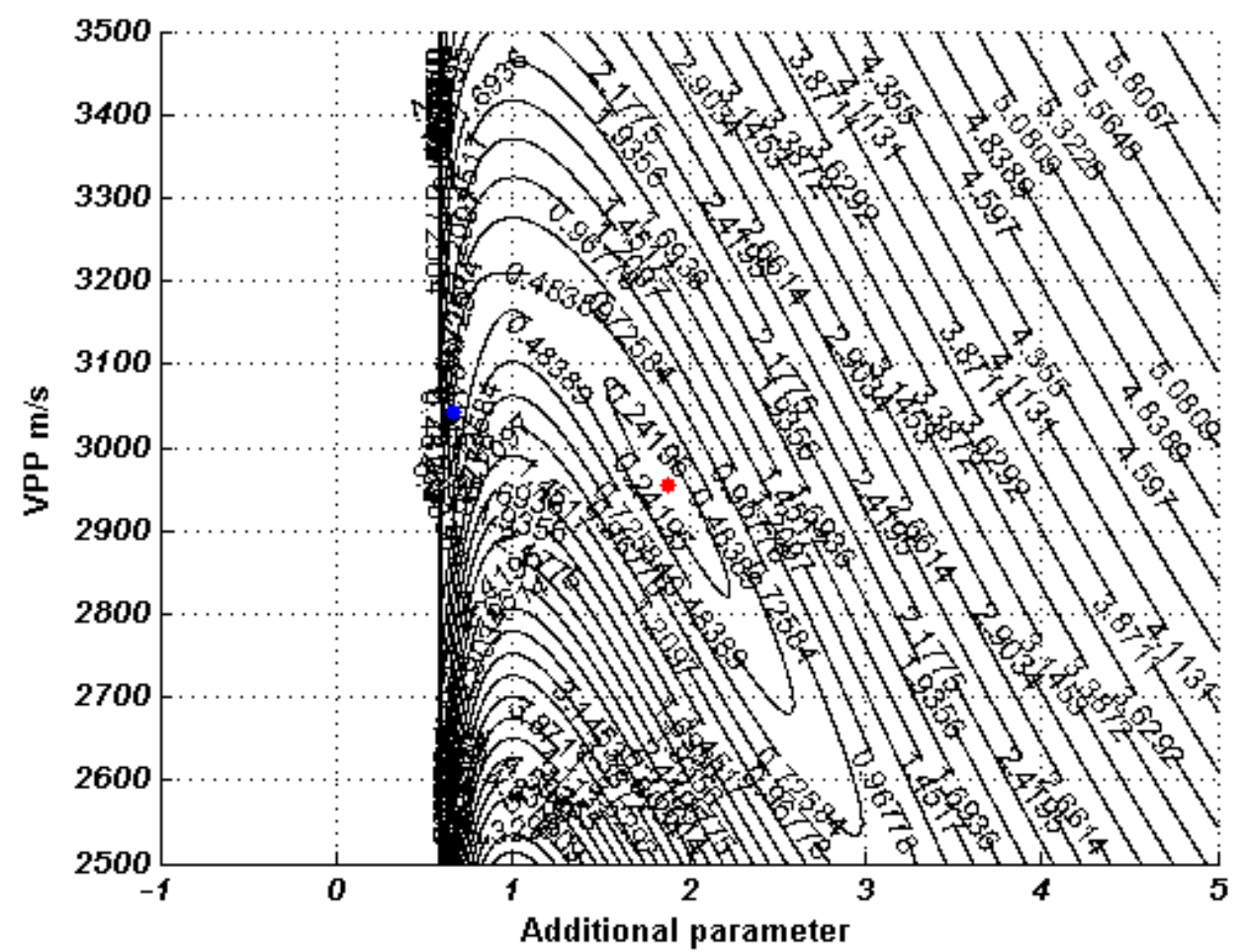

Figure 81: The residual function map which demonstrates the complexity of the Li and Yuan (2001) approximation of the PP reflection event with L2-norm. Red dispersions represent the global minimum region, and the blue dispersions, the local minimum regions.

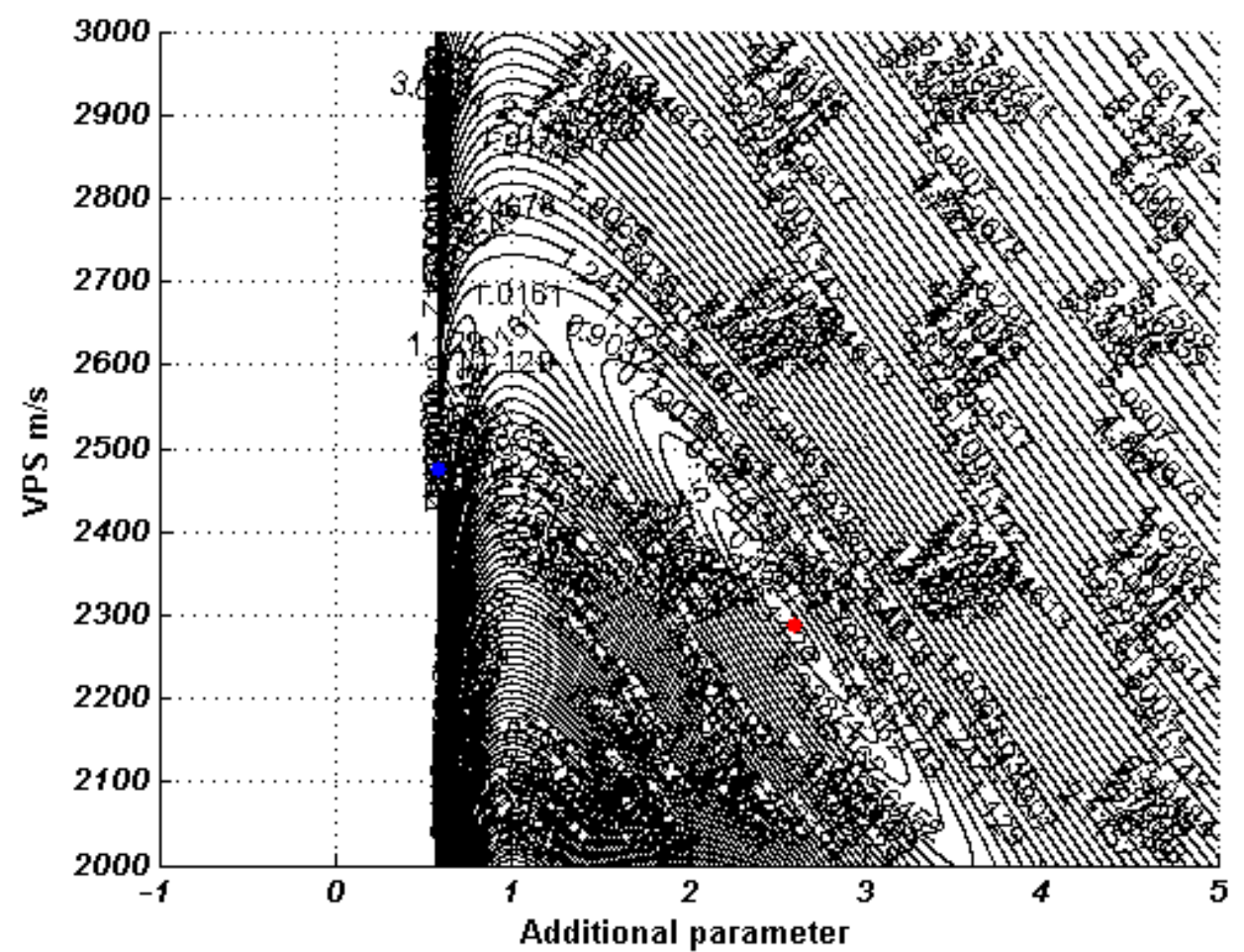

Figure 82: The residual function map which demonstrates the complexity of the Li and Yuan (2001) approximation of the PS reflection event with L2-norm. Red dispersions represent the global minimum region, and the blue dispersions, the local minimum regions. 


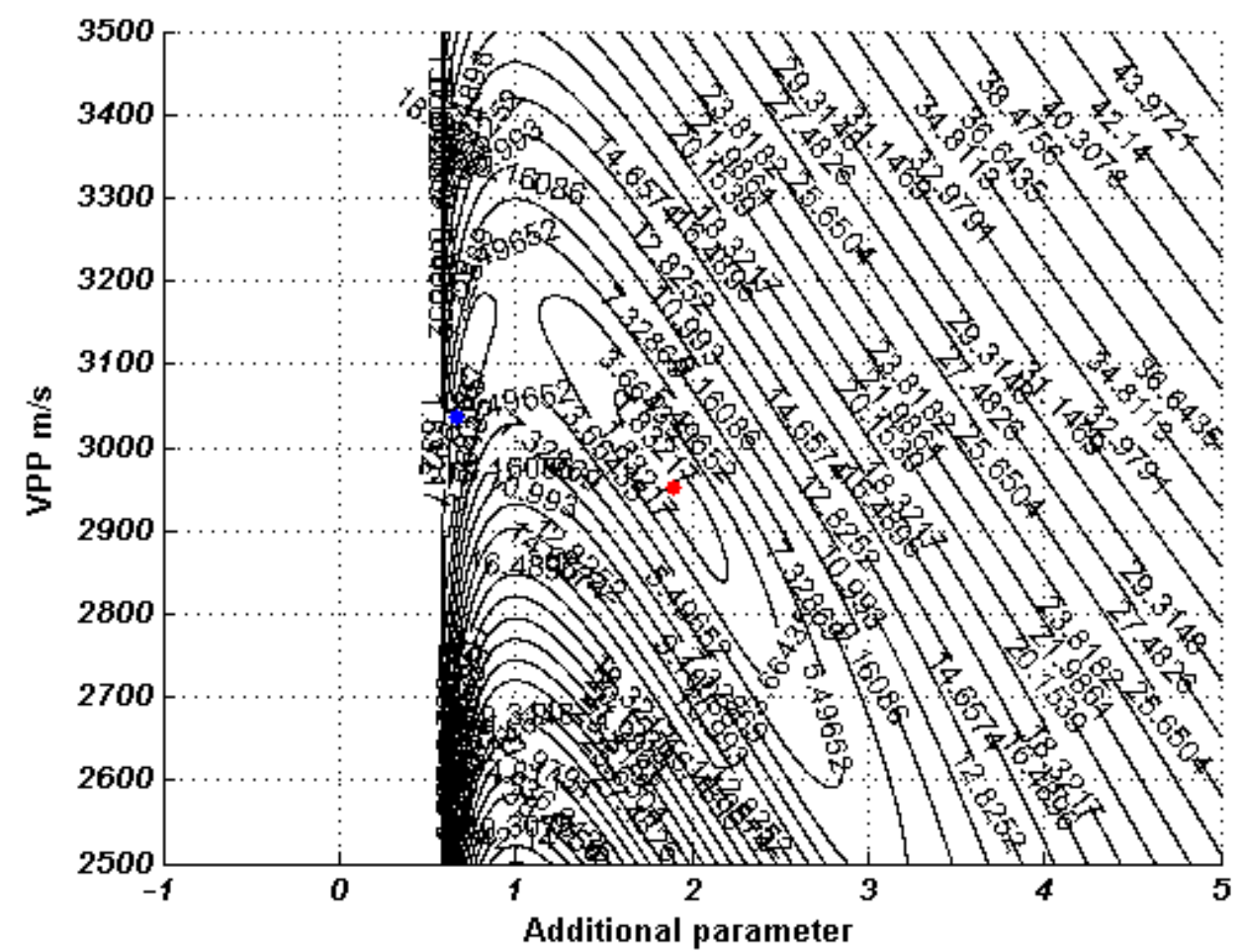

Figure 83: The residual function map which demonstrates the complexity of the Li and Yuan (2001) approximation of the PP reflection event with L1-norm. Red dispersions represent the global minimum region, and the blue dispersions, the local minimum regions.

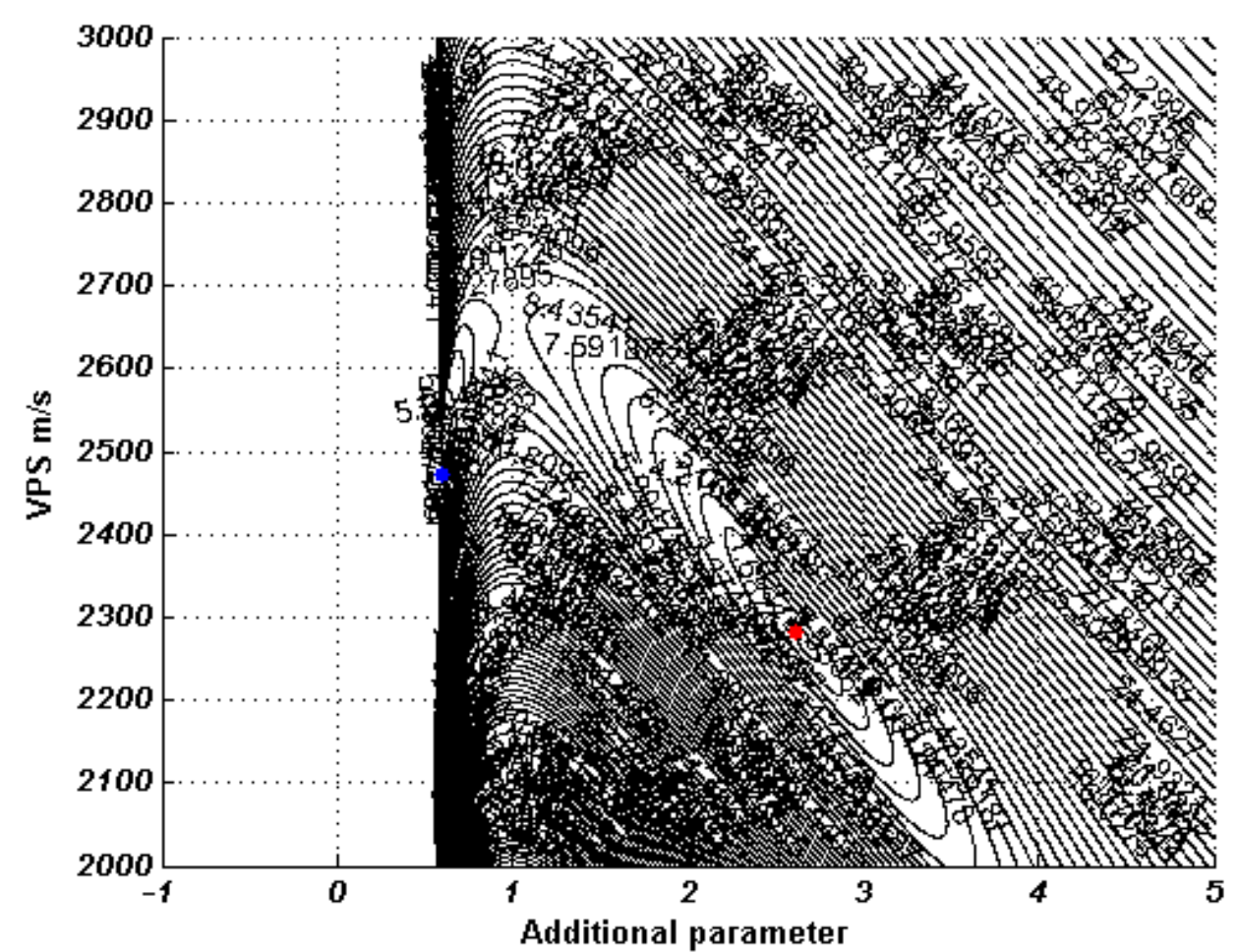

Figure 84: The residual function map which demonstrates the complexity of the Li and Yuan (2001) approximation of the PS reflection event with L1-norm. Red dispersions represent the global minimum region, and the blue dispersions, the local minimum regions. 


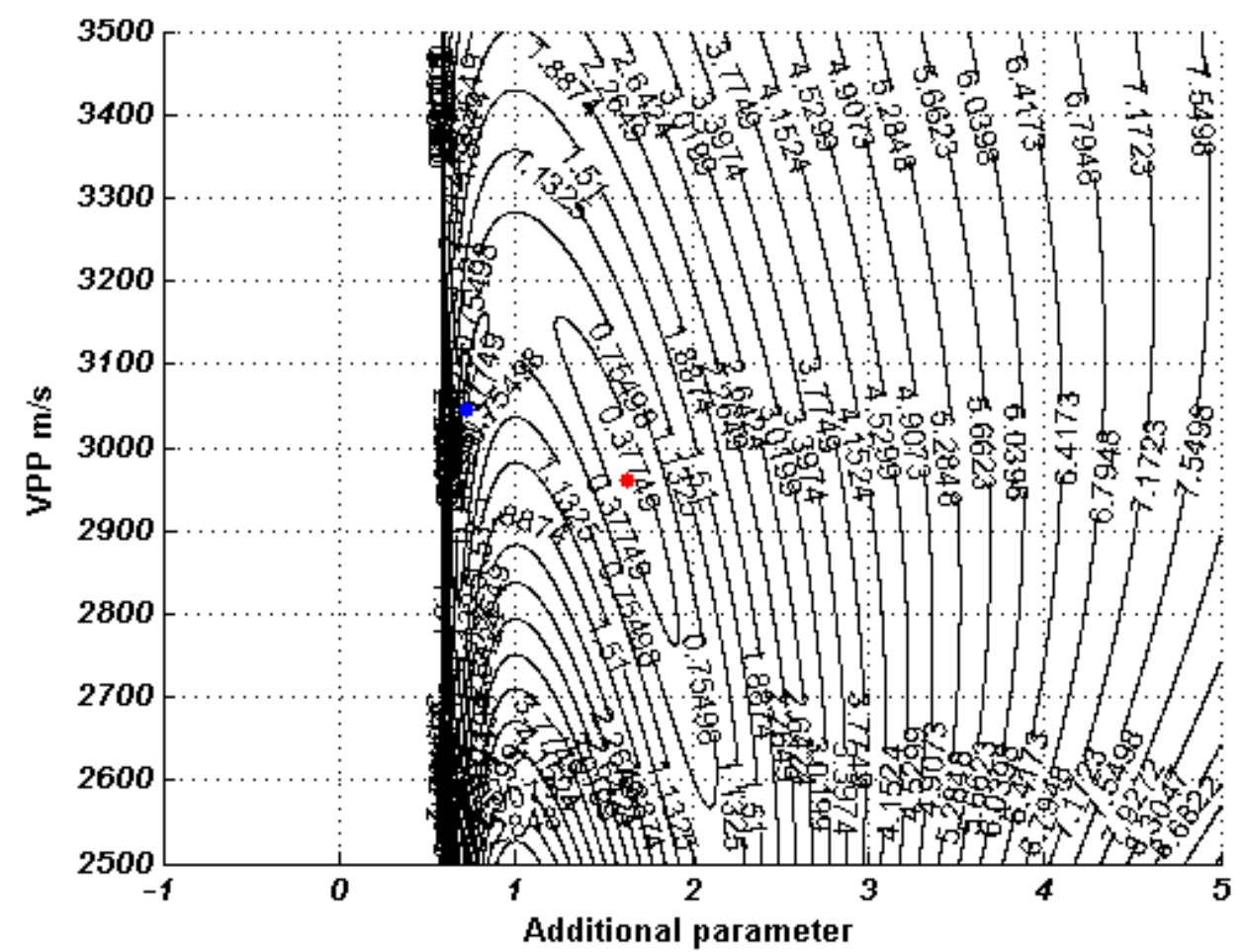

Figure 85: The residual function map which demonstrates the complexity of the approximation proposed in this work of the PP reflection event with L2-norm. Red dispersions represent the global minimum region, and the blue dispersions, the local minimum regions.

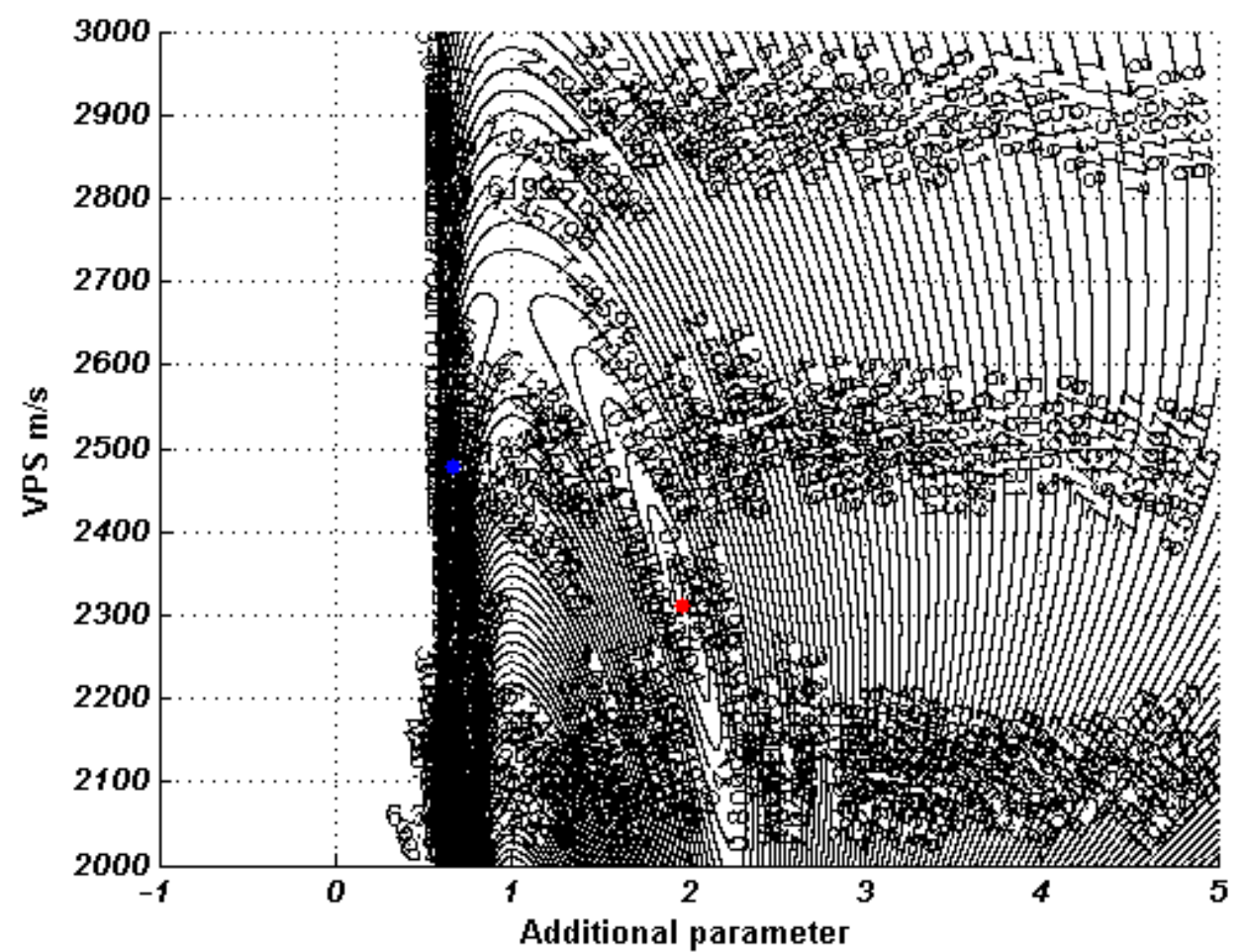

Figure 86: The residual function map which demonstrates the complexity of the approximation proposed in this work of the PS reflection event with L2-norm. Red dispersions represent the global minimum region, and the blue dispersions, the local minimum regions. 


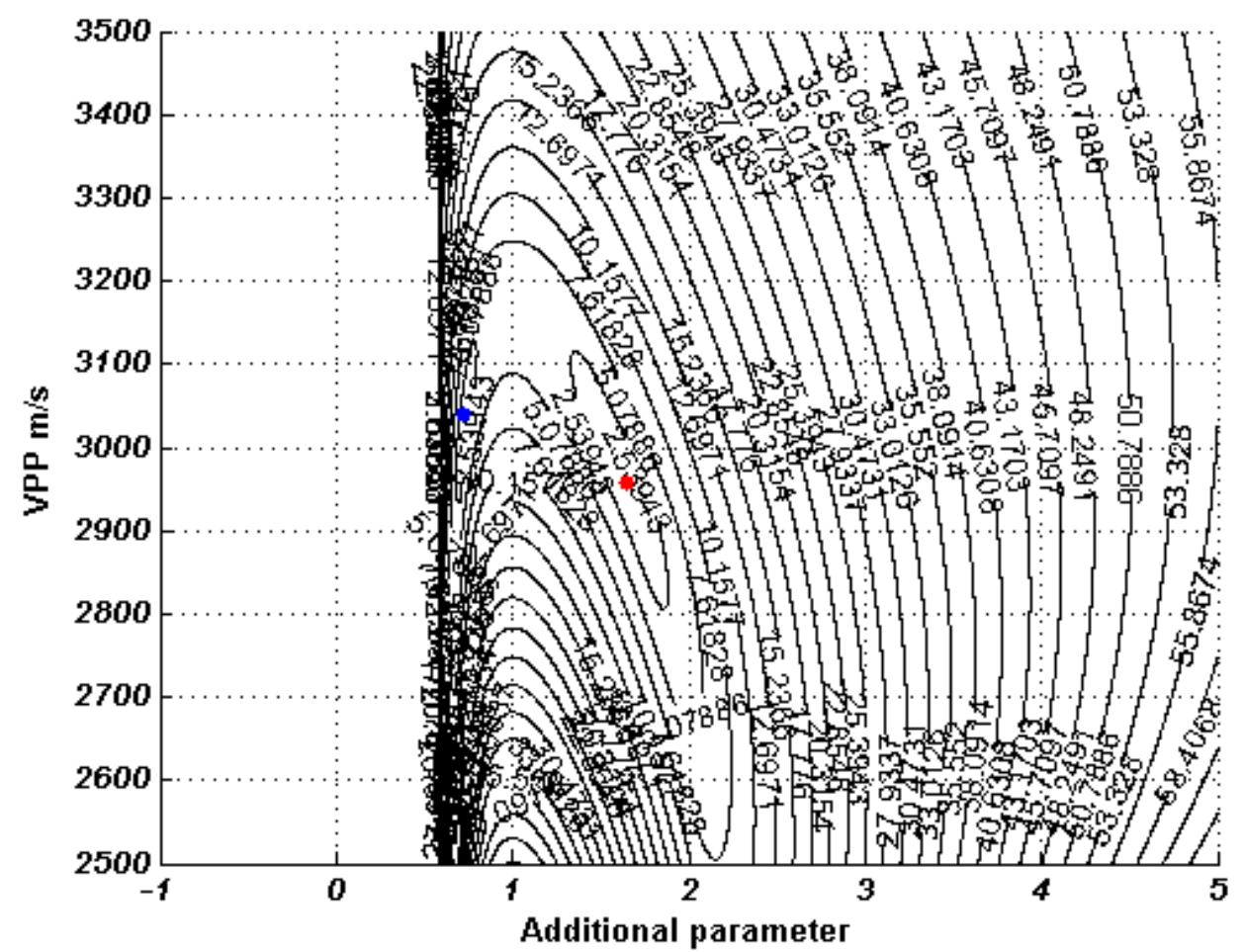

Figure 87: The residual function map which demonstrates the complexity of the approximation proposed in this work of the PP reflection event with L1-norm. Red dispersions represent the global minimum region, and the blue dispersions, the local minimum regions.

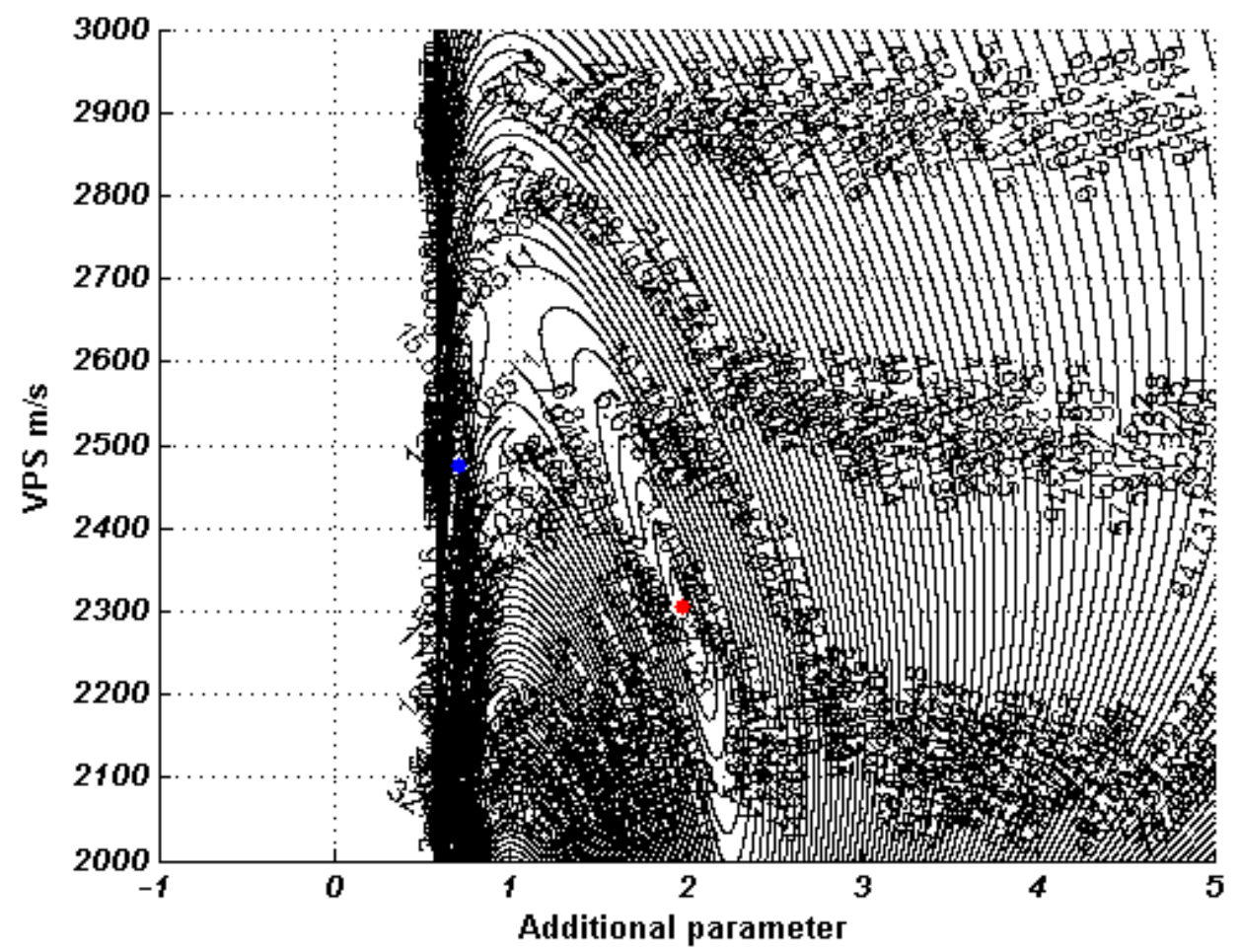

Figure 88: The residual function map which demonstrates the complexity of the approximation proposed in this work of the PS reflection event with L1-norm. Red dispersions represent the global minimum region, and the blue dispersions, the local minimum regions. 


\subsubsection{Model 3}

As it was shown, the third model has a different characteristic than the other Santos Basin structures, so it is significantly more complex than the previous models. However, nothing except for the subtle variation in the topological structure changed for the Malovichko (1978) approximation. The variations and characteristics that are similar to what already was observed in the Models 1 and 2 can be seen in the Figures 89, 90, 91 and 92.

The approximation proposed by Alkhalifah and Tsvankin (1995) behaved, concerning its variations, in a similar manner as before (Figures 93, 94, 95 and 96).

As it was shown before, the approximation proposed by Ursin and Stovas (2006) presented to be unimodal again, and with the same variations as in the previous models (Figures 97, 98, 99 and 100).

In a similar way as the Ursin and Stovas (2006) approximation, the one proposed by Blias (2009) showed the same kinds of variations (Figures 101, 102, 103 and 104).

Even with a model with a significantly different characteristic, the approximation proposed by Muir and Dellinger (1985) showed the same unusual characteristic concerning the variation between the conventional event to the converted event (Figures 105 and 106). Moreover, it showed the same multimodal behaviour as before and the same variations concerning the L2- and L1-norm (Figures 107 and 108).

The approximation proposed by Li and Yuan (2001), showed the same behaviour as before, being multimodal and with the already seen variations between PP to PS events and between L2- and L1-norm (Figures 109, 110, 111 and 112).

The proposed approximation in this work showed only subtle variations concerning the topology of the objective function. Concerning the other characteristics and variations, it behaved in a similar manner (Figure 113, 114, 115 and 116). 


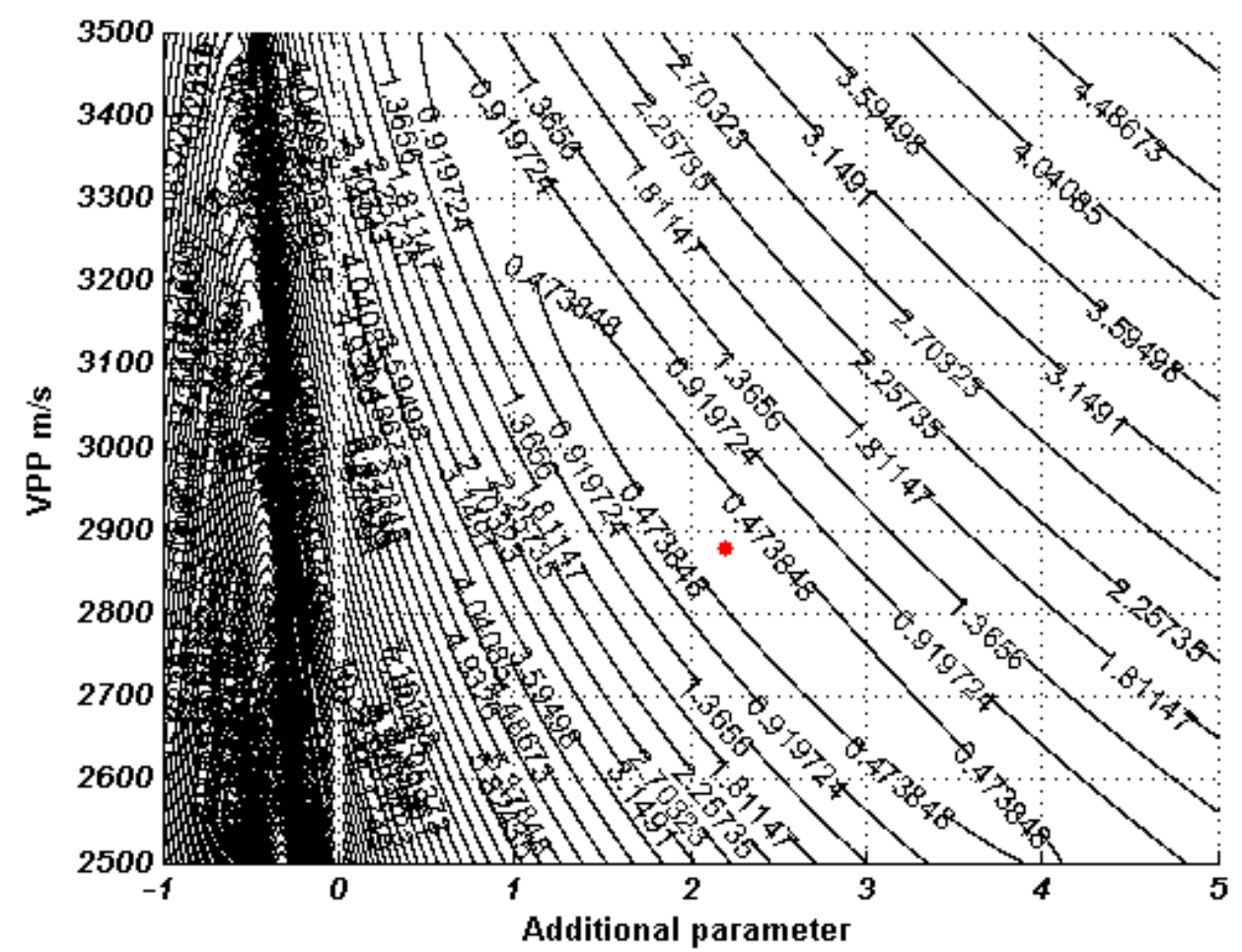

Figure 89: The residual function map which demonstrates the complexity of the Malovichko (1978) approximation of the PP reflection event with L2-norm. Red dispersions represent the global minimum region, and the blue dispersions, the local minimum regions.

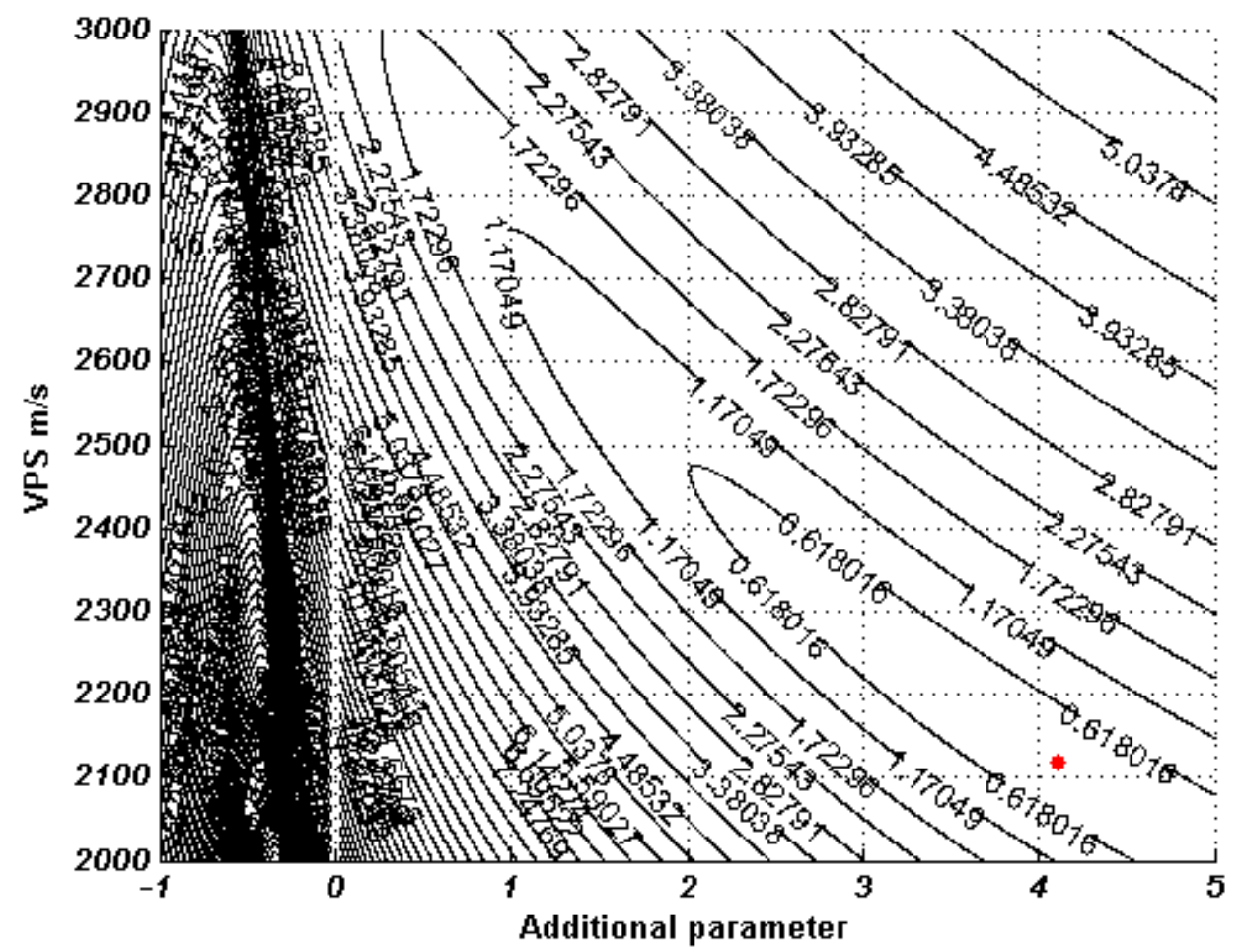

Figure 90: The residual function map which demonstrates the complexity of the Malovichko (1978) approximation of the PS reflection event with L2-norm. Red dispersions represent the global minimum region, and the blue dispersions, the local minimum regions. 


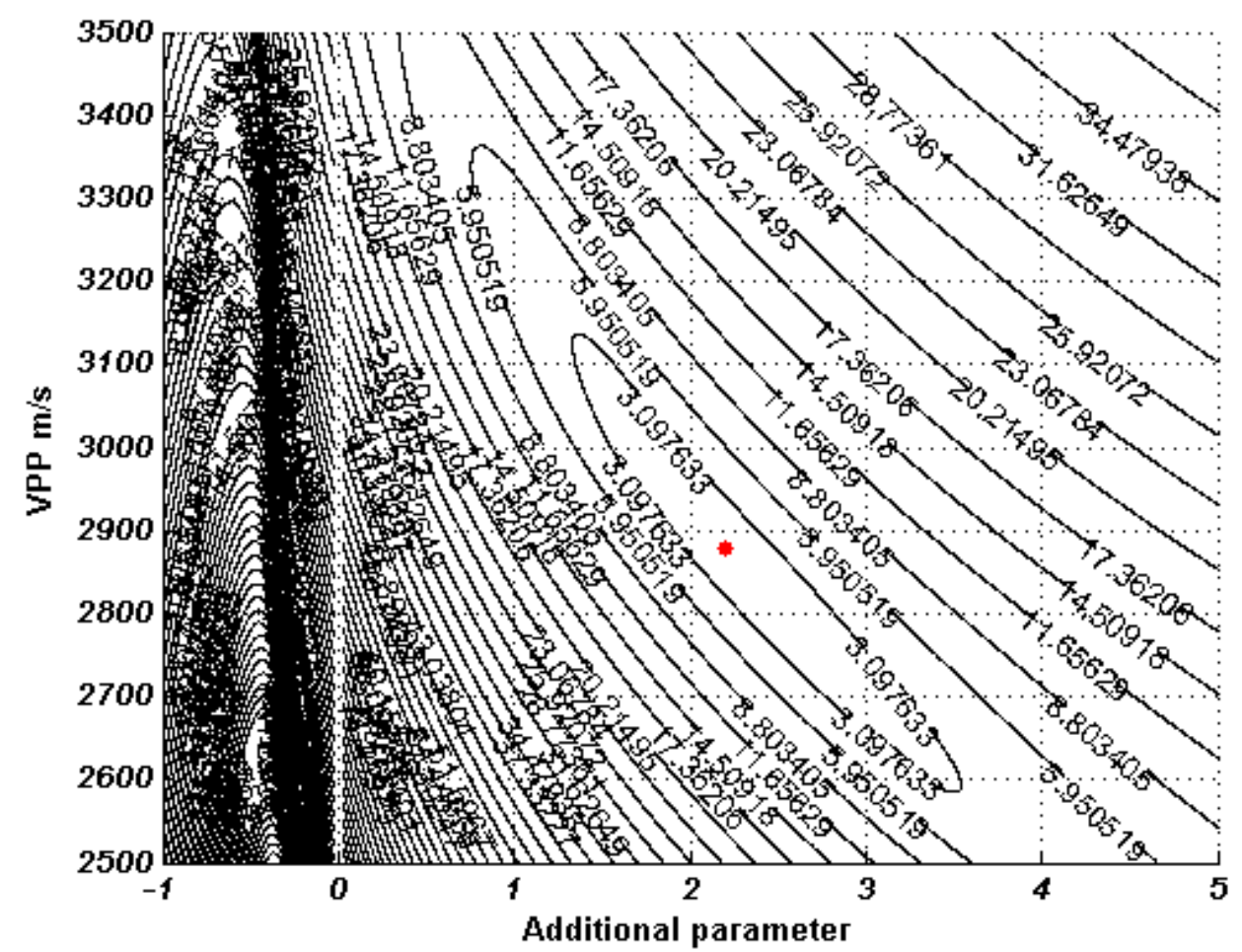

Figure 91: The residual function map which demonstrates the complexity of the Malovichko (1978) approximation of the PP reflection event with L1-norm. Red dispersions represent the global minimum region, and the blue dispersions, the local minimum regions.

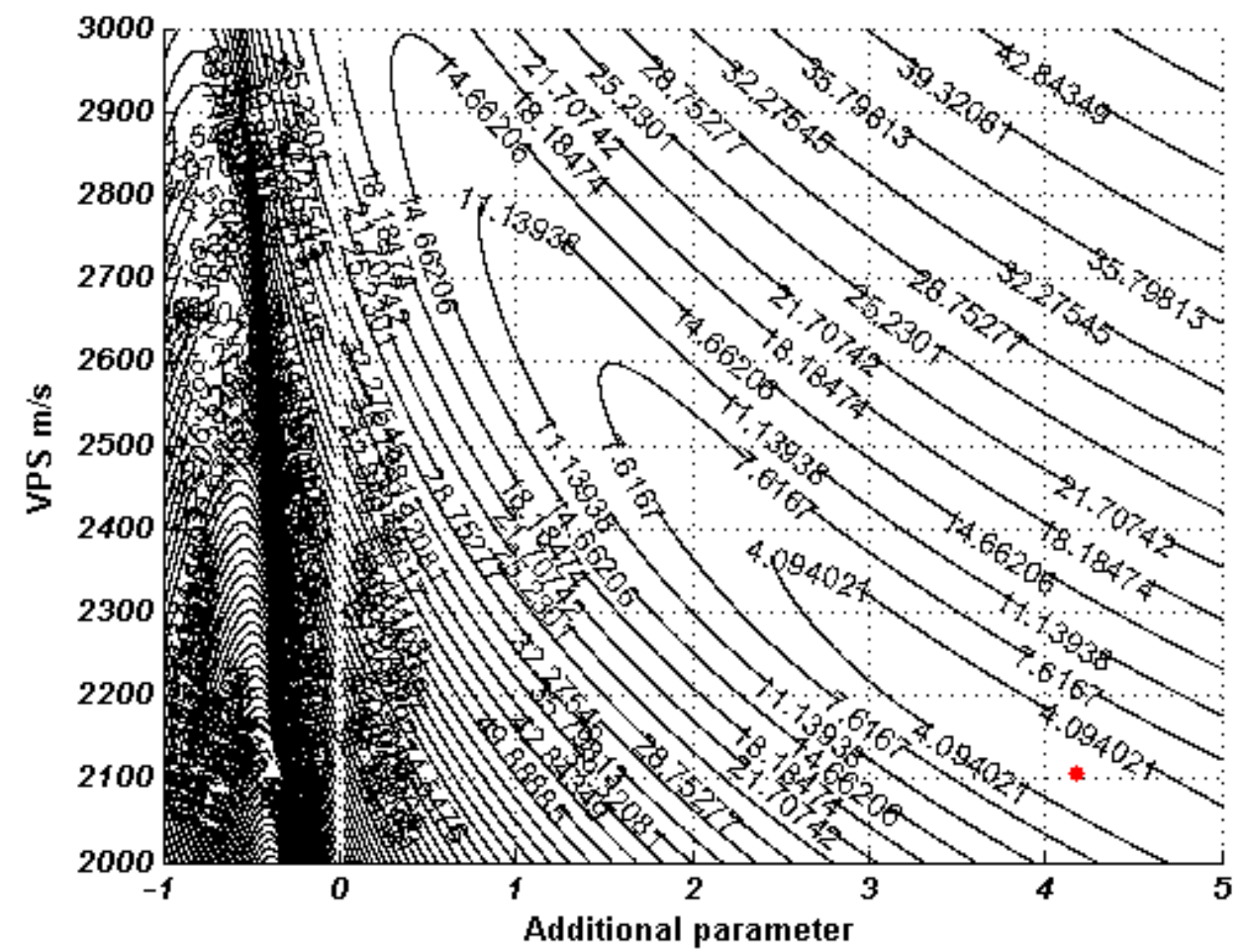

Figure 92: The residual function map which demonstrates the complexity of the Malovichko (1978) approximation of the PS reflection event with L1-norm. Red dispersions represent the global minimum region, and the blue dispersions, the local minimum regions. 


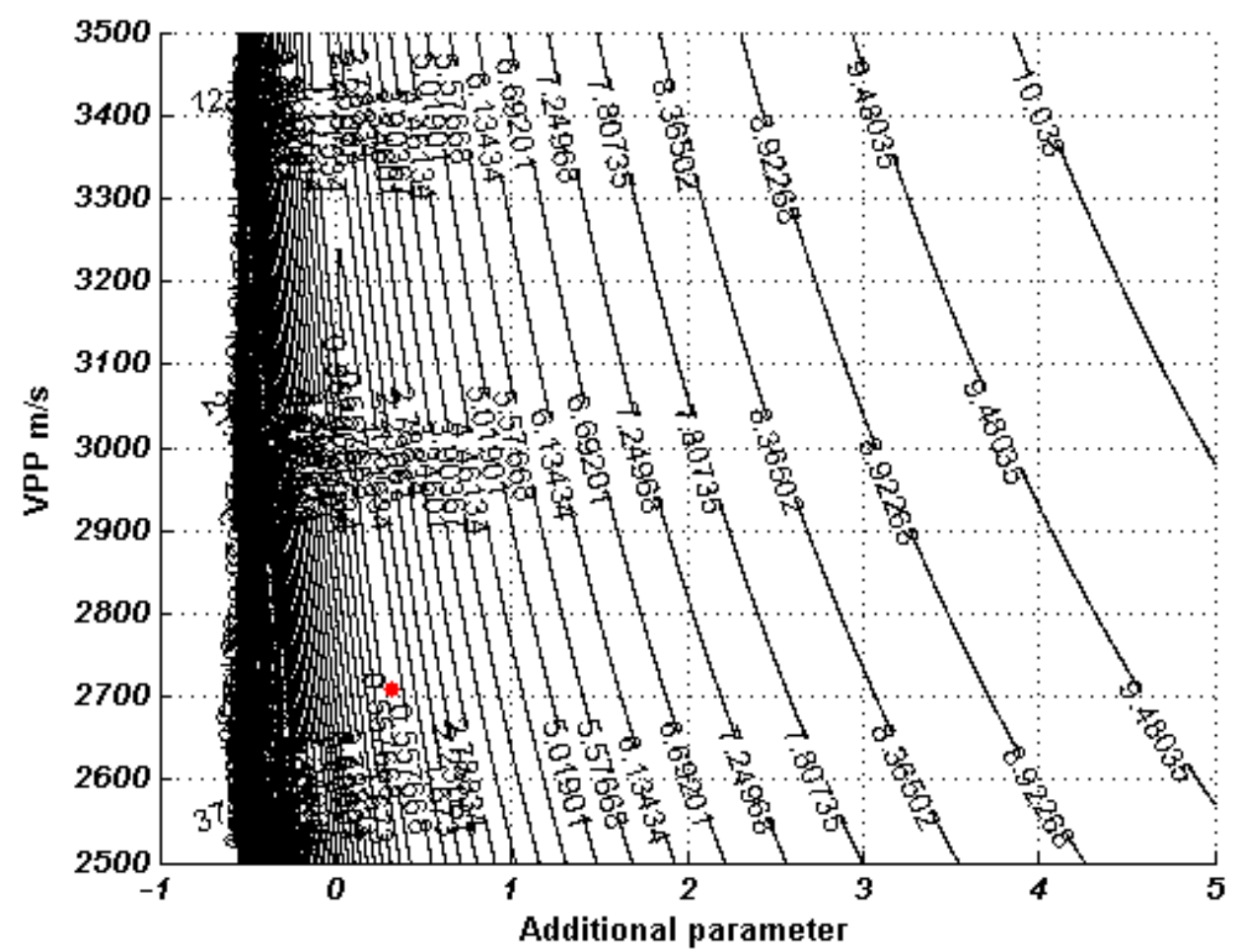

Figure 93: The residual function map which demonstrates the complexity of the Alkhalifah and Tsvankin (1995) approximation of the PP reflection event with L2-norm. Red dispersions represent the global minimum region, and the blue dispersions, the local minimum regions.

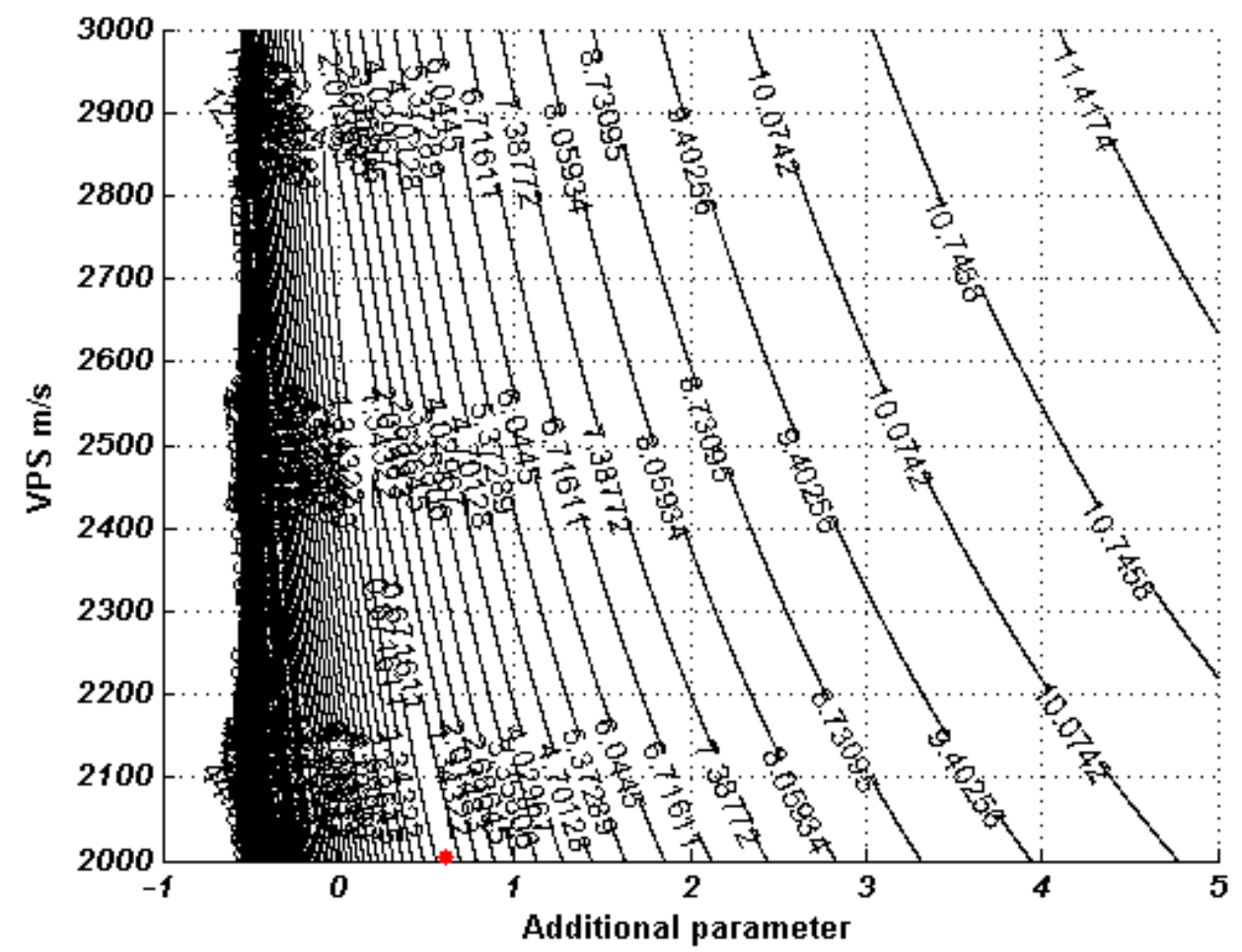

Figure 94: The residual function map which demonstrates the complexity of the Alkhalifah and Tsvankin (1995) approximation of the PS reflection event with L2-norm. Red dispersions represent the global minimum region, and the blue dispersions, the local minimum regions. 


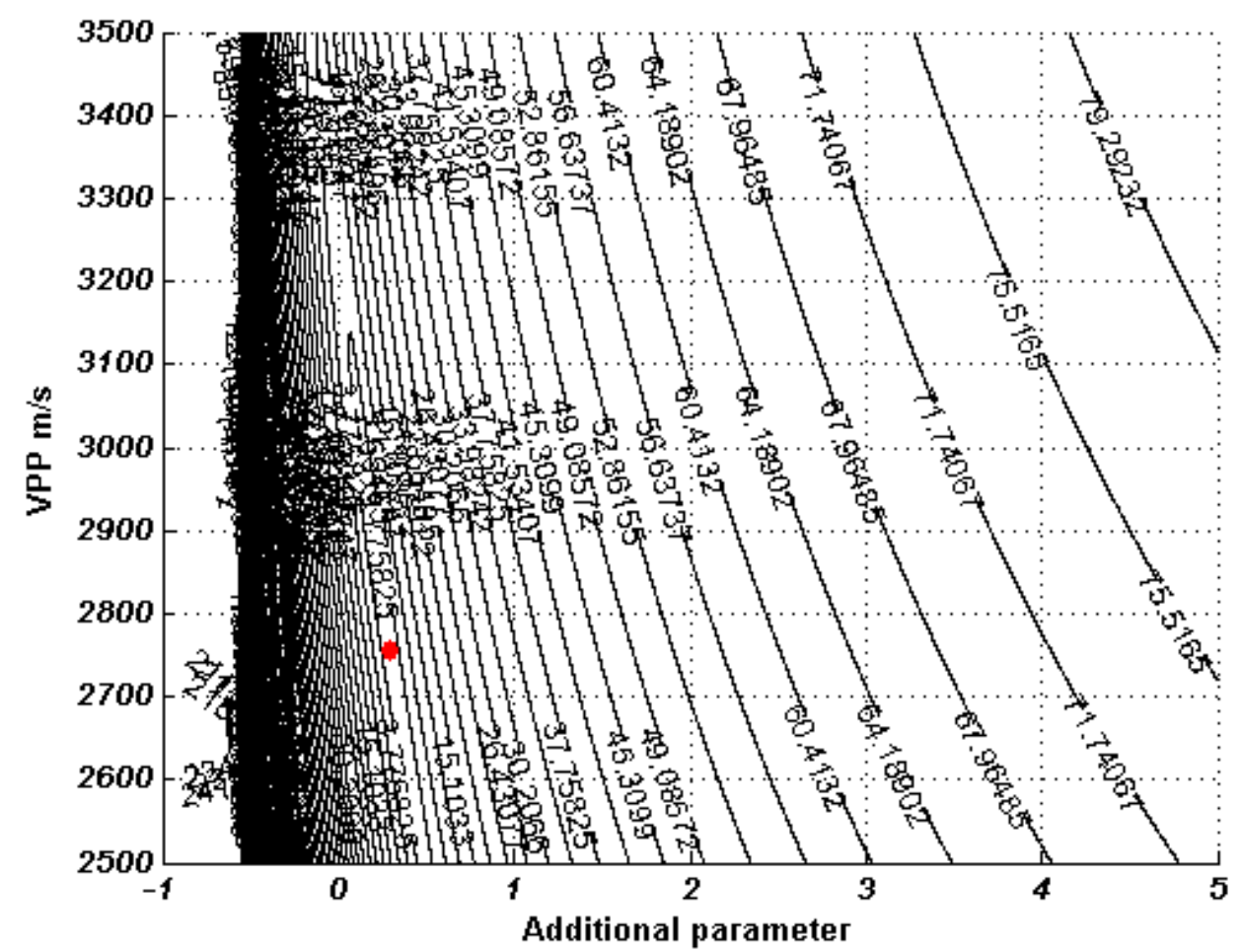

Figure 95: The residual function map which demonstrates the complexity of the Alkhalifah and Tsvankin (1995) approximation of the PP reflection event with L1-norm. Red dispersions represent the global minimum region, and the blue dispersions, the local minimum regions.

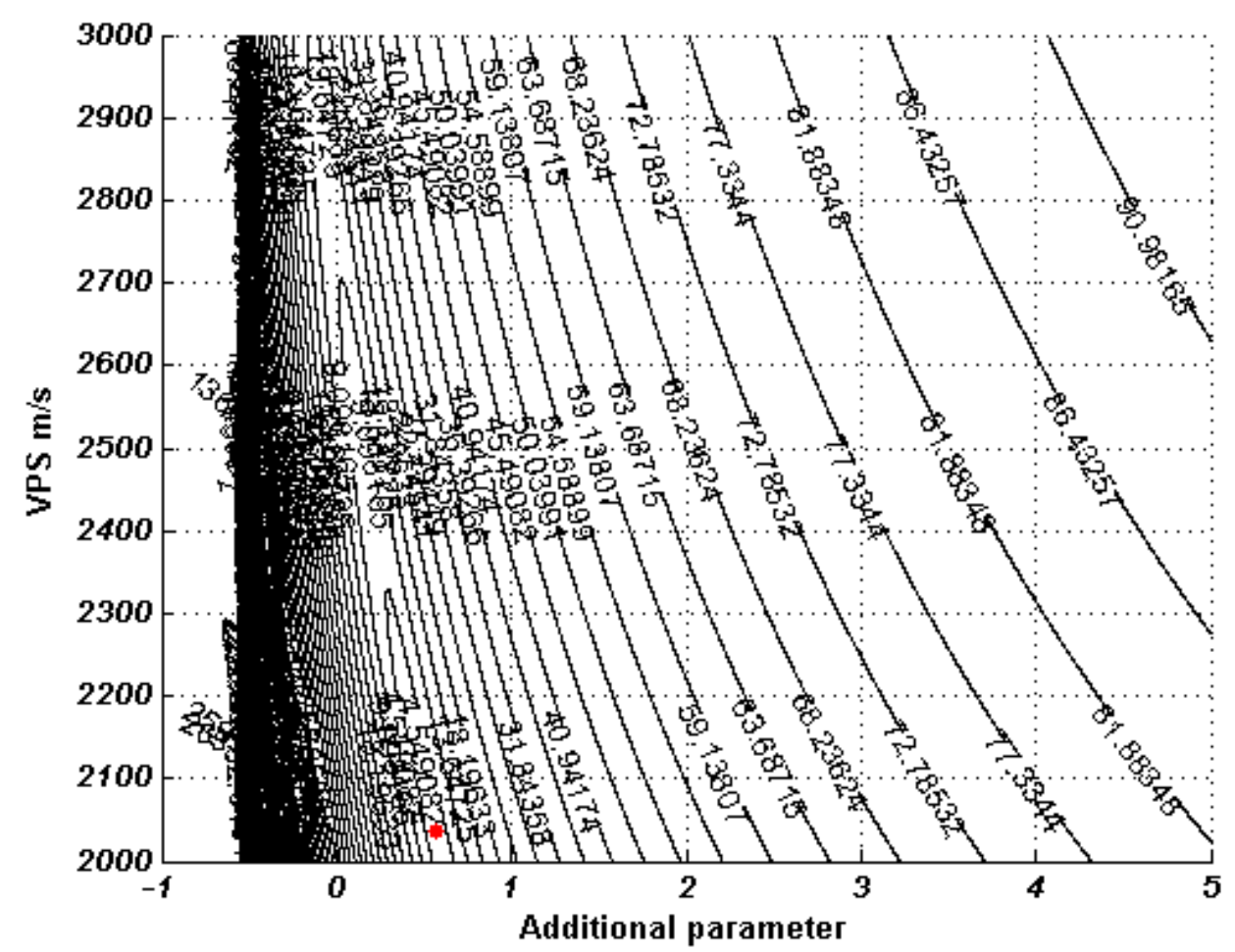

Figure 96: The residual function map which demonstrates the complexity of the Alkhalifah and Tsvankin (1995) approximation of the PS reflection event with L1-norm. Red dispersions represent the global minimum region, and the blue dispersions, the local minimum regions. 


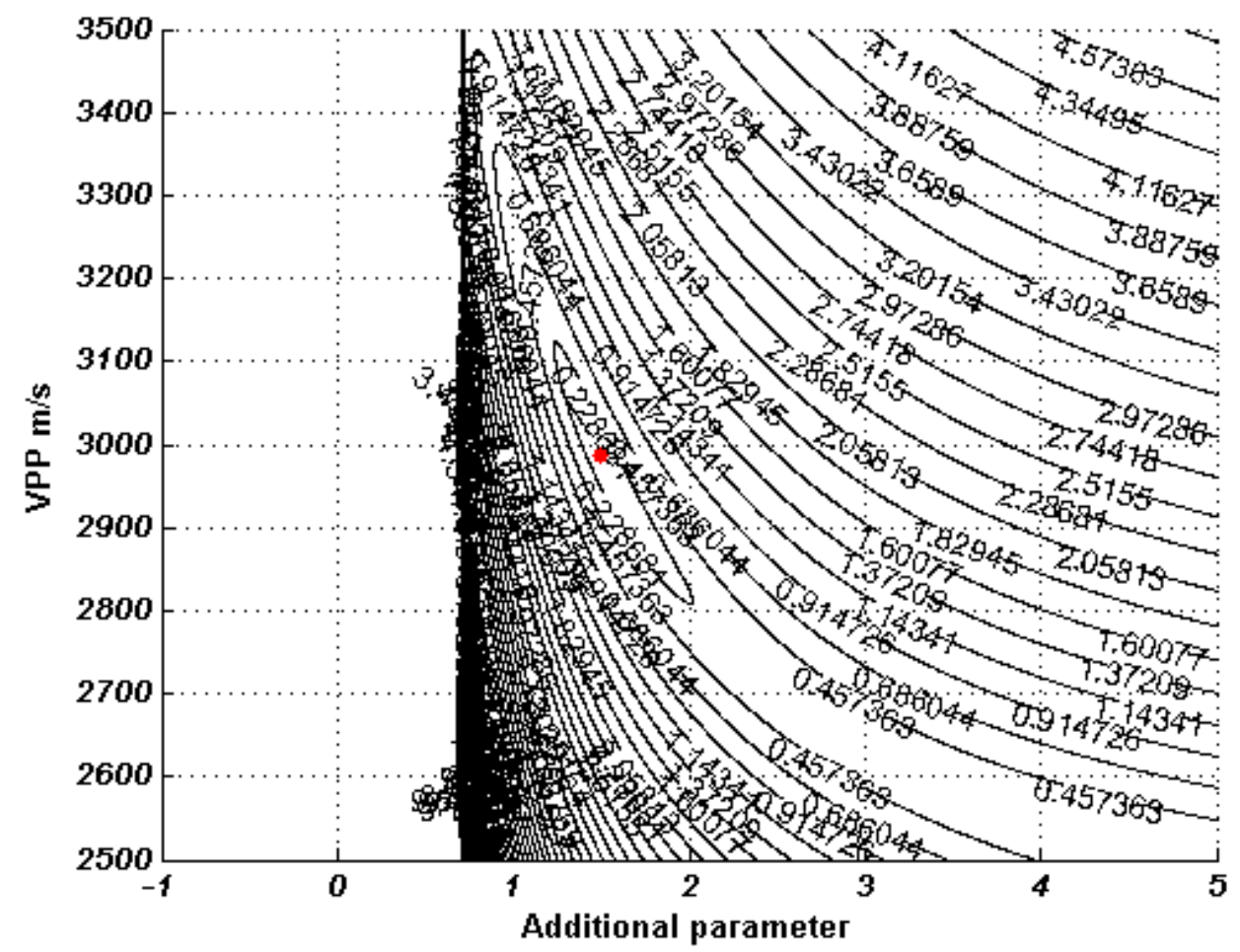

Figure 97: The residual function map which demonstrates the complexity of the Ursin and Stovas (2006) approximation of the PP reflection event with L2-norm. Red dispersions represent the global minimum region, and the blue dispersions, the local minimum regions.

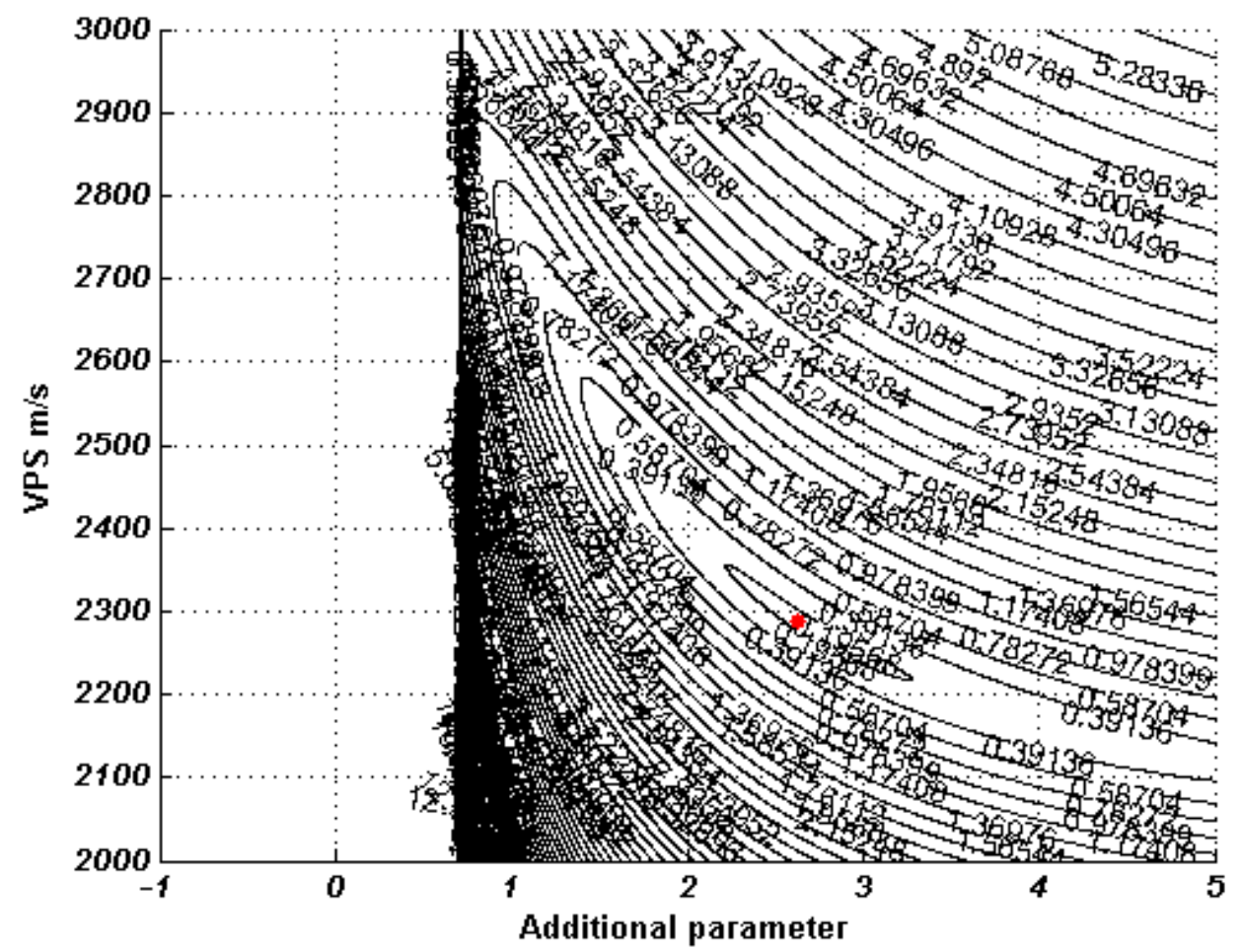

Figure 98: The residual function map which demonstrates the complexity of the Ursin and Stovas (2006) approximation of the PS reflection event with L2-norm. Red dispersions represent the global minimum region, and the blue dispersions, the local minimum regions. 


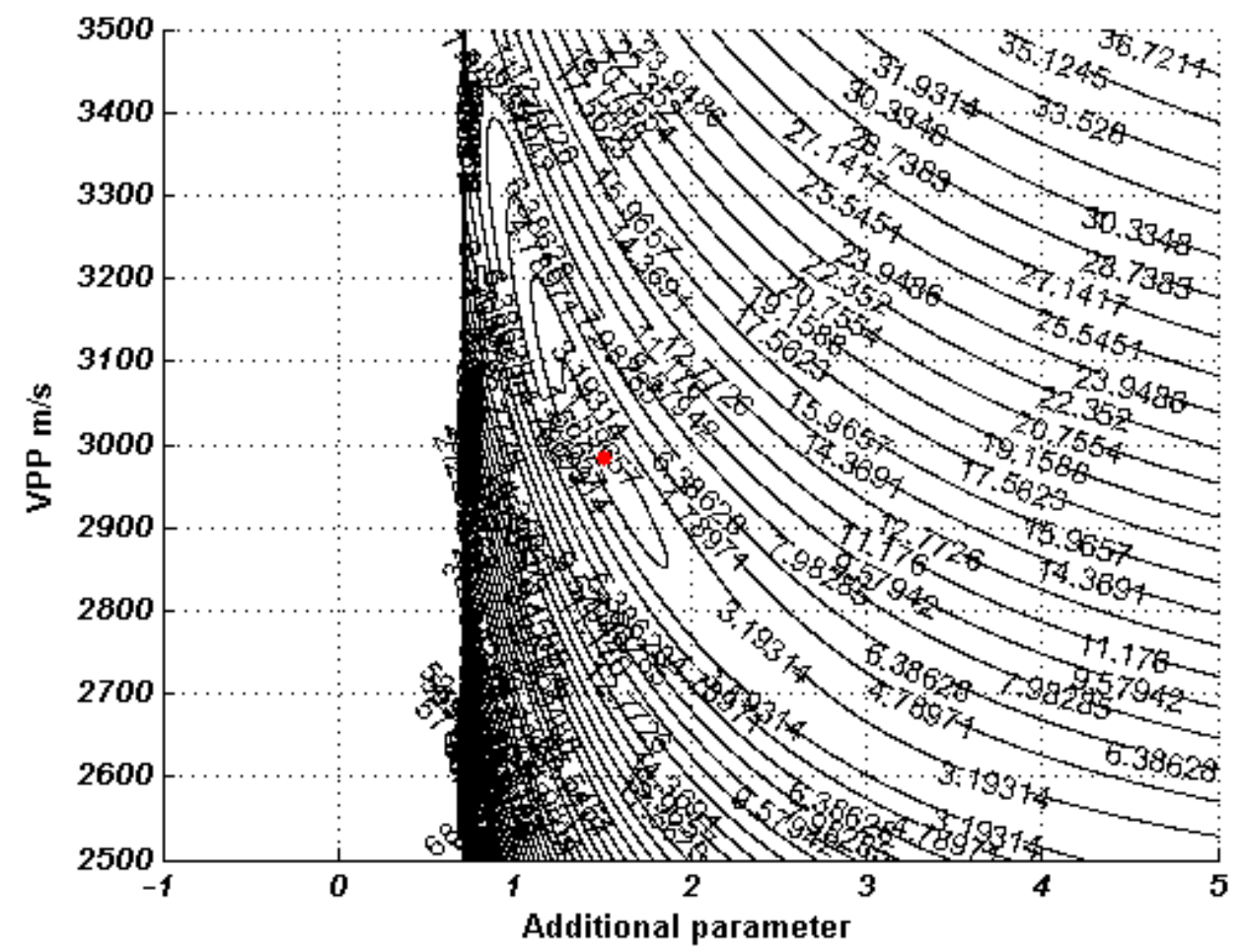

Figure 99: The residual function map which demonstrates the complexity of the Ursin and Stovas (2006) approximation of the PP reflection event with L1-norm. Red dispersions represent the global minimum region, and the blue dispersions, the local minimum regions.

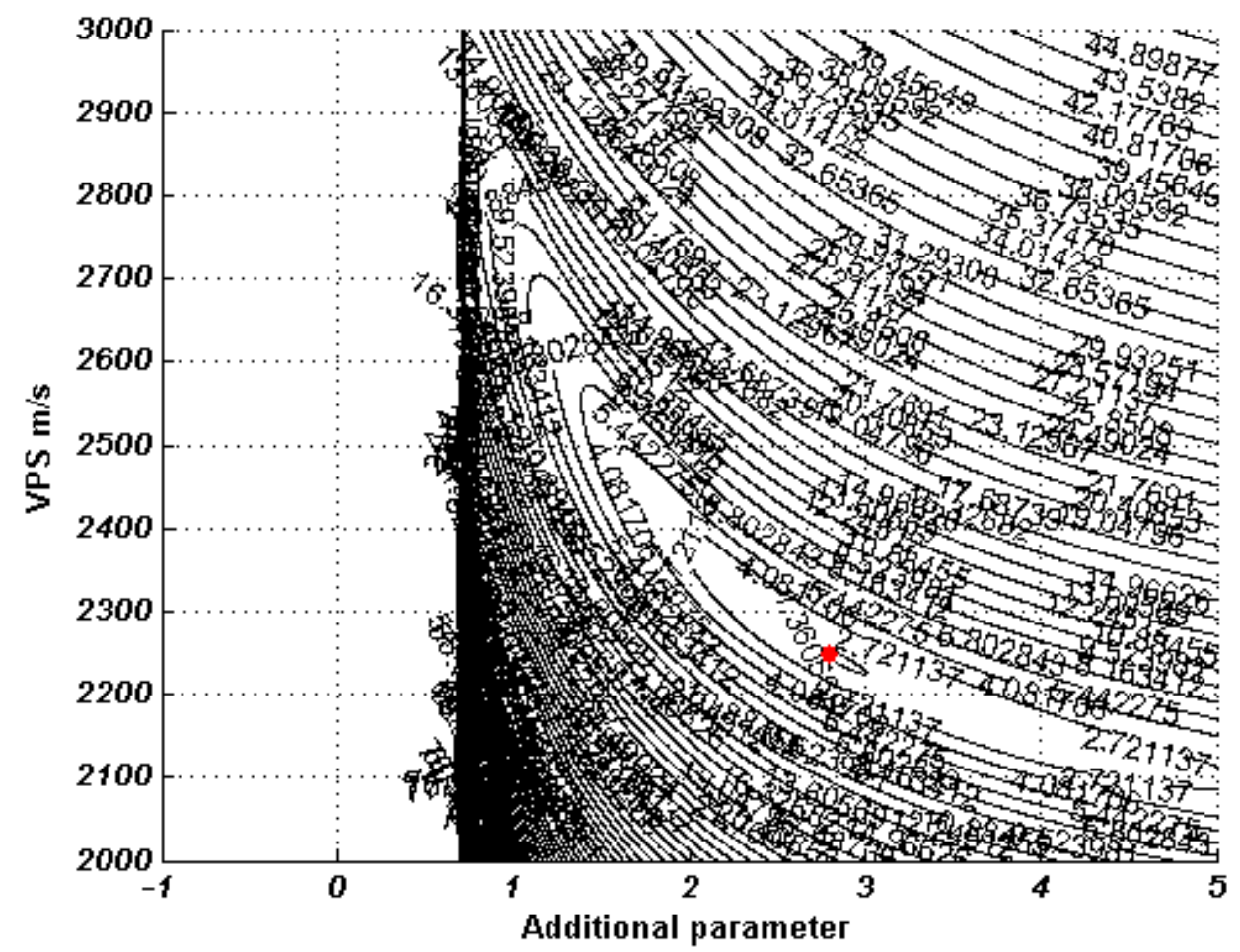

Figure 100: The residual function map which demonstrates the complexity of the Ursin and Stovas (2006) approximation of the PS reflection event with L1-norm. Red dispersions represent the global minimum region, and the blue dispersions, the local minimum regions. 


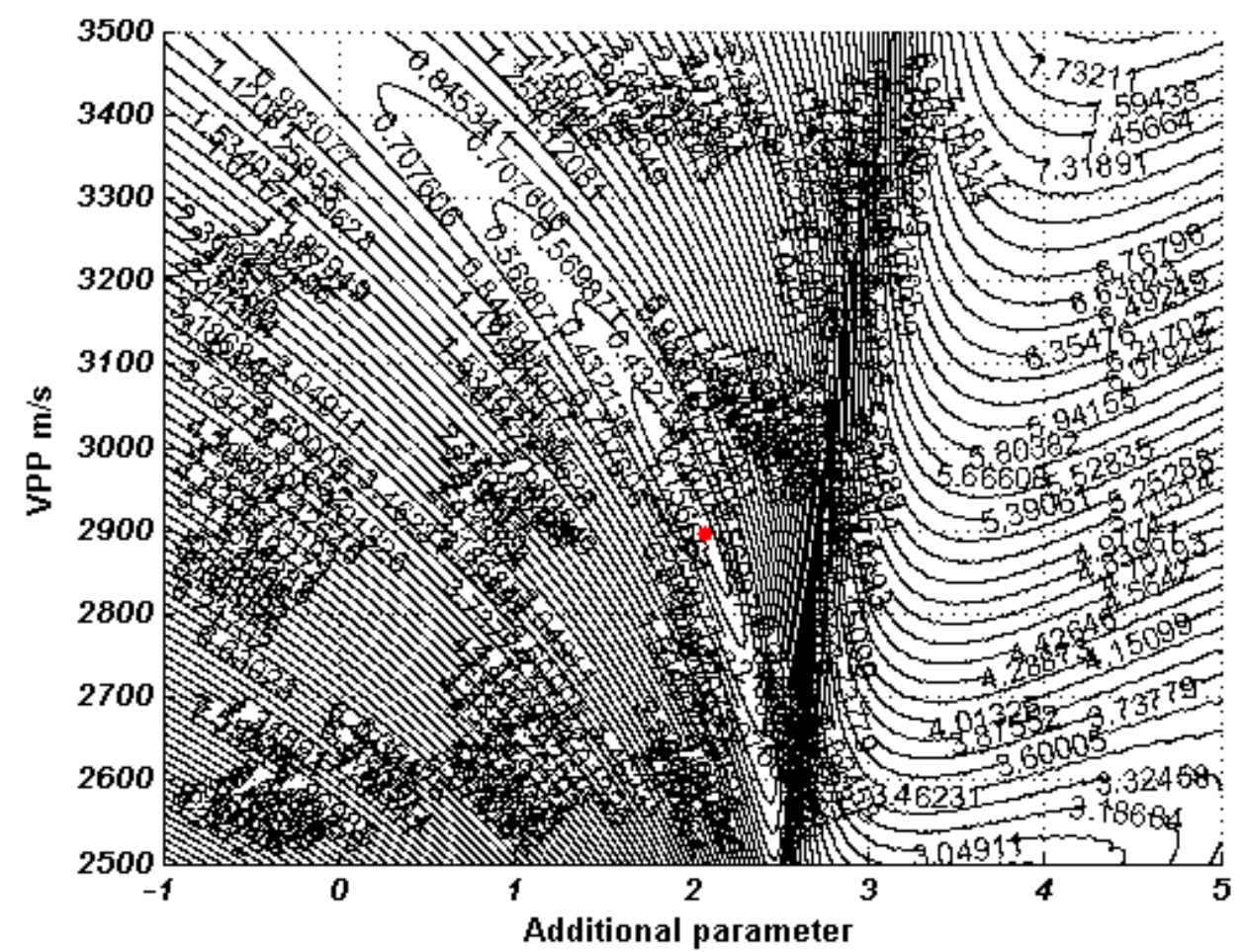

Figure 101: The residual function map which demonstrates the complexity of the Blias (2009) approximation of the PP reflection event with L2-norm. Red dispersions represent the global minimum region, and the blue dispersions, the local minimum regions.

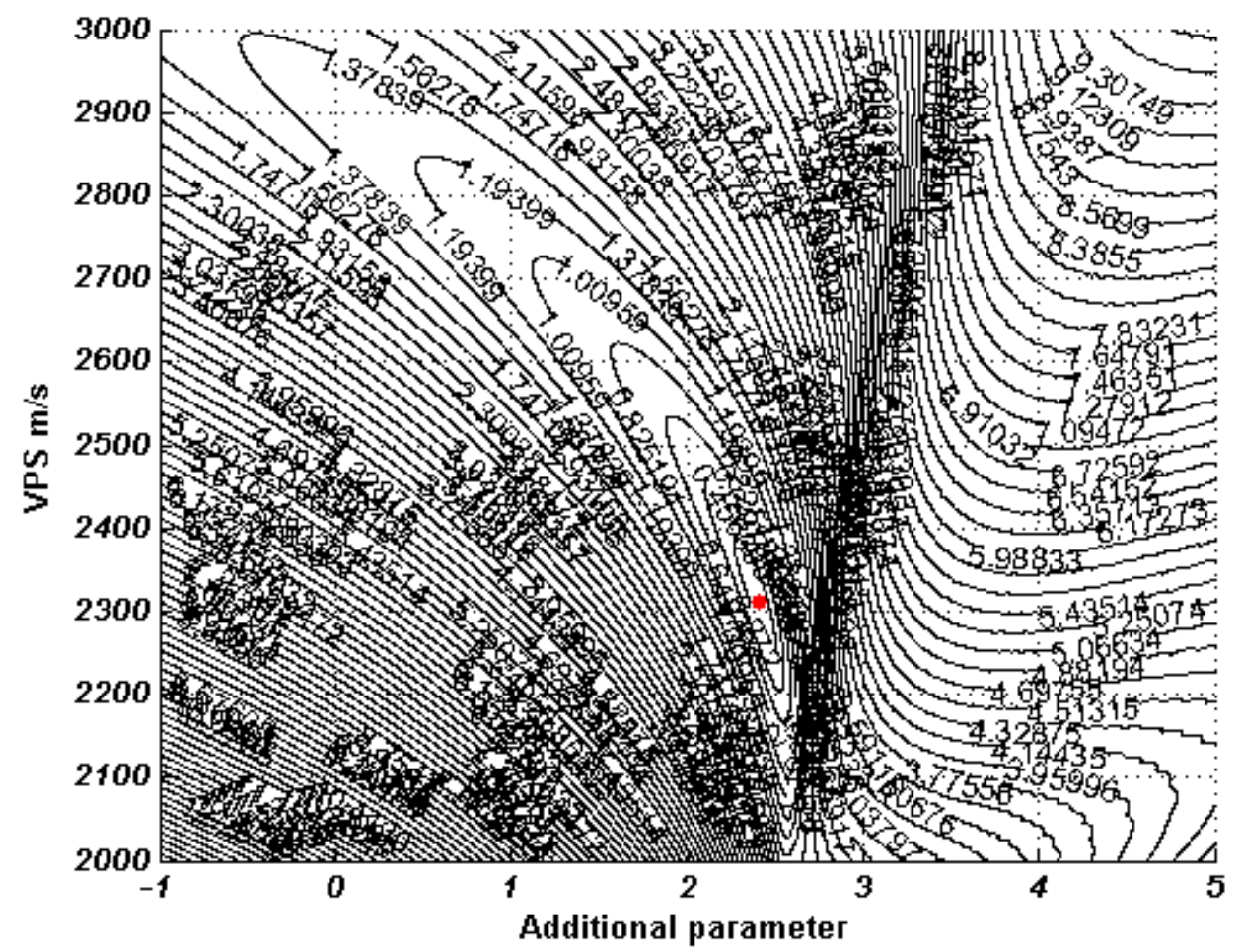

Figure 102: The residual function map which demonstrates the complexity of the Blias (2009) approximation of the PS reflection event with L2-norm. Red dispersions represent the global minimum region, and the blue dispersions, the local minimum regions. 


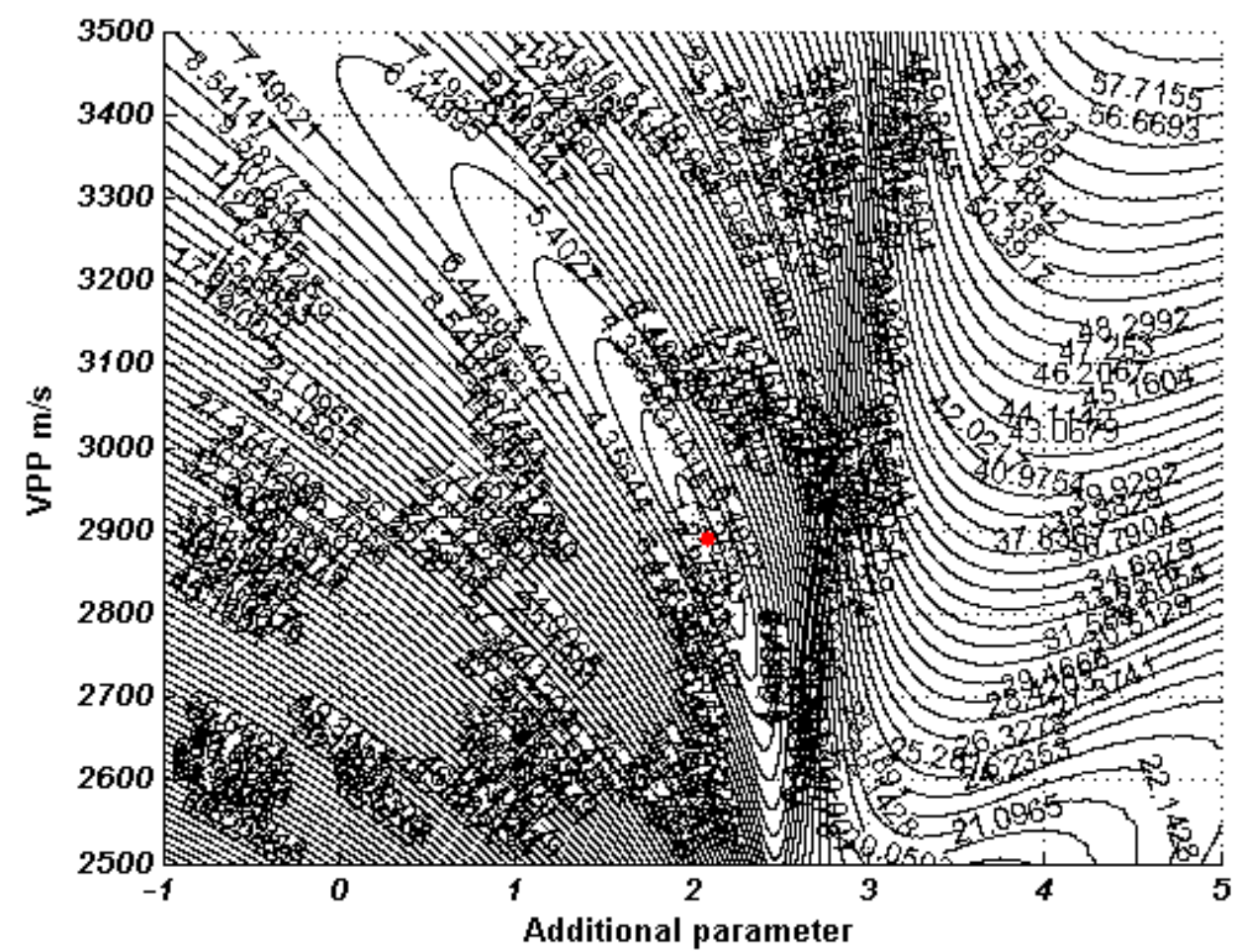

Figure 103: The residual function map which demonstrates the complexity of the Blias (2009) approximation of the PP reflection event with L1-norm. Red dispersions represent the global minimum region, and the blue dispersions, the local minimum regions.

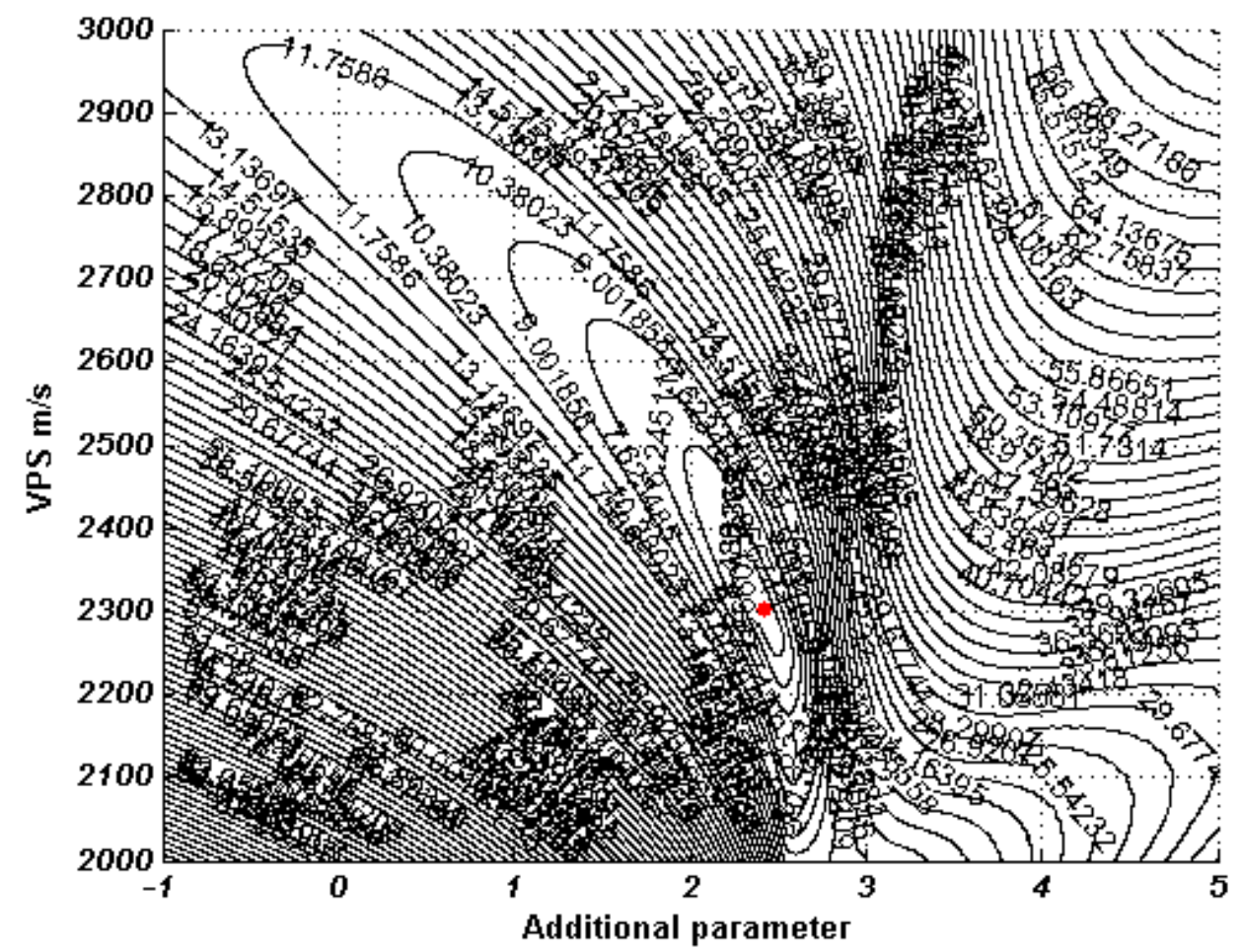

Figure 104: The residual function map which demonstrates the complexity of the Blias (2009) approximation of the PS reflection event with L1-norm. Red dispersions represent the global minimum region, and the blue dispersions, the local minimum regions. 


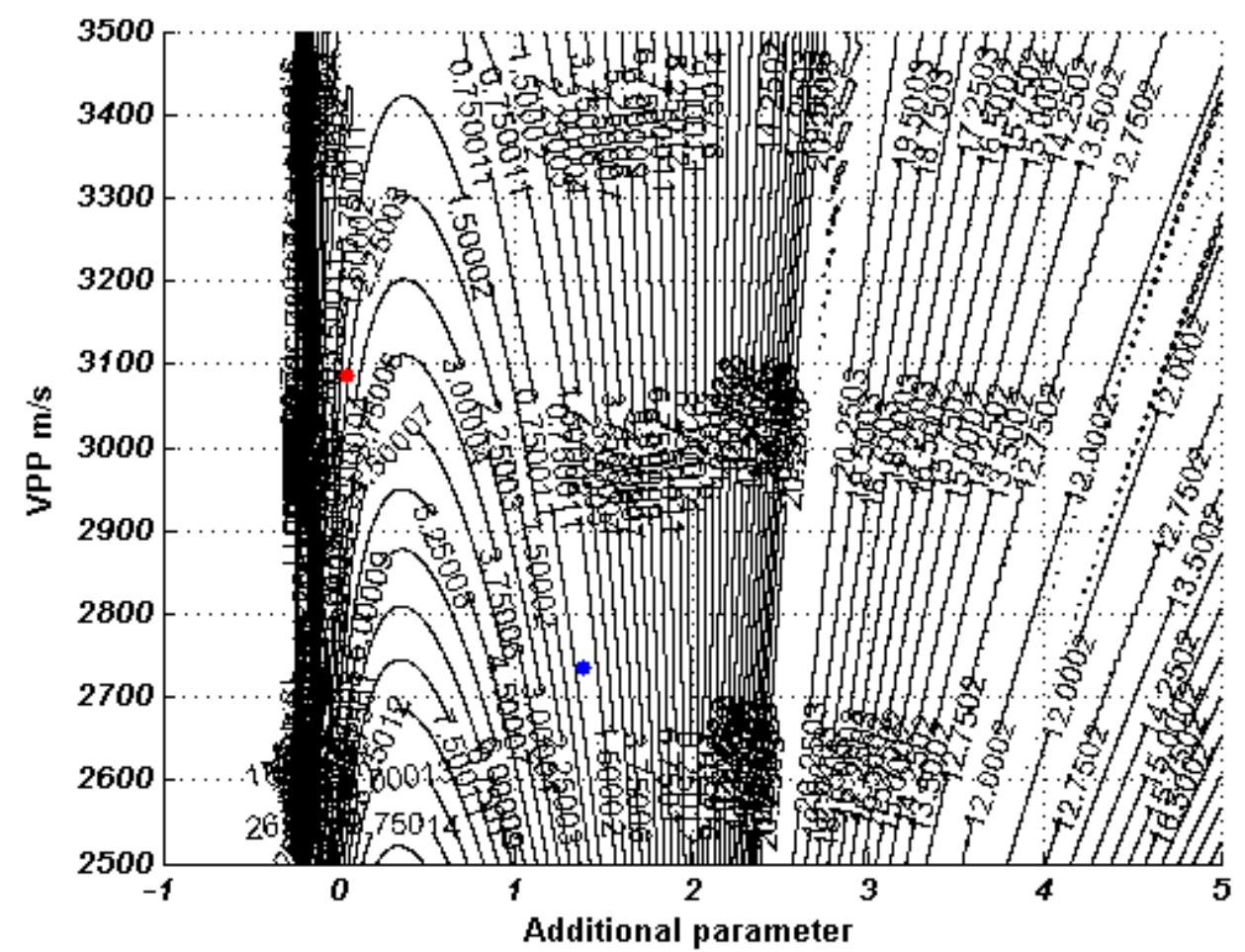

Figure 105: The residual function map which demonstrates the complexity of the Muir and Dellinger (1985) approximation of the PP reflection event with L2-norm. Red dispersions represent the global minimum region, and the blue dispersions, the local minimum regions.

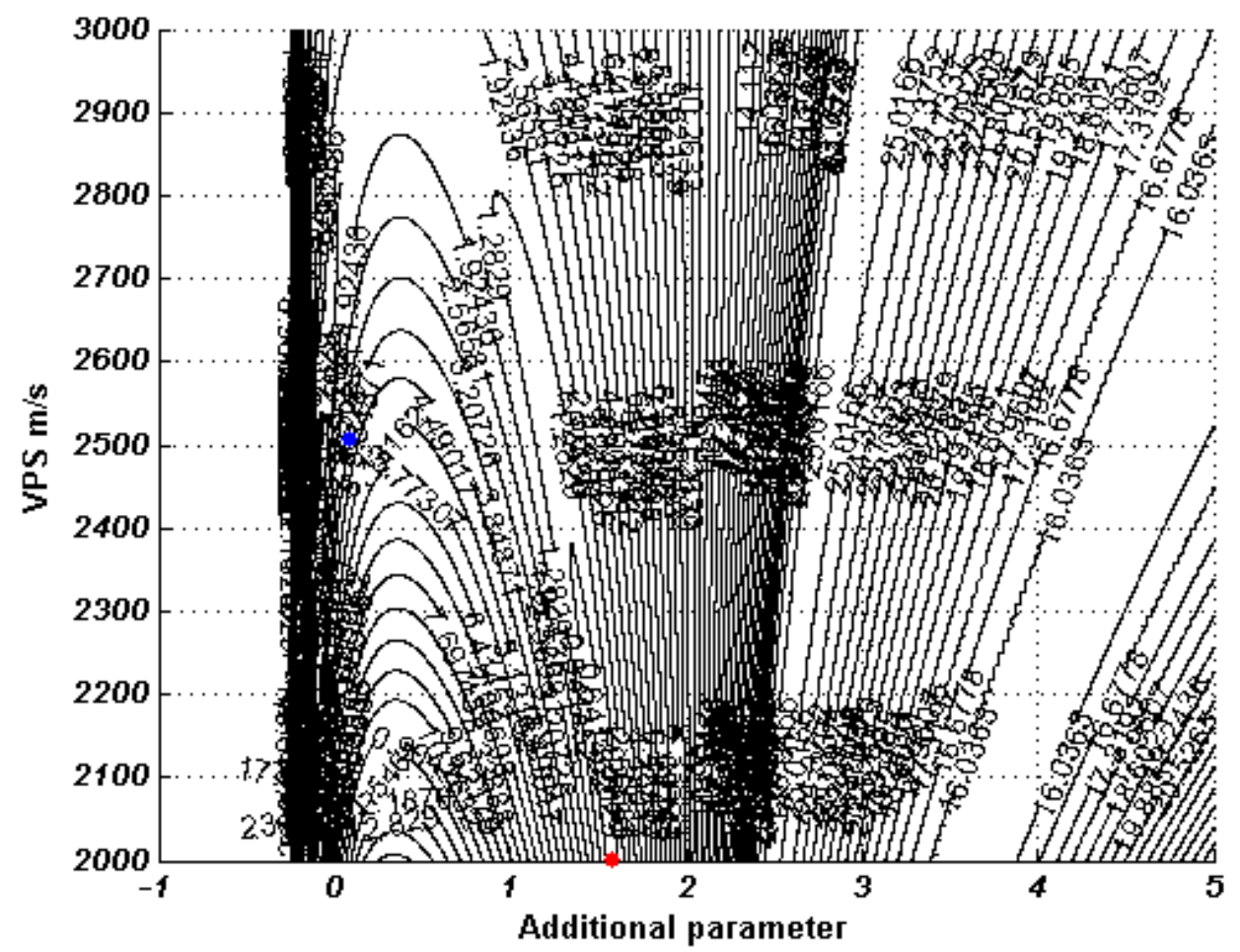

Figure 106: The residual function map which demonstrates the complexity of the Muir and Dellinger (1985) approximation of the PS reflection event with L2-norm. Red dispersions represent the global minimum region, and the blue dispersions, the local minimum regions. 


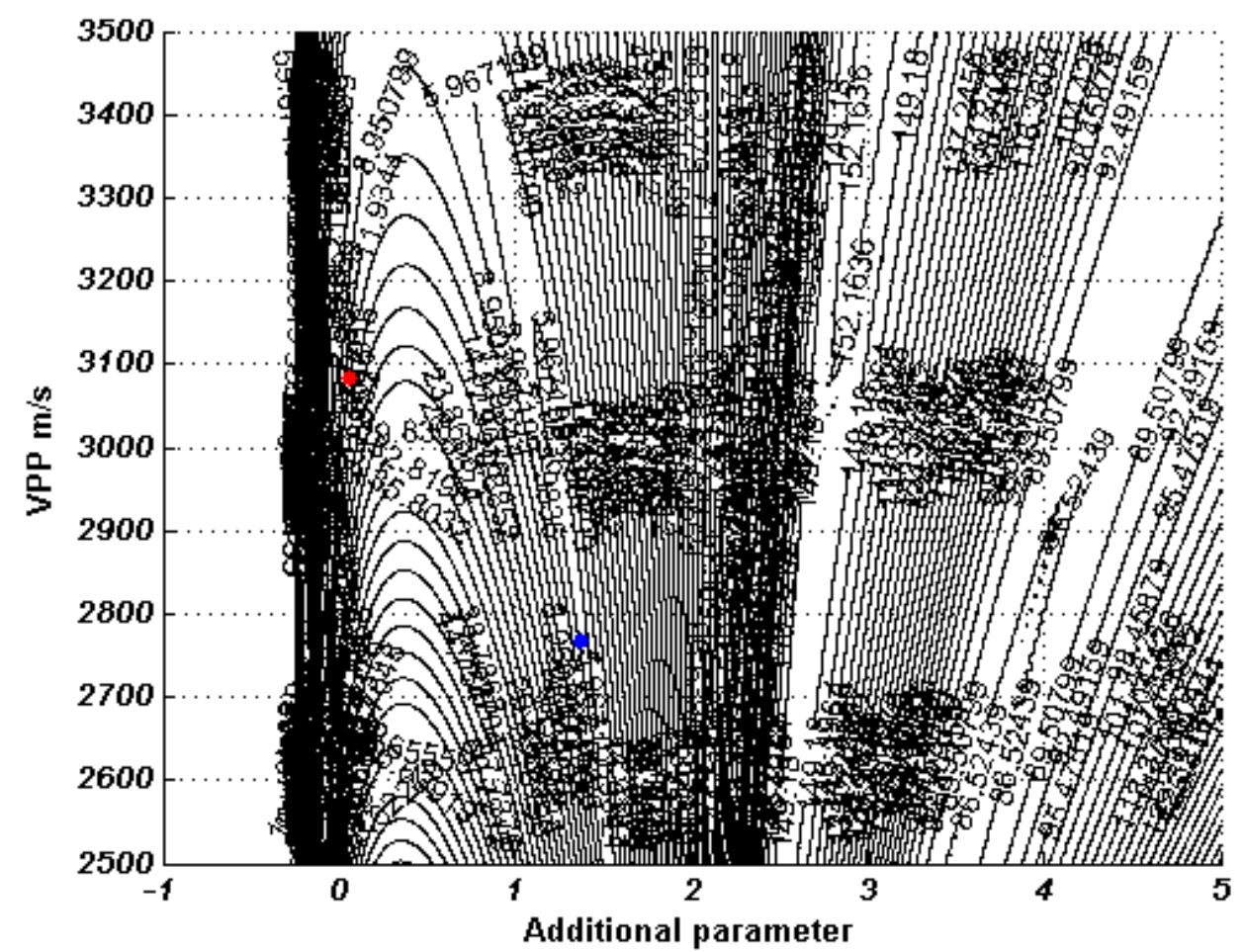

Figure 107: The residual function map which demonstrates the complexity of the Muir and Dellinger (1985) approximation of the PP reflection event with L1-norm. Red dispersions represent the global minimum region, and the blue dispersions, the local minimum regions.

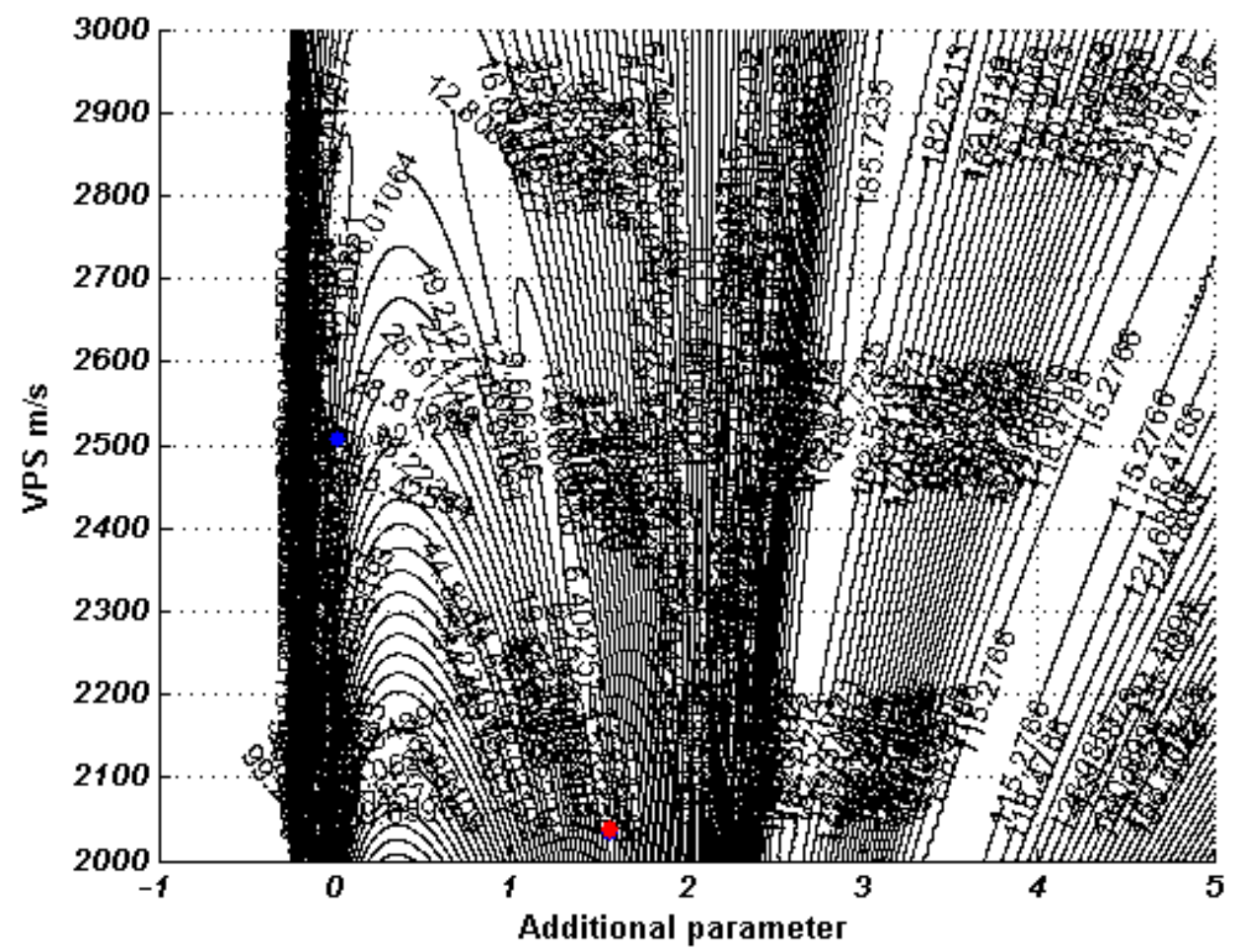

Figure 108: The residual function map which demonstrates the complexity of the Muir and Dellinger (1985) approximation of the PS reflection event with L1-norm. Red dispersions represent the global minimum region, and the blue dispersions, the local minimum regions. 


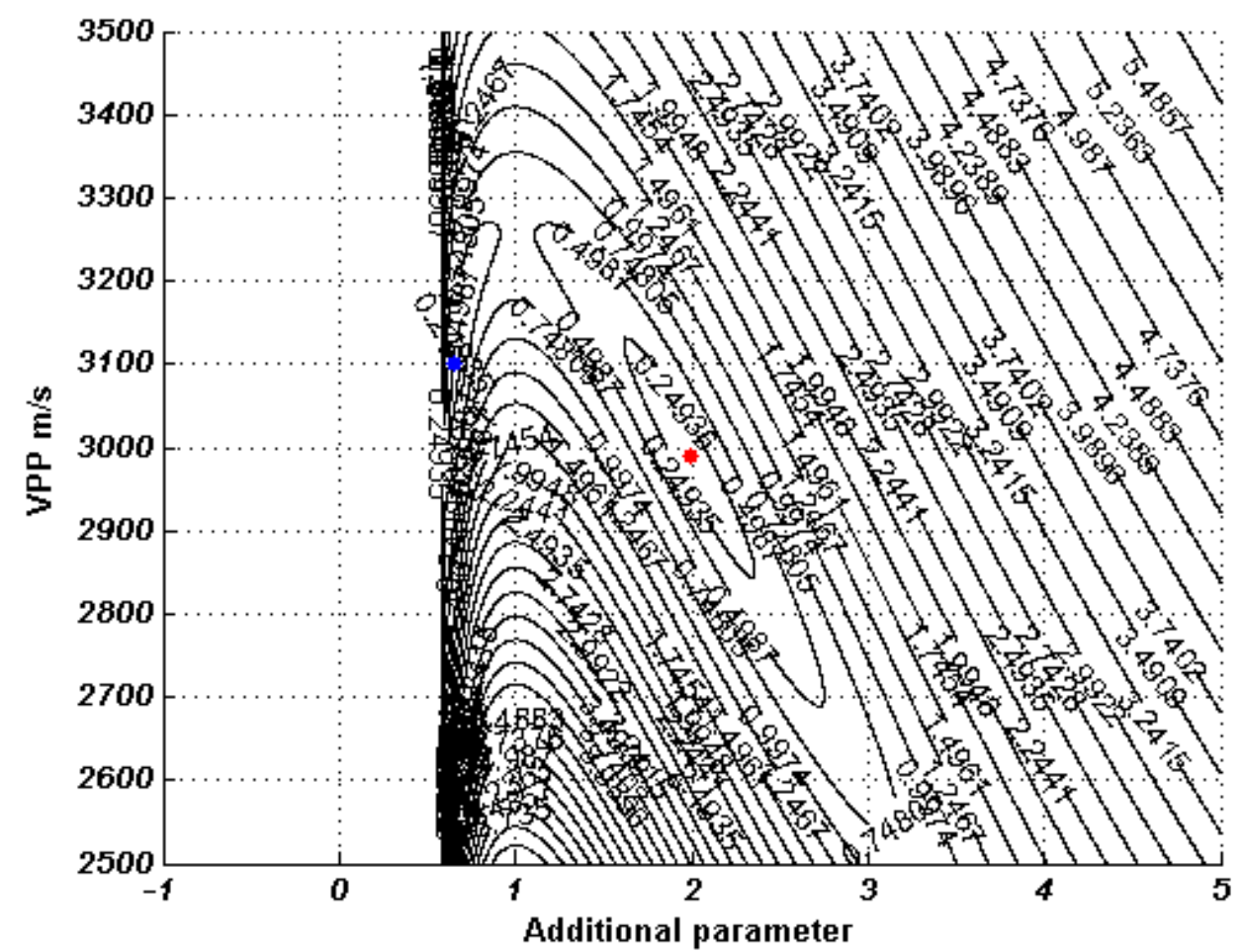

Figure 109: The residual function map which demonstrates the complexity of the Li and Yuan (2001) approximation of the PP reflection event with L2-norm. Red dispersions represent the global minimum region, and the blue dispersions, the local minimum regions.

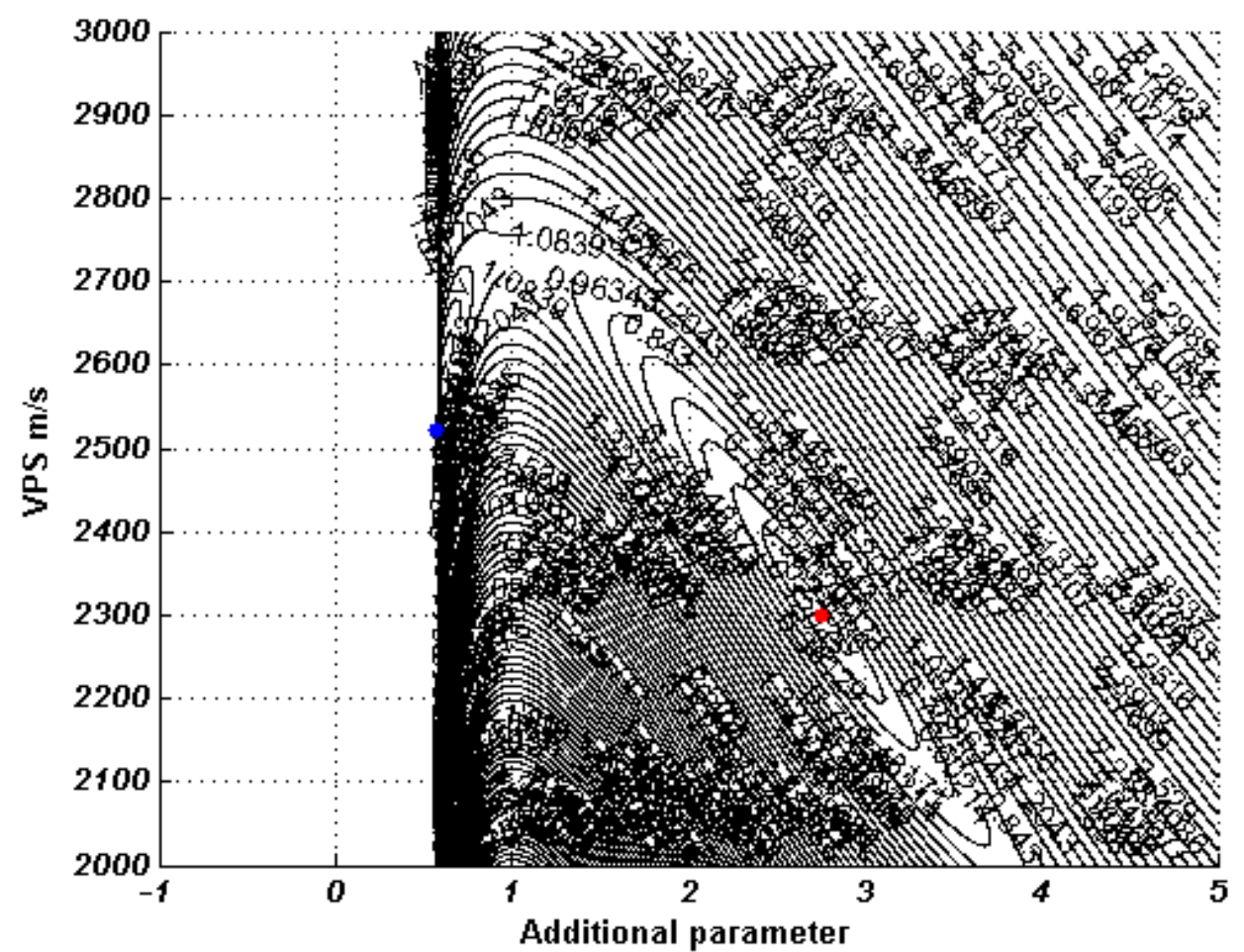

Figure 110: The residual function map which demonstrates the complexity of the Li and Yuan (2001) approximation of the PS reflection event with L2-norm. Red dispersions represent the global minimum region, and the blue dispersions, the local minimum regions. 


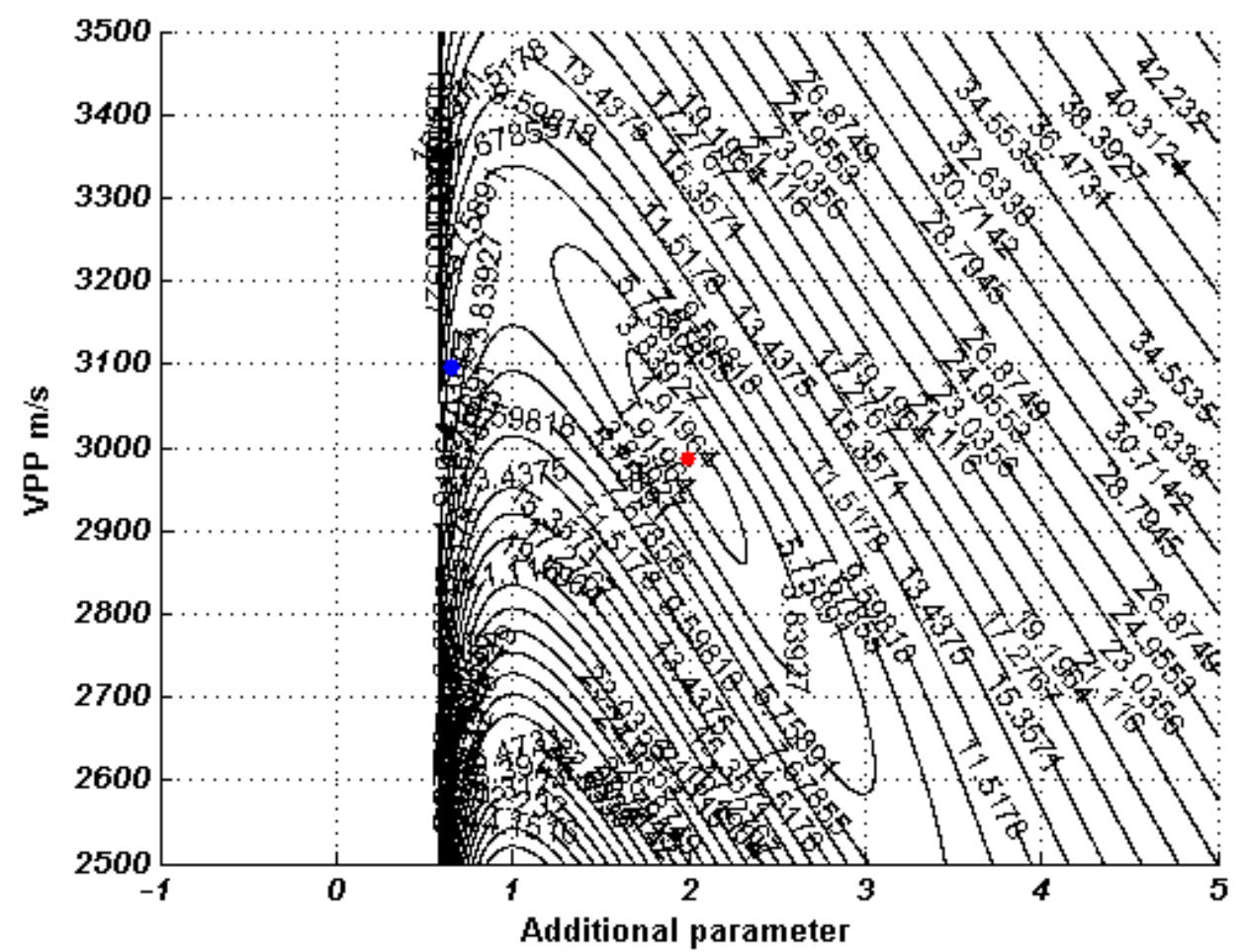

Figure 111: The residual function map which demonstrates the complexity of the Li and Yuan (2001) approximation of the PP reflection event with L1-norm. Red dispersions represent the global minimum region, and the blue dispersions, the local minimum regions.

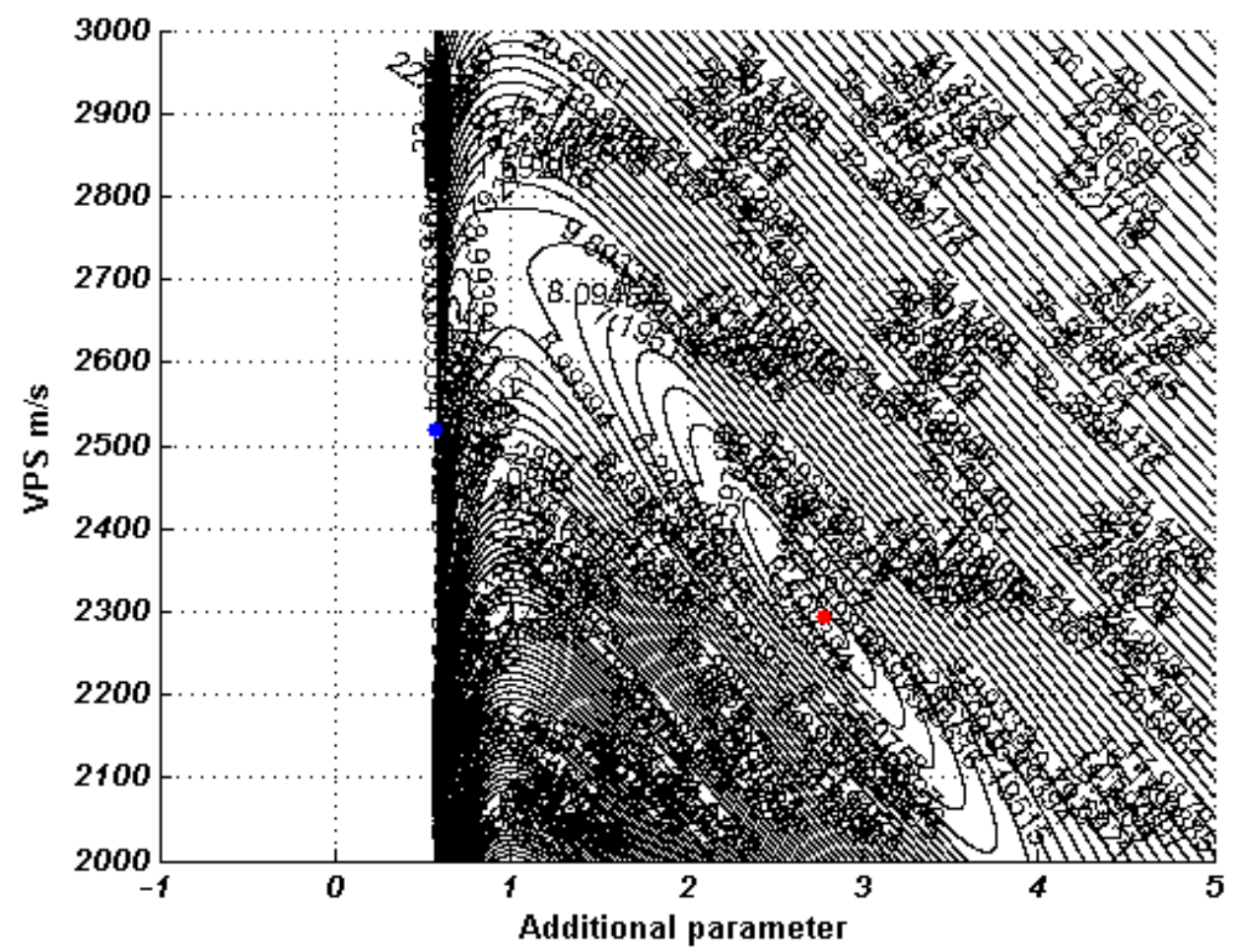

Figure 112: The residual function map which demonstrates the complexity of the Li and Yuan (2001) approximation of the PS reflection event with L1-norm. Red dispersions represent the global minimum region, and the blue dispersions, the local minimum regions. 


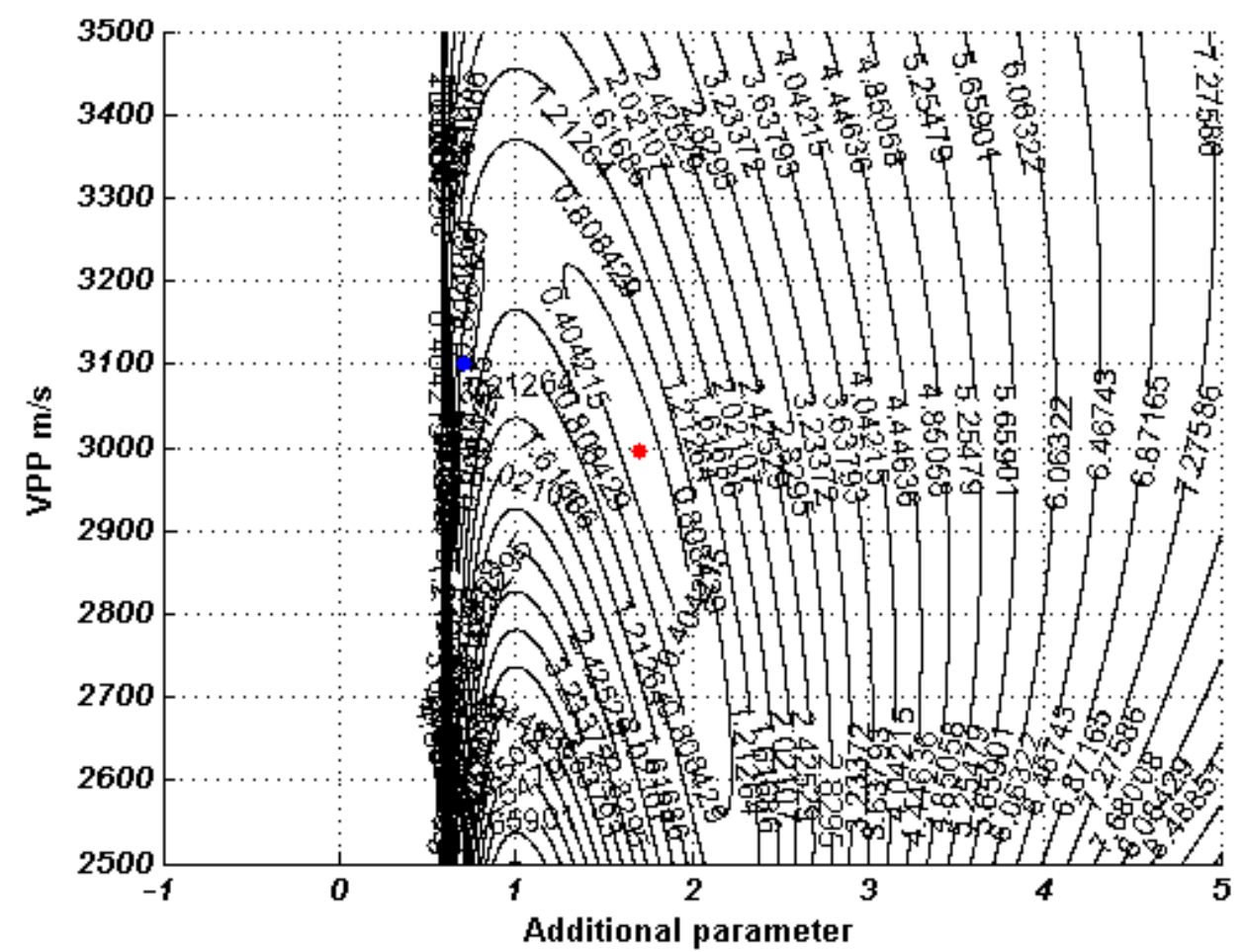

Figure 113: The residual function map which demonstrates the complexity of the approximation proposed in this work of the PP reflection event with L2-norm. Red dispersions represent the global minimum region, and the blue dispersions, the local minimum regions.

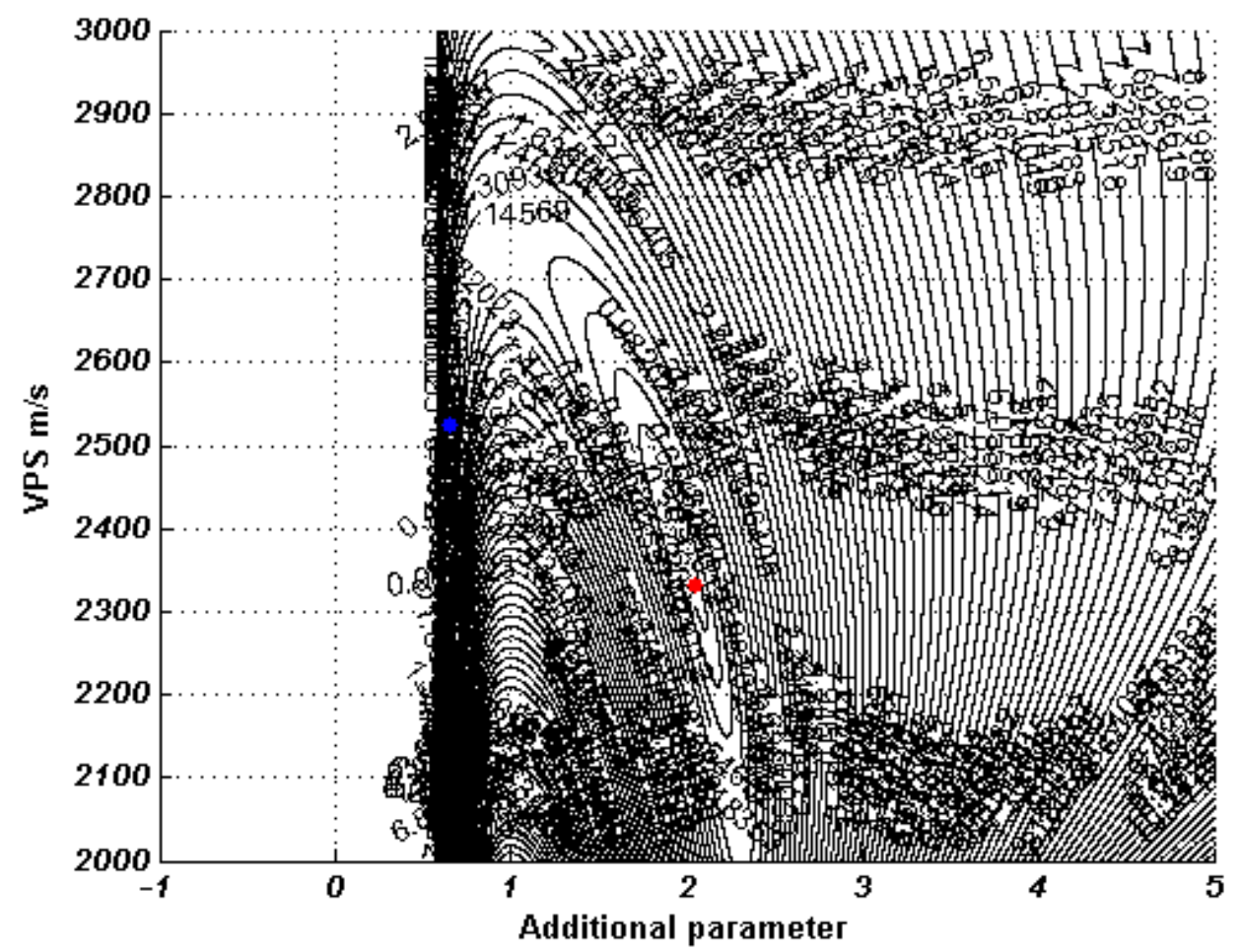

Figure 114: The residual function map which demonstrates the complexity of the approximation proposed in this work of the PS reflection event with L2-norm. Red dispersions represent the global minimum region, and the blue dispersions, the local minimum regions. 


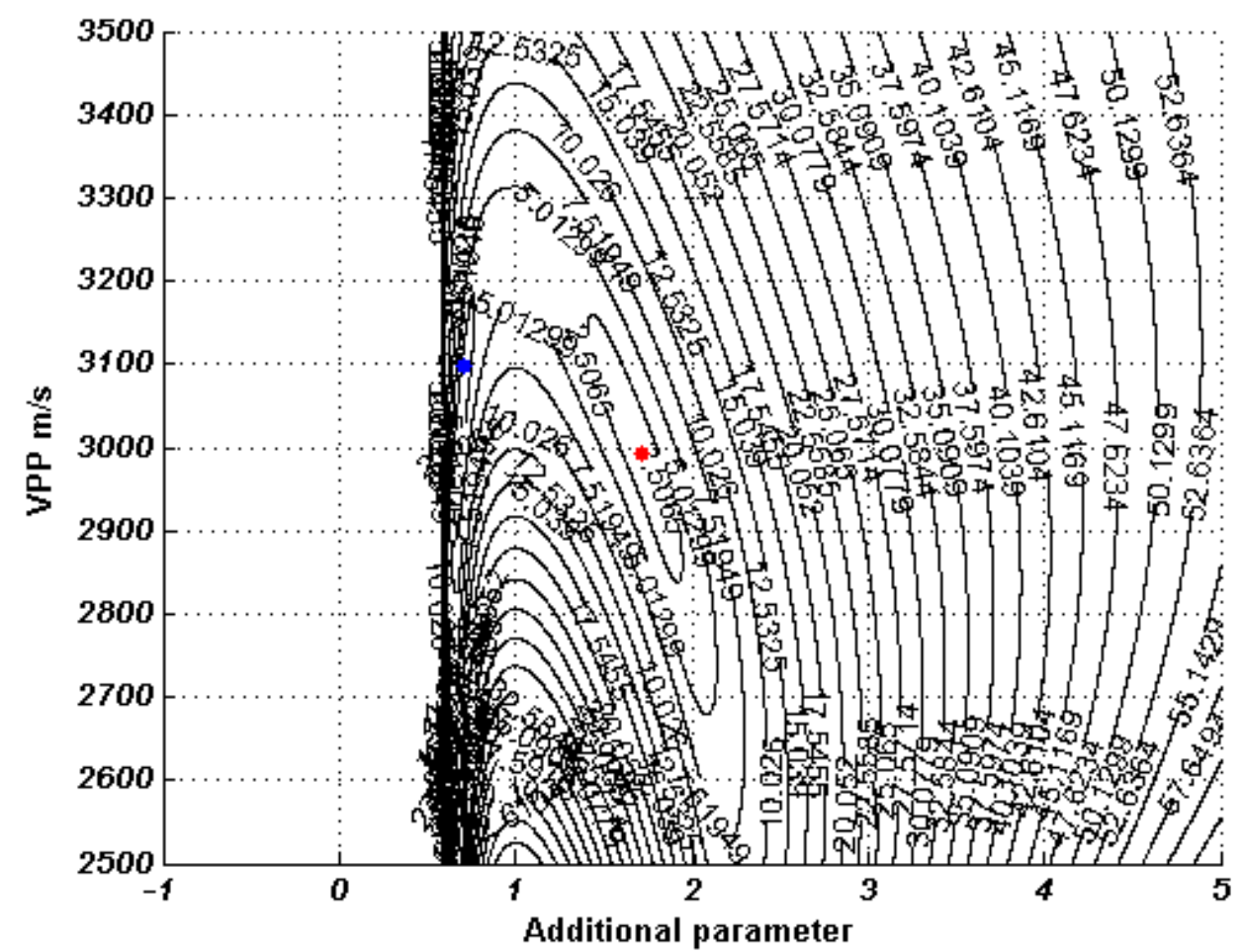

Figure 115: The residual function map which demonstrates the complexity of the approximation proposed in this work of the PP reflection event with L1-norm. Red dispersions represent the global minimum region, and the blue dispersions, the local minimum regions.

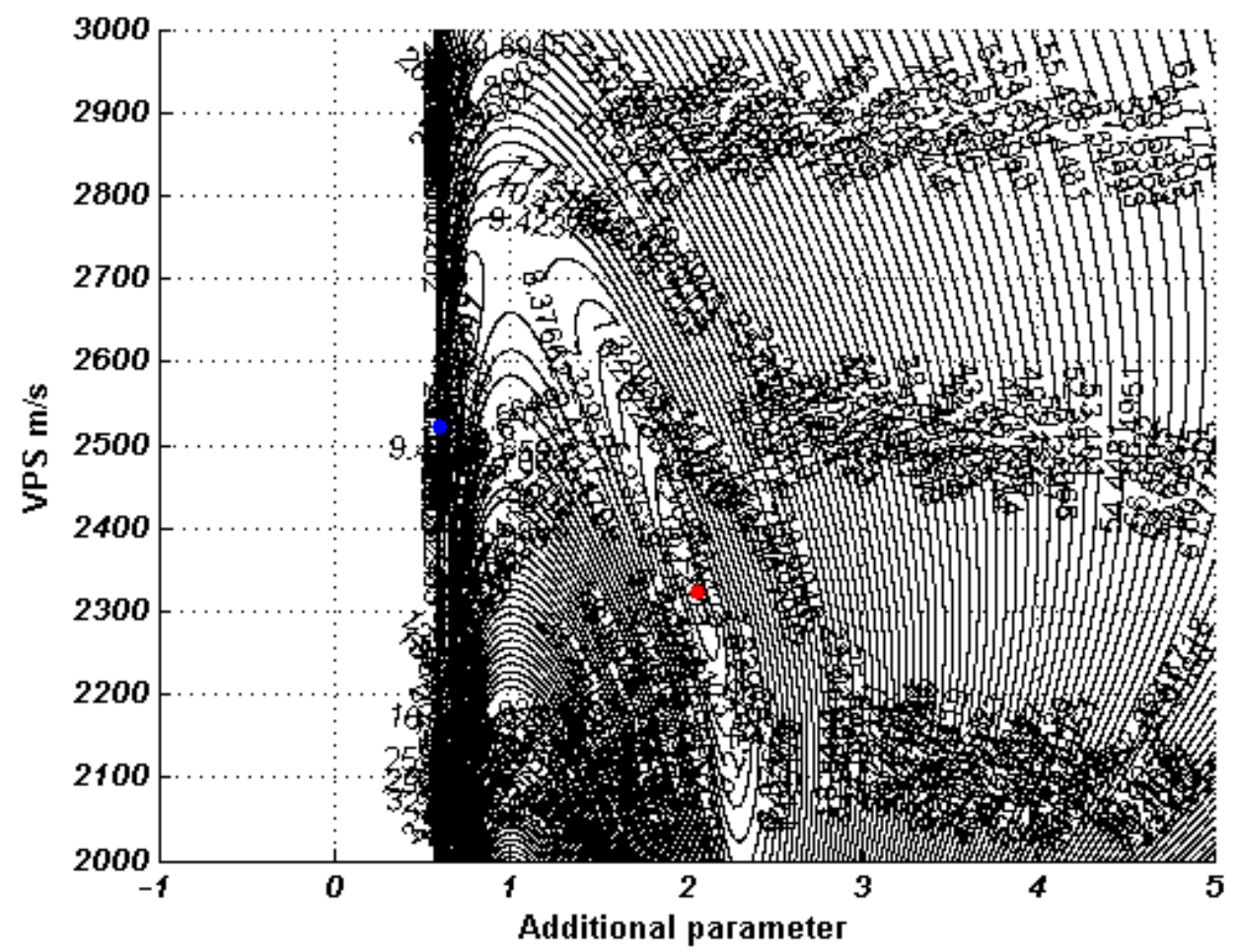

Figure 116: The residual function map which demonstrates the complexity of the approximation proposed in this work of the PS reflection event with L1-norm. Red dispersions represent the global minimum region, and the blue dispersions, the local minimum regions. 


\subsubsection{Model 4}

The fourth model is shallower than the three first ones, both in the water depth as in the rock structure where there is no complex structural characteristic. However, the acquisition geometry was maintained to force the limits of each approximation. Thus, even with a simple and shallow reservoir, the large maximum offset between source and receiver brings a significant complexity to this model. For this reason, it was possible to observe that the Malovichko (1978) approximation showed a more visible variation in the topological structure of the objective function. A more substantial variation was observed between the PP and PS events (Figures 117 and 118). However, the approximation was still unimodal and showed the same kinds of variations concerning the L2- and L1-norm (Figures 119 and 120).

The same strong variation could be seen between the PP and PS events of the Alkhalifah and Tsvankin (1995) approximation (Figures 121 and 122). However, the approximation maintained the unimodal behaviour and the variations between the L2- and L1norm (Figures 123 and 124).

In this case, the approximation proposed by Ursin and Stovas (2006) showed to be multimodal, differently than the three previous models. Furthermore, it showed a strong variation between the PP and PS events (Figures 125 and 126), similarly to the other previous approximations for this model. However, the most significant variation was concerning the one between the L2- and L1-norm, where the approximation changed its behaviour from multimodal to unimodal (Figures 127 and 128).

The same magnitude and kind of variations could be seen in this model for the approximation proposed by Blias (2009), which also had an important variation between the two norms (Figures 129, 130, 131 and 132).

The approximation proposed by Muir and Dellinger (1985) showed to be multimodal again. However, the variation between the two events, PP and PS, concerning the exchange in the global and local minimum region did not exist (Figures 133 and 134). Even with this difference, the variation concerning the norms was still similar to what was previously seen for this approximation (Figures 135 and 136).

The Li and Yuan (2001) approximation showed to be, again, multimodal, and showed, as the other approximations for this model, a visible variation between the PP and PS events 
(Figures 137 and 138). Concerning the variation between the norms, the same kind of variation seen before, for this approximation, is observed (Figures 139 and 140).

The proposed approximation showed similar variations and behaviour as the one proposed by Li and Yuan (2001). However, in this model, the topology of the objective function was strongly different than the topology in the first three models (Figures 141, 142, 143 and 144). And as consequence of this strong difference, the topological structure was narrower concerning the additional parameter. 


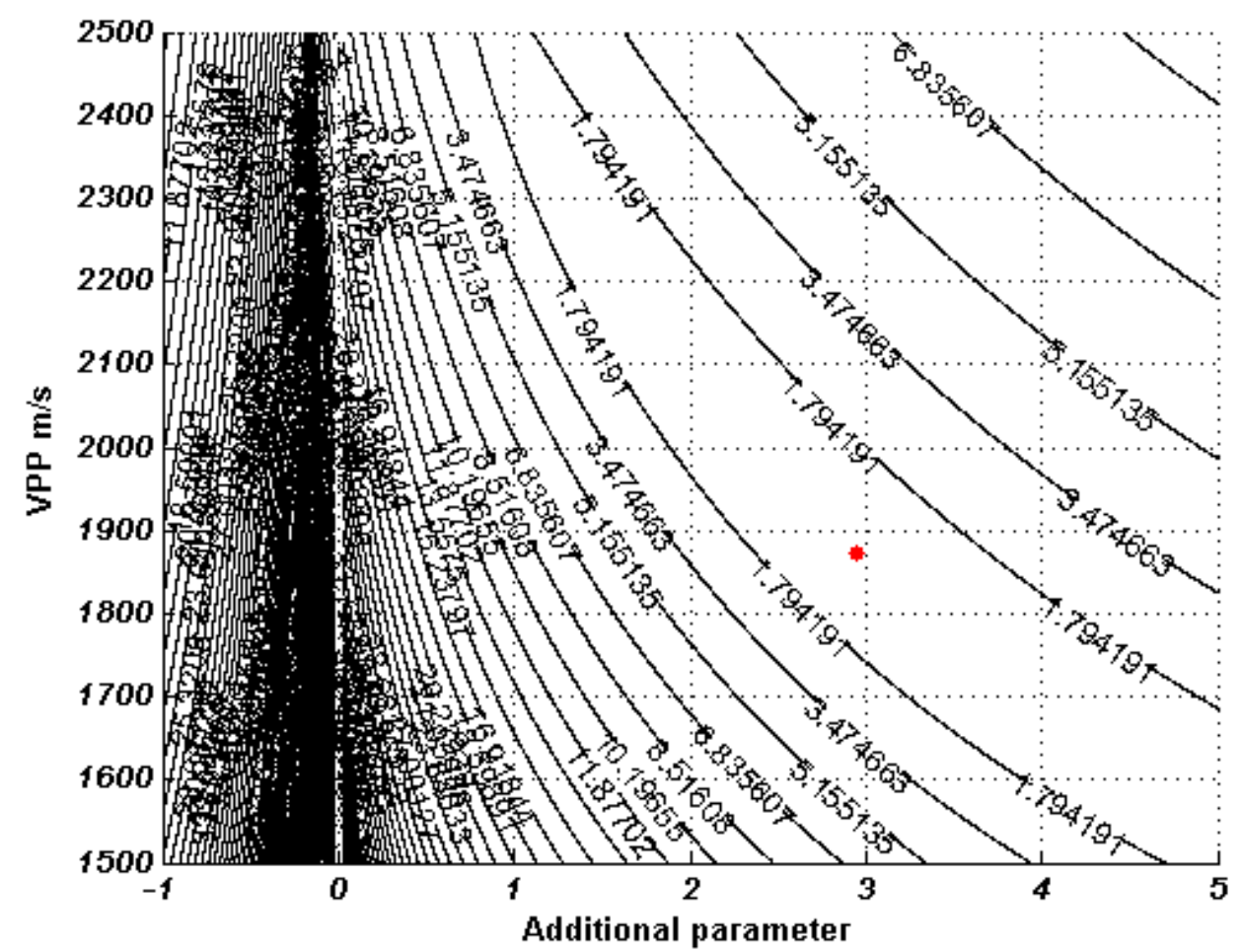

Figure 117: The residual function map which demonstrates the complexity of the Malovichko (1978) approximation of the PP reflection event with L2-norm. Red dispersions represent the global minimum region, and the blue dispersions, the local minimum regions.

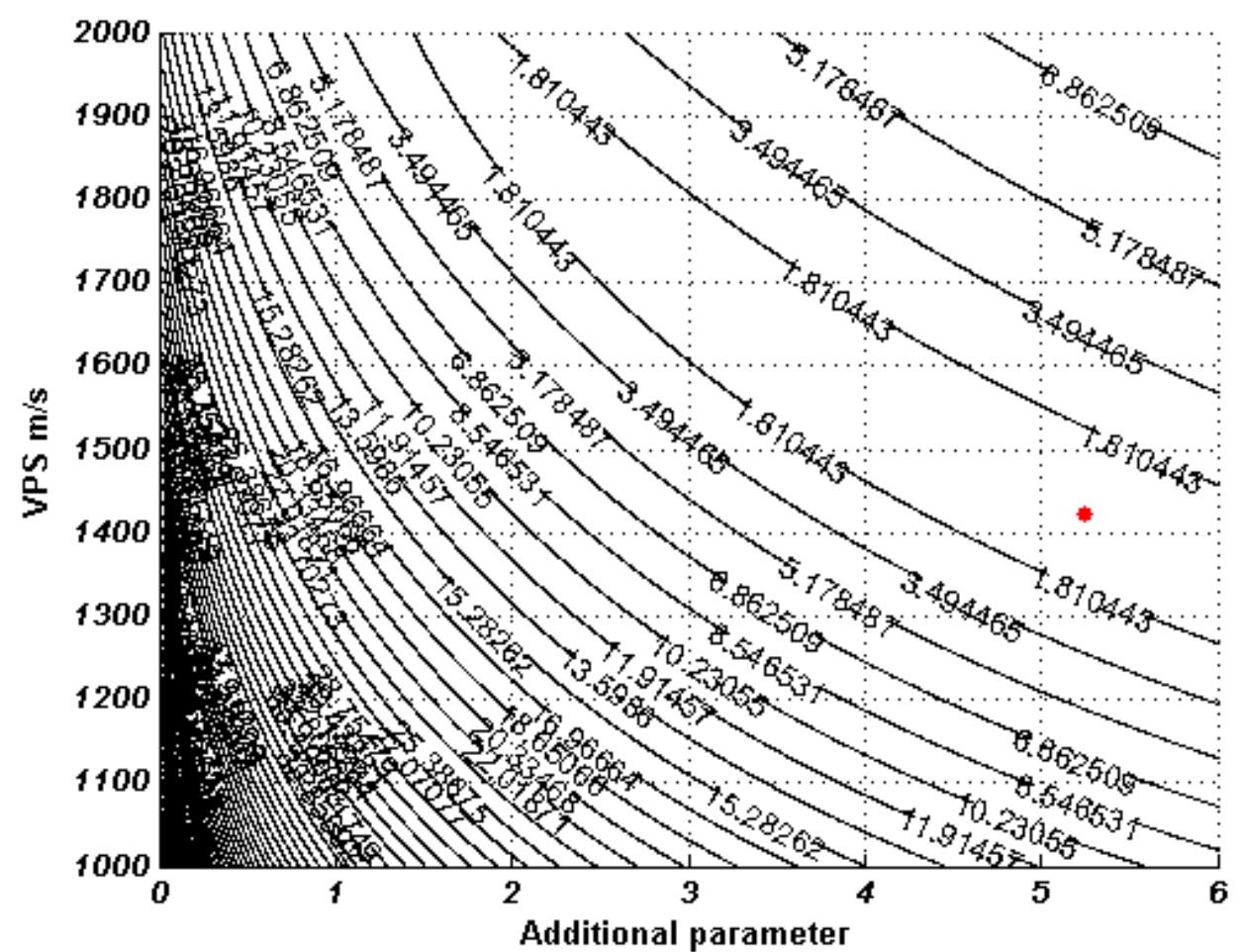

Figure 118: The residual function map which demonstrates the complexity of the Malovichko (1978) approximation of the PS reflection event with L2-norm. Red dispersions represent the global minimum region, and the blue dispersions, the local minimum regions. 


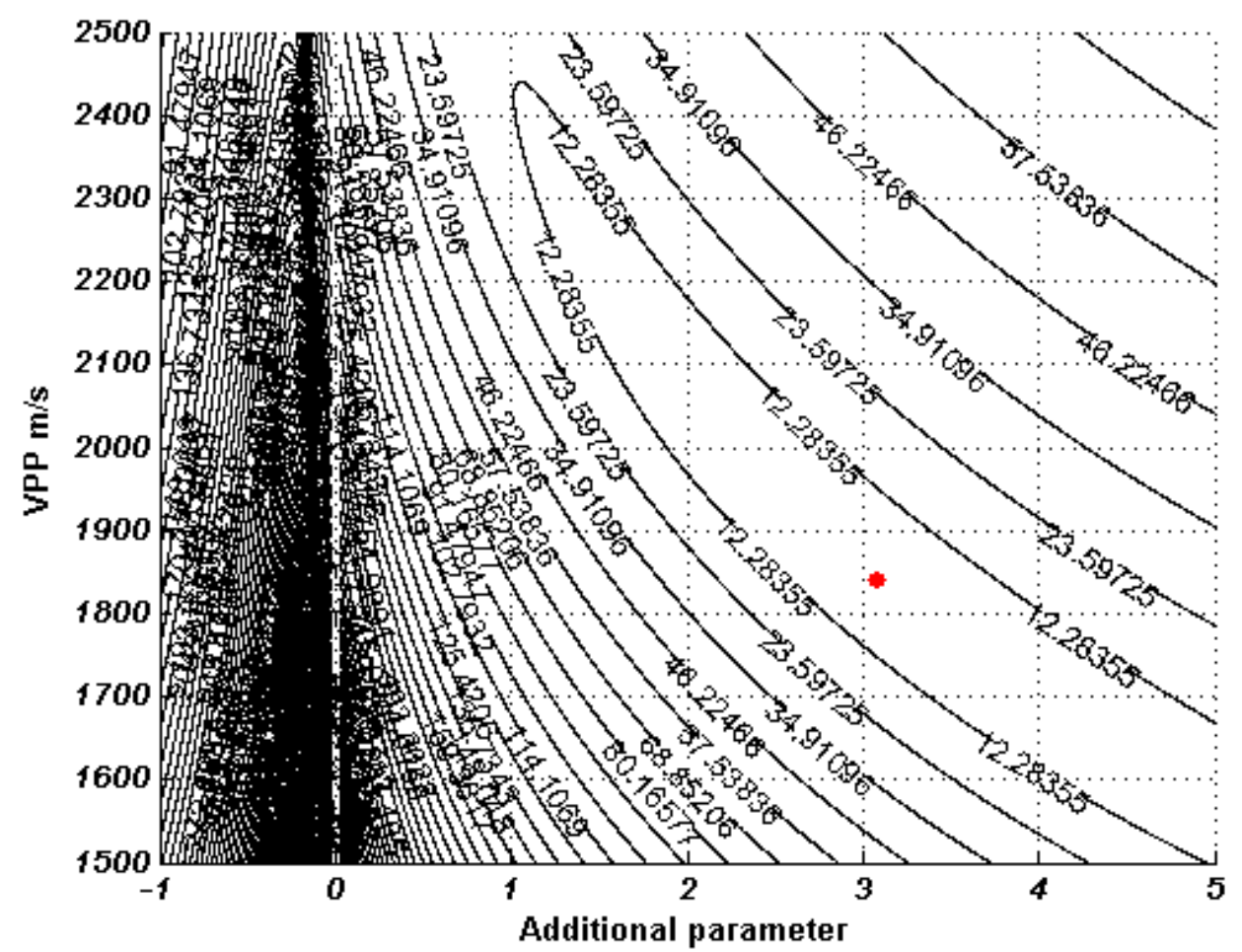

Figure 119: The residual function map which demonstrates the complexity of the Malovichko (1978) approximation of the PP reflection event with L1-norm. Red dispersions represent the global minimum region, and the blue dispersions, the local minimum regions.

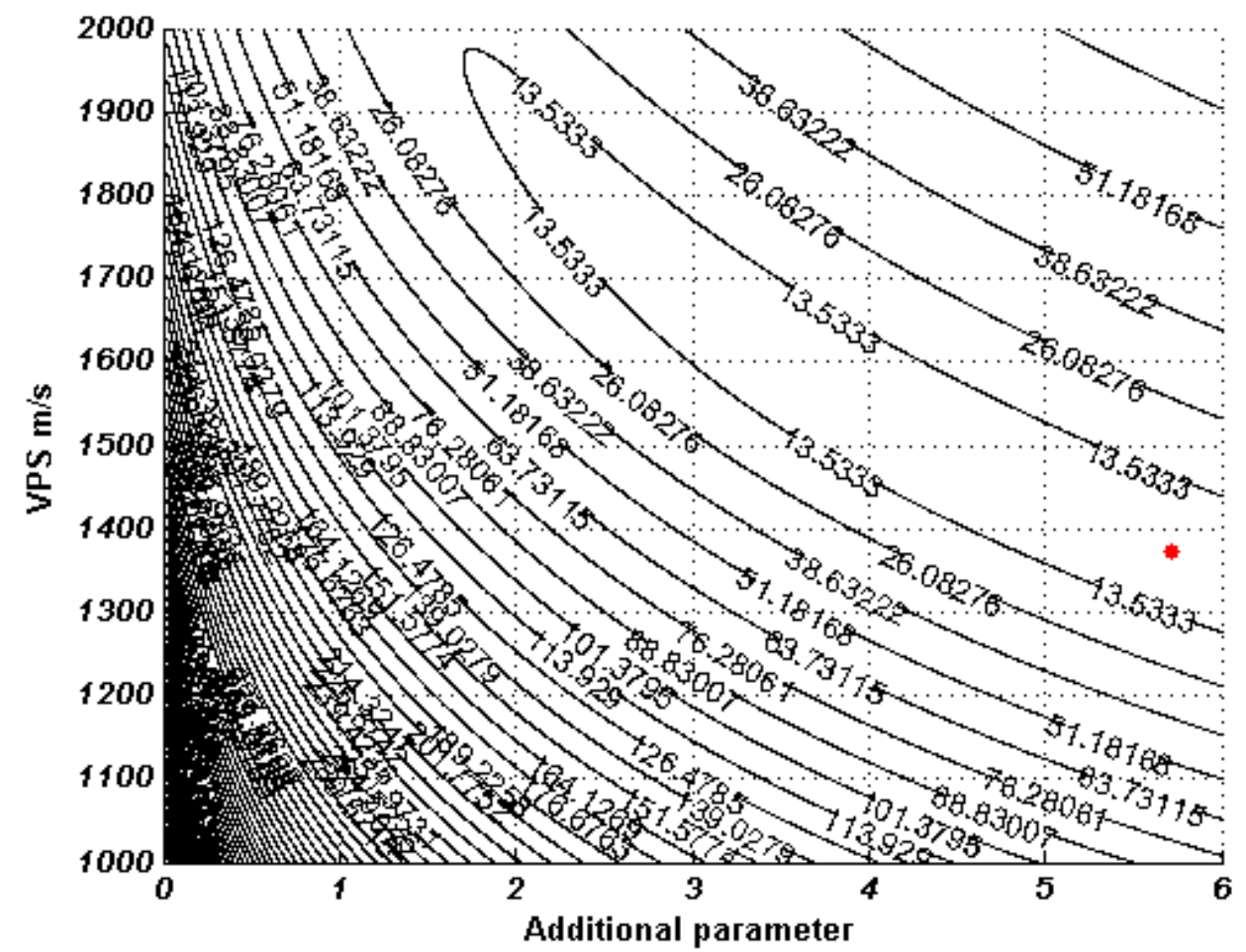

Figure 120: The residual function map which demonstrates the complexity of the Malovichko (1978) approximation of the PS reflection event with L1-norm. Red dispersions represent the global minimum region, and the blue dispersions, the local minimum regions. 


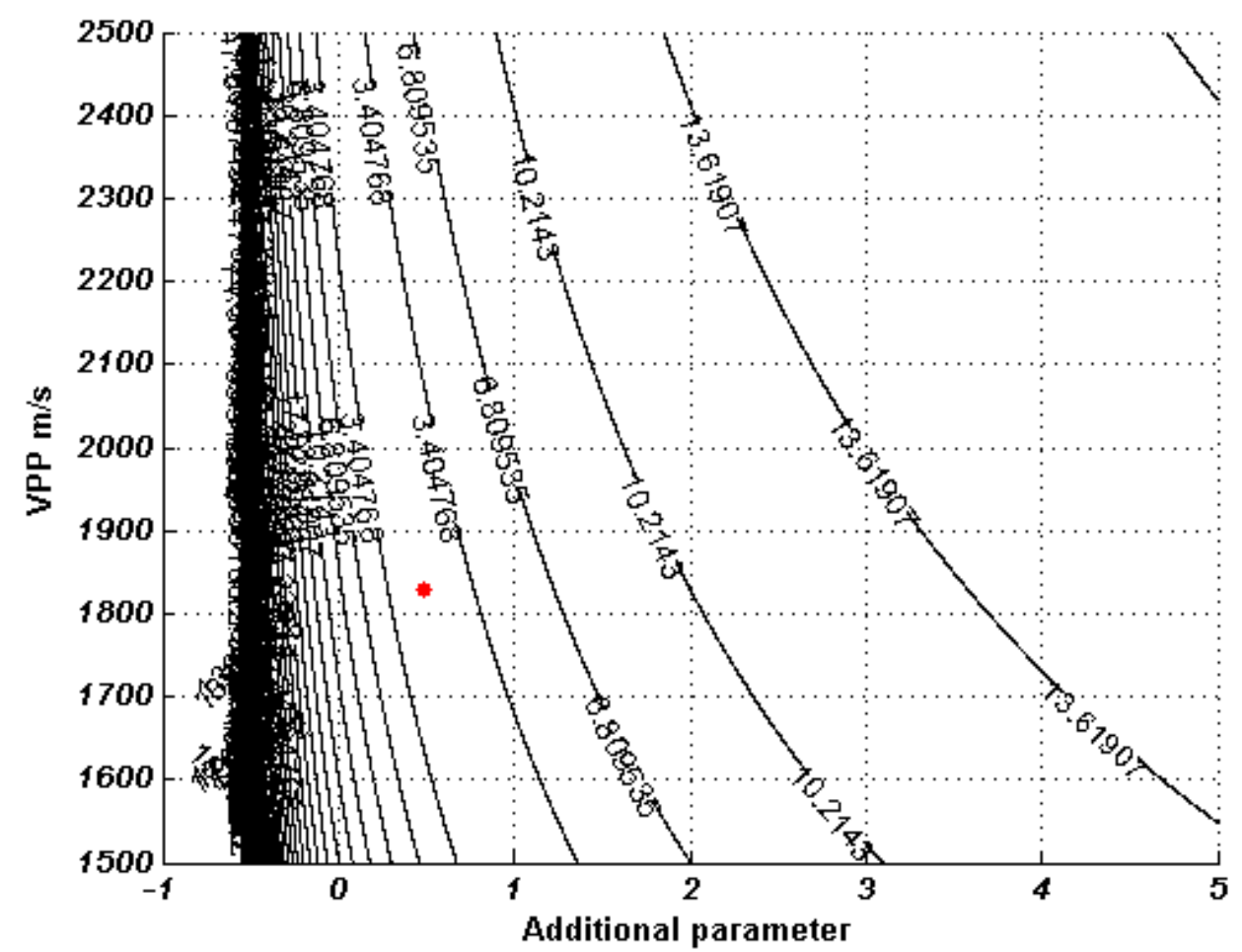

Figure 121: The residual function map which demonstrates the complexity of the Alkhalifah and Tsvankin (1995) approximation of the PP reflection event with L2-norm. Red dispersions represent the global minimum region, and the blue dispersions, the local minimum regions.

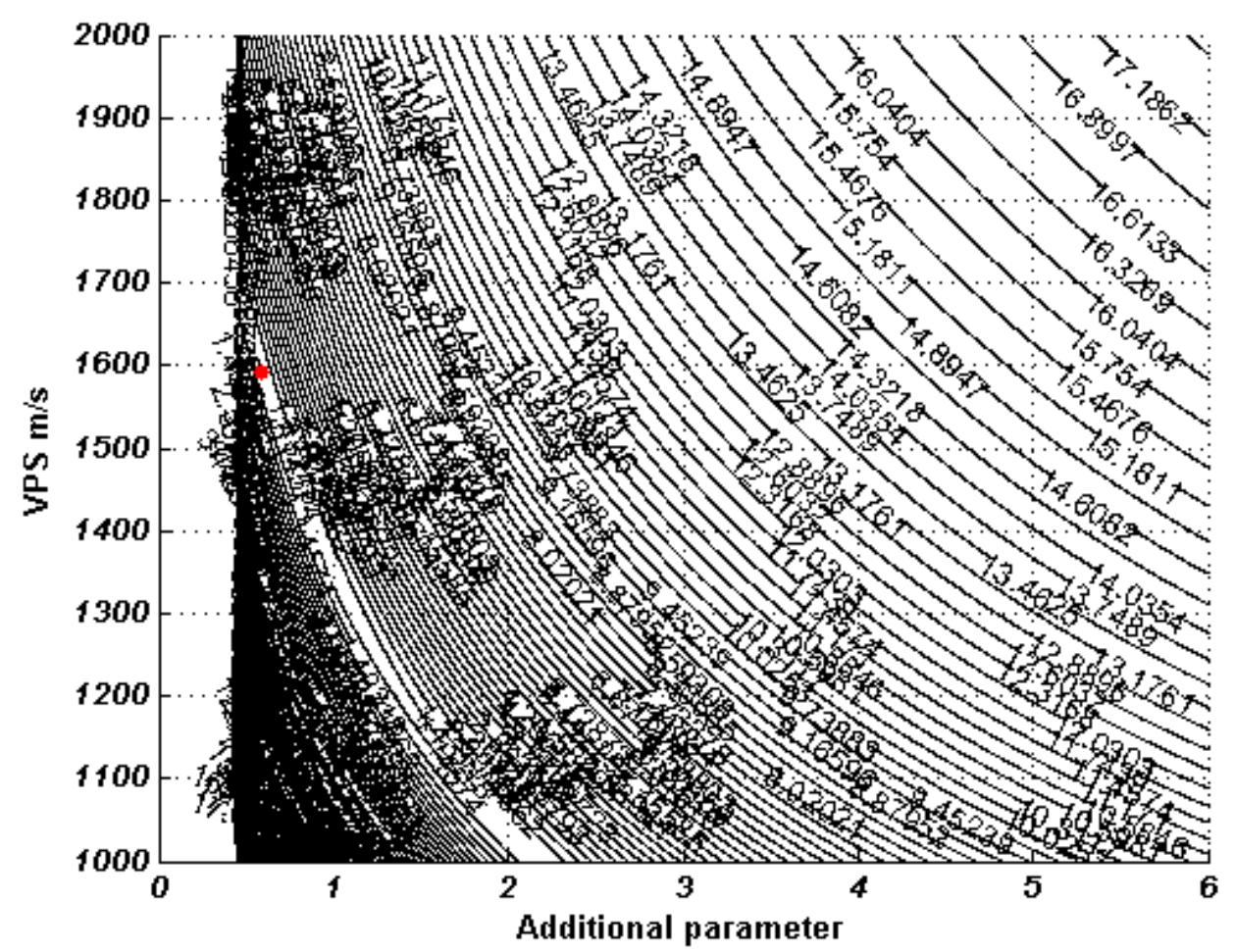

Figure 122: The residual function map which demonstrates the complexity of the Alkhalifah and Tsvankin (1995) approximation of the PS reflection event with L2-norm. Red dispersions represent the global minimum region, and the blue dispersions, the local minimum regions. 


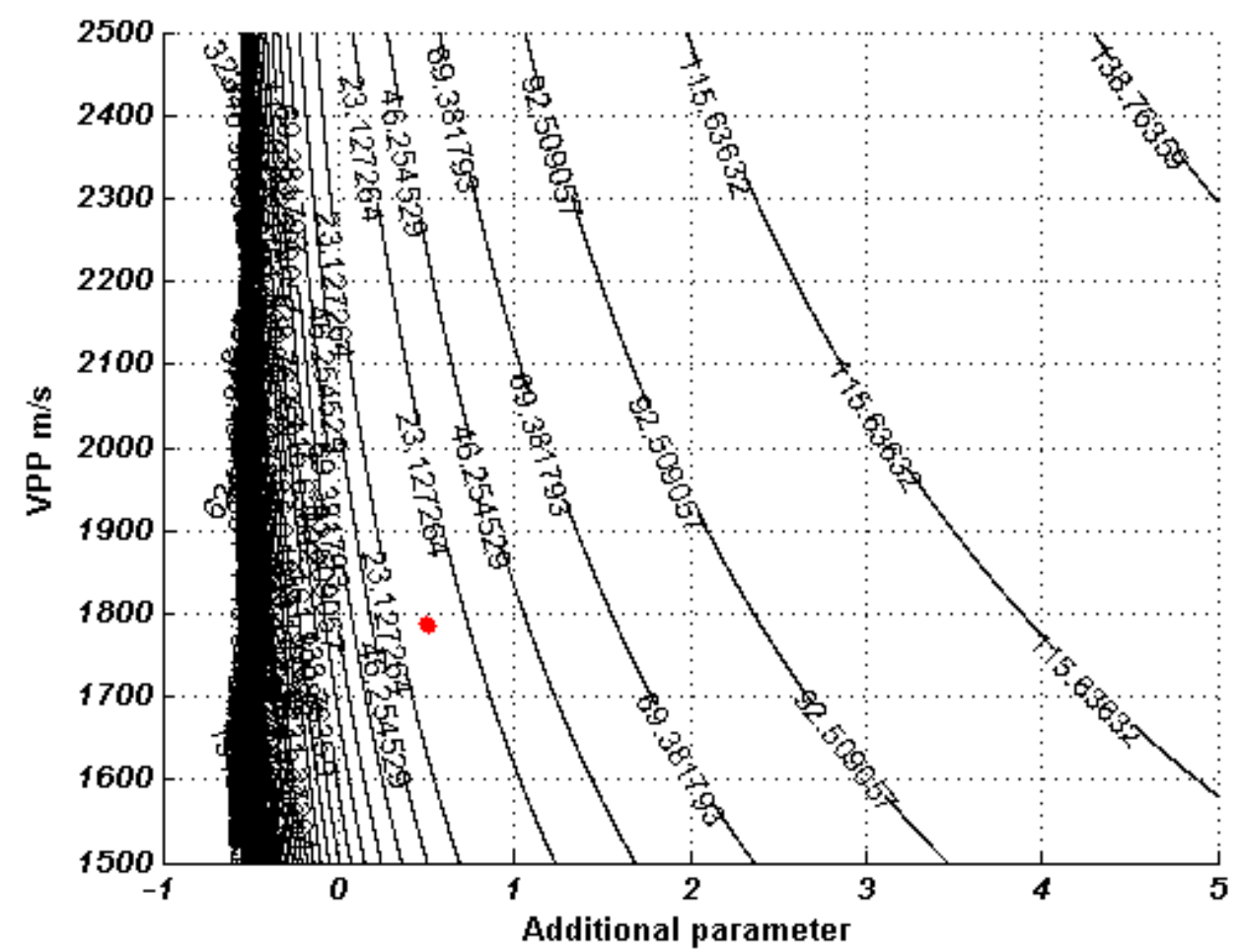

Figure 123: The residual function map which demonstrates the complexity of the Alkhalifah and Tsvankin (1995) approximation of the PP reflection event with L1-norm. Red dispersions represent the global minimum region, and the blue dispersions, the local minimum regions.

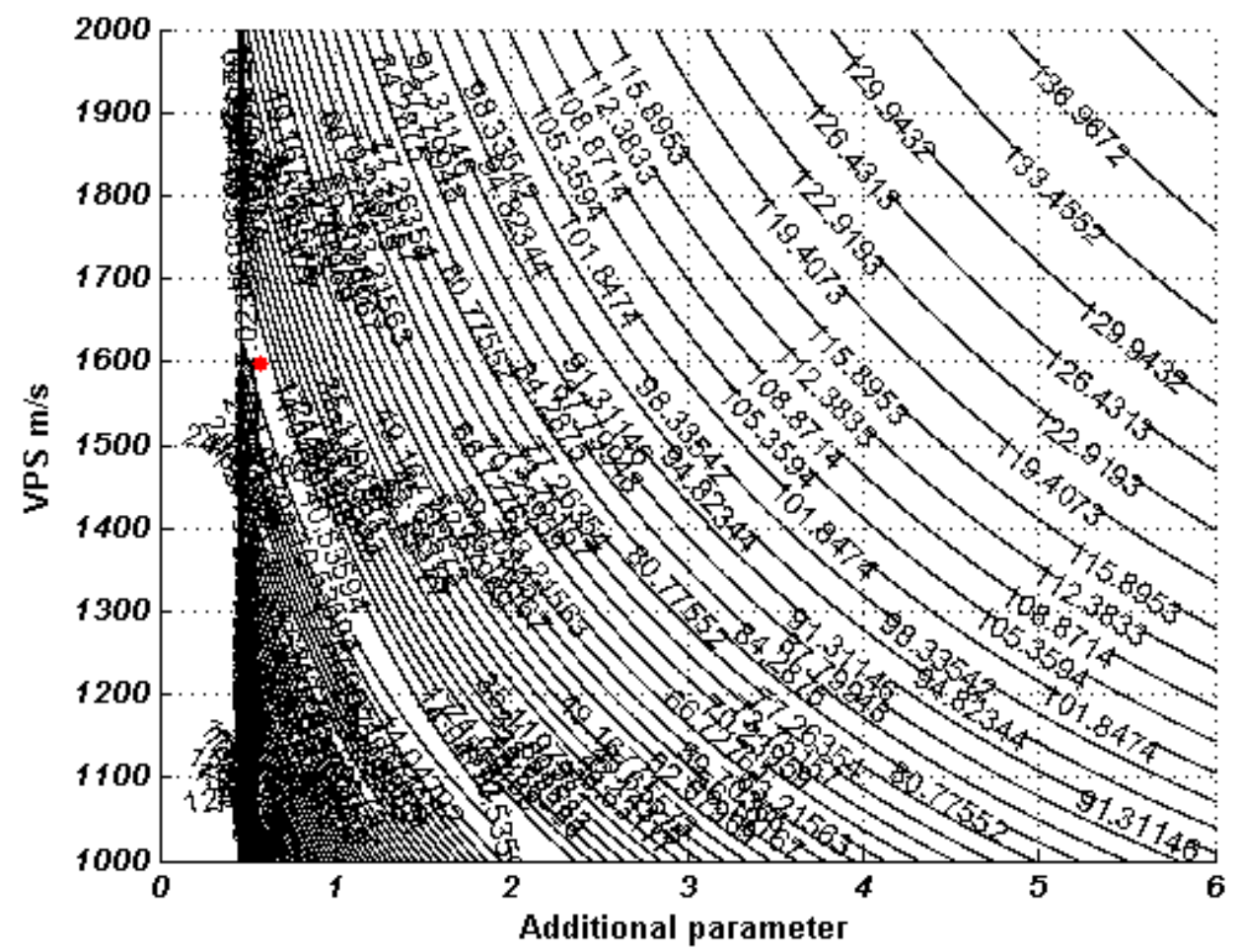

Figure 124: The residual function map which demonstrates the complexity of the Alkhalifah and Tsvankin (1995) approximation of the PS reflection event with L1-norm. Red dispersions represent the global minimum region, and the blue dispersions, the local minimum regions. 


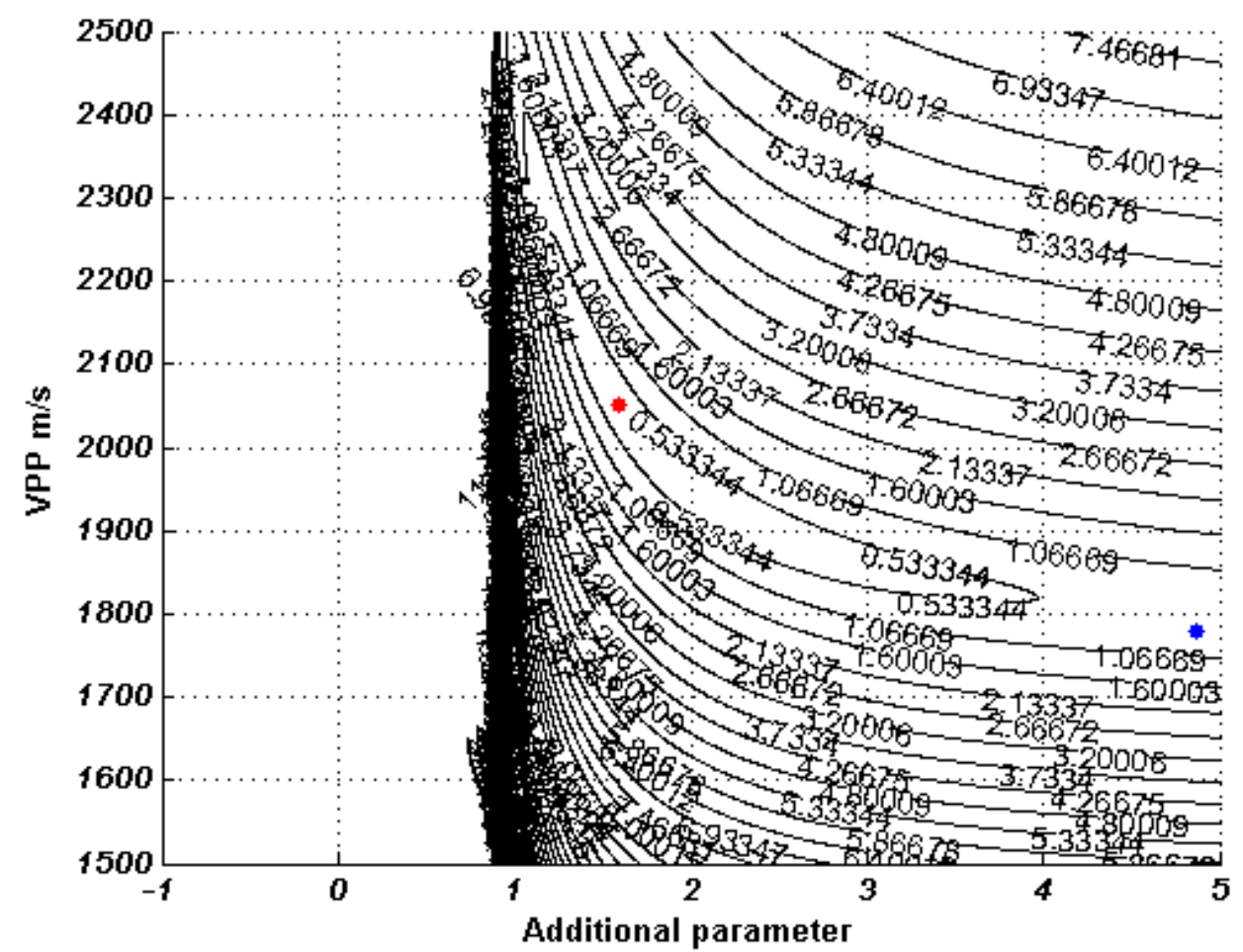

Figure 125: The residual function map which demonstrates the complexity of the Ursin and Stovas (2006) approximation of the PP reflection event with L2-norm. Red dispersions represent the global minimum region, and the blue dispersions, the local minimum regions.

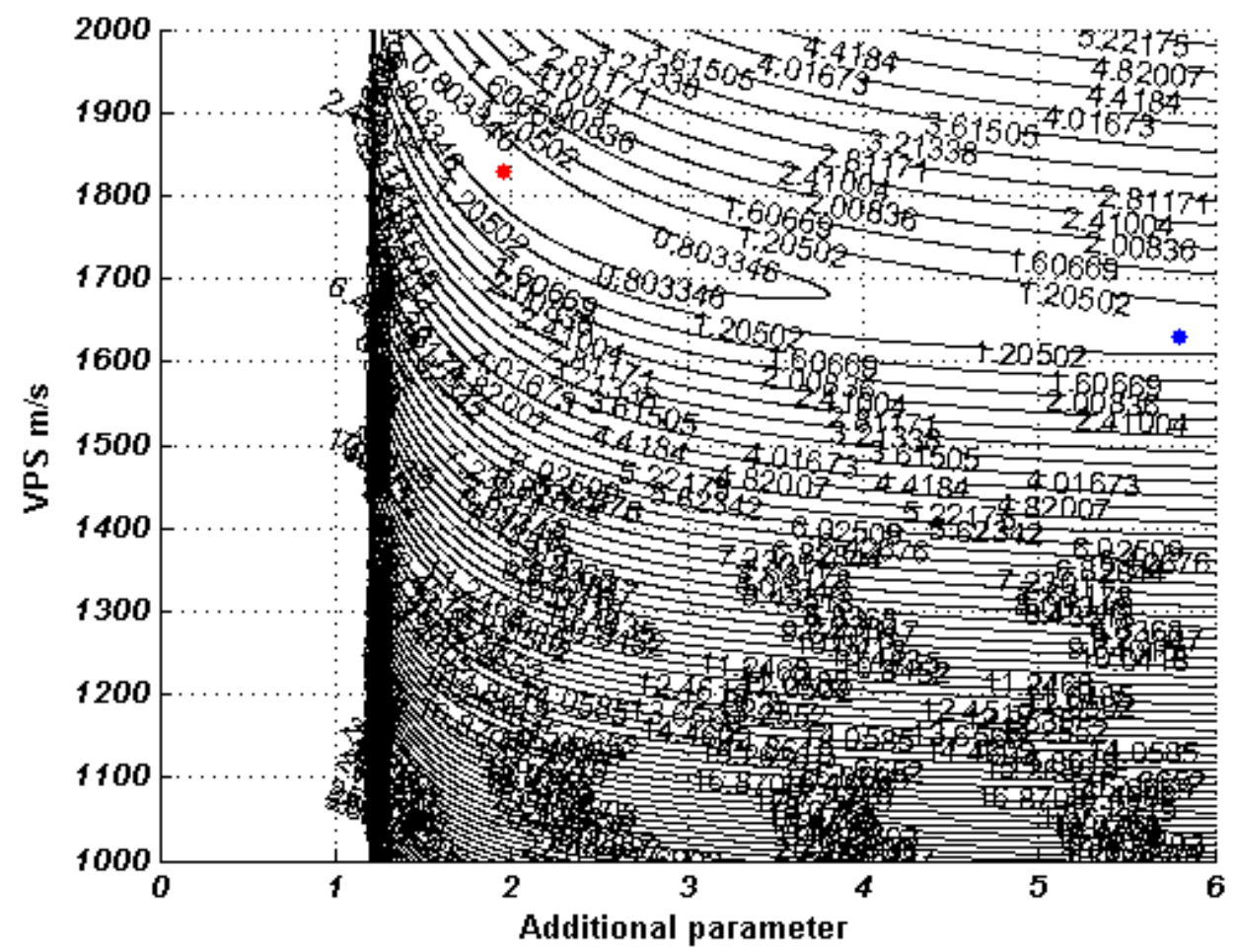

Figure 126: The residual function map which demonstrates the complexity of the Ursin and Stovas (2006) approximation of the PS reflection event with L2-norm. Red dispersions represent the global minimum region, and the blue dispersions, the local minimum regions. 


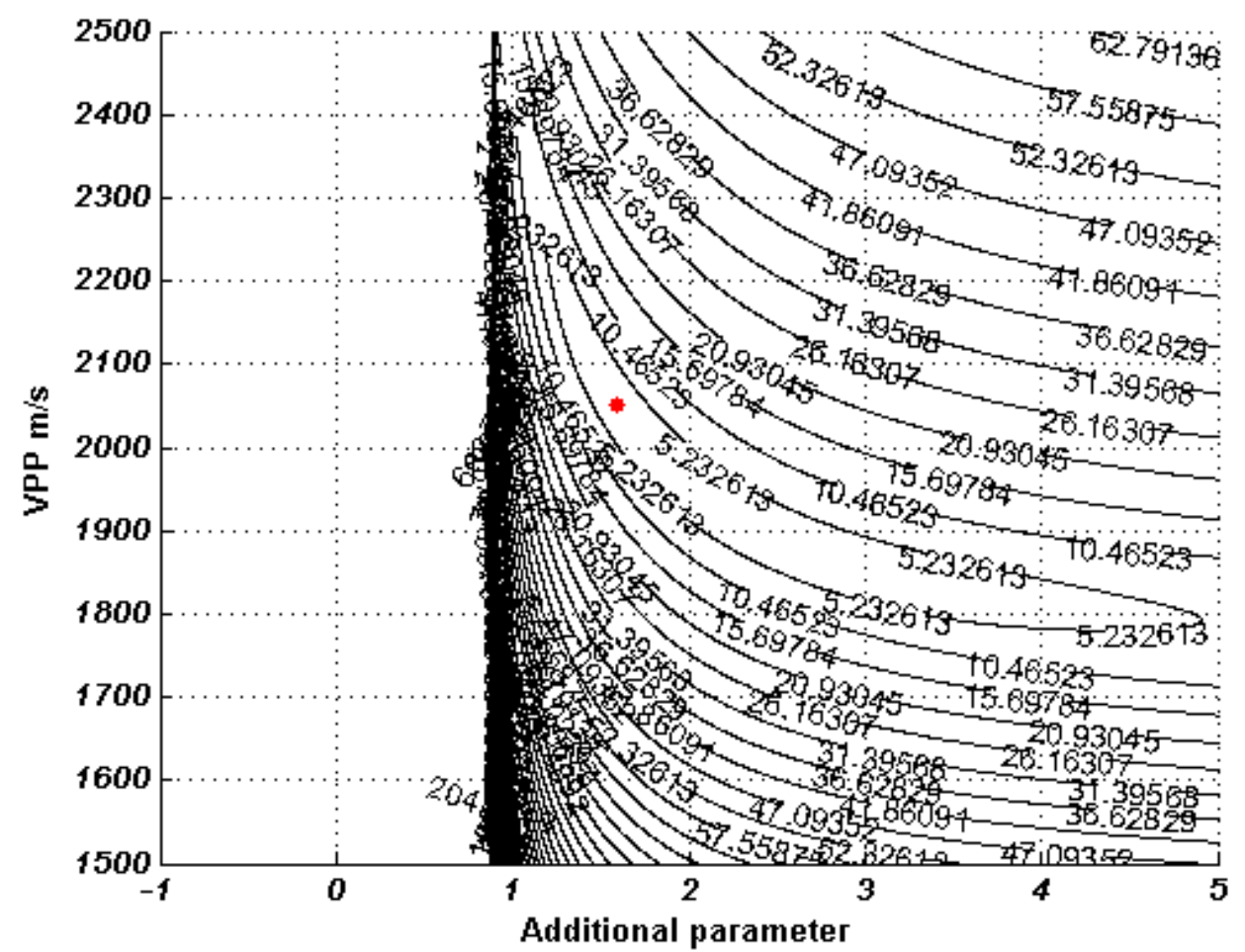

Figure 127: The residual function map which demonstrates the complexity of the Ursin and Stovas (2006) approximation of the PP reflection event with L1-norm. Red dispersions represent the global minimum region, and the blue dispersions, the local minimum regions.

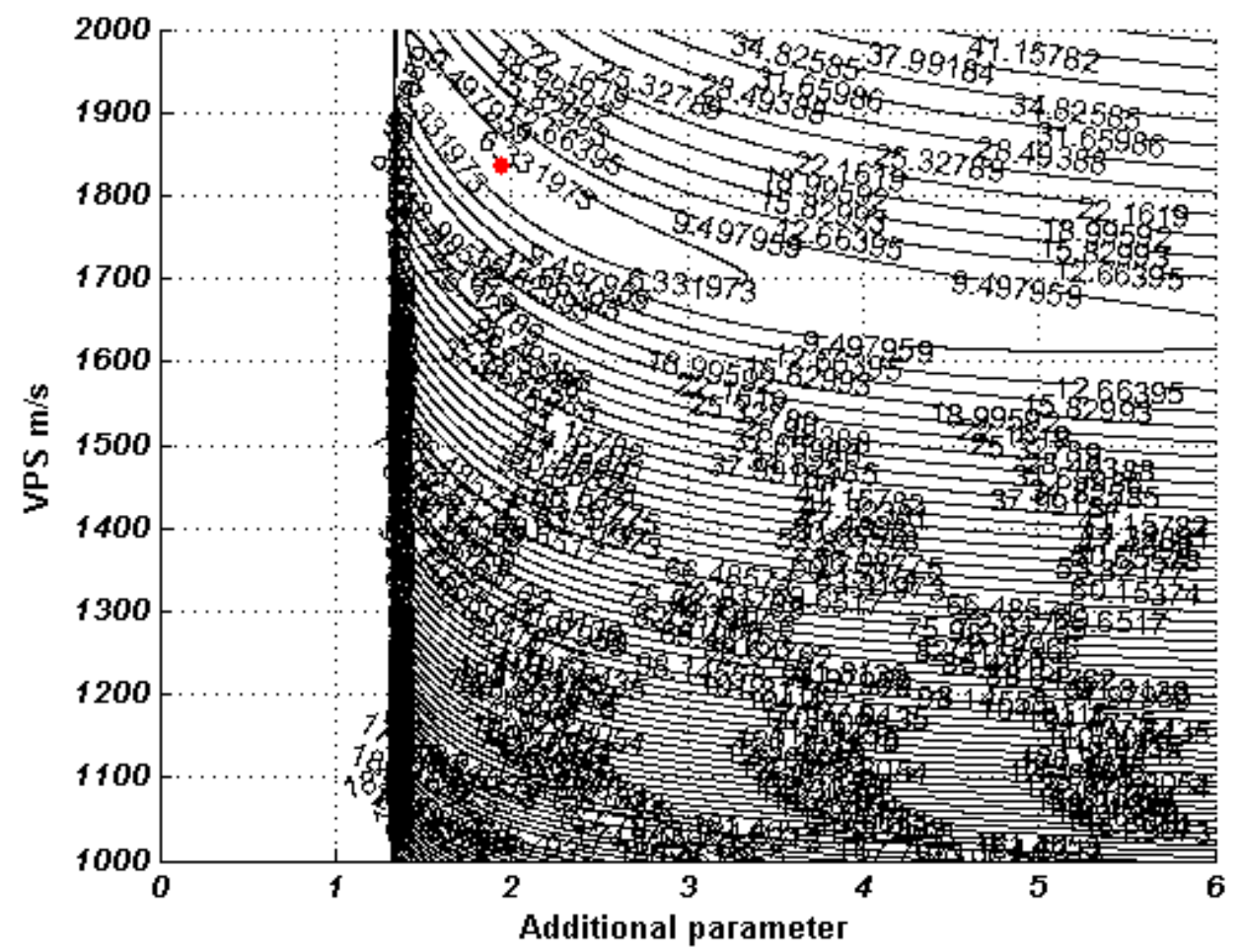

Figure 128: The residual function map which demonstrates the complexity of the Ursin and Stovas (2006) approximation of the PS reflection event with L1-norm. Red dispersions represent the global minimum region, and the blue dispersions, the local minimum regions. 


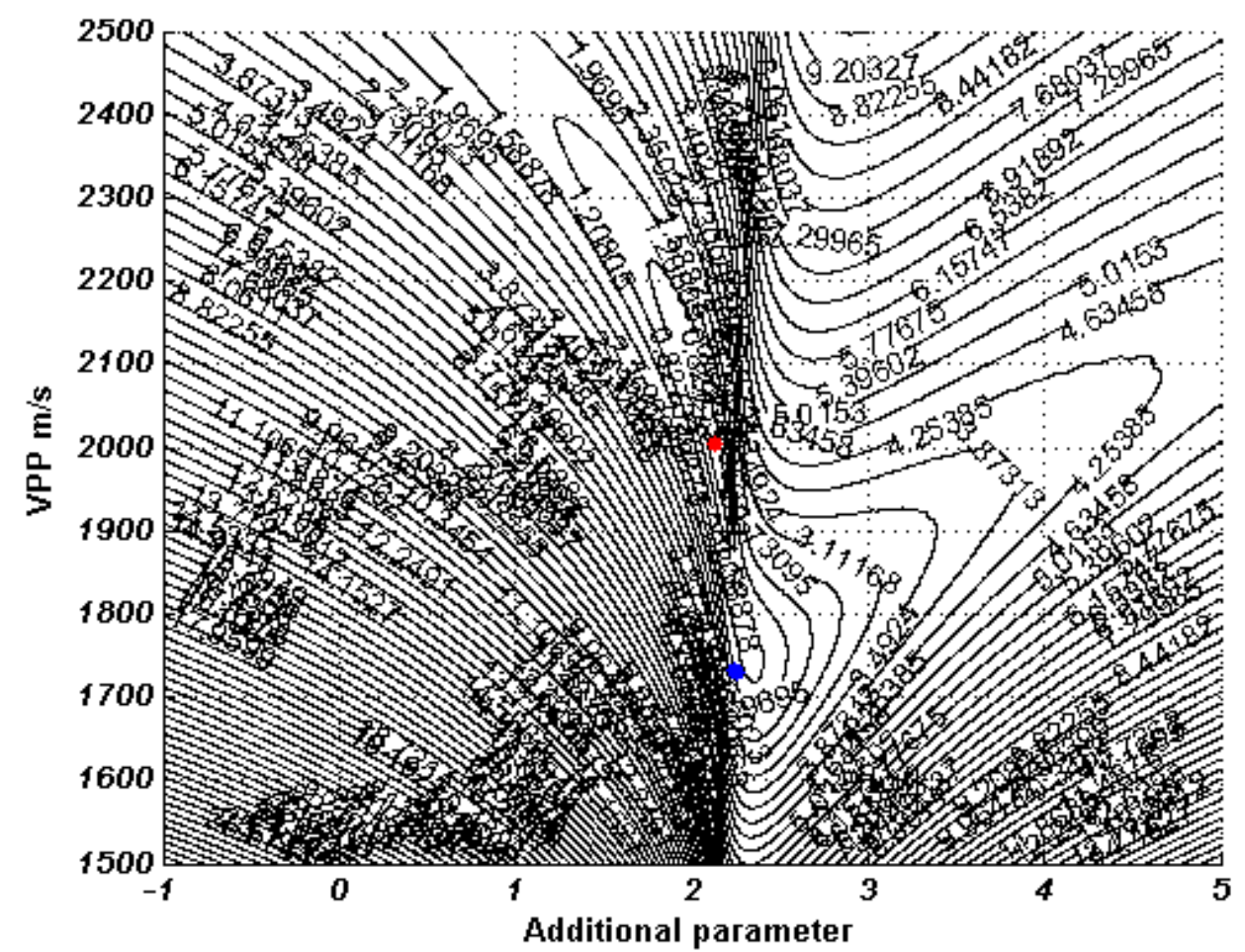

Figure 129: The residual function map which demonstrates the complexity of the Blias (2009) approximation of the PP reflection event with L2-norm. Red dispersions represent the global minimum region, and the blue dispersions, the local minimum regions.

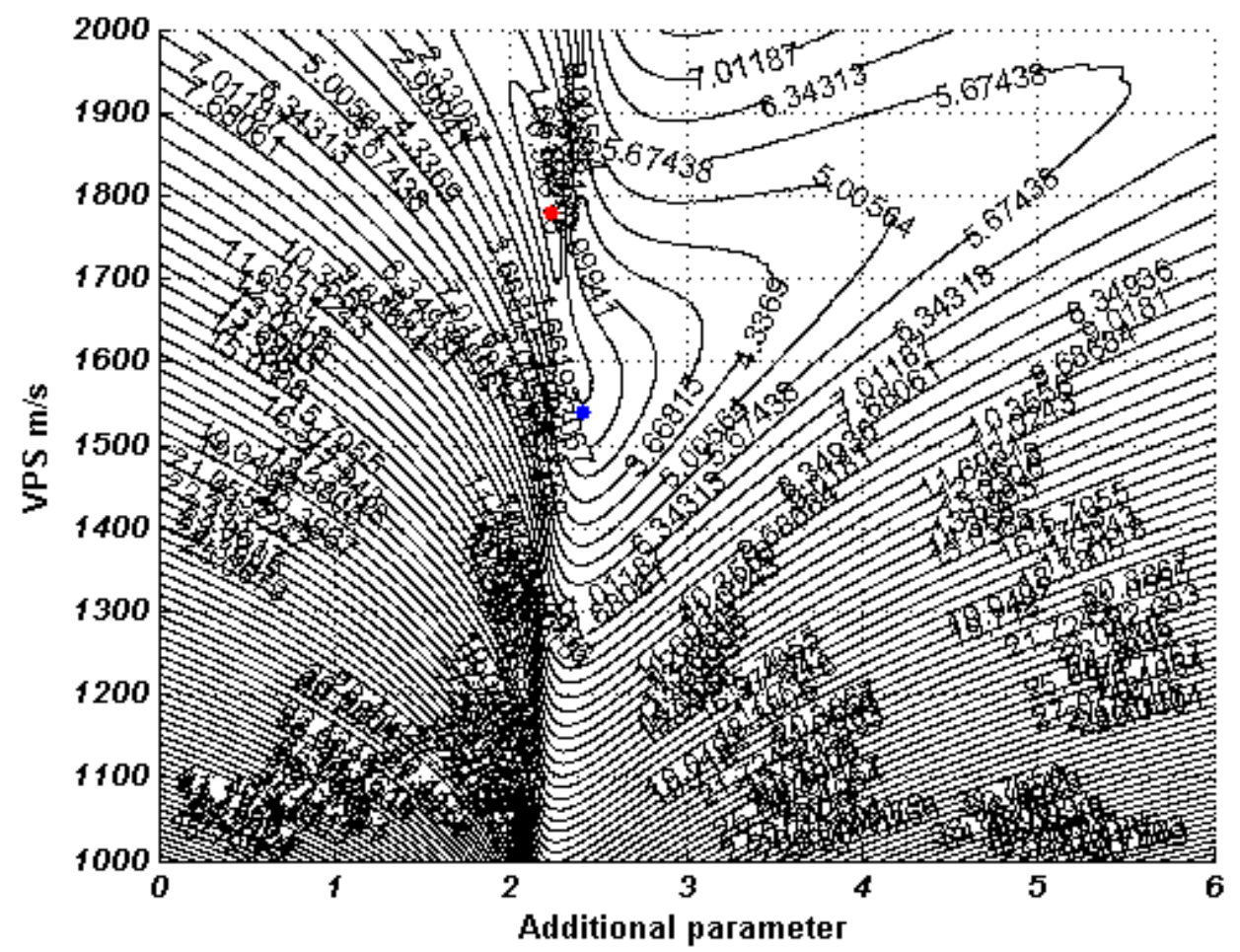

Figure 130: The residual function map which demonstrates the complexity of the Blias (2009) approximation of the PS reflection event with L2-norm. Red dispersions represent the global minimum region, and the blue dispersions, the local minimum regions. 


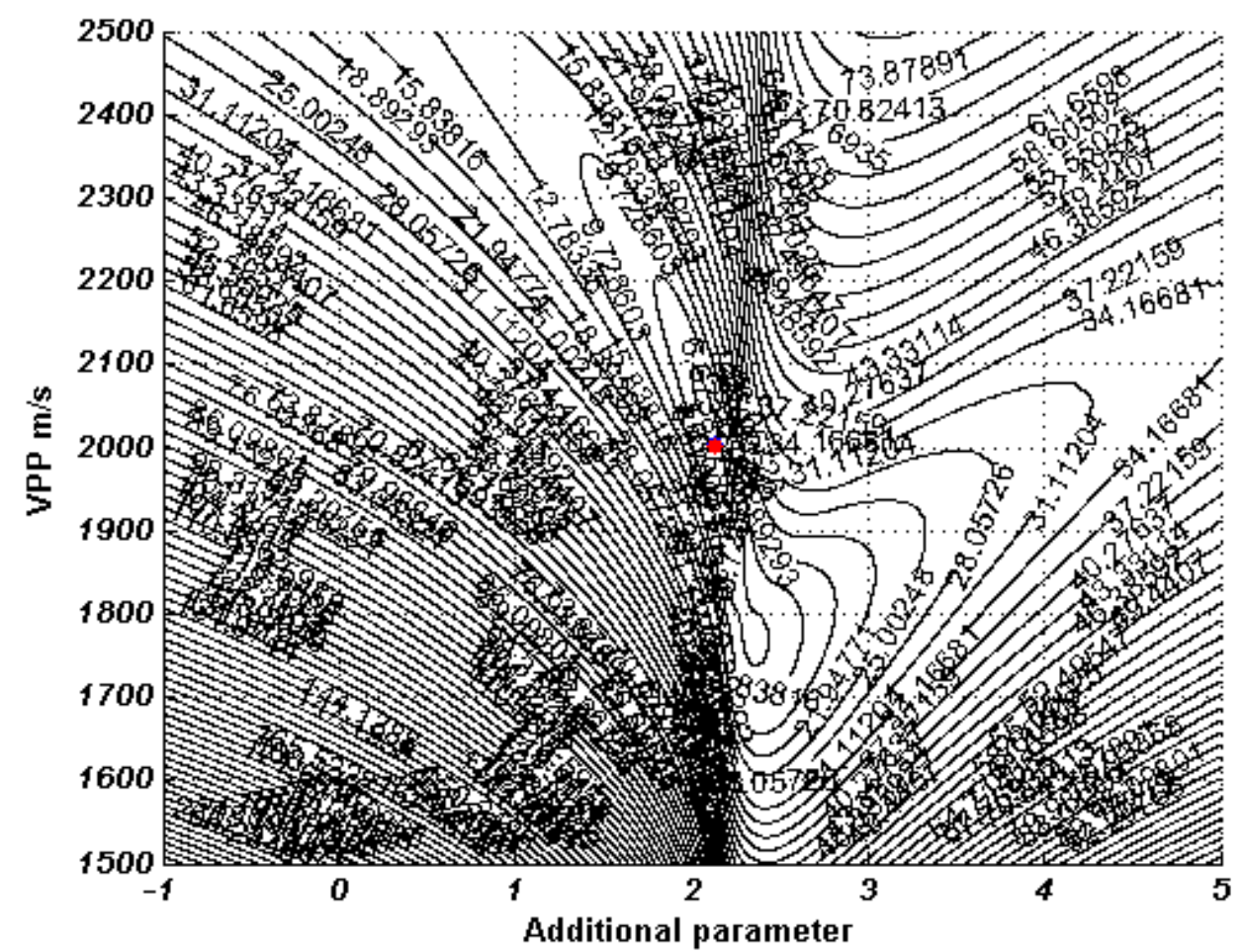

Figure 131: The residual function map which demonstrates the complexity of the Blias (2009) approximation of the PP reflection event with L1-norm. Red dispersions represent the global minimum region, and the blue dispersions, the local minimum regions.

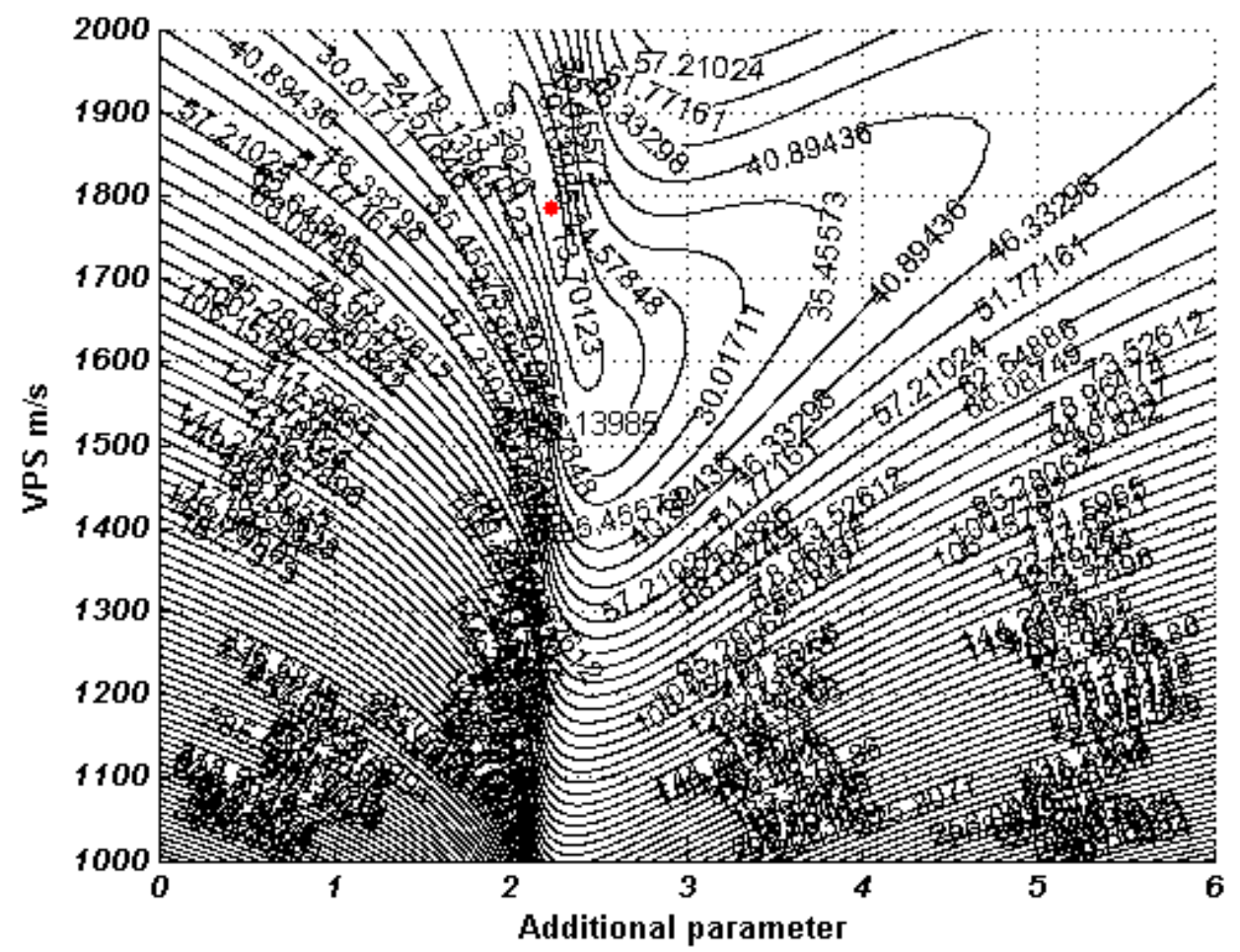

Figure 132: The residual function map which demonstrates the complexity of the Blias (2009) approximation of the PS reflection event with L1-norm. Red dispersions represent the global minimum region, and the blue dispersions, the local minimum regions. 


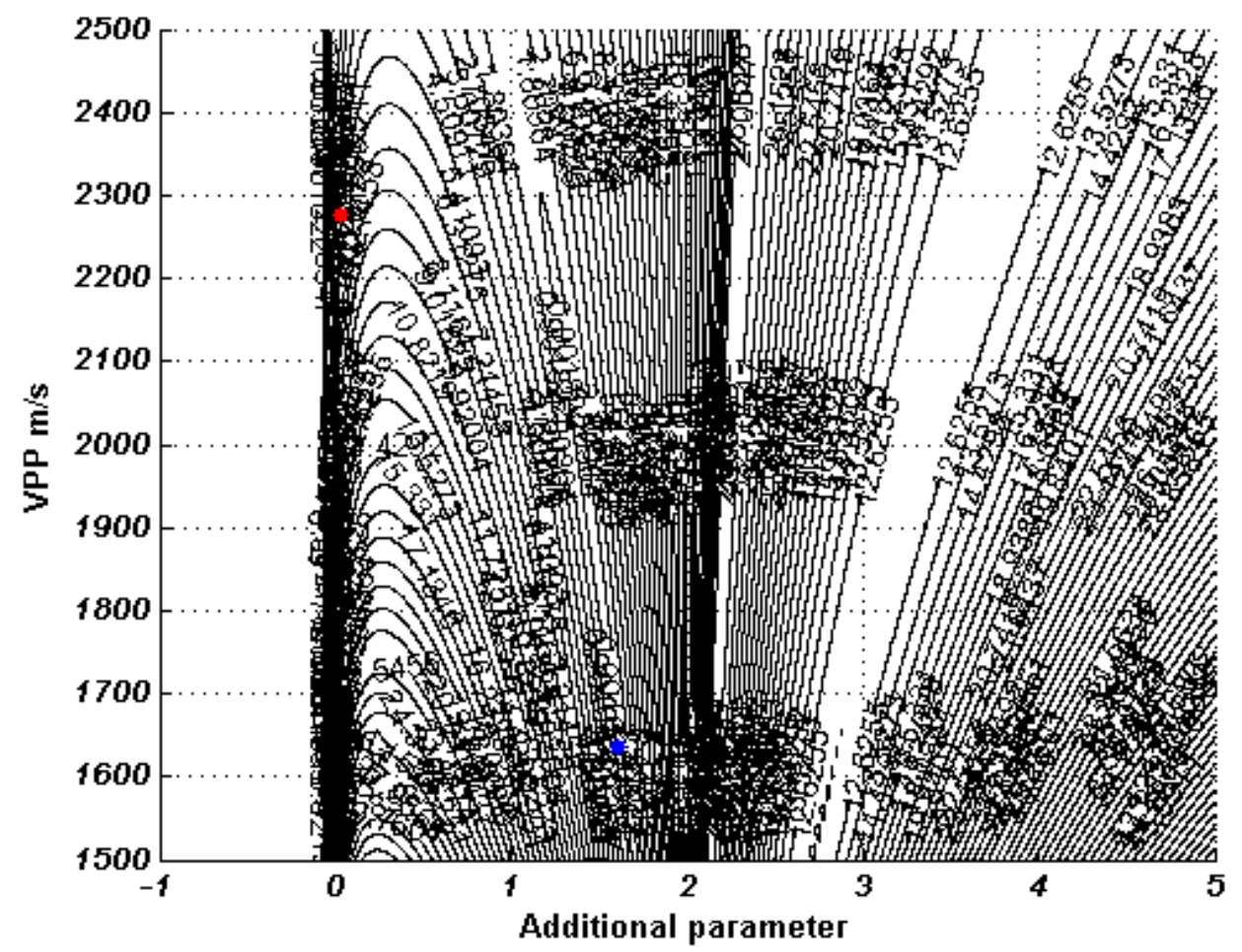

Figure 133: The residual function map which demonstrates the complexity of the Muir and Dellinger (1985) approximation of the PP reflection event with L2-norm. Red dispersions represent the global minimum region, and the blue dispersions, the local minimum regions.

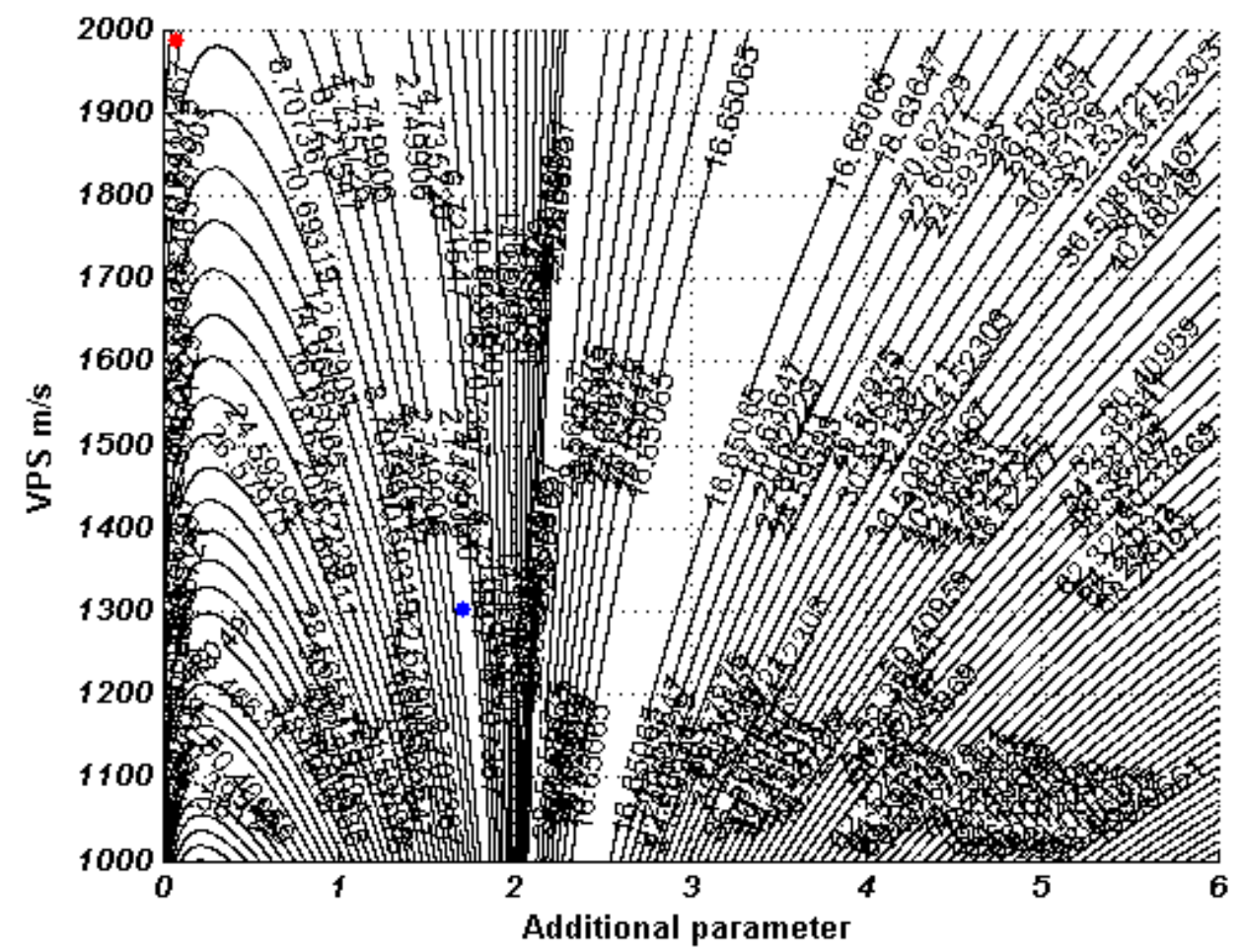

Figure 134: The residual function map which demonstrates the complexity of the Muir and Dellinger (1985) approximation of the PS reflection event with L2-norm. Red dispersions represent the global minimum region, and the blue dispersions, the local minimum regions. 


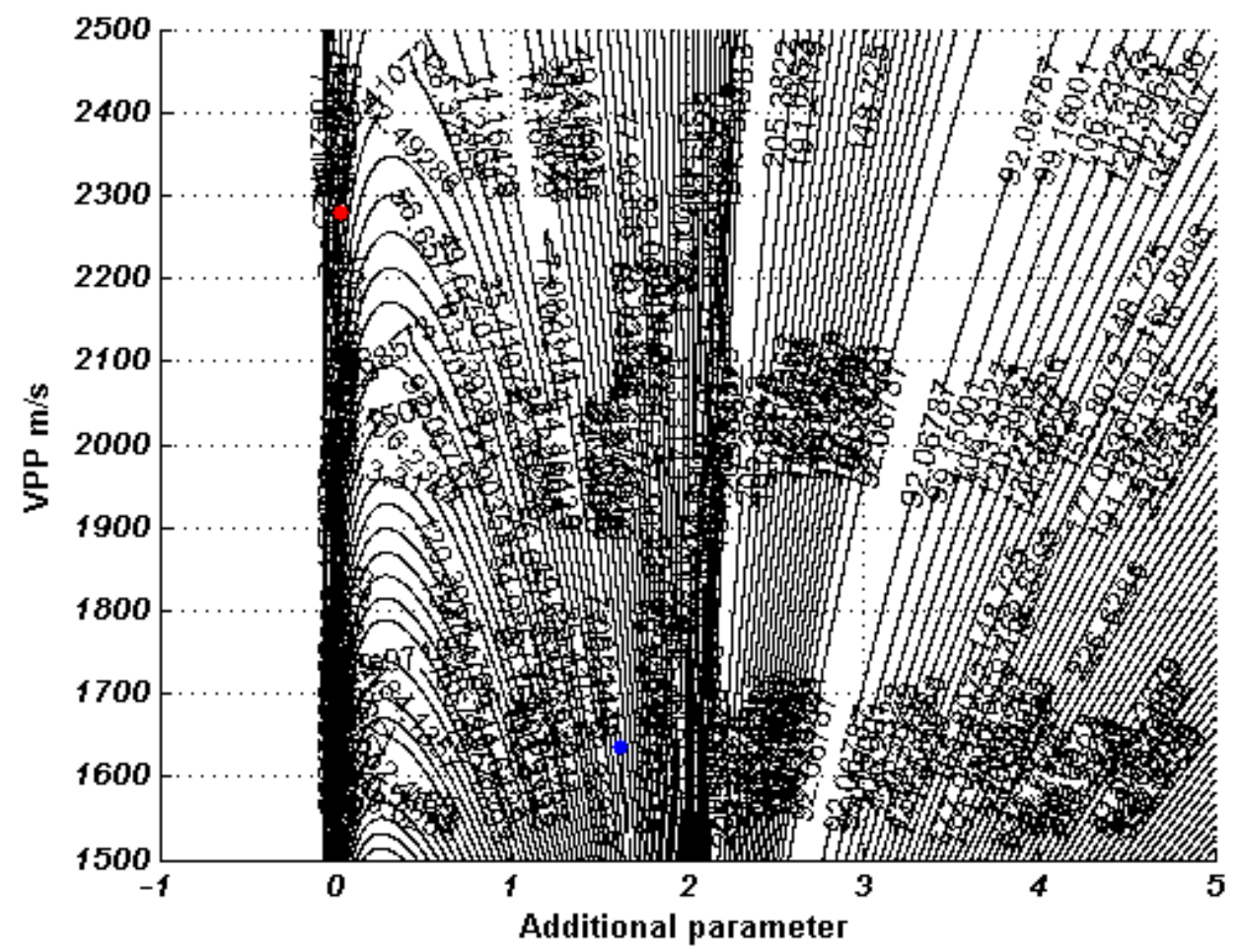

Figure 135: The residual function map which demonstrates the complexity of the Muir and Dellinger (1985) approximation of the PP reflection event with L1-norm. Red dispersions represent the global minimum region, and the blue dispersions, the local minimum regions.

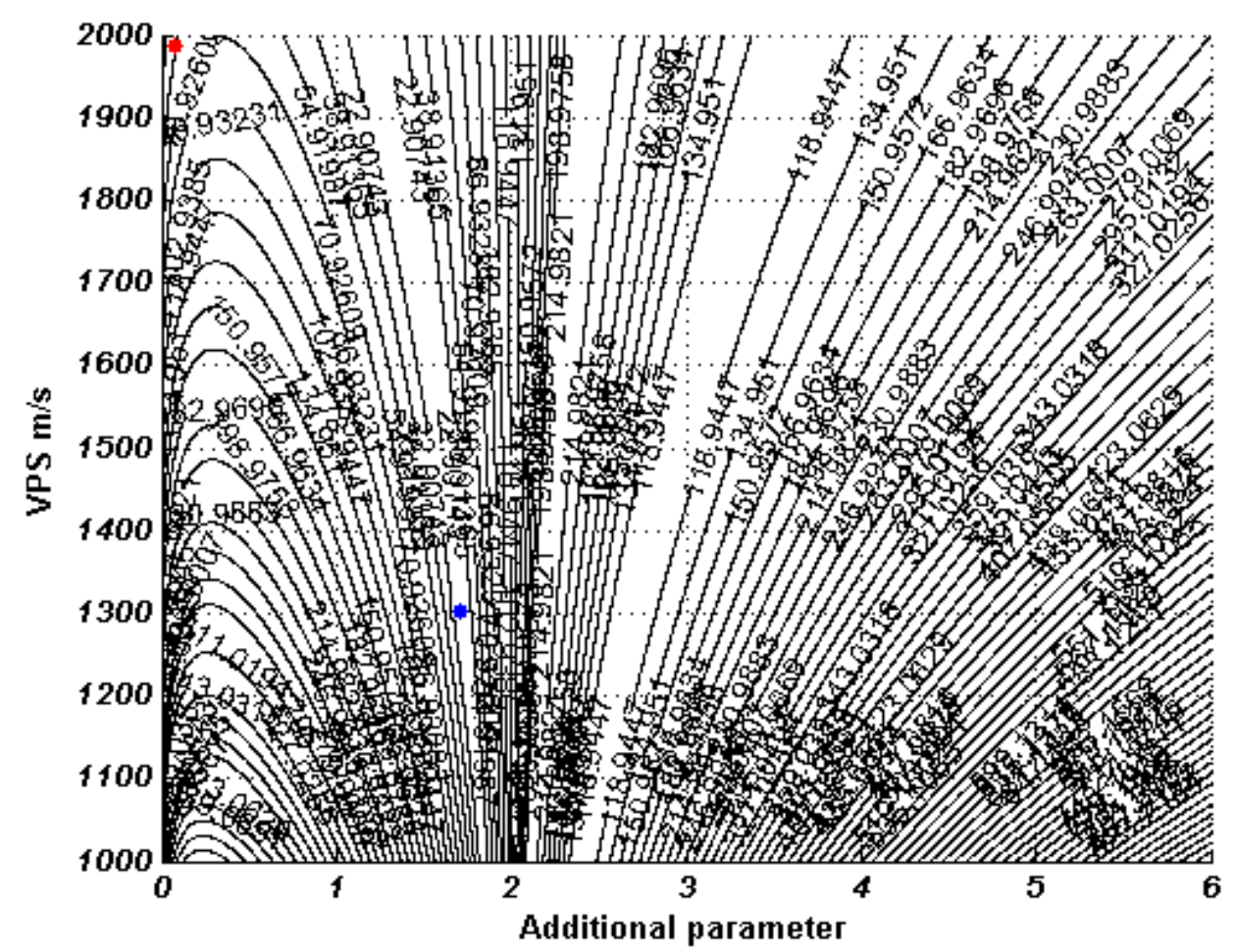

Figure 136: The residual function map which demonstrates the complexity of the Muir and Dellinger (1985) approximation of the PS reflection event with L1-norm. Red dispersions represent the global minimum region, and the blue dispersions, the local minimum regions. 


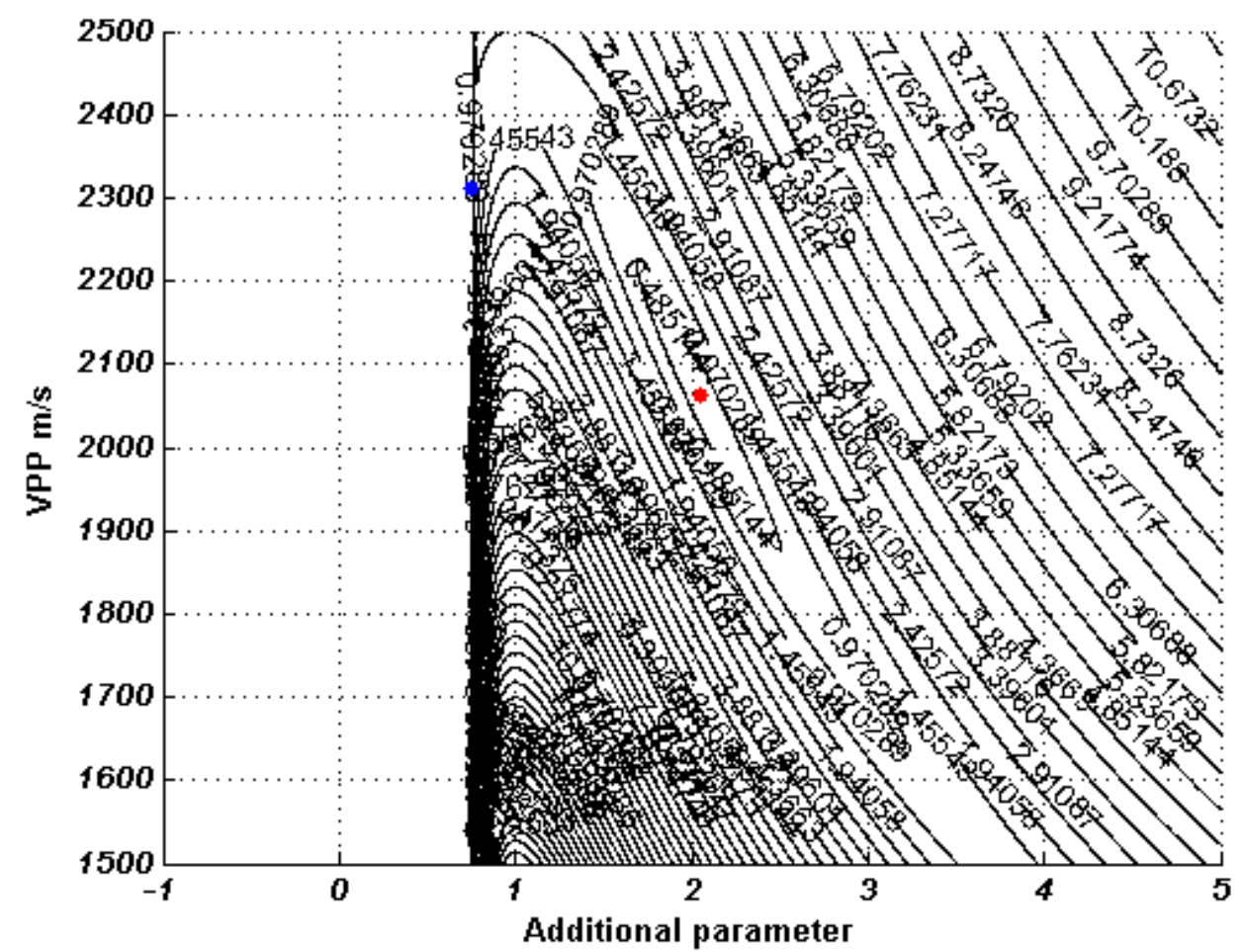

Figure 137: The residual function map which demonstrates the complexity of the Li and Yuan (2001) approximation of the PP reflection event with L2-norm. Red dispersions represent the global minimum region, and the blue dispersions, the local minimum regions.

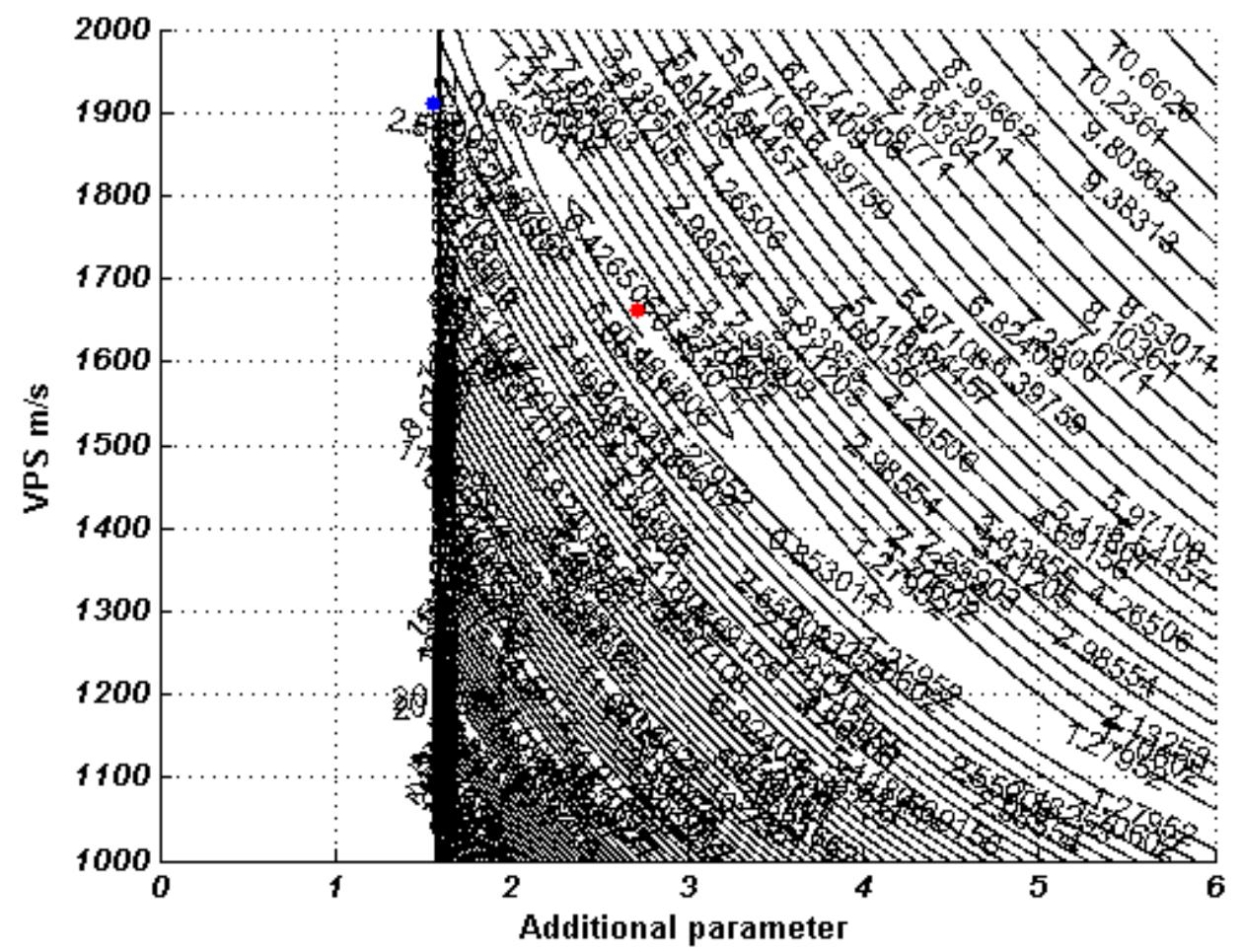

Figure 138: The residual function map which demonstrates the complexity of the Li and Yuan (2001) approximation of the PS reflection event with L2-norm. Red dispersions represent the global minimum region, and the blue dispersions, the local minimum regions. 


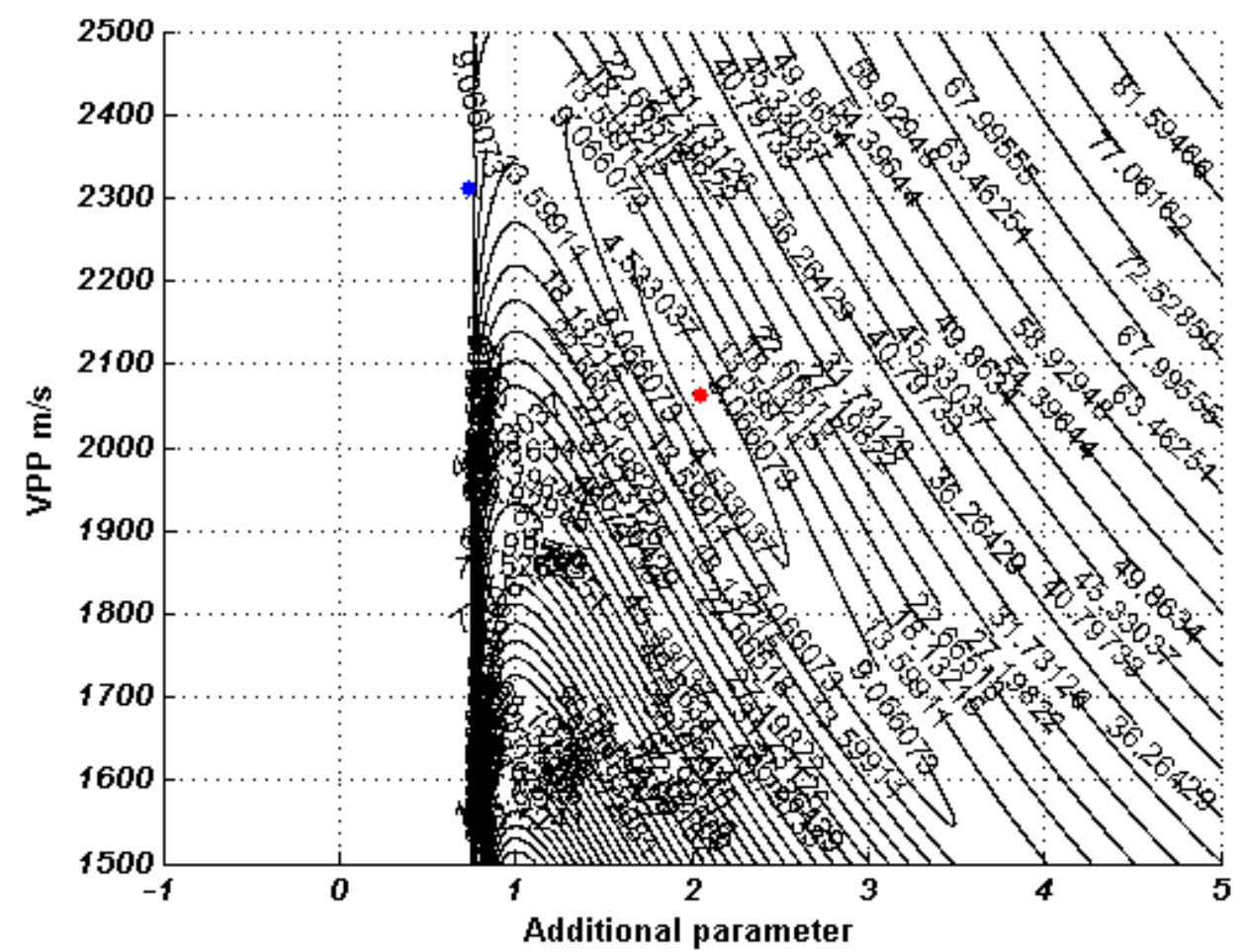

Figure 139: The residual function map which demonstrates the complexity of the Li and Yuan (2001) approximation of the PP reflection event with L1-norm. Red dispersions represent the global minimum region, and the blue dispersions, the local minimum regions.

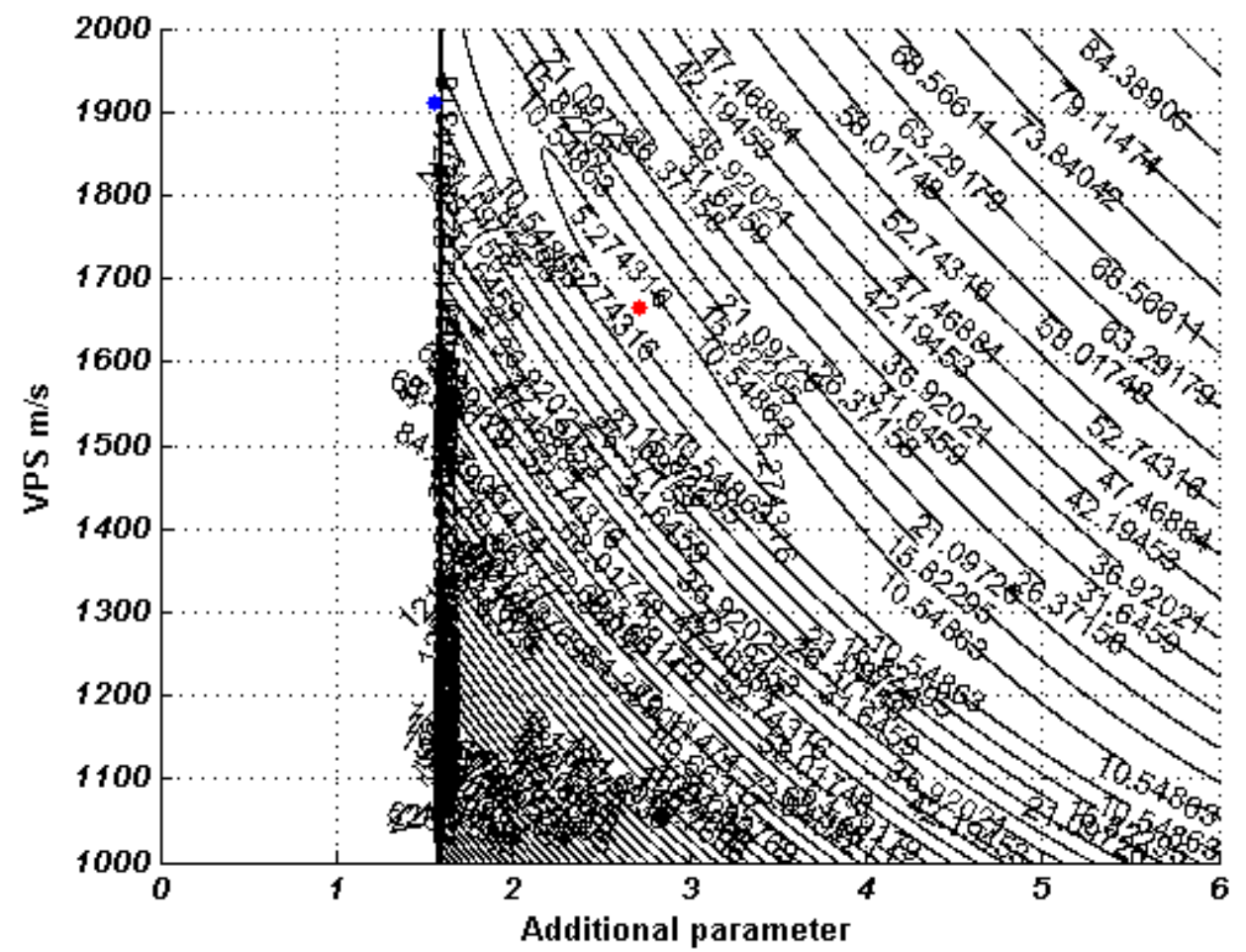

Figure 140: The residual function map which demonstrates the complexity of the Li and Yuan (2001) approximation of the PS reflection event with L1-norm. Red dispersions represent the global minimum region, and the blue dispersions, the local minimum regions. 


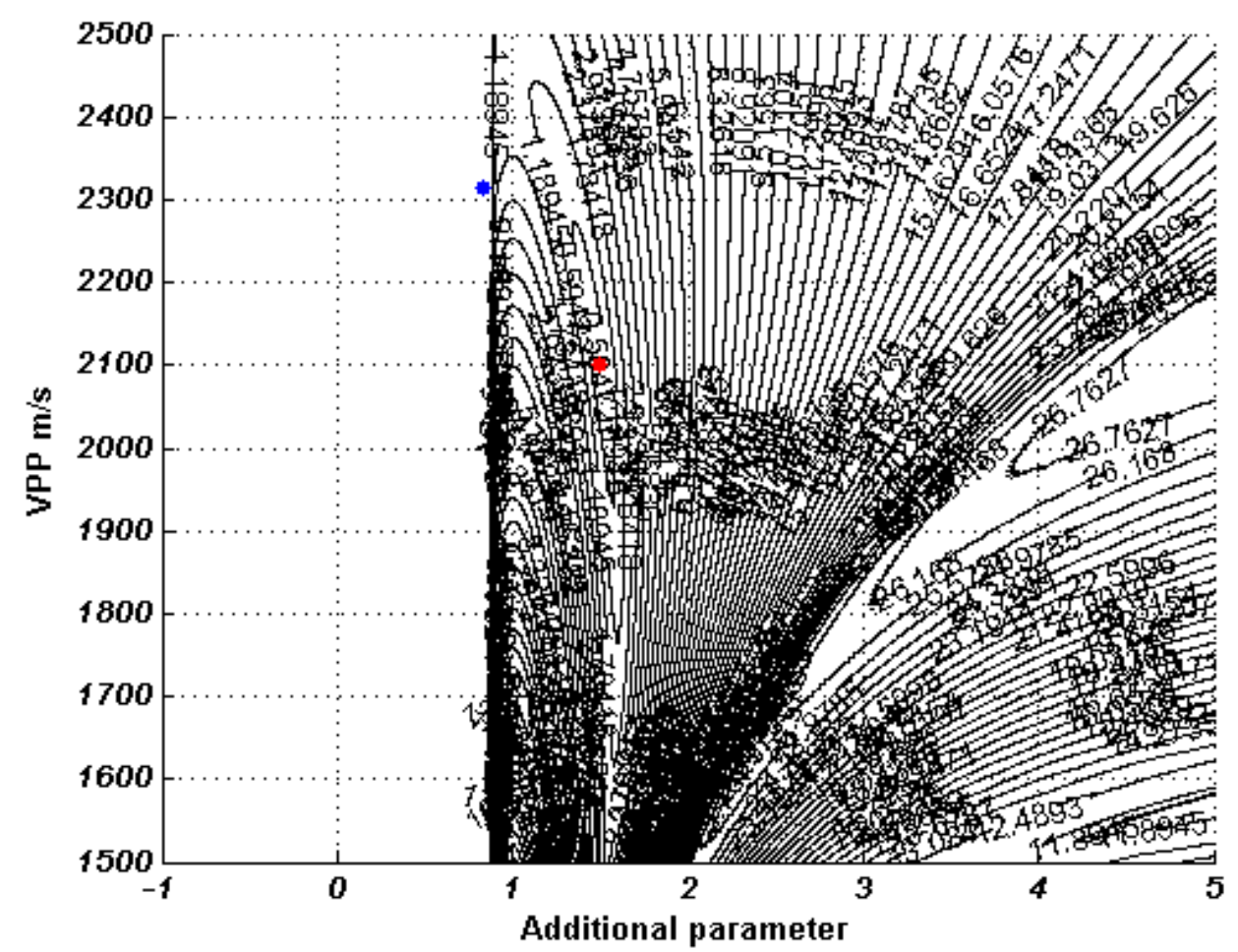

Figure 141: The residual function map which demonstrates the complexity of the approximation proposed in this work of the PP reflection event with L2-norm. Red dispersions represent the global minimum region, and the blue dispersions, the local minimum regions.

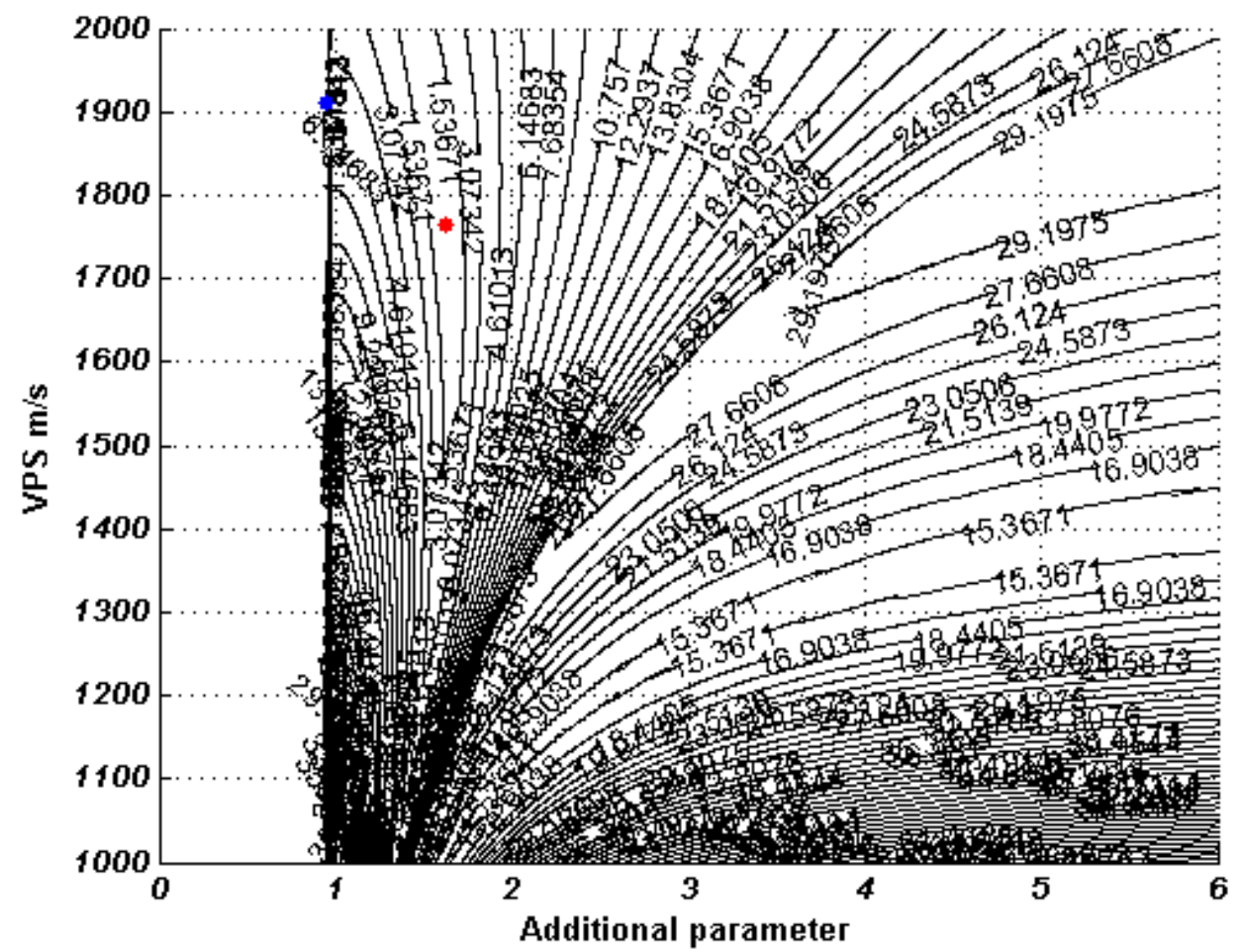

Figure 142: The residual function map which demonstrates the complexity of the approximation proposed in this work of the PS reflection event with L2-norm. Red dispersions represent the global minimum region, and the blue dispersions, the local minimum regions. 


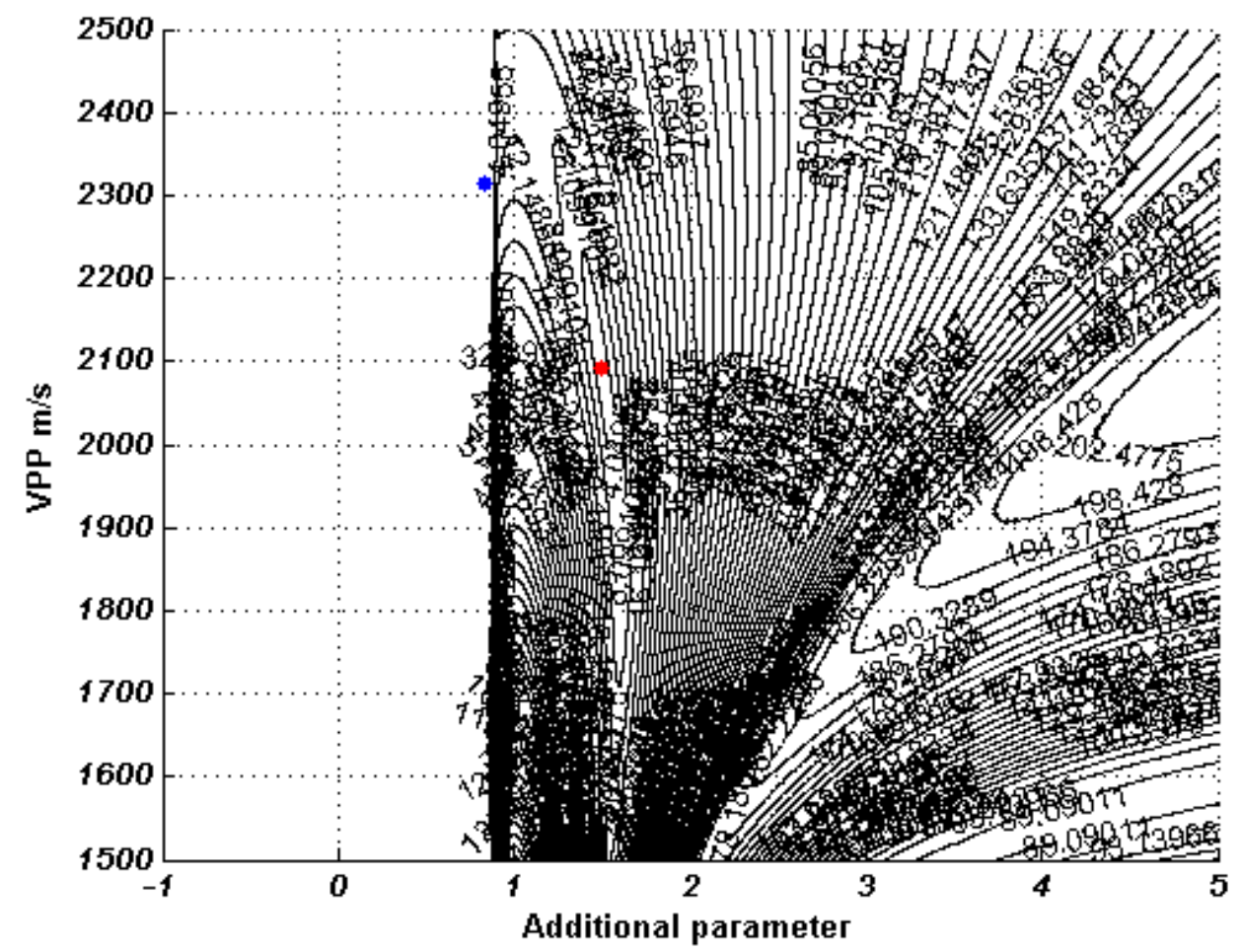

Figure 143: The residual function map which demonstrates the complexity of the approximation proposed in this work of the PP reflection event with L1-norm. Red dispersions represent the global minimum region, and the blue dispersions, the local minimum regions.

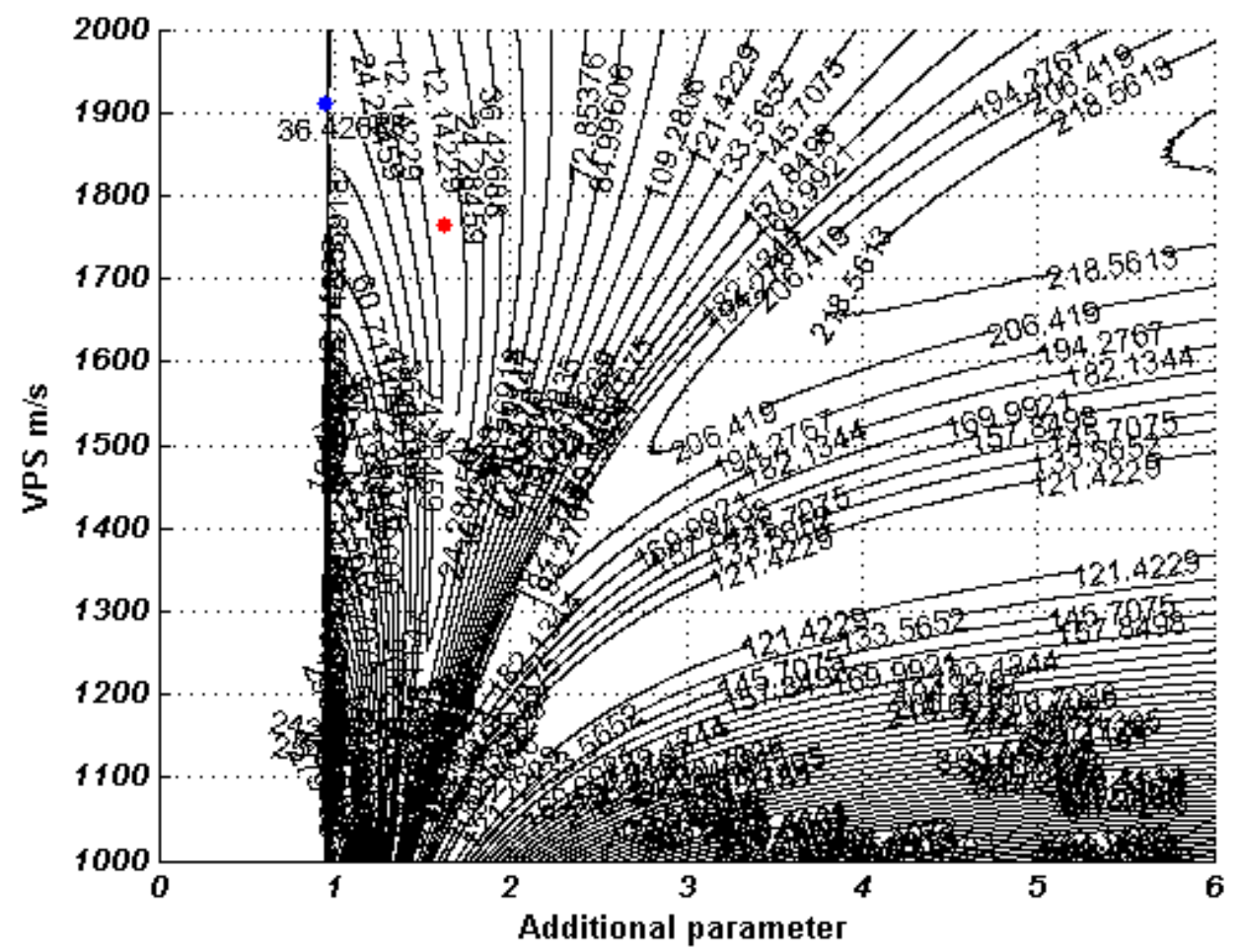

Figure 144: The residual function map which demonstrates the complexity of the approximation proposed in this work of the PS reflection event with L1-norm. Red dispersions represent the global minimum region, and the blue dispersions, the local minimum regions. 


\subsubsection{Model 5}

The Model 5 is a structure which is even shallower than the fourth model, which would make it a simpler model to work. However, as the previous one, the fifth model still uses the geometry of acquisition of the first three models, which increases the complexity, once the nonhyperbolicity due to the RMS velocity relations gets stronger. For this reason, the approximation proposed by Malovichko (1978) still showed a significant variation between the PP and PS events (Figures 145 and 146). It also showed the abrupt variation between the topology of the L2-norm and the L1-norm (Figures 147 and 148).

The same kind of variations could be observed for the Alkhalifah and Tsvankin (1995) approximation in this model (Figures 149, 150, 151 and 152).

The approximation proposed by Ursin and Stovas (2006) showed similar variations as the Model 4 between the PP and PS events (Figures 153 and 154), and also concerning the two norms (Figures 155 and 156).

The same could be observed in the approximation proposed by Blias (2006), which showed a significant variation between the two reflection events (Figures 157 and 158) and stopped being multimodal after the application of the L1-norm rather than the L2- (Figures 159 and 160).

Similarly to the Model 4, the Muir and Dellinger (1985) approximation showed only variations concerning the topology between the PP and PS events (Figures 161 and 162) and not concerning the exchange of the local and global minimum regions. Concerning the variation between the norms, it showed a similar behaviour to what was observed in the fourth model (Figures 163 and 164).

The Li and Yuan (2001) approximation showed the same kind of variations as in the previous model (Figures 165, 166, 167 and 168).

It can be observed that, in the Figures 169, 170, 171 and 172, the proposed approximation showed a similar more complex topological structure concerning the objective function, and also showed the same kind of variations and behaviour as it showed in the Model 4. 


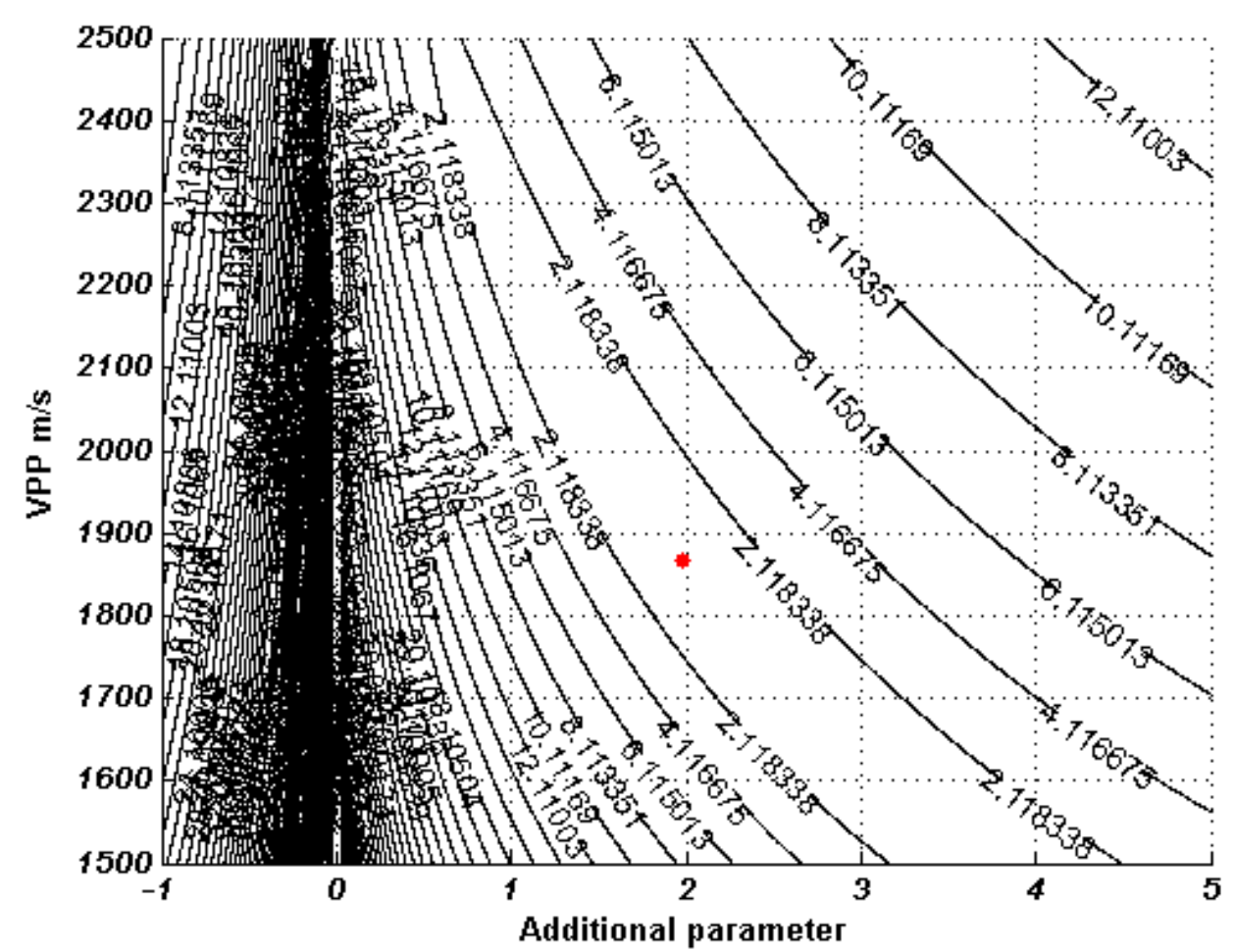

Figure 145: The residual function map which demonstrates the complexity of the Malovichko (1978) approximation of the PP reflection event with L2-norm. Red dispersions represent the global minimum region, and the blue dispersions, the local minimum regions.

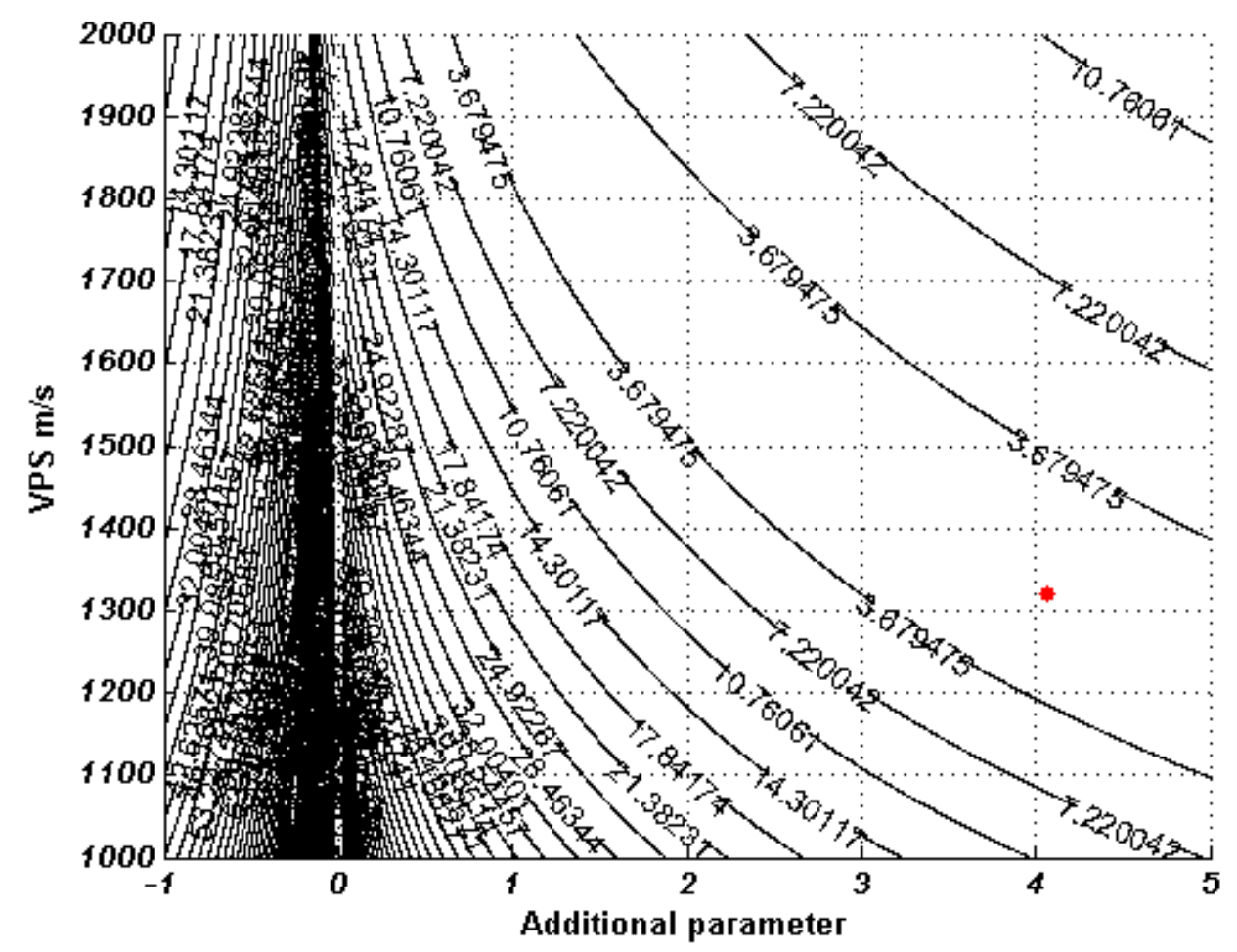

Figure 146: The residual function map which demonstrates the complexity of the Malovichko (1978) approximation of the PS reflection event with L2-norm. Red dispersions represent the global minimum region, and the blue dispersions, the local minimum regions. 


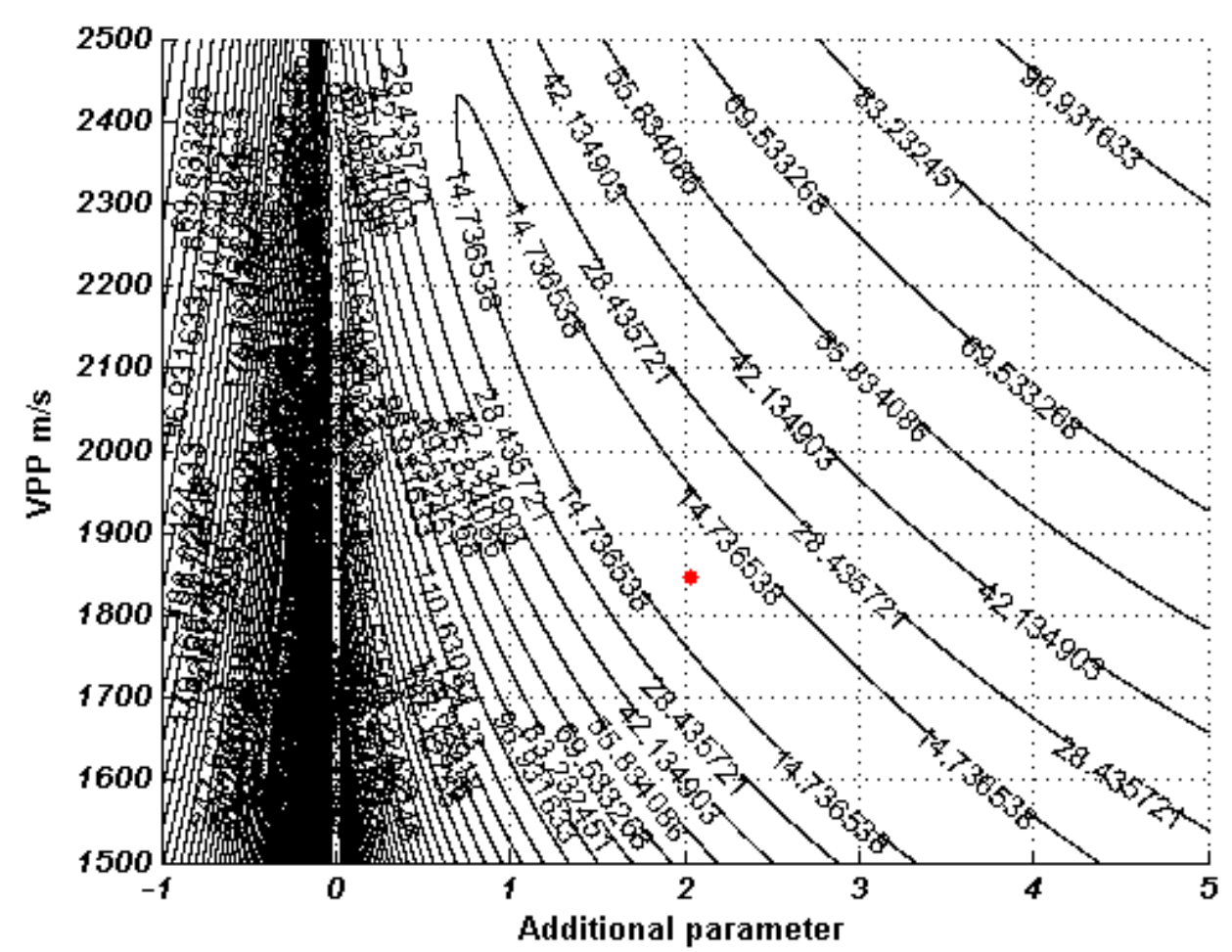

Figure 147: The residual function map which demonstrates the complexity of the Malovichko (1978) approximation of the PP reflection event with L1-norm. Red dispersions represent the global minimum region, and the blue dispersions, the local minimum regions.

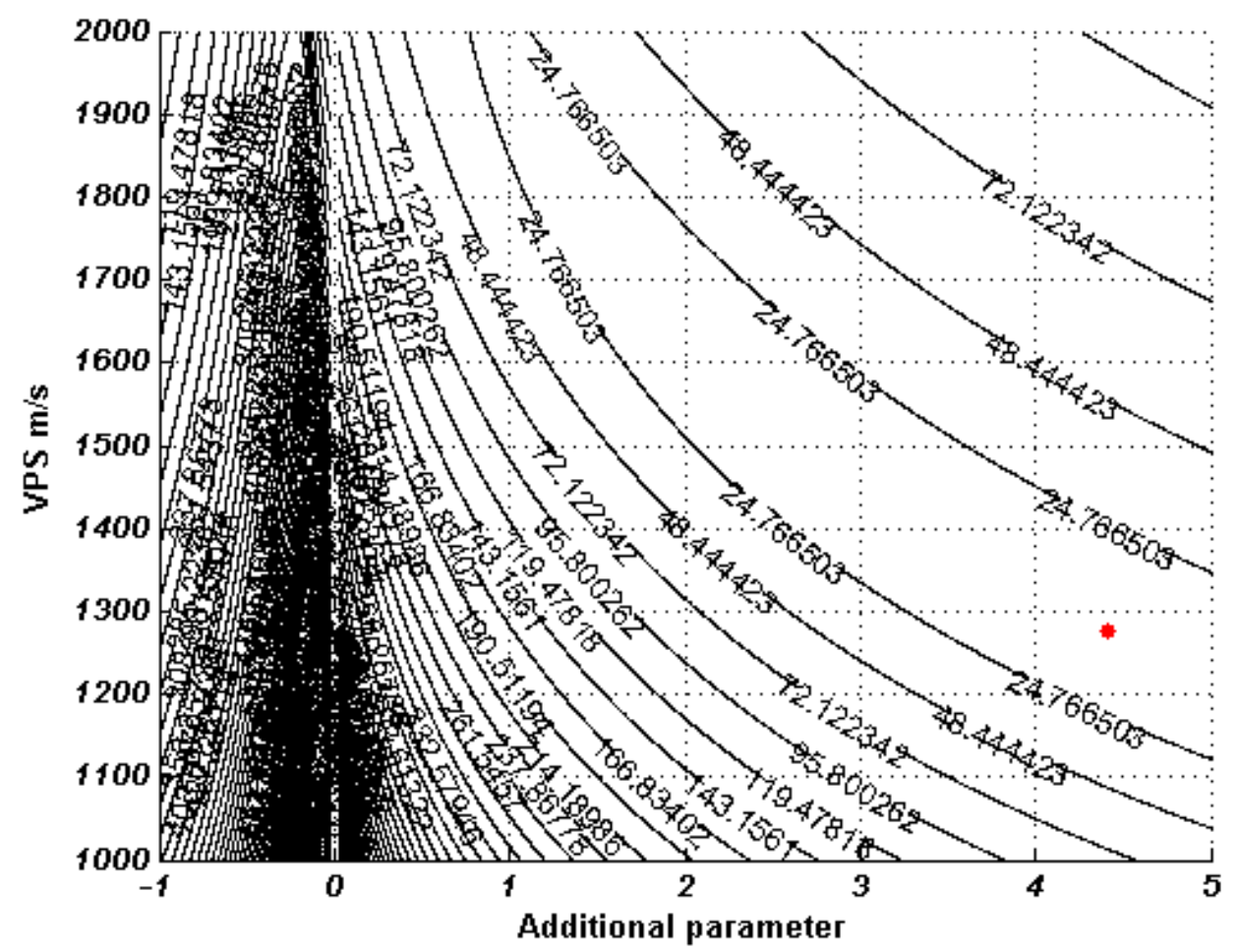

Figure 148: The residual function map which demonstrates the complexity of the Malovichko (1978) approximation of the PS reflection event with L1-norm. Red dispersions represent the global minimum region, and the blue dispersions, the local minimum regions. 


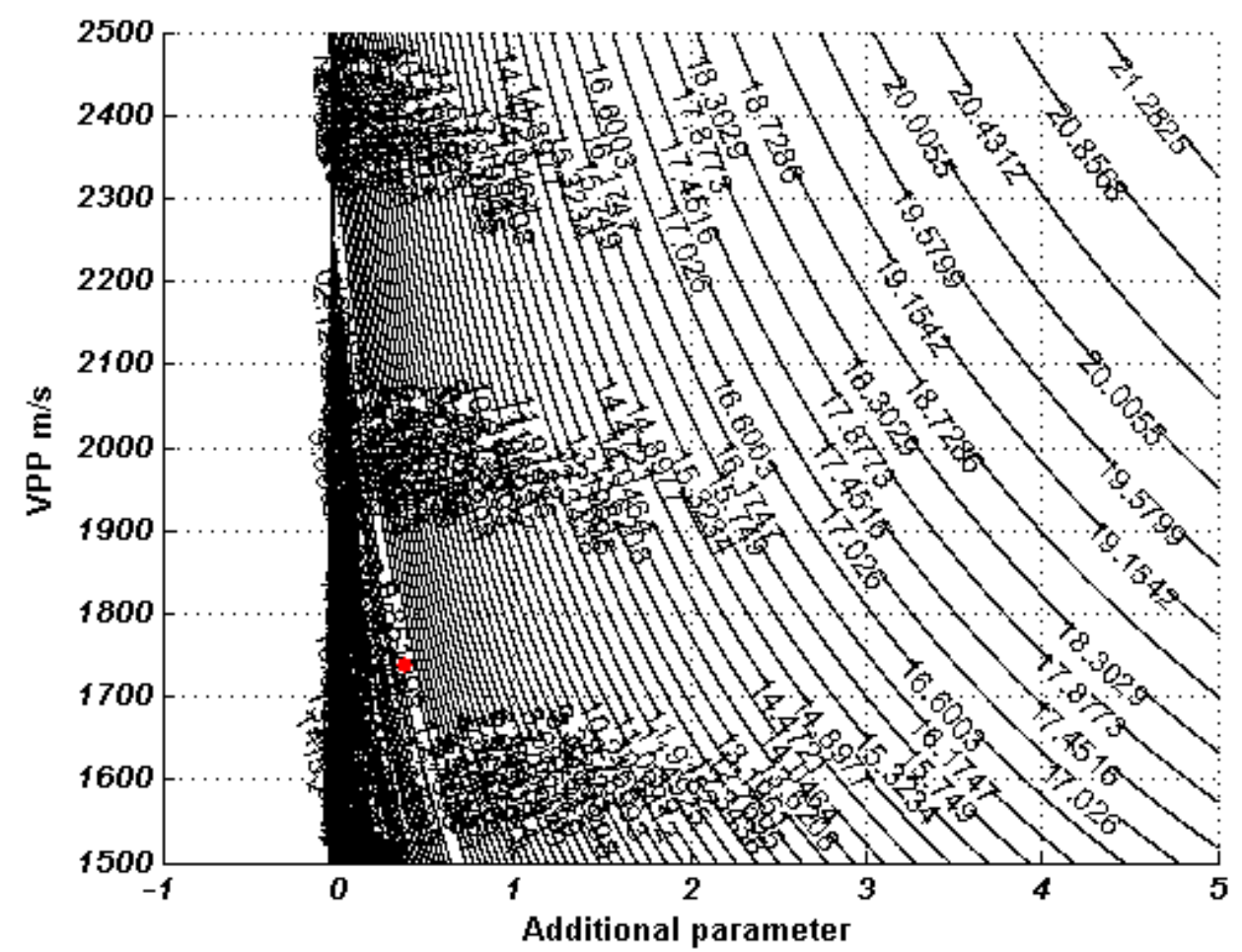

Figure 149: The residual function map which demonstrates the complexity of the Alkhalifah and Tsvankin (1995) approximation of the PP reflection event with L2-norm. Red dispersions represent the global minimum region, and the blue dispersions, the local minimum regions.

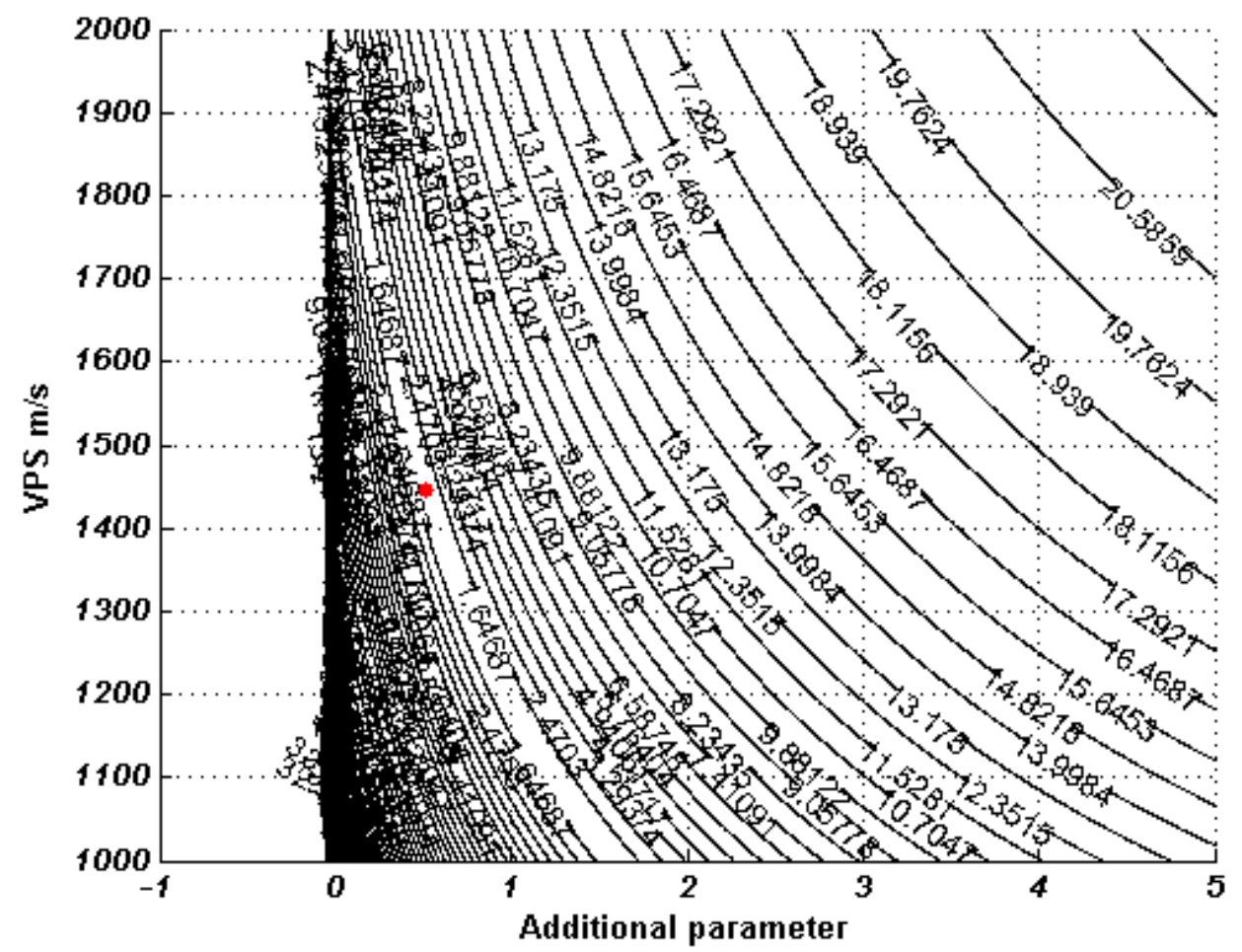

Figure 150: The residual function map which demonstrates the complexity of the Alkhalifah and Tsvankin (1995) approximation of the PS reflection event with L2-norm. Red dispersions represent the global minimum region, and the blue dispersions, the local minimum regions. 


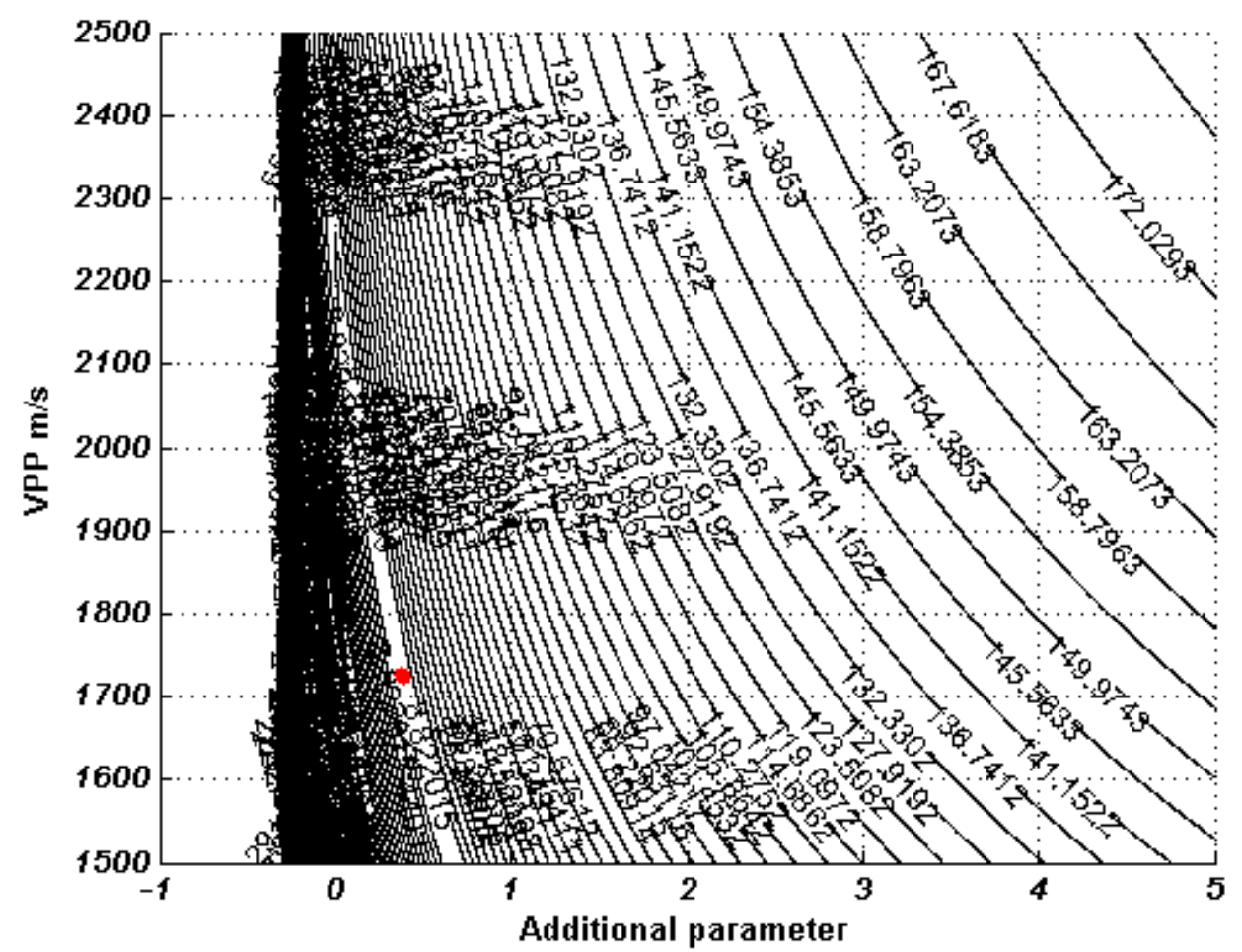

Figure 151: The residual function map which demonstrates the complexity of the Alkhalifah and Tsvankin (1995) approximation of the PP reflection event with L1-norm. Red dispersions represent the global minimum region, and the blue dispersions, the local minimum regions.

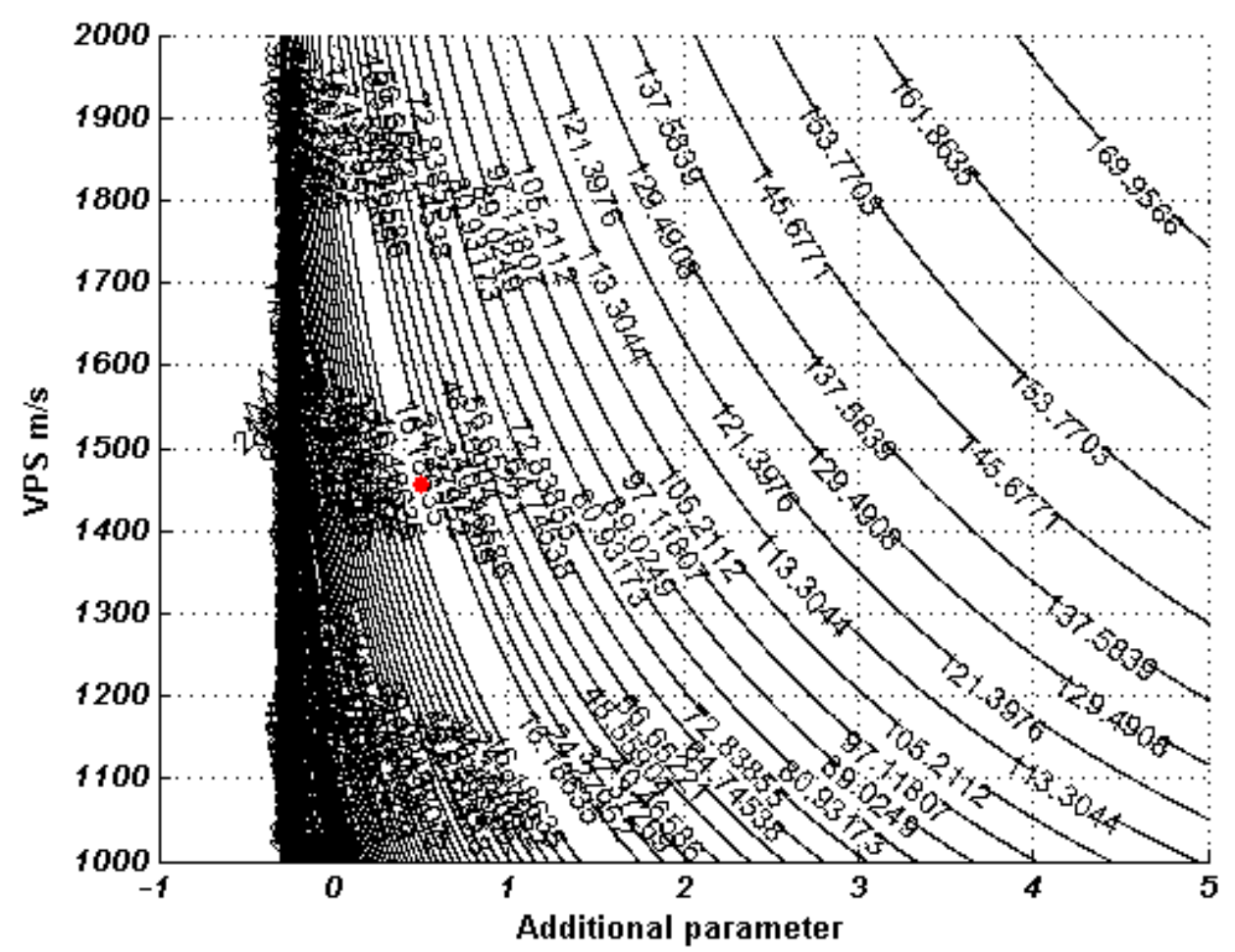

Figure 152: The residual function map which demonstrates the complexity of the Alkhalifah and Tsvankin (1995) approximation of the PS reflection event with L1-norm. Red dispersions represent the global minimum region, and the blue dispersions, the local minimum regions. 


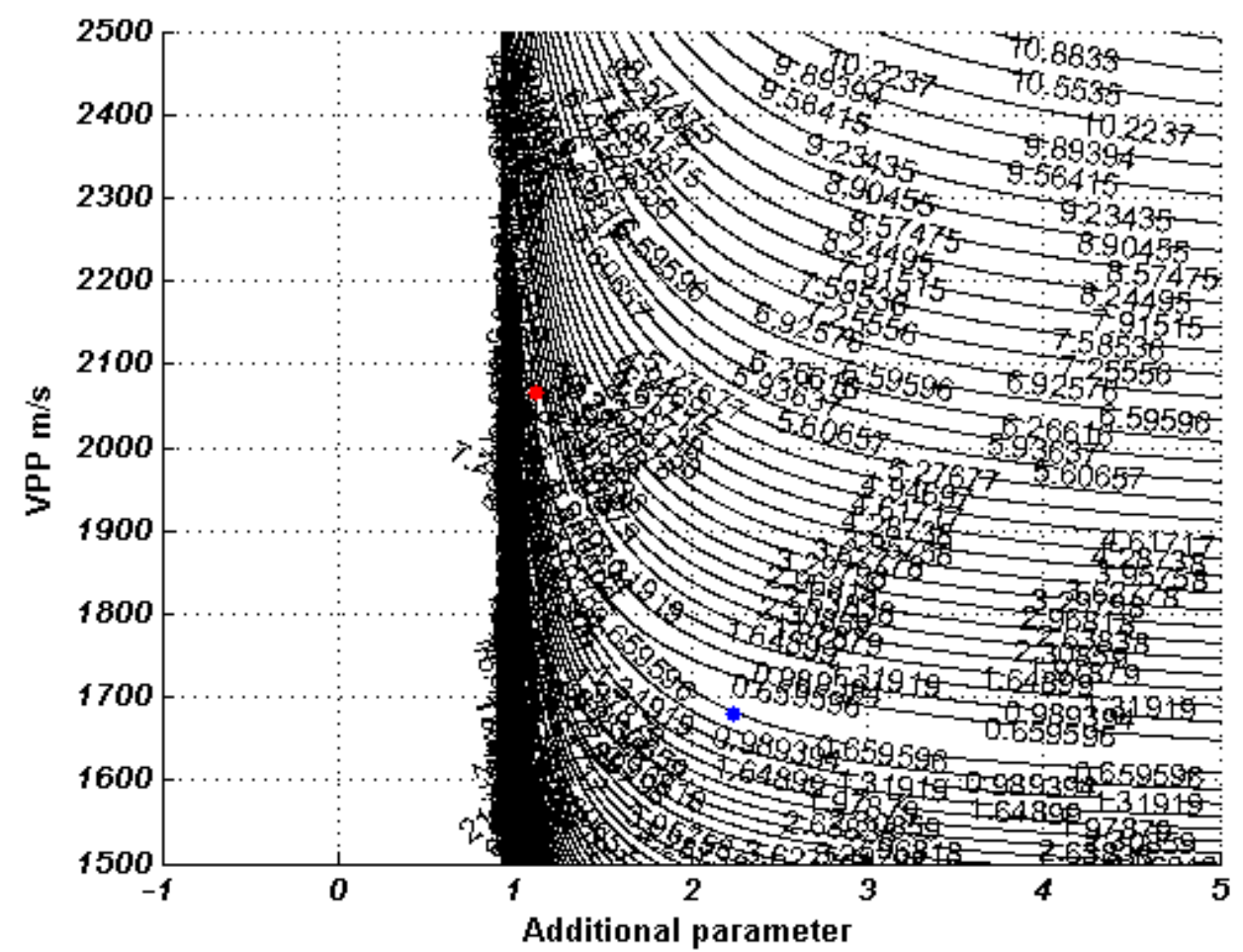

Figure 153: The residual function map which demonstrates the complexity of the Ursin and Stovas (2006) approximation of the PP reflection event with L2-norm. Red dispersions represent the global minimum region, and the blue dispersions, the local minimum regions.

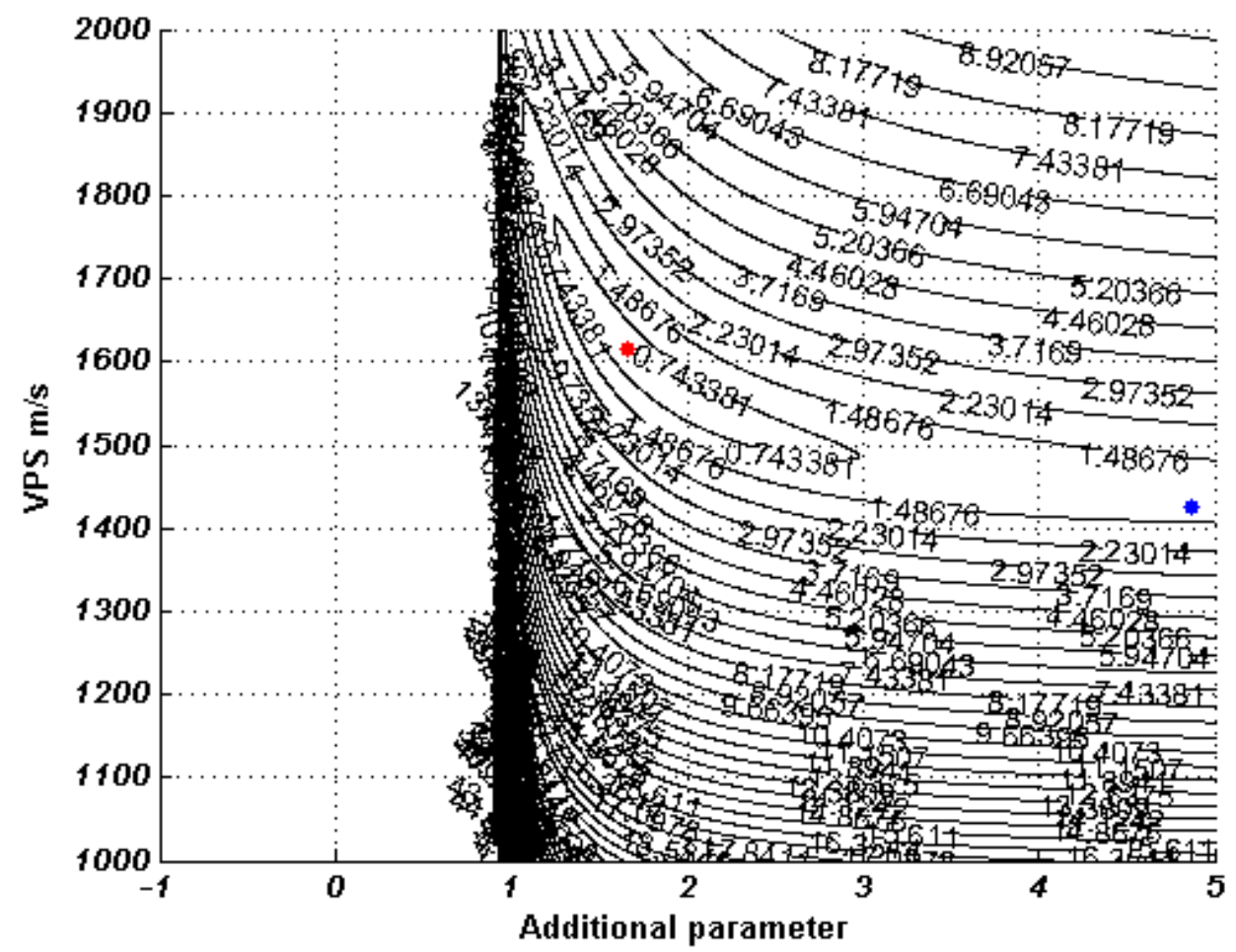

Figure 154: The residual function map which demonstrates the complexity of the Ursin and Stovas (2006) approximation of the PS reflection event with L2-norm. Red dispersions represent the global minimum region, and the blue dispersions, the local minimum regions. 


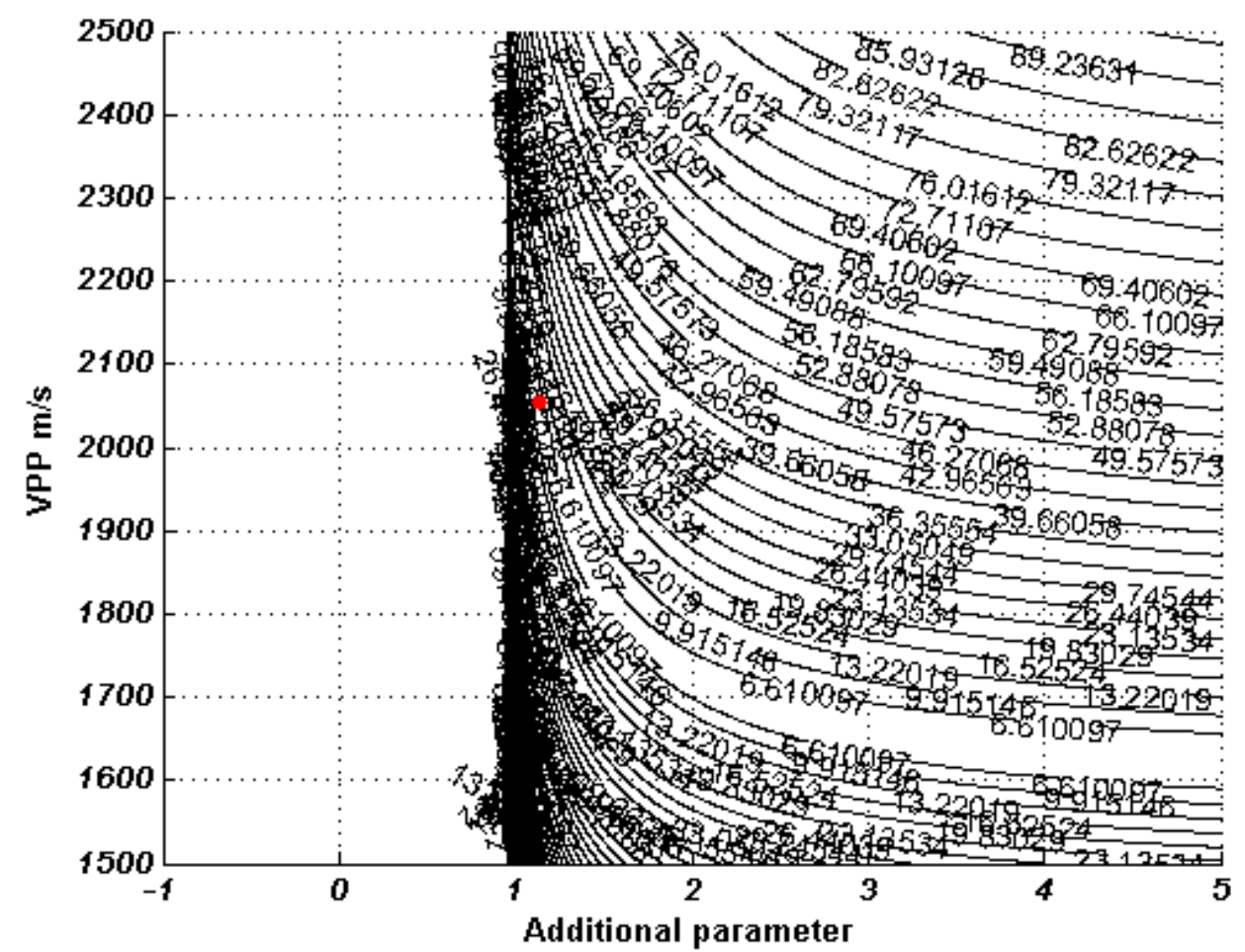

Figure 155: The residual function map which demonstrates the complexity of the Ursin and Stovas (2006) approximation of the PP reflection event with L1-norm. Red dispersions represent the global minimum region, and the blue dispersions, the local minimum regions.

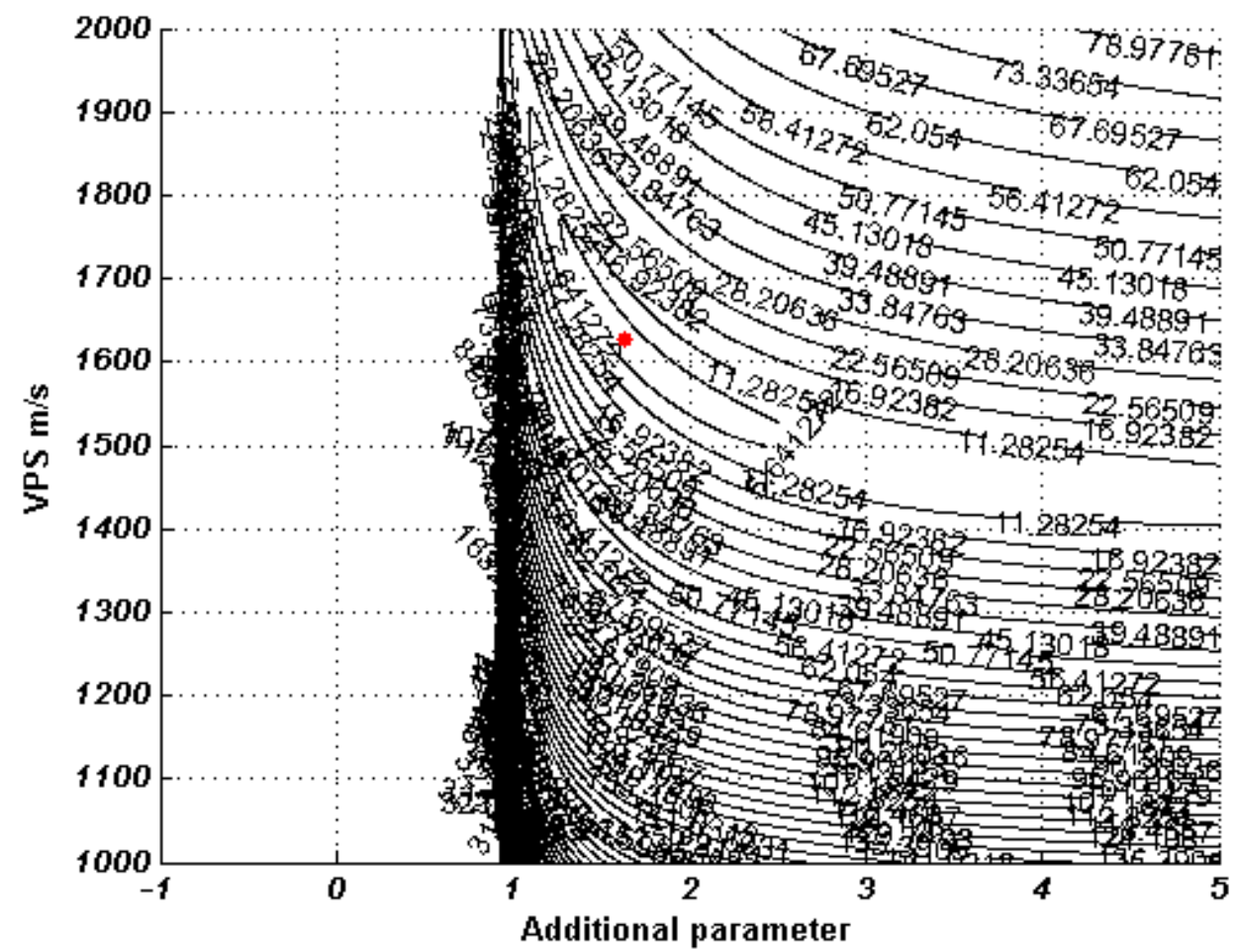

Figure 156: The residual function map which demonstrates the complexity of the Ursin and Stovas (2006) approximation of the PS reflection event with L1-norm. Red dispersions represent the global minimum region, and the blue dispersions, the local minimum regions. 


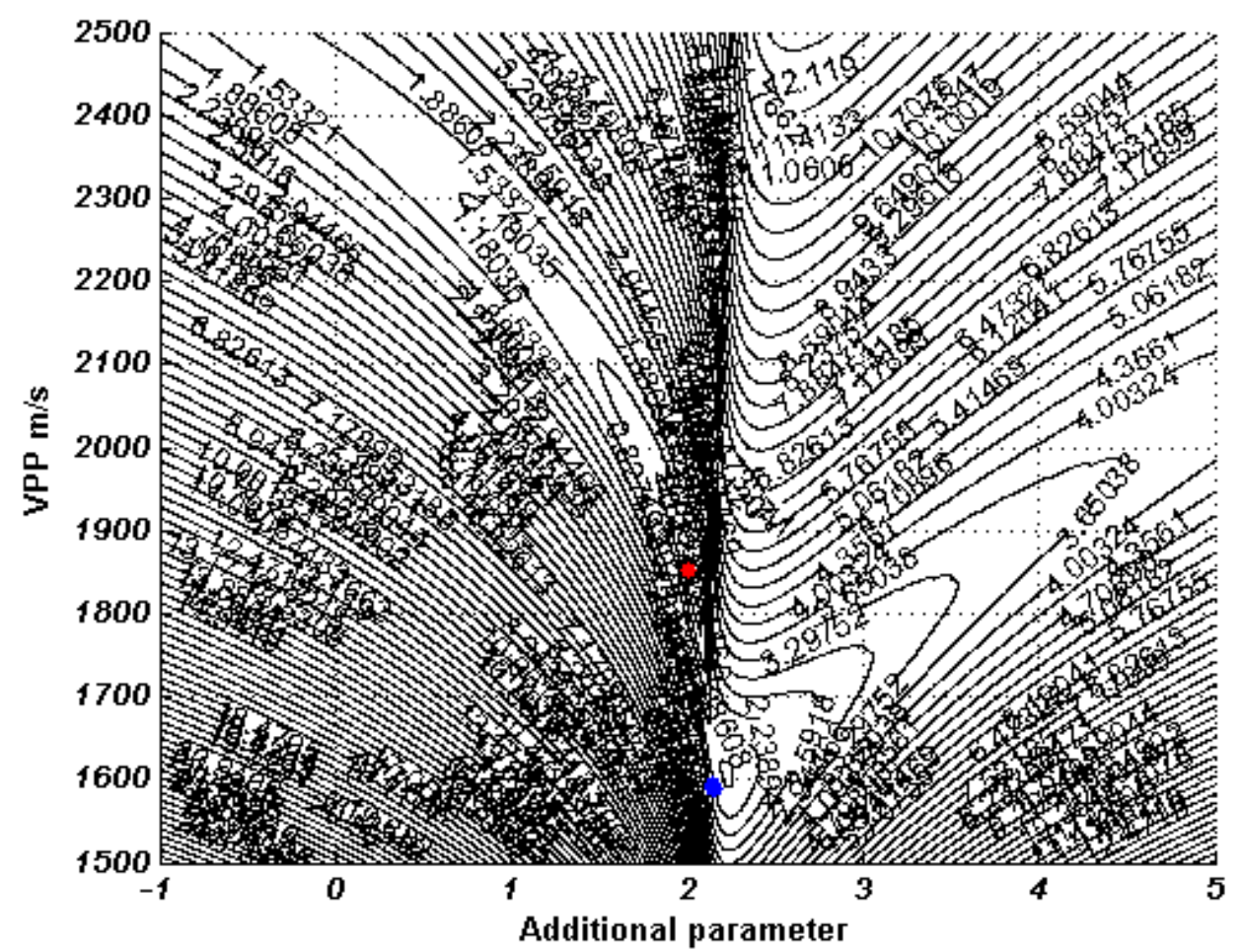

Figure 157: The residual function map which demonstrates the complexity of the Blias (2009) approximation of the PP reflection event with L2-norm. Red dispersions represent the global minimum region, and the blue dispersions, the local minimum regions.

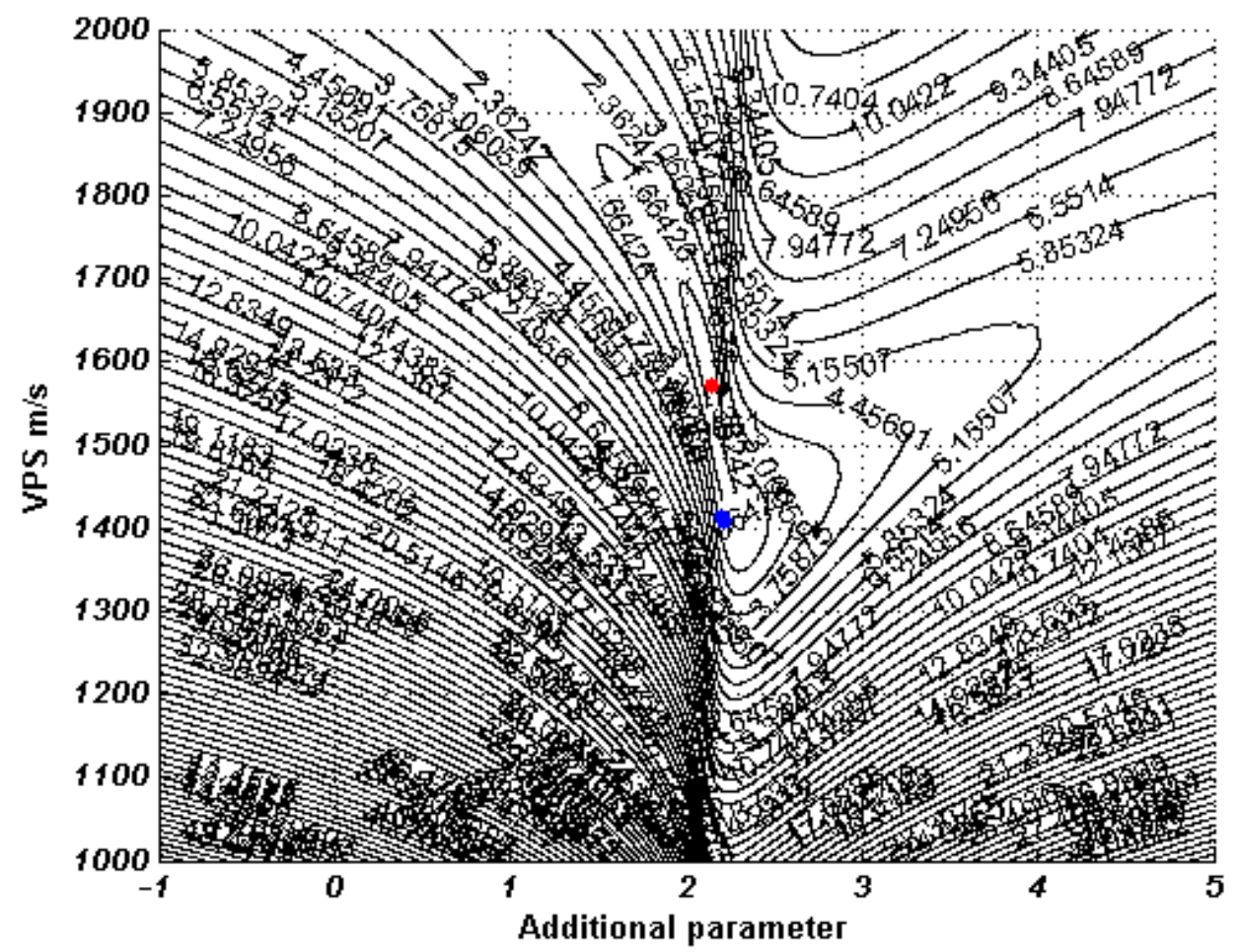

Figure 158: The residual function map which demonstrates the complexity of the Blias (2009) approximation of the PS reflection event with L2-norm. Red dispersions represent the global minimum region, and the blue dispersions, the local minimum regions. 


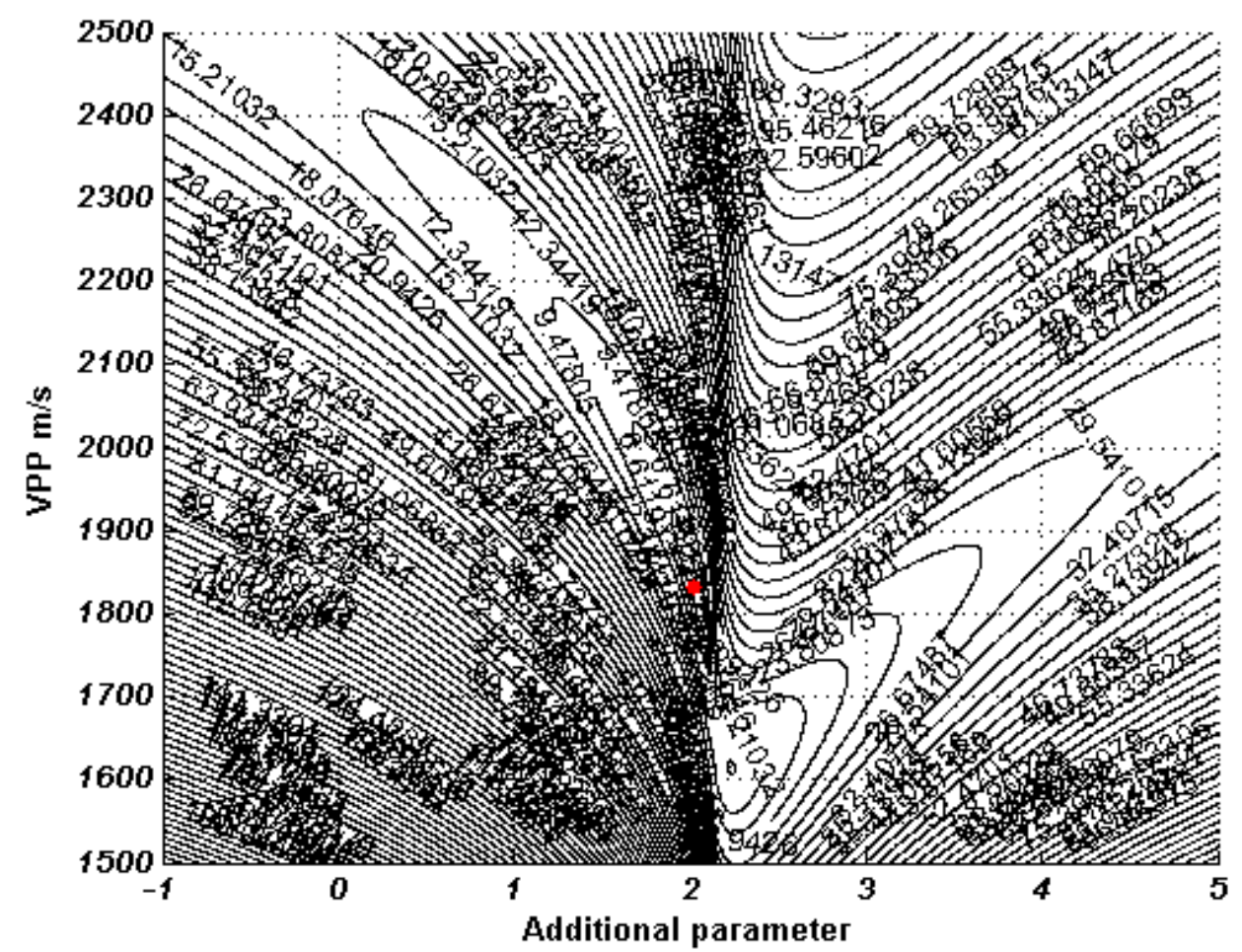

Figure 159: The residual function map which demonstrates the complexity of the Blias (2009) approximation of the PP reflection event with L1-norm. Red dispersions represent the global minimum region, and the blue dispersions, the local minimum regions.

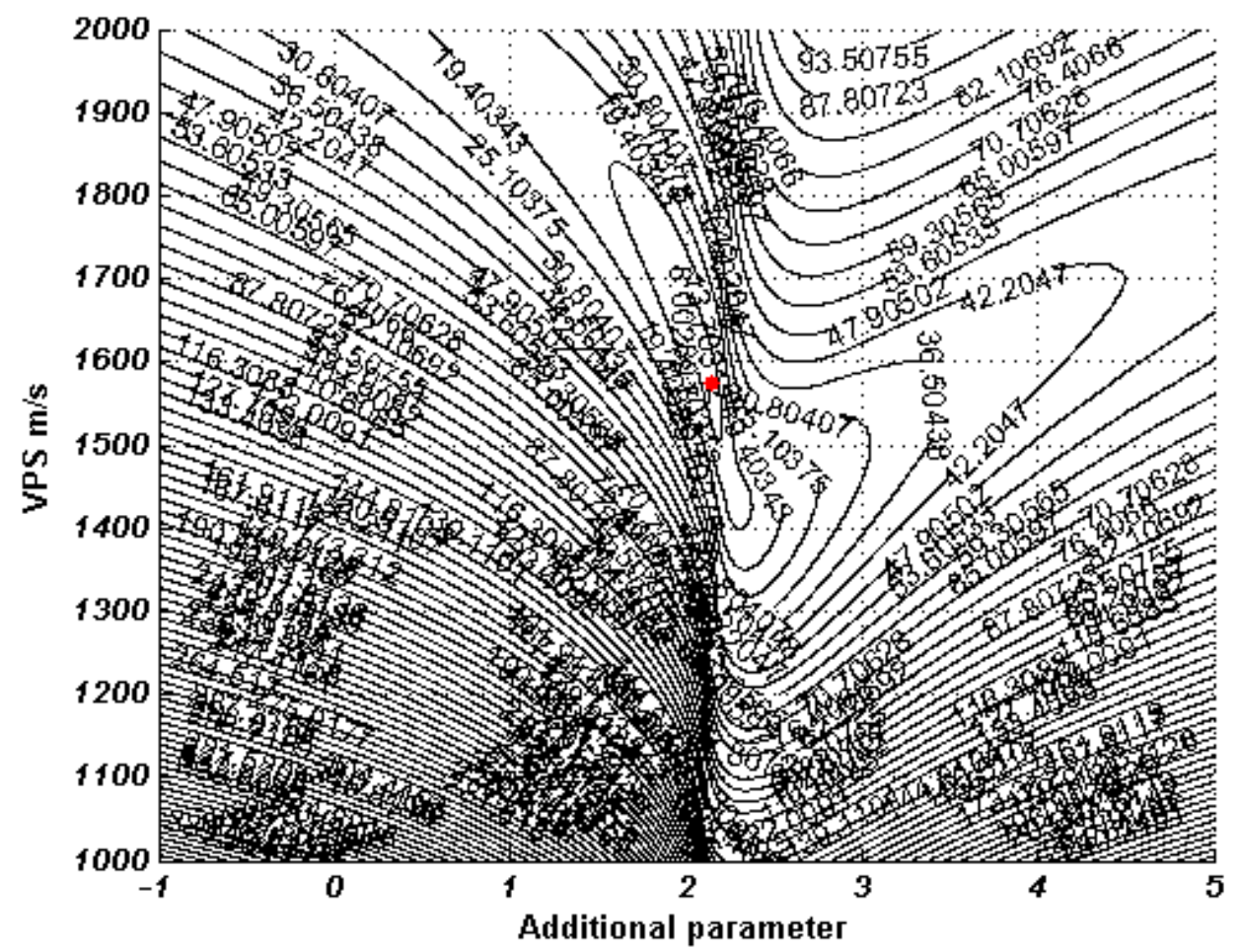

Figure 160: The residual function map which demonstrates the complexity of the Blias (2009) approximation of the PS reflection event with L1-norm. Red dispersions represent the global minimum region, and the blue dispersions, the local minimum regions. 


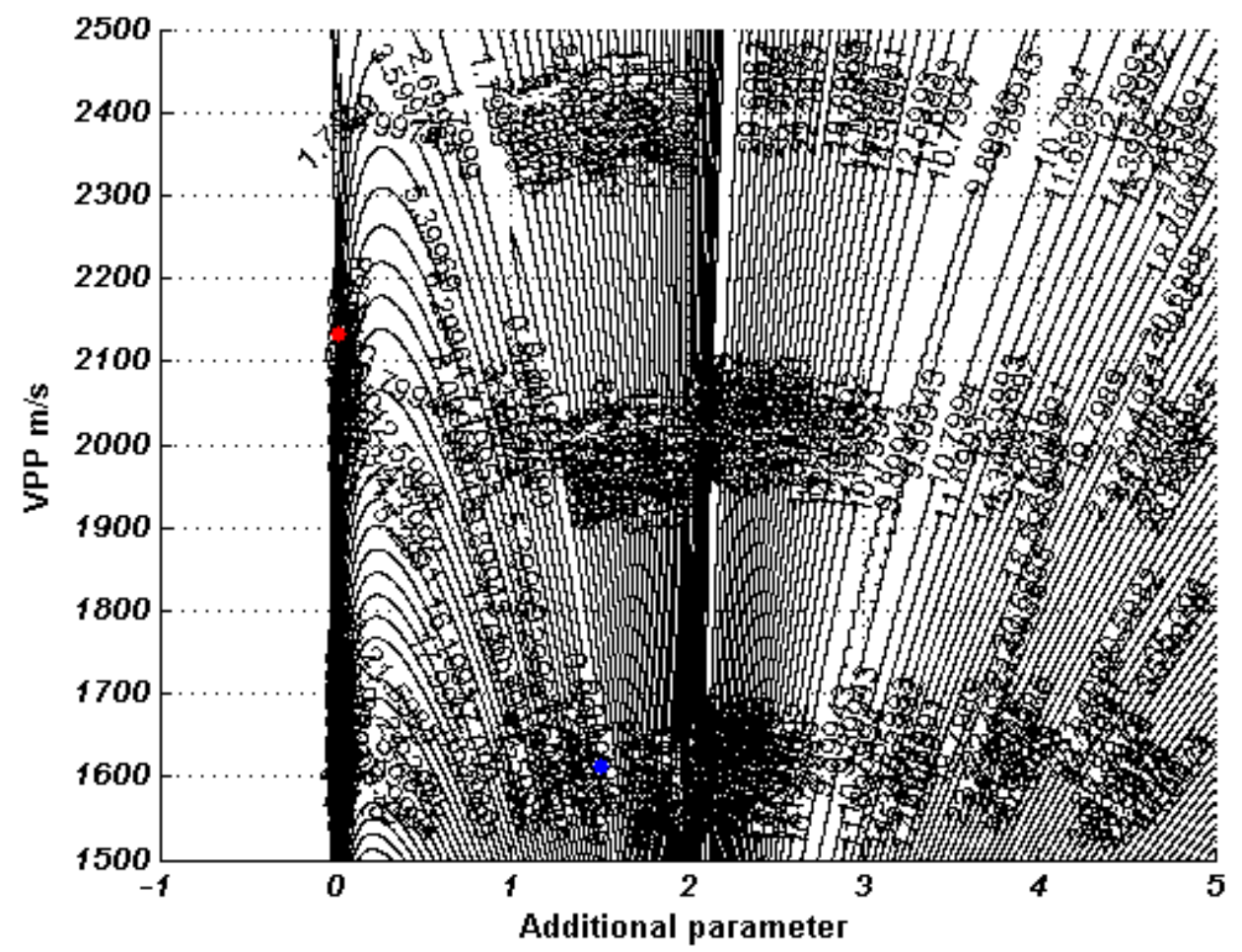

Figure 161: The residual function map which demonstrates the complexity of the Muir and Dellinger (1985) approximation of the PP reflection event with L2-norm. Red dispersions represent the global minimum region, and the blue dispersions, the local minimum regions.

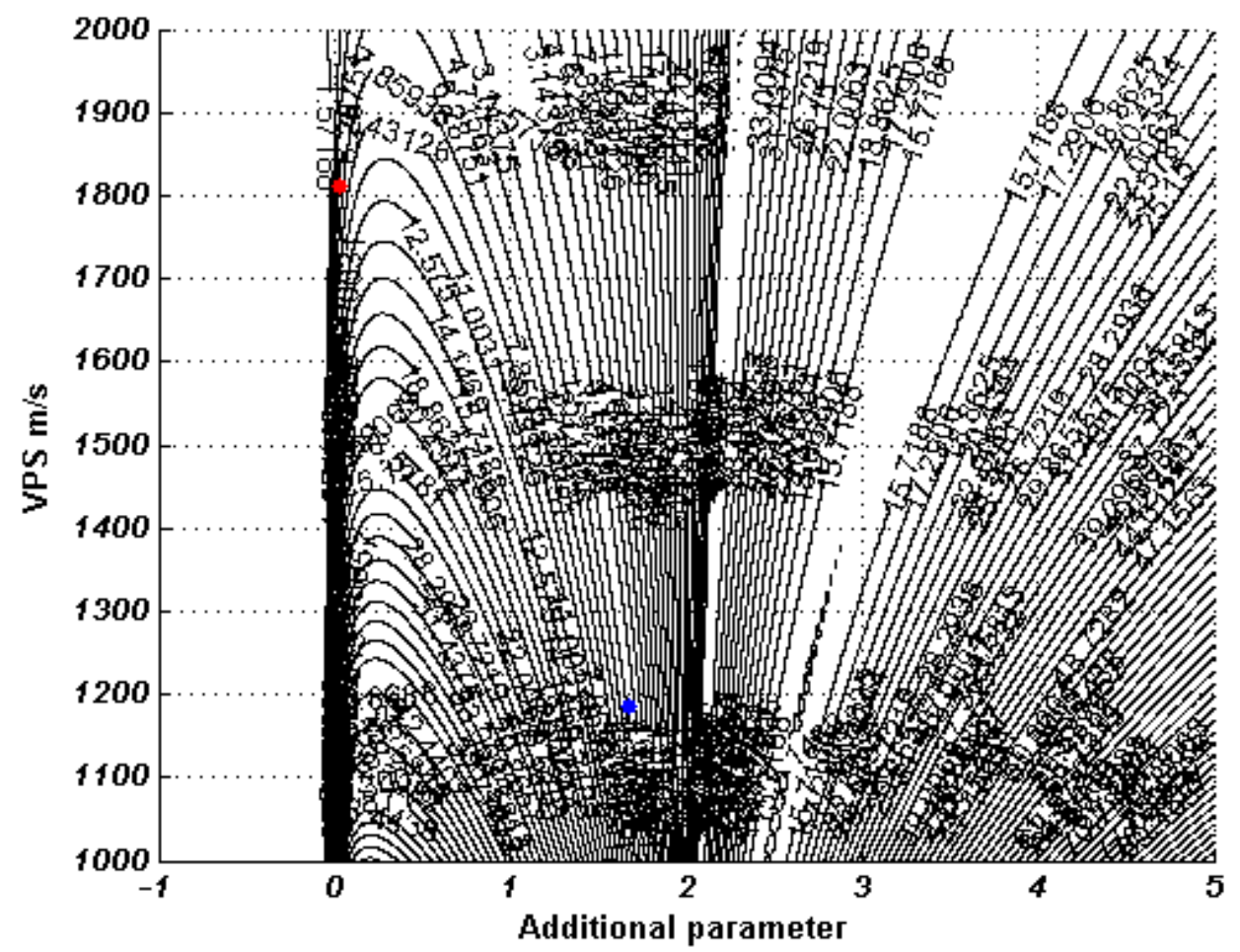

Figure 162: The residual function map which demonstrates the complexity of the Muir and Dellinger (1985) approximation of the PS reflection event with L2-norm. Red dispersions represent the global minimum region, and the blue dispersions, the local minimum regions. 


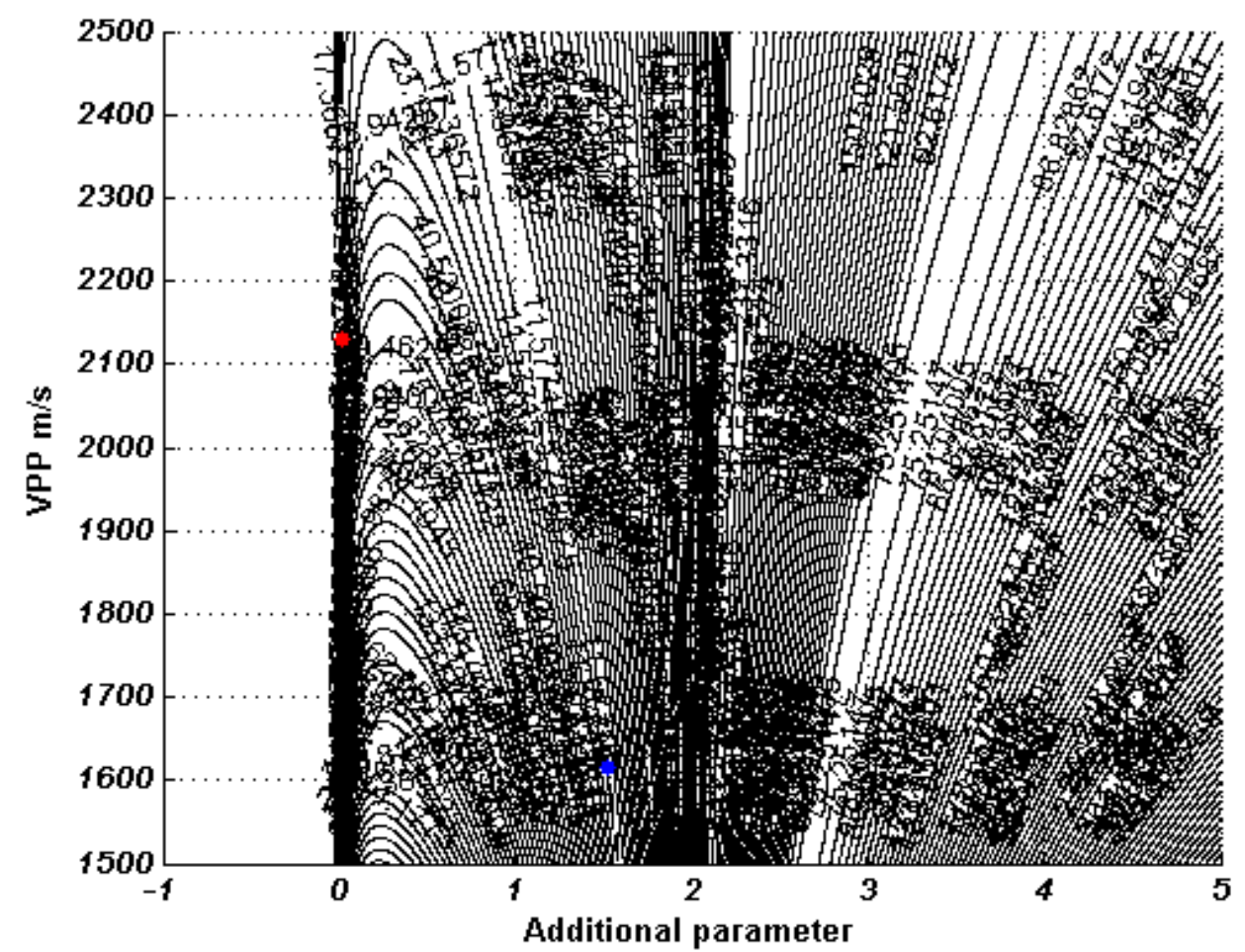

Figure 163: The residual function map which demonstrates the complexity of the Muir and Dellinger (1985) approximation of the PP reflection event with L1-norm. Red dispersions represent the global minimum region, and the blue dispersions, the local minimum regions.

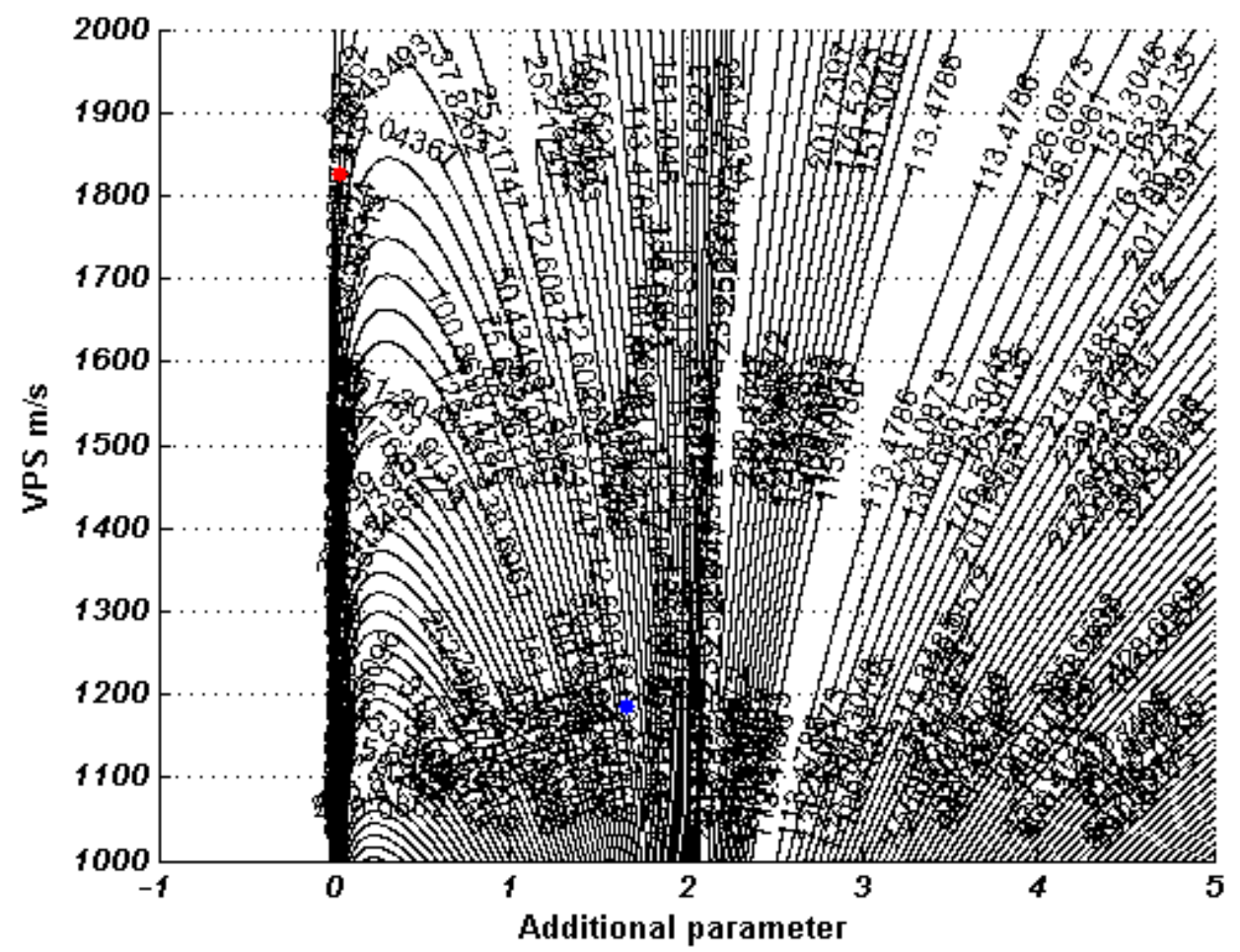

Figure 164: The residual function map which demonstrates the complexity of the Muir and Dellinger (1985) approximation of the PS reflection event with L1-norm. Red dispersions represent the global minimum region, and the blue dispersions, the local minimum regions. 


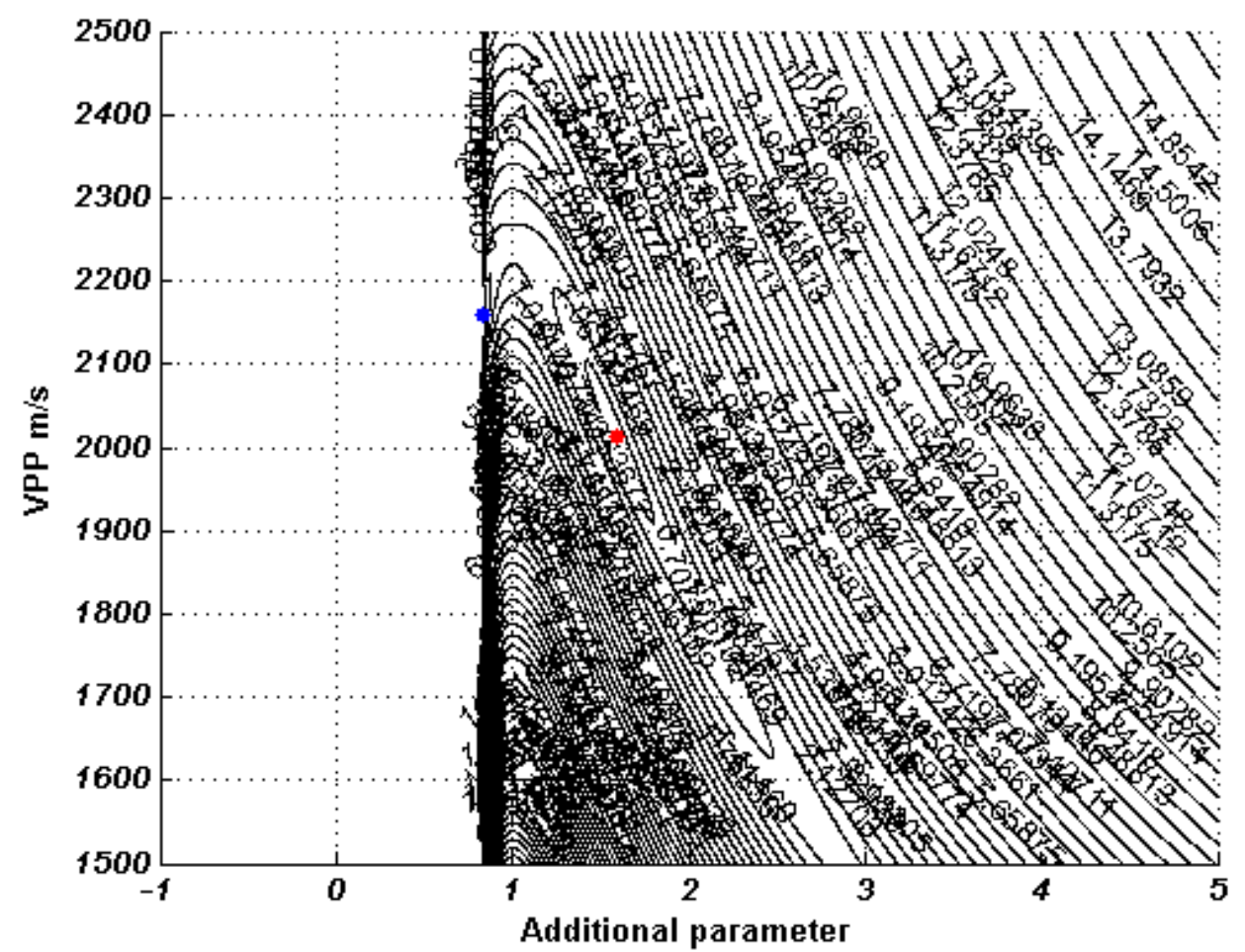

Figure 165: The residual function map which demonstrates the complexity of the Li and Yuan (2001) approximation of the PP reflection event with L2-norm. Red dispersions represent the global minimum region, and the blue dispersions, the local minimum regions.

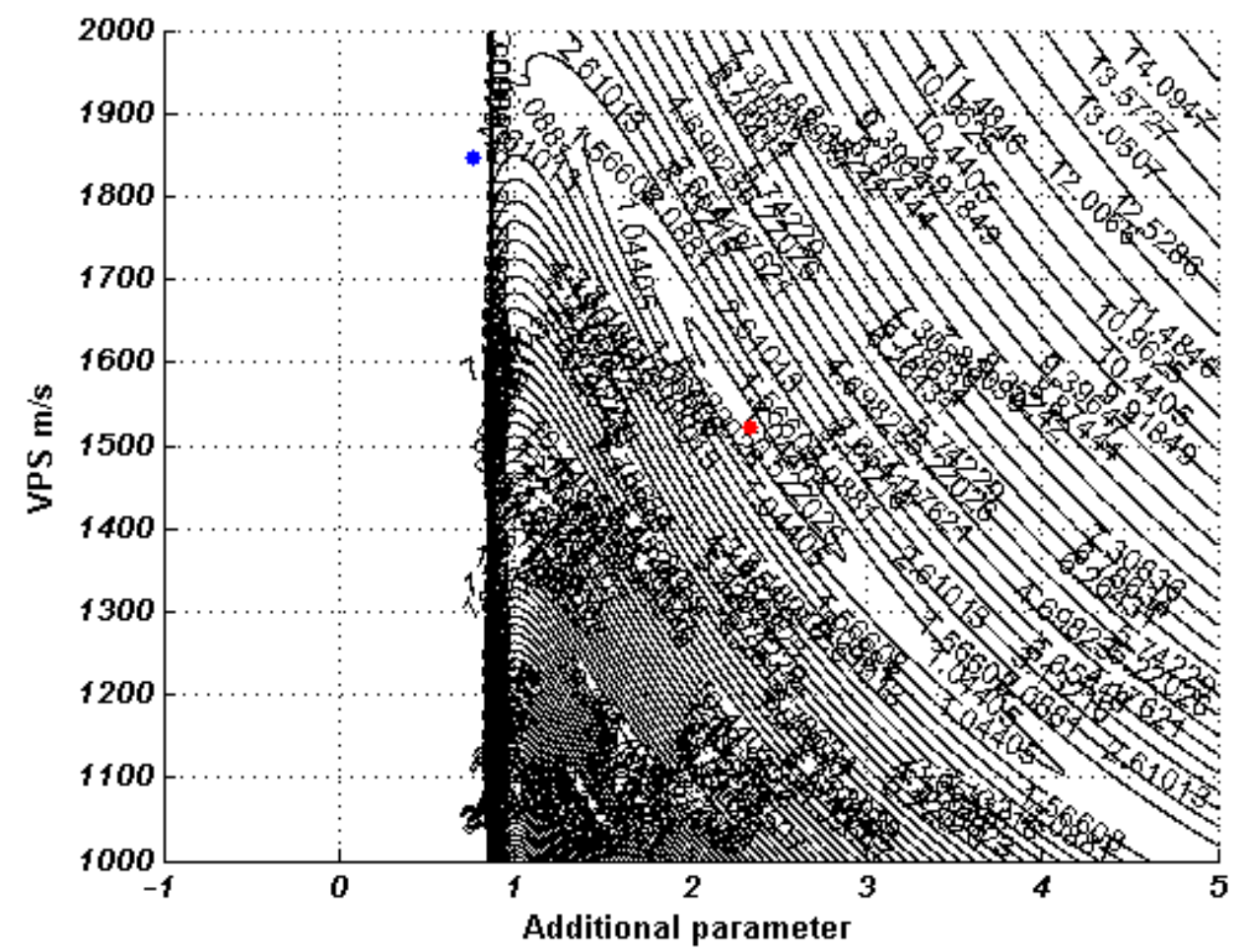

Figure 166: The residual function map which demonstrates the complexity of the Li and Yuan (2001) approximation of the PS reflection event with L2-norm. Red dispersions represent the global minimum region, and the blue dispersions, the local minimum regions. 


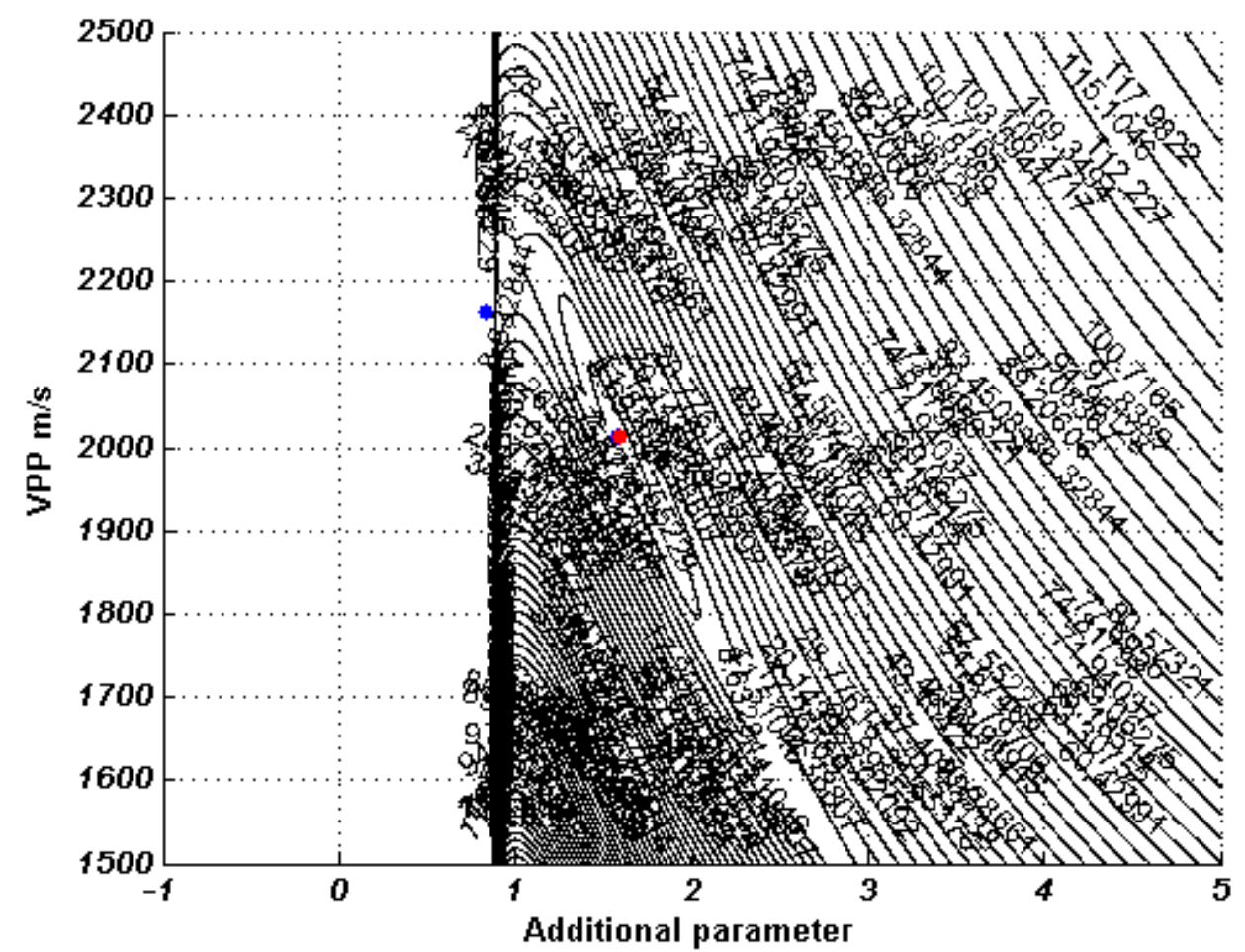

Figure 167: The residual function map which demonstrates the complexity of the Li and Yuan (2001) approximation of the PP reflection event with L1-norm. Red dispersions represent the global minimum region, and the blue dispersions, the local minimum regions.

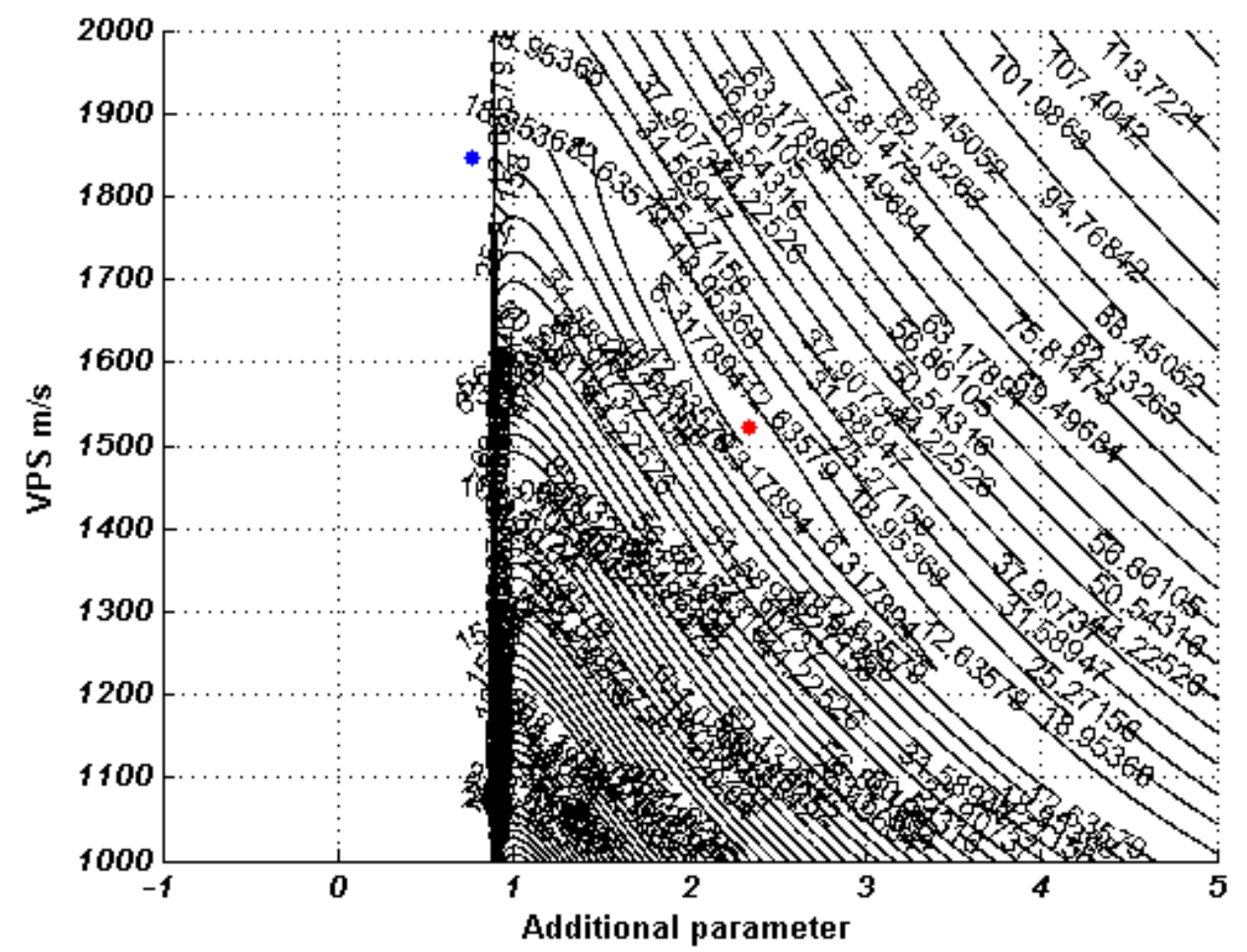

Figure 168: The residual function map which demonstrates the complexity of the Li and Yuan (2001) approximation of the PS reflection event with L1-norm. Red dispersions represent the global minimum region, and the blue dispersions, the local minimum regions. 


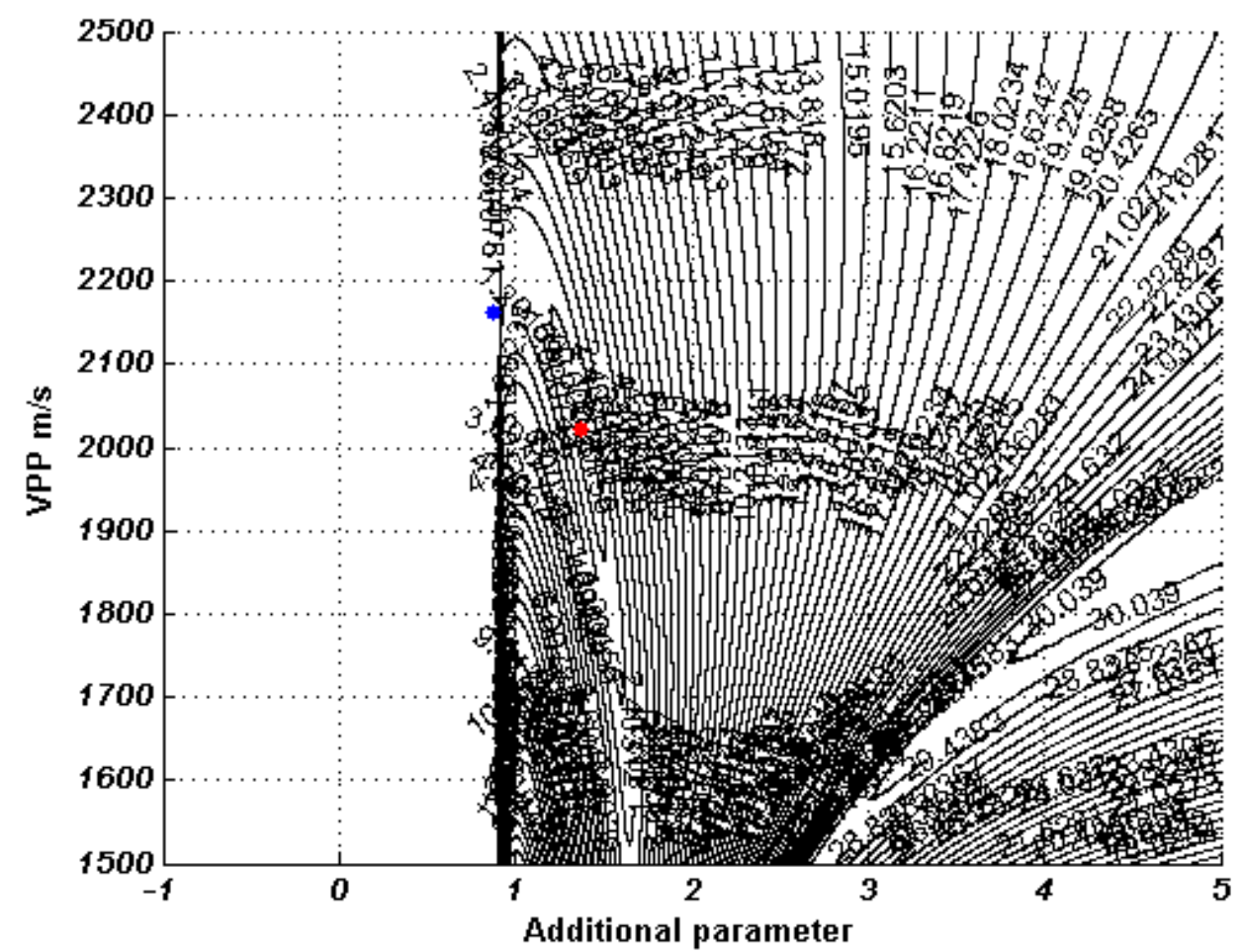

Figure 169: The residual function map which demonstrates the complexity of the approximation proposed in this work of the PP reflection event with L2-norm. Red dispersions represent the global minimum region, and the blue dispersions, the local minimum regions.

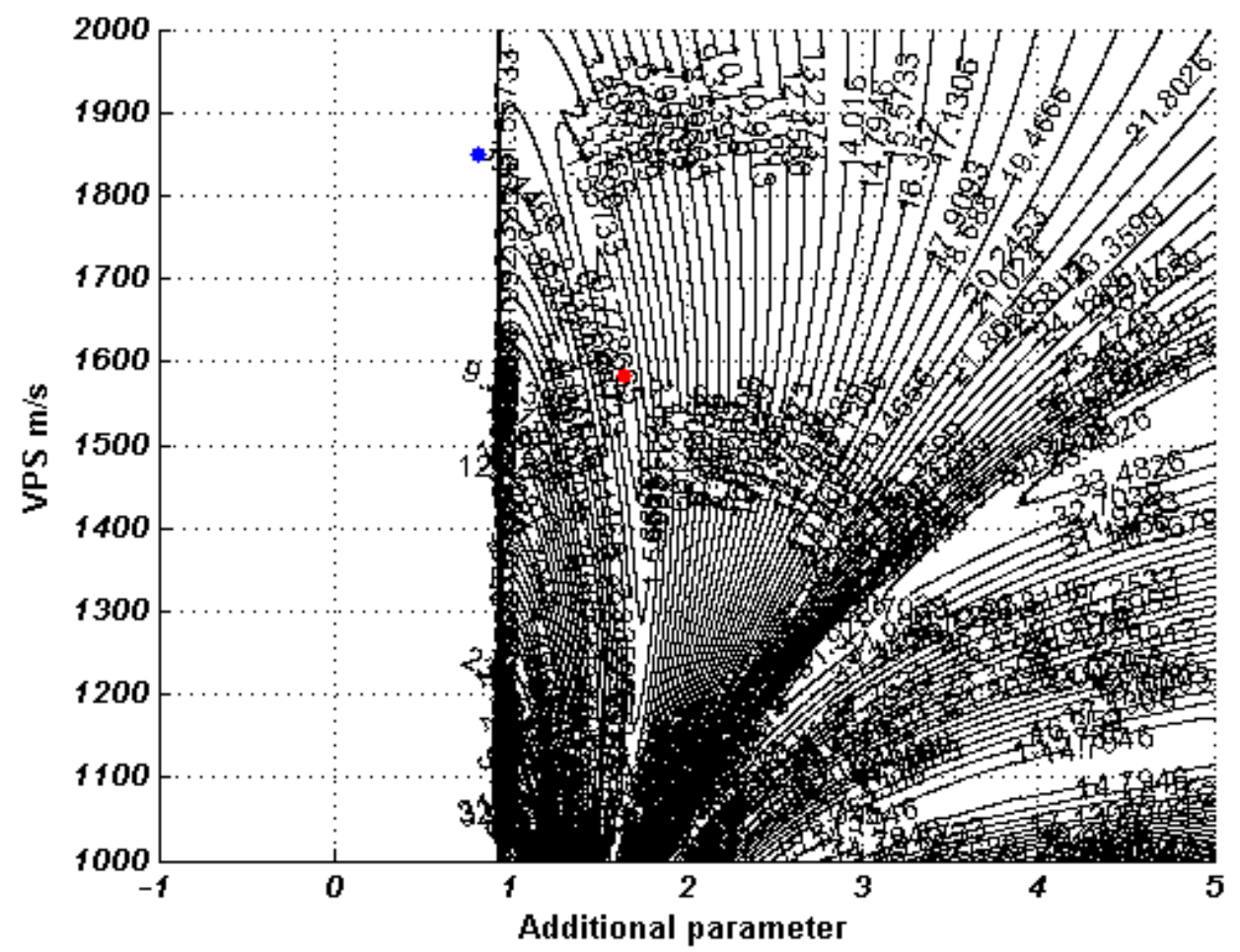

Figure 170: The residual function map which demonstrates the complexity of the approximation proposed in this work of the PS reflection event with L2-norm. Red dispersions represent the global minimum region, and the blue dispersions, the local minimum regions. 


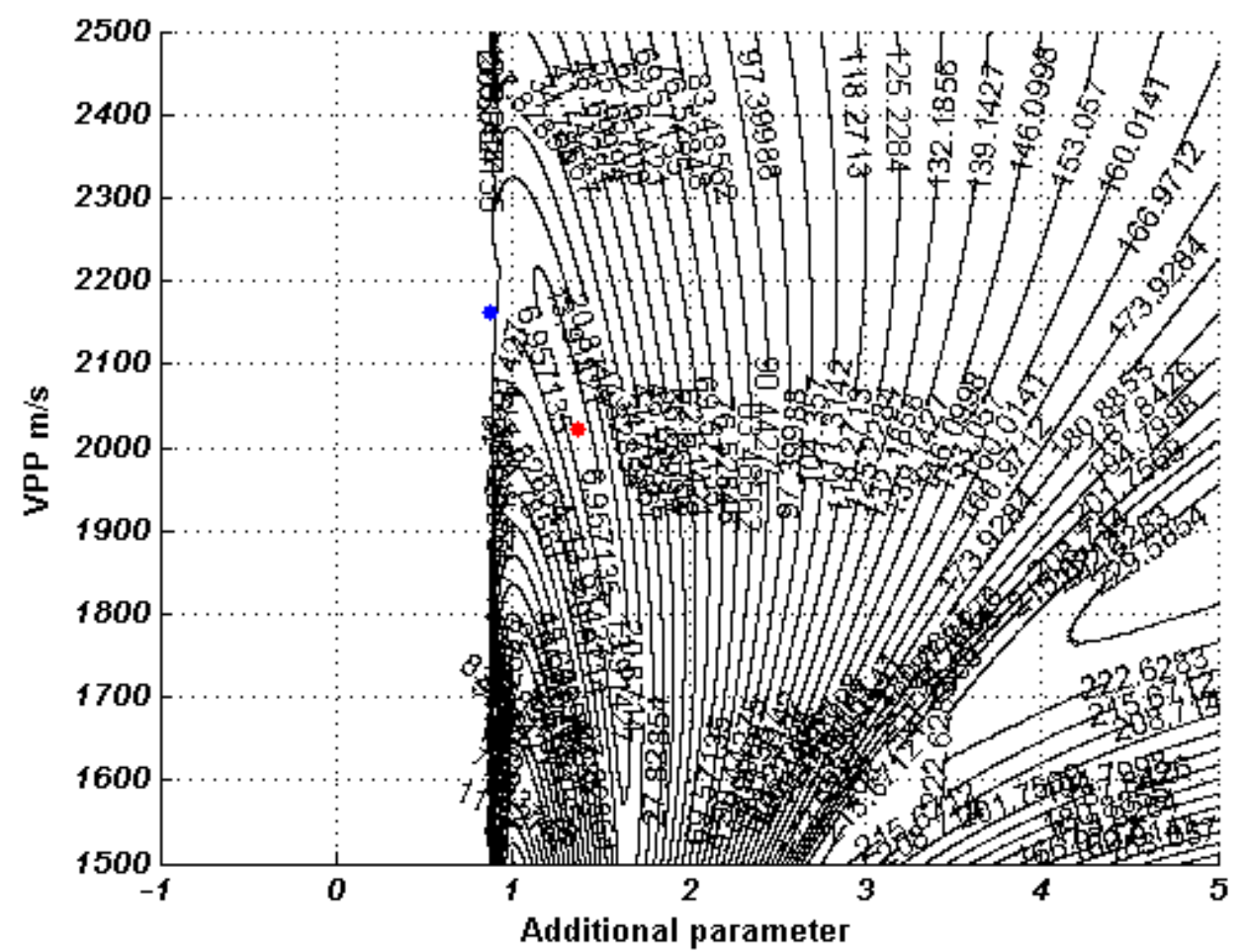

Figure 171: The residual function map which demonstrates the complexity of the approximation proposed in this work of the PP reflection event with L1-norm. Red dispersions represent the global minimum region, and the blue dispersions, the local minimum regions.

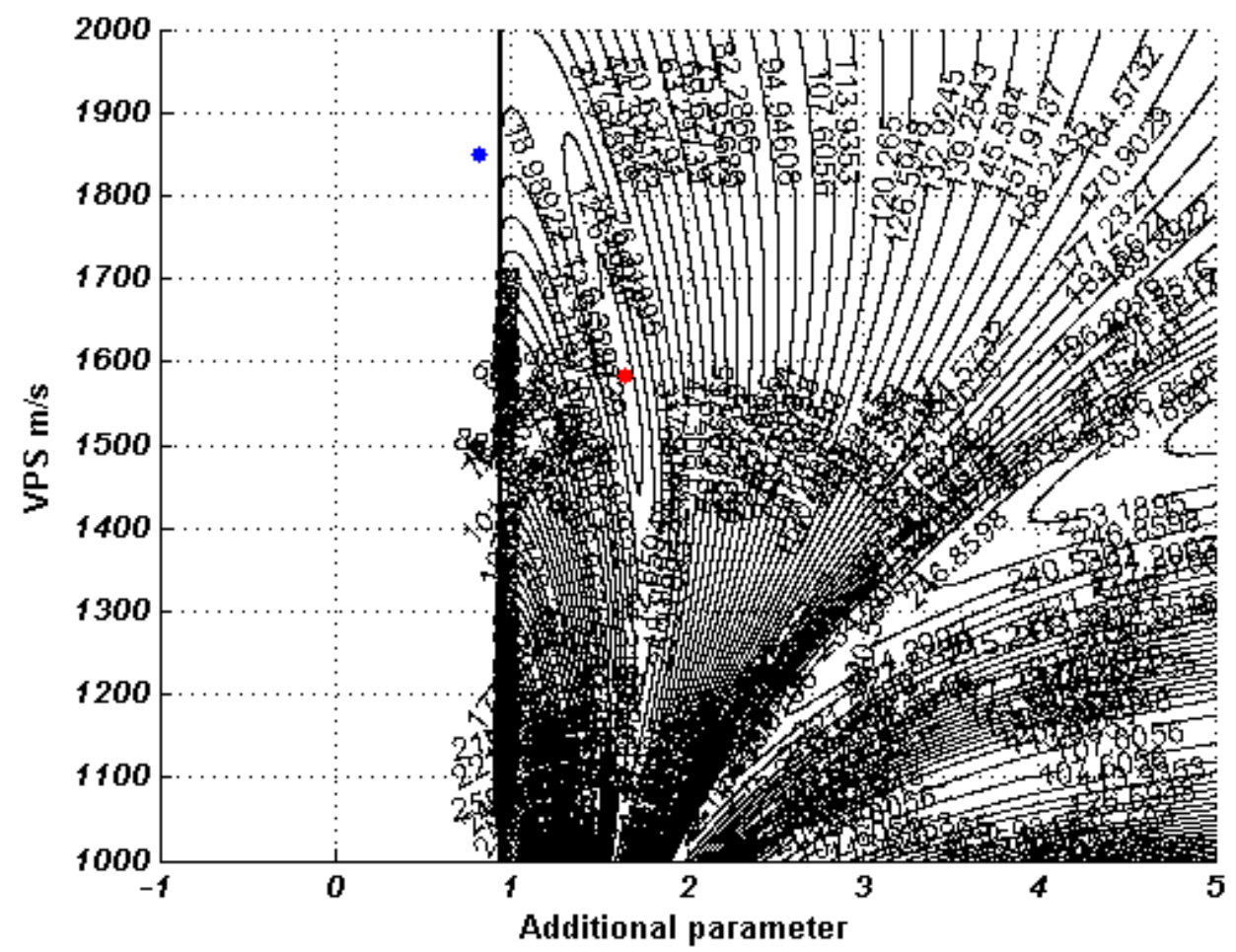

Figure 172: The residual function map which demonstrates the complexity of the approximation proposed in this work of the PS reflection event with L1-norm. Red dispersions represent the global minimum region, and the blue dispersions, the local minimum regions. 


\section{RESIDUAL TRAVEL-TIME CURVE ANALYSIS}

After the calculated curve is fitted to the observed one, there still is a residual error between the two curves. This error is associated to the objective function (minimum value), and it represents a solution related to the set of parameters that is the best characterization for the analysed model with the referent approximation. Therefore, the calculated curve, with the parameters related to the global minimum, represents the best possible solution among the set of inversions.

It is commonly difficult to compare two or more approximations in travel-time curves scale due to the quality of the obtained fit. For this reason, it is necessary to perform the residual analysis between the observed curve and the calculated one (the absolute difference between them). Thus, it is possible to observe the difference in the quality of the fit in a smaller scale. Then, it is possible to observe more accurately the regions in which the curve fits the best.

The most accurate curve is the one closer to the zero all along the offset; however, some approximations showed a better fitting in different regions of the curve. For this reason, it is necessary to consider not only the absolute quality of the fitting all along the offset, but also prioritize a better fitting closer to the time for zero offset, once it is one of the three parameters to be recovered during the inversion. So, an approximation that shows a very good fitting all along the curve, but not a good fitting closer to time for zero offset is not considered a good fitting, once the value of this parameter would be recovered with a higher error.

\subsection{Model 1}

At first, it is important to remember that the hyperbola equation (Dix, 1955) is being presented only for a comparison effect and due to its simplicity; the hyperbolic approach presented the worst results in all reflections events, for both norms, and all models. It was possible to observe the low quality of the curve fitting in the Figures 173 and 174, where for PS events it reached an error of almost $10 \%$ with the simplest optimization algorithm.

Another important confirmation concerns the results of the approximation proposed by Alkhalifah and Tsvankin (1995), which showed the worst results among the nonhyperbolic 
multiparametric travel-time approximations for all scenarios tested. It was possible to observe that, in the beginning of the curve, it was less accurate than the other nonhyperbolic approximations (Figure 176), even for the PS reflection event — when this approximation was showing a better fitting at the beginning of the curve - its accuracy had a very low quality for the rest of the curve (Figure 175).

A similar condition was observed in the behaviour of the Muir and Dellinger (1985) approximation, which presented the second worst set of results among the approximations with three parameters for all models, norms and reflection events tested in this work, and it was easily observable that, even with a better optimization algorithm, its accuracy was not so good (Figures 177 and 178). The use of the L1-norm increases the accuracy, but not enough to make this approximation reliable for this case (Figures 179 and 180).

In each wave reflection event, each norm and each model, the approximation proposed by Li and Yuan (2001) presented the second best set of results of all equations tested in this work. For the most accurate among the local search optimization algorithms tested, this approximation presented a very good fitting for both reflection events (Figures 181 and 182), and for the L1-norm, and it showed a 10\% decrease of the residual error (183 and 184).

The approximation proposed in this work presented to be the most accurate for all scenarios tested. This approximation for this model with the MCS optimization algorithm showed the highest efficiency, once it showed to be very accurate, and also a good processing time (Figures 185 and 186). For the L1-norm, it showed an even better accuracy (Figures 187 and 188).

In the Model 1, the Ursin and Stovas (2006) approximation showed the third best set of results for every PP wave reflection event of each optimization algorithm, while the Blias (2009) and Malovichko (1978) approximations presented, respectively, the fourth and the fifth best results, as it can be more clearly observed in Figure 189. In the case of the converted wave events for all optimization algorithms, the Figure 190 showed more easily that the Blias (2009) approximation presented the third best results; Malovichko (1978), the fourth best results; and Ursin and Stovas (2006), the fifth best results. Figures 191 and 192 showed the same variation, but with an increase of accuracy of around $10 \%$. 


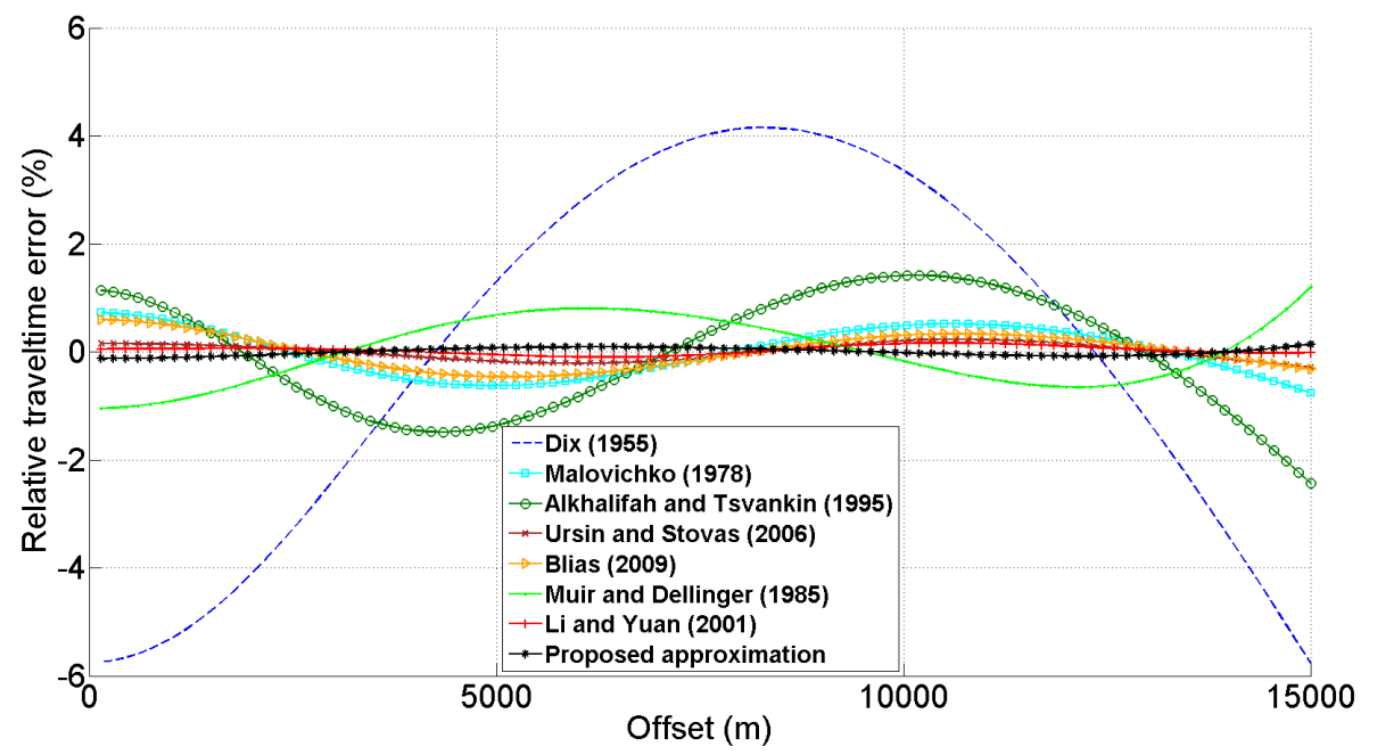

Figure 173: Relative error in travel-time between the observed curve and the calculated curve with each approximation of the PP reflection event with L2-norm and IMFIL optimization algorithm.

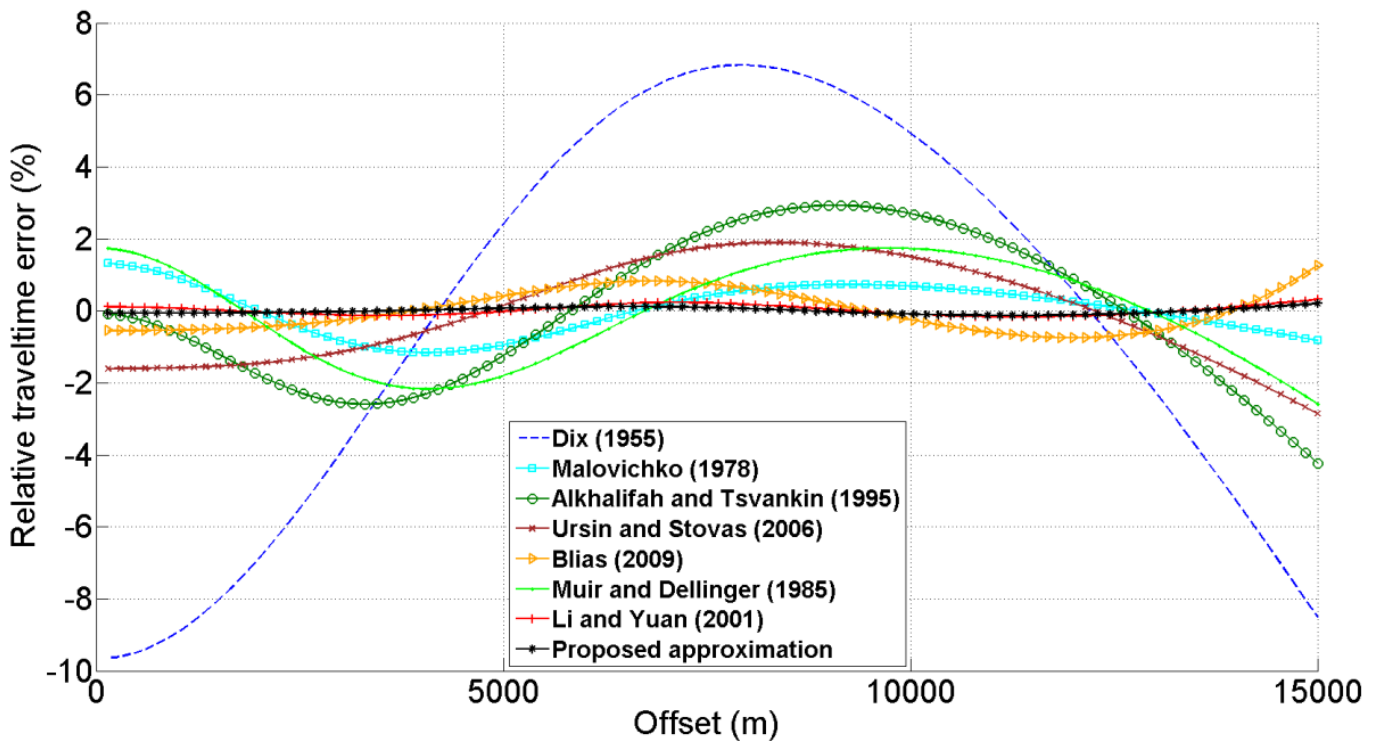

Figure 174: Relative error in travel-time between the observed curve and the calculated curve with each approximation of the PS reflection event with L2-norm and IMFIL optimization algorithm. 


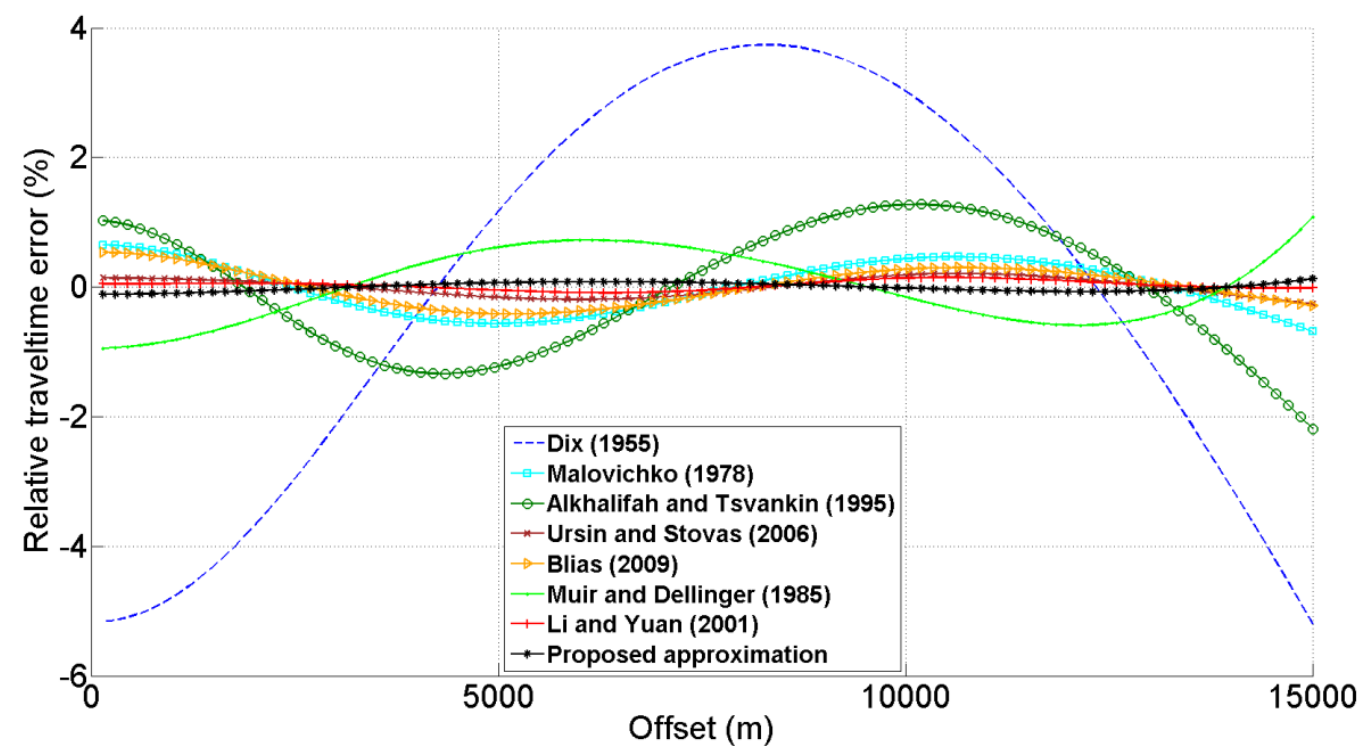

Figure 175: Relative error in travel-time between the observed curve and the calculated curve with each approximation of the PP reflection event with L1-norm and IMFIL optimization algorithm.

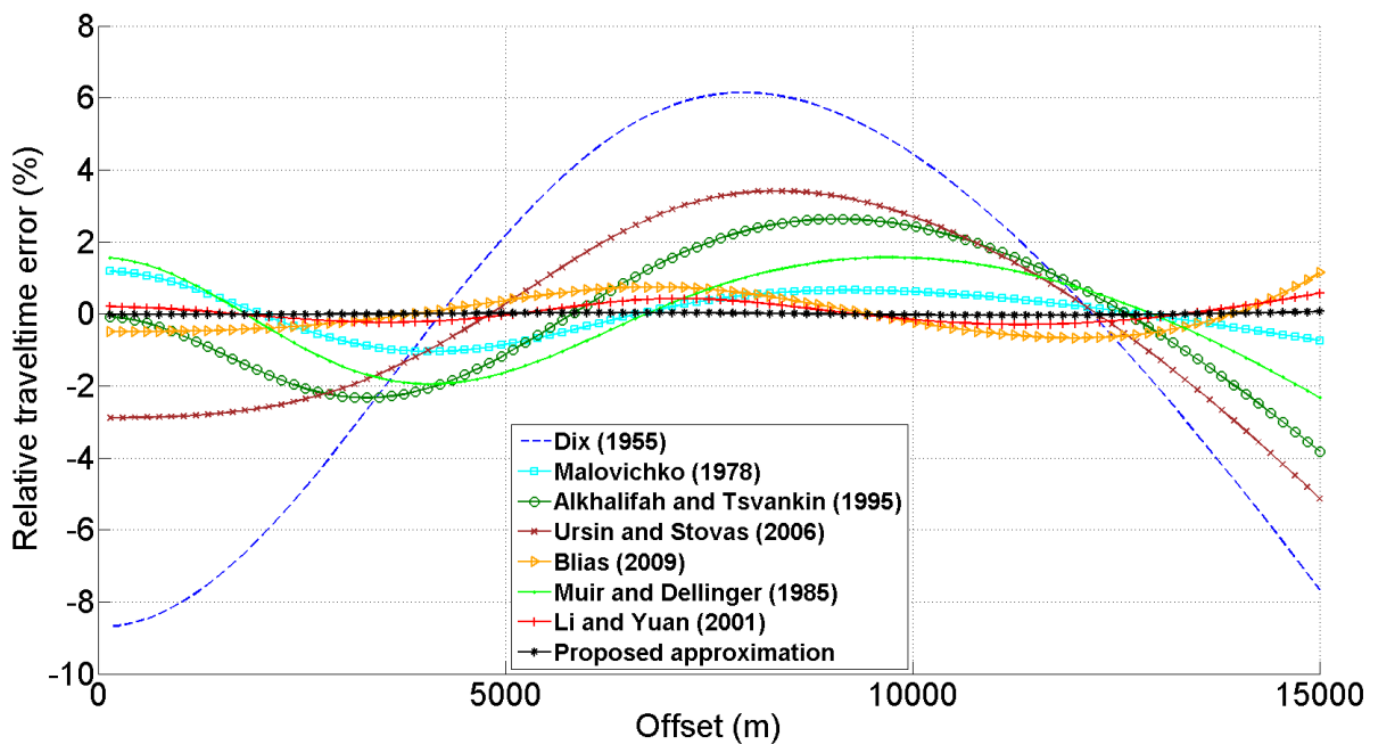

Figure 176: Relative error in travel-time between the observed curve and the calculated curve with each approximation of the PS reflection event with L1-norm and IMFIL optimization algorithm. 


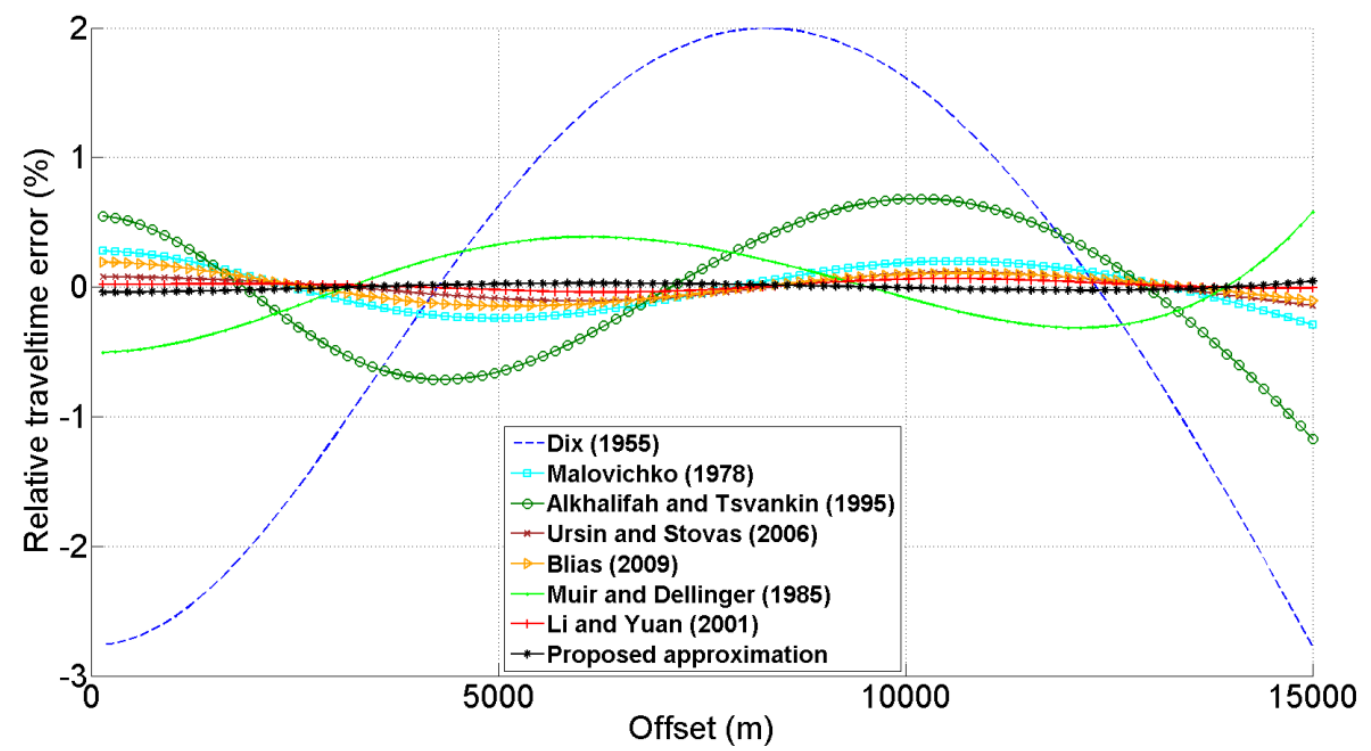

Figure 177: Relative error in travel-time between the observed curve and the calculated curve with each approximation of the PP reflection event with L2-norm and FMINSEARCH optimization algorithm.

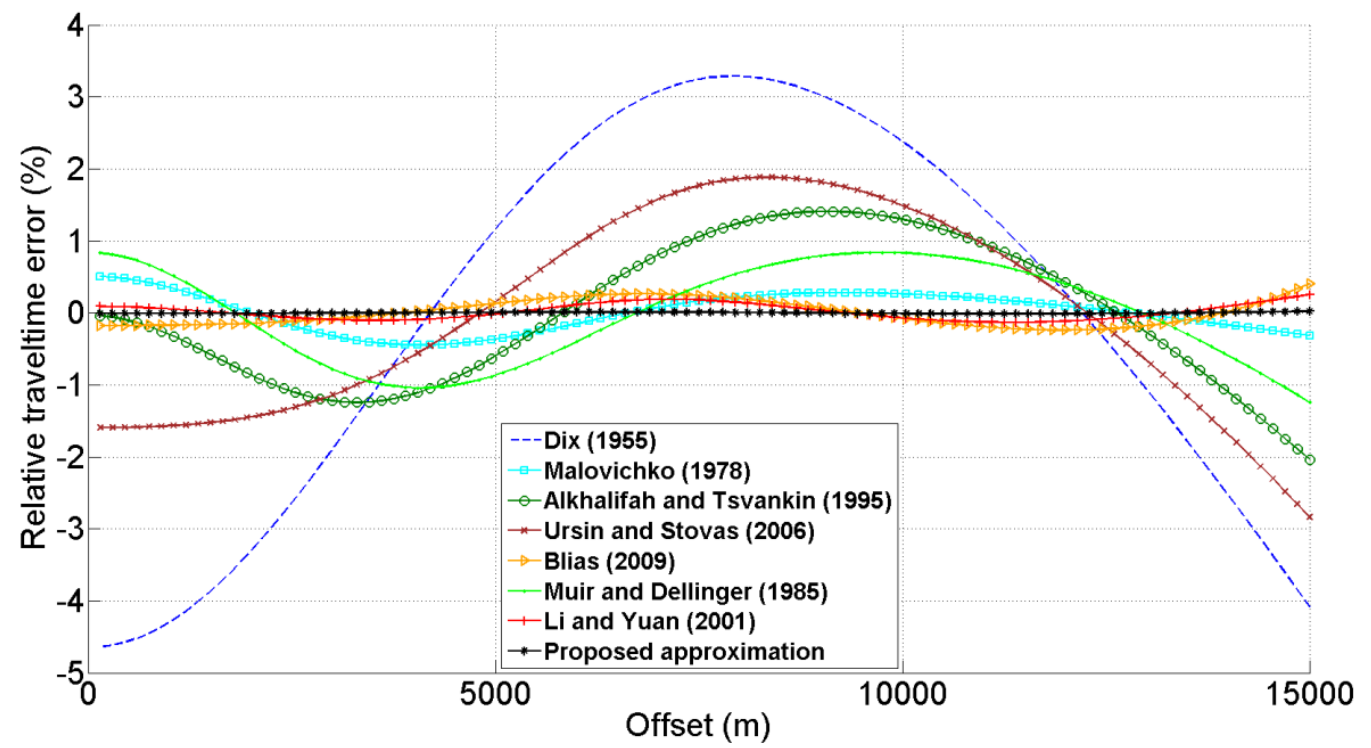

Figure 178: Relative error in travel-time between the observed curve and the calculated curve with each approximation of the PS reflection event with L2-norm and FMINSEARCH optimization algorithm. 


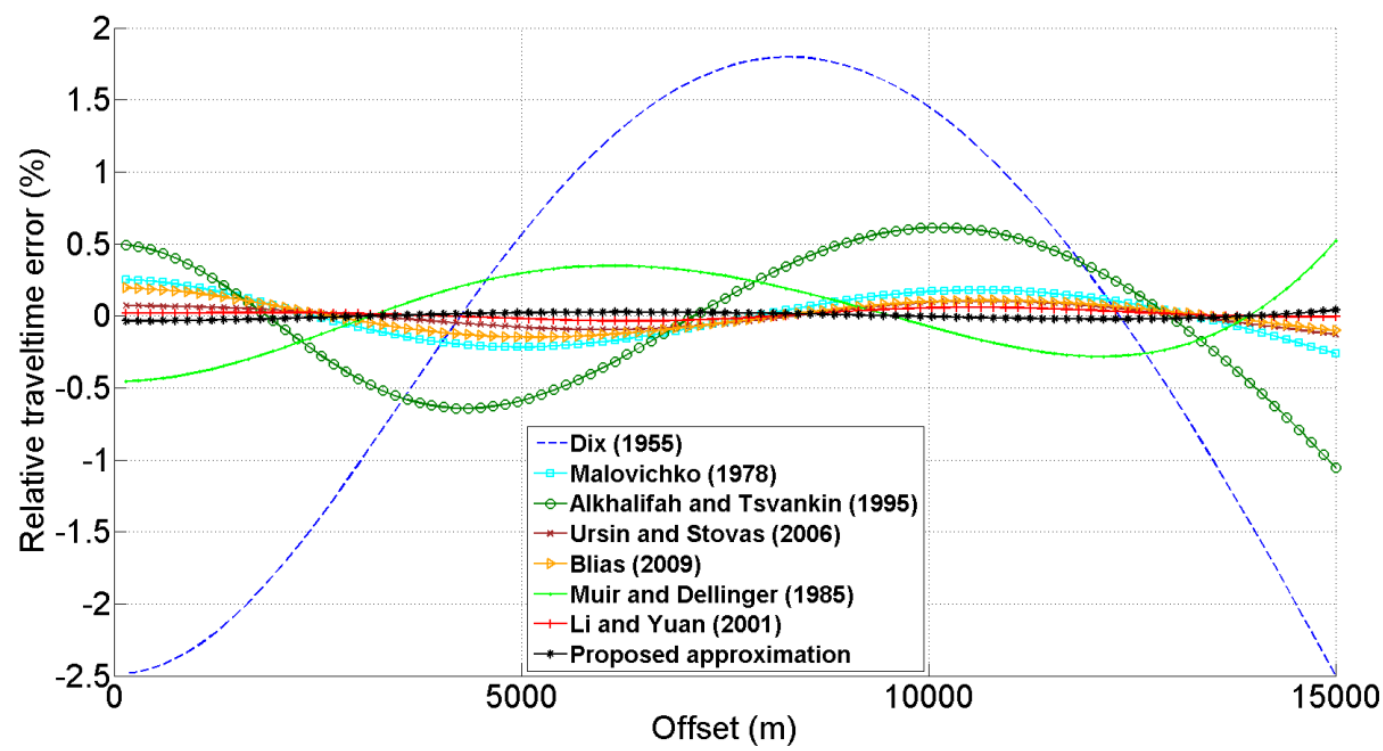

Figure 179: Relative error in travel-time between the observed curve and the calculated curve with each approximation of the PP reflection event with L1-norm and FMINSEARCH optimization algorithm.

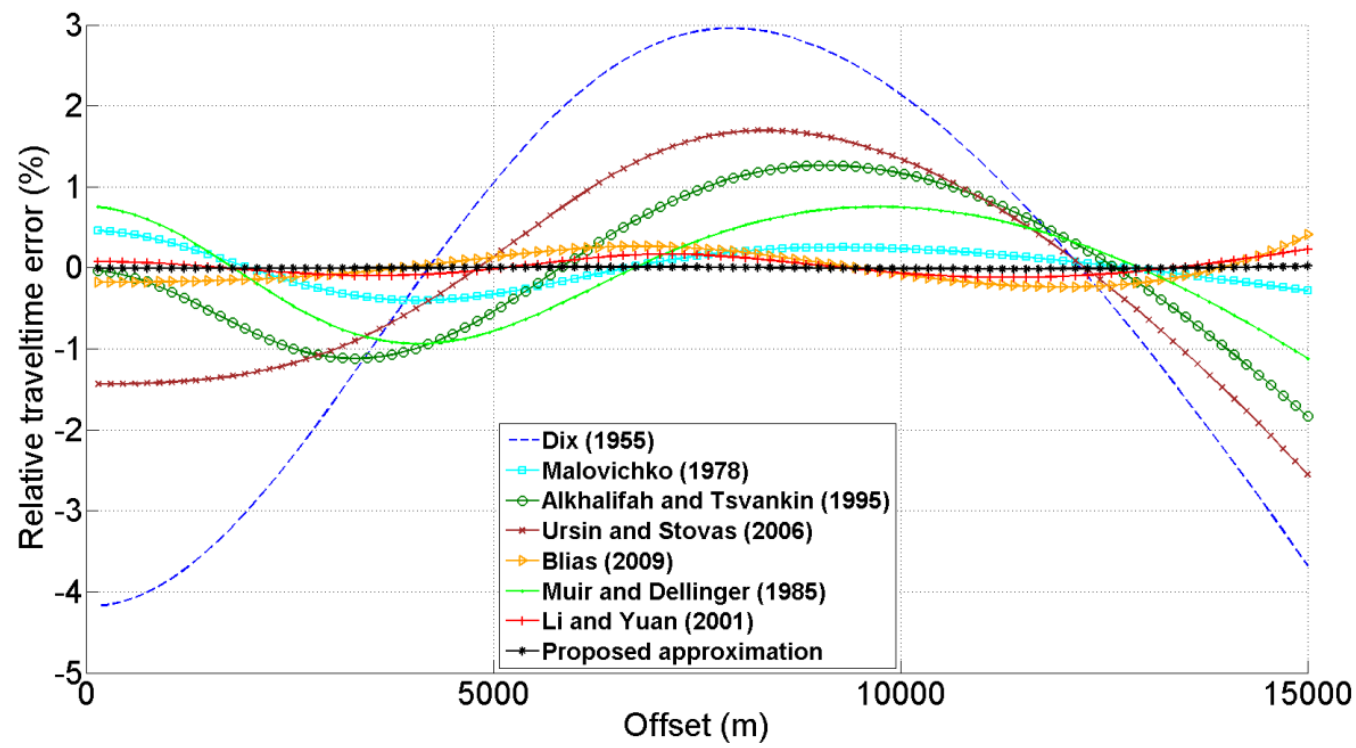

Figure 180: Relative error in travel-time between the observed curve and the calculated curve with each approximation of the PS reflection event with L1-norm and FMINSEARCH optimization algorithm. 


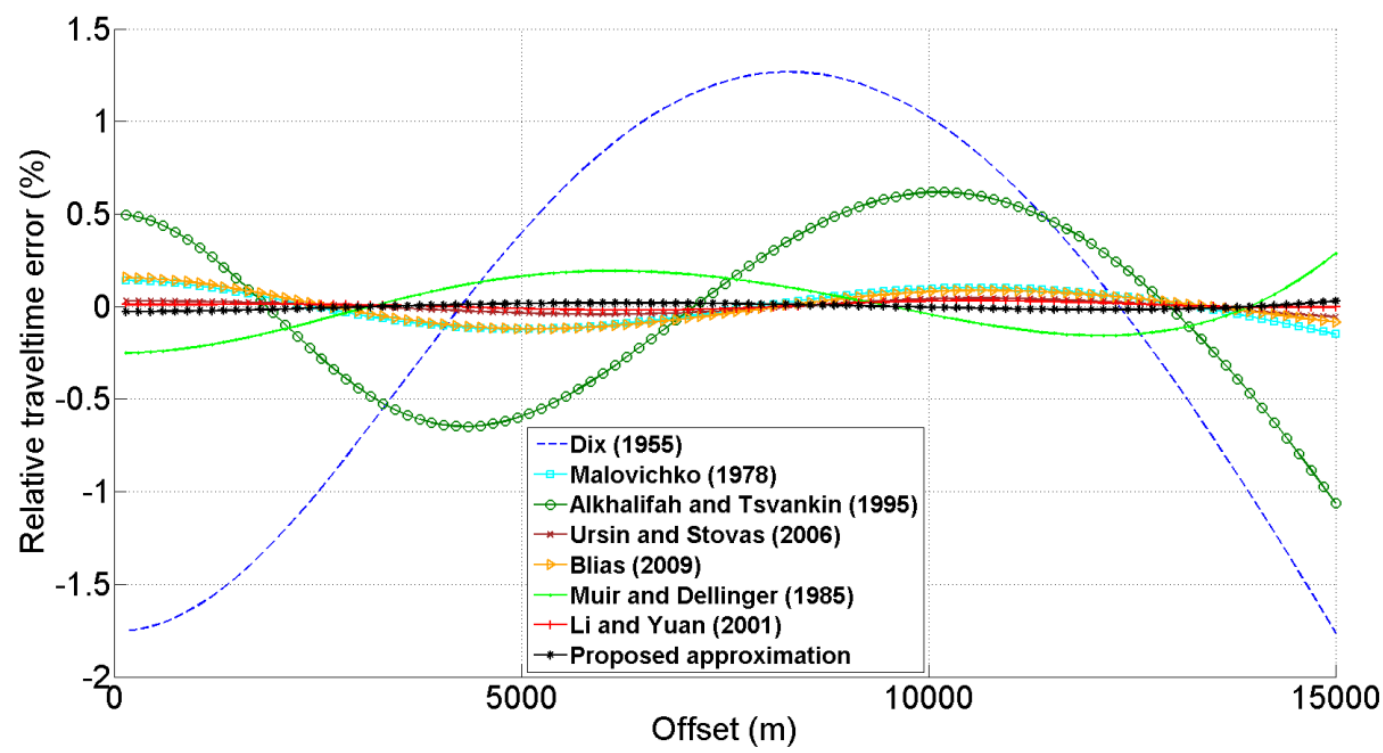

Figure 181: Relative error in travel-time between the observed curve and the calculated curve with each approximation of the PP reflection event with L2-norm and SID-PSM optimization algorithm.

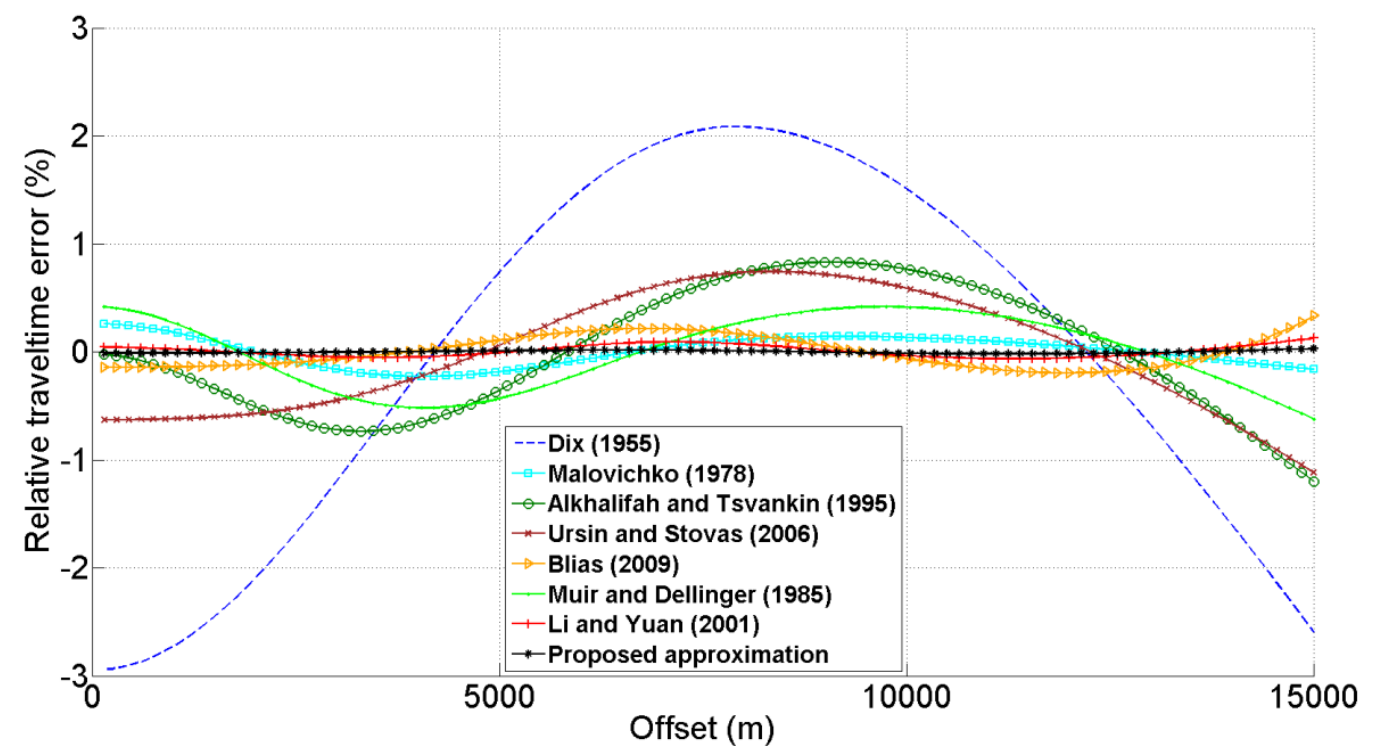

Figure 182: Relative error in travel-time between the observed curve and the calculated curve with each approximation of the PS reflection event with L2-norm and SID-PSM optimization algorithm. 


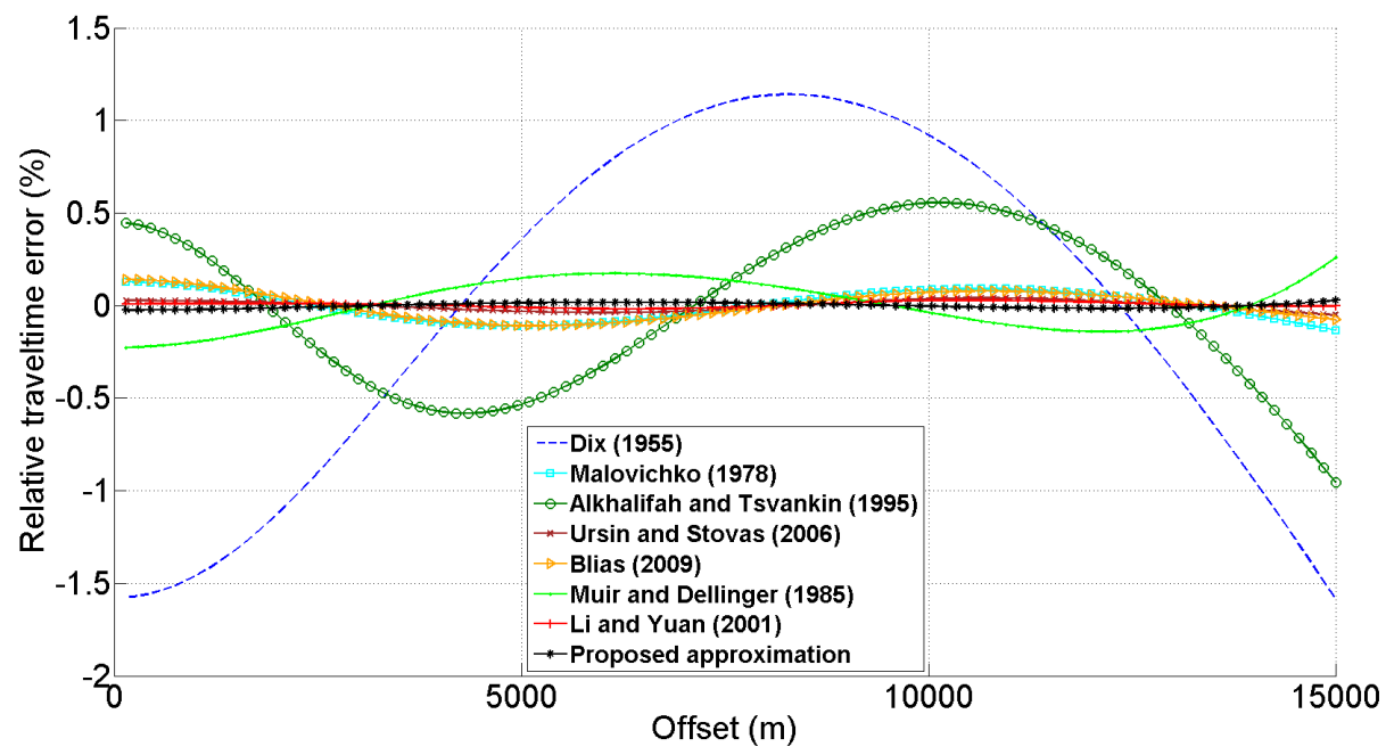

Figure 183: Relative error in travel-time between the observed curve and the calculated curve with each approximation of the PP reflection event with L1-norm and SID-PSM optimization algorithm.

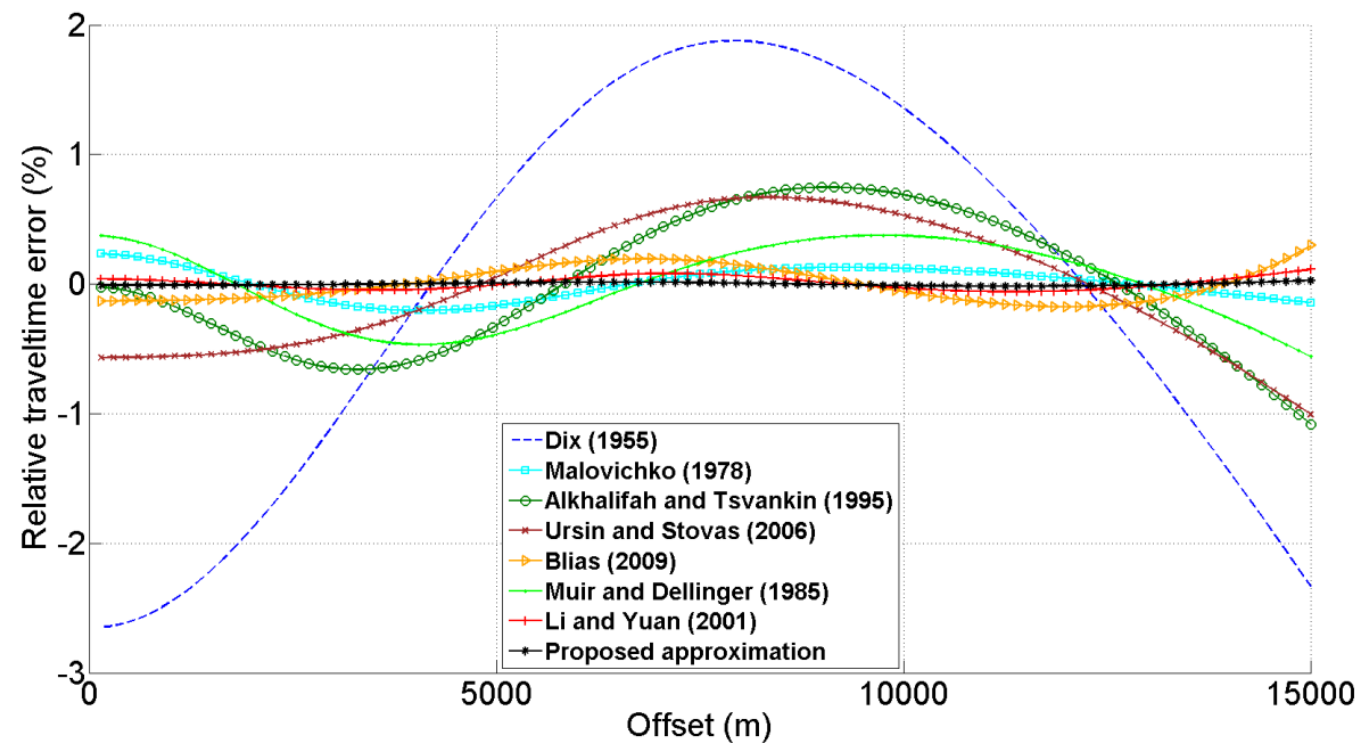

Figure 184: Relative error in travel-time between the observed curve and the calculated curve with each approximation of the PS reflection event with L1-norm and SID-PSM optimization algorithm. 


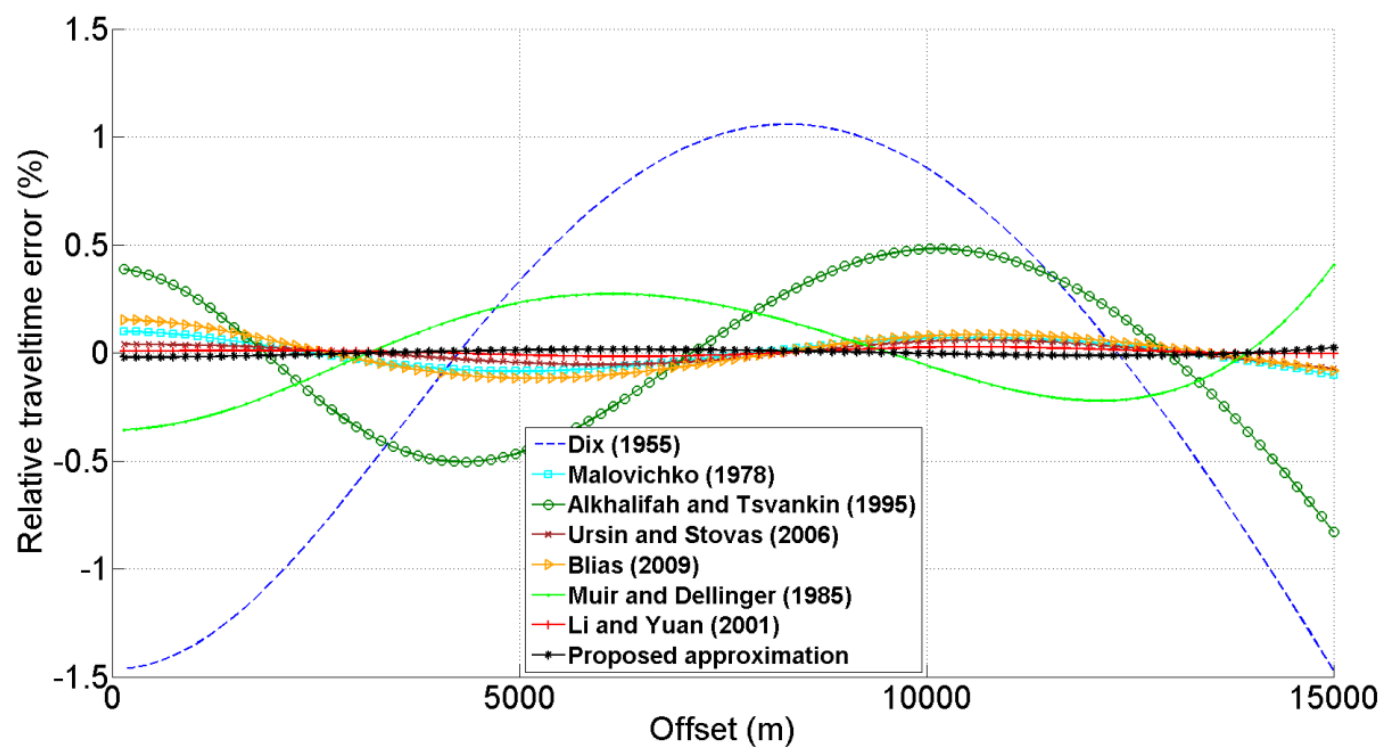

Figure 185: Relative error in travel-time between the observed curve and the calculated curve with each approximation of the PP reflection event with L2-norm and MCS optimization algorithm.

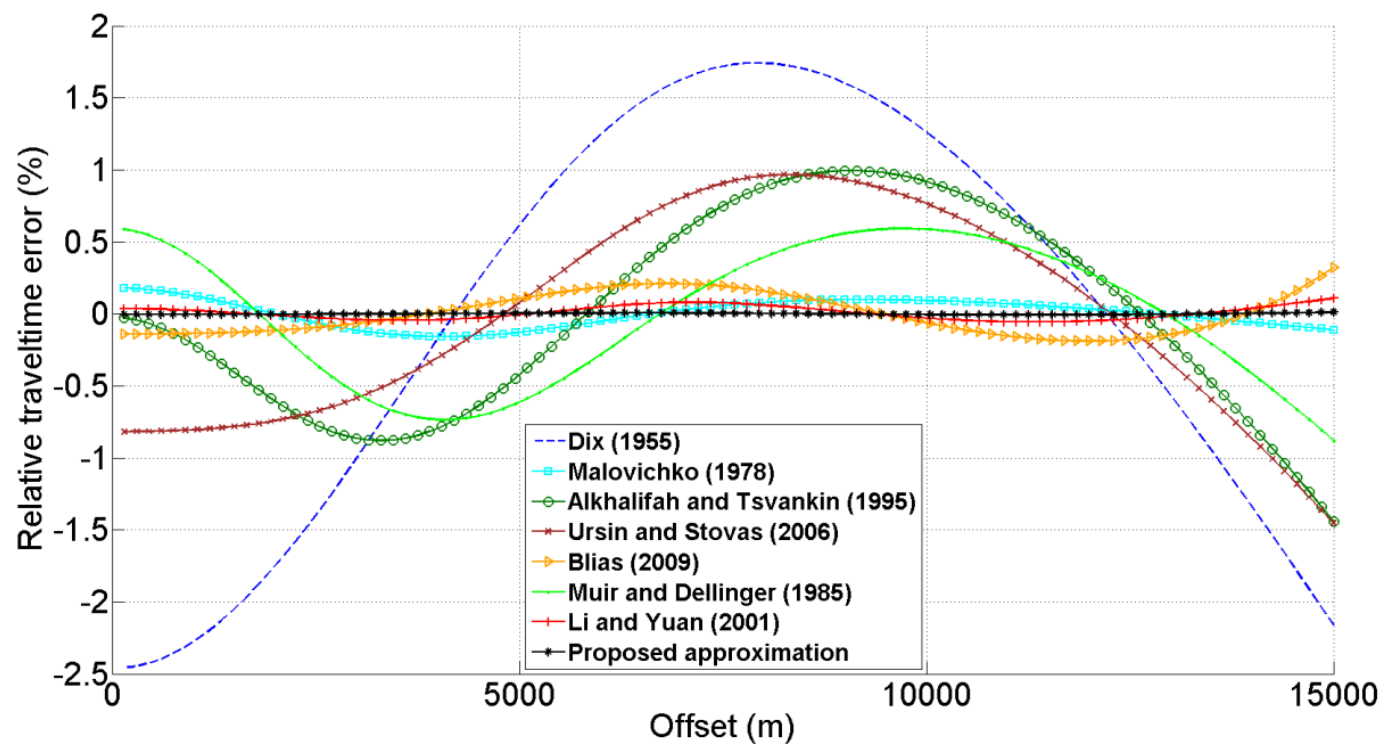

Figure 186: Relative error in travel-time between the observed curve and the calculated curve with each approximation of the PS reflection event with L2-norm and MCS optimization algorithm. 


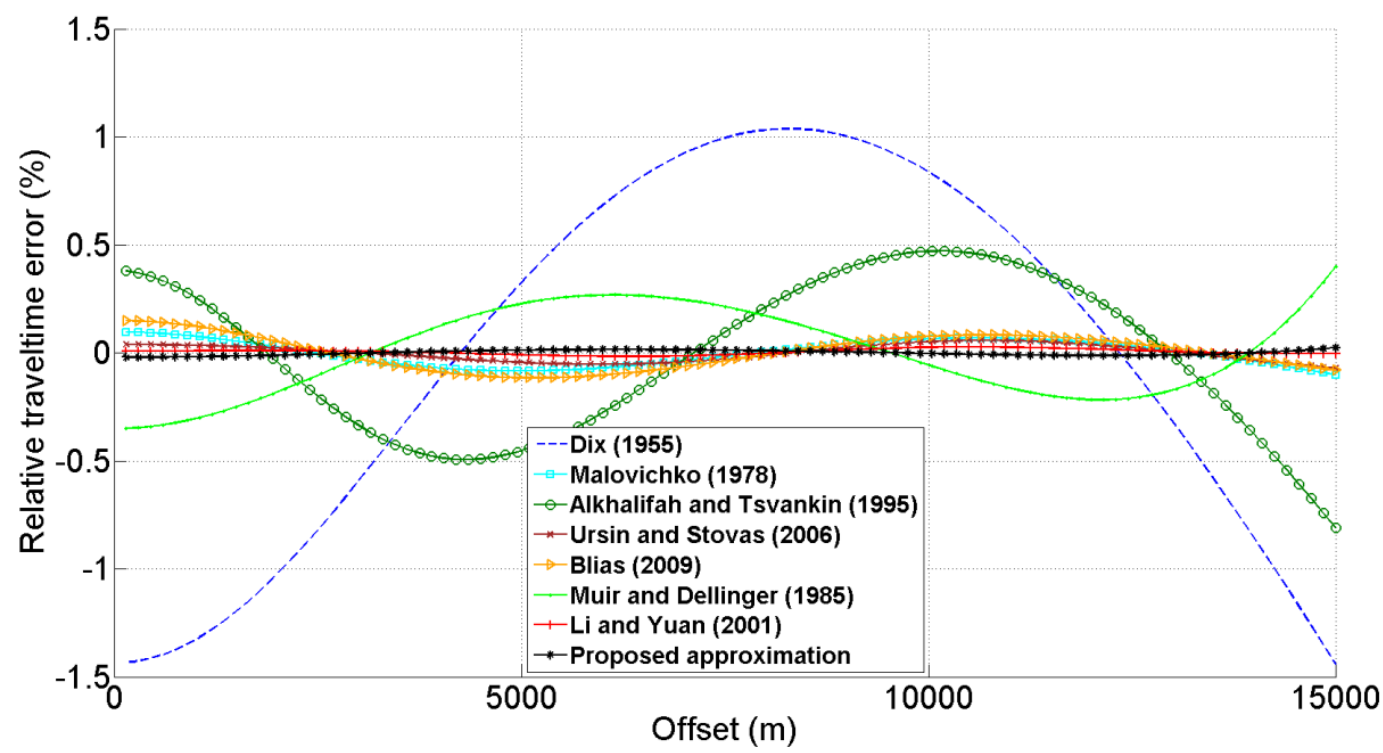

Figure 187: Relative error in travel-time between the observed curve and the calculated curve with each approximation of the PP reflection event with L1-norm and MCS optimization algorithm.

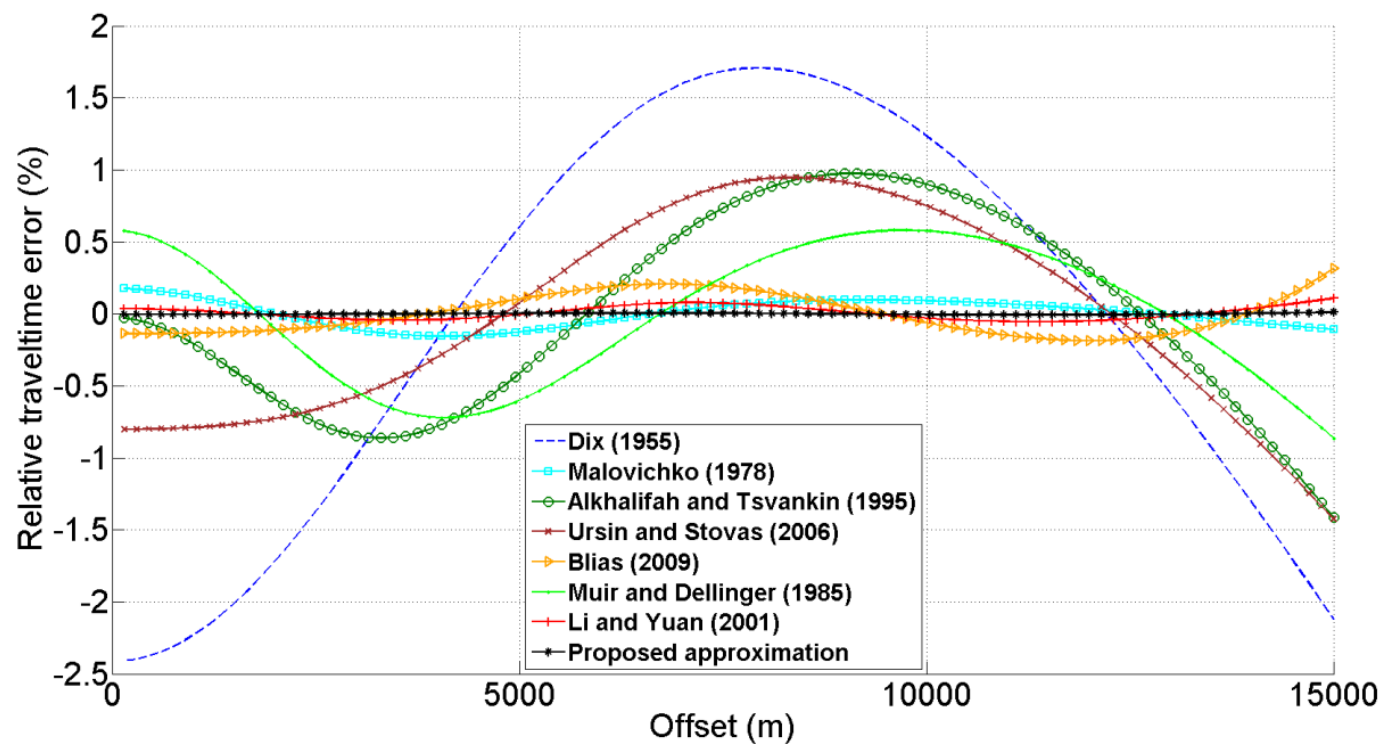

Figure 188: Relative error in travel-time between the observed curve and the calculated curve with each approximation of the PS reflection event with L1-norm and MCS optimization algorithm. 


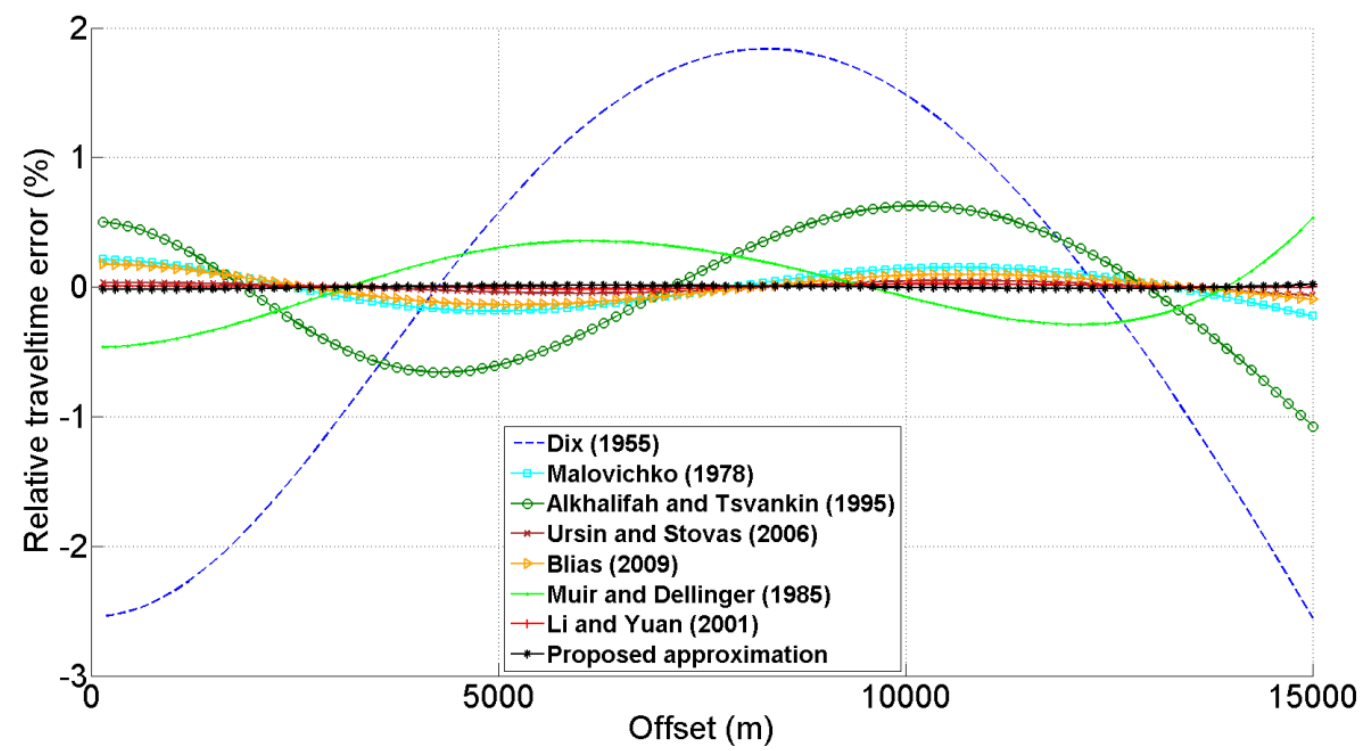

Figure 189: Relative error in travel-time between the observed curve and the calculated curve with each approximation of the PP reflection event with L2-norm and TOMLAB/LGO optimization algorithm.

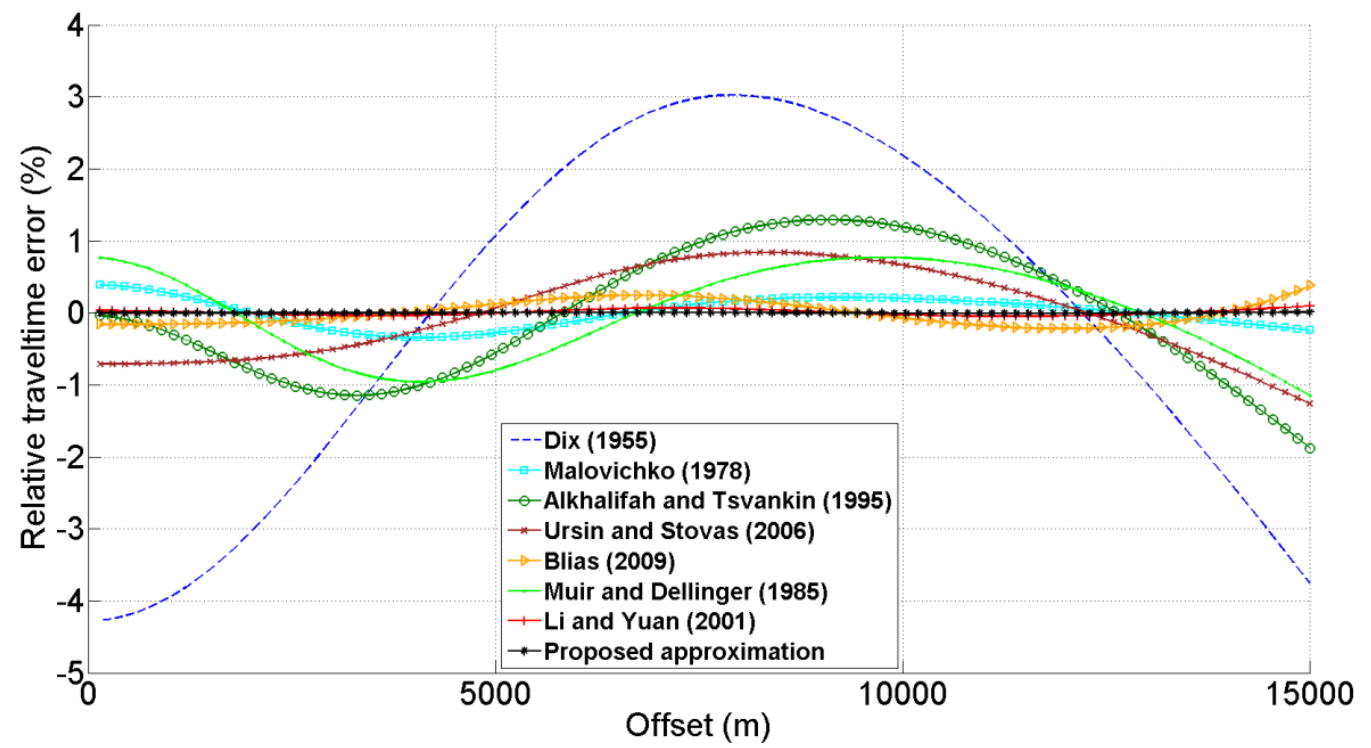

Figure 190: Relative error in travel-time between the observed curve and the calculated curve with each approximation of the PS reflection event with L2-norm and TOMLAB/LGO optimization algorithm. 


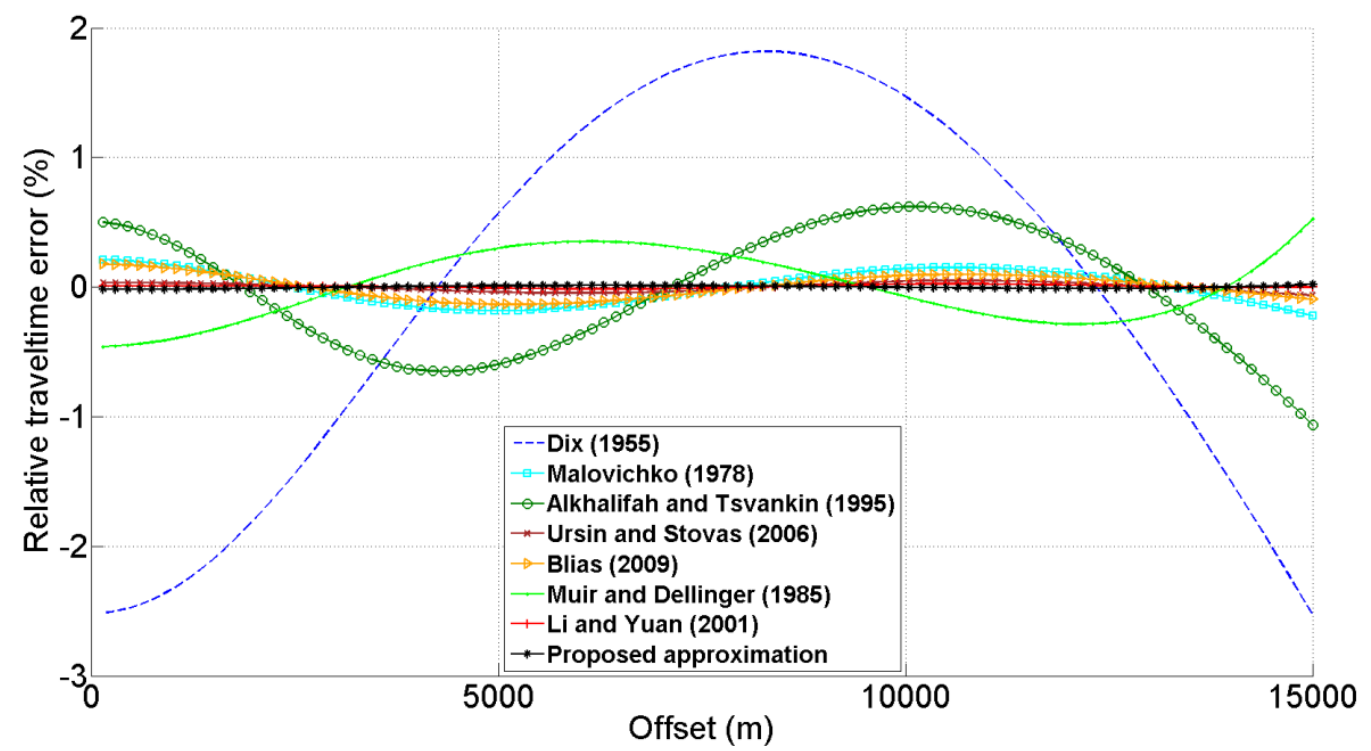

Figure 191: Relative error in travel-time between the observed curve and the calculated curve with each approximation of the PP reflection event with L1-norm and TOMLAB/LGO optimization algorithm.

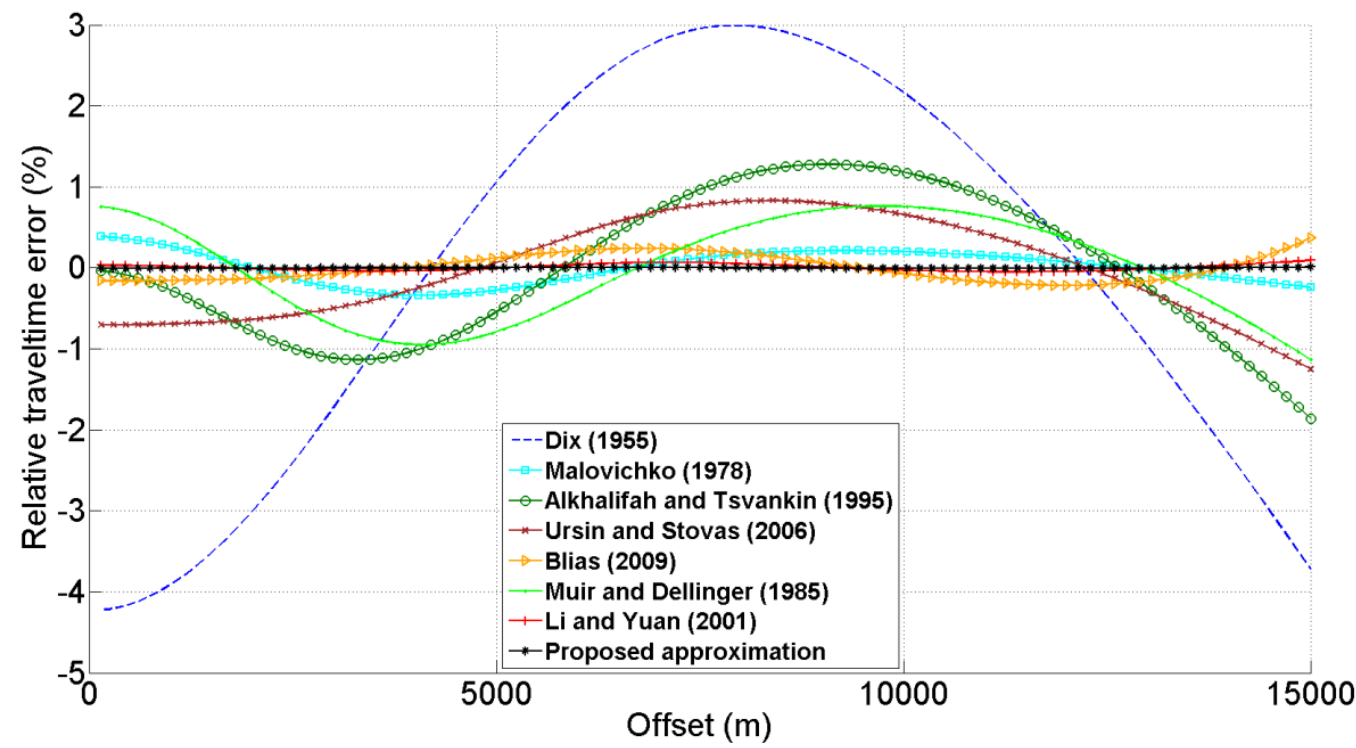

Figure 192: Relative error in travel-time between the observed curve and the calculated curve with each approximation of the PS reflection event with L1-norm and TOMLAB/LGO optimization algorithm. 


\subsection{Model 2}

For the PP event with L2-norm using the IMFL optimization algorithm (Figure 193), the approximation of Ursin and Stovas (2006) showed the third best result, while the one proposed by Blias (2009) showed the fourth best. The one proposed by Malovichko showed the fifth best result for this case. It was possible to observe that, for the PS event, Blias (2009) showed the third best result; Malovichko (1978), the fourth best; and Ursin and Stovas (2006), the fifth best (Figure 194). However, applying the L1-norm (Figures 195 and 196), the result showed to be very similar, with the exception of the decrease of the error in around $10 \%$.

With the FMINSEARCH algorithm, the PP event using L2-norm (Figure 197) showed the third best result for the Ursin and Stovas (2006) approximation, the fourth best for Blias (2009) and the fifth best for Malovichko (1978). For the converted event (Figure 198), it was observed that the third best result was for Blias (2009), the fourth best for Malovichko (1978) and the fifth best for Ursin and Stovas (2006). These results presented an increase of around $1 \%$ concerning the accuracy when the L1- norm was used (Figures 199 and 200).

The SID-PSM optimization algorithm along with the L2-norm presented that, for the conventional reflection event, Ursin and Stovas (2006) had the third, Blias (2009) the fourth, and Malovichko (1978) the fifth best results (Figure 201). For the PS reflection event (Figure 202), Blias (2009) showed to be the third, Malovichko (1978) the fourth and Ursin and Stovas (2006) the fifth best. For the L1-norm, an increase of the quality in the result could be observed (Figures 203 and 204), but still showing the same sequence of results.

The MCS algorithm along with the L2-norm used for the inversion of the PP event showed the Ursin and Stovas (2006) approximation as the third best result; Blias (2009), the fourth; and Malovichko (1978), the fifth (Figure 205). For the converted wave event, Blias (2009) showed to be the third, Malovichko (1978) the fourth and Ursin and Stovas (2006) the fifth best result (Figure 206). When the L1- norm is used for this case (Figures 207 and 208) a similar result can be observed, however, with a small increase of accuracy.

For the TOMLAB/LGO algorithm, when the L2-norm was used for the PP event (Figure 209), Ursin and Stovas (2006) showed the third best result; Blias (2009), the fourth; and Malovichko (1978), the fifth. For the PS event, Blias (2009) showed the third best result; Malovichko (1978), the fourth; and Ursin and Stovas (2006), the fifth (Figure 210). The same small increase as before could be observed for the L1-norm (Figures 211 and 212). 


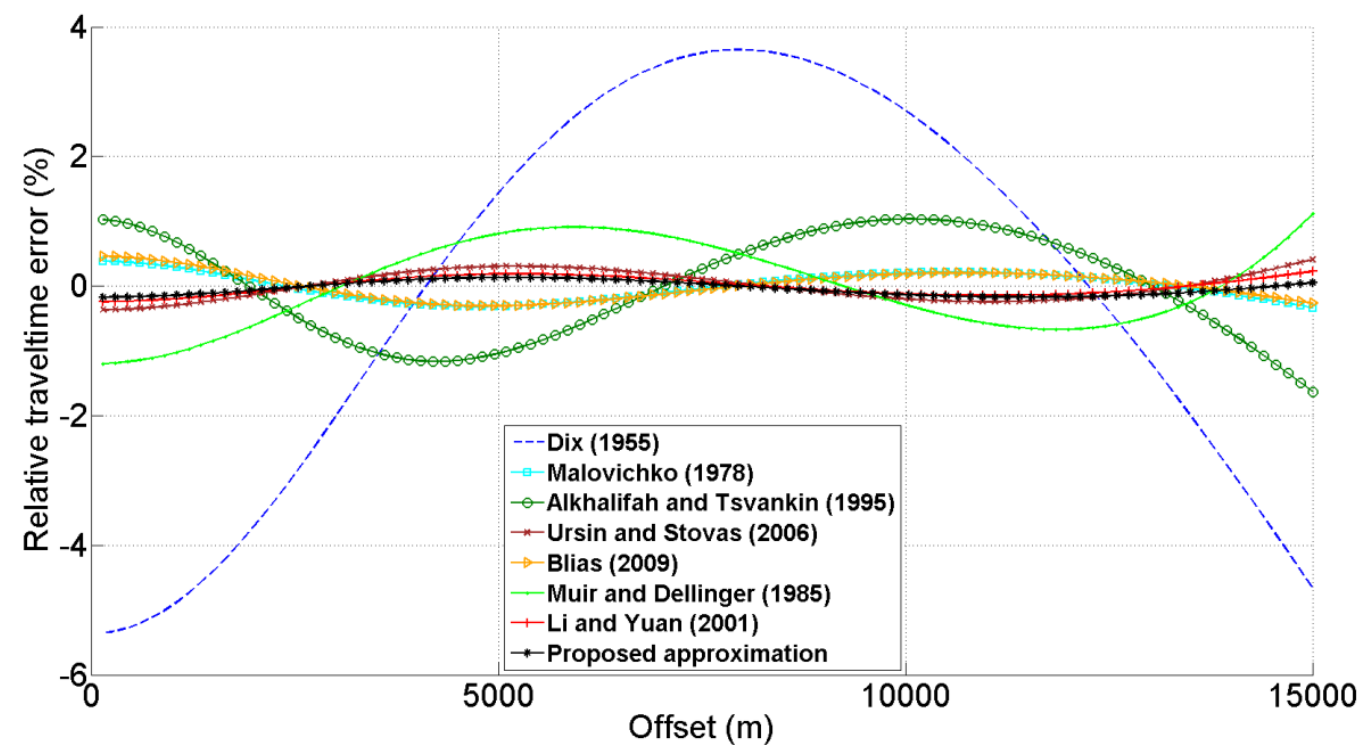

Figure 193: Relative error in travel-time between the observed curve and the calculated curve with each approximation of the PP reflection event with L2-norm and IMFIL optimization algorithm.

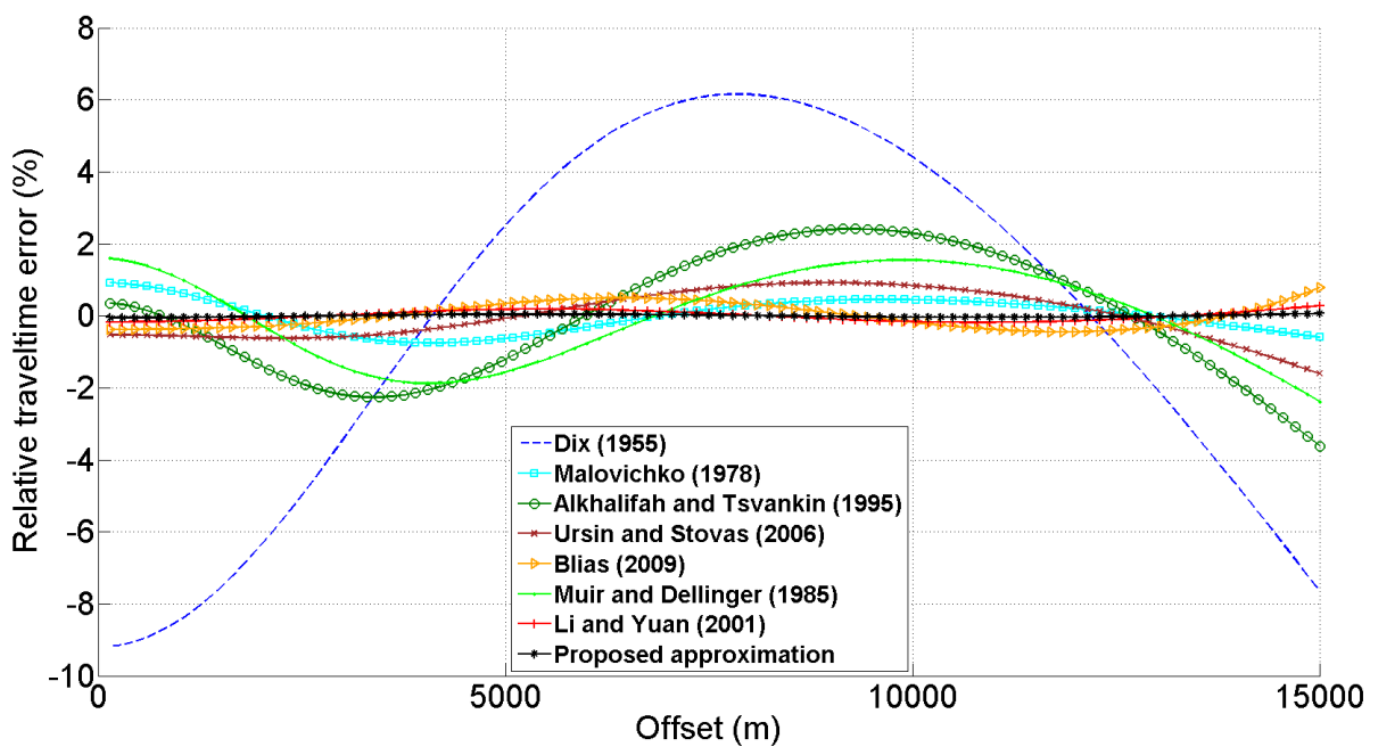

Figure 194: Relative error in travel-time between the observed curve and the calculated curve with each approximation of the PS reflection event with L2-norm and IMFIL optimization algorithm. 


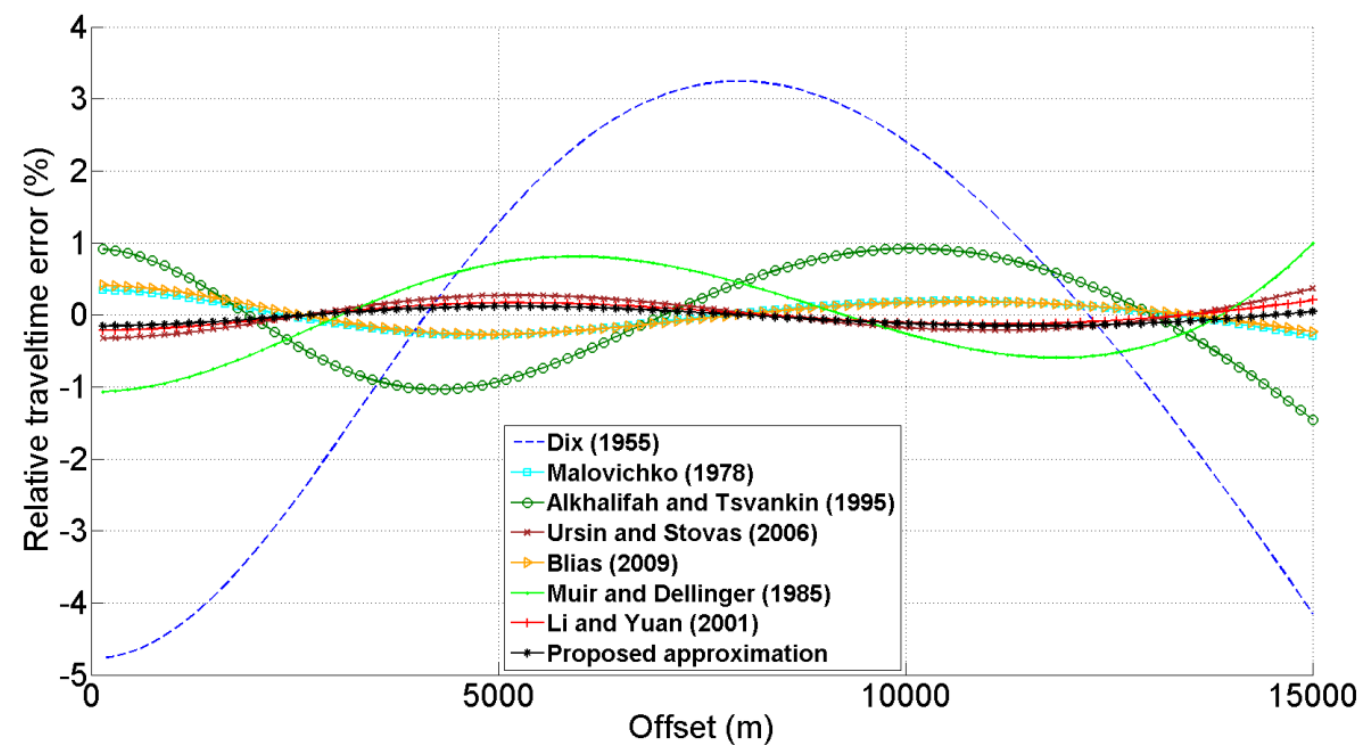

Figure 195: Relative error in travel-time between the observed curve and the calculated curve with each approximation of the PP reflection event with L1-norm and IMFIL optimization algorithm.

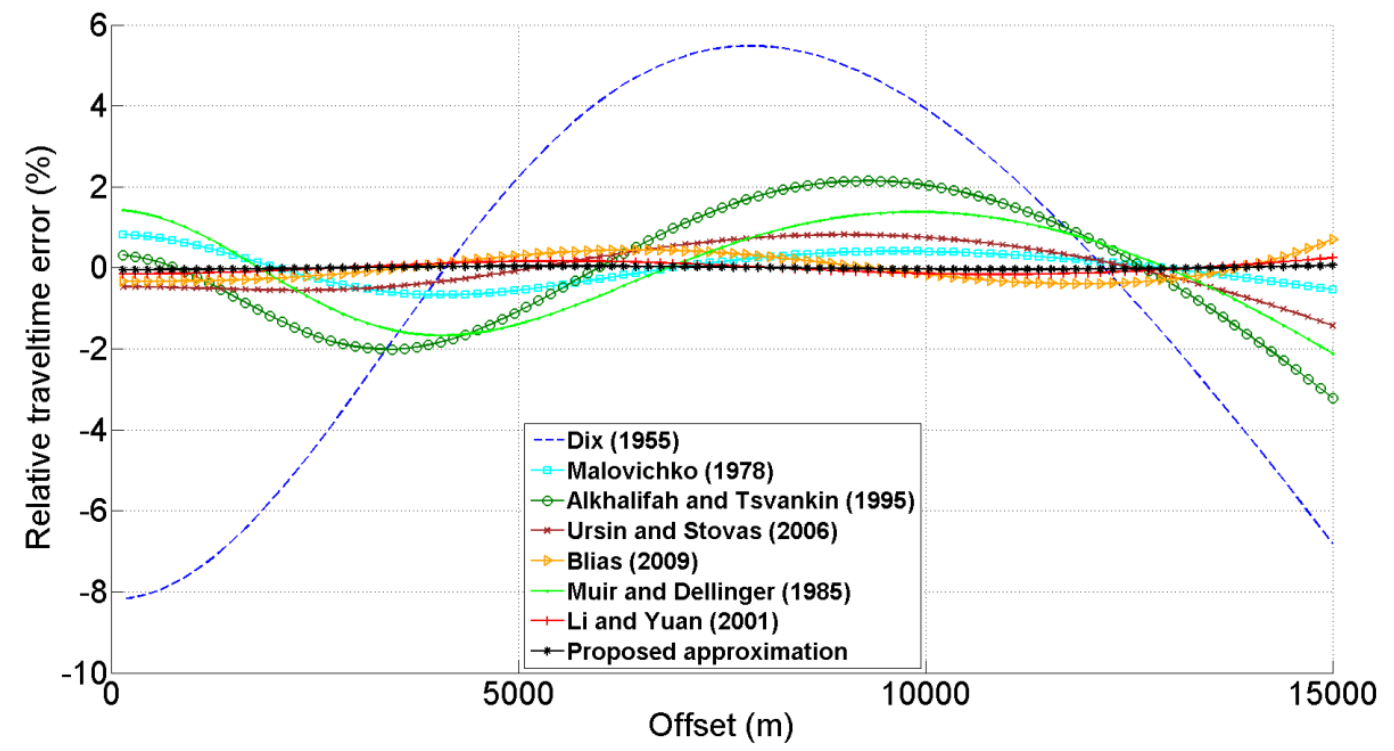

Figure 196: Relative error in travel-time between the observed curve and the calculated curve with each approximation of the PS reflection event with L1-norm and IMFIL optimization algorithm. 


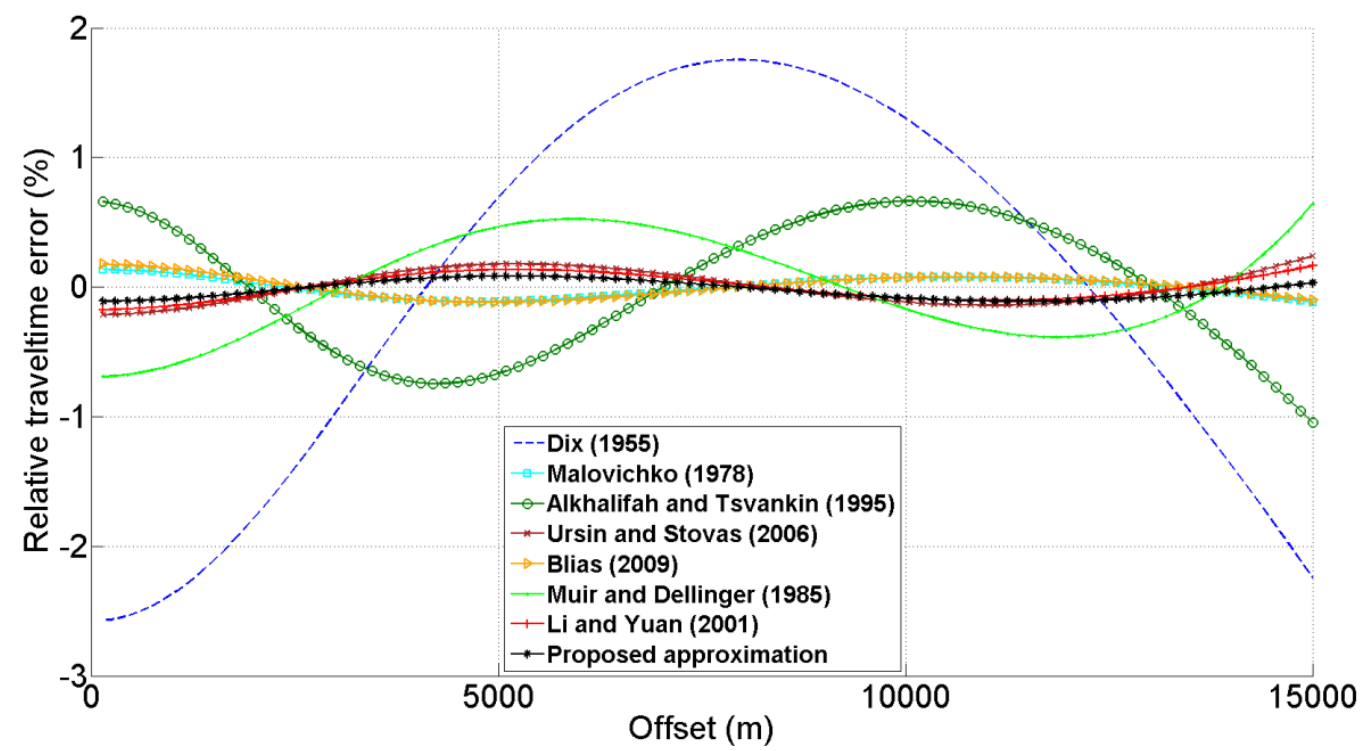

Figure 197: Relative error in travel-time between the observed curve and the calculated curve with each approximation of the PP reflection event with L2-norm and FMINSEARCH optimization algorithm.

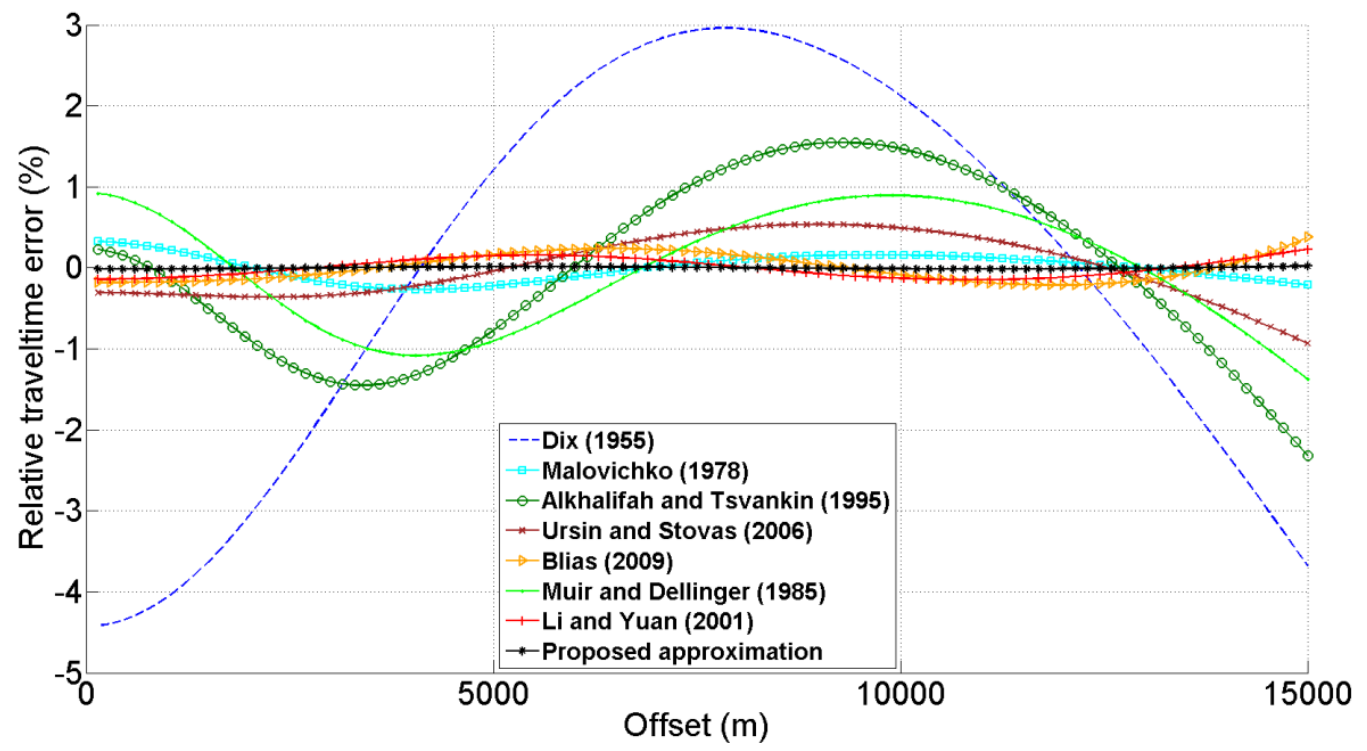

Figure 198: Relative error in travel-time between the observed curve and the calculated curve with each approximation of the PS reflection event with L2-norm and FMINSEARCH optimization algorithm. 


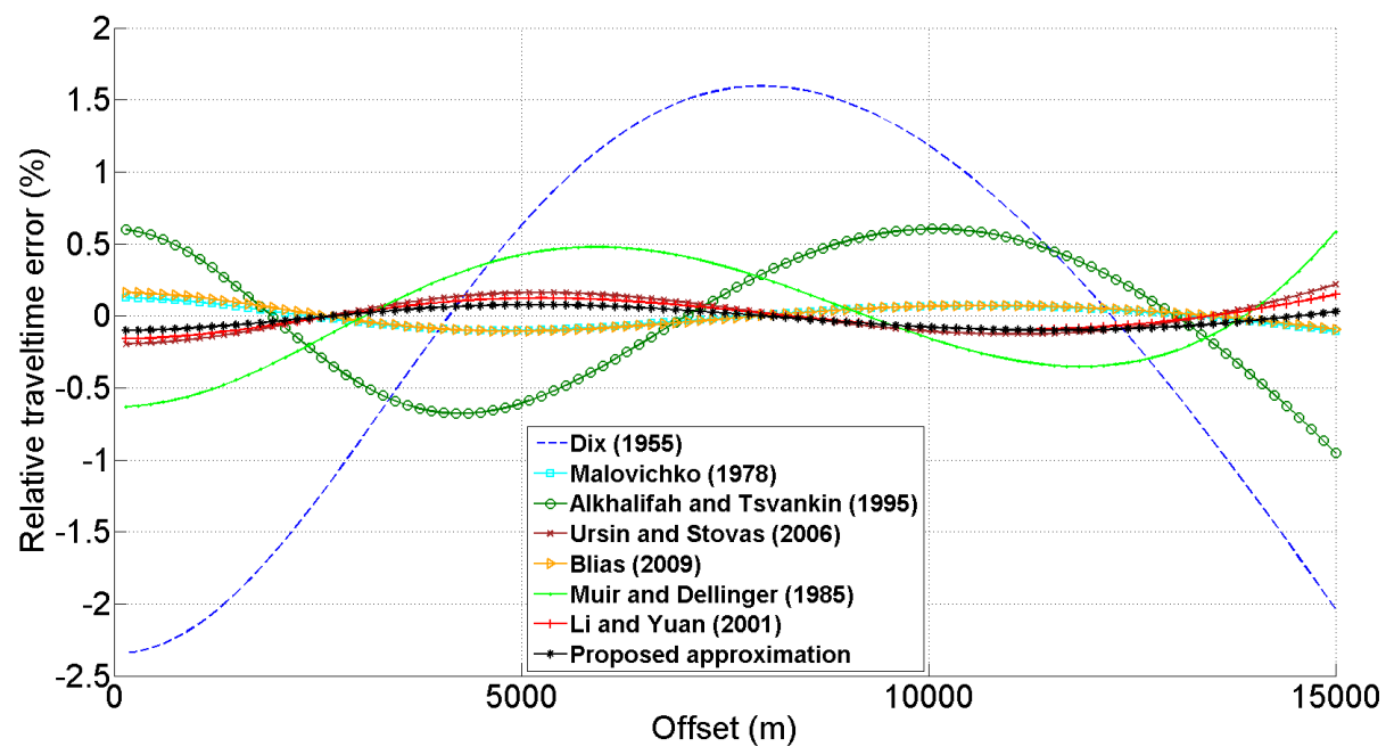

Figure 199: Relative error in travel-time between the observed curve and the calculated curve with each approximation of the PP reflection event with L1-norm and FMINSEARCH optimization algorithm.

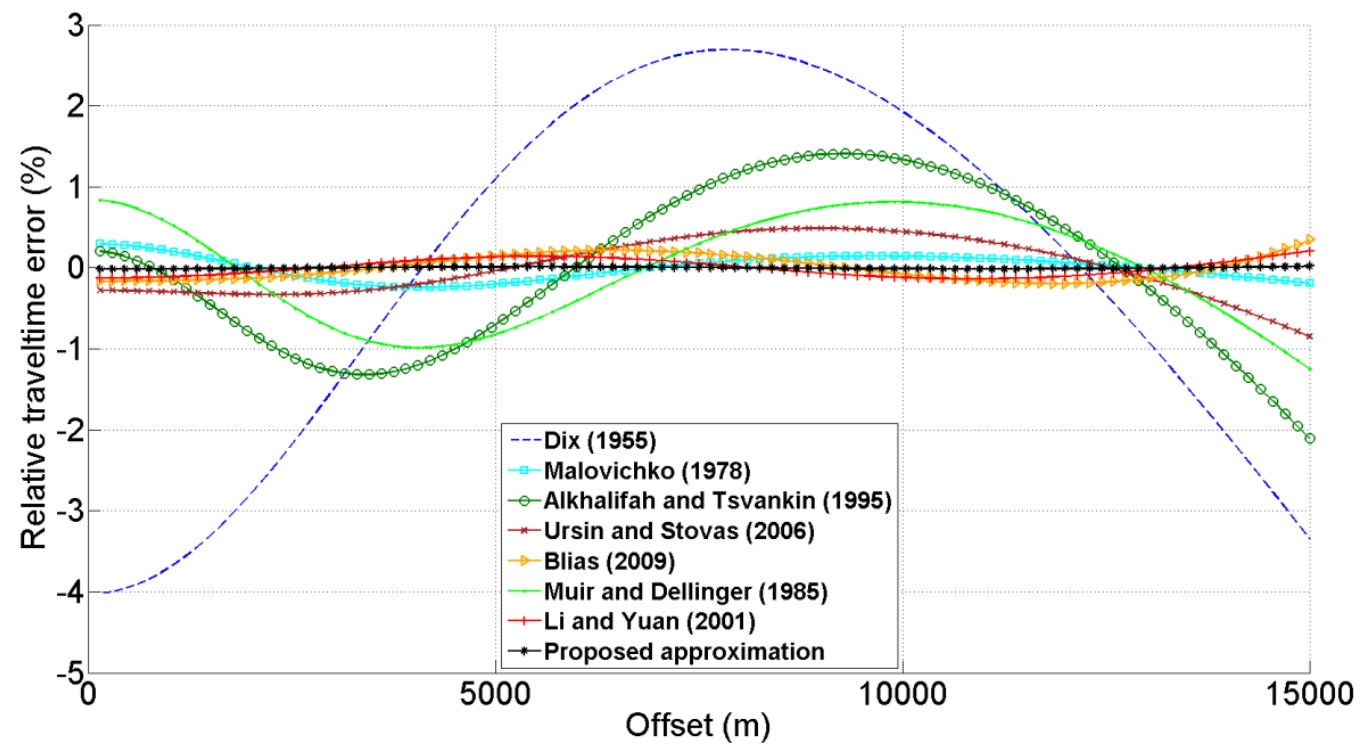

Figure 200: Relative error in travel-time between the observed curve and the calculated curve with each approximation of the PS reflection event with L1-norm and FMINSEARCH optimization algorithm. 


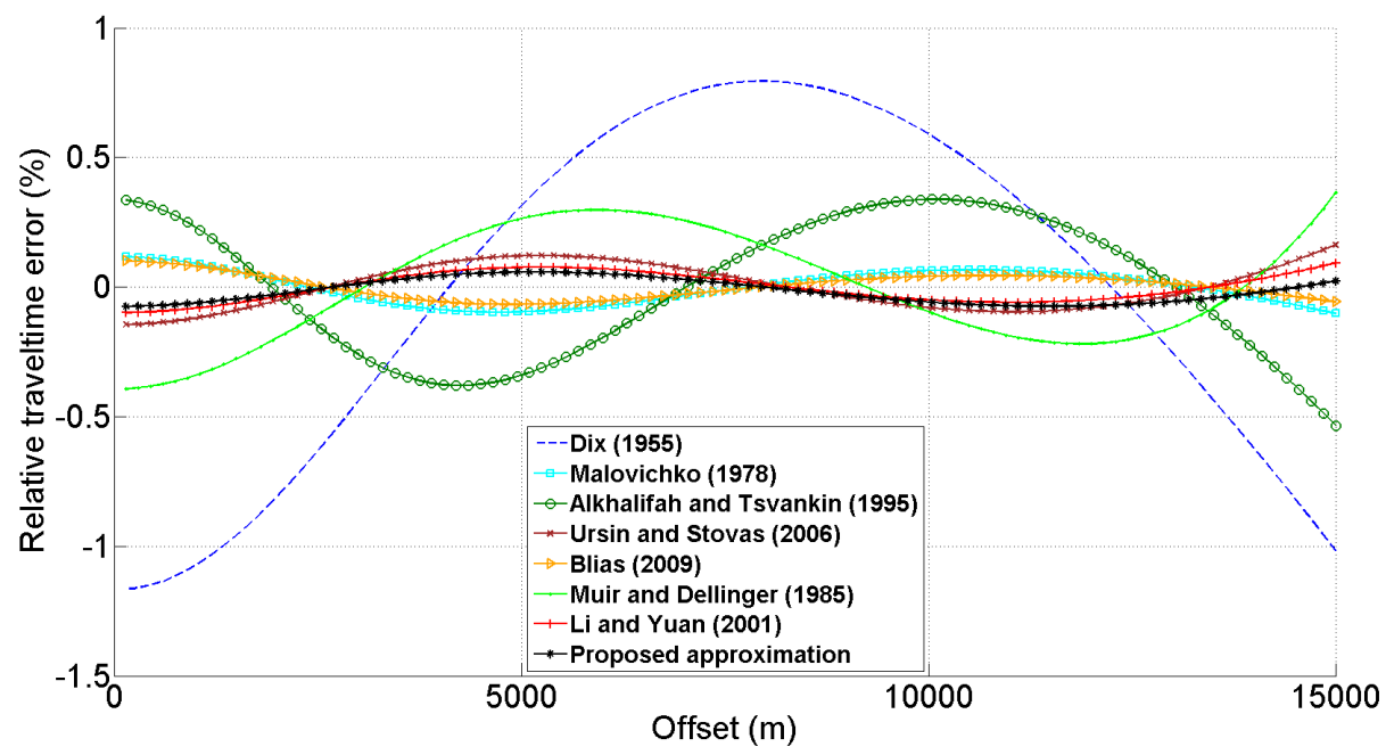

Figure 201: Relative error in travel-time between the observed curve and the calculated curve with each approximation of the PP reflection event with L2-norm and SID-PSM optimization algorithm.

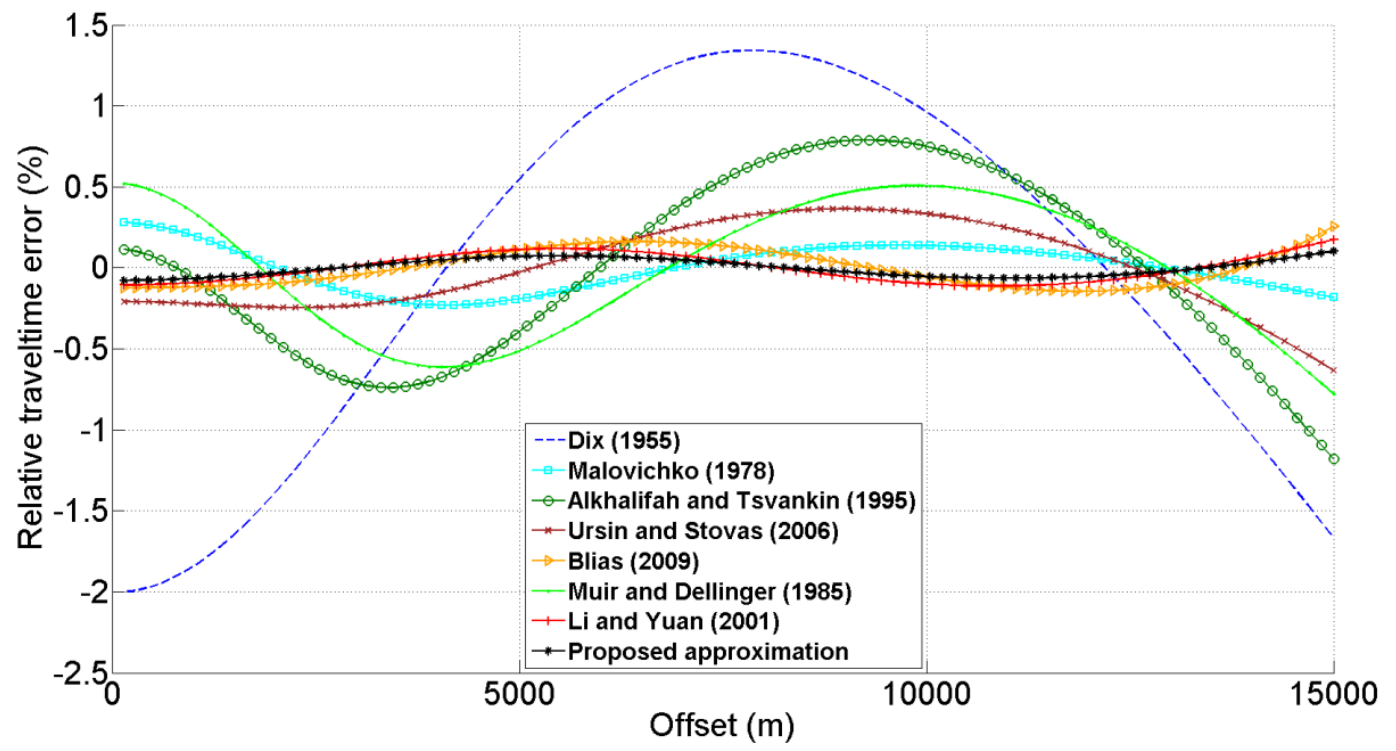

Figure 202: Relative error in travel-time between the observed curve and the calculated curve with each approximation of the PS reflection event with L2-norm and SID-PSM optimization algorithm. 


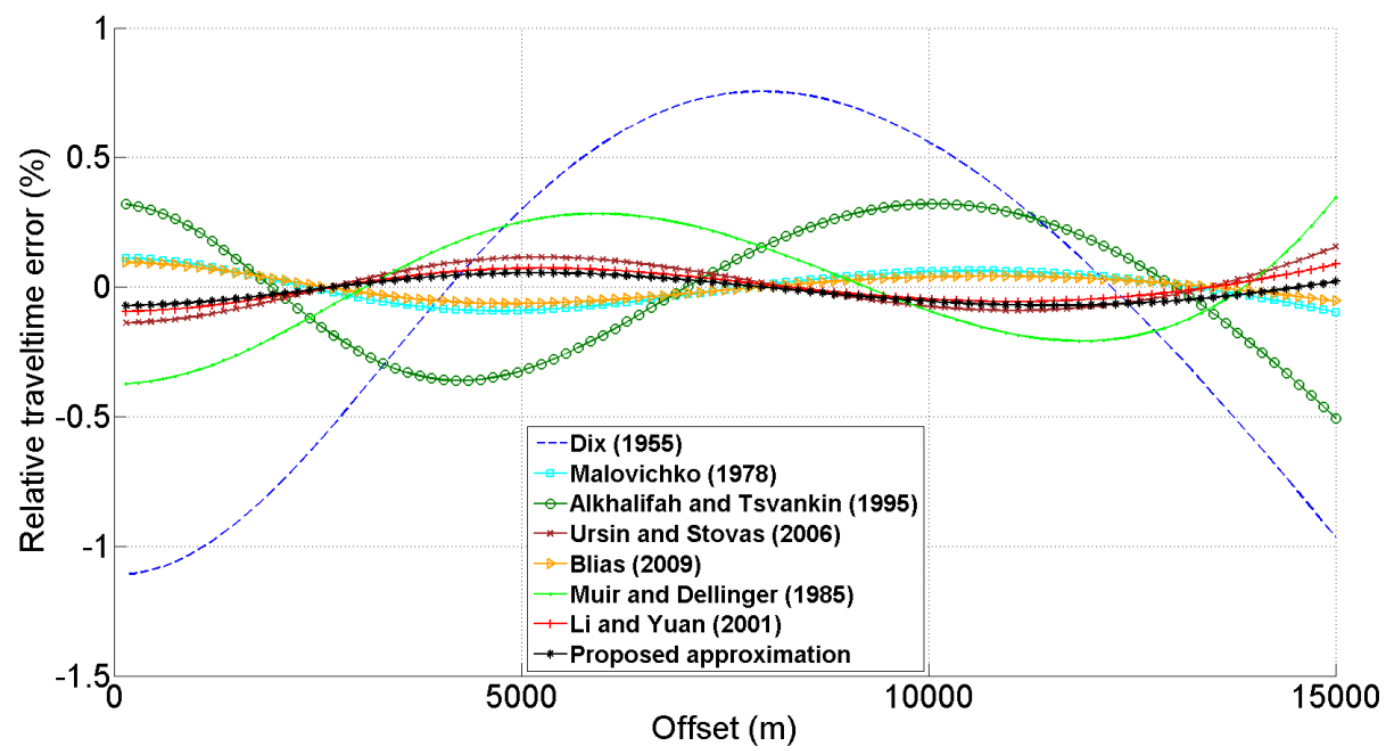

Figure 203: Relative error in travel-time between the observed curve and the calculated curve with each approximation of the PP reflection event with L1-norm and SID-PSM optimization algorithm.

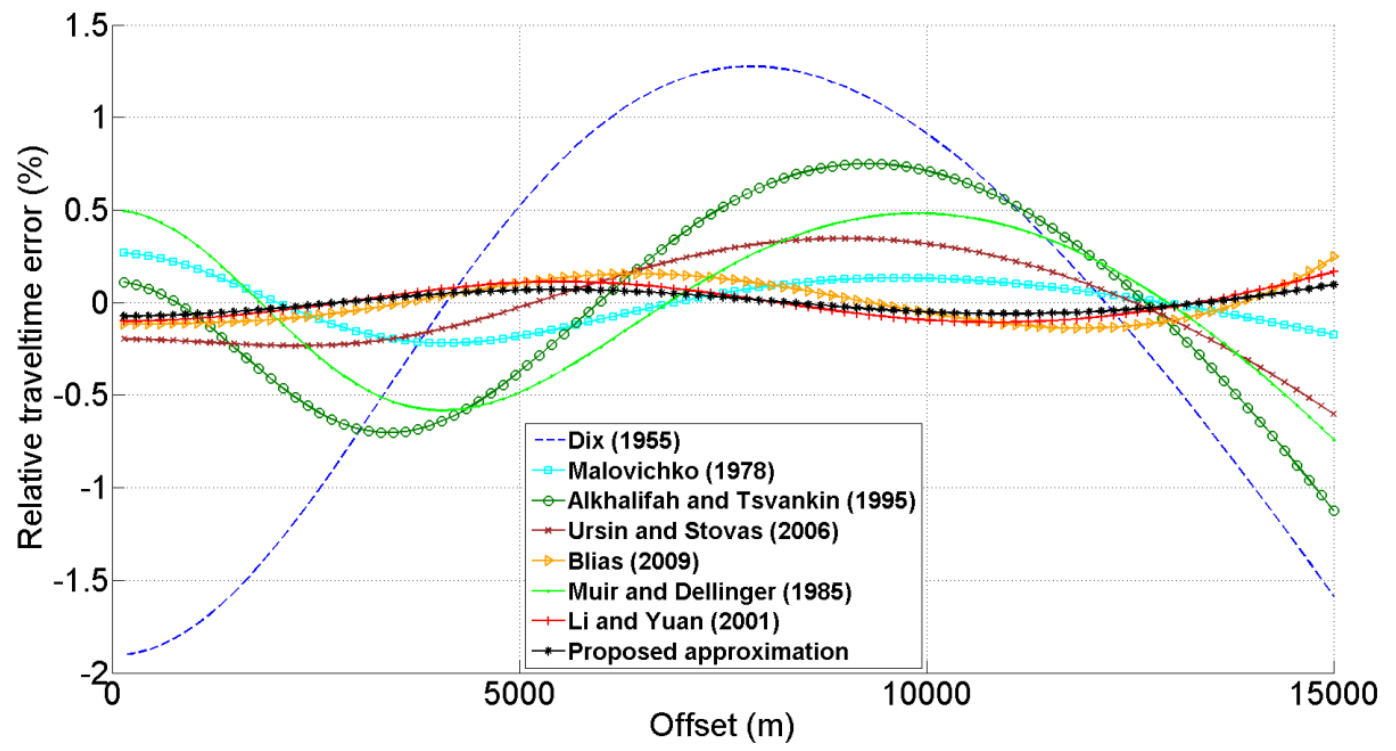

Figure 204: Relative error in travel-time between the observed curve and the calculated curve with each approximation of the PS reflection event with L1-norm and SID-PSM optimization algorithm. 


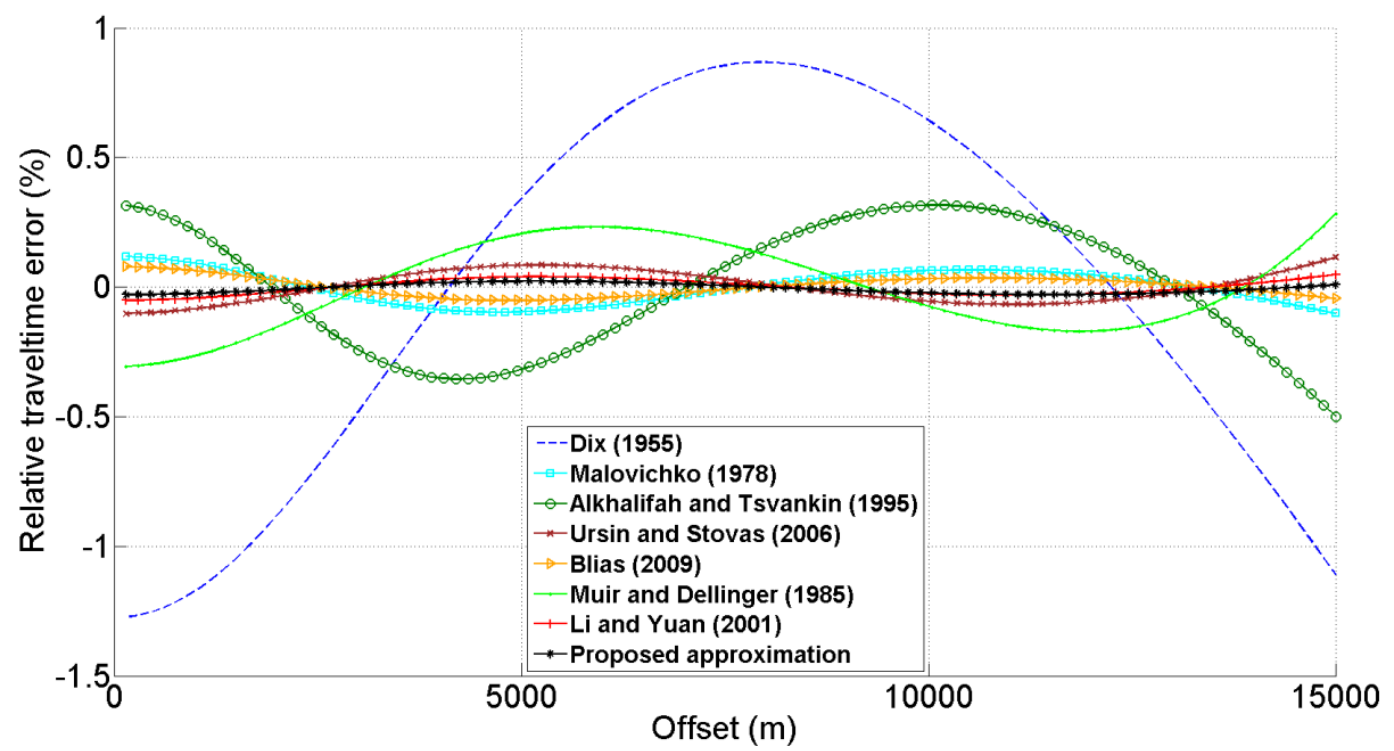

Figure 205: Relative error in travel-time between the observed curve and the calculated curve with each approximation of the PP reflection event with L2-norm and MCS optimization algorithm.

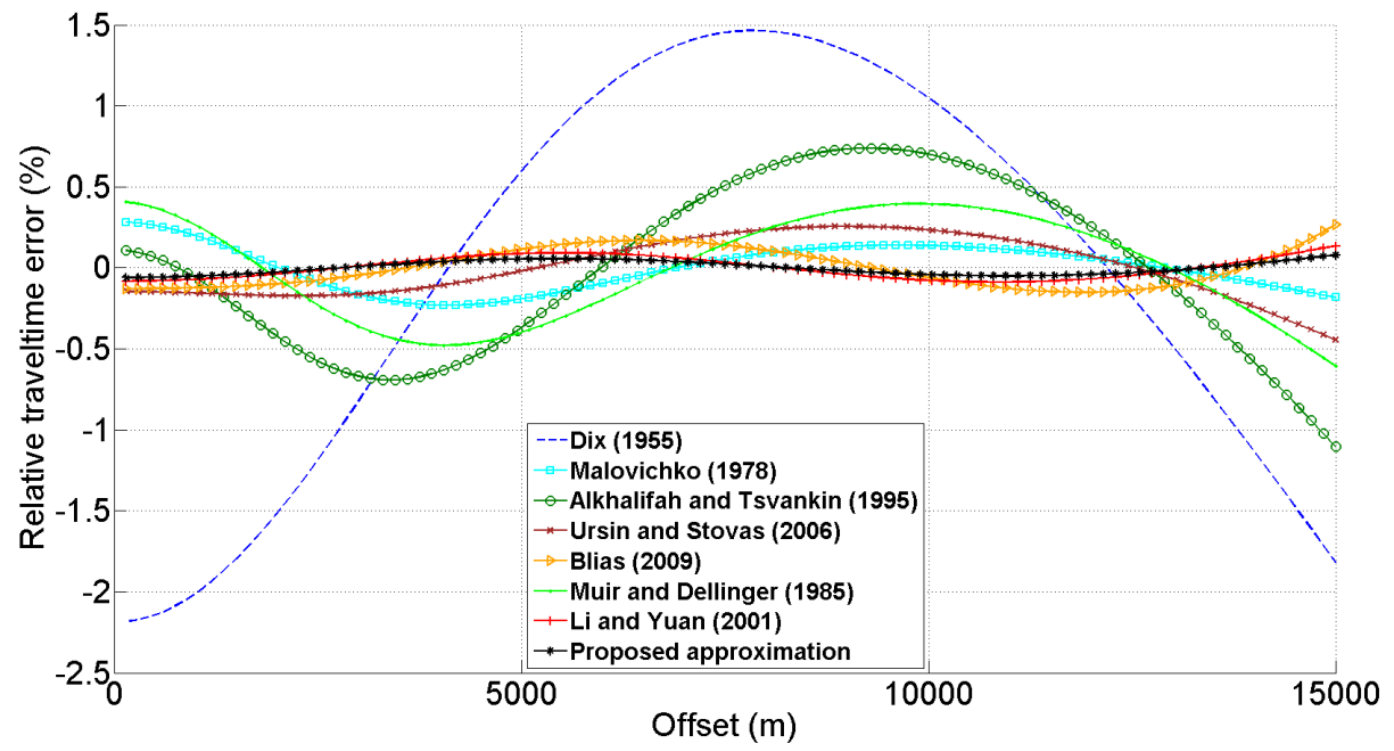

Figure 206: Relative error in travel-time between the observed curve and the calculated curve with each approximation of the PS reflection event with L2-norm and MCS optimization algorithm. 


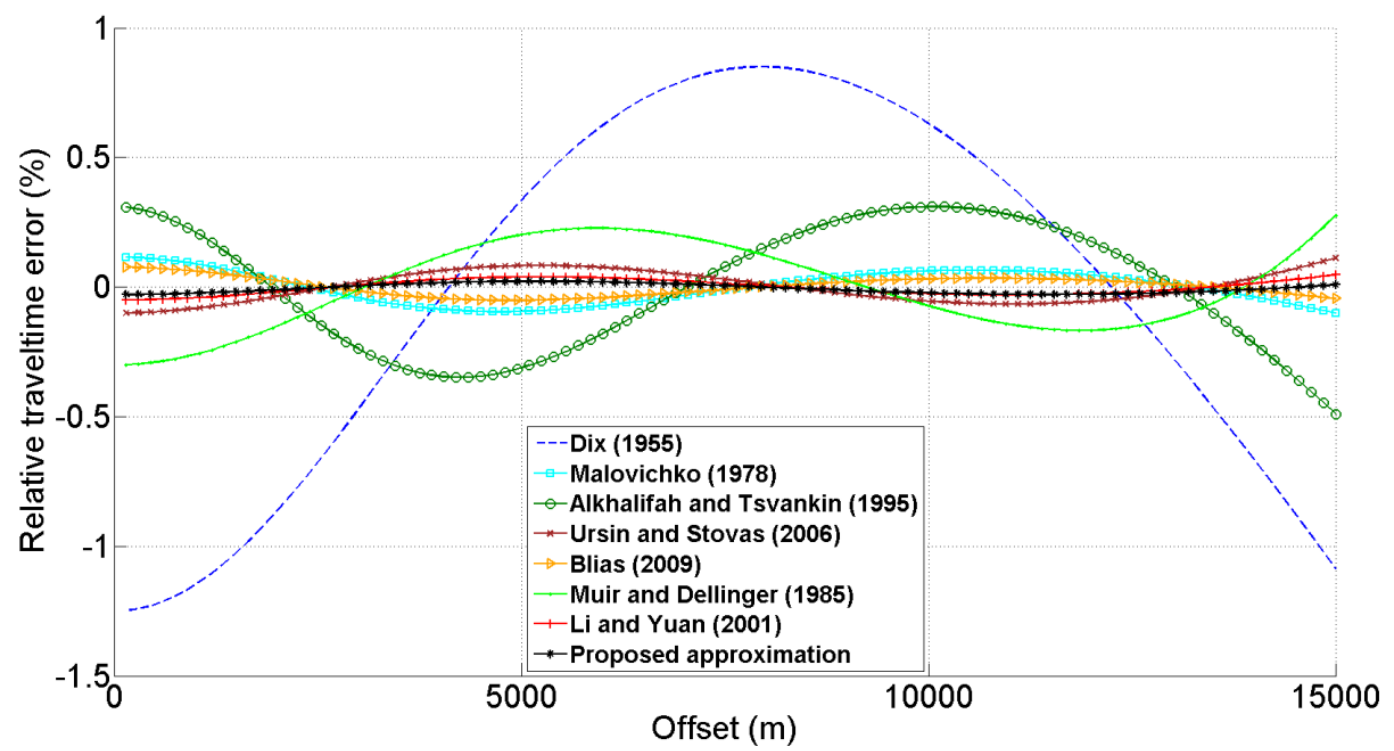

Figure 207: Relative error in travel-time between the observed curve and the calculated curve with each approximation of the PP reflection event with L1-norm and MCS optimization algorithm.

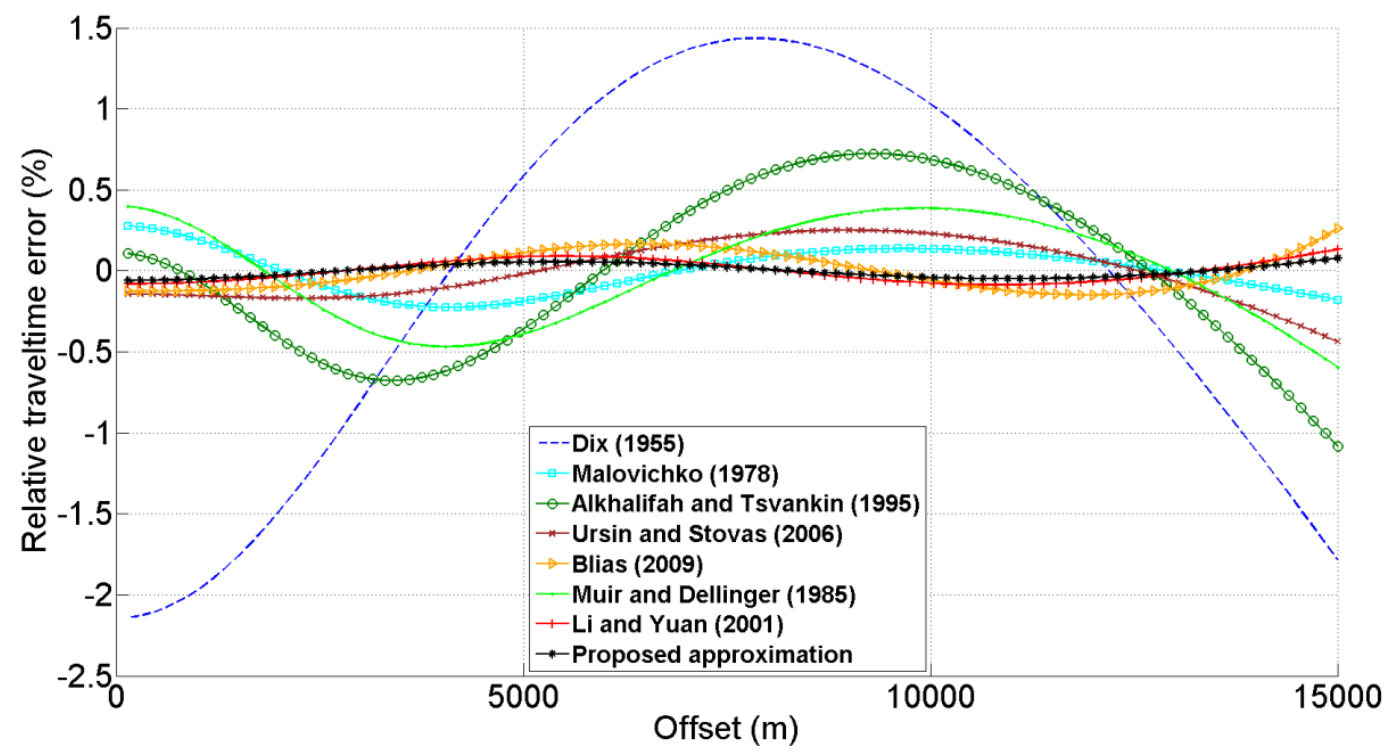

Figure 208: Relative error in travel-time between the observed curve and the calculated curve with each approximation of the PS reflection event with L1-norm and MCS optimization algorithm. 


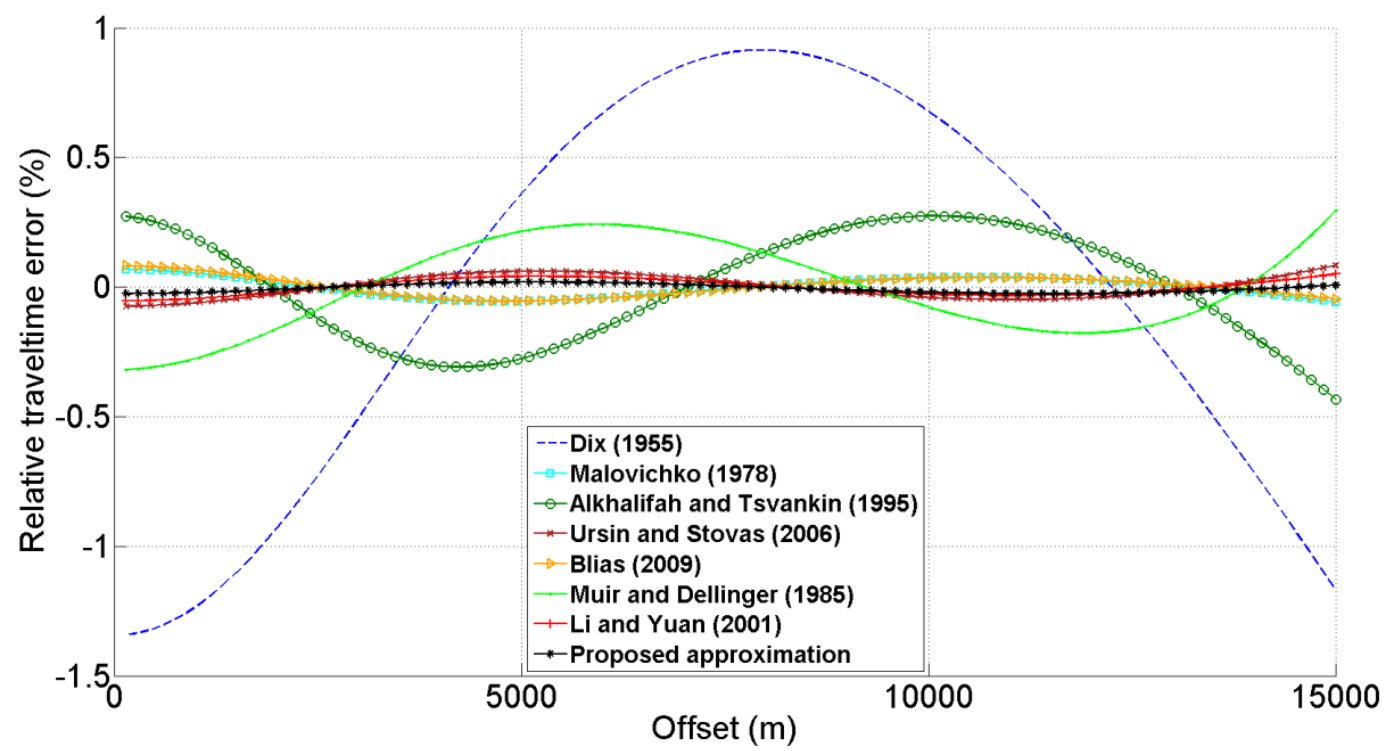

Figure 209: Relative error in travel-time between the observed curve and the calculated curve with each approximation of the PP reflection event with L2-norm and TOMLAB/LGO optimization algorithm.

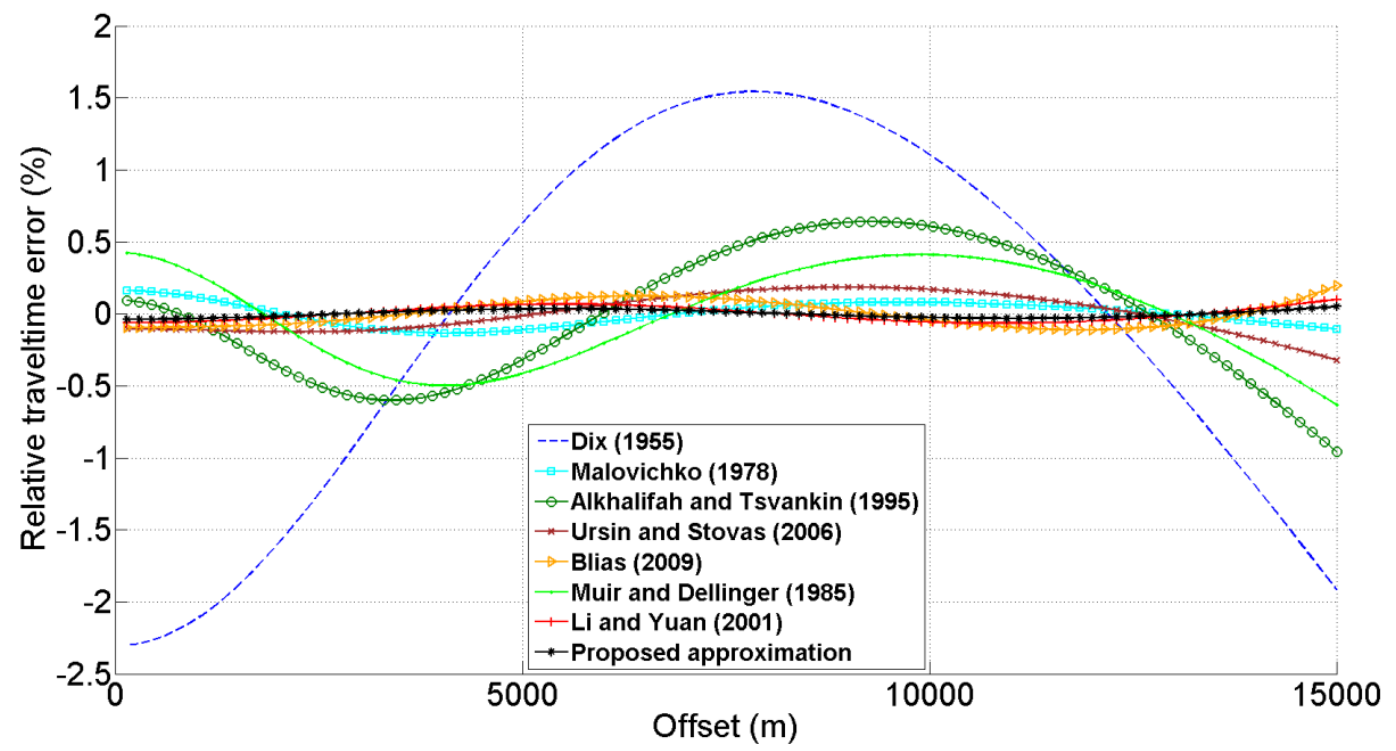

Figure 210: Relative error in travel-time between the observed curve and the calculated curve with each approximation of the PS reflection event with L2-norm and TOMLAB/LGO optimization algorithm. 


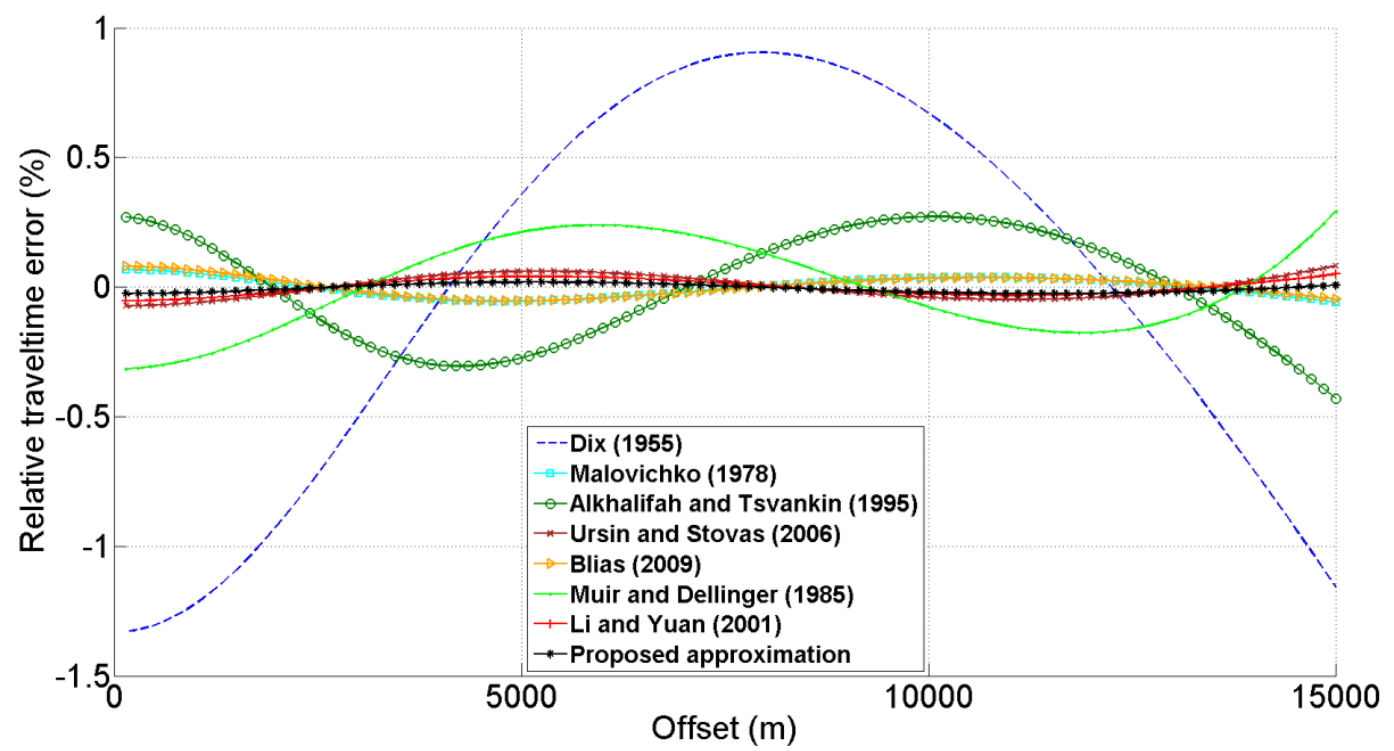

Figure 211: Relative error in travel-time between the observed curve and the calculated curve with each approximation of the PP reflection event with L1-norm and TOMLAB/LGO optimization algorithm.

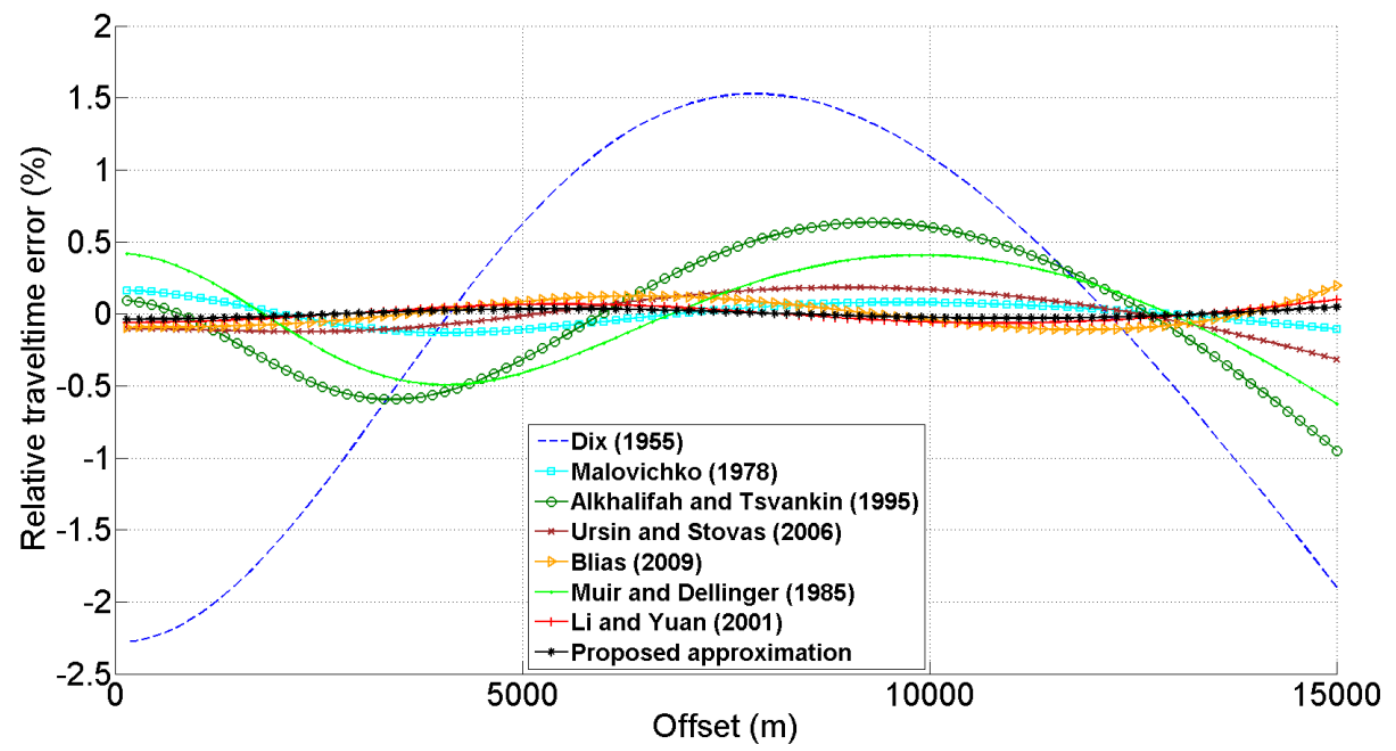

Figure 212: Relative error in travel-time between the observed curve and the calculated curve with each approximation of the PS reflection event with L1-norm and TOMLAB/LGO optimization algorithm. 


\subsection{Model 3}

In the third model, using the IMFL optimization algorithm with the L2-norm for PP event resulted in Ursin and Stovas (2006) showing the third best result; Malovichko (1978), the fourth; and Blias (2009), the fifth (Figure 213). For the PS reflection event, Malovichko (1978) showed the third best result; Blias (2009), the fourth; and Ursin and Stovas (2006), the fifth (Figure 214). Similarly to the previous two models, the L1-norm presented a better result than the L2-norm (Figures 215 and 216).

The FMINSEARCH optimization algorithm presented Ursin and Stovas (2006) as the third, Malovichko (1978) as the fourth and Blias (2009) as the fifth best results using L2-norm for the conventional event (Figure 217). For the converted wave event, Malovichko (1978) showed to be the third most accurate, while Blias (2009) showed to be the fourth and Ursin and Stovas (2006) were the fifth (Figure 218). The use of the L1-norm resulted in less than $10 \%$ of increase for the accuracy (Figures 219 and 220).

As shown in Figure 221, the SID-PSM optimization algorithm along with the use of L2-norm showed Ursin and Stovas (2006) as the third best result; Malovichko (1978), the fourth; and Blias (2009), the fifth for the PP event. On the other hand, for the PS event, Malovichko (1978) presented the third best result; Blias (2009), the fourth; and Ursin and Stovas (2006), the fifth (Figure 222). As it was seen before for the other two local search algorithms with the use of L1-norm, it was possible to observe a similar increase concerning the accuracy (Figures 223 and 224).

Using the global search optimization algorithm MCS with the L2-norm for the PP event, it was found that the approximation proposed by Ursin and Stovas (2006) had the third, Malovichko (1978) the fourth, and Blias (2009) the fifth best results (Figure 225). For the converted wave event, Malovichko (1978) showed the third best result, Blias (2009) showed the fourth and Ursin and Stovas (2006) were the fifth (Figure 226). Using the L1-norm resulted in a decrease of the error of around 1\% (Figure 227 and 228).

The TOMLAB/LGO optimization algorithm presented the same sequence of results shown by the MCS algorithm for the PP event using the L2-norm (Figure 229) and for PS using the same norm (Figure 230). Using the L1-norm (Figures 231 and 232) also showed a decrease of the error of around $10 \%$. 


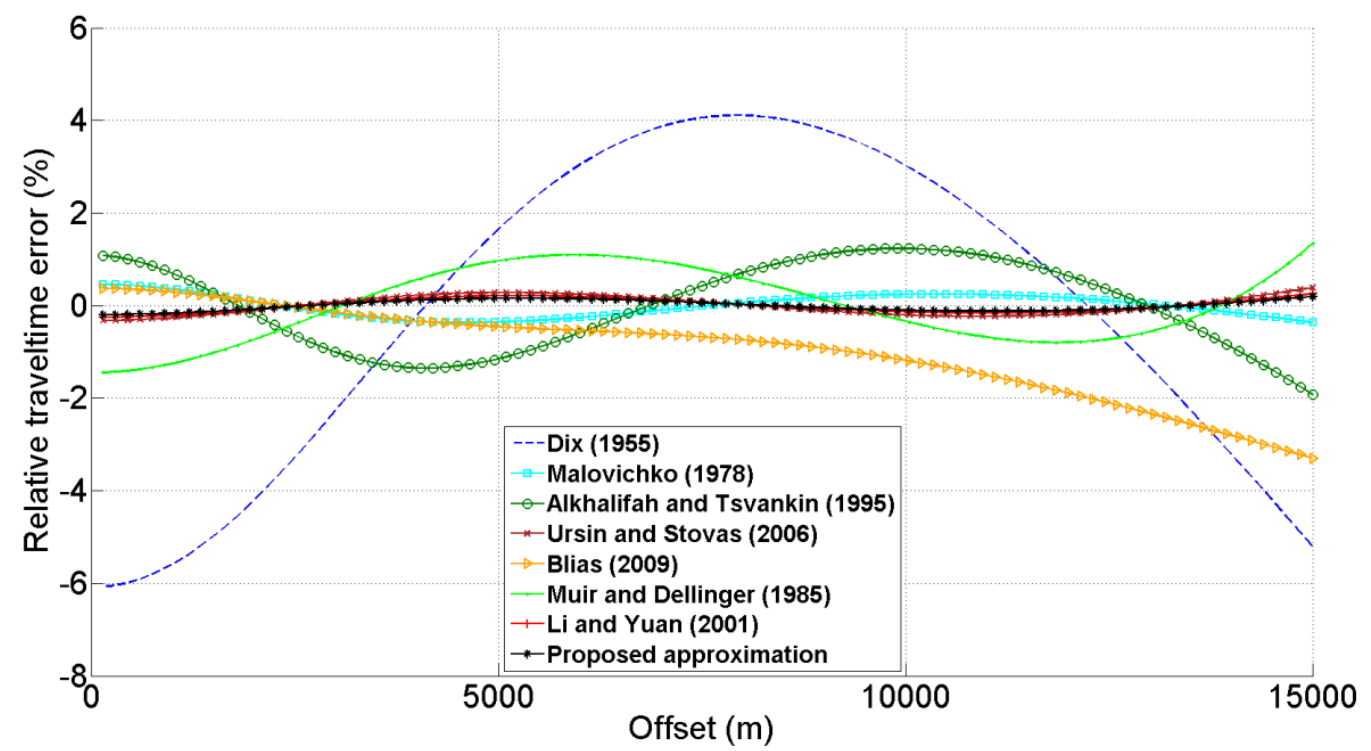

Figure 213: Relative error in travel-time between the observed curve and the calculated curve with each approximation of the PP reflection event with L2-norm and IMFIL optimization algorithm.

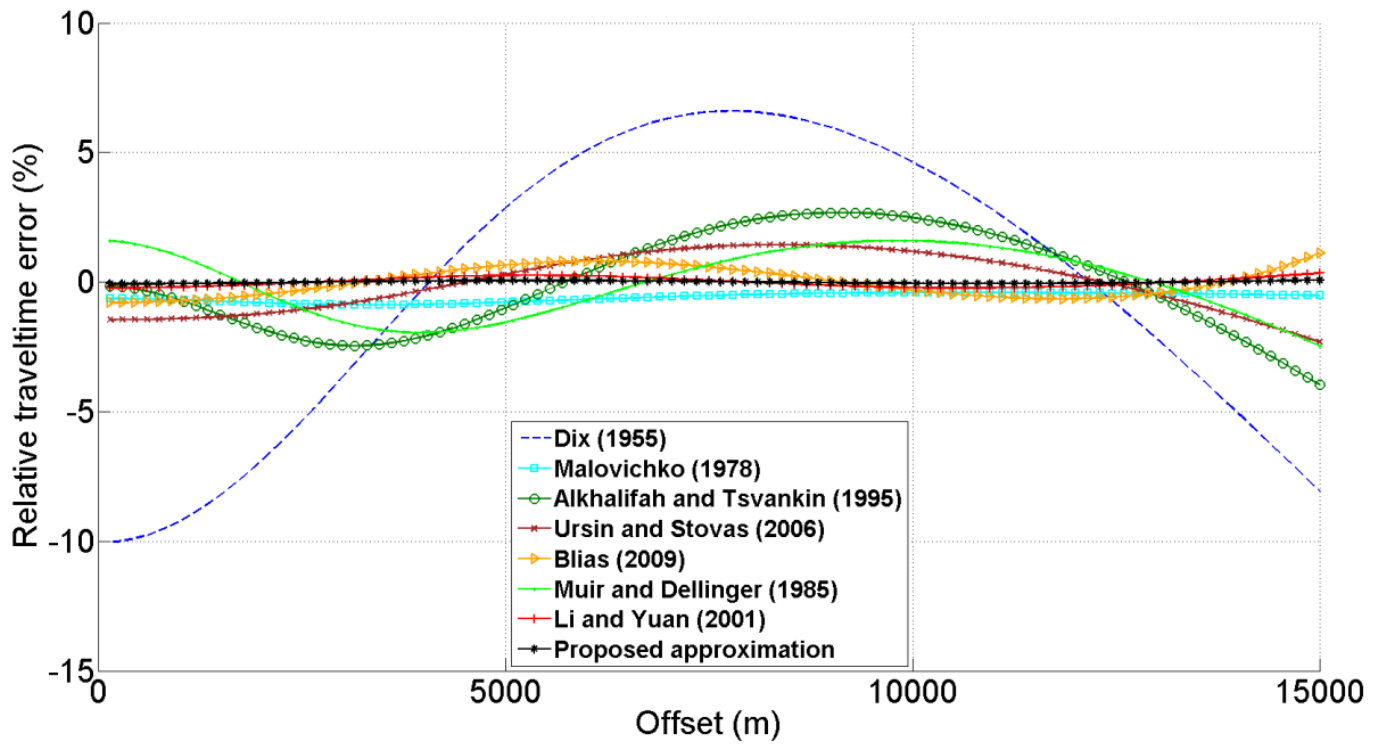

Figure 214: Relative error in travel-time between the observed curve and the calculated curve with each approximation of the PS reflection event with L2-norm and IMFIL optimization algorithm. 


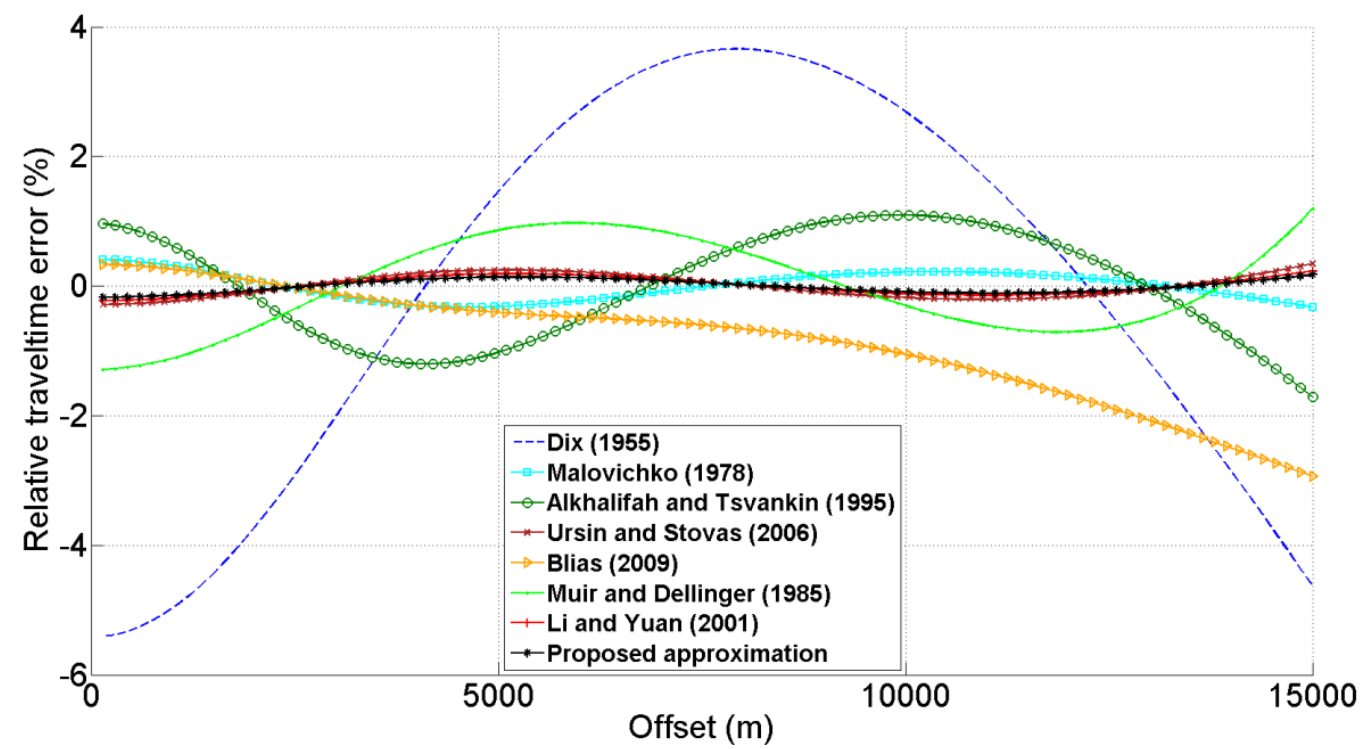

Figure 215: Relative error in travel-time between the observed curve and the calculated curve with each approximation of the PP reflection event with L1-norm and IMFIL optimization algorithm.

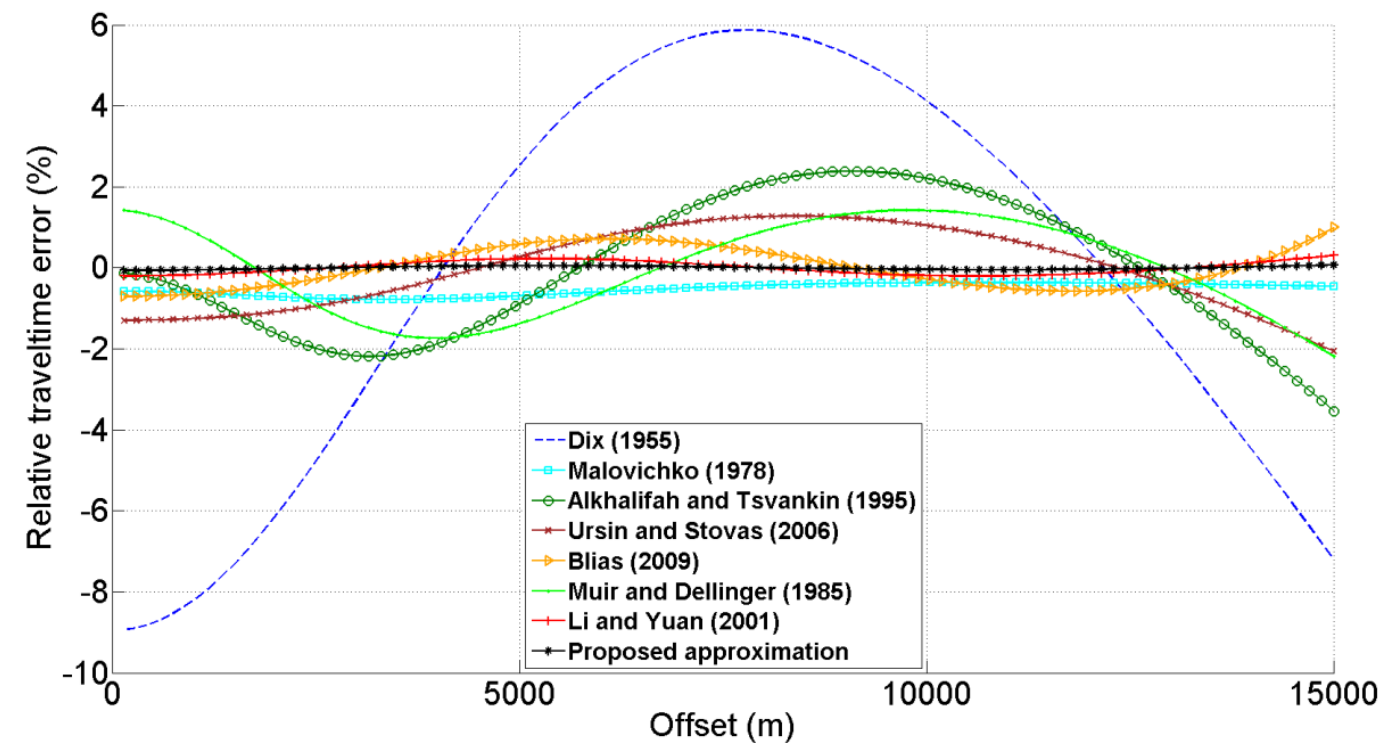

Figure 216: Relative error in travel-time between the observed curve and the calculated curve with each approximation of the PS reflection event with L1-norm and IMFIL optimization algorithm. 


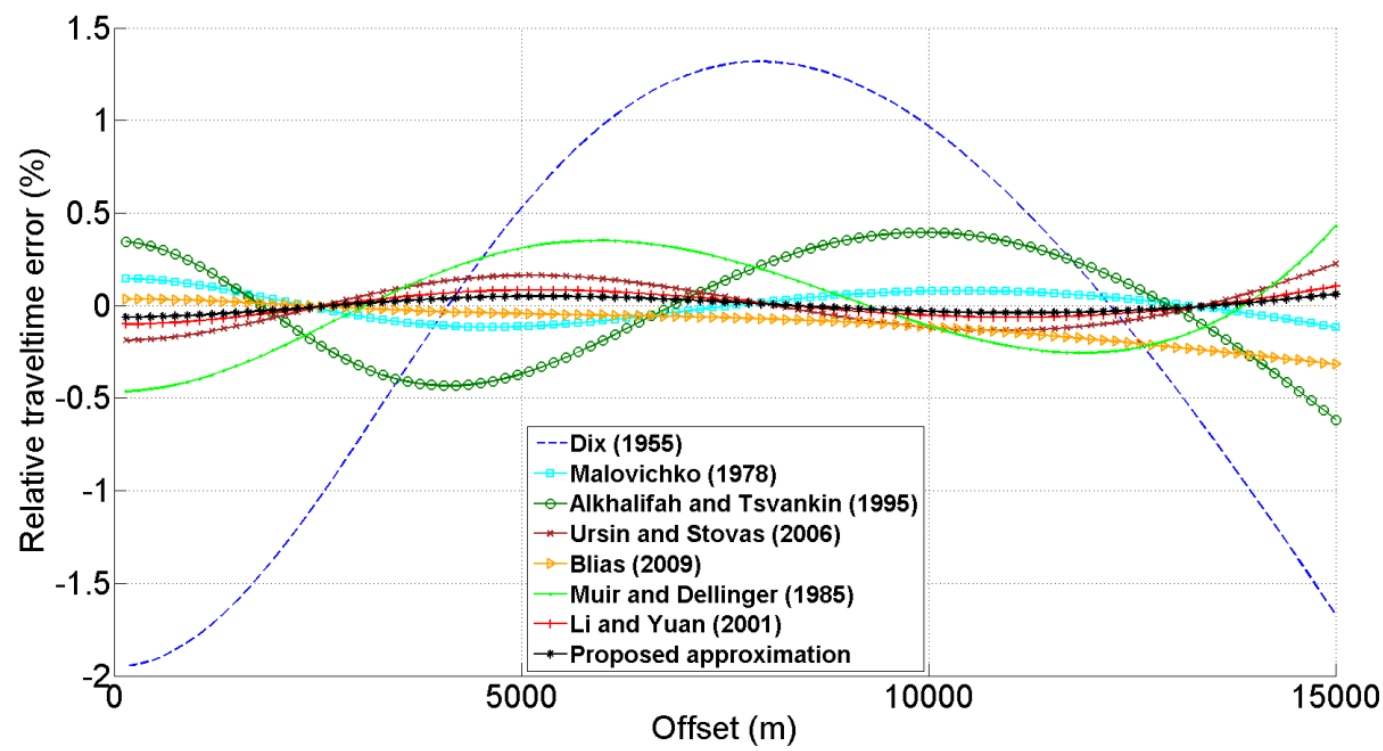

Figure 217: Relative error in travel-time between the observed curve and the calculated curve with each approximation of the PP reflection event with L2-norm and FMINSEARCH optimization algorithm.

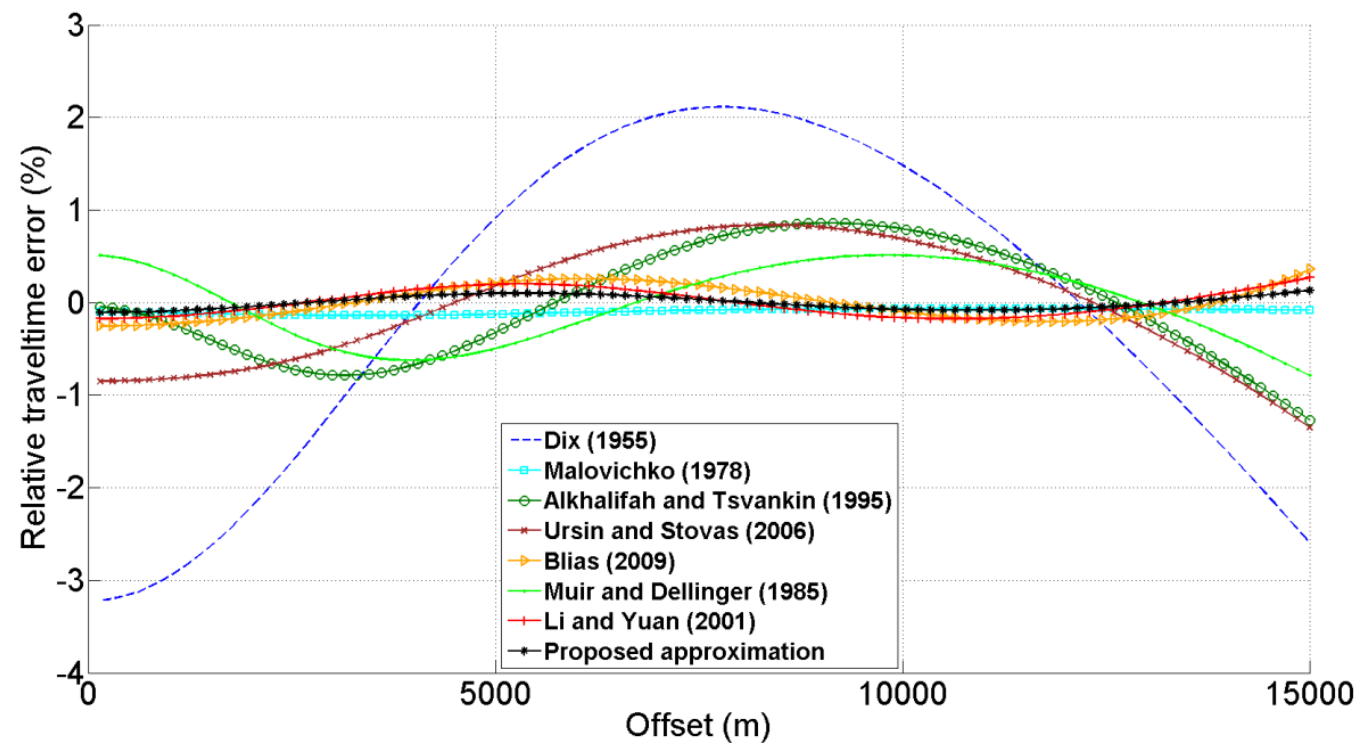

Figure 218: Relative error in travel-time between the observed curve and the calculated curve with each approximation of the PS reflection event with L2-norm and FMINSEARCH optimization algorithm. 


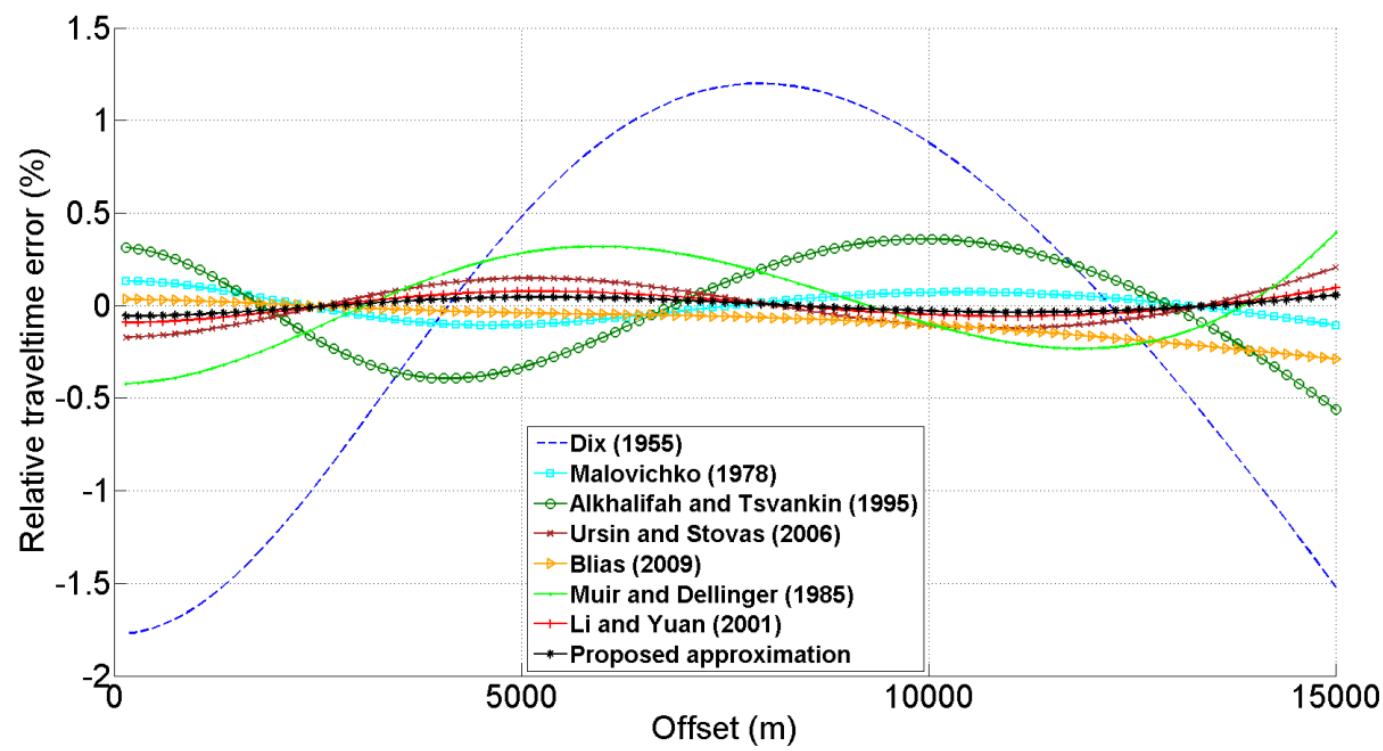

Figure 219: Relative error in travel-time between the observed curve and the calculated curve with each approximation of the PP reflection event with L1-norm and FMINSEARCH optimization algorithm.

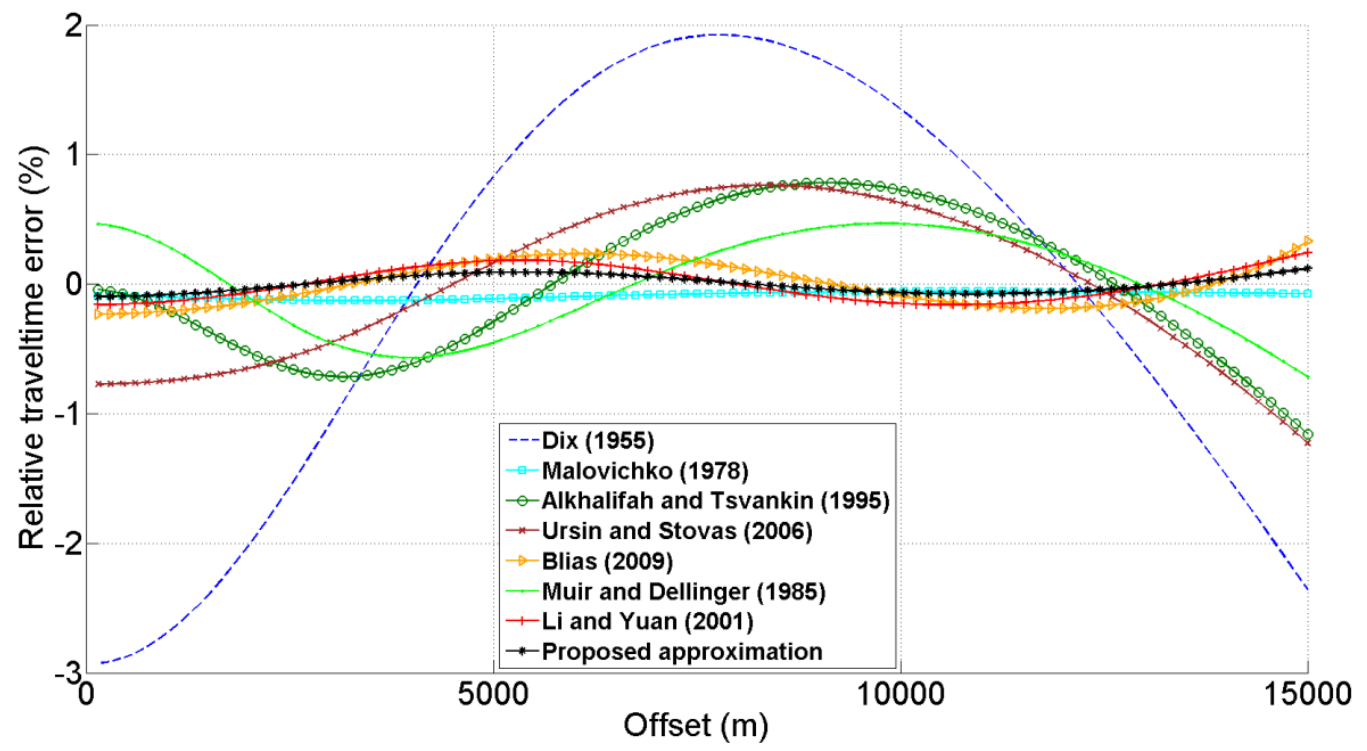

Figure 220: Relative error in travel-time between the observed curve and the calculated curve with each approximation of the PS reflection event with L1-norm and FMINSEARCH optimization algorithm. 


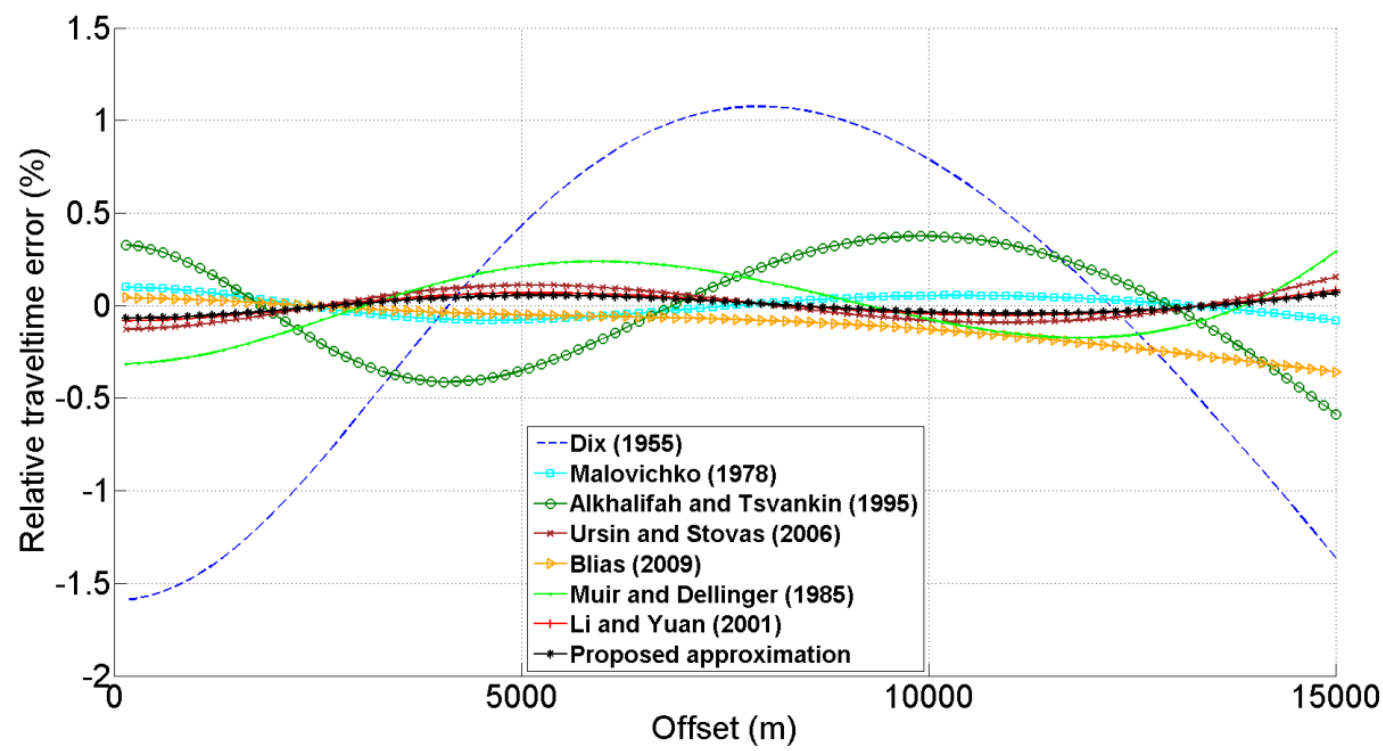

Figure 221: Relative error in travel-time between the observed curve and the calculated curve with each approximation of the PP reflection event with L2-norm and SID-PSM optimization algorithm.

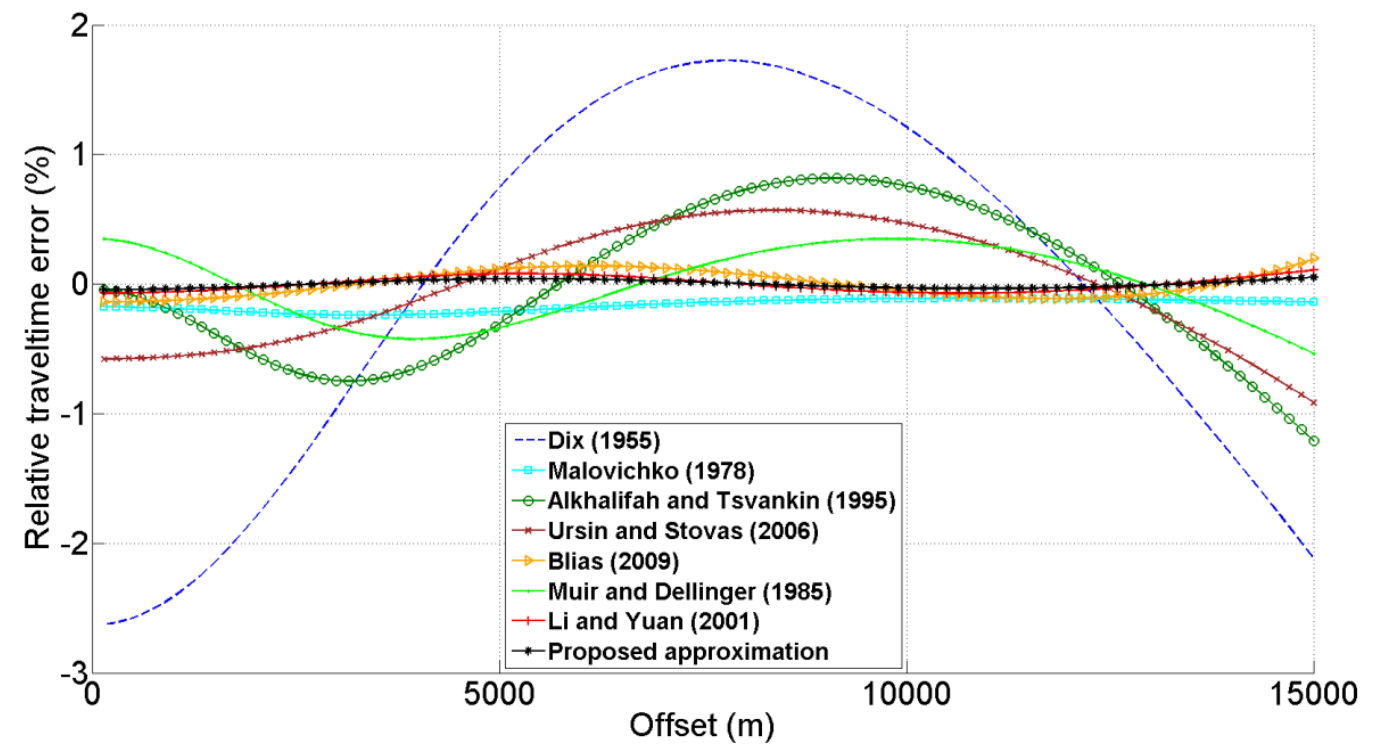

Figure 222: Relative error in travel-time between the observed curve and the calculated curve with each approximation of the PS reflection event with L2-norm and SID-PSM optimization algorithm. 


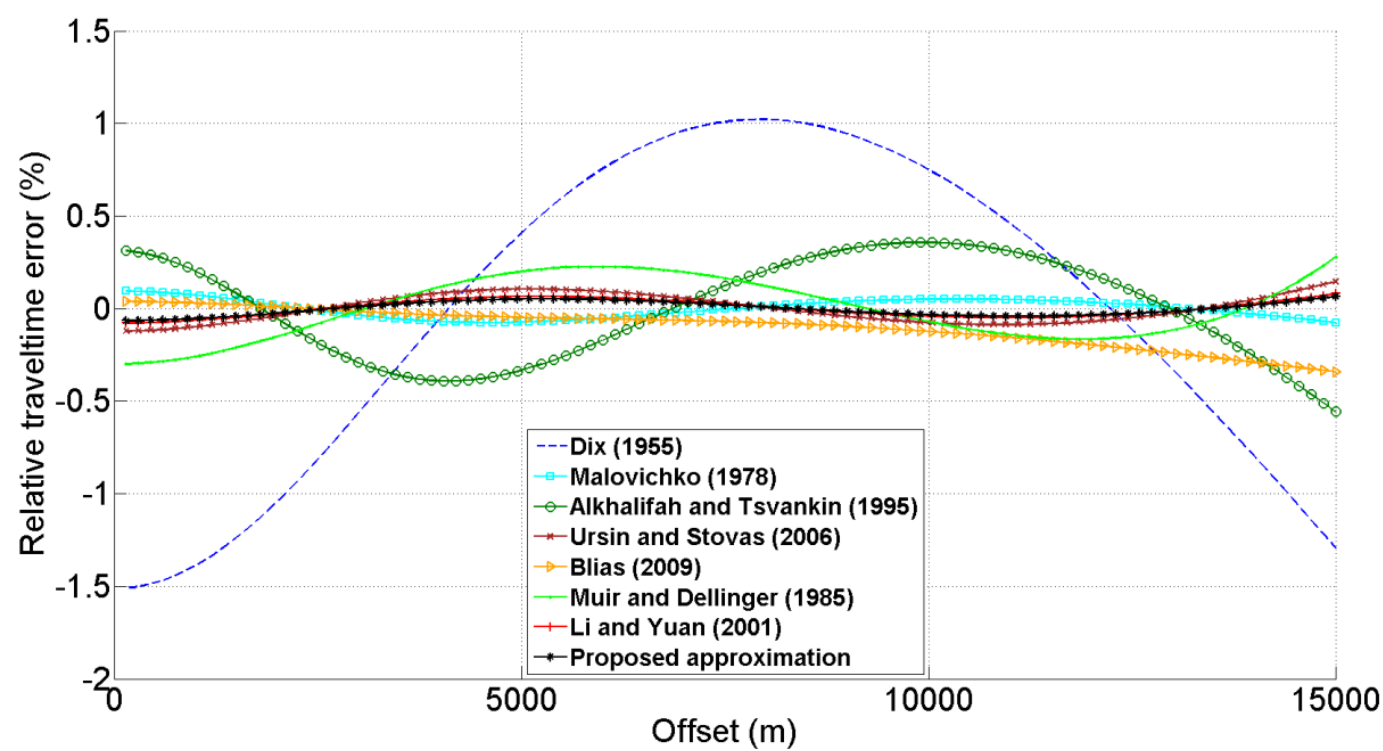

Figure 223: Relative error in travel-time between the observed curve and the calculated curve with each approximation of the PP reflection event with L1-norm and SID-PSM optimization algorithm.

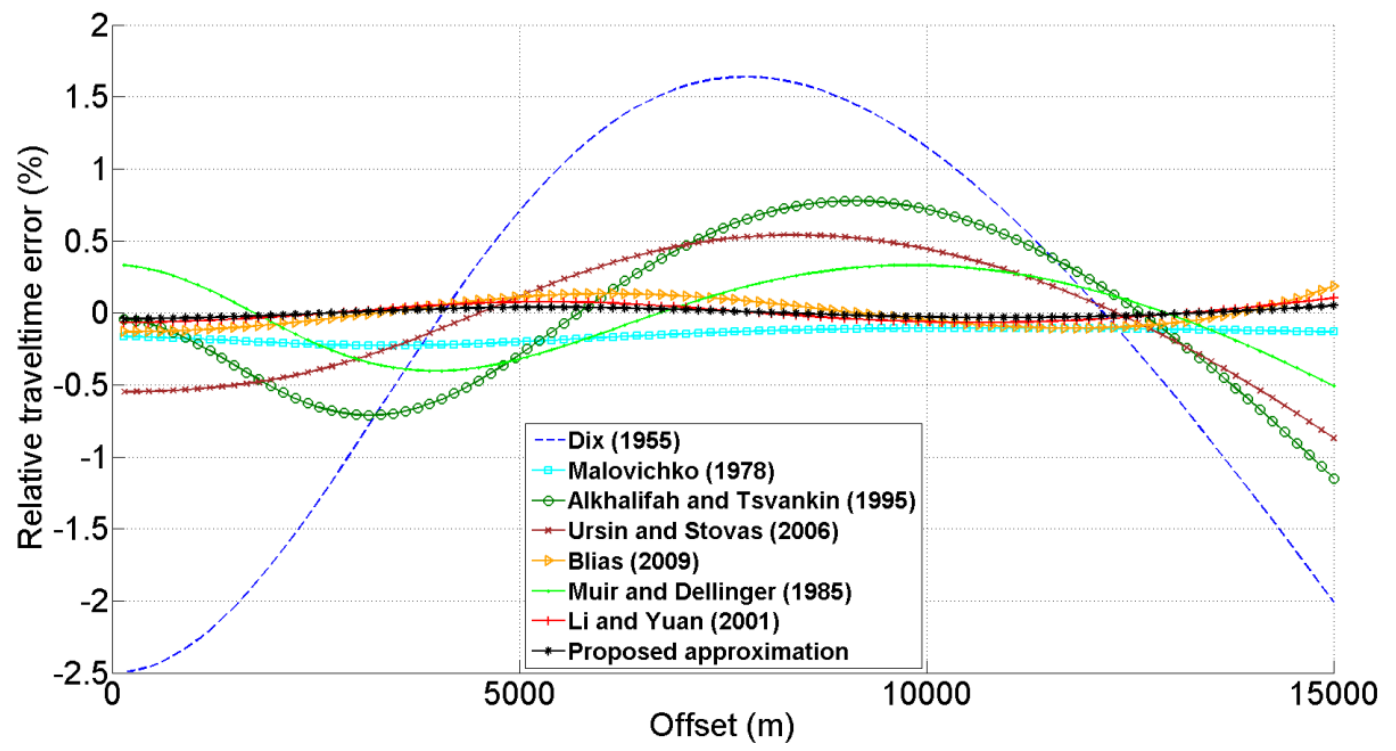

Figure 224: Relative error in travel-time between the observed curve and the calculated curve with each approximation of the PS reflection event with L1-norm and SID-PSM optimization algorithm. 


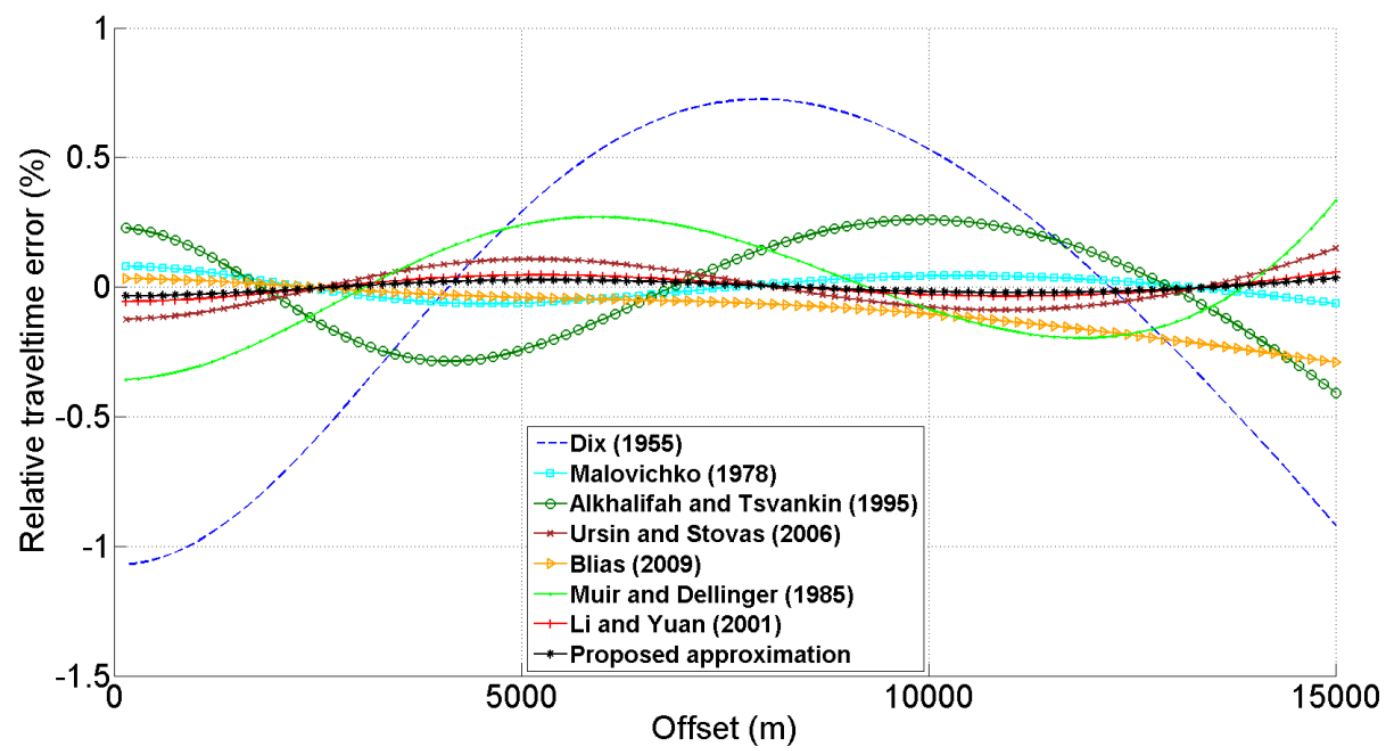

Figure 225: Relative error in travel-time between the observed curve and the calculated curve with each approximation of the PP reflection event with L2-norm and MCS optimization algorithm.

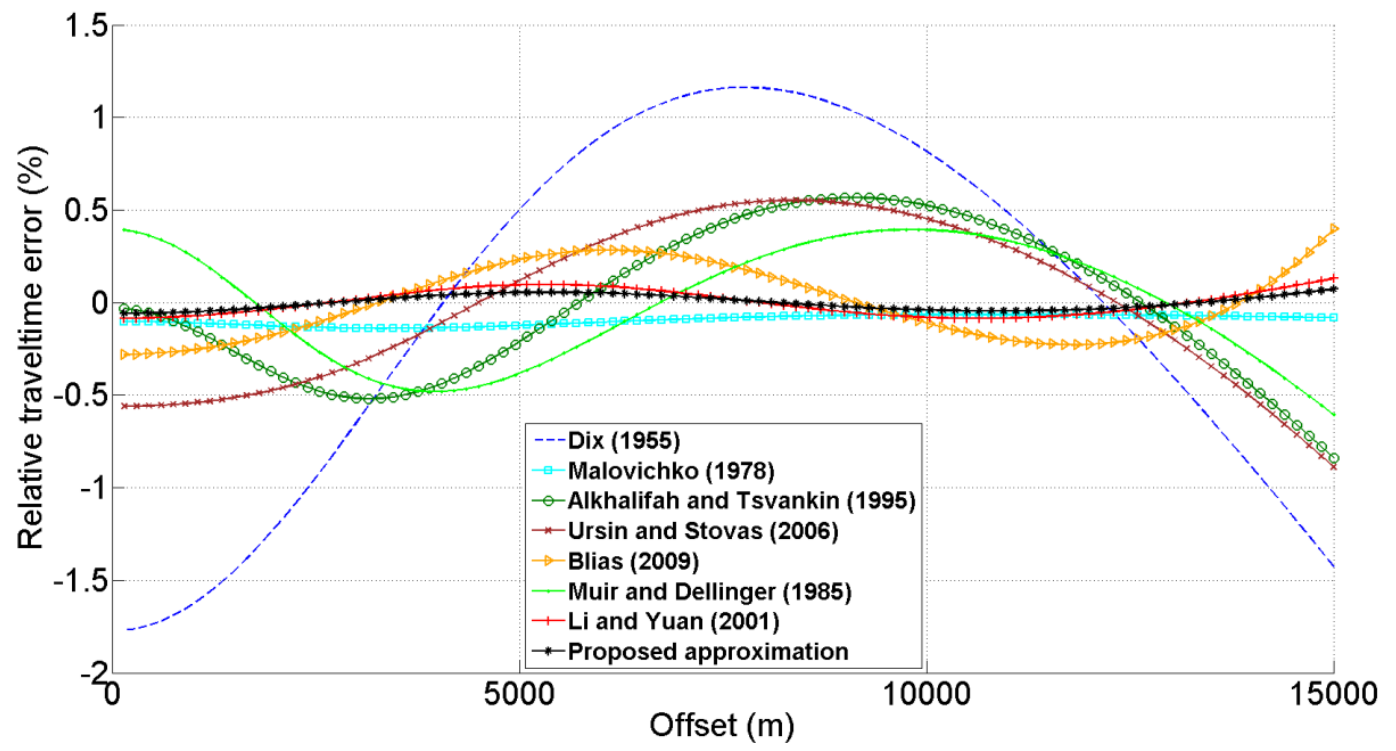

Figure 226: Relative error in travel-time between the observed curve and the calculated curve with each approximation of the PS reflection event with L2-norm and MCS optimization algorithm. 


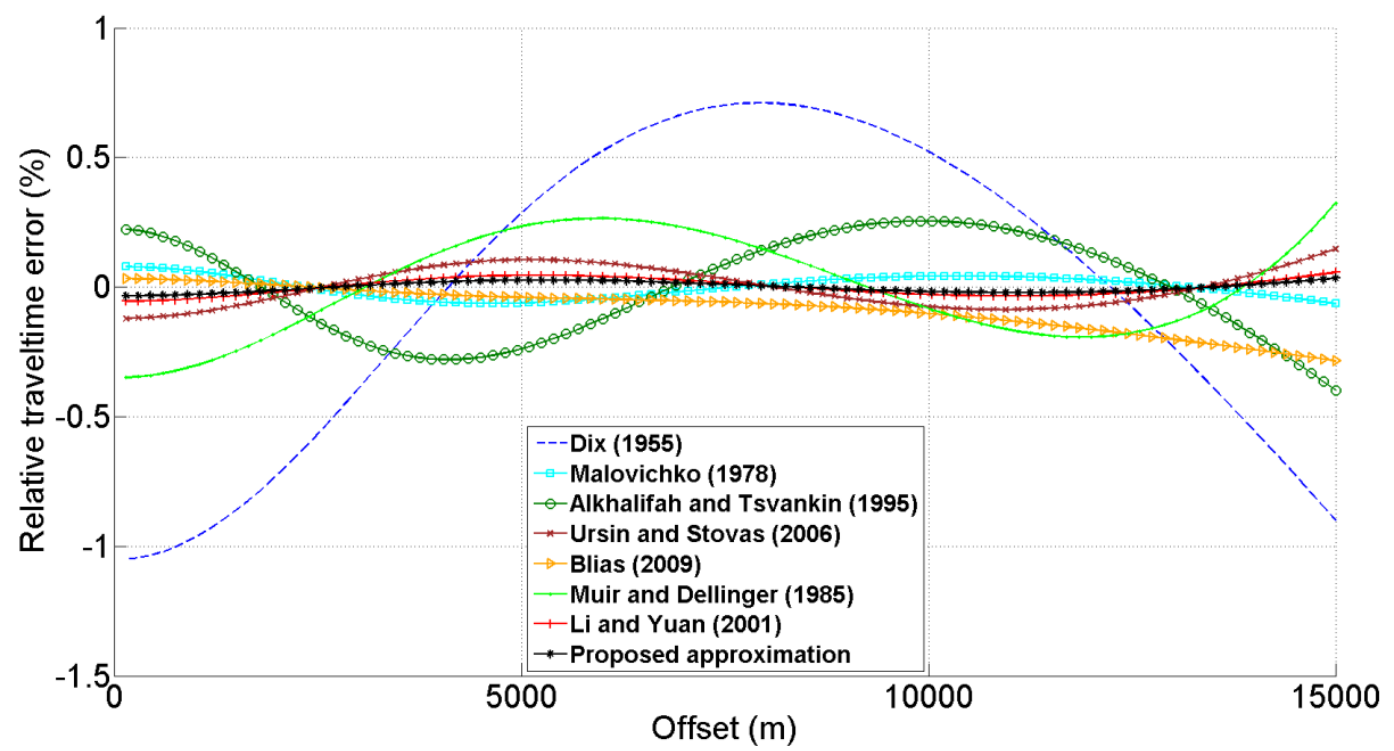

Figure 227: Relative error in travel-time between the observed curve and the calculated curve with each approximation of the PP reflection event with L1-norm and MCS optimization algorithm.

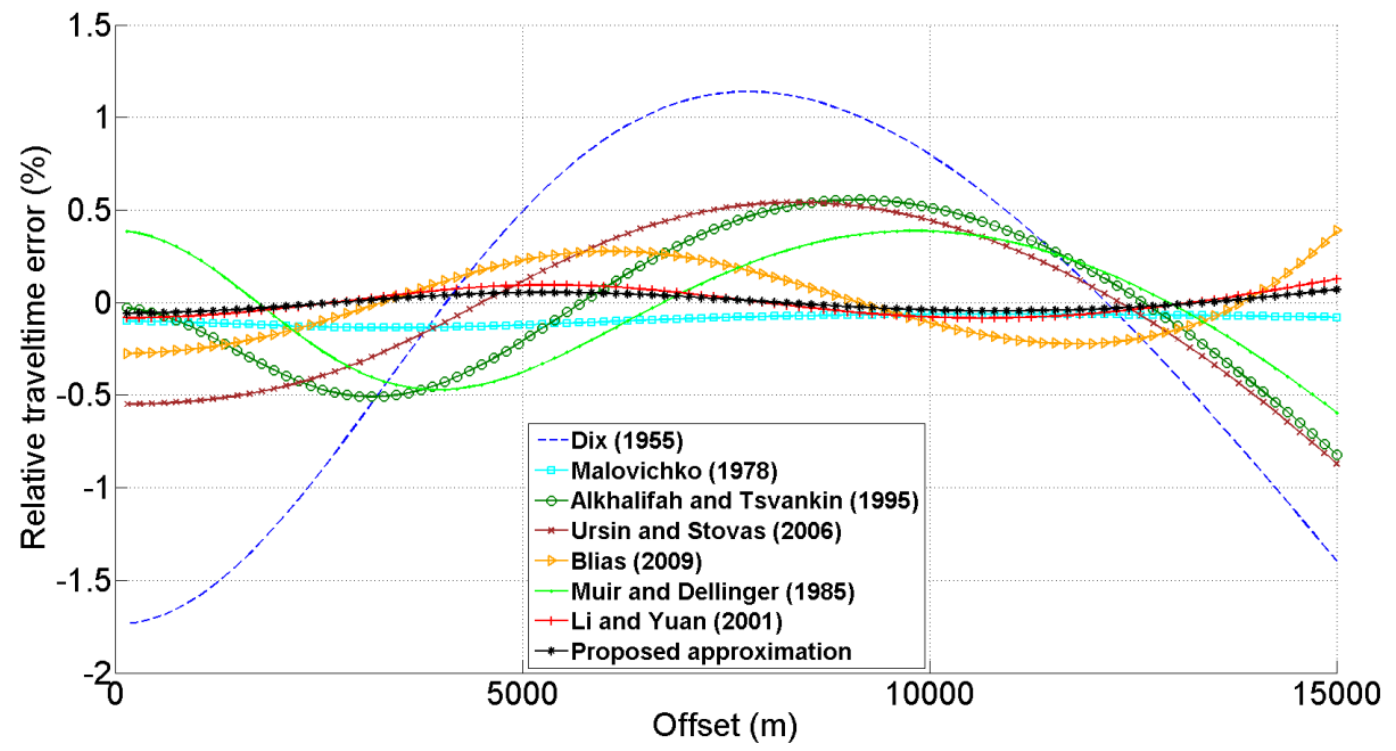

Figure 228: Relative error in travel-time between the observed curve and the calculated curve with each approximation of the PS reflection event with L1-norm and MCS optimization algorithm. 


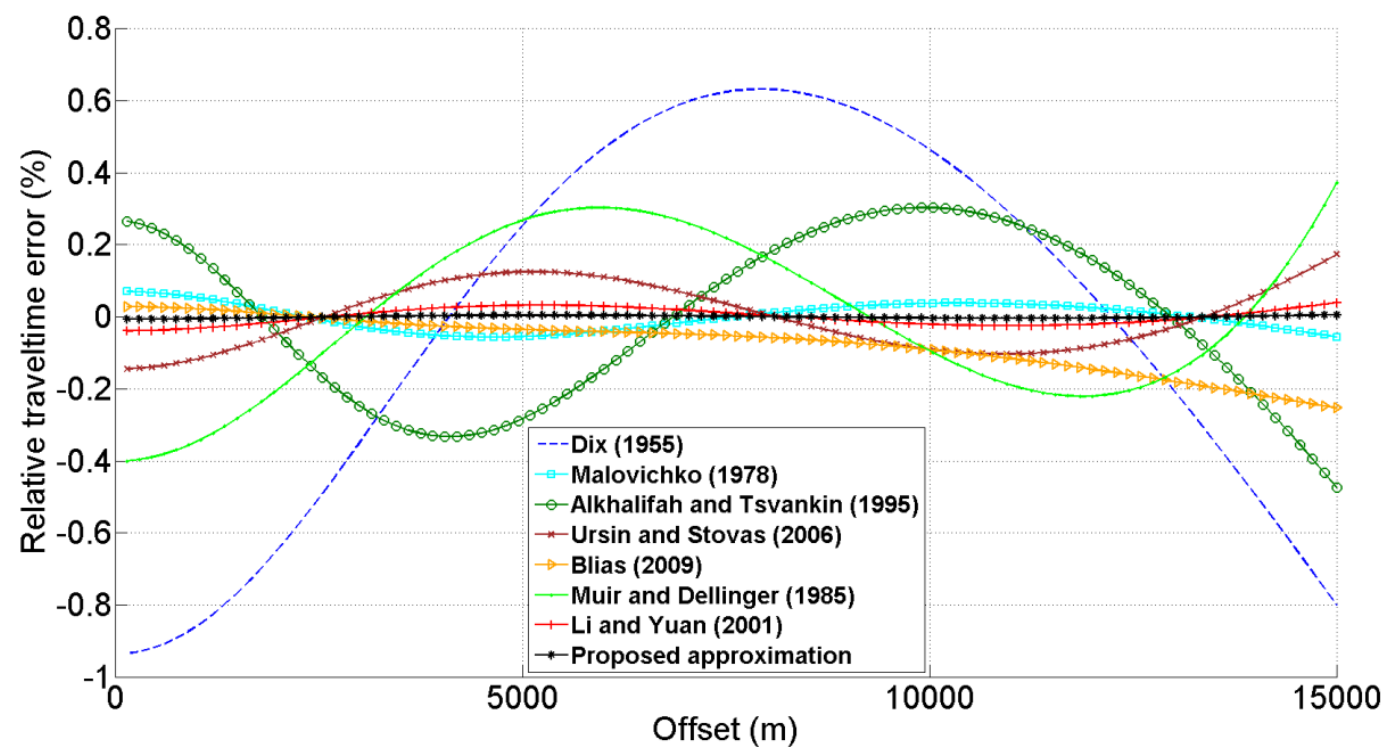

Figure 229: Relative error in travel-time between the observed curve and the calculated curve with each approximation of the PP reflection event with L2-norm and TOMLAB/LGO optimization algorithm.

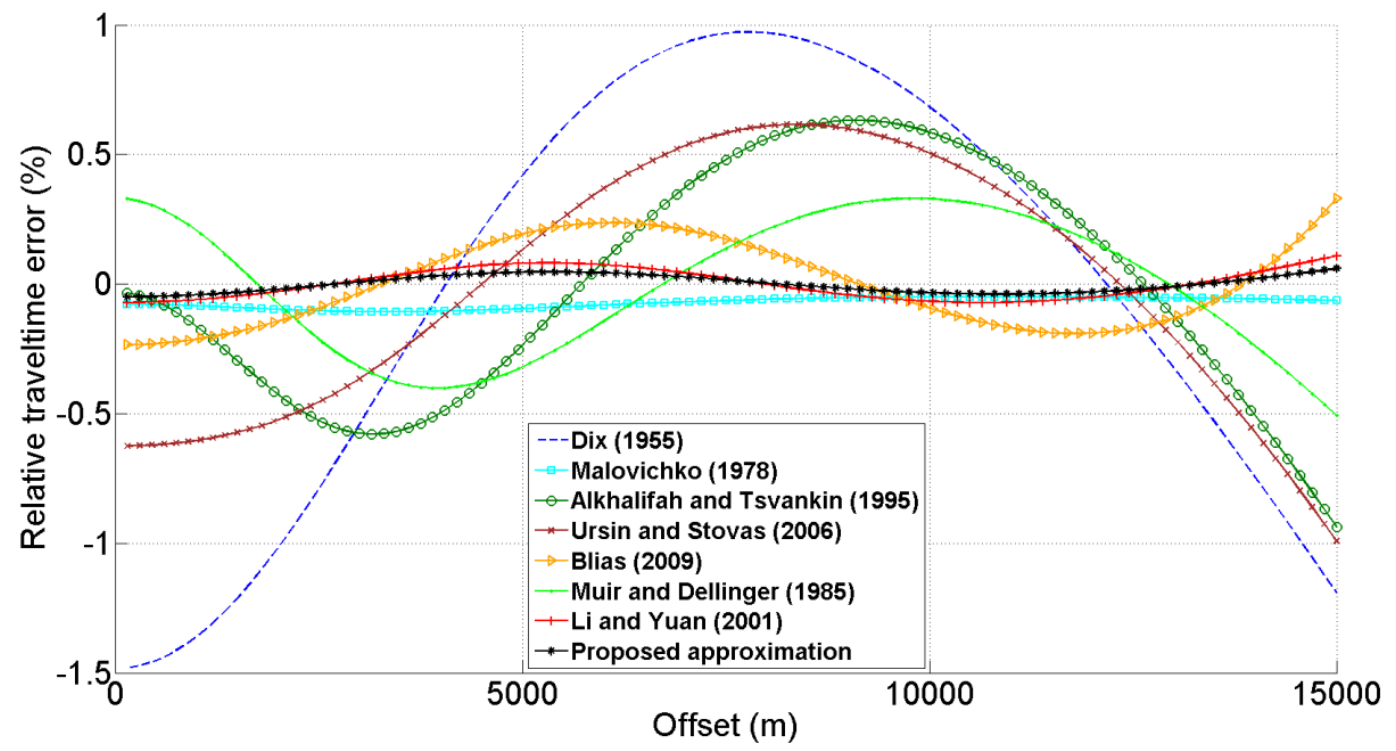

Figure 230: Relative error in travel-time between the observed curve and the calculated curve with each approximation of the PS reflection event with L2-norm and TOMLAB/LGO optimization algorithm. 


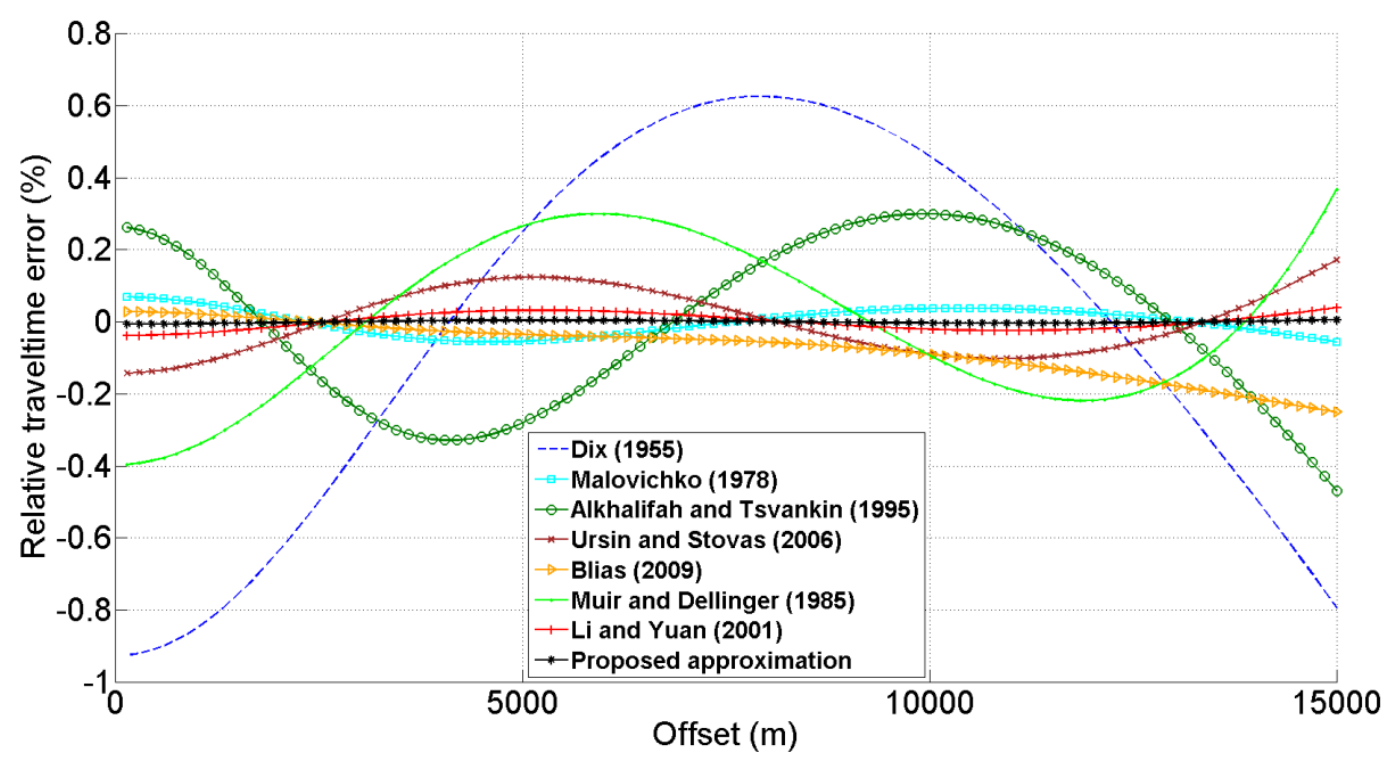

Figure 231: Relative error in travel-time between the observed curve and the calculated curve with each approximation of the PP reflection event with L1-norm and TOMLAB/LGO optimization algorithm.

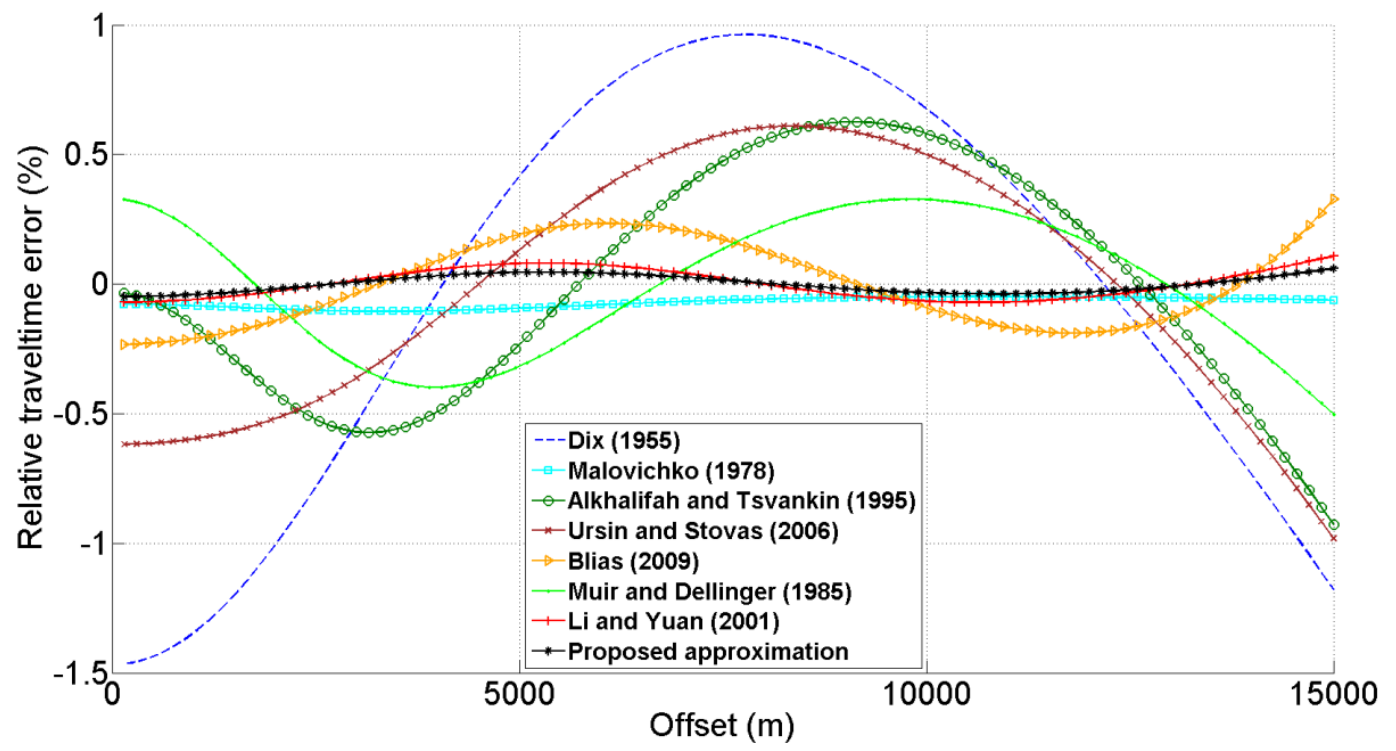

Figure 232: Relative error in travel-time between the observed curve and the calculated curve with each approximation of the PS reflection event with L1-norm and TOMLAB/LGO optimization algorithm. 


\subsection{Model 4}

In Model 4, the proposed approximation and the one proposed by Li and Yuan (2001) presented respectively the best and the second best results, even when this model did not represent a carbonate reservoir from pre salt, which showed that both approximations are very stable for controlling the nonhyperbolicity in different kinds of problems. However, all approximations showed much more difficulty, once the RMS velocity was much stronger than the previous models. This problem can be clearly observed in Figures 233 and 234, where the Dix (1955) approximation presented the highest error of around 25\%, while other approximations showed 5 to $10 \%$ of maximum error. Even though both approximations showed the best set of results, the error was the second highest presented in this work, reaching more than $2 \%$. For the L1-norm, all the approximations showed an average increase of the accuracy of more than 10\% (Figures 235 and 236).

For the FMINSEARCH algorithm, the error decreased significantly, but there was also a variation among Blias (2009), Malovichko (1978) and Ursin and Stovas (2006) concerning the quality of the fitting. With the L2-norm for PP event (Figure 237), Blias (2009) showed the third best curve fitting; Malovichko (1978), the fourth; and Ursin and Stovas (2006), the fifth. For the PS event (Figure 238), Malovichko (1978) showed the third, Ursin and Stovas (2006) the fourth and Blias (2009) the fifth best result. In Figures 239 and 240, it is possible to observe that the use of L1-norm provides an increase higher than $10 \%$.

Using the SID-PSM algorithm with L2-norm for the PP event, the approximation proposed by Blias (2009) showed the third best result, while Malovichko (1978) showed the fourth best, and Ursin and Stovas (2006) the fifth best one (Figure 241). For the converted event, Malovichko (1978) showed the third best result; Ursin and Stovas (2006), the fourth; and Blias (2009), the fifth (Figure 242). It could be observed that the decrease of the error was of around 10\% when the L1-norm was used (Figures 243 and 244).

Using the MCS, the results increased significantly for this case, showing a lower than $2 \%$ error for the nonhyperbolic equations using the L2-norm for PP (Figure 245) and for PS (Figure 246). Using the L1-norm resulted again in a decrease of $10 \%$ of the error (Figures 247 and 248). The same condition could be observed for the PP event using the TOMLAB/LGO algorithm (Figure 249) and for PS (Figure 250). For the L1-norm, the same increase and the same sequence of results for other algorithms were observed (Figures 251 and 252). 


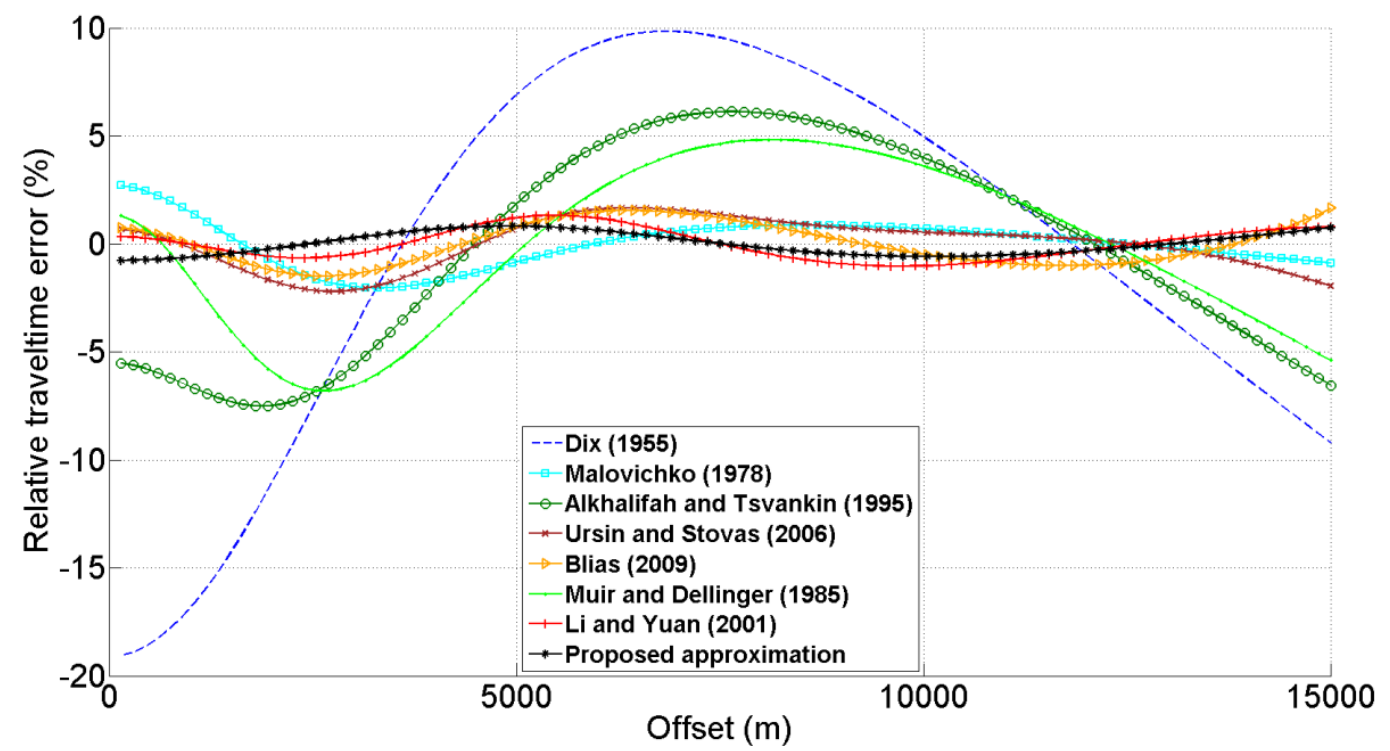

Figure 233: Relative error in travel-time between the observed curve and the calculated curve with each approximation of the PP reflection event with L2-norm and IMFIL optimization algorithm.

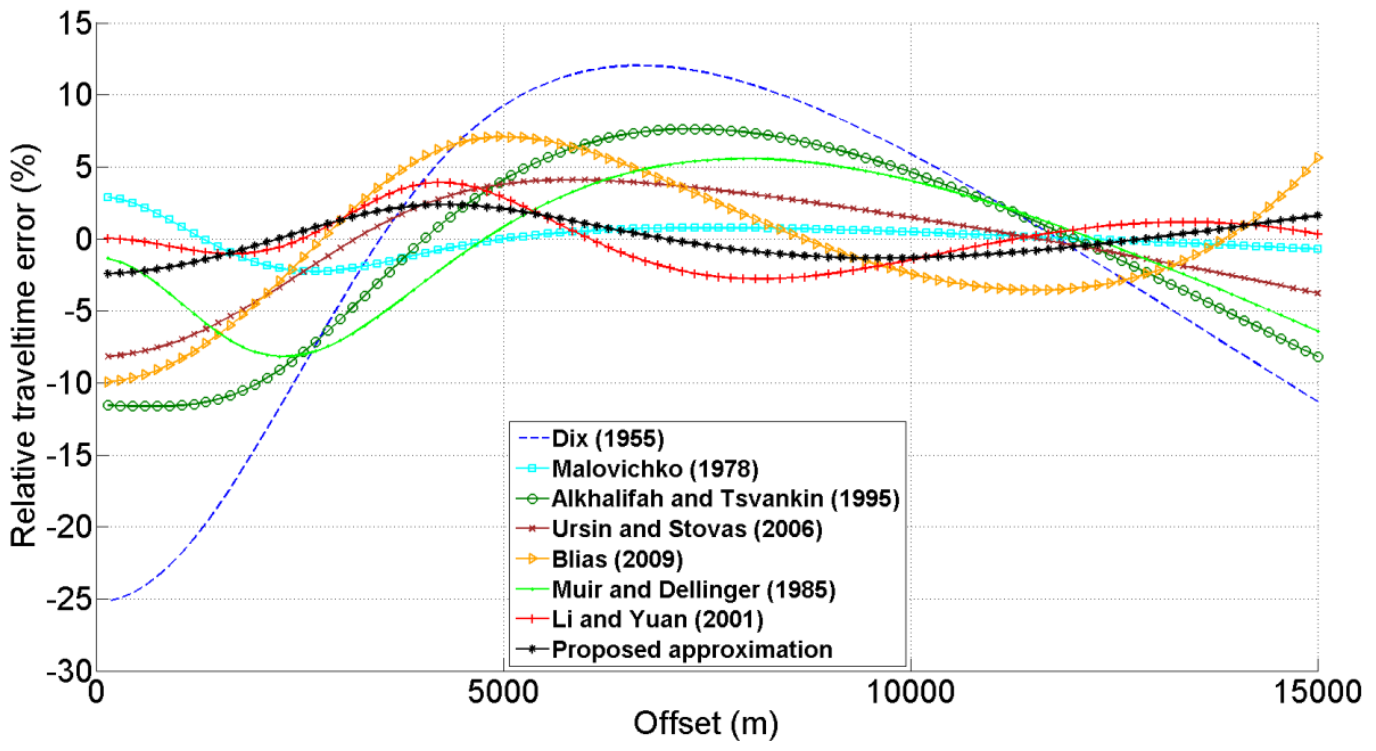

Figure 234: Relative error in travel-time between the observed curve and the calculated curve with each approximation of the PS reflection event with L2-norm and IMFIL optimization algorithm. 


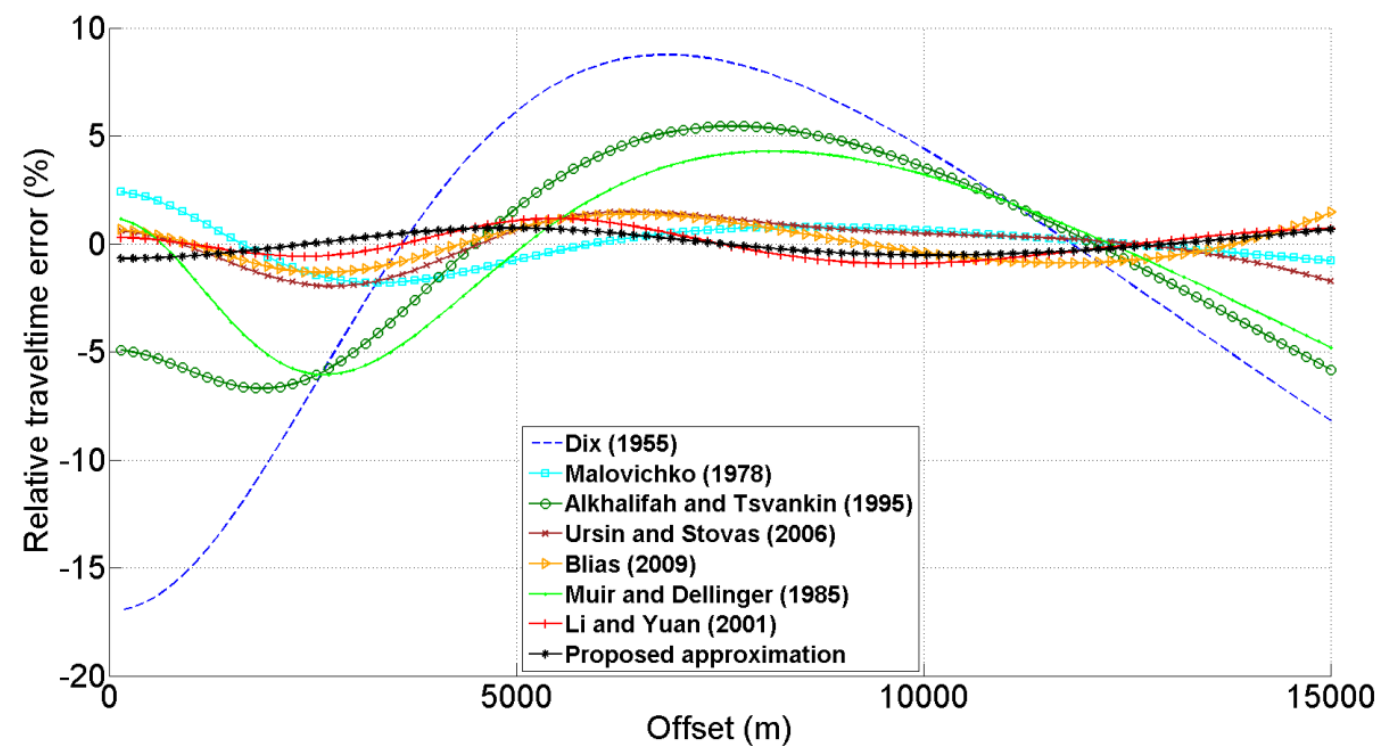

Figure 235: Relative error in travel-time between the observed curve and the calculated curve with each approximation of the PP reflection event with L1-norm and IMFIL optimization algorithm.

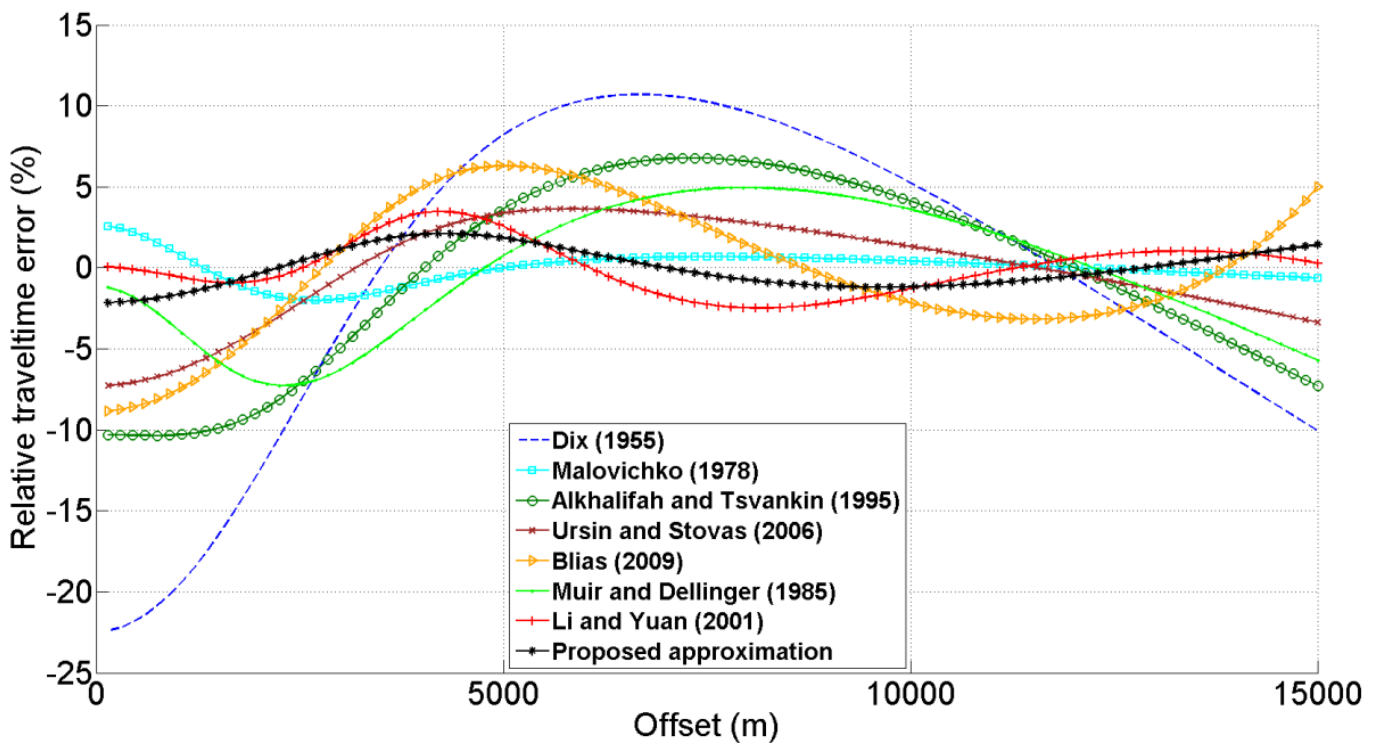

Figure 236: Relative error in travel-time between the observed curve and the calculated curve with each approximation of the PS reflection event with L1-norm and IMFIL optimization algorithm. 


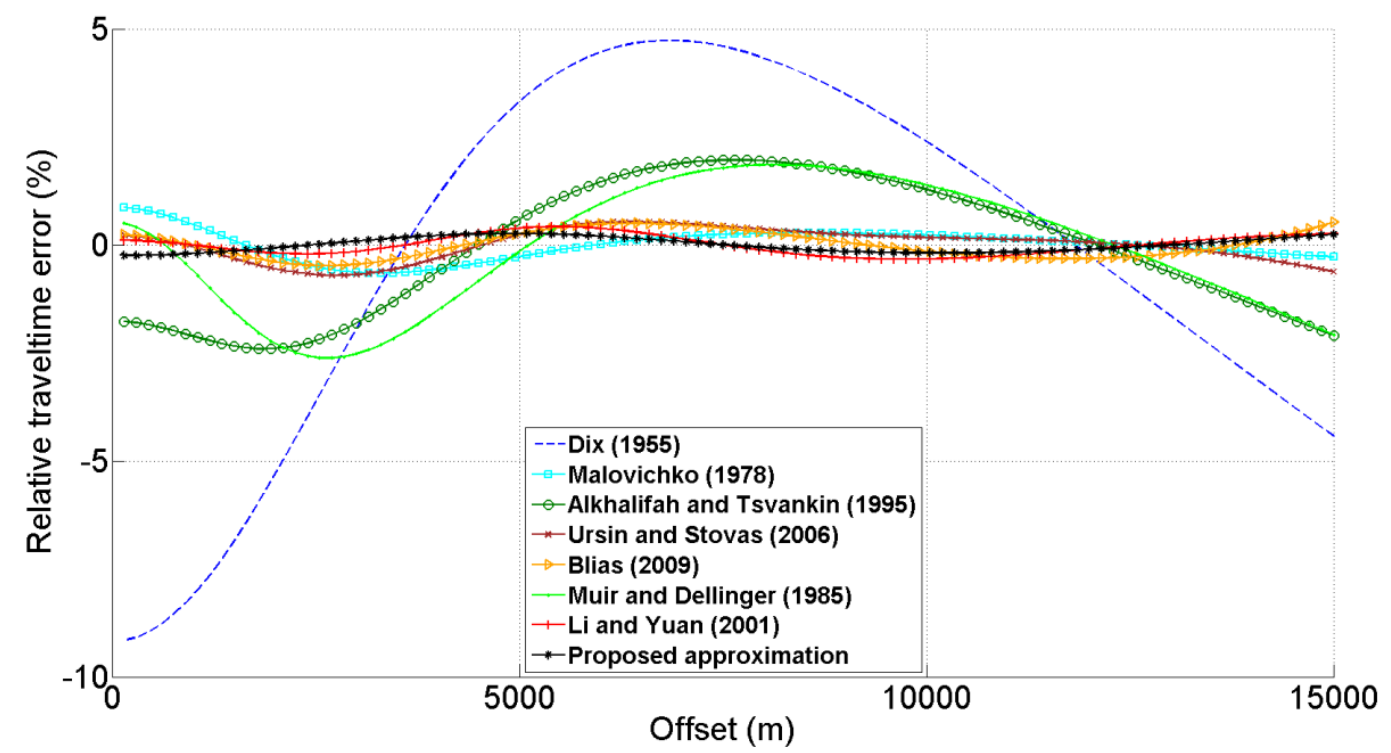

Figure 237: Relative error in travel-time between the observed curve and the calculated curve with each approximation of the PP reflection event with L2-norm and FMINSEARCH optimization algorithm.

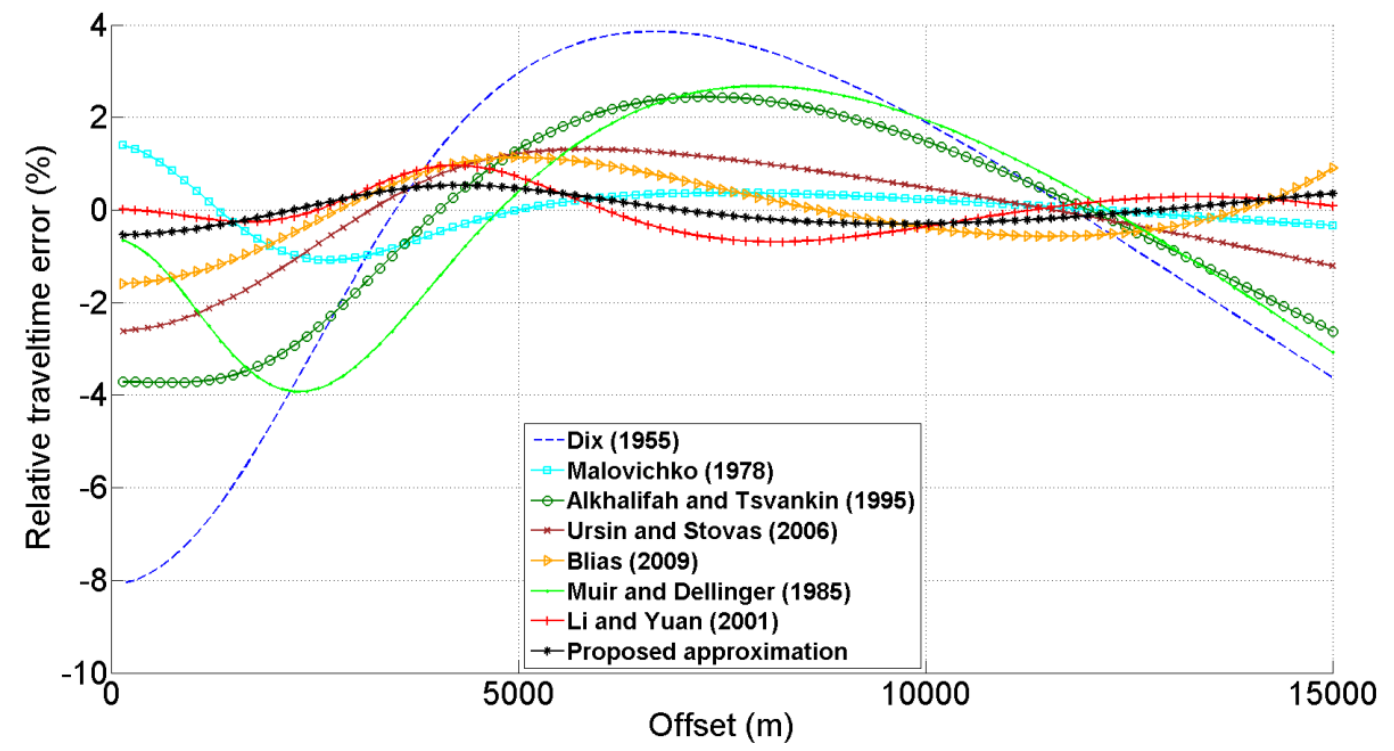

Figure 238: Relative error in travel-time between the observed curve and the calculated curve with each approximation of the PS reflection event with L2-norm and FMINSEARCH optimization algorithm. 


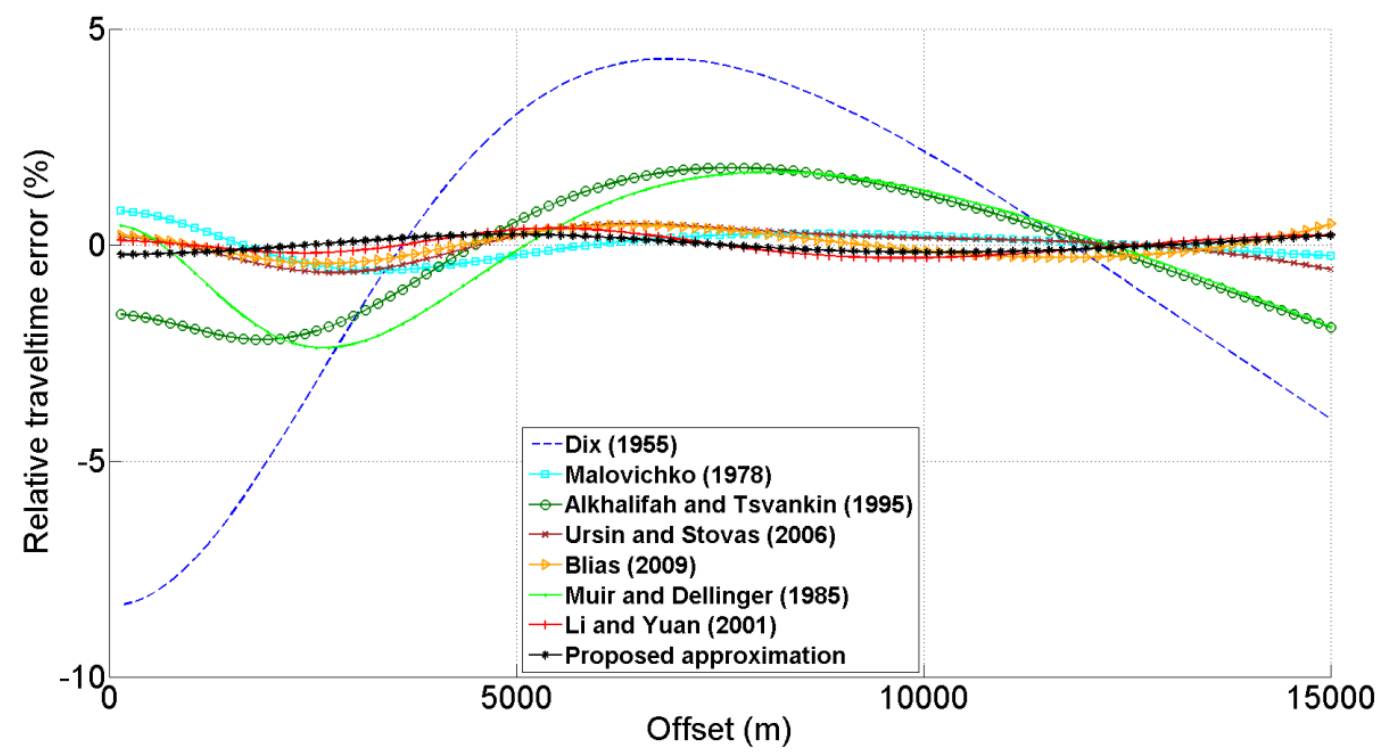

Figure 239: Relative error in travel-time between the observed curve and the calculated curve with each approximation of the PP reflection event with L1-norm and FMINSEARCH optimization algorithm.

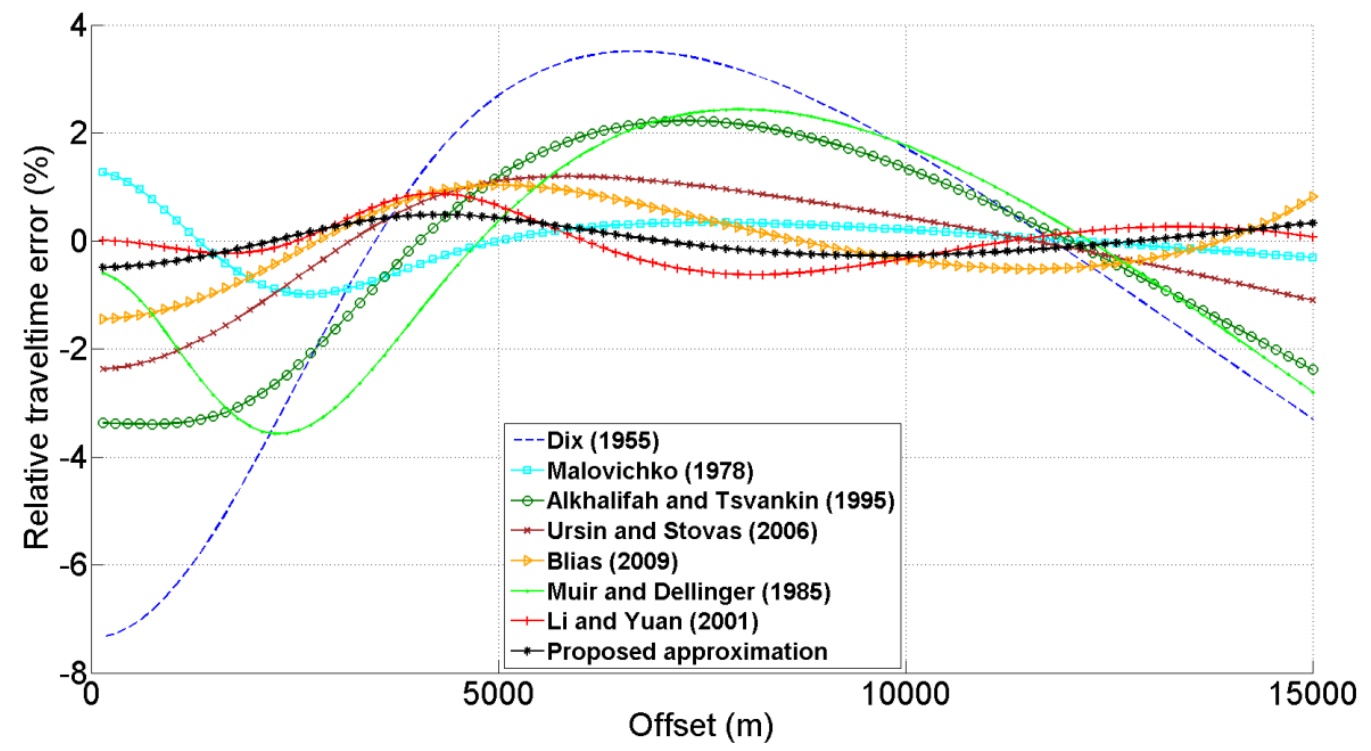

Figure 240: Relative error in travel-time between the observed curve and the calculated curve with each approximation of the PS reflection event with L1-norm and FMINSEARCH optimization algorithm. 


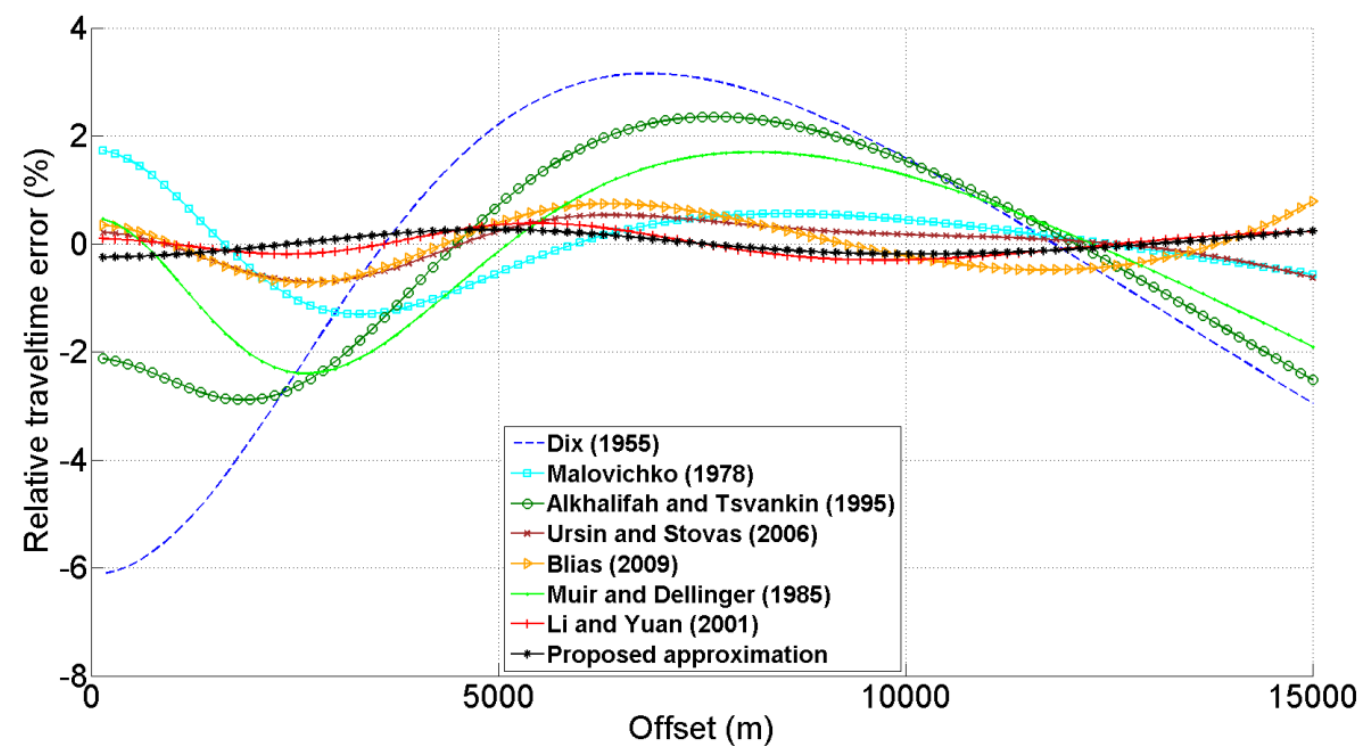

Figure 241: Relative error in travel-time between the observed curve and the calculated curve with each approximation of the PP reflection event with L2-norm and SID-PSM optimization algorithm.

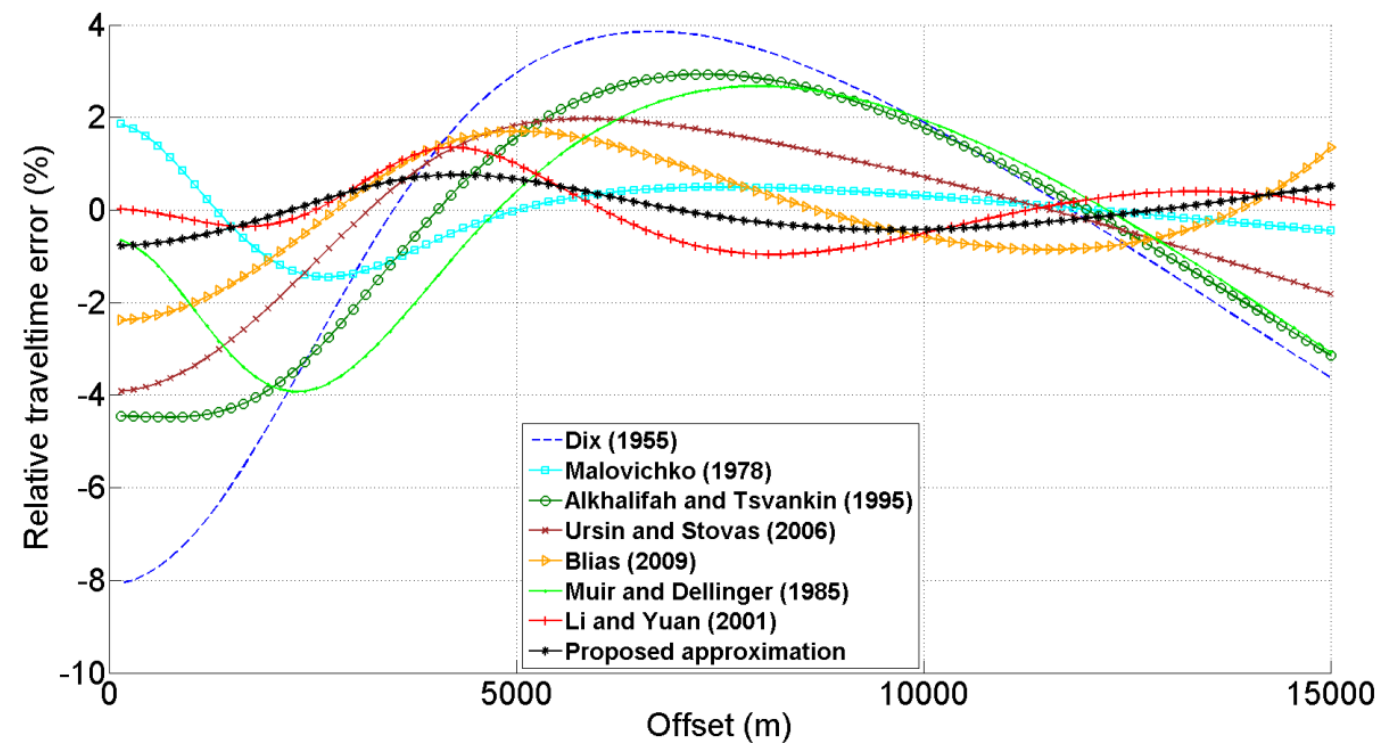

Figure 242: Relative error in travel-time between the observed curve and the calculated curve with each approximation of the PS reflection event with L2-norm and SID-PSM optimization algorithm. 


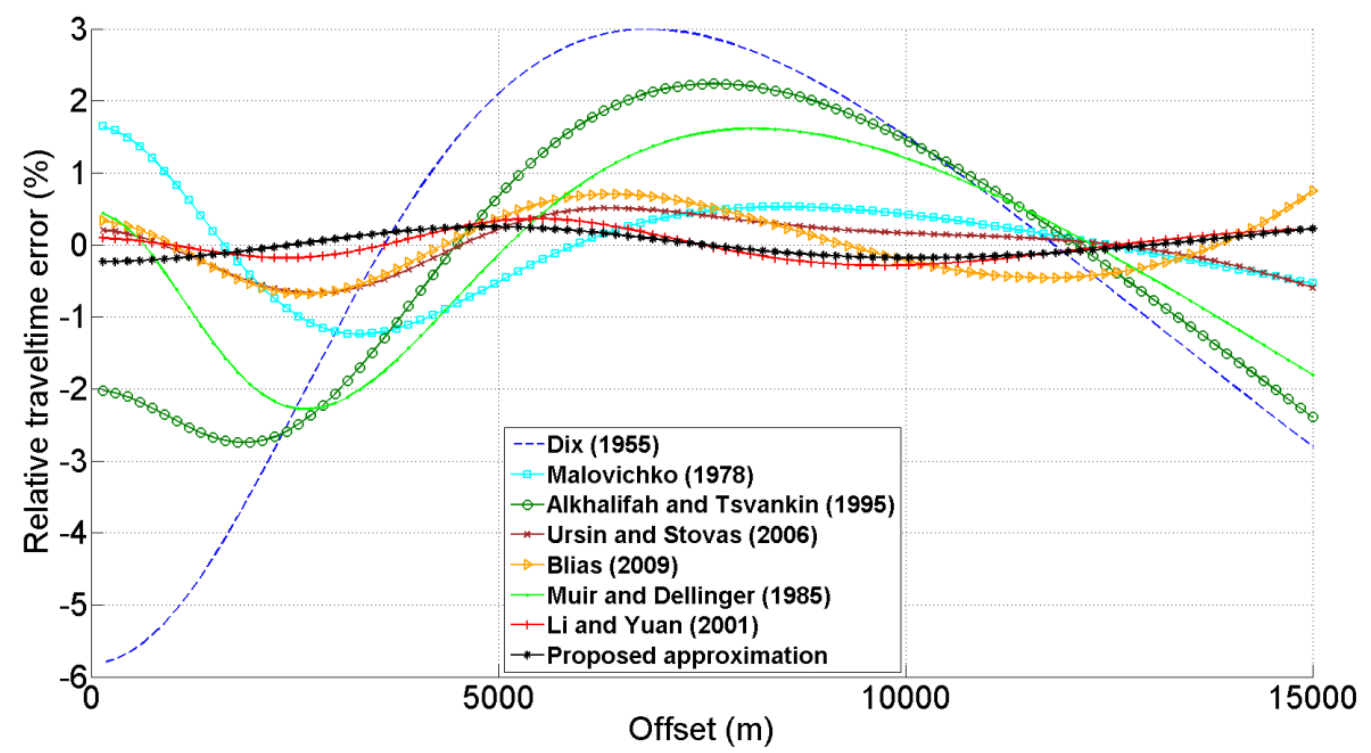

Figure 243: Relative error in travel-time between the observed curve and the calculated curve with each approximation of the PP reflection event with L1-norm and SID-PSM optimization algorithm.

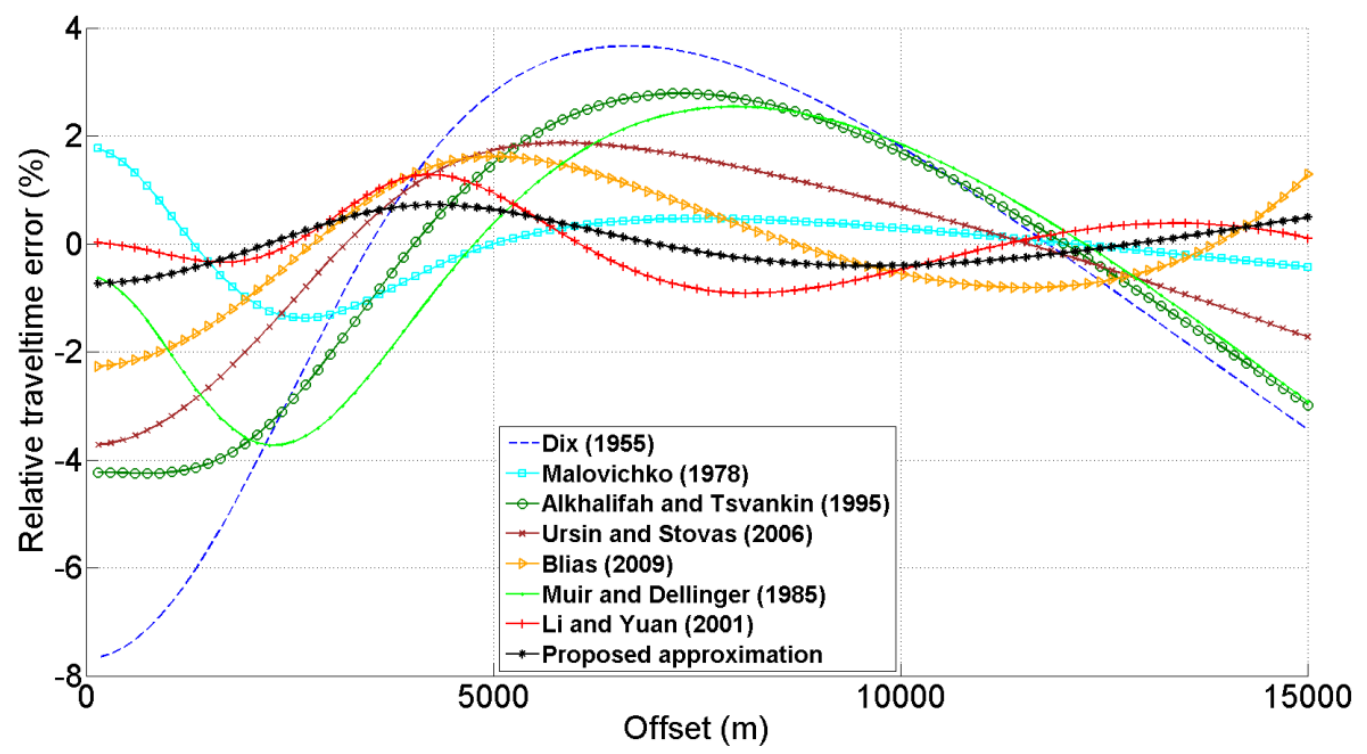

Figure 244: Relative error in travel-time between the observed curve and the calculated curve with each approximation of the PS reflection event with L1-norm and SID-PSM optimization algorithm. 


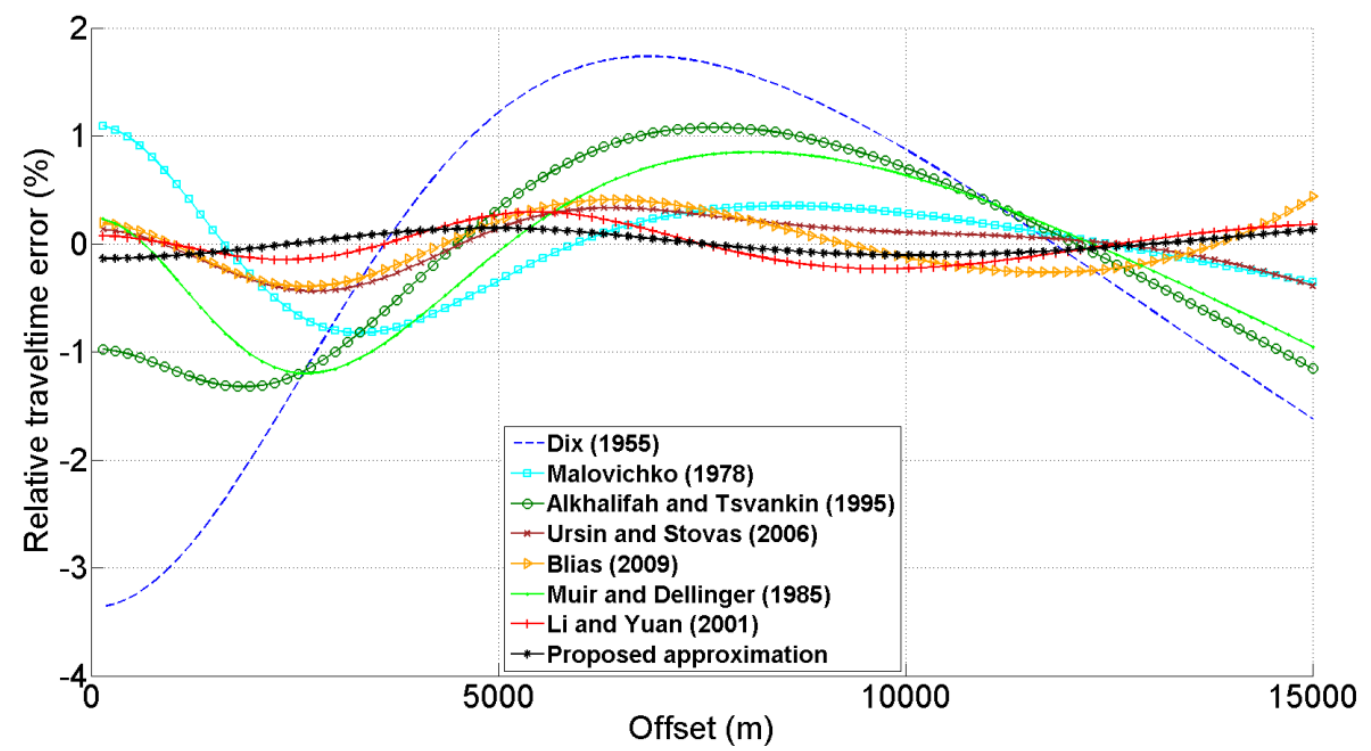

Figure 245: Relative error in travel-time between the observed curve and the calculated curve with each approximation of the PP reflection event with L2-norm and MCS optimization algorithm.

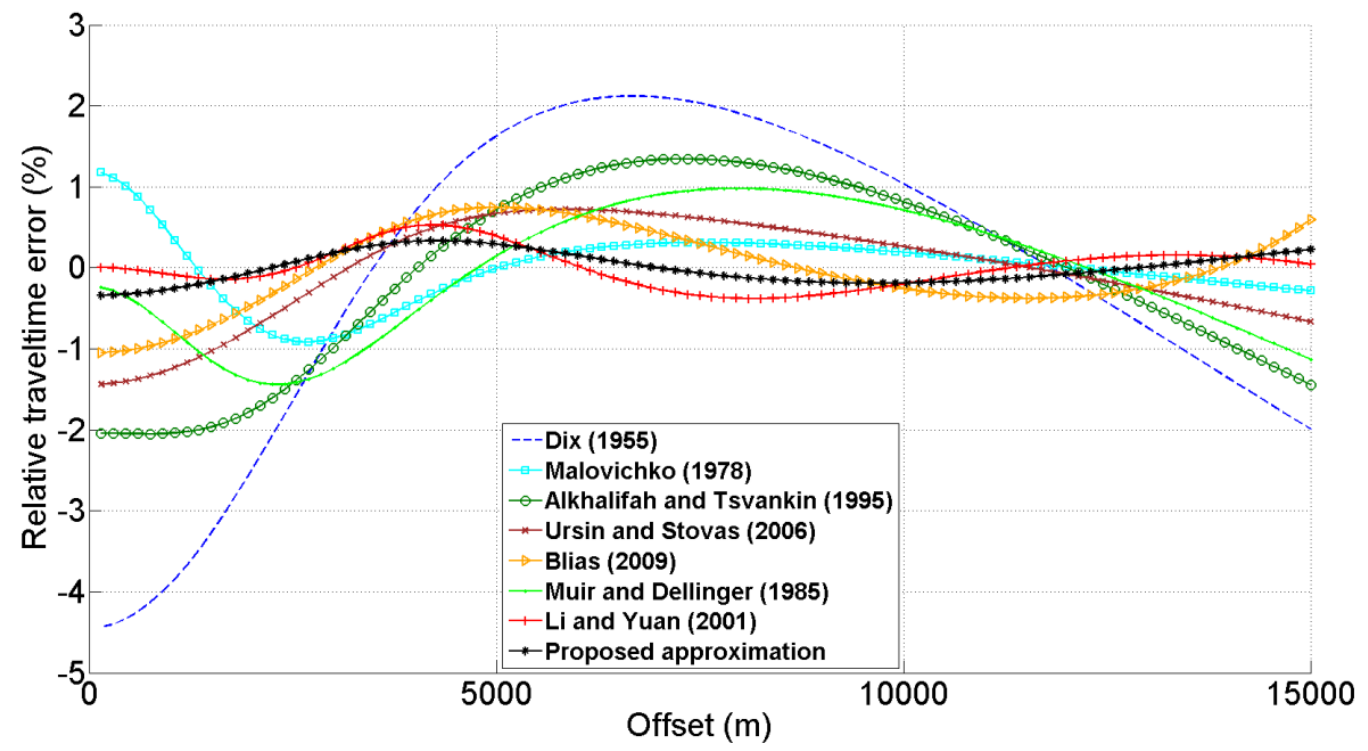

Figure 246: Relative error in travel-time between the observed curve and the calculated curve with each approximation of the PS reflection event with L2-norm and MCS optimization algorithm. 


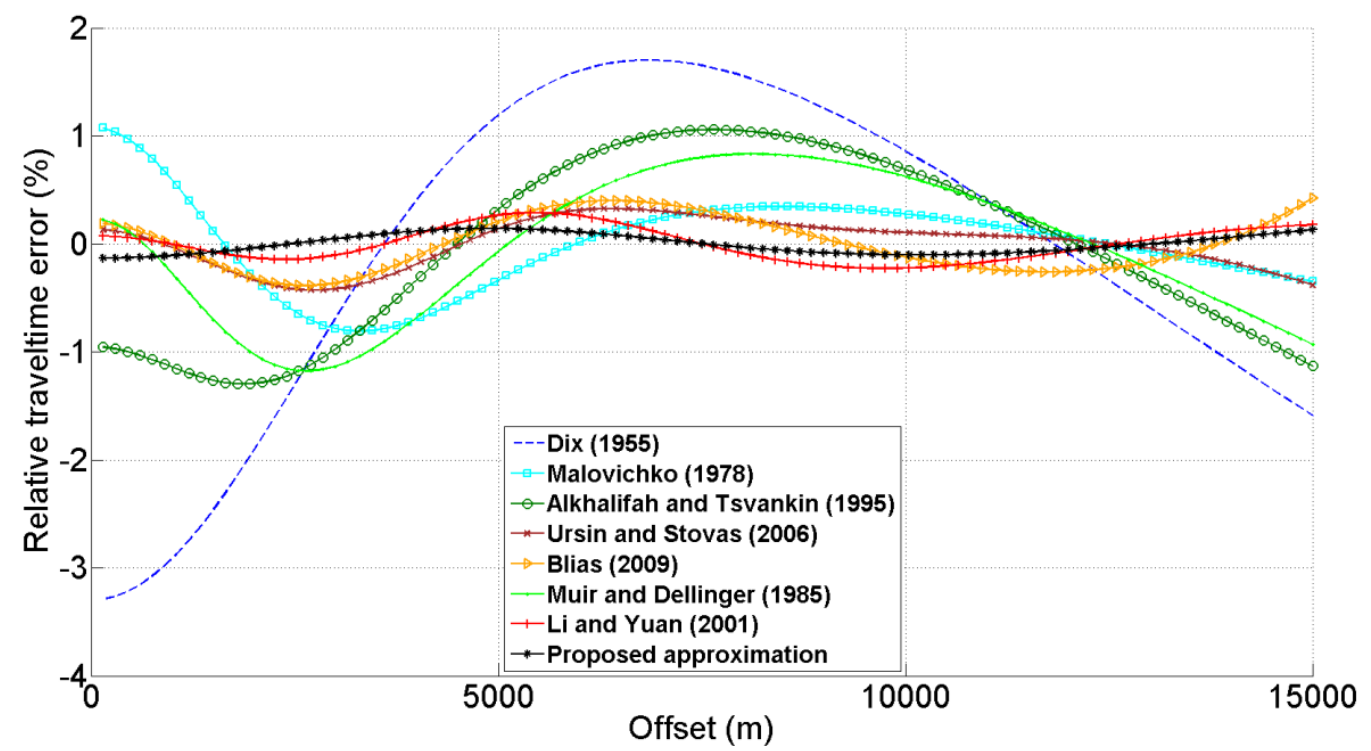

Figure 247: Relative error in travel-time between the observed curve and the calculated curve with each approximation of the PP reflection event with L1-norm and MCS optimization algorithm.

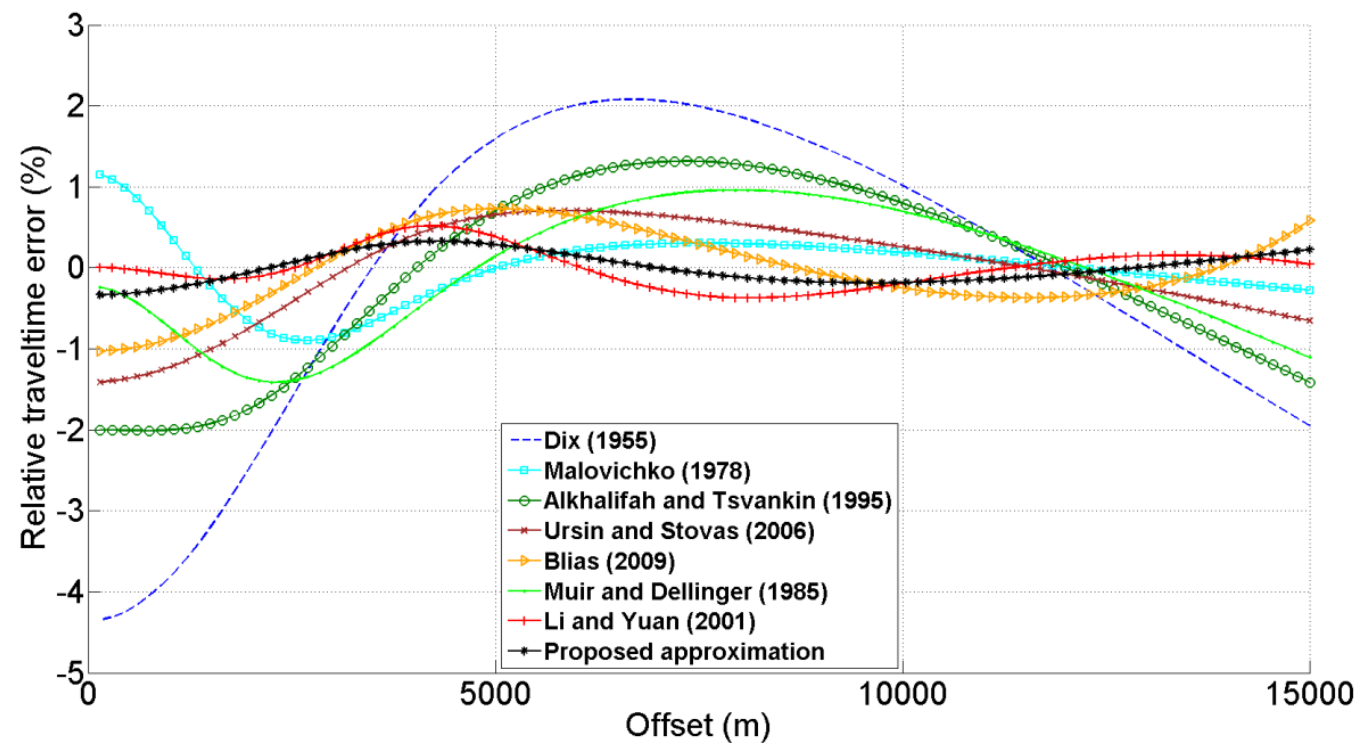

Figure 248: Relative error in travel-time between the observed curve and the calculated curve with each approximation of the PS reflection event with L1-norm and MCS optimization algorithm. 


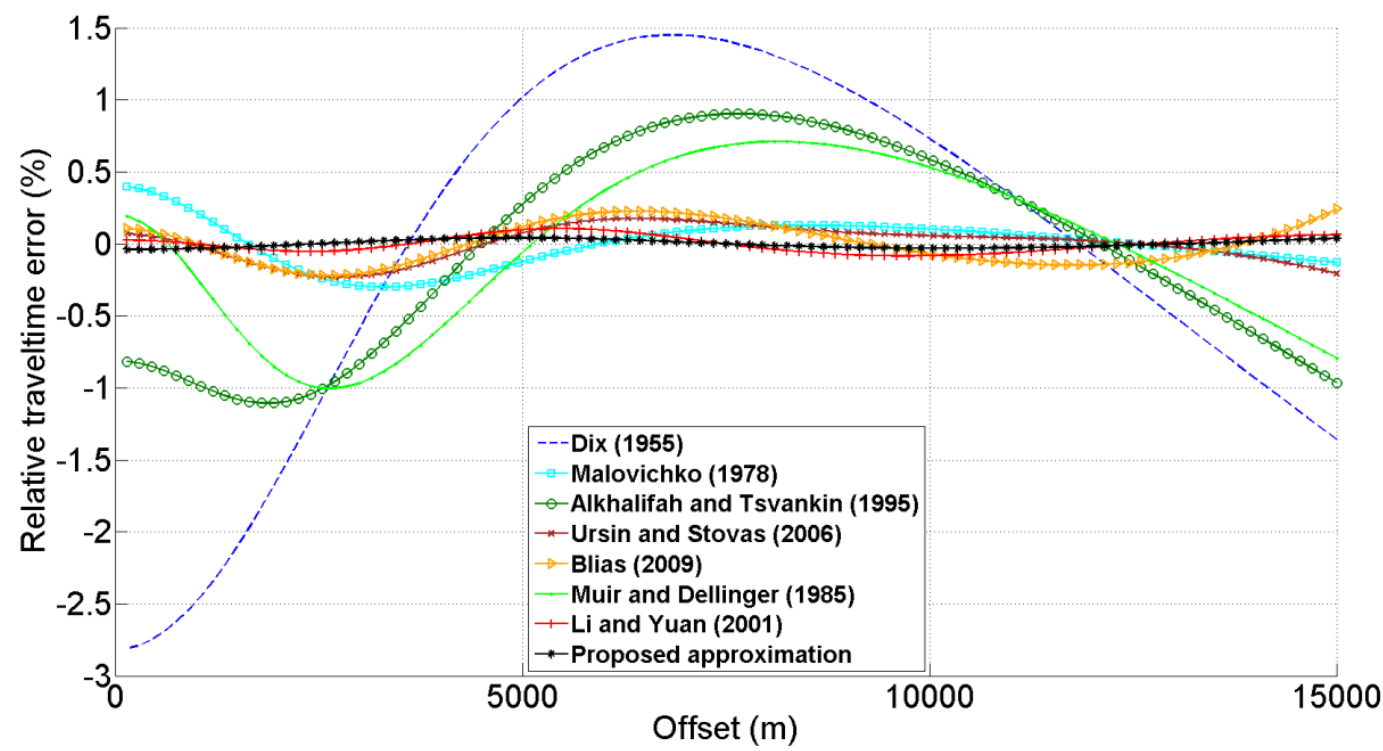

Figure 249: Relative error in travel-time between the observed curve and the calculated curve with each approximation of the PP reflection event with L2-norm and TOMLAB/LGO optimization algorithm.

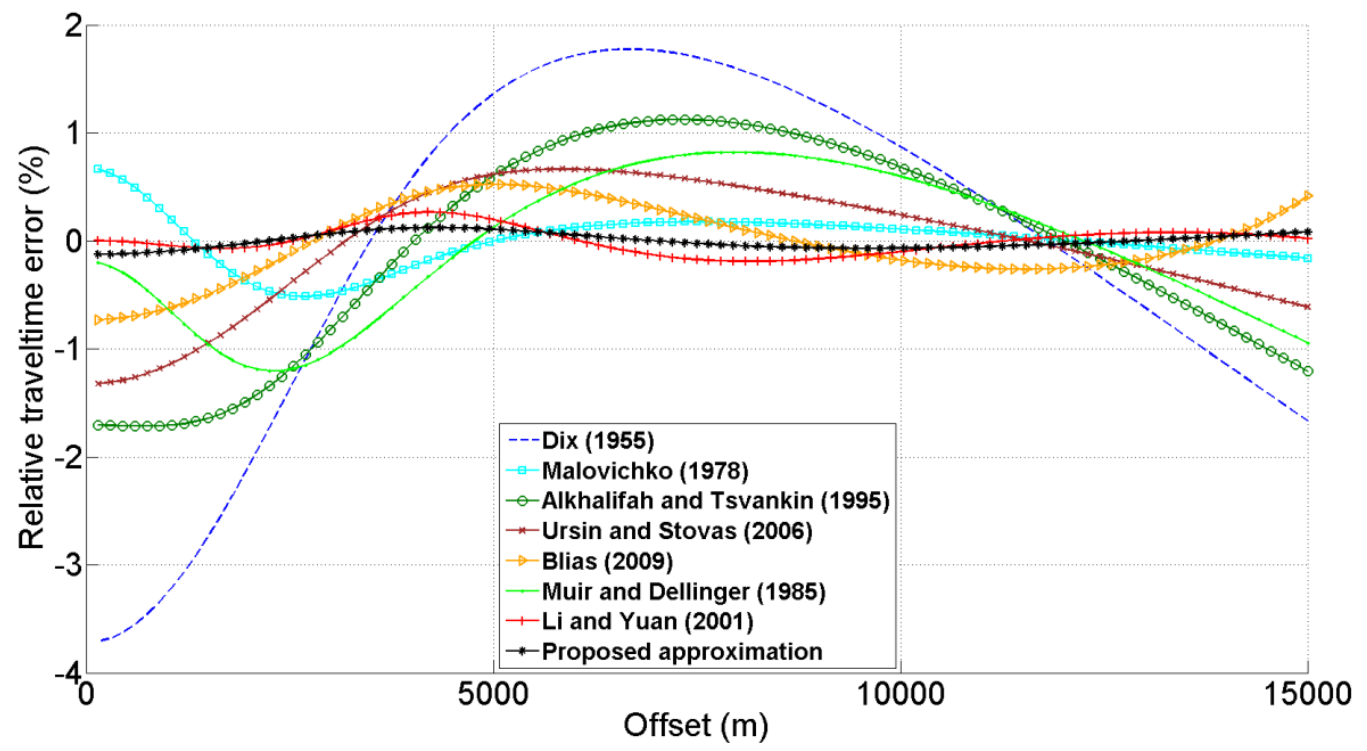

Figure 250: Relative error in travel-time between the observed curve and the calculated curve with each approximation of the PS reflection event with L2-norm and TOMLAB/LGO optimization algorithm. 


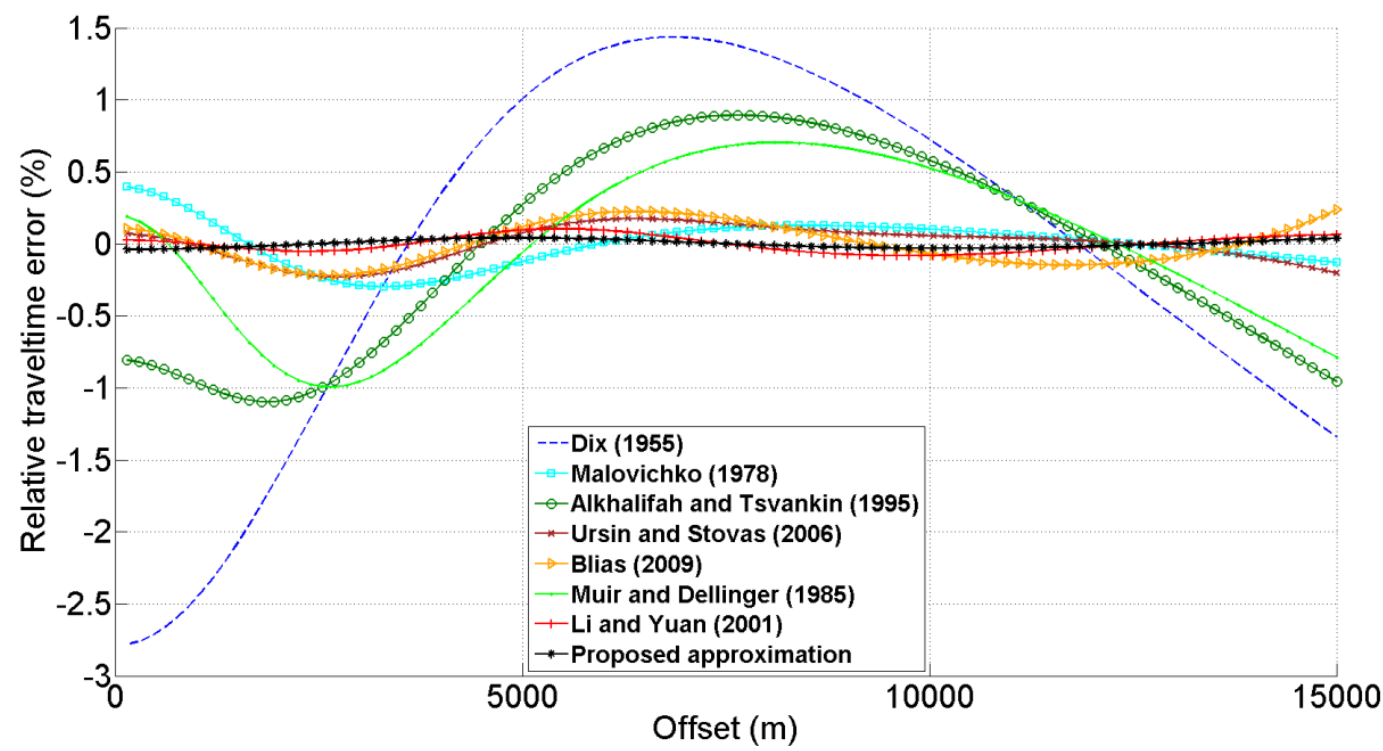

Figure 251: Relative error in travel-time between the observed curve and the calculated curve with each approximation of the PP reflection event with L1-norm and TOMLAB/LGO optimization algorithm.

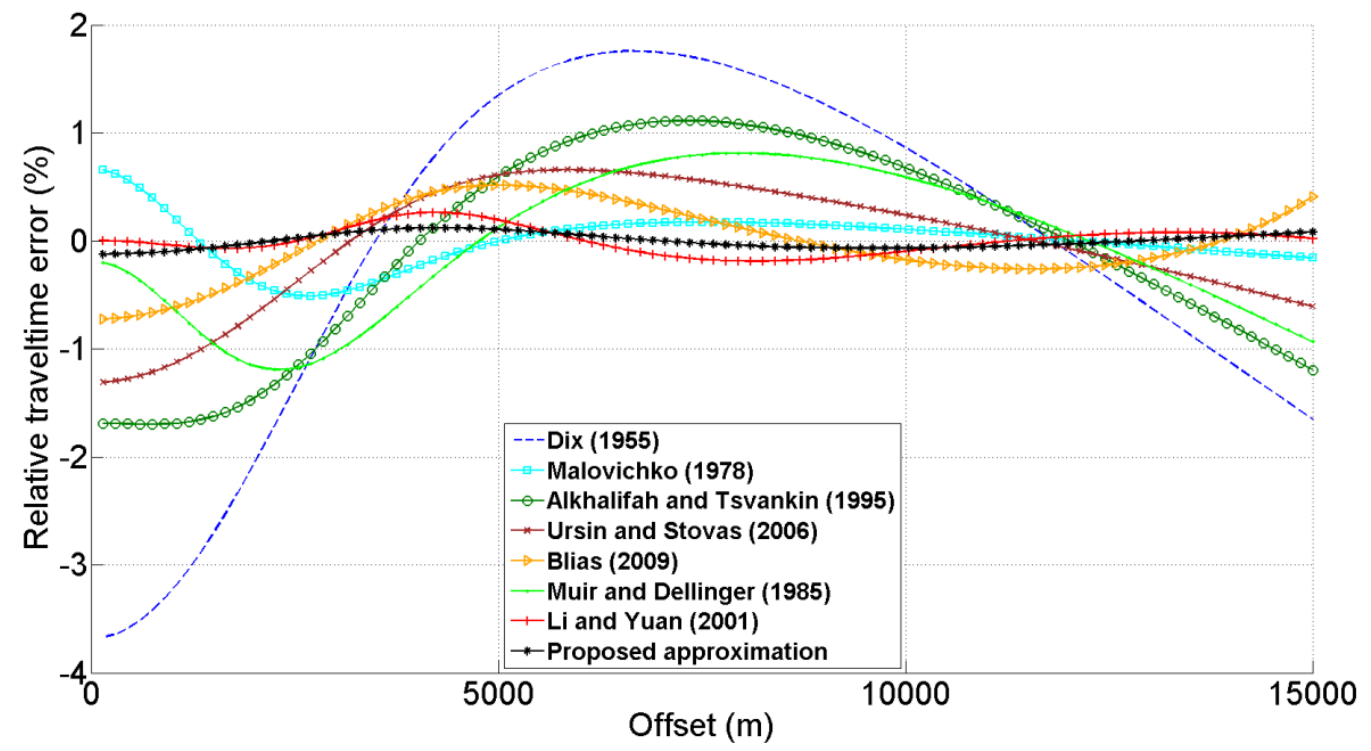

Figure 252: Relative error in travel-time between the observed curve and the calculated curve with each approximation of the PS reflection event with L1-norm and TOMLAB/LGO optimization algorithm. 


\subsection{Model 5}

For the last model tested in this work, it was possible to observe the highest error among all the five models; and for the IMFL algorithm (Figure 253), it was clear that the converted event (Figure 254) presented as the hardest condition to perform the curve fitting, showing almost $30 \%$ of residual of maximum error. Even when it decreases the error in around $10 \%$, the use of the L1-norm showed a high error for the conventional event (Figure 255 ), and a maximum error higher than $25 \%$ (Figure 256).

Using the FMINSEARCH along with the L2-norm, the average error decreased from $5 \%$ to $3 \%$ for the PP event (Figure 257) and from 5.5\% to $2.5 \%$ for the converted-wave event (Figure 258). The L1-norm helped to decrease the error in between $0.5 \%$ (Figure 259) and $0.2 \%$ (Figure 260) for the PP and PS events, respectively.

The last local search optimization algorithm, the SID-PSM, showed a good result for L2-norm, where it was possible to observe that, as it was in the two previous algorithms, the approximation proposed by Ursin and Stovas (2006) showed the third best result, Blias (2009) showed the fourth best, and Malovichko (1978) showed the fifth best one for the conventional reflection event (Figure 261). For the PS event, the approximation proposed by Malovichko (1978) showed the third best result; Blias (2009), the fourth; and Ursin and Stovas (2006), the fifth (Figure 262). For the L1-norm, it was observed that this condition also showed an increase of the accuracy of around 10\% (Figures 263 and 264).

For the MCS optimization algorithm, it was possible to observe, using the L2-norm, which, for the conventional event, the same sequence of results shown by the previous algorithms (Figure 265). The same could be said for the converted wave event (Figure 266), even when showing a higher and more variable error. For the L1-norm, the error decreased in the same manner as it was shown before (Figures 267 and 268), related to an increase of around $10 \%$ of accuracy.

For the TOMLAB/LGO algorithm using the L2-norm, Ursin and Stovas (2006) showed the third best result, Blias (2009) showed the fourth best result and Malovichko (1978) showed the fifth best one for the PP event (Figure 269). For the PS event, Malovichko (1978) showed the third best result, while Blias (2009) showed the fourth best and Ursin and Stovas (2006) presented the fifth best one (Figure 270). Using the L1-norm, it was possible to observe the increase of the accuracy, again for both events (Figures 271 and 272). 


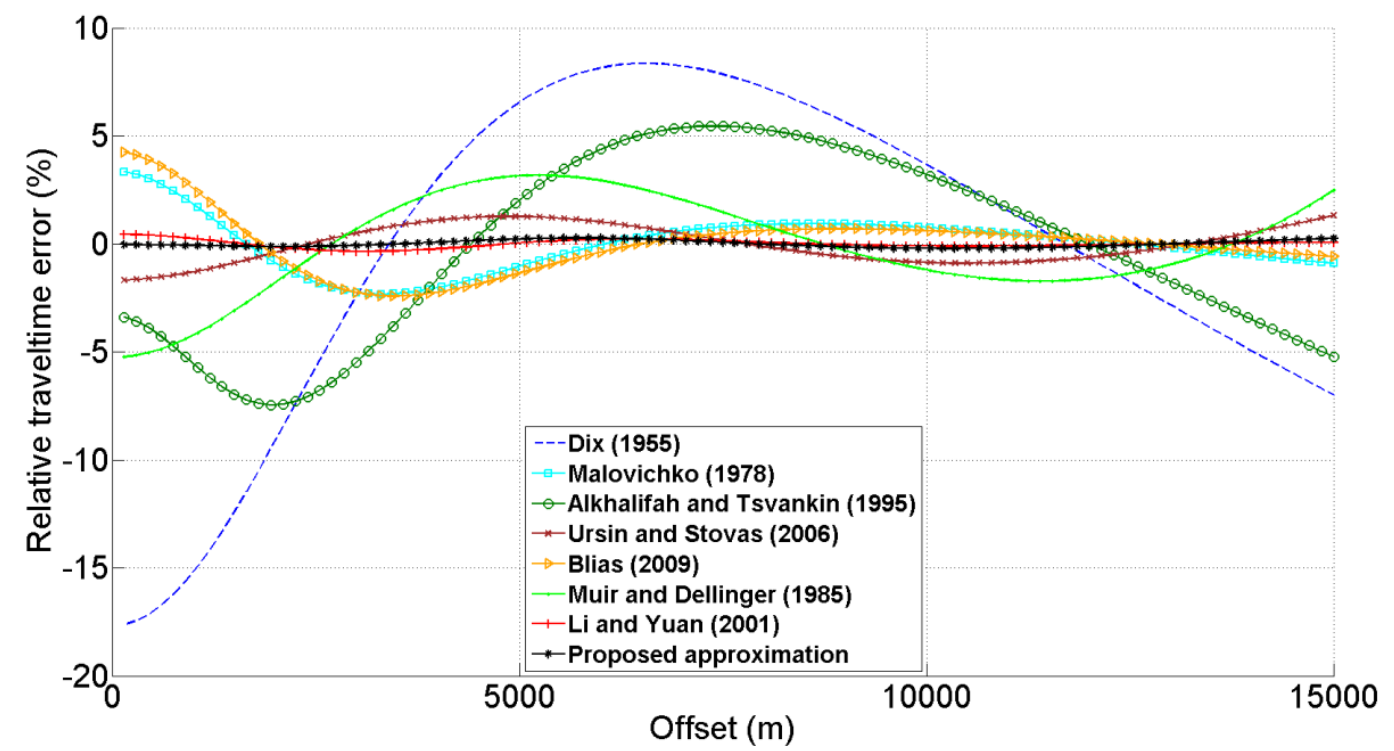

Figure 253: Relative error in travel-time between the observed curve and the calculated curve with each approximation of the PP reflection event with L2-norm and IMFIL optimization algorithm.

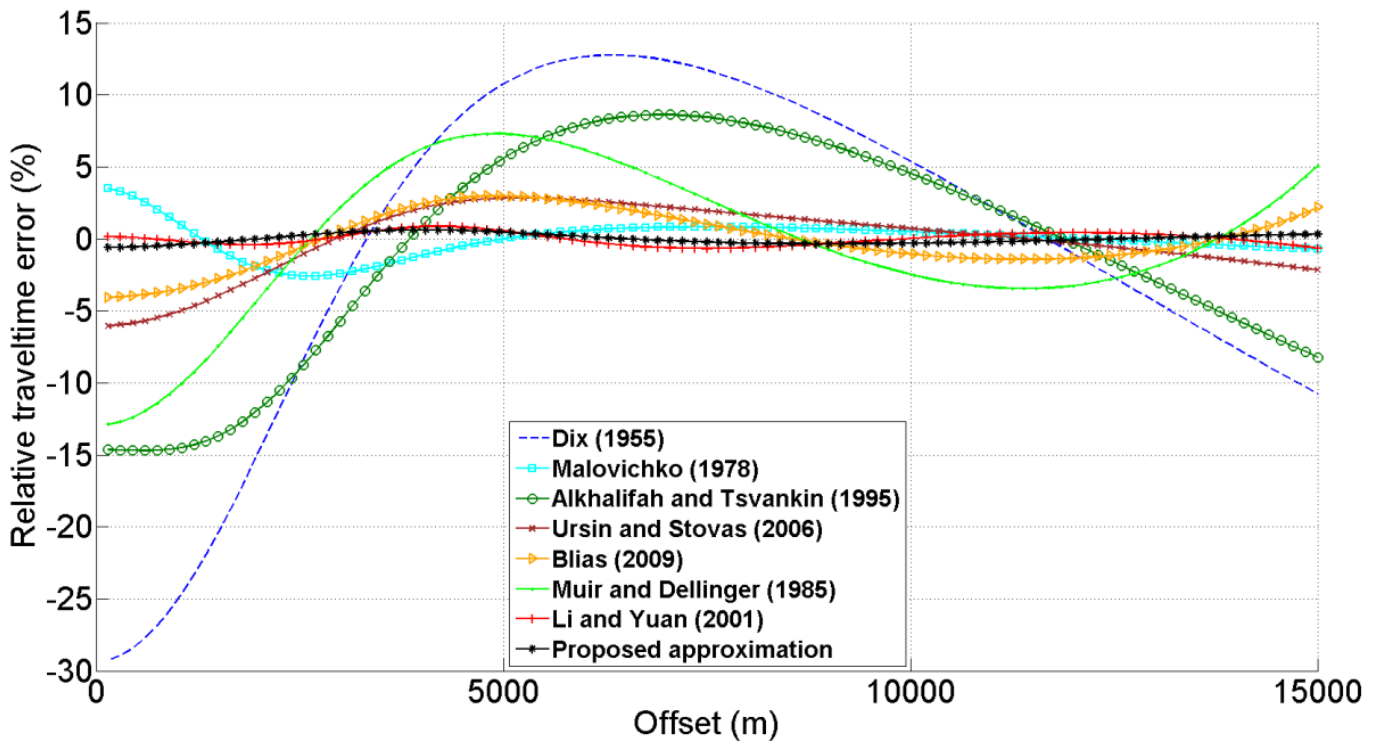

Figure 254: Relative error in travel-time between the observed curve and the calculated curve with each approximation of the PS reflection event with L2-norm and IMFIL optimization algorithm. 


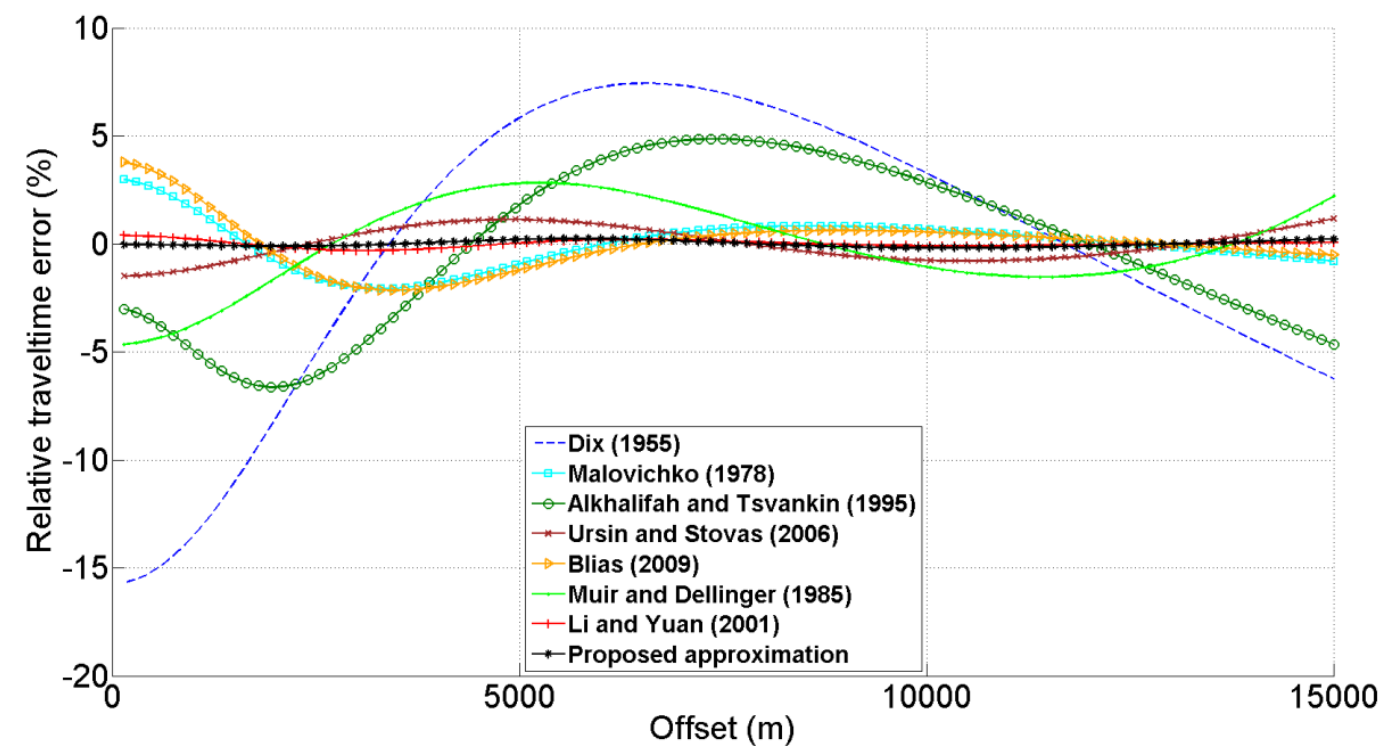

Figure 255: Relative error in travel-time between the observed curve and the calculated curve with each approximation of the PP reflection event with L1-norm and IMFIL optimization algorithm.

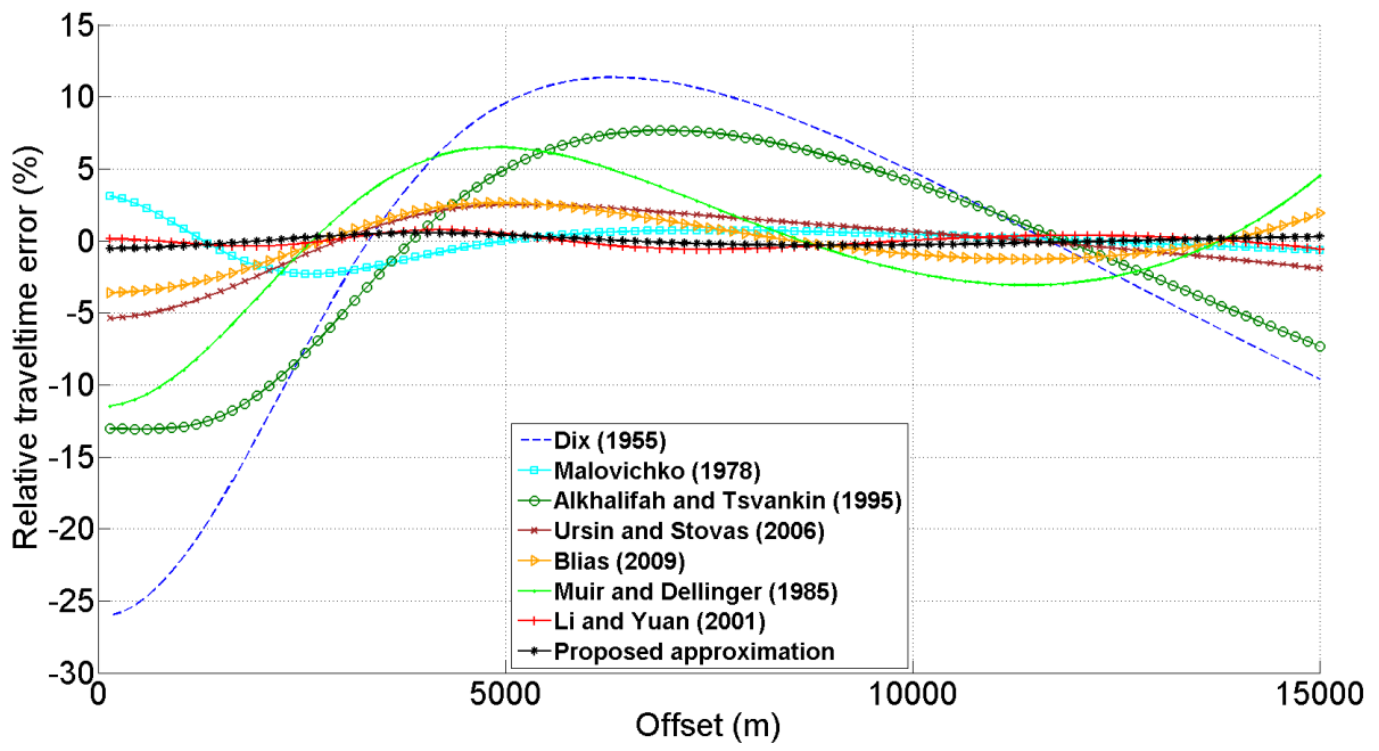

Figure 256: Relative error in travel-time between the observed curve and the calculated curve with each approximation of the PS reflection event with L1-norm and IMFIL optimization algorithm. 


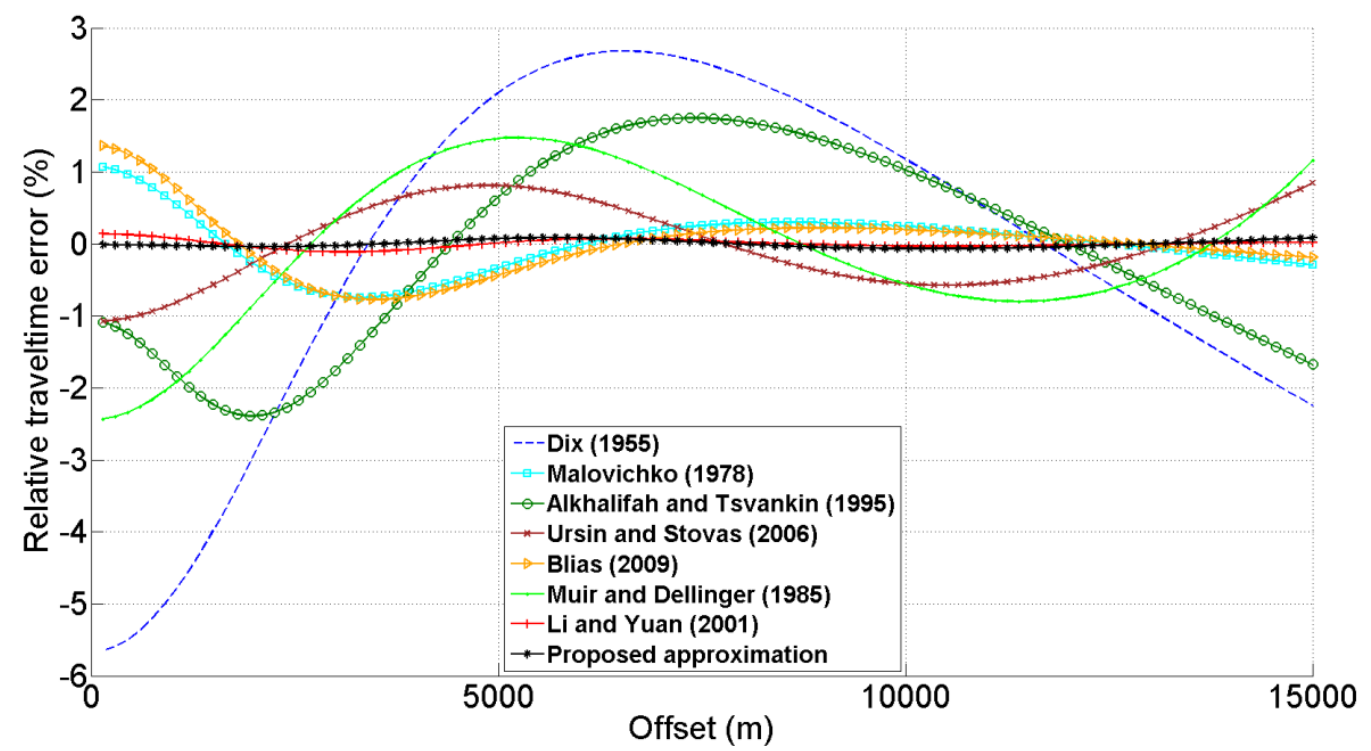

Figure 257: Relative error in travel-time between the observed curve and the calculated curve with each approximation of the PP reflection event with L2-norm and FMINSEARCH optimization algorithm.

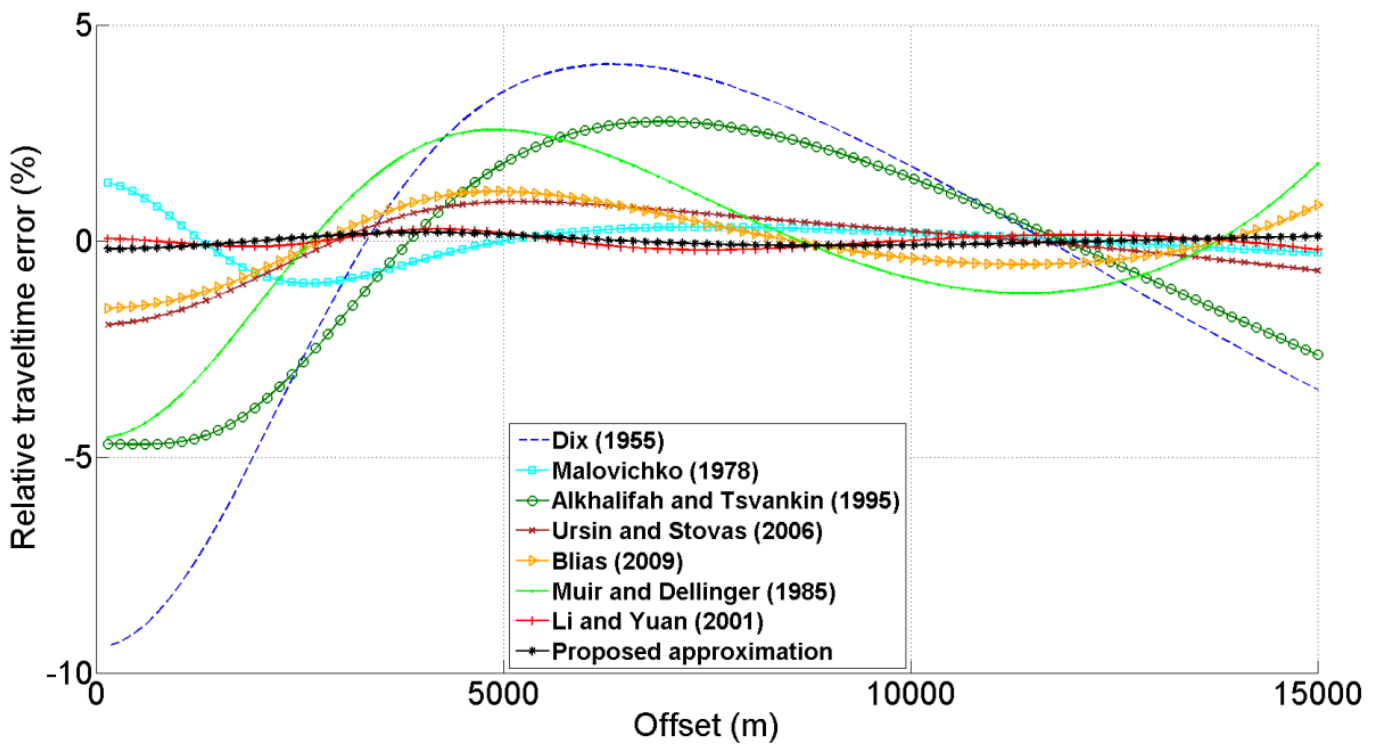

Figure 258: Relative error in travel-time between the observed curve and the calculated curve with each approximation of the PS reflection event with L2-norm and FMINSEARCH optimization algorithm. 


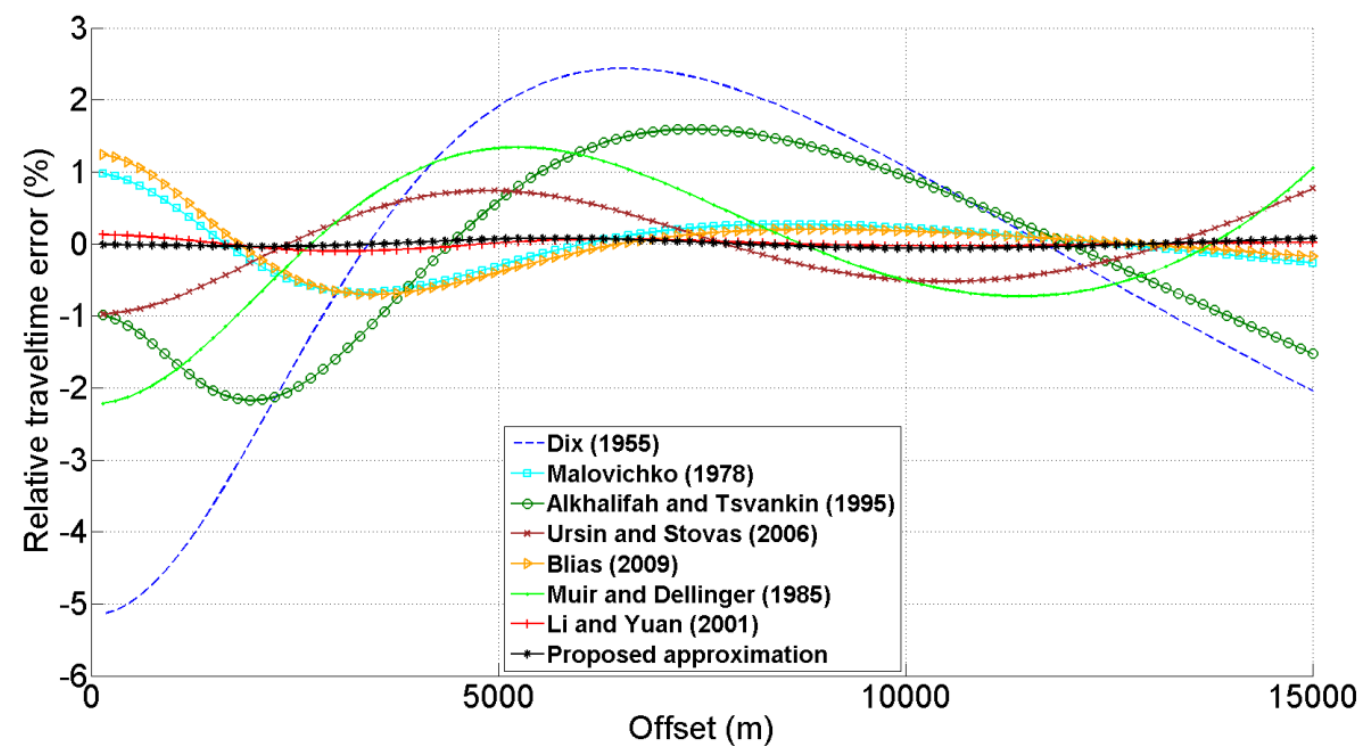

Figure 259: Relative error in travel-time between the observed curve and the calculated curve with each approximation of the PP reflection event with L1-norm and FMINSEARCH optimization algorithm.

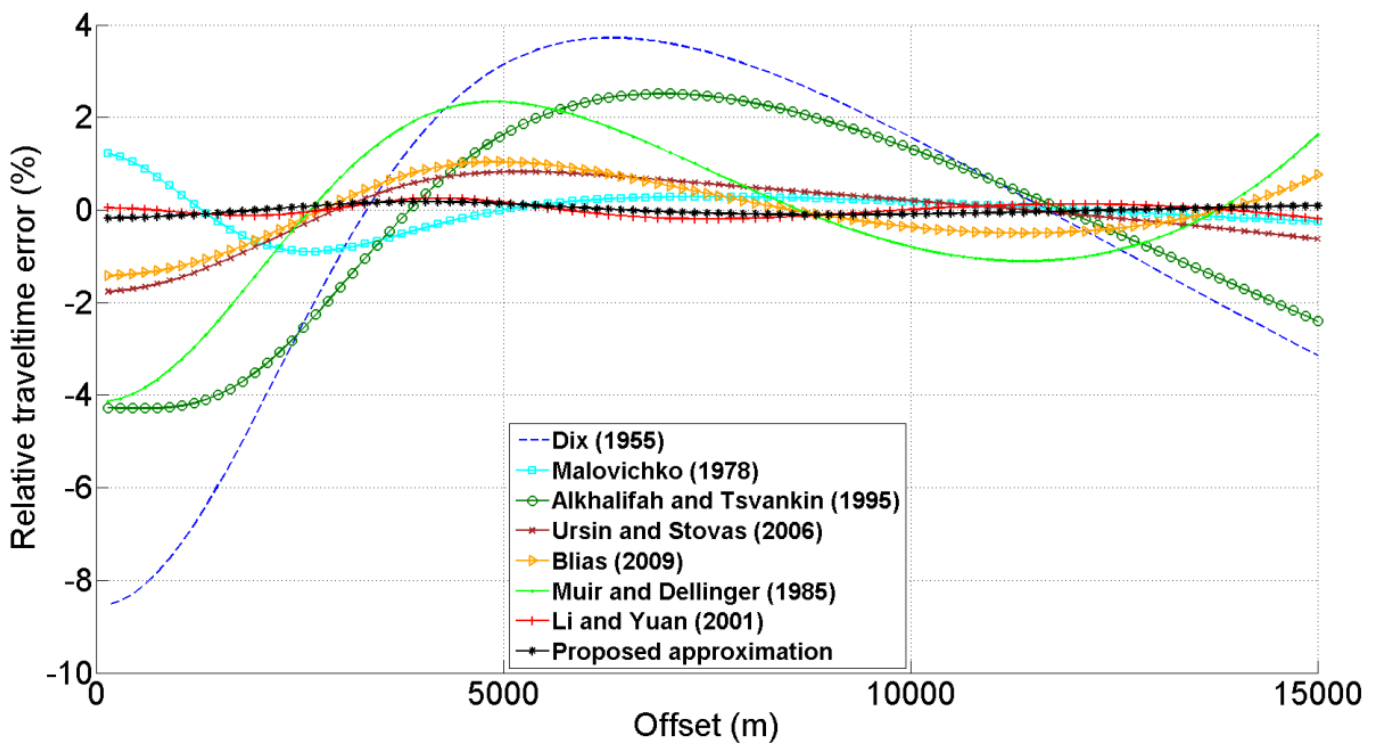

Figure 260: Relative error in travel-time between the observed curve and the calculated curve with each approximation of the PS reflection event with L1-norm and FMINSEARCH optimization algorithm. 


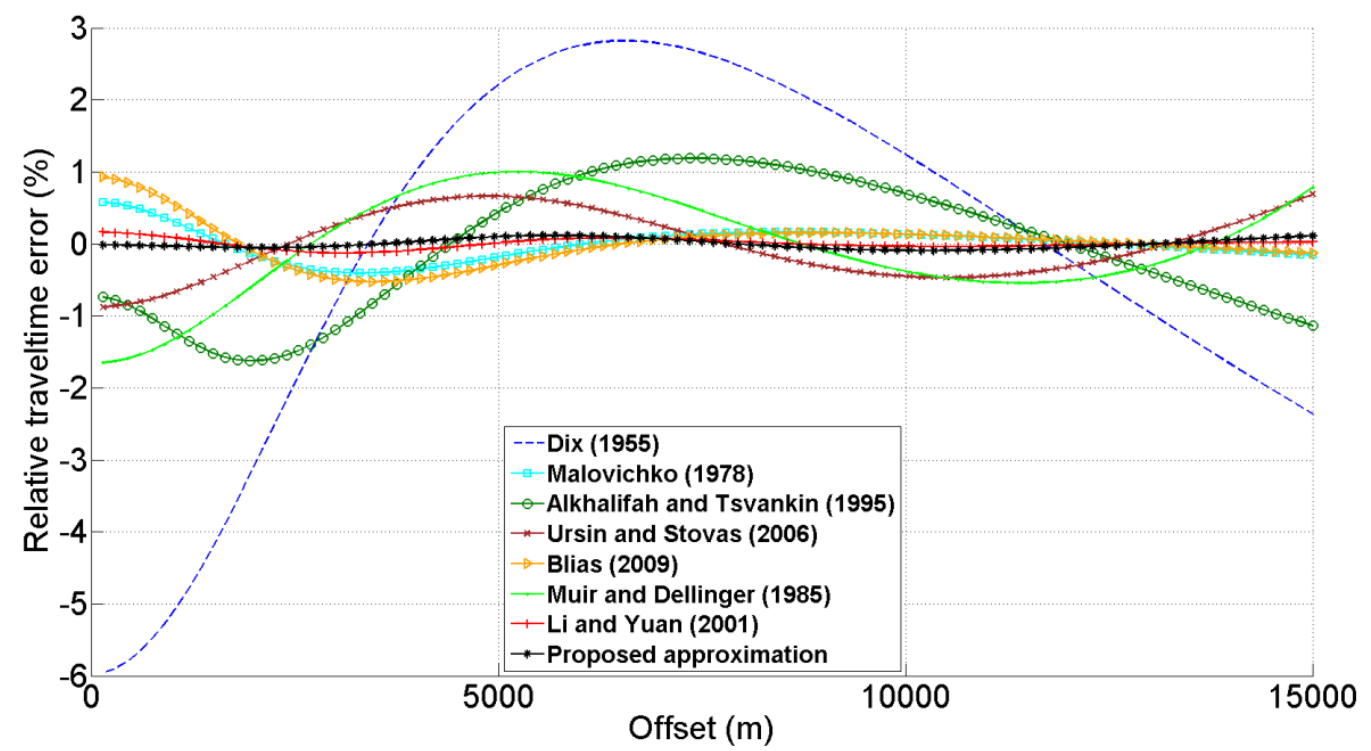

Figure 261: Relative error in travel-time between the observed curve and the calculated curve with each approximation of the PP reflection event with L2-norm and SID-PSM optimization algorithm.

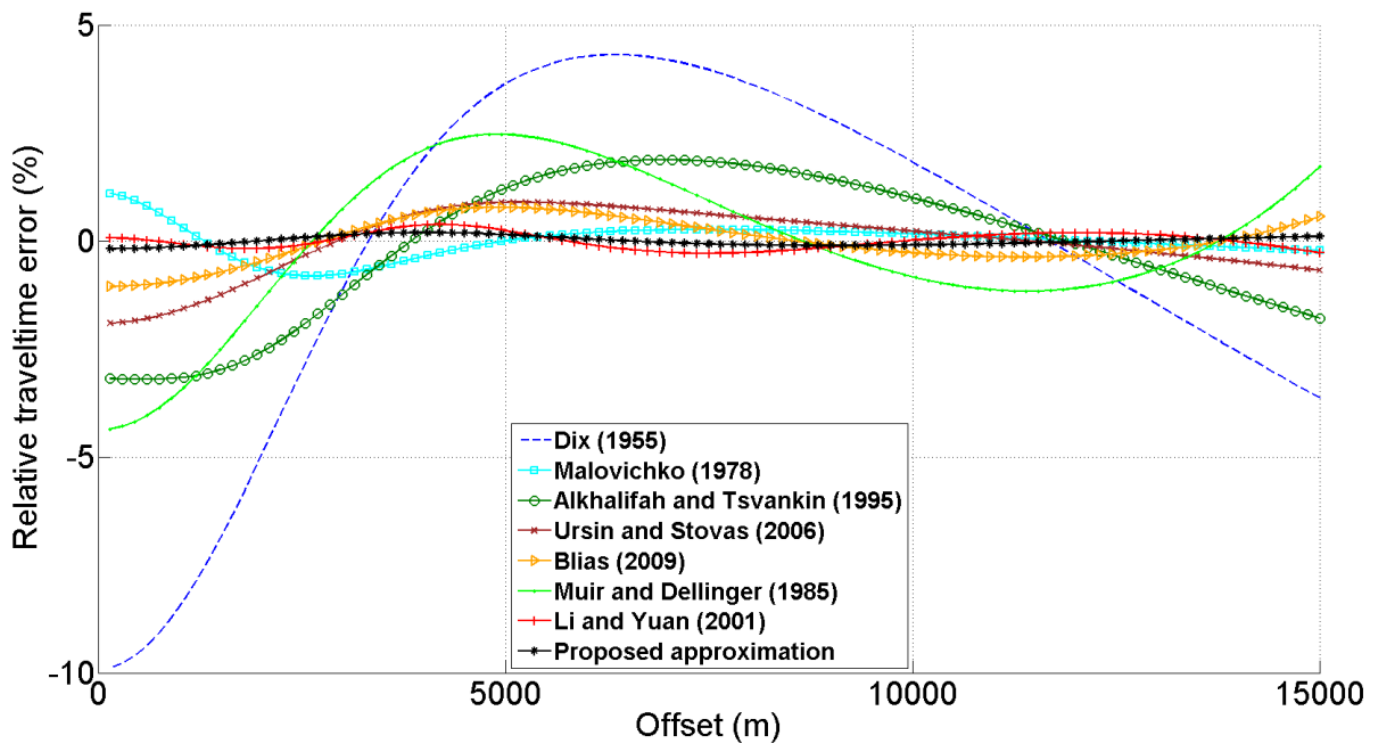

Figure 262: Relative error in travel-time between the observed curve and the calculated curve with each approximation of the PS reflection event with L2-norm and SID-PSM optimization algorithm. 


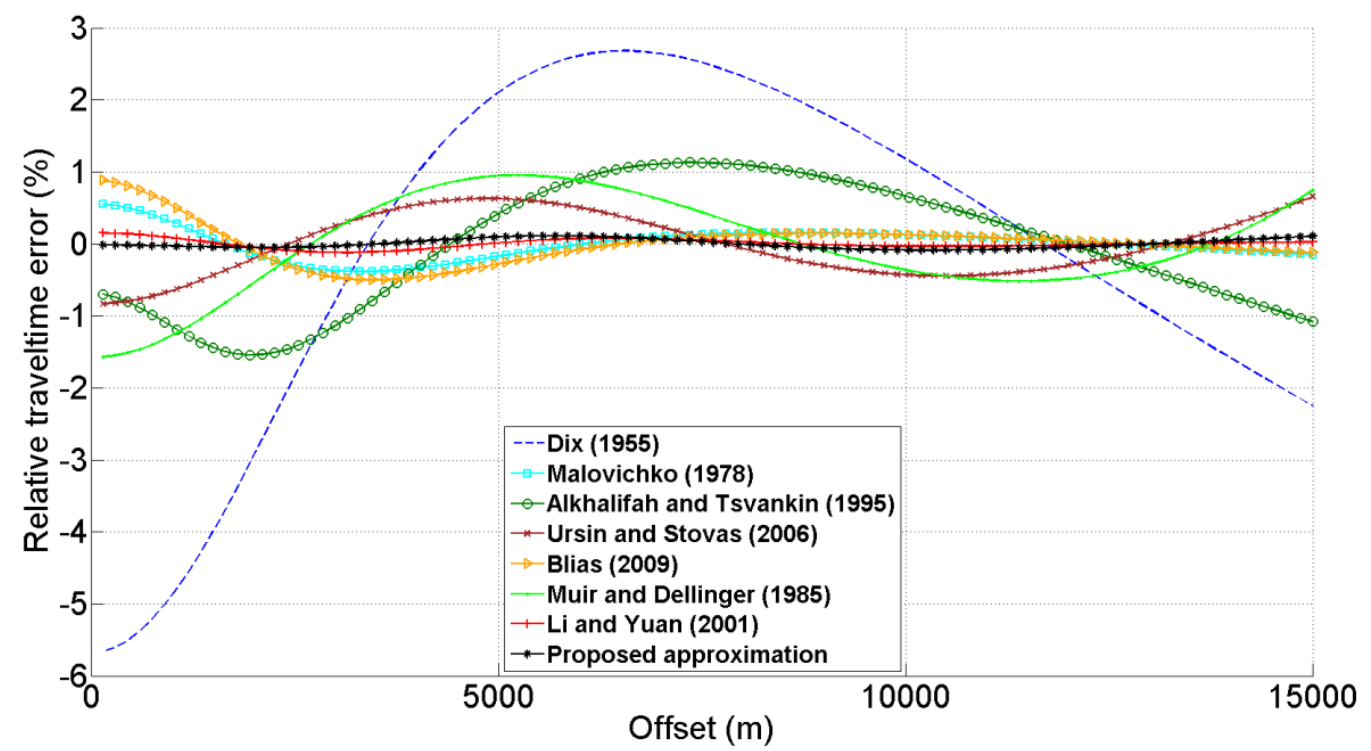

Figure 263: Relative error in travel-time between the observed curve and the calculated curve with each approximation of the PP reflection event with L1-norm and SID-PSM optimization algorithm.

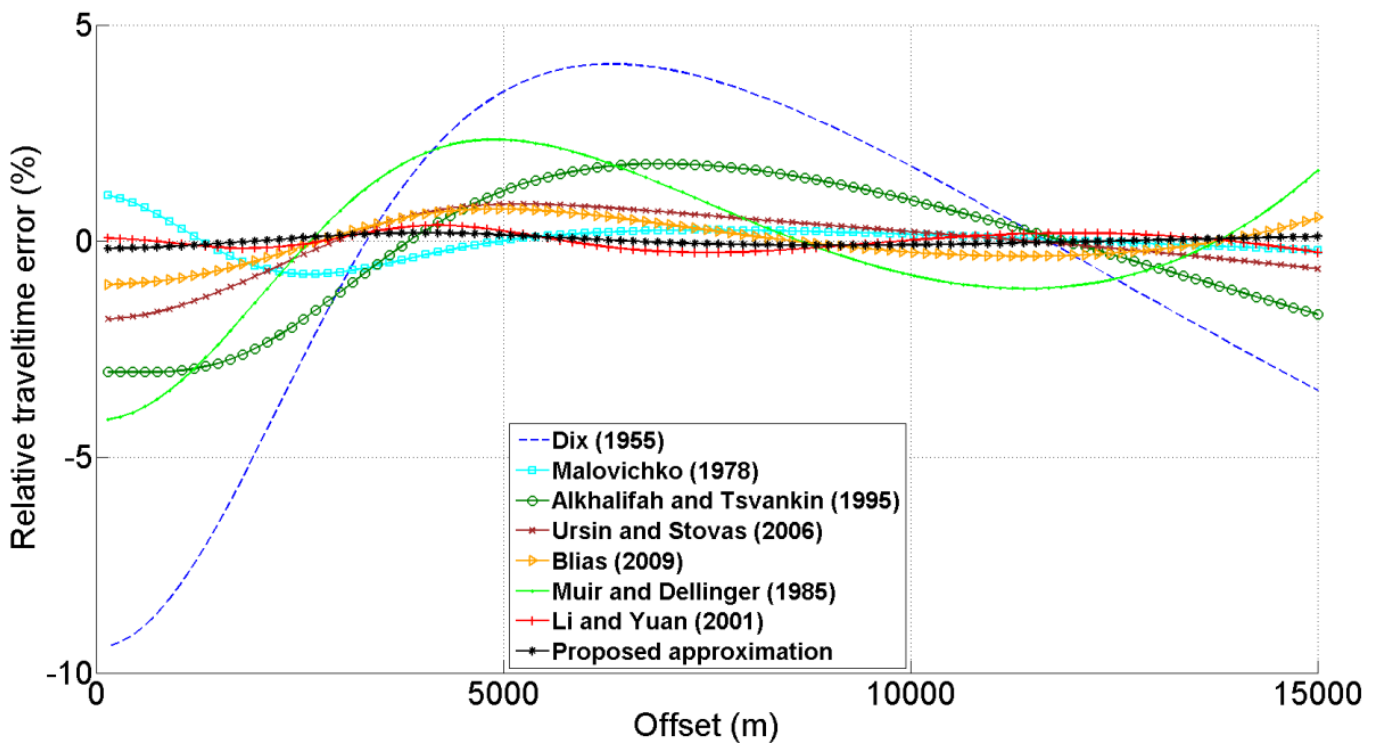

Figure 264: Relative error in travel-time between the observed curve and the calculated curve with each approximation of the PS reflection event with L1-norm and SID-PSM optimization algorithm. 


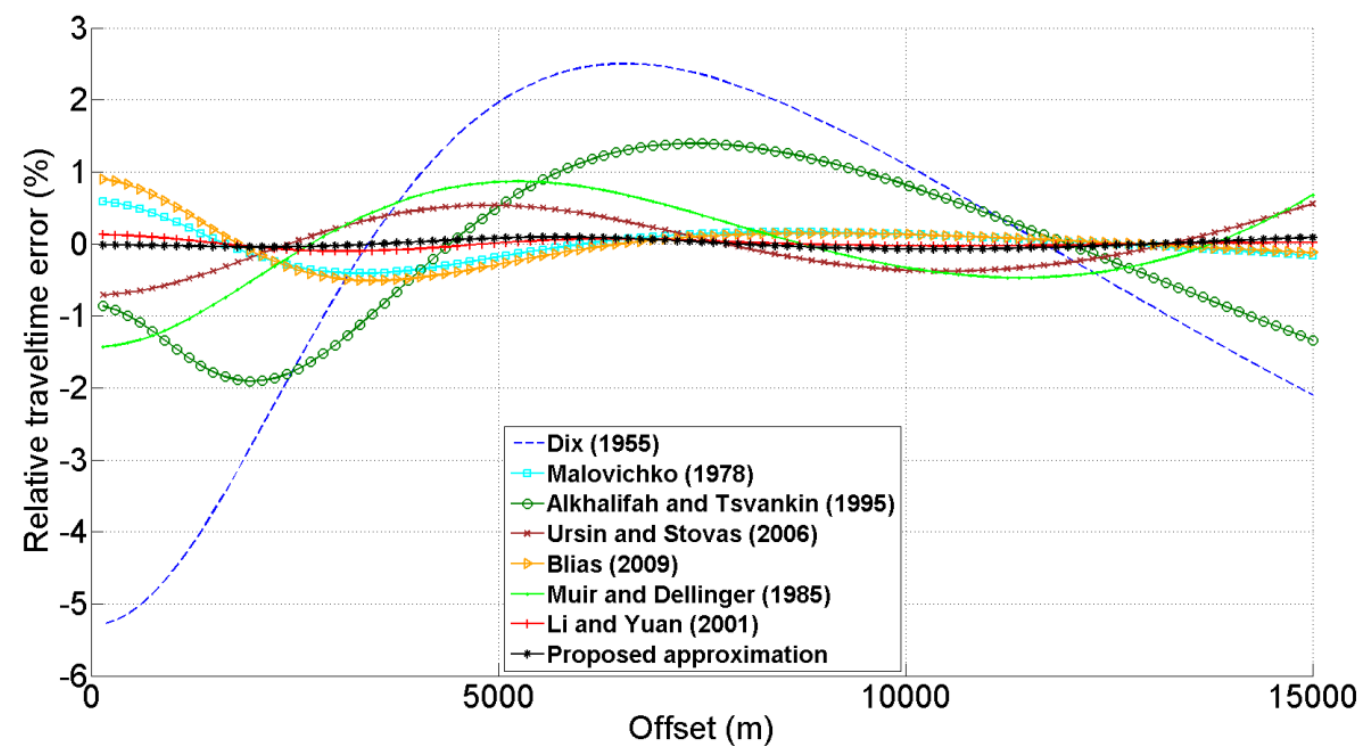

Figure 265: Relative error in travel-time between the observed curve and the calculated curve with each approximation of the PP reflection event with L2-norm and MCS optimization algorithm.

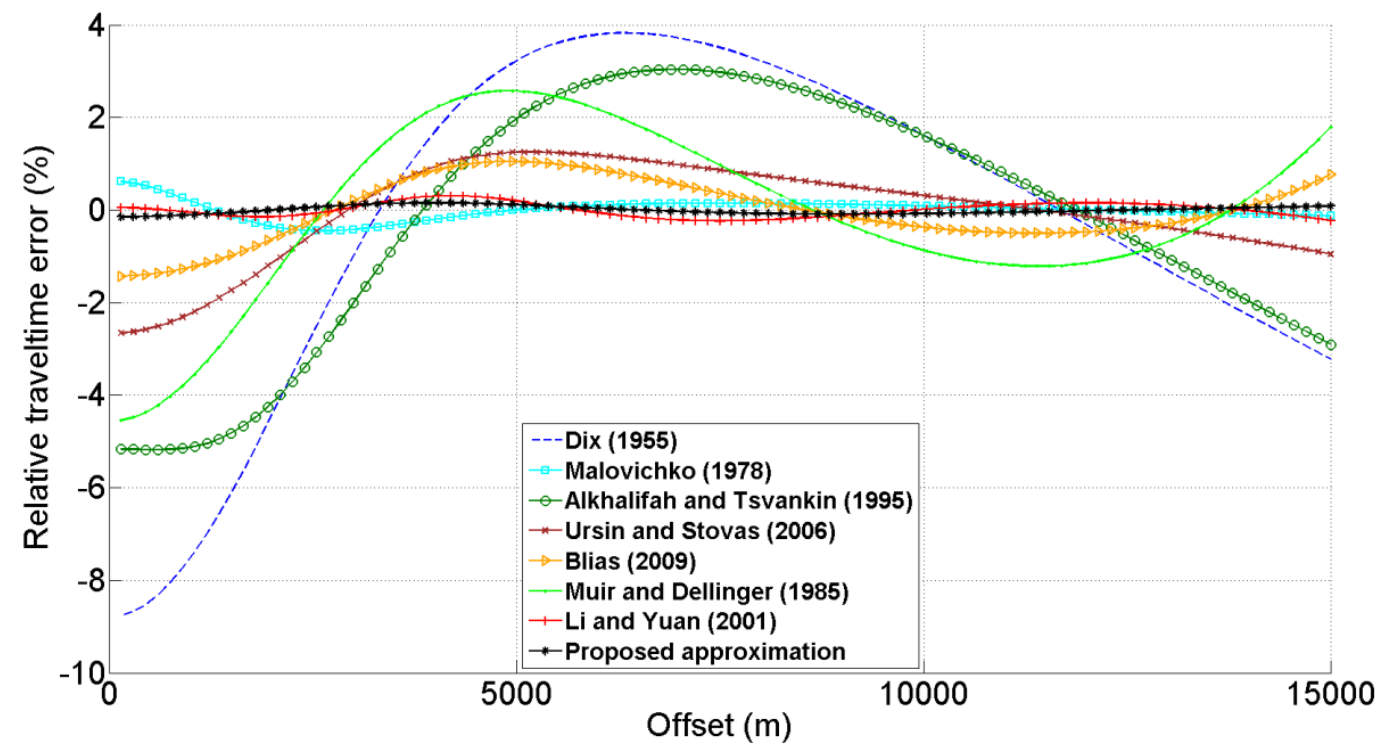

Figure 266: Relative error in travel-time between the observed curve and the calculated curve with each approximation of the PS reflection event with L2-norm and MCS optimization algorithm. 


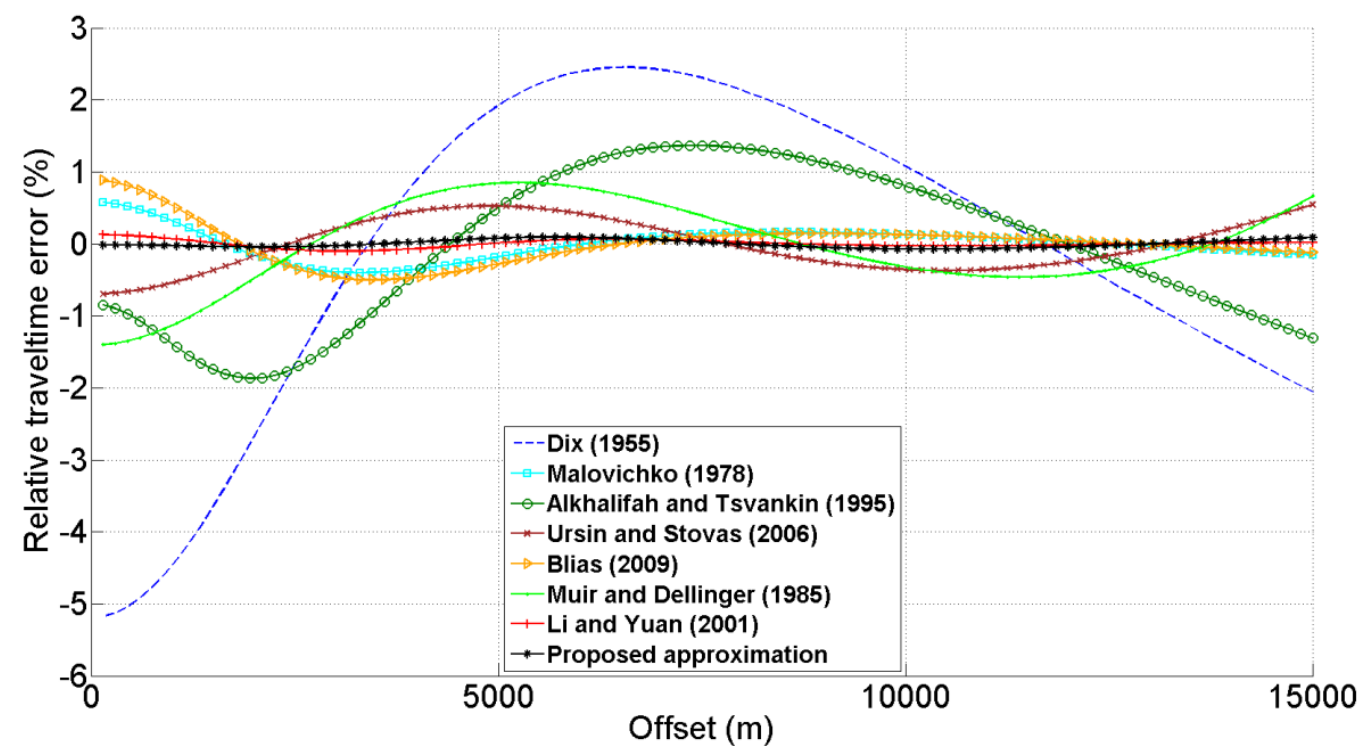

Figure 267: Relative error in travel-time between the observed curve and the calculated curve with each approximation of the PP reflection event with L1-norm and MCS optimization algorithm.

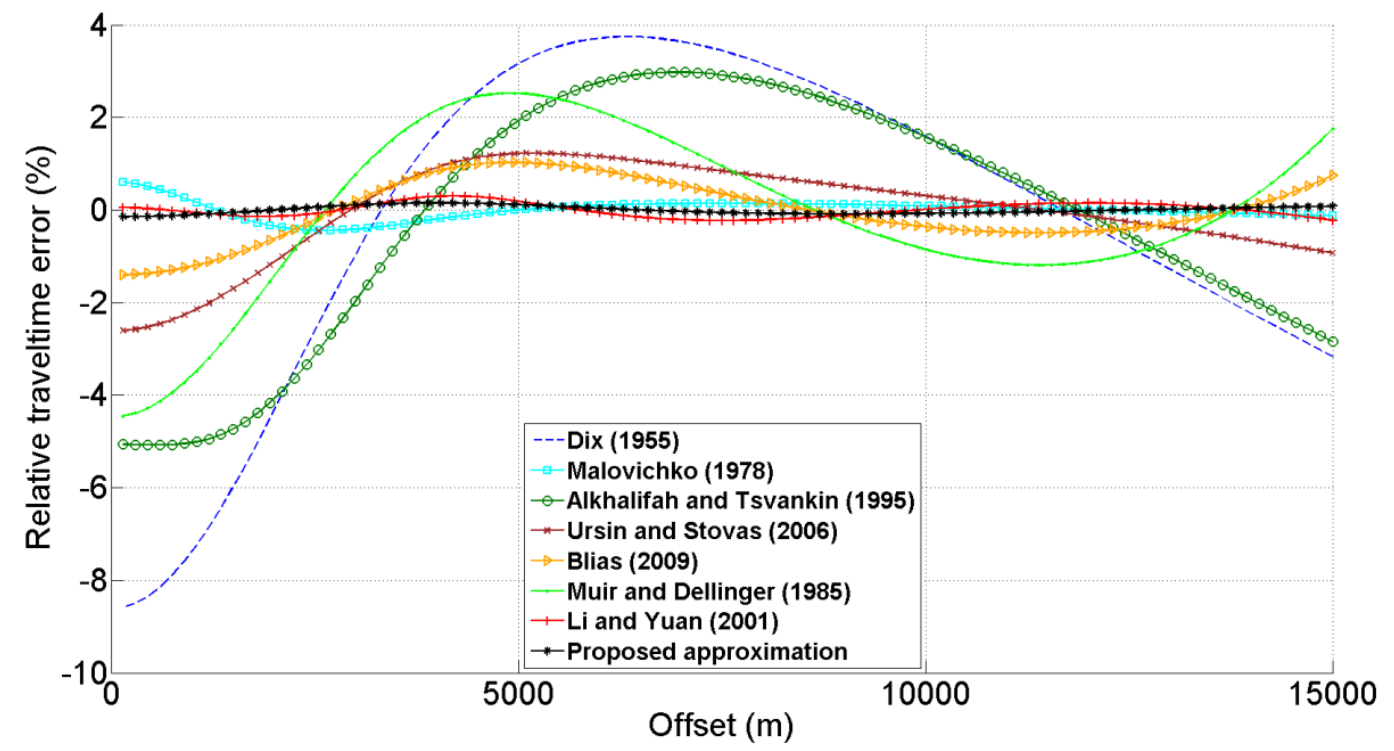

Figure 268: Relative error in travel-time between the observed curve and the calculated curve with each approximation of the PS reflection event with L1-norm and MCS optimization algorithm. 


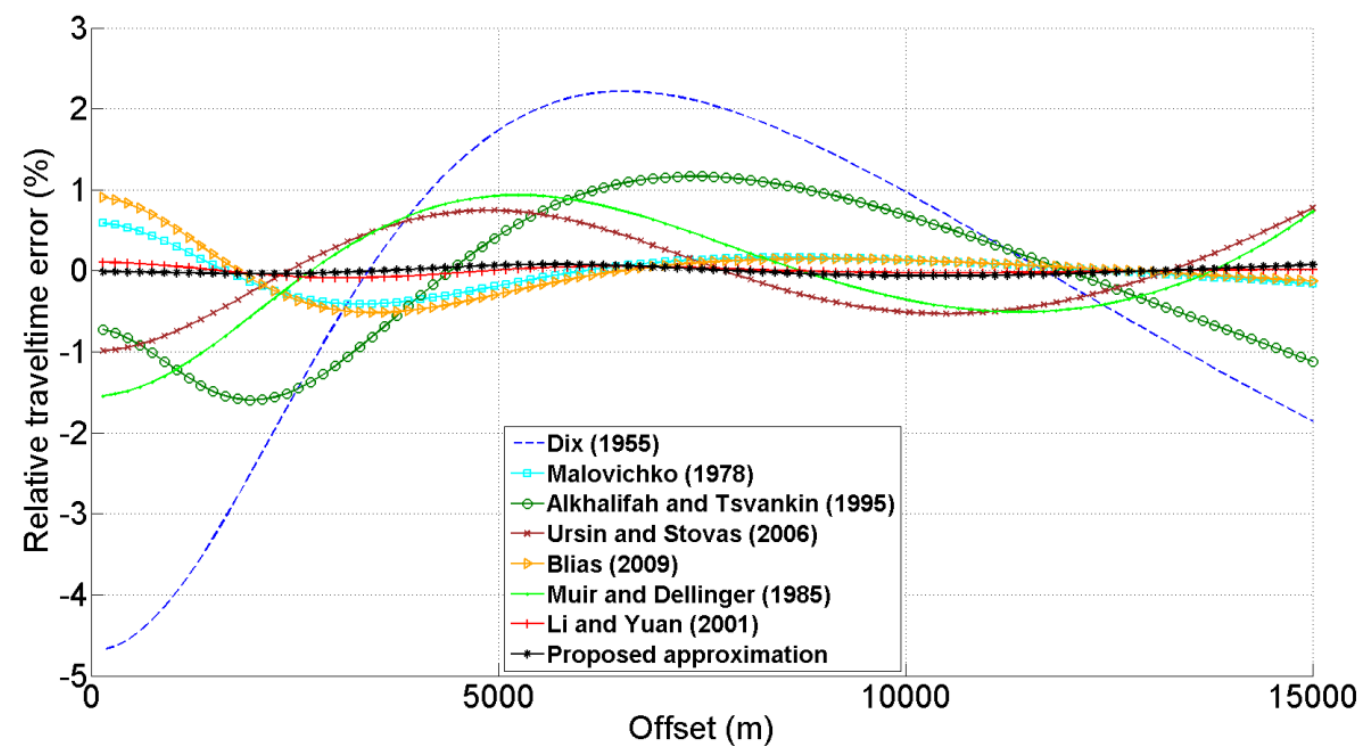

Figure 269: Relative error in travel-time between the observed curve and the calculated curve with each approximation of the PP reflection event with L2-norm and TOMLAB/LGO optimization algorithm.

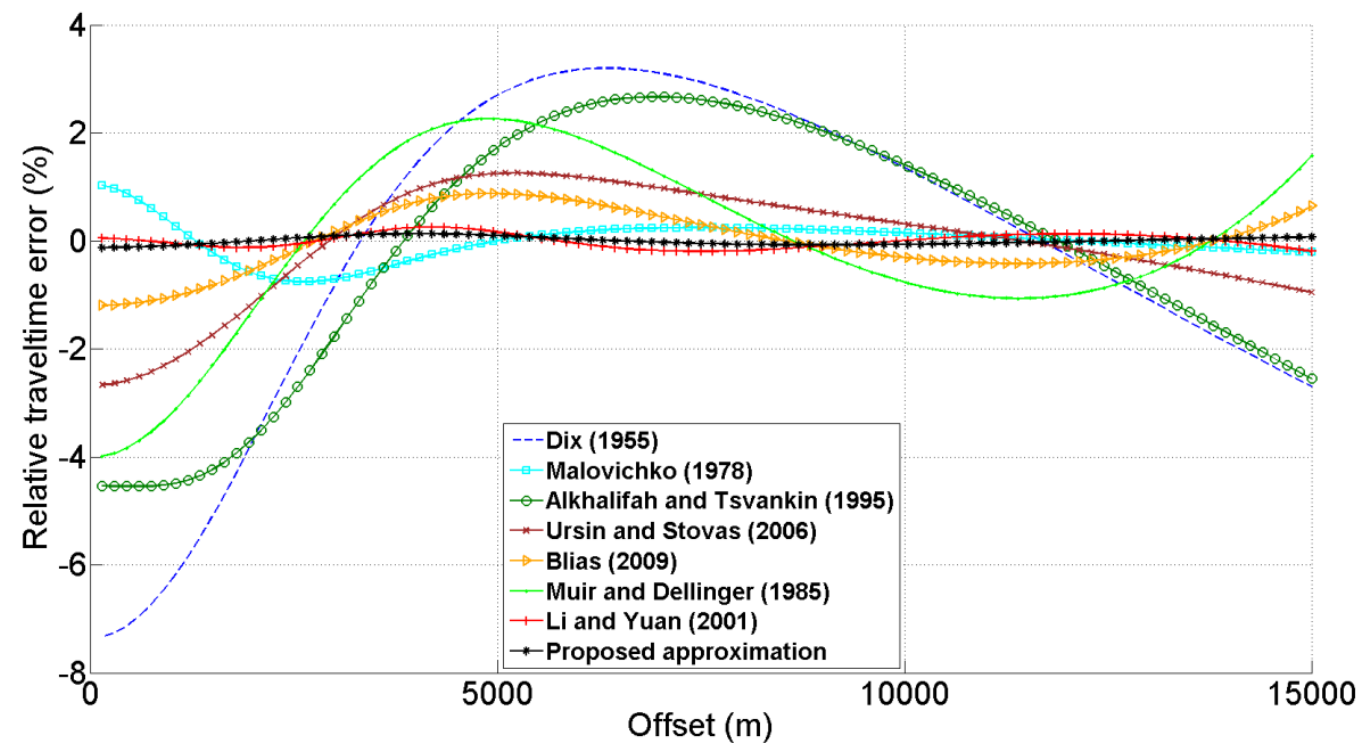

Figure 270: Relative error in travel-time between the observed curve and the calculated curve with each approximation of the PS reflection event with L2-norm and TOMLAB/LGO optimization algorithm. 


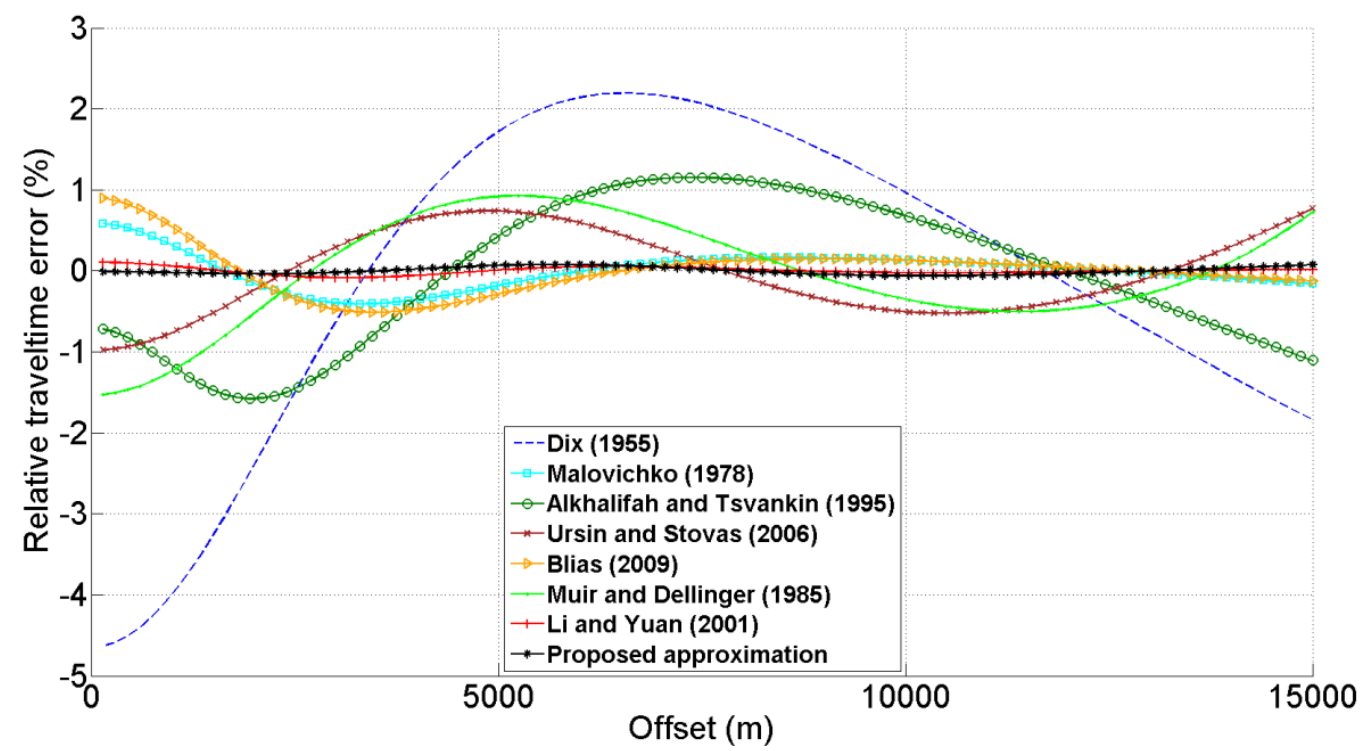

Figure 271: Relative error in travel-time between the observed curve and the calculated curve with each approximation of the PP reflection event with L1-norm and TOMLAB/LGO optimization algorithm.

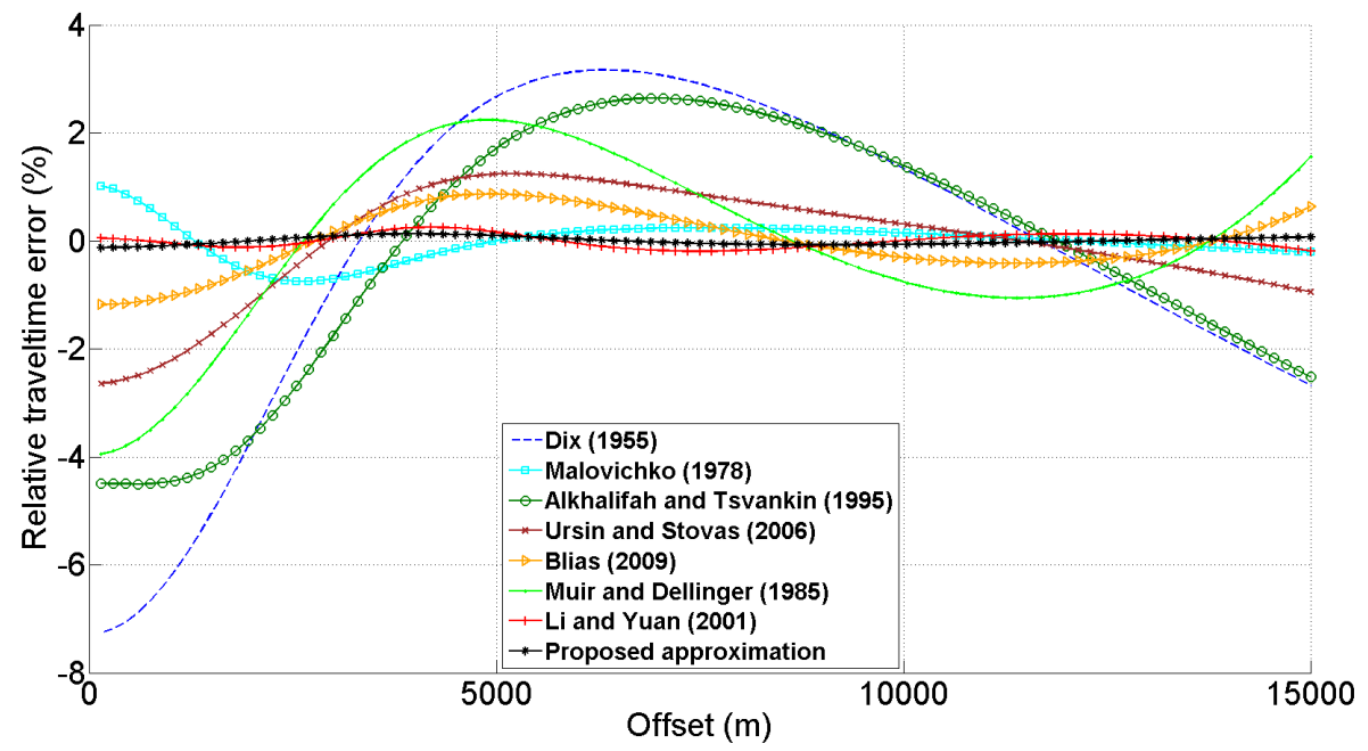

Figure 272: Relative error in travel-time between the observed curve and the calculated curve with each approximation of the PS reflection event with L1-norm and TOMLAB/LGO optimization algorithm. 


\subsection{General accuracy analysis}

As it was previously observed, the proposed approximation presented the best set of results, while Li and Yuan (2001) approximation showed the second best set of results analysed in this work.

In general, the approximation proposed by Blias (2009) showed the third best results. However, the approximation proposed by Malovichko (1978) showed almost the same quality in the results as the Blias (2009) approximation. The Ursin and Stovas (2006) approximation presented the fifth best results in a general way.

The approximations presented by Muir and Dellinger (1985) and Alkhalifah and Tsvankin (1995) presented, respectively, the second worst and the worst results, generically, in this work. And as it was already mentioned, all nonhyperbolic approximations tested in this thesis yield better results than the standard hyperbolic approximation of Dix (1955)..

The quality of the results decayed significantly in the converted wave events in comparison to the conventional PP events, due to the higher complexity of the PS events.

The application of L1-norm had a mean increase of accuracy of around $10 \%$ in comparison with the L2-norm considering the same optimization algorithm.

Concerning the variation from the L2-norm to L1-norm, the optimization algorithm IMFIL showed a mean increase of accuracy of around 11\%. The FMINSEARCH showed a mean increase of $9 \%$ for all approximations. The algorithm SID-PSM presented a mean increase of $5 \%$ for all scenarios which were tested. A mean increase of $2 \%$ was shown by MCS algorithm. The lesser difference after applying the L1-norm was shown by the TOMLAB/LGO optimization algorithm, a mean increase of accuracy of around $1 \%$.

Considering the IMFIL optimization algorithm as the reference, the FMINSEARCH algorithm presented a mean increase of $68 \%$ concerning the accuracy. SID-PSM optimization algorithm showed a mean increase of $78 \%$; while the MCS algorithm, a mean increase of $83 \%$. The TOMLAB/LGO optimization algorithm presented a mean increase of accuracy of around $85 \%$. 


\subsection{Processing time analysis}

Dix (1955) hyperbola equation presented the lowest processing time for every inversion, in which the parameters were recovered. The approximation proposed by Li and Yuan (2001) presented the second best processing time. The approximation proposed in this work presented the third best processing time, generically. The Malovichko (1978) approximation showed the fourth best processing time, while the approximation proposed by Blias (2009) showed the fifth lowest processing time. The approximation proposed by Alkhalifah and Tsvankin (1995) presented the third worst processing time, while the approximation proposed by Muir and Dellinger (1985) presented the second worst processing time. The highest processing time was shown by the approximation proposed by Ursin and Stovas (2006).

Considering the IMFIL optimization algorithm as the reference, the FMINSEARCH algorithm showed a mean increase in processing time of around $12 \%$. The mean increase of processing time which was shown by SID-PSM algorithm was of around 103\%. The MCS optimization algorithm showed a mean increase of $209 \%$ concerning the relative processing time. A mean increase of $411 \%$ was observed in the processing time of the TOMLAB/LGO optimization algorithm.

The processing times, concerning the models, have a mean increase between $2.1 \%$ and $5.4 \%$, considering the first model as the reference, due to its simplicity.

All approximations, concerning the variation from the conventional PP wave event to the converted PS wave event, presented a mean increase in processing time of around $11 \%$.

Concerning the application of the L1-norm, the eight approximations had a mean increase in processing time of $11 \%$ comparing to the L2-norm. 


\section{CONCLUSIONS}

The proposed automated picking technique was very important to conclude the main objectives in this work. With this, it was possible, for each model, to find the target traveltime curve of each reflection event. The other proposed approach was also important to find other kinds of information concerning the phase variation, which were very important to understand how the phase changes and how to correct it, to perform a better NMO correction without muting a large part of the horizontalized event. It was also important to find the critical point data-driven, which was important to obtain information about elastic parameters.

The variations between PP wave reflection events and the PS converted wave reflection events was clearly observed concerning the displacement of the contour lines, which is associated to the variation in the composition of the parameters. In the cases of Models 4 and 5, the variation between the reflection events is associated to the fact that the maximum offsets between source and receiver increase the distortion of the raytracing.

Concerning the approximations, each one presents a different structure of topology of the objective function intrinsically related to their complexities.

The gradient of the contour lines became much more abrupt after the L1-norm was applied rather than the L2-norm, which made a narrower global minimum region and attenuated some features.

The variation in the topology concerning the models is associated to the fact that each model presents different composition of parameters. Furthermore, the variation in the model interferes significantly in some approximations, making them change their statistical distribution (e.g. Ursin and Stovas, 2006; Blias, 2009) or making exchanges between the global and local minimum regions (e.g. Muir and Dellinger, 1985). The second example is also affected by the variation between PP and PS wave reflection events.

The approximations which presented unimodal behaviour were the one proposed by Malovichko (1978) and the one proposed by Alkhalifah and Tsvankin (1995), due to their simple topological structure of the objective function. The approximations mentioned before (Ursin and Stovas, 2006; Blias, 2009) behaved as unimodal for the Models 1 to 3, which presented a higher RMS velocity, while for the Models 4 and 5, which presented significantly 
lower RMS velocities, these approximations showed to be multimodal. Thus, it led to understand that the increase of the RMS velocity of a model made the topological structure of the objective function more abrupt. Additionally, the approximations proposed by Muir and Dellinger (1985), Li and Yuan (2001) and the one proposed in this work showed to always be multimodal. Furthermore, the exchanges between the global and local minimum regions of the approximation proposed by Muir and Dellinger (1985) are associated to its idiosyncratic structure of the objective function that has local and global minimum regions very close to each other. Thus, the topology of the objective function was much more susceptible to varying with the approximation than with the models, reflection events or the norms.

The proposed approximation and the one proposed by Li and Yuan (2001) share not only the multimodality, but also the similarities concerning the objective function. However, the approximation proposed by Li and Yuan (2001) was almost equally sensible concerning the RMS velocity and the $\gamma$ parameter. On the other hand, the proposed approximation presented an RMS velocity parameter which is much more sensible than the additional one, which increases the precision to recover the RMS velocity, even increasing the time to perform the curve fitting. Another important behaviour of the proposed approximation is about the strong variation from the Models 1, 2 and 3 to the Models 4 and 5. The observed distortion made the objective function topology even narrower, which made this approximation reliable even for these kinds of models.

Once the hyperbola equation (Dix, 1955) is the simplest approximation being compared, it presented the worst set of results. Among the nonhyperbolic multiparametric approximations, the ones proposed by Alkhalifah and Tsvankin (1995) and Muir and Dellinger (1985) were, respectively, the least and the second least accurate. The Ursin and Stovas (2006) approximation presented the fifth best set of results, while the approximation proposed by Malovichko (1978) showed the fourth best results. The approximation proposed by Blias (2009) showed a set of results slightly more accurate than the one proposed by Malovichko (1978). The approximation proposed by Li and Yuan (2001) presented as the second most accurate. The approximation proposed in this work showed the best set of results.

The accuracy decreased for the converted wave event due to its higher complexity, which also happened with the complexity of the models. However, the L1-norm showed increases concerning the accuracy for all approximations and all optimization algorithms 
tested. About the algorithms, the IMFIL showed to be the simplest one tested, while there were increases in the accuracy for all other optimization algorithms. The one which presented as the most precise is the TOMLAB/LGO, while the MCS presented results of almost the same quality. The third and the fourth most accurate optimization algorithms are, respectively, the SID-PSM and the FMINSEARCH.

The processing time of the hyperbola approximation (Dix, 1955) was, by far, the lowest one, due to its simplicity. Among the nonhyperbolic multiparametric approximations, the one proposed by $\mathrm{Li}$ and Yuan (2001) presented the best processing time, while the proposed approximation appeared as the second best processing time. The Malovichko (1978) approximation showed the third best processing time among the multiparametric approximations. The Blias (2009) approximation presented the fourth lowest processing time among the nonhyperbolic approximations. The approximations proposed by Ursin and Stovas (2006), Muir and Dellinger (1985), and Alkhalifah and Tsvankin (1995) presented the highest processing times, respectively.

The IMFIL was the optimization algorithm with the lowest processing time, while the FMINSEARCH was the second one. The SID-PSM algorithm presented the third best processing time. The highest processing time was shown by the TOMLAB/LGO and the second highest, by the MCS optimization algorithm.

As it was expected, the conventional wave reflection events presented a lower processing time than the converted wave reflection events. However, there was a significant decrease in the processing time when the L1-norm was applied, rather than the L2-norm.

Among what was tested, it was possible to conclude that the residual function maps were important to understand the behaviour and its variations for each scenario, which brought significant information about which optimization algorithm and nonhyperbolic approximation must be used.

The application of the L1-norm showed to be an important option to perform a good processing, presenting faster and more accurate results than the L2-norm. However, it can only be used with derivative-free optimization algorithms.

The optimization algorithm which presented the best results with an acceptable processing time was the MCS algorithm, once the TOMLAB/LGO was too slow. As well, the 
FMINSEARCH and the IMFIL can be used with the unimodal approximation proposed by Malovichko (1978), once the inversion can be performed only once, even with a local search algorithm. Certainly, this situation should be applied in conditions where there is enough knowledge about the model, and not for exploratory conditions. For more complex conditions, it is important to give more attention to the accuracy than to the processing time. Thus, the proposed approximation along with the MCS optimization algorithm should be the best option to obtain information in an exploratory field.

The approximation proposed in this thesis not only works very well, as it also presents a good processing time for a multimodal approximation, and is the most accurate among the approximations tested here for those kinds of models. 


\section{REFERENCES}

ABEDI, M. M; RIAHI, M. A. Nonhyperbolic stretch-free normal moveout correction. Geophysics, 81, 87-95, 2016.

AGUDELO, W; PINEDA, E; GOMEZ, R; GUERRERO, J; ROJAS, N; STEWART, R. Using converted- wave seismic data for lithology discrimination in a complex fluvial setting: Tenerife oil field, Middle Magdalena Valley, Colombia. The Leading Edge, 32, 72-78, 2013.

ALEIXO, R; SCHLEICHER, J. Traveltime approximations for q-P waves in vertical transversely isotropic media. Geophys. Prospect, 58, 191-201, 2010.

ALIFANOV, O. M. Inverse Heat Transfer Problems. Berlin: Springer, p. 348. 1995.

ALKHALIFAH, T; TSVANKIN, I. Velocity analysis for transversely isotropic Media. Geophysics, 60, 1550-1566, 1995.

ASTER, R. C; BORCHERS, B; THURBER, C. H. Parameter estimation and inverse problems. $2^{\mathrm{a}}$ ed. Oxford: Elsevier Science \& Tecnology, 2012.

BLANGY, J. P. Integrated seismic lithology interpretation: The petrophysical basis. Ph.D. Dissertation, Stanford University, 1992.

BLIAS, E. Reflected waves travel-time curve in flat-bedded medium with transverse layers and their interpretation: Soviet Geology and Geophysics, N2, 91-95, 1983.

BLIAS, E. Long-offset NMO approximations for a layered VTI model: Model study. In: 79th Annual International Meeting: Society of Exploration Geophysics. Expanded Abstract, 2009.

BOKHONOK, O. Sísmica de reflexão rasa multicomponente: Aquisição e inversão de tempos de trânsito e amplitudes. Ph. D. Dissertation, Universidade de São Paulo, 2010.

BOURBAKI, N. Topological Vector Spaces. 1st ed. Berlin, Springer-Verlag Berlin and Heidelberg, 1987.

CADORET, T. Effet de la saturation eau/gaz sur les proprie'te's acoustiques des roches. Ph.D. Dissertation, University of Paris, 1993.

CAI, Y; FOMEL, S; ZENG, H. Automated spectral recomposition with application in stratigraphic interpretation. Interpretation, 1(1), 109-116, 2013.

CASTLE, R. J. Shifted hyperbolas and normal moveout. In: 58 th Annual International Meeting: Society of Exploration Geophysics, 1988. Expanded Abstracts, 894-896. 1988.

CASTLE, R. J. A theory of normal moveout. Geophysics, 59, 983-999, 1994.

CAUSSE, E; HAUGEN, G. U; ROMMEL. B. E. Large-offset approximation to seismic reflection traveltimes. Geophysical Prospecting, 48, 763-778, 2000. 
CHERET, T; BALE, R; LEANEY, S. Parameterization of polar anisotropic moveout for converted wave. In: 70th Annual Internat. Mtg., Soc. Expl. Expanded Abstract, 1181-1184, 2000 .

COXETER, H. S. M. Regular Polytopes. London: Methuen, p. 321, 1948.

CUSTÓDIO, A. L; ROCHA, H; VICENTE, L. N. Incorporating minimum Frobenius norm models in direct search. Computational Optimizing and Applications, 46, 265-278, 2010.

CUSTÓDIO, A. L; VICENTE, L. N. Using sampling and simplex derivatives in pattern search methods. SIAN Journal on Optimization, 537-555, 2007.

CUSTÓDIO, A. L; VICENTE, L. N. SID-PSM: A pattern search method guided by simplex derivatives for use in derivative-free optimization. Coimbra, Departamento de Matemática de Coimbra, 2008.

DE BAZELAIRE, E. Normal moveout revisited: Inhomogeneous media and curved interfaces. Geophysics, 53, 143-157, 1988.

DIX, C. H. Seismic velocities from surface measurements. Geophysics, 20, 68-86, 1955.

DAVIS, T. L. Multicomponent Seismology - The next wave. Geophysics, 66, 49, 2001.

DUARTE, O. O. Dicionário enciclopédico inglês-português de geofísica e geologia. 4th ed. Rio de Janeiro: Sociedade Brasileira de Geofísica-SBGf, 2010.

FOMEL, S; GRECHKA, V. On nonhyperbolic reflection moveout in anisotropic media. Standford Exploration Project, 617-640, 2000.

FOMEL, S; GRECHKA, V. Nonhyperbolic reflection moveout of P waves. An overview and comparison of reason: Colorado School of Report CWP-372, 2001.

GILMORE, P; KELLEY, C. T. An implicit filtering algorithmfor optimization of functions with many local minima. SIAM Journal on Optimization, 5, 269-285, 1995.

GOLIKOV, P; STOVAS, A. Accuracy comparison of nonhyperbolic moveout approximations for qP-waves in VTI media. Journal of Geophysics and Engineering. 9, 428-432, 2012.

GONZALEZ-SERRANO, A; CLAERBOUT, J. F. Wave-function velocity analysis. Geophysics, 49, 1432-1456, 1984.

GRANLI, J. R; ARNTSEN, B; SOLLID, A; HILDE, E. Imaging through gas-filled sediments with marine S-wave data. $65^{\text {th }}$ Ann. Intl. Mtg., SEG, Expanded Abstract, 1995.

GEERTSMA, J. Velocity-log interpretation: The effect of rock bulk compressibility. Soc. Pet.Eng, 1, 235-248, 1961.

HAN, D. H. Effects of porosity and clay content on acoustic properties of sandstones and unconsolidated sediments. Ph.D. Dissertation. Stanford University, 1986.

HANSEN, N. The CMA evolution strategy: A computing review. Springer. 2006.

HANSEN, N. The CMA evolution strategy: A tutorial. 2011. 
HARDAGE, B. A; MURRAY, P. E; ANGELO, M; SAVA, D; ROBERTS, H. H; SHEDD, W; HURT, J. Multicomponent Seismic Technology. Tulsa, Oklahoma: Society of Exploration Geophysicists, 2011.

HOLMSTRÖM, K; QUTTINEH, N. H; EDVALL, M. M. An adaptive radial basis algorithm (ARBF) for expensive black-box mixed-integer constrained global optimization. Optimization and Engineering, 9, 311-339, 2008, 2008.

HOLMSTRÖM, K; GÖRAN, A. O; EDVALL, M. M. User's Guide for TOMLAB/CGO. TOMLAB Optimization, 2007a.

HOLMSTRÖM, K; GÖRAN, A. O; EDVALL, M. M. User's Guide for TOMLAB/OQNLP. TOMLAB Optimization, 2007b.

HORST, R; PARDALOS, P. M; THOAI, N. V. Introduction to global optimization. 2nd ed. Dordrecht: Kluwer Academic Publusher, 2000.

HUYER, W; NEUMAIER, A. Global optmization by multilevel coordinate search. Journal of Global Optimization, 14, 331-355, 1999.

HUYER, W; NEUMAIER, A. SNOBFIT - Stable Noisy optimization by branch and fit. ACM Transactions on Mathematical Software, 35, 1-25, 2008.

JIZBA, D. L. Mechanical and acoustical properties of sandstones and shales. Ph.D. Dissertation, Stanford University, 1991.

JONES, D. R. The DIRECT global optimization algorithm. Boston, 2001.

KAN, H. A. H. G; TIMMER, G. T. Stochastic global optimization method part I: Clustering methods. Mathematical Programing, 39, 27-56, 1987a.

KAN, H. A. H. G; TIMMER, G. T. Stochastic global optimization method part II: Multi level methods. Mathematical Programing, 39, 57-78, $1987 \mathrm{~b}$.

KEAREY, P; BROOKS, M; HILL, I. An introduction to geophysical exploration. 3rd ed. Blackwell Science, 2002.

KELLEY, C. T. Implicit Filtering. 1st ed, Raleigh. 2011.

KENDALL, R. Advances in Land Multicomponent Seismic Acquisition, Processing and Interpretation. CSEG Recorder, Special Edition, 65-75, 2006.

KHALEELULLA, S. M. Counterexample in Topological Vector Spaces. 2nd ed. Springer, 1982.

KURT, H. Joint inversion of AVA data for elastic parameters by bootstrapping. Computers \& Geosciences, 33, 367-382, 2007.

LAGARIAS, J. C; REEDS, J. A; WRIGHT, P. E. Convergence properties of the Nelder Mead simplex method in low dimensions. SIAM Journal on Optimization, 9, 112-147, 1998.

LARSEN, J. A. AVO Inversion by Simultaneous P-P and P-S Inversion. 1999. M.Sc. Dissertation, University of Calgary, 1999. 
LEIDERMAN, R; ARTOLA, F. A. V; SILVA, M. B. C; FONTOURA, S. A. B. Exemplo de modelagem sísmica dos modos PP e PS a partir de dados de poço e perfil litológico. $2^{\circ}$ Congresso Brasileiro de P\&D em Petróleo \& Gás, Expanded Abstract, 2003.

LEWIS, R. M; TORCZON, V; TROSSET, M. W. Direct search methods: Then and now. ICASE Report, 2000.

LI, X. Y. Converted-wave moveout analysis revisited: The search for a standard approach. 73rd Annual internat. Mtg. Soc. Expl. Geophysics, Expanded Abstract, 805-808, 2003.

LI, X. Y; YUAN, J. Converted-waves moveout and parameter estimation for transverse isotropy. 61st EAGE Conference, Expanded Abstract, 1, 4-35, 1999.

LI, X. Y; YUAN, J. Converted wave imaging in inhomogeneous, anisotropic media: Part I. Parameter estimation. In: 63rdEAGE conference, Expanded Abstract, 1, 109, 2001.

LI, X. Y; YUAN, J. Converted-wave moveout and conversion-point equations in layered VTI media: theory and applications. Journal of Applied Geophysics, 54, 297-318, 2003.

LUCET, N. Vitesse et Attenuation des ondes e'lastiques soniques et ultrasoniques dans les roches sous pression de confinement. Ph.D. Dissertation, University of Paris, 1989.

MALOVICHKO, A. A. A new representation of the traveltime curve of reflected waves in horizontally layered media. Applied Geophysics (in Russian), 91, 47-53, 1978.

MARGRAVE, G. F. New seismic modellingfacilities in Matlab. CREWES Research Report, 12, 2000.

MARGRAVE, G. F. Numerical methods exploration seismology with algorithms in MATLAB. CREWES Research Report, 2003.

MAVKO, G; MUKERJI, T; DVORKIN, J. The rock physics handbook: Tools for seismic analysis of porous media. 2nd ed. Cambridge, Cambridge University Press, 2009.

MEIER, M. A; LEE, P. J. Converted-wave resolution. Geophysics, 74, Q1-Q16, 2009.

MUIR, F; DELLINGER, J. A practical anisotropic system, in SEP-44. Stanford Exploration Project, 55-58, 1985.

NELDER, J. A; MEAD, R. A simplex method for function minimization. The Computer Journal, 7, 308-313, 1965.

NEUMAIER, A; SHCHERBINA, R; HUYER, W; VINKÓ, T. A comparison of complete global optimization solvers. Mathematical Programming, 103, 335-356, 2005.

NOWROOZI, A. A. Generalized form of the Dix equation for the calculation of the interval velocities and layer thicknesses. Geophysics, 54, 659-661, 1989.

PICKETT, G. R. Acoustic character logs and their application in formation evaluation. Journal of Petroleum technology, 15, 659-667, 1963. 
PINTÉR, J. D. Global optimization in action: Continuous and Lipschitz optimization. Algorithms, Implementations and Applications. 1995.

PINTÉR, J. D; HOLMSTRÖM, K; GÖRAN, A. O; EDVALL, M. M. User's Guide for TOMLAB/LGO. TOMLAB Optimization, 2006.

PURNELL, M. A. Conodonts of the Lower Border Group and equivalent strata (Lower Carboniferous) in northern Cumbria and the Scottish Borders. Royal Ontario Museum, Life Science Contributions, 156, 1-79, 1992.

QIAN, Z; CHAPMAN, M; LI, X. A; DAI, H. C; LIU, E; ZHANG, Y; WANG, Y. Use of multicomponent seismic data for oil-water discrimination in fractures reservoirs. The Leading Edge, 26, 1176-1184, 2007.

RIOS, L. M; SAHINIDIS, N. V. Derivative-free optimization: A review of algorithms and comparison of softwares implementations. Journal of Global Optimization, 56, 1247-1293, 2013.

SAIN, K; KAILA, K. L. Direct calculation of interval velocities and layer thicknesses from seismic wide-angle reflection times. Geophysical Journal International, 125, 30-38, 1996.

SHERIFF, R. E; GELDART, L. P. Exploration seismology. 2nd ed. Cambridge: Cambridge University Press. p. 628, 1995.

SILVA, M. B. C; FILHO, O. K; GUIMARÃES, M. A. G; WINDELS, R; LEIDERMAN, R; ARTOLA, F. A. V; FONTOURA, S. A. B; SOBREIRA, J. F. F. 4-C seismic data processing experience in Brazil. 8th International Congress of the Brazilian Geophysical Society, Expanded Abstract, 2003.

SLOTBOOM, R. T. Converted wave moveout estimation. In: 60 th Annual International Meeting: Society of Exploration Geophysics, Expanded Abstracts, 104-1106, 1990.

SPENDLEY, W; HEXT, G. R; HIMSWORTH, F. R. Sequential Application of Simplex Designs in Optimazation and Evolutionary Operation. Technometrics, 4, 441-461, 1962.

SPITZ, S; GRATACOS, B; HAGGARD, W; VUILLERMOZ, C. Reservoir monitoring using multicomponent seismic: Processing the Teal South 4D-4C. SEG/EAGE Summer Research Workshop, Expanded Abstracts, 2000.

STEWART, R. R; GAISER, J. E; BROWN, R. J; LAWTON, D. C. Converted-wave seismic exploration: Methods. Geophysics, 67, 1348-1363, 2002.

STEWART, R. R; GAISER, J. E; BROWN, R. J; LAWTON, D. C. Converted-wave seismic exploration: Applications. Geophysics, 68, 40-57, 2003.

STIGLER, S. M. Gauss and the invention of least squares. Ann Statist, 9, 465-474, 1981.

STRANDENES, S. Rock physics analysis of the Brent Group reservoir in the Oseberg field. Stanford Rockphysics and Borehole Geophysics Project, Special volume, 1991.

TARANTOLA, A. Inverse problem theory and methods for model parameters estimation. 1st ed. Paris: Society for Industrial and Applied Mathematics, 2005. 
TERLAKY, T; SOTIROV, R. Multi-start approach to global conic optimization.ISE Archives of Working Papers, 2010.

TERLAKY, T; SOTIROV, R. Multi-start approach for an integer determinant maximization problem. Optimization, 61, 101-114, 2013.

THOMSEN, L. Weak elastic anisotropy. Geophysics, 51, 1954-1966, 1986.

THOMSEN, L. Converted-wave reflection seismology over inhomogeneous, anisotropic media. Geophysics, 64, 678-690, 1999.

THORBECKE, J. W; DRAGANOV, D. Finite-difference modeling experiments for seismic interferometry. Geophysics, 76, H1-H18, 2012.

TOMASSO, M; BOUROULLEC, R; PYLES, D. R. The use of spectral recomposition in tailored forward seismic modeling of outcrop analogs. AAPG Bulletin, 94(4), 457-474, 2010.

TREVES, F. Topological Vector Spaces, Distributions and Kernels. 1st ed. Academic Press, 1967.

TSVANKIN, I. Seismic Signatures and analysis of reaction data in anisotropic media. Elsevier, 2001.

TSVANKIN, I; GRECHKA, V. Dip moveout of converted waves and parameter estimation in transversely isotropic media. Geophysics, 48, 257-292, 2000a.

TSVANKIN, I; GRECHKA, V. Two approaches to anisotropic velocity analysis of converted waves. In: 70th Annual Internat. Mtg., Soc. Expl. Expanded Abstract, 1193-1196, 2000b.

TSVANKIN, I; THOMSEN, L. Nonhyperbolic reflection moveout in anisotropic media. Geophysics, 59, 1290-1304, 1994.

URSENBACH, C; CARY, P; PERZ, M. Limits on resolution enhancement for PS data mapped to PP time. The Leading Edge, 32, 64-71, 2013.

URSIN, B; STOVAS, A. Traveltime approximations for a layered transversely isotropic medium. Geophysics, 71, 23-33, 2006.

VAZ, A. I. F; VICENTE, L. N. A particle swarm pattern search method for bound constrained global optimization. Journal of Global Optimization, 39, 197-219, 2007.

WANG, W; PHAM, L. D. Converted-wave prestack imaging and velocity analysis by pseudo-offset migration. 63th EAGE conference, Expanded Abstrdact, L-12, 2001.

WANG, P. L; JUNFENG, C. Y; HU, T. Converted-wave imaging technology and application for complex structures. Exploration Geophysics, 2014.

WINSLOW, T. A; TREW, R. J; GILMORE, P; KELLEY, C. T. Simulated performance optimization of gas mesfet amplifiers. Conference on Advanced Concepts in High Speed Semiconductor Devices and Circuits, Expanded Abstract, 1991. 
YALE, D. P; JAMEISON, W. H., Jr. Static and dynamic rock mechanical properties in the Hugoton and Panoma fields. In: Society of Petroleum Engineers Mid-Continent Gas Symposium, Expanded Abstract, 1994.

YILMAZ, O. Seismic data analysis: Processing, inversion, and interpretation of seismic data. 2nd ed. Tulsa: Society of Exploration Geophysicists. Vol. 1, 2001.

YUAN, J. Analysis of four-component seaoor seismic data for seismic anisotropy. Ph.D. Dissertation, University of Edinburgh, 2002.

YUAN, J; LI, X. Y. Converted wave anisotropic parameter estimation from conversion point. In: 64rd EAGE conference, Expanded Abstract, 2, 253, 2002.

ZUNIGA, N. R. C. F. Comparação de aproximações não hiperbólicas multiparamétricas dos tempos de trânsito para correção de NMO dos dados sísmicos multicomponente. Bachelor's Thesis, Catholic University of Santos, 2015.

ZUNIGA, N. R. C. F. Análise comparativa de aproximações não-hiperbólicas dos tempos de trânsito de dados sísmicos multicomponente utilizando tecnologia OBN. Master's Thesis, University of São Paulo, 2017.

ZUNIGA, N. R. C. F. Análise de velocidade de dados sísmicos multicomponente da bacia de Santos utilizando tecnologia OBN e técnica SWD. HOLOS, 2, 39-48, 2018.

ZUNIGA, N. R. C. F. Comparison of global search optimization algorithms to perform the velocity analysis with a converted wave moveout equation. International Journal of Research, 7, 1-9, 2019a.

ZUNIGA, N. R. C. F. Local search optimization: A comparison of algorithms for nonhyperbolic travel-time analysis. International Journal of Research, 7, 321-328, 2019b.

ZUNIGA, N. R. C. F; BOKHONOK, O; DIOGO, L. A. Comparison of nonhyperbolic traveltime approximations for multicomponent seismic data. $14^{\text {th }}$ International Congress of the Brazilian Geophysical Society \& EXPOGEF, Expanded Abstract, 1176, 2015.

ZUNIGA, N. R. C. F, MOLINA, E. C, PRADO, R. L. Inversion of multicomponent seismic data of the Santos Basin. Far East Hydrocarbons, Expanded Abstract, 2016.

ZUNIGA, N. R. C. F; MOLINA, E. C; PRADO, R. L. Comparison of travel-time approximation for unconventional reservoir from Santos basin. Revista Brasileira de Geofísica, 35, 273-286, 2017.

ZUNIGA, N. R. C. F; MOLINA, E. C; PRADO, R. L. Comparative analysis of nonhyperbolic multiparametric travel-time approximations of multicomponent seismic data considering difference of depth between source and receiver using OBN technology. Revista Brasileira de Geofísica, 37, 397-407, 2019a.

ZUNIGA, N. R. C. F; RIBEIRO, F. B; PRIIMENKO, V. I. Relation between the model and the topography of the objective function in a velocity analysis using a nonhyperbolic multicomponent travel-time approximation. Revista Brasileira de Geofísica, 36, 1-10, 2018.

ZUNIGA, N. R. C. F; RIBEIRO, F. B; PRIIMENKO, V. I. Comparison of L2- and L1-norm to perform the inversion of travel-time curves using nonhyperbolic multiparametric 
approximations with unimodal and multimodal behavior. Revista Brasileira de Geofísica, 37, 291-297, 2019b.

ZUNIGA, N. R. C. F; RIBEIRO, F. B; PRIIMENKO, V. I. L2- and L1-norm applied to inversion of nonhyperbolic travel-times. Revista Brasileira de Geofísica, 37, 155-161, 2019c. 
9 APPENDIX

9.1 Appendix A - Seismogram of the pre salt models 


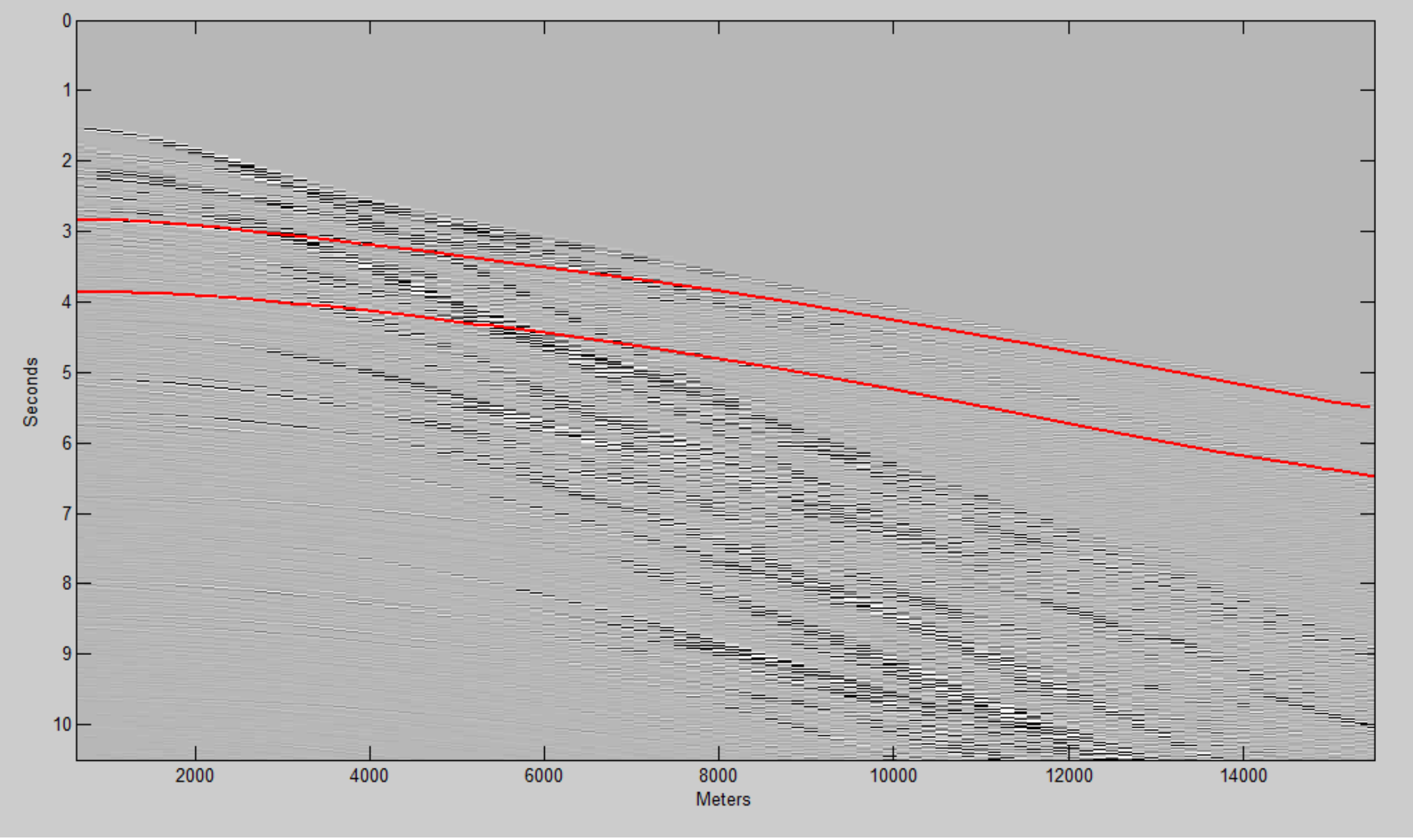

Figure 273: Seismogram of the Model 1 with no noise. The red lines represent the automated picks for the PP and PSv events. 


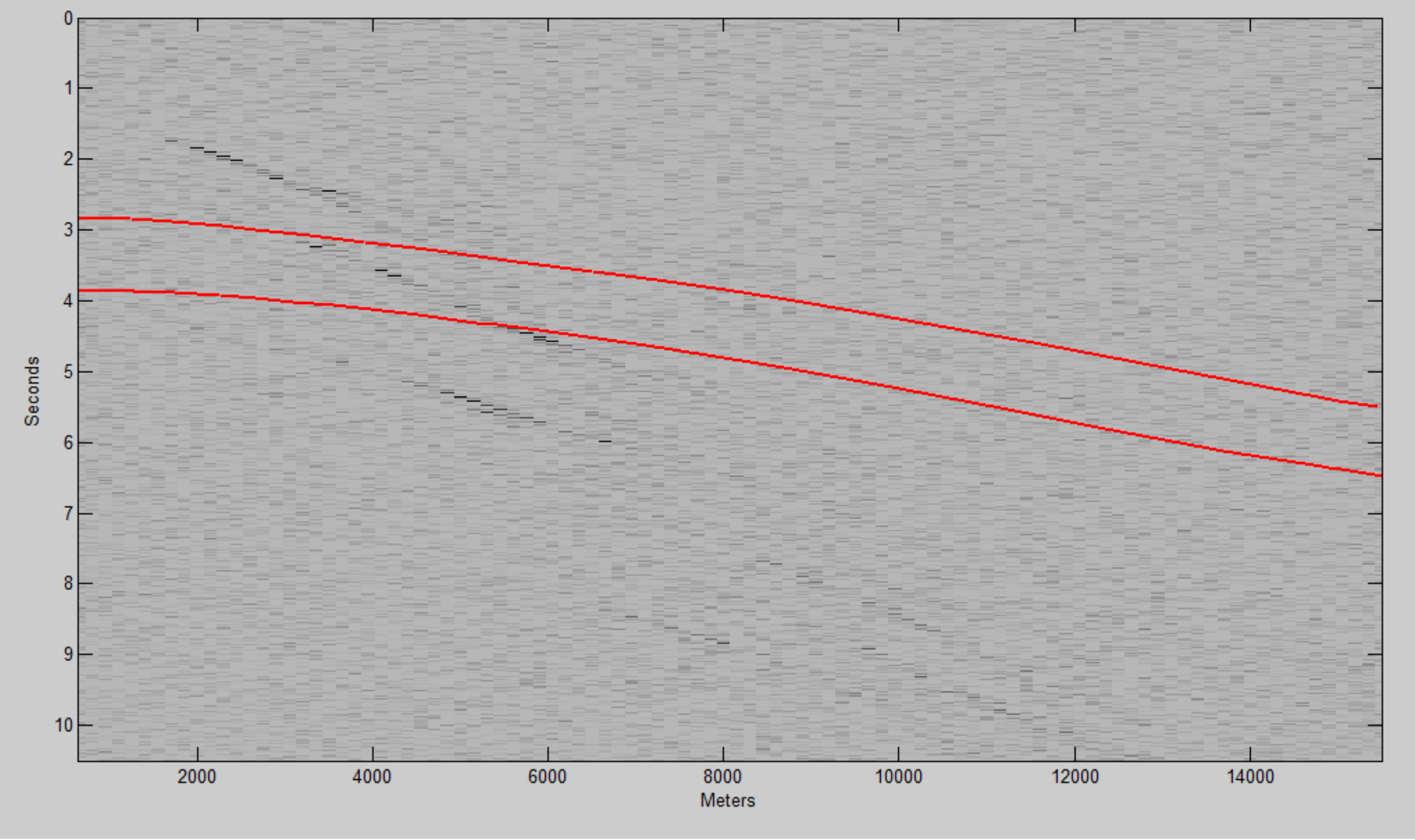

Figure 274: Seismogram of the Model 1 with signal-noise ratio of 90\%. The red lines represent the automated picks for the PP and PSv events. 


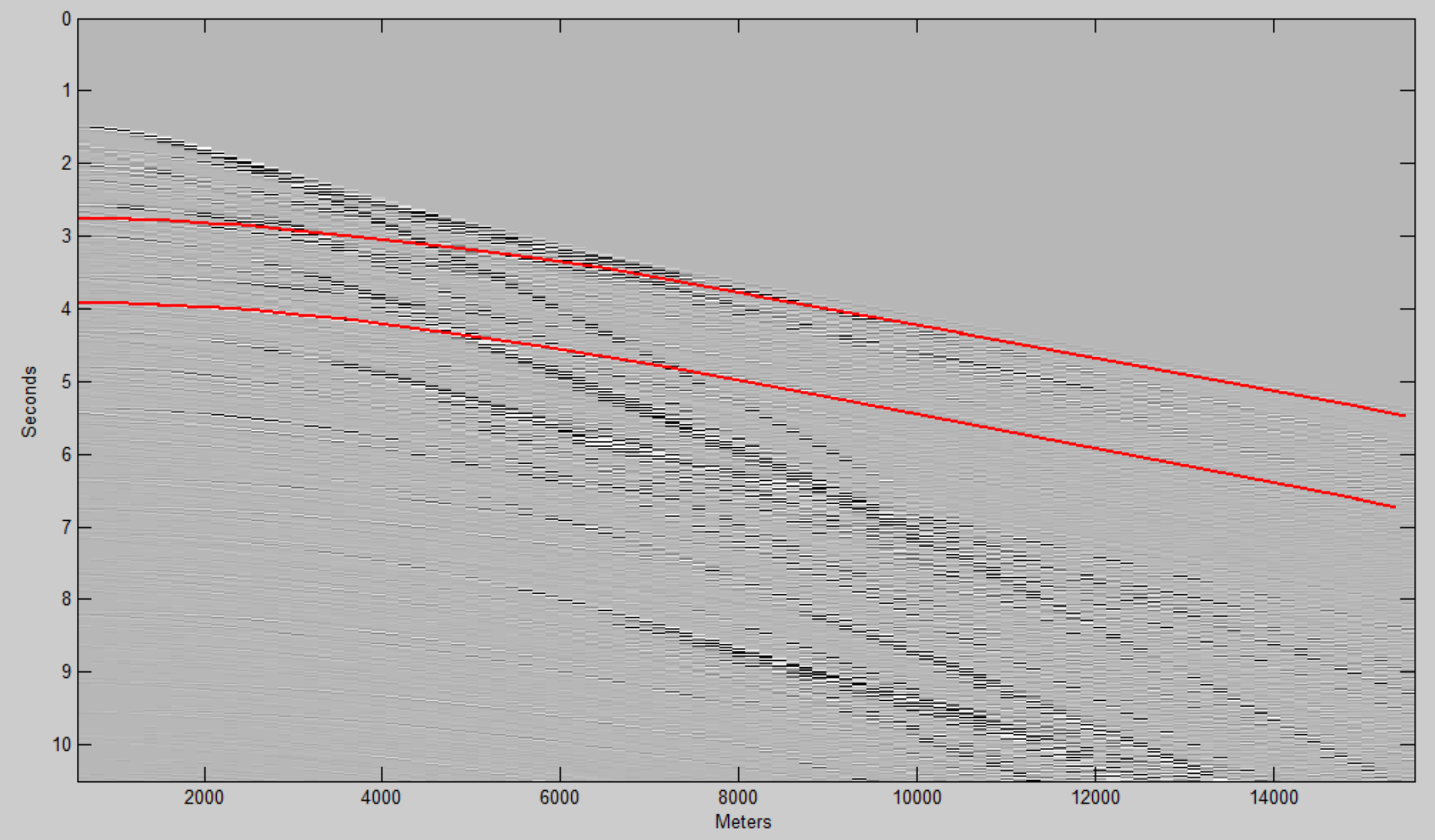

Figure 275: Seismogram of the Model 2 with no noise. The red lines represent the automated picks for the PP and PSv events. 


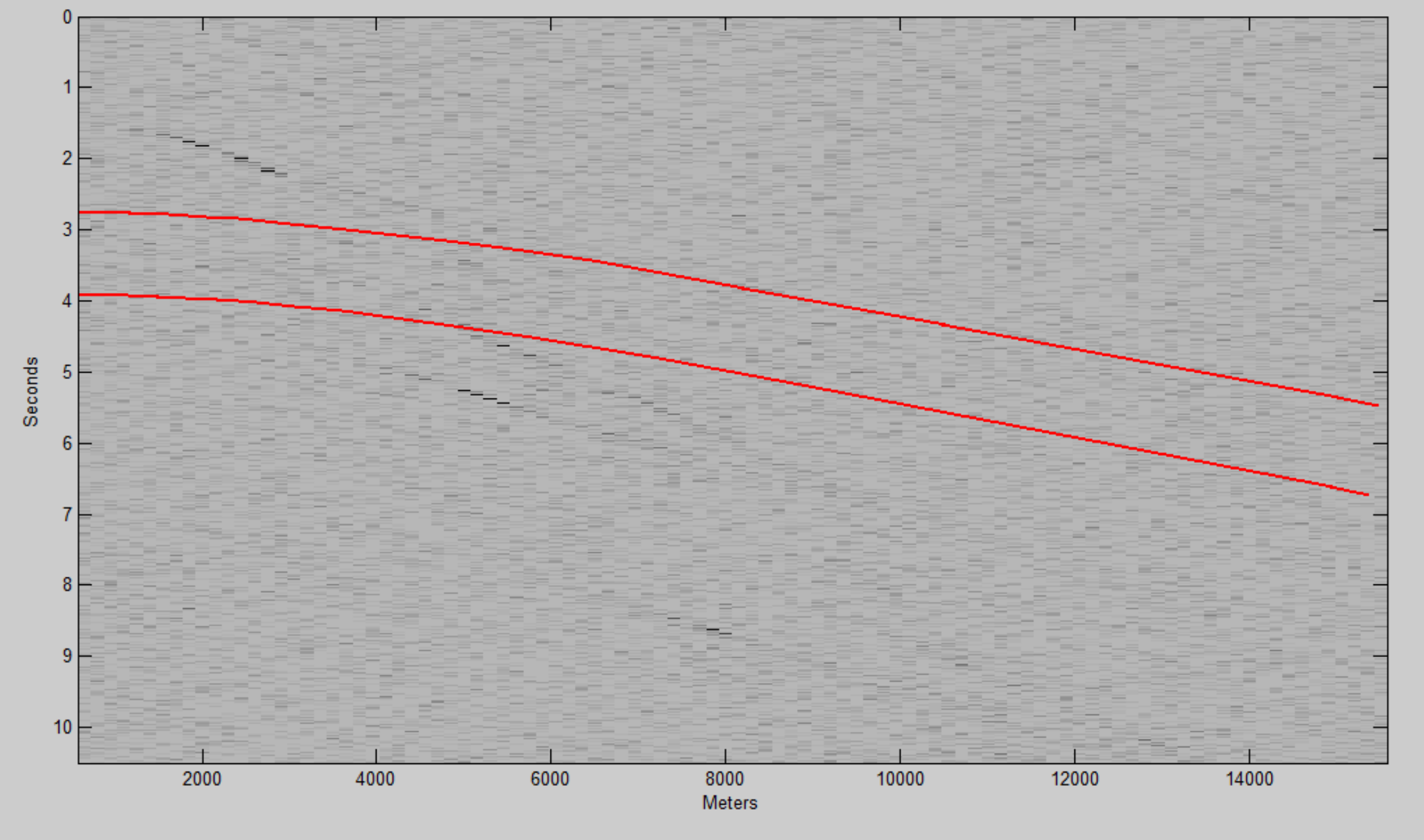

Figure 276: Seismogram of the Model 2 with signal-noise ratio of 90\%. The red lines represent the automated picks for the PP and PSv events. 


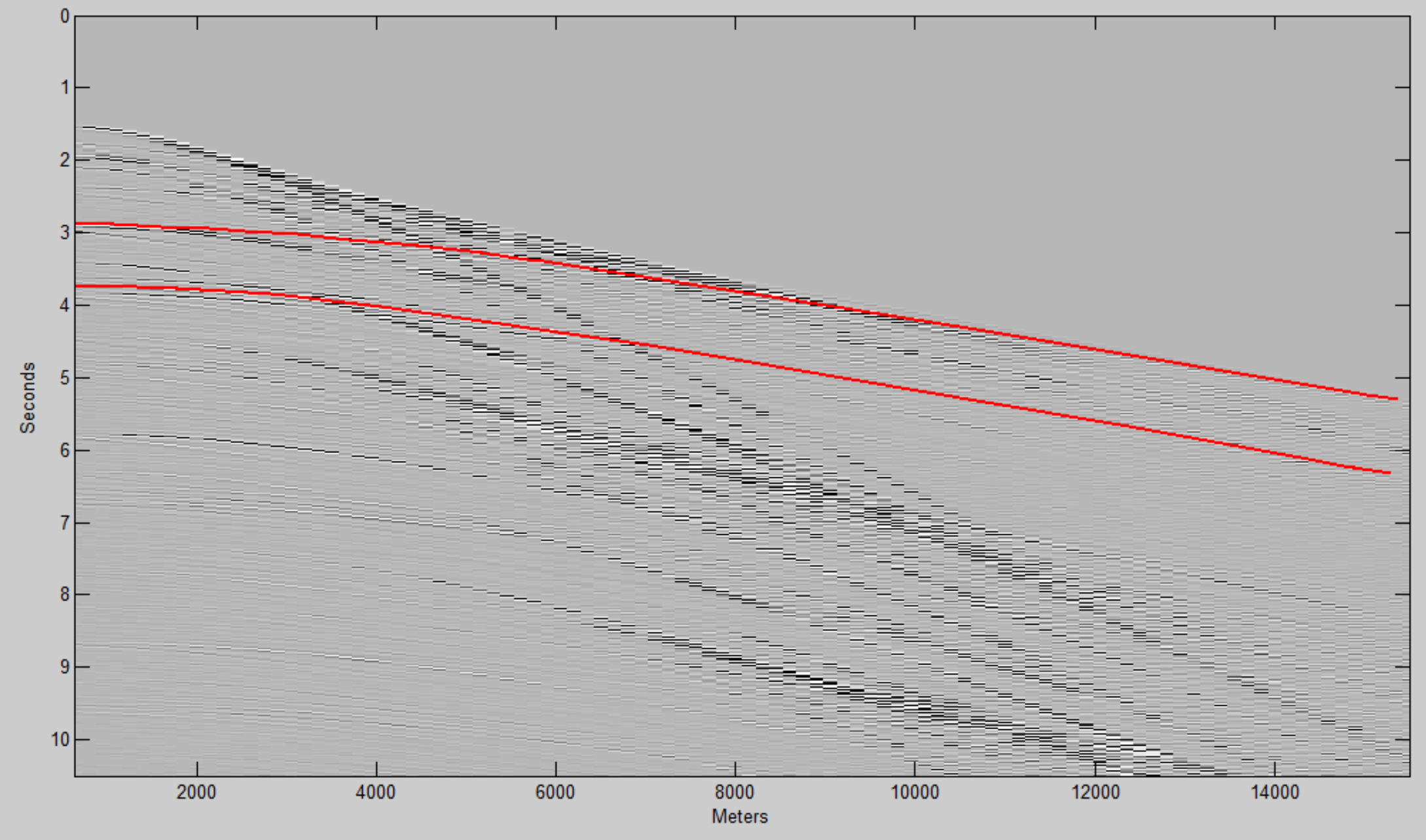

Figure 277: Seismogram of the Model 3 with no noise. The red lines represent the automated picks for the PP and PSv events. 


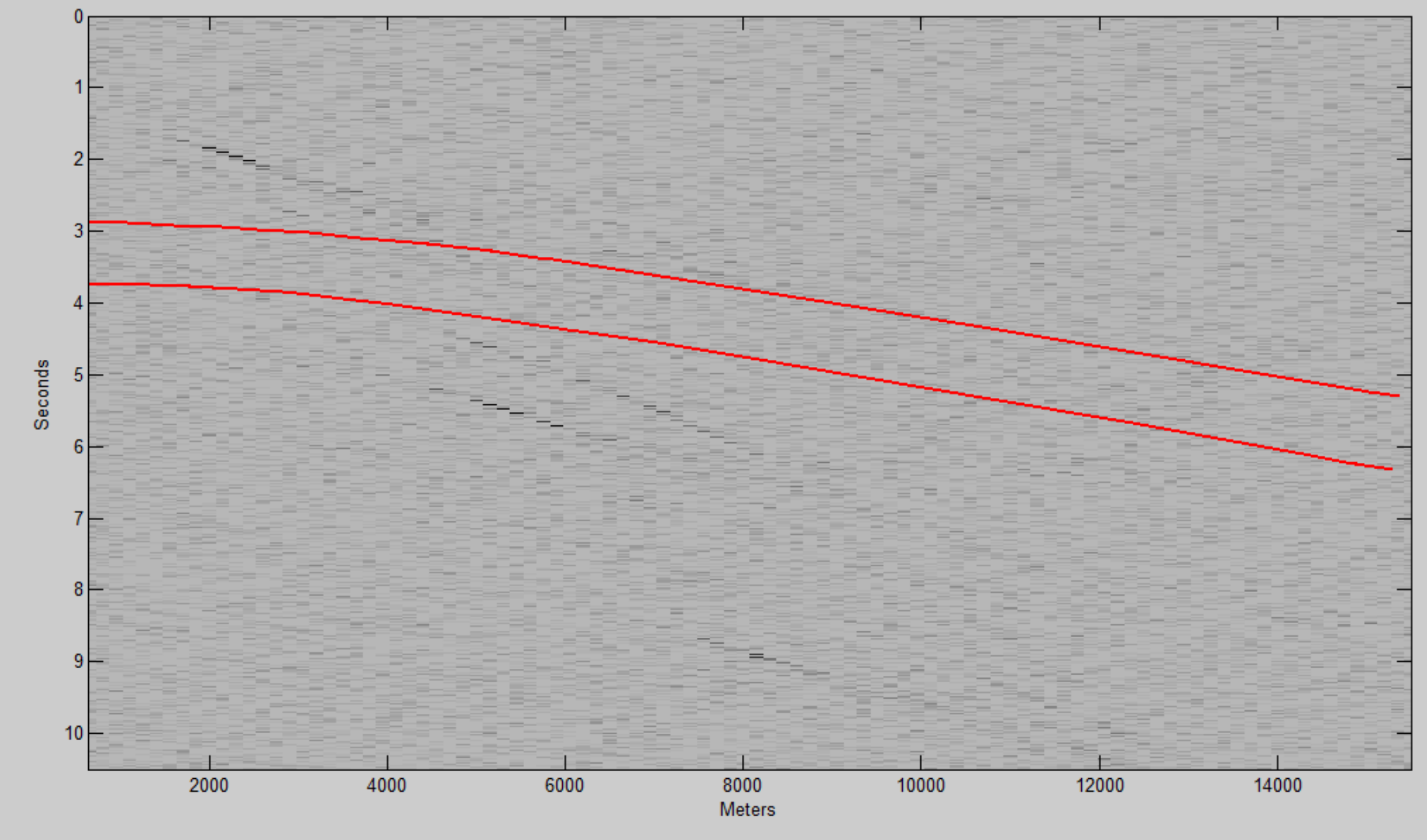

Figure 278: Seismogram of the Model 3 with signal-noise ratio of 90\%. The red lines represent the automated picks for the PP and PSv events. 


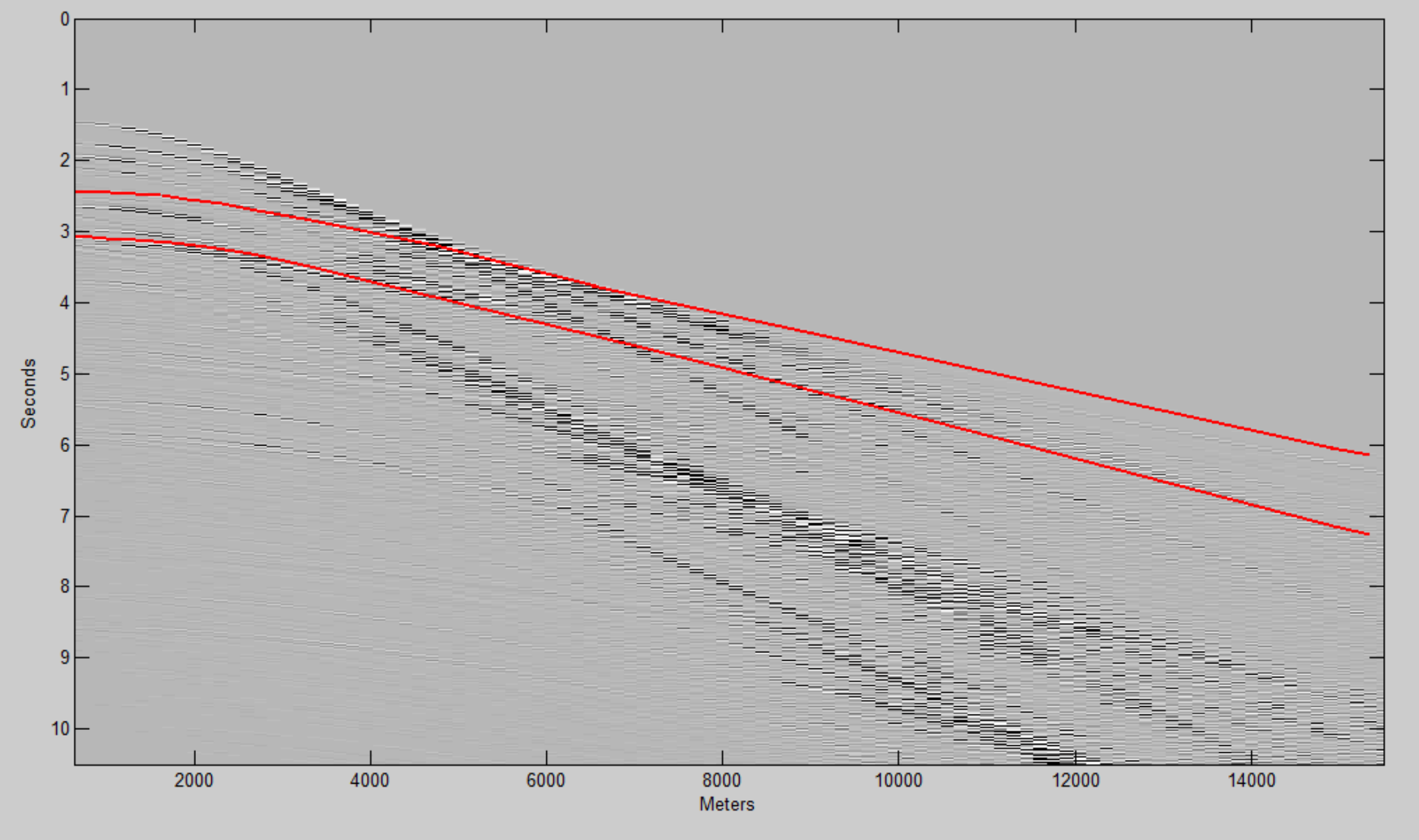

Figure 279: Seismogram of the Model 4 with no noise. The red lines represent the automated picks for the PP and PSv events. 


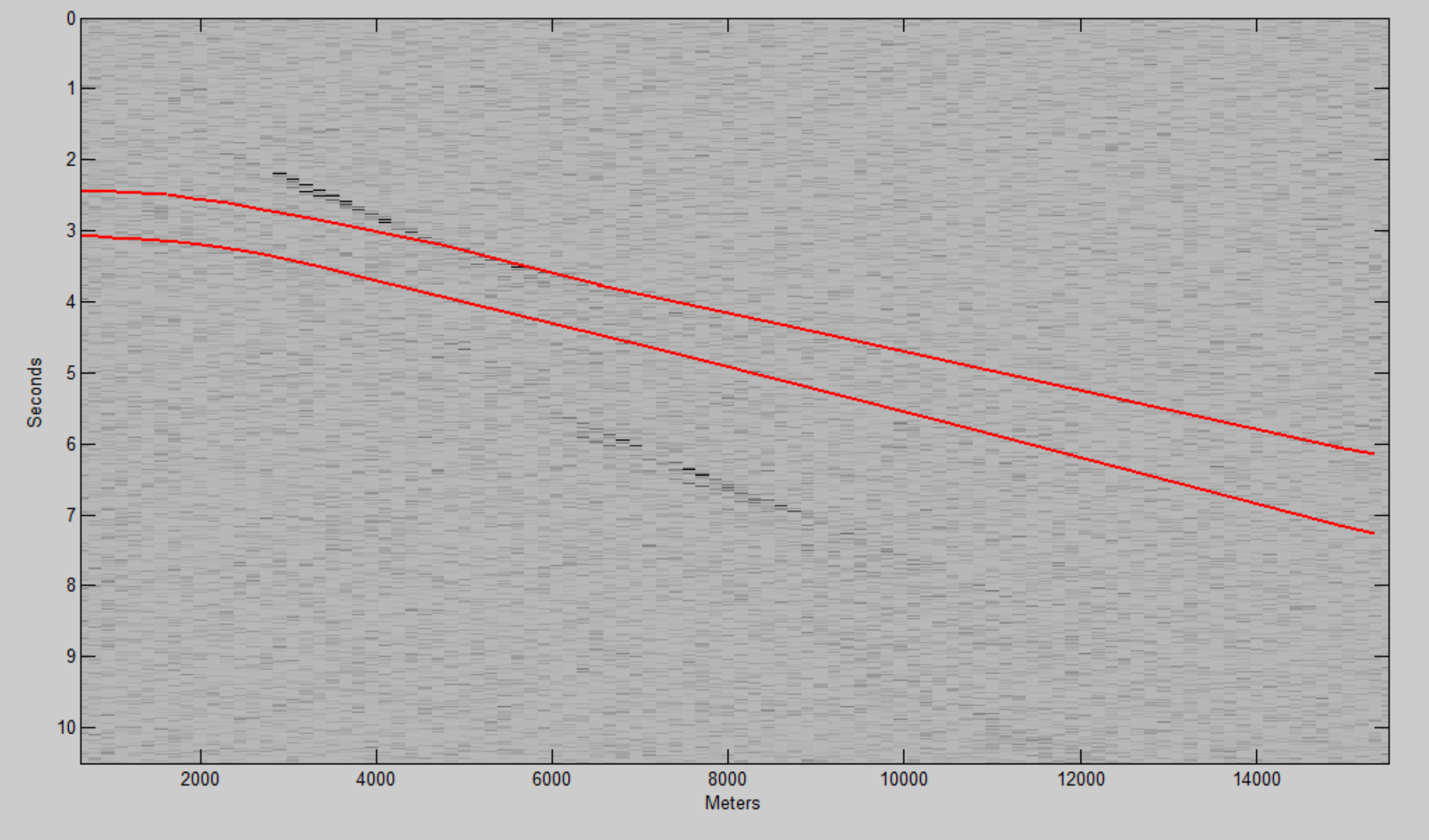

Figure 280: Seismogram of the Model 4 with no noise with signal-noise ratio of $90 \%$. The red lines represent the automated picks for the PP and PSv events. 


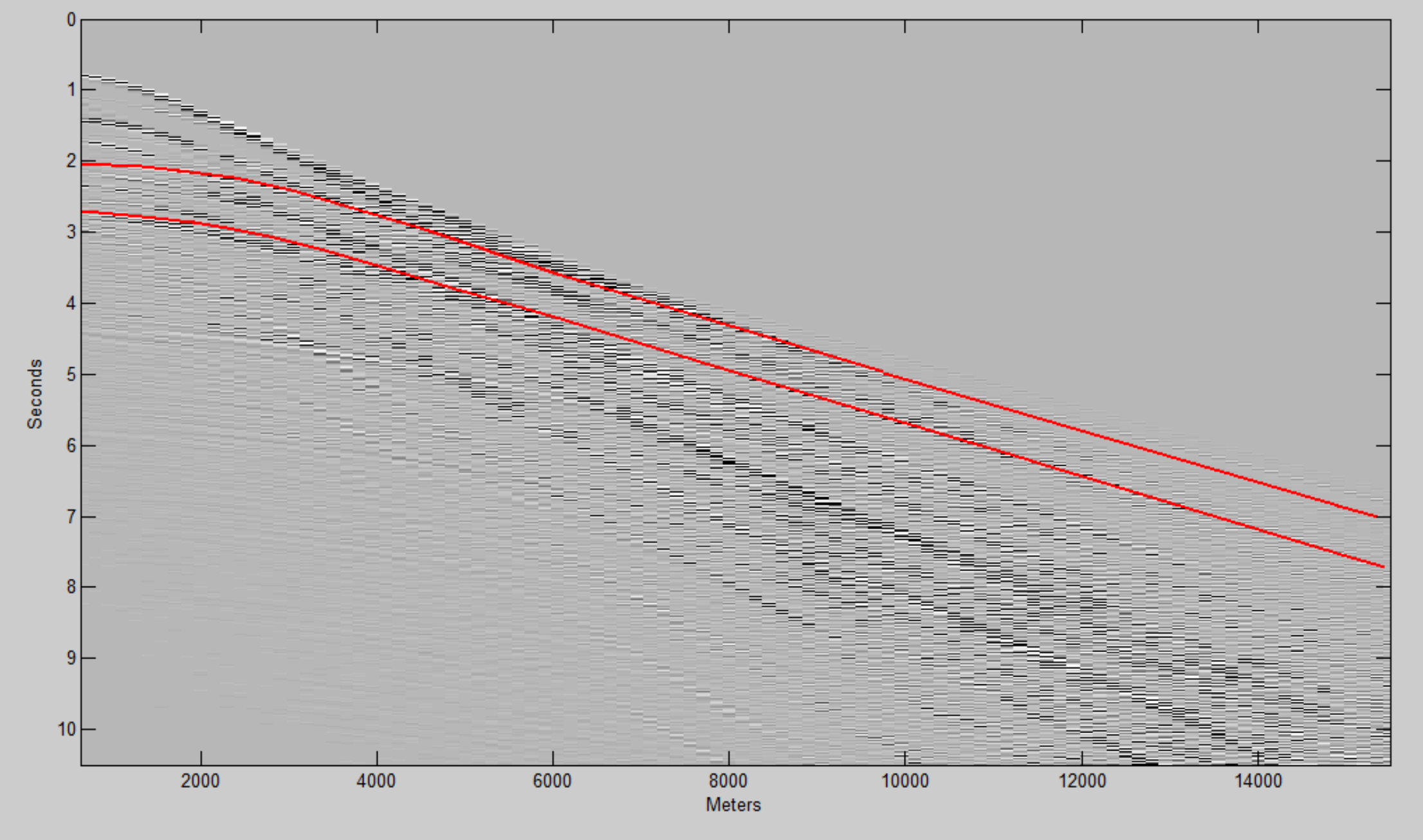

Figure 281: Seismogram of the Model 5 with no noise. The red lines represent the automated picks for the PP and PSv events. 


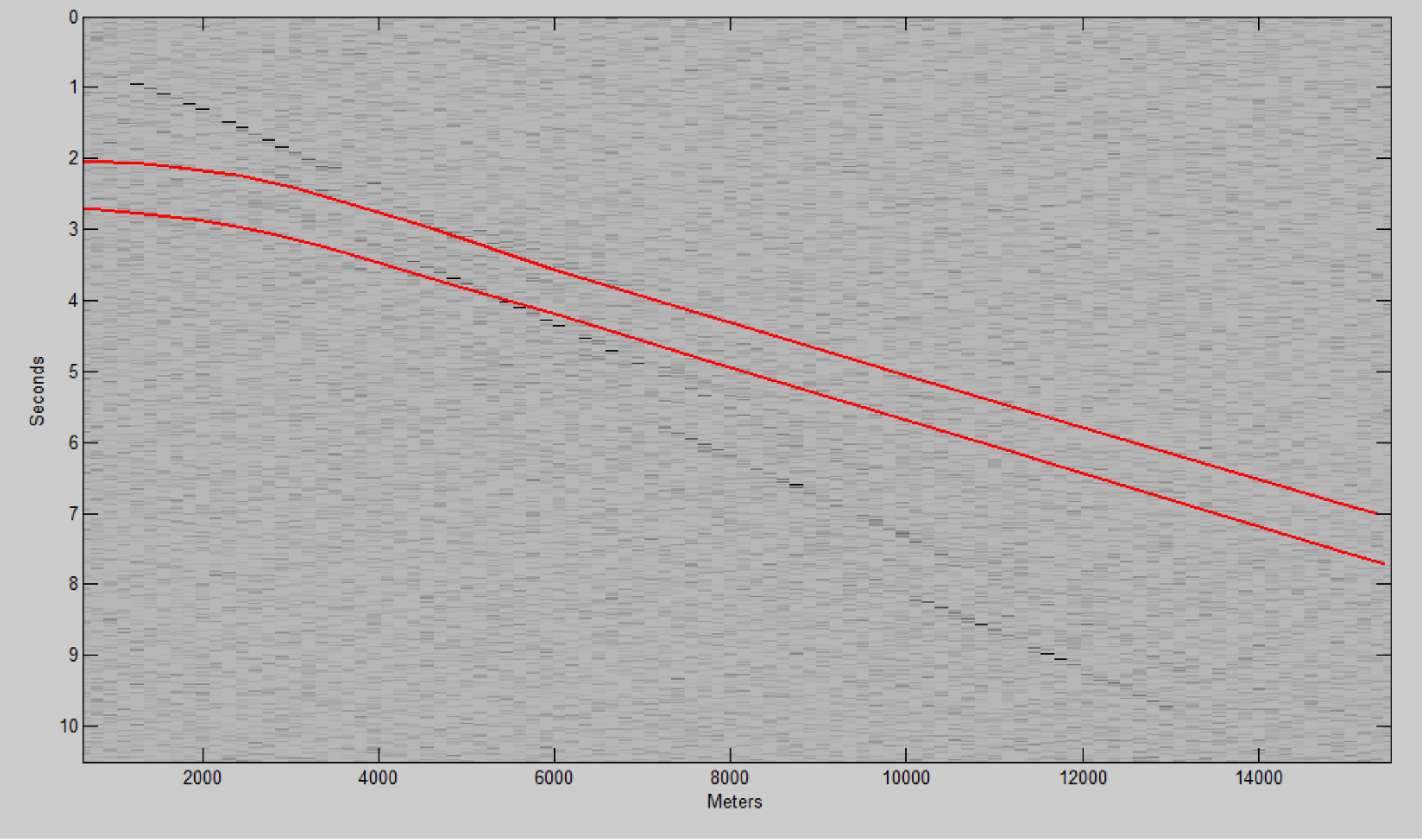

Figure 282: Seismogram of the Model 5 with signal-noise ratio of $90 \%$. The red lines represent the automated picks for the PP and PSv events. 


\subsection{Appendix B - PVO analysis of SvSv reflection events}
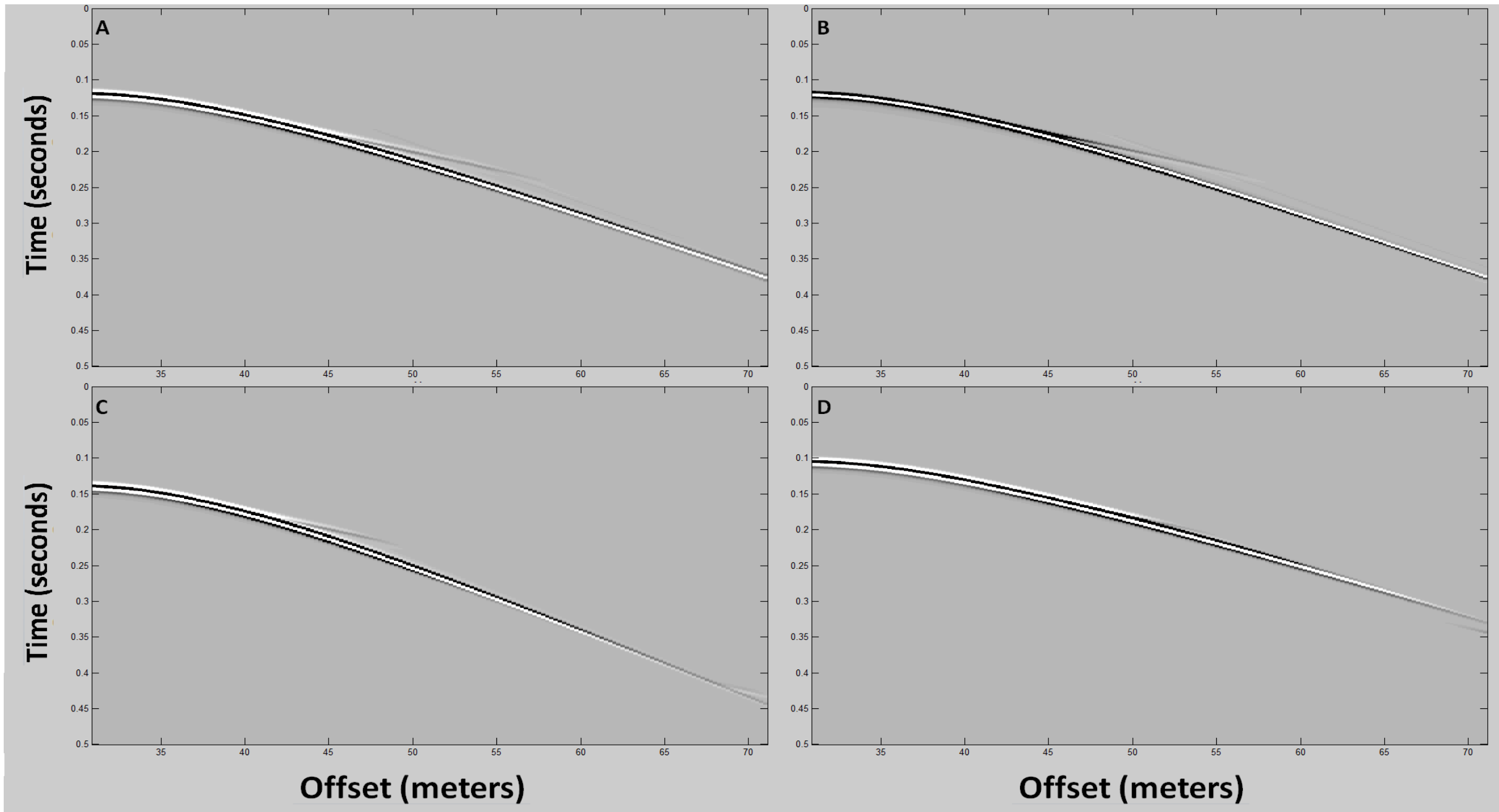

Figure 283: Seismograms of the four models used to perform the tests of PVO analysis. (A) Critical angle of around 37 degrees, (B) same critical angle, but with semiGaussian wavelet, (C) critical angle of around 30 degrees and (D) critical angle of around 44 degrees. 
A
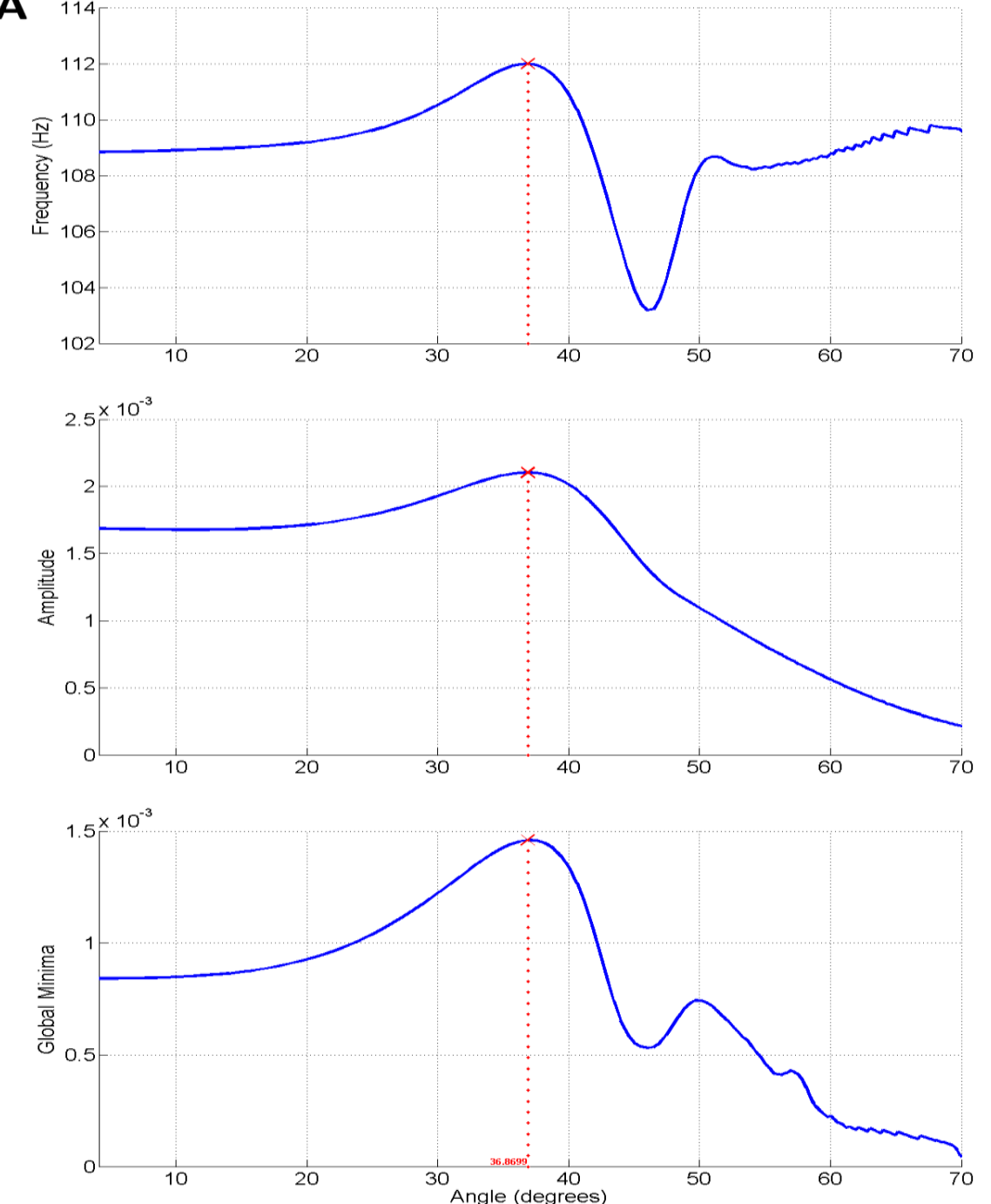

B
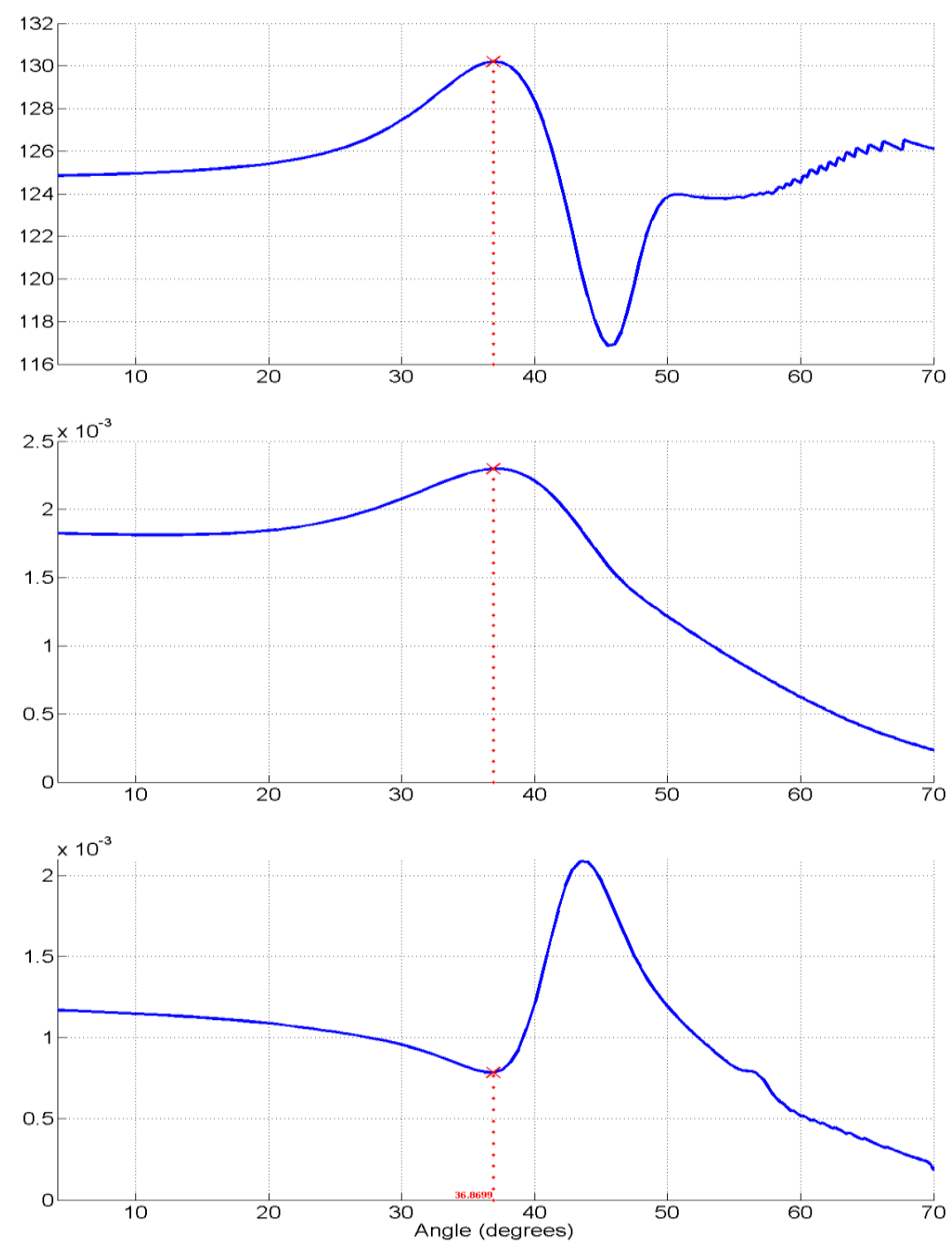

Figure 284: The variation for each trace/angle shown versus the global minimum, amplitude and peak frequency (A) of the first and (B) of the second models from the PVO analysis. 
A
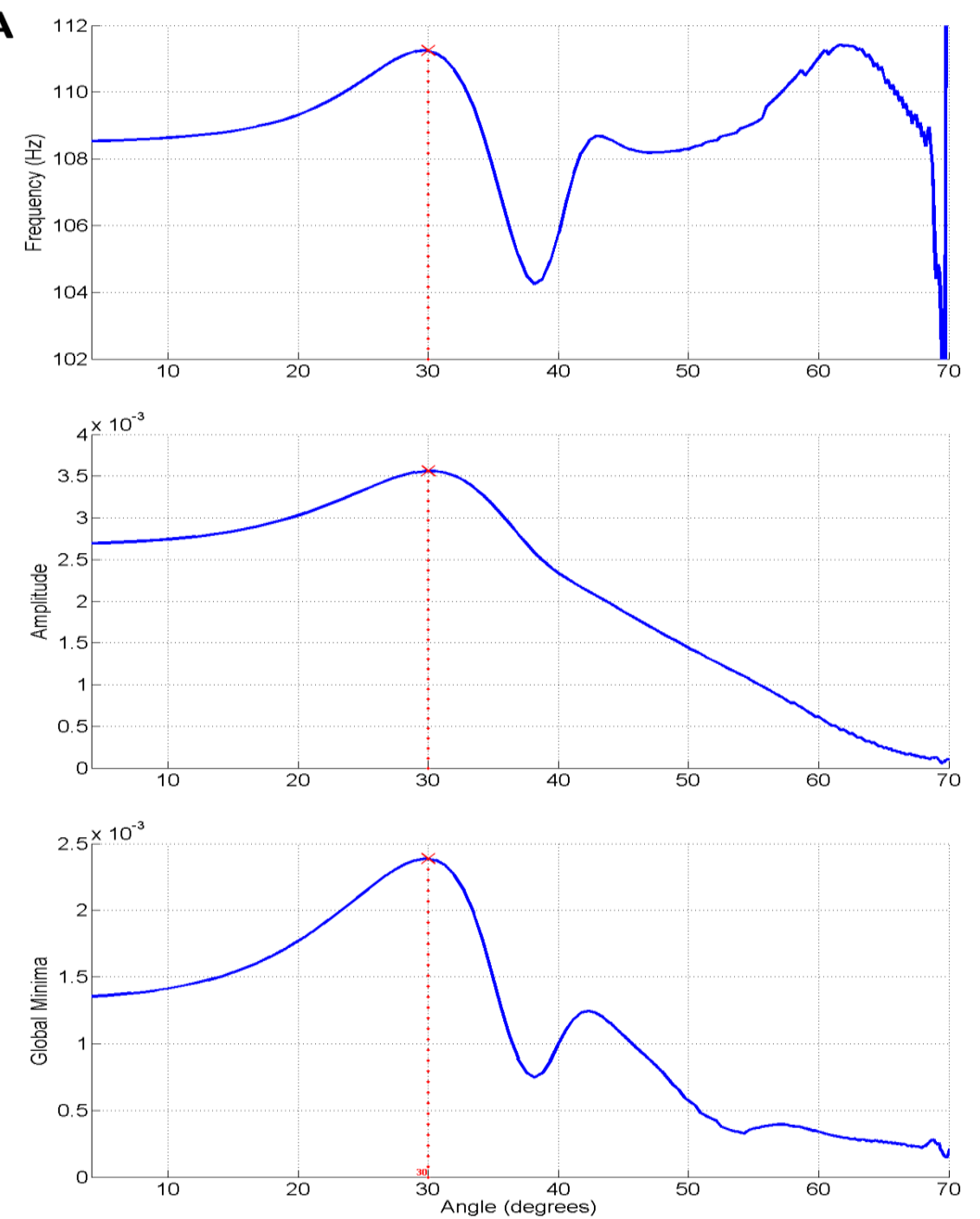

B
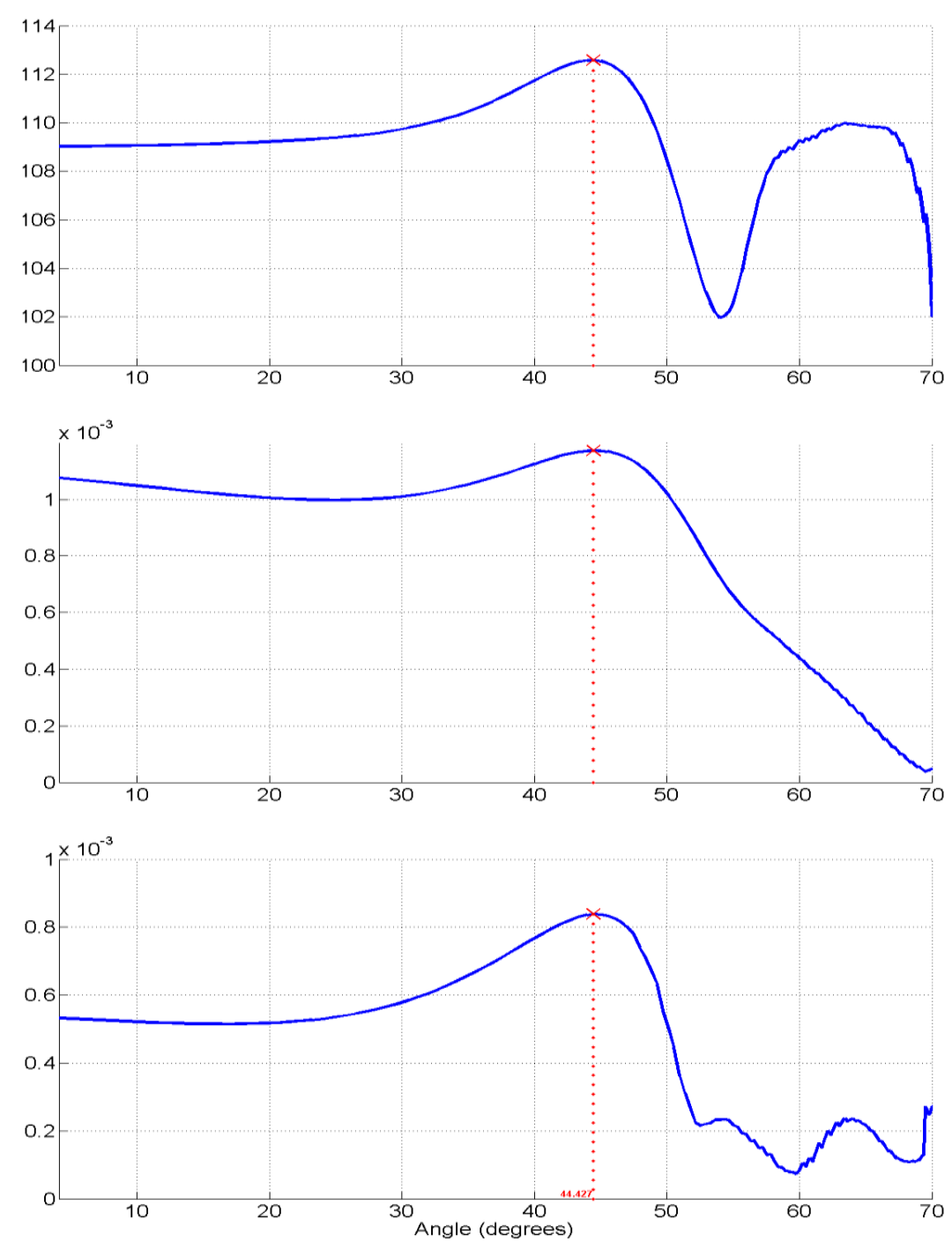

Figure 285: The variation for each trace/angle shown versus the global minimum, amplitude and peak frequency (A) of the third and (B) of the fourth models from the PVO analysis. 


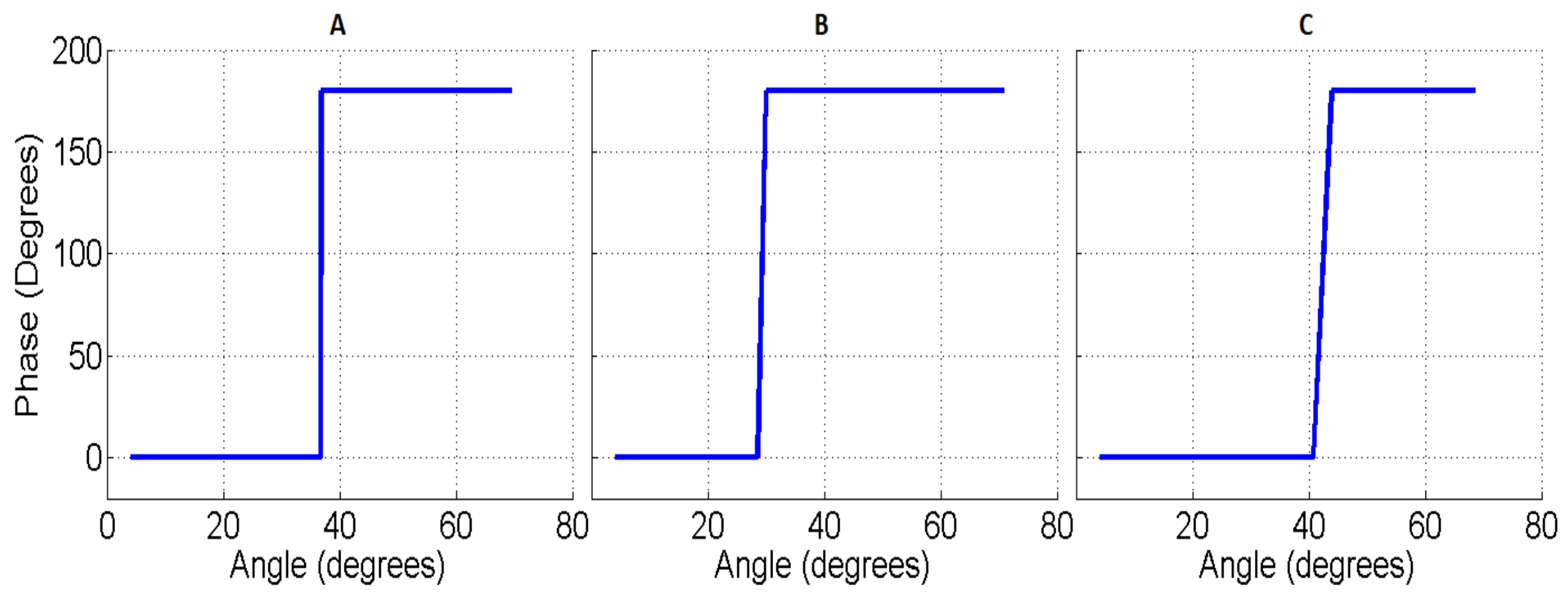

Figure 286: The analytically calculated instantaneous phase versus the incident angle of (A) the first and second models, (B) third model and (C) the fourth model. 

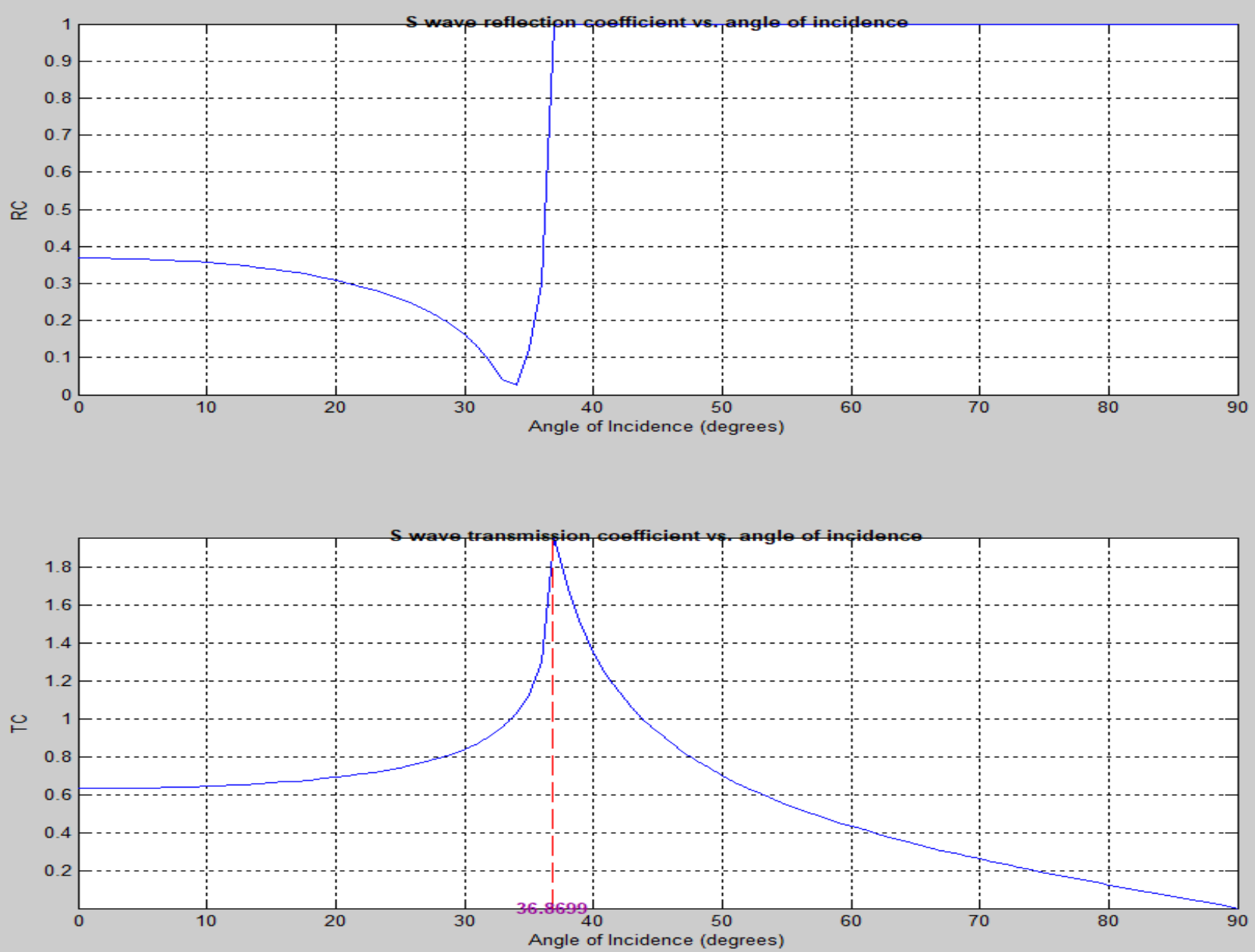

Figure 287: The reflection coefficient and the transmission coefficient versus the incidence angle. The red dashed line represents the critical angle of the first and second models. 

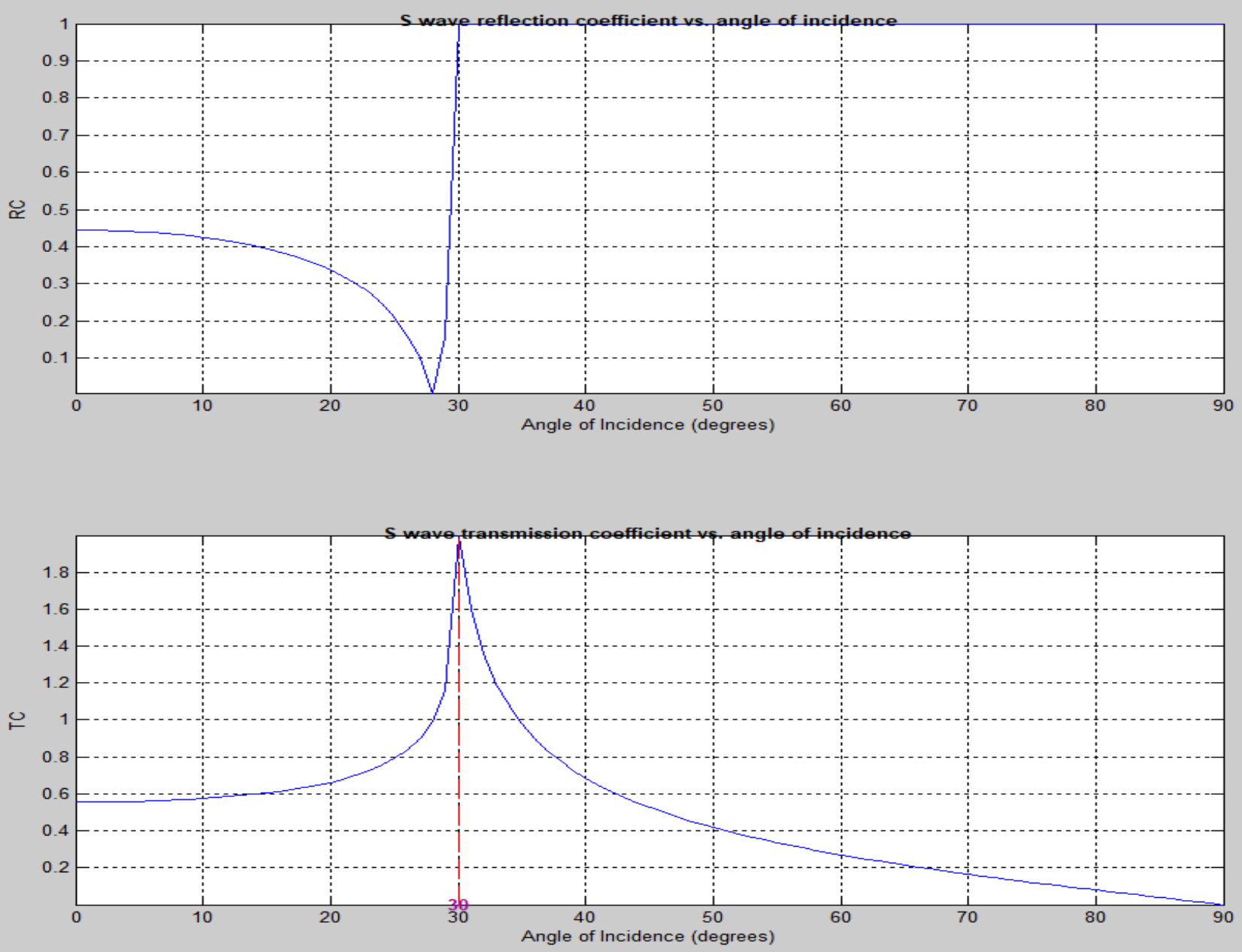

Figure 288: The reflection coefficient and the transmission coefficient versus the incidence angle. The red dashed line represents the critical angle of the third model. 

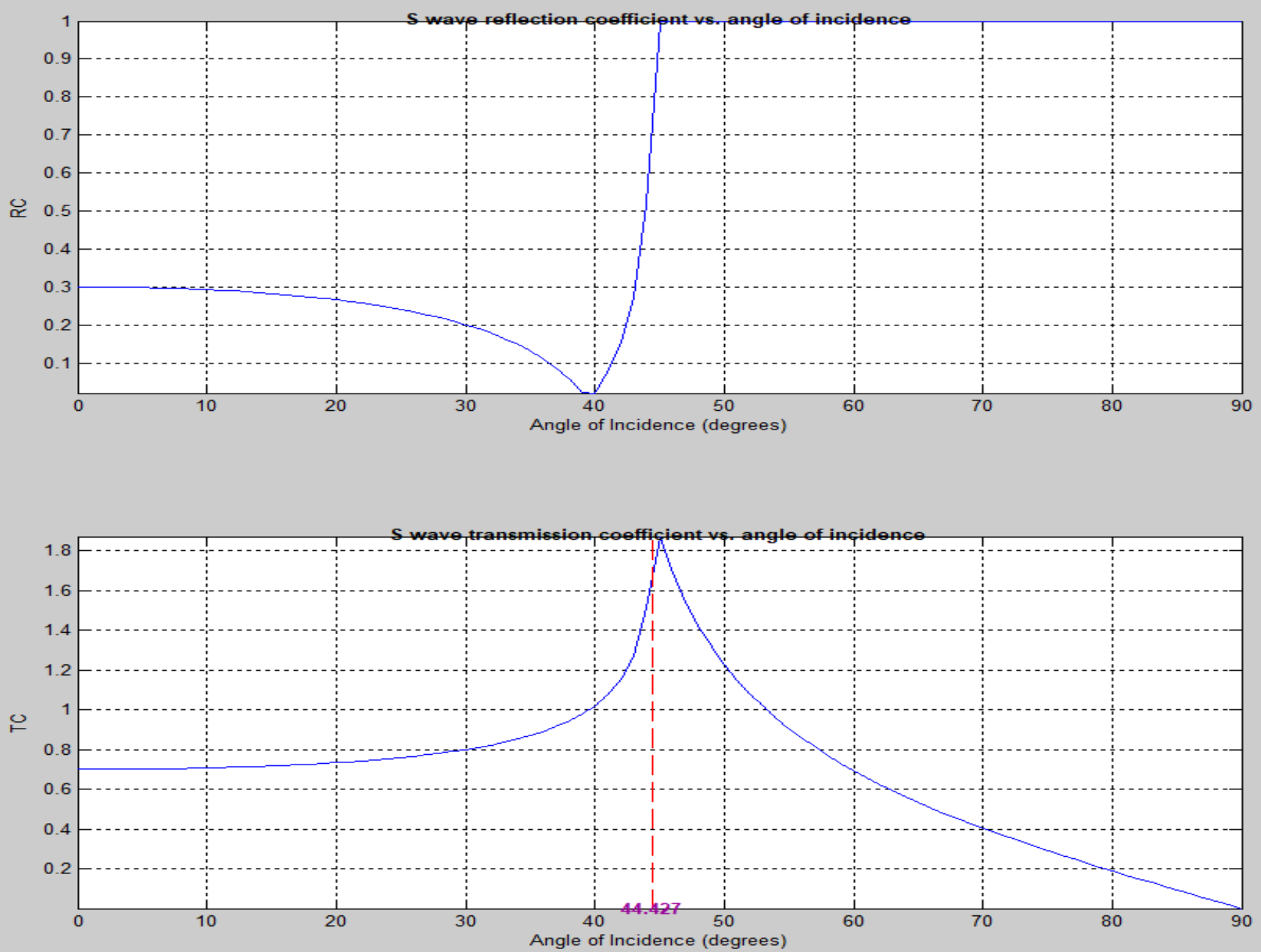

Figure 289: The reflection coefficient and the transmission coefficient versus the incidence angle. The red dashed line represents the critical angle of the fourth model. 


\subsection{Appendix C - Processing times}

Table 1: Processing time (in seconds) of each inversion routine of the Model 1.

\begin{tabular}{|c|c|c|c|c|c|c|c|c|c|c|c|c|c|c|c|c|c|c|c|c|}
\hline \multirow{3}{*}{ EQUATIONS } & \multicolumn{4}{|c|}{ IMFIL } & \multicolumn{4}{|c|}{ FMINSEARCH } & \multicolumn{4}{|c|}{ SID-PSM } & \multicolumn{4}{|c|}{ MCS } & \multicolumn{4}{|c|}{ TOMLAB/LGO } \\
\hline & \multicolumn{2}{|c|}{ L2-norm } & \multicolumn{2}{|c|}{ L1-norm } & \multicolumn{2}{|c|}{ L2-norm } & \multicolumn{2}{|c|}{ L1-norm } & \multicolumn{2}{|c|}{ L2-norm } & \multicolumn{2}{|c|}{ L1-norm } & \multicolumn{2}{|c|}{ L2-norm } & \multicolumn{2}{|c|}{ L1-norm } & \multicolumn{2}{|c|}{ L2-norm } & \multicolumn{2}{|c|}{ L1-norm } \\
\hline & PP & PS & PP & PS & PP & PS & PP & PS & PP & PS & PP & PS & PP & PS & PP & PS & PP & PS & PP & PS \\
\hline Dix & 34.10 & 37.51 & 30.72 & 33.79 & 38.19 & 42.01 & 34.41 & 37.85 & 69.22 & 76.15 & 62.36 & 68.60 & 105.4 & 115.9 & 94.93 & 104.4 & 174.3 & 191.7 & 157.0 & 172.7 \\
\hline Malovichko & 71.12 & 74.18 & 62.39 & 65.07 & 79.65 & 83.08 & 69.87 & 72.88 & 144.4 & 150.6 & 126.6 & 132.1 & 219.8 & 229.2 & 192.8 & 201.1 & 363.4 & 379.1 & 318.8 & 332.5 \\
\hline Alkhalifah and Tsvankin & 75.50 & 84.47 & 67.41 & 75.42 & 84.56 & 94.61 & 75.50 & 84.47 & 153.3 & 171.5 & 136.8 & 153.1 & 233.3 & 261.0 & 208.3 & 233.1 & 385.8 & 431.6 & 344.5 & 385.4 \\
\hline Ursin and Stovas & 79.81 & 86.50 & 68.80 & 74.57 & 89.39 & 96.88 & 77.06 & 83.52 & 162.0 & 175.6 & 139.7 & 151.4 & 246.6 & 267.3 & 212.6 & 230.4 & 407.8 & 442.0 & 351.6 & 381.1 \\
\hline Blias & 68.69 & 79.37 & 59.22 & 68.42 & 76.93 & 88.89 & 66.32 & 76.63 & 139.4 & 161.1 & 120.2 & 138.9 & 212.3 & 245.3 & 183.0 & 211.4 & 351.0 & 405,6 & 302.6 & 349.6 \\
\hline Muir and Dellinger & 76.94 & 89.19 & 68.09 & 78.93 & 86.17 & 99.89 & 76.26 & 88.40 & 156.2 & 181.1 & 138.2 & 160.2 & 237.8 & 275.6 & 210.4 & 243.9 & 393.2 & 455.8 & 347.9 & 403.3 \\
\hline Li and Yuan & 61.79 & 72.29 & 51.49 & 60.24 & 69.20 & 80.96 & 57.67 & 67.47 & 125.4 & 145.8 & 104.5 & 122.3 & 190.9 & 223.4 & 159.1 & 186.2 & 315.8 & 369.4 & 263.1 & 307.8 \\
\hline Proposed approximation & 67.55 & 74.99 & 56.29 & 62.49 & 75.66 & 83.99 & 63.05 & 69.99 & 137.1 & 152.2 & 114.3 & 126.9 & 208.7 & 231.7 & 173.9 & 193.1 & 345.2 & 383.2 & 287.7 & 319.3 \\
\hline
\end{tabular}


Table 2: Processing time (in seconds) of each inversion routine of the Model 2.

\begin{tabular}{|c|c|c|c|c|c|c|c|c|c|c|c|c|c|c|c|c|c|c|c|c|}
\hline \multirow{3}{*}{ EQUATIONS } & \multicolumn{4}{|c|}{ IMFIL } & \multicolumn{4}{|c|}{ FMINSEARCH } & \multicolumn{4}{|c|}{ SID-PSM } & \multicolumn{4}{|c|}{ MCS } & \multicolumn{4}{|c|}{ TOMLAB/LGO } \\
\hline & \multicolumn{2}{|c|}{ L2-norm } & \multicolumn{2}{|c|}{ L1-norm } & \multicolumn{2}{|c|}{ L2-norm } & \multicolumn{2}{|c|}{ L1-norm } & \multicolumn{2}{|c|}{ L2-norm } & \multicolumn{2}{|c|}{ L1-norm } & \multicolumn{2}{|c|}{ L2-norm } & \multicolumn{2}{|c|}{ L1-norm } & \multicolumn{2}{|c|}{ L2-norm } & \multicolumn{2}{|c|}{ L1-norm } \\
\hline & $\mathrm{PP}$ & PS & PP & PS & $\mathrm{PP}$ & PS & PP & PS & $\mathrm{PP}$ & PS & PP & PS & PP & PS & $\mathrm{PP}$ & PS & $\mathrm{PP}$ & PS & PP & PS \\
\hline Dix & 34.82 & 38.30 & 31.37 & 34.50 & 38.99 & 42.89 & 35.13 & 38.64 & 70.68 & 77.74 & 63.67 & 70.04 & 107.6 & 118.3 & 96.92 & 106.6 & 177.9 & 195.7 & 160.3 & 176.3 \\
\hline Malovichko & 72.61 & 75.74 & 63.70 & 66.44 & 81.33 & 84.83 & 71.34 & 74.41 & 147.4 & 153.8 & 129.3 & 134.9 & 224.4 & 234.0 & 196.8 & 205.3 & 371.1 & 387.0 & 325.5 & 339.5 \\
\hline Alkhalifah and Tsvankin & 77.09 & 86.24 & 68.83 & 77.00 & 86.34 & 96.59 & 77.09 & 86.24 & 156.5 & 175.1 & 139.7 & 156.3 & 238.2 & 266.5 & 212.7 & 237.9 & 393.9 & 440.7 & 351.7 & 393.5 \\
\hline Ursin and Stovas & 81.49 & 88.32 & 70.25 & 76.13 & 91.26 & 98.91 & 78.68 & 85.27 & 165.4 & 179.3 & 142.6 & 154.6 & 251.8 & 272.9 & 217.1 & 235.3 & 416.4 & 451.3 & 359.0 & 389.1 \\
\hline Blias & 70.13 & 81.04 & 60.46 & 69.86 & 78.55 & 90.76 & 67.71 & 78.24 & 142.4 & 164.5 & 122.7 & 141.8 & 216.7 & 250.4 & 186.8 & 215.9 & 358.4 & 414.1 & 309.0 & 357.0 \\
\hline Muir and Dellinger & 78.56 & 91.06 & 69.52 & 80.59 & 87.98 & 102.0 & 77.86 & 90.26 & 159.5 & 184.9 & 141.1 & 163.6 & 242.7 & 281.4 & 241.8 & 249.0 & 401.4 & 465.3 & 355.2 & 411.8 \\
\hline Li and Yuan & 63.09 & 73.81 & 52.57 & 61.51 & 70.66 & 82.67 & 58.88 & 68.89 & 128.1 & 149.8 & 106.7 & 124.9 & 194.9 & 228.1 & 162.5 & 190.1 & 322.4 & 377.2 & 268.7 & 314.3 \\
\hline Proposed approximation & 68.97 & 76.56 & 57.47 & 63.80 & 77.24 & 85.75 & 64.37 & 71.46 & 140.0 & 155.4 & 116.7 & 129.5 & 213.1 & 236.6 & 177.6 & 197.2 & 352.4 & 391.3 & 293.7 & 326.0 \\
\hline
\end{tabular}


Table 3: Processing time (in seconds) of each inversion routine of the Model 3.

\begin{tabular}{|c|c|c|c|c|c|c|c|c|c|c|c|c|c|c|c|c|c|c|c|c|}
\hline \multirow{3}{*}{ EQUATIONS } & \multicolumn{4}{|c|}{ IMFIL } & \multicolumn{4}{|c|}{ FMINSEARCH } & \multicolumn{4}{|c|}{ SID-PSM } & \multicolumn{4}{|c|}{ MCS } & \multicolumn{4}{|c|}{ TOMLAB/LGO } \\
\hline & \multicolumn{2}{|c|}{ L2-norm } & \multicolumn{2}{|c|}{ L1-norm } & \multicolumn{2}{|c|}{ L2-norm } & \multicolumn{2}{|c|}{ L1-norm } & \multicolumn{2}{|c|}{ L2-norm } & \multicolumn{2}{|c|}{ L1-norm } & \multicolumn{2}{|c|}{ L2-norm } & \multicolumn{2}{|c|}{ L1-norm } & \multicolumn{2}{|c|}{ L2-norm } & \multicolumn{2}{|c|}{ L1-norm } \\
\hline & $\mathrm{PP}$ & PS & PP & PS & $\mathrm{PP}$ & PS & PP & PS & $\mathrm{PP}$ & PS & PP & PS & PP & PS & $\mathrm{PP}$ & PS & $\mathrm{PP}$ & PS & PP & PS \\
\hline Dix & 35.98 & 39.57 & 32.41 & 35.65 & 40.29 & 44.32 & 36.30 & 39.93 & 73.03 & 80.33 & 65.79 & 72.37 & 110.2 & 122.3 & 100.2 & 110.2 & 183.8 & 202.2 & 165.6 & 182.2 \\
\hline Malovichko & 75.03 & 78.26 & 65.82 & 68.65 & 84.04 & 87.65 & 73.72 & 76.89 & 152.3 & 158.9 & 113.6 & 139.4 & 231.9 & 241.8 & 203.4 & 212.1 & 383.4 & 399.9 & 336.3 & 350.8 \\
\hline Alkhalifah and Tsvankin & 79.65 & 89.12 & 71.12 & 79.57 & 89.21 & 99.81 & 79.65 & 89.12 & 161.7 & 180.9 & 144.4 & 161.5 & 246.1 & 275.4 & 219.8 & 245.9 & 407.0 & 455.4 & 363.4 & 406.6 \\
\hline Ursin and Stovas & 84.20 & 91.26 & 72.59 & 78.67 & 94.30 & 102.2 & 81.30 & 88.11 & 170.9 & 185.3 & 147.4 & 159.7 & 260.2 & 282.9 & 224.3 & 243.1 & 430.3 & 466.3 & 370.9 & 402.0 \\
\hline Blias & 72.47 & 83.74 & 62.47 & 72.19 & 81.16 & 93.78 & 69.97 & 80.85 & 147.1 & 170.0 & 126.8 & 146.5 & 223.9 & 258.7 & 193.0 & 223.1 & 370.3 & 427.9 & 319.2 & 368.9 \\
\hline Muir and Dellinger & 81.17 & 94.10 & 71.83 & 83.29 & 90.91 & 105.4 & 80.45 & 93.26 & 164.8 & 191.0 & 145.8 & 169.0 & 250.8 & 290.8 & 221.9 & 257.3 & 414.8 & 480.8 & 367.1 & 425.5 \\
\hline Li and Yuan & 65.19 & 76.27 & 54.32 & 63.55 & 73.01 & 85.42 & 60.84 & 71.18 & 132.3 & 154.8 & 110.3 & 129.0 & 201.4 & 235.7 & 167.9 & 196.4 & 333.1 & 389.7 & 277.6 & 324.8 \\
\hline Proposed approximation & 71.27 & 79.11 & 59.39 & 65.93 & 79.82 & 88.61 & 66.51 & 73.84 & 144.7 & 160.6 & 120.6 & 133.8 & 220.2 & 244.5 & 183.5 & 203.7 & 364.2 & 404.3 & 303.5 & 336.9 \\
\hline
\end{tabular}


Table 4: Processing time (in seconds) of each inversion routine of the Model 4.

\begin{tabular}{|c|c|c|c|c|c|c|c|c|c|c|c|c|c|c|c|c|c|c|c|c|}
\hline \multirow{3}{*}{ EQUATIONS } & \multicolumn{4}{|c|}{ IMFIL } & \multicolumn{4}{|c|}{ FMINSEARCH } & \multicolumn{4}{|c|}{ SID-PSM } & \multicolumn{4}{|c|}{ MCS } & \multicolumn{4}{|c|}{ TOMLAB/LGO } \\
\hline & \multicolumn{2}{|c|}{ L2-norm } & \multicolumn{2}{|c|}{ L1-norm } & \multicolumn{2}{|c|}{ L2-norm } & \multicolumn{2}{|c|}{ L1-norm } & \multicolumn{2}{|c|}{ L2-norm } & \multicolumn{2}{|c|}{ L1-norm } & \multicolumn{2}{|c|}{ L2-norm } & \multicolumn{2}{|c|}{ L1-norm } & \multicolumn{2}{|c|}{ L2-norm } & \multicolumn{2}{|c|}{ L1-norm } \\
\hline & PP & PS & PP & PS & PP & PS & PP & PS & PP & PS & PP & PS & PP & PS & PP & PS & PP & PS & PP & PS \\
\hline Dix & 35.63 & 39.20 & 32.10 & 35.31 & 39.91 & 43.90 & 35.96 & 39.55 & 72.34 & 79.57 & 65.17 & 71.69 & 110.1 & 121.1 & 99.20 & 109.1 & 182.1 & 200.3 & 164.1 & 180.5 \\
\hline Malovichko & 74.32 & 77.52 & 65.19 & 68.00 & 83.24 & 86.82 & 73.02 & 76.16 & 150.9 & 157.4 & 132.3 & 138.0 & 229.7 & 239.5 & 201.5 & 210.1 & 379.8 & 396.1 & 333.1 & 347.5 \\
\hline Alkhalifah and Tsvankin & 78.90 & 88.27 & 70.44 & 78.81 & 88.37 & 98.68 & 78.90 & 88.27 & 160.2 & 179.2 & 143.0 & 160.0 & 243.8 & 272.8 & 217.7 & 243.5 & 403.2 & 451.1 & 360.0 & 402.7 \\
\hline Ursin and Stovas & 83.40 & 90.39 & 71.90 & 77.92 & 93.41 & 101.2 & 80.53 & 87.28 & 169.3 & 183.5 & 146.0 & 158.2 & 257.7 & 279.3 & 222.2 & 240.8 & 426.2 & 461.9 & 367.4 & 398.2 \\
\hline Blias & 71.78 & 82.94 & 61.88 & 71.50 & 80.39 & 92.89 & 69.31 & 80.08 & 145.7 & 168.4 & 125.6 & 145.2 & 221.8 & 256.3 & 191.2 & 220.9 & 366.8 & 423.8 & 316.2 & 365.4 \\
\hline Muir and Dellinger & 80.40 & 93.20 & 71.15 & 82.48 & 90.05 & 140.4 & 79.69 & 92.38 & 163.2 & 189.2 & 144.4 & 167.4 & 248.4 & 288.0 & 219.9 & 254.9 & 410.9 & 476.3 & 363.6 & 421.5 \\
\hline $\mathrm{Li}$ and Yuan & 64.57 & 75.54 & 53.81 & 62.95 & 72.32 & 84.61 & 60.27 & 70.51 & 131.1 & 153.4 & 109.2 & 127.8 & 199.5 & 233.4 & 166.3 & 194.5 & 330.0 & 386.0 & 275.0 & 321.7 \\
\hline Proposed approximation & 70.59 & 78.36 & 58.82 & 65.30 & 79.06 & 87.77 & 65.88 & 73.14 & 143.3 & 159.1 & 119.4 & 132.6 & 218.1 & 242.2 & 181.8 & 201.8 & 360.7 & 400.4 & 300.6 & 333.7 \\
\hline
\end{tabular}


Table 5: Processing time (in seconds) of each inversion routine of the Model 5.

\begin{tabular}{|c|c|c|c|c|c|c|c|c|c|c|c|c|c|c|c|c|c|c|c|c|}
\hline \multirow{3}{*}{ EQUATIONS } & \multicolumn{4}{|c|}{ IMFIL } & \multicolumn{4}{|c|}{ FMINSEARCH } & \multicolumn{4}{|c|}{ SID-PSM } & \multicolumn{4}{|c|}{ MCS } & \multicolumn{4}{|c|}{ TOMLAB/LGO } \\
\hline & \multicolumn{2}{|c|}{ L2-norm } & \multicolumn{2}{|c|}{ L1-norm } & \multicolumn{2}{|c|}{ L2-norm } & \multicolumn{2}{|c|}{ L1-norm } & \multicolumn{2}{|c|}{ L2-norm } & \multicolumn{2}{|c|}{ L1-norm } & \multicolumn{2}{|c|}{ L2-norm } & \multicolumn{2}{|c|}{ L1-norm } & \multicolumn{2}{|c|}{ L2-norm } & \multicolumn{2}{|c|}{ L1-norm } \\
\hline & PP & PS & PP & PS & PP & PS & PP & PS & PP & PS & PP & PS & PP & PS & PP & PS & PP & PS & PP & PS \\
\hline Dix & 35.57 & 39.12 & 32.04 & 35.25 & 39.83 & 43.82 & 35.89 & 39.48 & 72.20 & 79.42 & 65.04 & 71.55 & 109.9 & 120.9 & 99.01 & 108.9 & 181.7 & 199.9 & 163.7 & 180.1 \\
\hline Malovichko & 74.18 & 77.37 & 65.07 & 67.87 & 83.08 & 86.65 & 72.88 & 76.01 & 150.6 & 157.1 & 132.1 & 137.8 & 229.2 & 239.1 & 201.1 & 209.7 & 379.1 & 395.4 & 332.5 & 346.8 \\
\hline Alkhalifah and Tsvankin & 78.75 & 88.10 & 70.31 & 78.66 & 88.20 & 98.67 & 78.75 & 88.10 & 159.9 & 178.9 & 142.7 & 159.7 & 243.3 & 227.2 & 217.3 & 243.1 & 402.4 & 450.2 & 359.3 & 402.0 \\
\hline Ursin and Stovas & 83.24 & 90.22 & 71.76 & 77.78 & 93.23 & 101.1 & 80.37 & 87.11 & 169.0 & 183.2 & 145.7 & 157.9 & 257.2 & 278.8 & 221.7 & 240.3 & 425.4 & 461.0 & 366.7 & 397.4 \\
\hline Blias & 71.64 & 82.78 & 61.76 & 71.36 & 80.24 & 92.72 & 69.17 & 79.93 & 145.4 & 168.1 & 125.4 & 144.9 & 221.4 & 255.8 & 190.8 & 220.5 & 366.1 & 423.0 & 315.6 & 364.7 \\
\hline Muir and Dellinger & 80.25 & 93.03 & 71.02 & 82.32 & 89.88 & 104.2 & 79.54 & 92.20 & 162.9 & 188.8 & 144.2 & 167.1 & 248.0 & 287.5 & 219.4 & 254.4 & 410.1 & 475.4 & 362.9 & 420.7 \\
\hline $\mathrm{Li}$ and Yuan & 64.45 & 75.40 & 53.71 & 62.83 & 72.18 & 84.45 & 60.15 & 70.37 & 130.8 & 153.1 & 109.0 & 127.6 & 199.1 & 233.0 & 166.0 & 194.2 & 329.3 & 385.3 & 274.4 & 321.1 \\
\hline Proposed approximation & 70.45 & 78.21 & 58.71 & 65.18 & 78.91 & 87.60 & 65.76 & 73.00 & 143.0 & 158.8 & 119.2 & 132.3 & 217.7 & 241.7 & 181.4 & 201.4 & 360.0 & 399.7 & 300.0 & 333.1 \\
\hline
\end{tabular}

